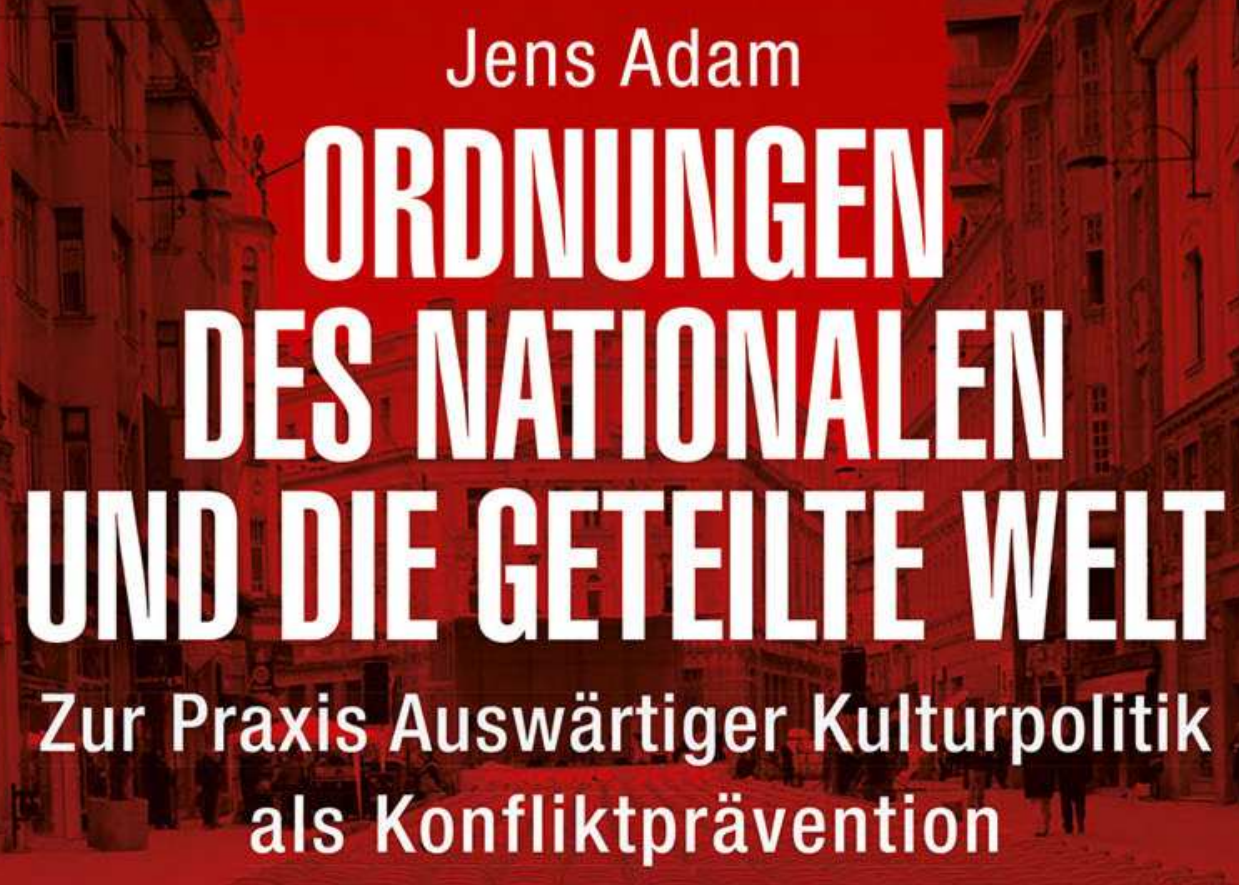

[transcript] Edition P olitik 
Jens Adam

Ordnungen des Nationalen und die geteilte Welt

Edition Politik | Band 6o 
Jens Adam (Dr. phil.) ist wissenschaftlicher Mitarbeiter am Institut für Europäische Ethnologie der Humboldt-Universität zu Berlin. Seine Arbeitsschwerpunkte liegen in den Bereichen der Anthropologie des Politischen, der Stadtanthropologie sowie der Europäisierungs- und Kosmopolitisierungsforschung. 
JENS ADAM

\section{Ordnungen des Nationalen und die geteilte Welt}

Zur Praxis Auswärtiger Kulturpolitik als Konfliktprävention 
Diese Studie entstand als Dissertation an der Philosophischen Fakultät der Humboldt-Universität zu Berlin und wurde dort am 18. November 2015 verteidigt.

Gutachter/innen: Prof. Dr. Wolfgang Kaschuba und Prof. Dr. Beate Binder. Dekan: Prof. Michael Seadle, PhD.

Gefördert durch die Heinrich-Böll-Stiftung.

\section{(2) $(\mathbb{D} \Theta \Theta$}

Dieses Werk ist lizenziert unter der Creative Commons Attribution-NonCommercial-NoDerivs 4.0 Lizenz (BY-NC-ND). Diese Lizenz erlaubt die private Nutzung, gestattet aber keine Bearbeitung und keine kommerzielle Nutzung. Weitere Informationen finden Sie unter https://creativecommons.org/licenses/by-nc-nd/4.o/deed.de/. Um Genehmigungen für Adaptionen, Übersetzungen, Derivate oder Wiederverwendung zu kommerziellen Zwecken einzuholen, wenden Sie sich bitte an rights@transcript-verlag.de

\section{(C) 2018 transcript Verlag, Bielefeld}

\section{Bibliografische Information der Deutschen Nationalbibliothek}

Die Deutsche Nationalbibliothek verzeichnet diese Publikation in der Deutschen Nationalbibliografie; detaillierte bibliografische Daten sind im Internet über http://dnb.d-nb.de abrufbar.

\section{Umschlaggestaltung: Maria Arndt, Bielefeld}

Umschlagabbildung: Jens Adam: Sarajevo Red Line, Gedenkveranstaltung zum 20. Jahrestag des Beginns der Belagerung der Stadt, 6. April 2012.

Satz: Harry Adler

Druck: Majuskel Medienproduktion GmbH, Wetzlar

Print-ISBN 978-3-8376-4262-9

PDF-ISBN 978-3-8394-4262-3

Gedruckt auf alterungsbeständigem Papier mit chlorfrei gebleichtem Zellstoff. Besuchen Sie uns im Internet: http://www.transcript-verlag.de Bitte fordern Sie unser Gesamtverzeichnis und andere Broschüren an unter: info@transcript-verlag.de 


\section{Inhalt}

\section{Einleitung $\mid 7$}

I. Rahmungen: Ethnografische Zugänge - theoretische Bezüge | 25

I. Erste Einsichten: Auf der Suche nach der Konfliktprävention $\mid 25$

2. Konzeptionelle Grundlagen: Politiken als anthropologische Forschungsfelder | 3I

3. Leitmotiv: Feldforschungen im Nationalen $\mid 43$

II. Ein politisches Feld: Imaginäre Dimensionen, policy community, Übersetzungsketten | 6 I

I. Menschen bewegen: Akteursfeld und politisches Narrativ auf zwei Berliner Konferenzen $\mid 6_{3}$

2. Diskursive Formationen: Schlüsselbegriffe, Konfigurationen kultureller Bedeutungen, politische Rationalitäten | 68

3. Entwicklungslinien: Begründungsfiguren Auswärtiger Kulturpolitik in Regierungsdokumenten 185

4. Policy takes place: Urbane Bühnen, politische Materialitäten, Formierungsmacht | IO3

5. Dichotomien: Der innere und der äußere Raum der Kultur | I24

6. Asymmetrien und Handlungsspielräume: Relationen zwischen politischen Zentren und Kulturmittlern | I35

III. Translokale Formationen: Wissens- und Übersetzungsarbeit in Ramallah und Sarajevo | 147

I. Lokale Kontexte: Ramallah und Sarajevo aus der Perspektive eines Anreisenden | I48

2. Analyserahmen: Translokale Praxis- und Übersetzungsfelder | I53

3. Subjektpositionen und Wissenshierarchien: Entsendungen als Strategien des Verortens | I7I 
4. Deutschlandbezüge und ihre Wirkungsketten: Verankern und Anschlüsse schaffen als Strategien kulturpolitischer Übersetzung | I97

5. Konfliktprävention und ihre Reibungsflächen: Verknüpfen als navigierende Wissensarbeit | 222

IV. Schluss $\mid 27 \mathrm{I}$

V. Anhang $\mid 287$

I. Feldtagebücher und Interviews $\mid 287$

2. Materialien $\mid 288$

3. Literatur 292

Danksagung | 303 


\section{Einleitung}

\section{"Lindenstrasse in Palästina"}

Ende August 2008 berichten die Tagesthemen von der »Palästinensersoap Matabb «, die wenige Tage zuvor im Al-Kasaba-Theater in Ramallah Premiere feierte. Als treibende Kraft hinter dieser Fernsehserie wird das örtliche Goethe-Institut vorgestellt, das hierzu deutsche und europäische Fördergelder akquirieren konnte. Unter dem Titel Lindenstraße in Palästina erzählt der Bericht von dem geringen Budget, den einfachen Produktionsbedingungen, den positiven Reaktionen des lokalen Publikums und rückt »Tabus« in der palästinensischen Gesellschaft, welche Matabb zu bearbeiten versuche, ins Zentrum. So erläutert der Leiter des Goethe-Instituts in Ramallah, Fareed Majari, dass durch die Serie »soziale und politische Themen, die sonst unter den Teppich gekehrt werden« und »brennende Inhalte« thematisiert würden, »etwa wie über sein eigenes Liebesleben zu entscheiden oder Gewalt gegen Frauen oder auch das berufliche Fortkommen von Frauen oder Arbeitsteilung in der Familie«. ${ }^{1}$

Bemerkenswert ist hierbei zunächst der offen formulierte Anspruch eines deutschen Akteurs, mit kulturpolitischen Instrumenten in lokale Diskussionen und Problemlagen in den besetzten Palästinensischen Gebieten zu intervenieren. Zugleich wird anhand des Berichts der Charakter dieser Intervention sichtbar: Indem sie das Auf und Ab im Alltag der Mitarbeiter einer Nichtregierungsorganisation mit grundlegenden politischen und sozialen Problemen verknüpft, orientiert sich die Serie Matabb in ihrer zentralen narrativen Technik an dem deutschen Pendant der Lindenstraße. Die ambivalente Haltung vieler Palästinenser zu solchen, von externen Geldgebern abhängigen NGOs, die permanente Unterbrechung des Alltags durch die israelische Besatzung und

1 | "Lindenstraße in Palästina: Matabb: Die Palästinensersoap ", in: Tagesthemen vom 31.8.2008. "Matabb" wird von den Machern als "speed bumb", also "Bremsschwelle" übersetzt.

2 | Die zehn Episoden der Serie können auch im Internet gesehen werden: www.goethe. de/ins/ps/ram/prj/mat/enindex.htm (letzter Zugriff am 11. November 2017). 
ihre Checkpoints, Fragen nach adäquaten Formen des Widerstandes oder nach angemessenen Umgangsformen zwischen den Geschlechtern bilden durchgängige Motive in dieser Serie, die somit - als Effekt einer deutschen Policy eine spezifische Version des Lokalen schafft und als narrativen Rahmen für die alltäglichen Probleme und Verwicklungen der Protagonisten setzt.

\section{Das Forschungsfeld: AusWÄrtige Kulturpolitik in Konfliktregionen}

Am Abend der Ausstrahlung dieses Berichts befand ich mich auf der Reise nach Tel Aviv, um zwei Tage später eine Feldforschung in Ramallah zu beginnen. Hier wollte ich mich mit der Umsetzung deutscher Auswärtiger Kulturpolitik in Konfliktregionen beschäftigen und somit den Politikbereich fokussieren, dessen Zielvorstellungen, Infrastrukturen, Ressourcen und Akteure - neben vielen anderen Projekten - auch die Produktion von Matabb ermöglicht hatten.

Im Kern interessierten mich Fragen nach den Mechanismen der Übersetzung von politischen Zielvorstellungen in Arbeitsweisen, Programmschwerpunkte und Projektformate: Über welche Wege wandern in Deutschland formulierte politische Konzepte in die Zielregionen Auswärtiger Kulturpolitik? Wie eng ist die Arbeitspraxis einer Kulturmittlerin in Ramallah an solche Papiere gekoppelt? Und welche Effekte lassen sich durch die Einarbeitung kulturpolitischer Zielsetzungen in solche lokalen Handlungsfelder hervorrufen? Zwei Übersetzungsvorgänge standen somit im Zentrum meines Interesses: einerseits die translokale Übersetzung innerhalb eines Netzwerkes, das Berlin mit einer Vielzahl von Orten weltweit verbindet; andererseits die transformative Übersetzung eher allgemein gehaltener politischer Absichtserklärungen und Grundsatzpapiere in lokal situierte Arbeitsweisen und Kooperationsformen. Diesen Fragen wollte ich im Rahmen von ethnografischen Feldforschungen zunächst in Ramallah und später in Sarajevo nachgehen. An beiden Orten arbeitete ich mehrere Monate in den Programmabteilungen der Goethe-Institute mit, um die Praxis kulturpolitischer Übersetzung aus einer Alltagsperspektive in den Blick zu nehmen. ${ }^{3}$

Mein Forschungsfeld bildete somit die deutsche Auswärtige Kultur-und Bildungspolitik. ${ }^{4}$ In Selbstdarstellungen und Beschreibungen des Politikbereichs

3 | Meine Feldforschung in Ramallah führte ich zwischen September und Dezember 2008 durch; in Sarajevo hielt ich mich zwischen Mai und Juli, im November 2009 sowie im April 2012 auf.

4 | "Auswärtige Kultur- und Bildungspolitik" ist der offizielle Name des Politikbereichs. In der Regel wird in Selbstdarstellungen oder Berichten die Bezeichnung "Auswärtige Kulturpolitik“ - abgekürzt AKP - genutzt. Im Folgenden ist auch in dieser Arbeit 
wird häufig auf die Formulierung Willy Brandts von der »Dritten Säule der deutschen Außenpolitik« zurückgegriffen, um die gleichwertige Bedeutung der staatlicherseits geförderten Beziehungen Deutschlands in den Bereichen Kultur, Bildung und Wissenschaft im Verhältnis zur politischen Diplomatie und den Außenwirtschaftsbeziehungen zu behaupten. Zur Realisierung ihrer kulturpolitischen Zielsetzungen im Ausland stellt die Bundesregierung jährlich finanzielle Mittel im Bundeshaushalt bereit: Im Jahr $2009^{5}$ standen hierzu über I,4 Milliarden Euro zur Verfügung, von denen knapp 60\% dem Auswärtigen Amt zuflossen. ${ }^{6}$

Für den weiteren Argumentationsgang sind an dieser Stelle drei grundlegende Informationen wichtig: Erstens hat sich zur Realisierung Auswärtiger Kulturpolitik ein weltumspannendes Netzwerk aus unterschiedlichen Institutionen, Organisationen, Akteuren und Einsatzorten entwickelt, in dessen Mittelpunkt die Abteilung Kultur und Kommunikation des Auswärtigen Amtes steht. Zweitens hat sich eine spezifische Struktur herausgebildet, die auf kontinuierlichen und zugleich asymmetrischen Beziehungen zwischen den politischen Entscheidungszentren und den formell unabhängigen Mittlerorganisationen beruht: Das Goethe-Institut, der Deutsche Akademische Austauschdienst, die deutschen Auslandsschulen, das Institut für Auslandsbeziehungen oder das Deutsche Archäologische Institut befinden sich somit bei allen inhaltlichen und organisatorischen Unterschieden in einer ähnlichen Spannung zwischen

von "Auswärtiger Kulturpolitik" die Rede. Siehe hierzu etwa den Bericht der Bundesregierung zur Auswärtigen Kultur- und Bildungspolitik 2009/2010 unter: www.ifa.de/ fileadmin/pdf/aa/akbp_bericht2009-10.pdf (letzter Zugriff am 11. November 2017).

5 | Das Ende der Regierungskoalition aus CDU und SPD im Jahr 2009 bildete den Schlusspunkt meiner empirischen Arbeit für diese Forschung. Neben Aspekten der Pragmatik gibt es hierfür konzeptionelle Gründe: Das Ende der ersten Amtszeit des sozialdemokratischen Außenministers Frank-Walter Steinmeier lässt sich als Abschluss einer Phase (1998-2009) verstehen, die mit dem Antritt der rotgrünen Regierung begonnen hatte und durch den Anspruch auf außenpolitische Veränderungen und Neupositionierungen geprägt war. Trotz gewisser Justierungen durch den Wechsel von "rotgrün « zu "schwarzrot" im Jahr 2005 zeigen sich über dieses Jahr hinaus personelle und konzeptionelle Kontinuitäten. Eine Ausnahme von dieser Regel stellen meine Beobachtungen und Gespräche in Sarajevo aus Anlass der Gedenkfeierlichkeiten zum 20. Jahrestag des Beginns der Belagerung der Stadt im April 2012 dar, die geeignet waren, empirische Befunde aus meinen Feldforschungen im Jahr 2009 abzurunden.

6 | Die restlichen Mittel verteilten sich vor allem auf die Haushalte des Beauftragten für Kultur und Medien und des Bundesministeriums für Bildung und Forschung, die in diesem Jahr über 19,9\%, beziehungsweise 14,6\% der Gesamtmittel verfügten. Siehe hierzu den Bericht der Bundesregierung zur Auswärtigen Kultur- und Bildungspolitik 2009/2010, S. 11. 
ihrem Anspruch auf Autonomie und ihrer Abhängigkeit von der Zuweisung öffentlicher Gelder. Und drittens etabliert der Politikbereich zu seiner Legitimierung offizielle Zielsetzungen. Einen Kerngedanken bildet hierbei - neben der Förderung der deutschen Sprache im Ausland - seit den frühen Jahren der Bundesrepublik der Wunsch, über Formen der kulturellen Vermittlung und Selbstdarstellung Reputation und Sympathie für Deutschland zu erwerben. Gerade nach Zweitem Weltkrieg und Nationalsozialismus wuchs die Hoffnung, durch »Kultur« ein anderes Bild vom nunmehr demokratischen Deutschland zu mobilisieren und somit die Aufnahme der jungen Bundesrepublik in die Staatengemeinschaft $\mathrm{zu}$ unterstützen. Diese zentrale Intention der Vermittlung von Deutschlandbildern wurde im Laufe der Jahrzehnte ergänzt, reformuliert und mit neuen Diskussionsfeldern verknüpft. Veränderungen in der öffentlichen Begründung Auswärtiger Kulturpolitik, die sich etwa um die Jahrtausendwende zeigten, bilden den zentralen Ausgangspunkt dieses Buches.

\section{Diskursive Verschiebungen: AusWÄrtige Kulturpolitik als KonfliktPRÄVEntion?}

Im Jahr 200I erscheint eine Ausgabe der Zeitschrift für Kulturaustausch mit dem Titel Mit Kultur gegen Krisen. Kulturdialog als Mittel der Konfliktprävention ${ }^{7}$ und verweist hiermit auf das Auftauchen einer neuen »diskursiven Formation $\aleph^{8}$ zur Legitimierung des Politikbereichs. Das Cover zeigt eine Fotografie aus einem südosteuropäischen Flüchtlingslager und knüpft somit allein ikonografisch an eine wesentliche Bruchstelle europäischer Politik der I990er Jahre an: Kriege und das Auseinanderbrechen Jugoslawiens, Flucht, Vertreibung, ethnisch begründete politische Gewalt, Völkermord, der Makel des langandauernden Unvermögens der Westeuropäer, sich auf eine Reaktionsweise zu einigen sowie damit verbundene Diskussionen um mögliche Interventionen und die Grenzen staatlicher Souveränität in »Konfliktgebieten«. In Deutschland führen diese Ereignisse zu Debatten um die Beteiligung des Landes an einer militärischen Intervention im Kosovo und letztlich zu der ersten aktiven Teilnahme an einem Kampfeinsatz nach Ende des Zweiten Weltkrieges. In diesem historischen Moment verbinden sich Fragen nach dem nationalstaatlichen Selbstverständnis, der neuen geopolitischen Ordnung nach Ende des Kalten Krieges, dem Auftauchen »neuer Kriege « auch in Europa, ${ }^{9}$ der Funktion inter-

7 | Zeitschrift für Kulturaustausch 2001, 2 (im Folgenden: ZfKA).

8 | Michel Foucault: Archäologie des Wissens. Frankfurt 1981, S. 58.

9 | Mary Kaldor: Neue und alte Kriege: Organisierte Gewalt im Zeitalter der Globalisierung. Frankfurt 2000; Herfried Münkler: Die neuen Kriege. Reinbek bei Hamburg 2002. 
nationalen Rechts und nach möglichen Handlungsoptionen der »internationalen Gemeinschaft« im Umgang mit gewalttätigen Konflikten.

Dies ist der Kontext, in dem die Zeitschrift die Positionen und Diskussionen einer Konferenz, zu der Bundestagsabgeordnete der damaligen Regierungskoalition, der Bundesaußenminister, Mitarbeiterinnen des Goethe-Instituts und des British Councils, Praktiker der Konfliktbearbeitung, Journalisten und Wissenschaftlerinnen ${ }^{10}$ im Mai 200I in Stuttgart zusammengekommen waren, dokumentiert. Sichtbar werden hierbei die Bestandteile und Argumentationslinien einer neuen Begründungsfigur Auswärtiger Kulturpolitik, die sich um den Schlüsselbegriff der Konfliktprävention herum gruppiert. So heißt es in der Einleitung:

"Deutschlands Außenpolitik verändert sich. So sehen es zumindest die sie beobachtenden Theoretiker in den Sozialwissenschaften. Sie machen immer mehr Ansätze für eine an Werten orientierte Außenpolitik aus, die auf eine ,Verrechtlichung der internationaIen Beziehungen als Teil ihrer Zivilisierung', so der Politikwissenschaftler Volker Rittberger, ausgerichtet ist. Die deutsche Außenpolitik unternimmt Initiativen, um durch Aufbau oder Unterstützung von Strukturen der Krisenvorbeugung oder Konfliktbearbeitung die friedliche oder gewaltfreie Lösung von Konflikten zu ermöglichen.

Erstmalig hat auch die Auswärtige Kulturpolitik ihren möglichen Beitrag zu diesem neuen Ansatz formuliert. In der ,Konzeption 2000، des Auswärtigen Amtes heißt es: 'Die Auswärtige Kulturpolitik [...] ist an den allgemeinen Zielen und Interessen der deutschen Außenpolitik - Sicherung des Friedens, Konfliktverhütung, Verwirklichung der Menschenrechte, partnerschaftliche Zusammenarbeit - ausgerichtet und unterstützt sie. [...] Der Bedarf nach Stabilisierung und Konfliktprävention durch Dialog, Förderung demokratischer Entwicklungsprozesse und Verwirklichung der Menschenrechte wächst.`

Was aber kann Kultur als ein möglicher Faktor internationaler Krisenprävention wirklich leisten? «11

Bereits anhand dieses Zitates zeigen sich drei zentrale Argumentationsebenen innerhalb der Diskussionen zu einer »kulturellen Krisenprävention als Mittel deutscher Außenpolitik « ${ }^{12}$, die auch im Rahmen dieser Studie aufgegriffen werden:

10 | Aus Gründen der Pragmatik und Lesbarkeit nutze ich in dieser Arbeit in der Regel nur eine geschlechtsanzeigende Endung und verzichte gleichfalls auf ein großes "| " oder einen Unterstrich. Ich wechsle die Endungen aber durchgängig an hierfür geeigneten Stellen und meine im Zweifelsfall jeweils alle Geschlechter.

11 | ZfKA 2001, 2, S. 23.

12 | So die Formulierung in Joschka Fischers Beitrag: ZfKA 2001, 2, S. 24. 
Eine erste Ebene - und gleichzeitig die diskursive Grundlage für die Neujustierung des Politikbereichs - stellen Verweise auf die Veränderungen geopolitischer Rahmenbedingungen dar: Globalisierung, Diskussionen zur Entstehung neuer Konfliktlinien oder die Betonung der wachsenden Bedeutung bestimmter, universal ausgerichteter politischer Projekte - etwa der »Herrschaft des Rechts« oder der »Achtung der Menschenrechte« - bilden Bezugspunkte für die Begründung der Suche nach neuen kulturpolitischen Herangehensweisen. Hinzu treten Verweise auf eine »neue Rolle«, die Deutschland nach Ende des Kalten Krieges zukomme: So schreibt etwa Joschka Fischer von den »wachsenden internationalen Erwartungen an das wiedervereinte Deutschland «. Die Artikel dokumentieren somit die Ausarbeitung und Mobilisierung eines politischen Narrativs von einer sich rapide transformierenden Welt, die eine Anpassung deutscher Außenpolitik erforderlich mache.

Auf einer zweiten Ebene lassen sich vier Schlüsselbegriffe identifizieren, die das Gerüst der neuen diskursiven Formation darstellen:

\begin{tabular}{r|l} 
Konflikt/Krise/Krieg & Kultur \\
\hline Prävention & (Außen-)Politik
\end{tabular}

Anhand der Beiträge wird sichtbar, wie diese vier Begriffe in ein neues Verhältnis zueinander rücken, sich aber zugleich mit etablierten Argumentationslinien verknüpfen. Im Ergebnis entsteht ein Geflecht von Bedeutungen, das eine Vielzahl von Anschlussmöglichkeiten bietet. Ich möchte dies anhand von zwei Begriffen verdeutlichen:

So werden in den Beiträgen unterschiedliche, sich zum Teil widersprechende Vorstellungen von Kultur aktiviert. Hier finden sich Positionen, die Kultur eine zivilisierende, Verständigung fördernde Wirkung zuschreiben neben Stellungnahmen, die auf »kulturelle Unterschiede« als mögliche Ursache von Konflikten verweisen. Kultur wird teilweise als ein freier, unabhängiger Artikulationsraum mit einer notwendigen Distanz zur Politik dargestellt; in anderen Stellungnahmen ist hingegen von der möglichen Indienstnahme von Kultur zur Umsetzung politischer Zielsetzungen die Rede. Einzelne Beiträge konzentrieren sich auf die kreative Arbeit von Künstlerinnen und Intellektuellen, andere Positionen verknüpfen Kultur hingegen mit »Werten« wie etwa der »Förderung von Demokratie und Menschenrechten « oder dem »Schutz der natürlichen Ressourcen«. Manche Autoren sprechen von den Potenzialen der Kultur, Räume zu öffnen und Begegnungen zu ermöglichen; andere Positionen verweisen auf Tendenzen »kultureller Abgrenzungen« etwa als Reaktion auf Globalisierungsprozesse. Neben der Betonung einer transformativen Kraft 
von Kultur in »Konfliktgebieten« oder autoritär regierten Staaten finden sich Beiträge, die daran zweifeln, dass mit kulturellen Aktivitäten nachweisbare politische Effekte zu erzielen seien. Dem positiven Bild von Künstlern als »geistigem Roten Kreuz« in Kriegsgebieten stehen Verweise auf die negative Rolle von Intellektuellen als »geistigen Brandstiftern« in Konflikten und Diktaturen gegenüber. Diese Aufzählung verdeutlicht zunächst, dass sehr unterschiedliche, historisch gewachsene und miteinander sogar im Konflikt stehende Verständnisweisen von Kultur in diese neue diskursive Formation eingebracht werden können. Bemerkenswert ist weiterhin, dass sich kein Hinweis findet, dass dieses Nebeneinander durch die Diskussionsteilnehmer als erklärungsbedürftig eingeschätzt worden wäre. ${ }^{13}$

Für den Begriff Prävention lassen sich gleichfalls verschiedene Verbindungslinien herausarbeiten, auch wenn die Bedeutungen in diesem Fall nicht ebenso vielfältig sind. Hier sticht erstens der Verweis auf ein Prestigeprojekt rotgrüner Außen-, Entwicklungs- und Sicherheitspolitik heraus, das im Koalitionsvertrag von 1998 festgeschrieben worden war und sich auf den »Aufbau einer Infrastruktur für Krisenprävention und zivile Konfliktbearbeitung « konzentrieren sollte. So verabschiedete die Bundesregierung im Jahr 2000 ein ressortübergreifendes »Gesamtkonzept Krisenprävention und Konfliktbeilegung «, richtete im Auswärtigen Amt die »Stelle eines Krisenbeauftragten« ein, gründete die »Deutsche Stiftung für Friedensforschung « und förderte den »Aufbau des Zivilen Friedensdienstes in der bilateralen Entwicklungszusammenarbeit«. ${ }^{14}$ Über die Verknüpfung der Begriffe Prävention und Kultur wird somit zumindest diskursiv eine Verbindung Auswärtiger Kulturpolitik zu dieser politisch gesetzten »Querschnittsaufgabe« hergestellt. Zweitens zeigen sich Verbindungen zu Diskussionen innerhalb der Vereinten Nationen, wie sich insbesondere anhand der mehrfachen Bezüge zu Kofi Annans Formulierung »Kultur der Prävention $\aleph^{15}$ nachzeichnen lässt. Der klassischerweise nationalstaatlichen Auswärtigen Kulturpolitik werden somit Anschlussmöglichkeiten an die Diskurse und Handlungsfelder einer entstehenden globalen Regierungsführung zur Bearbeitung internationaler Problemlagen eröffnet. Die Frage nach den Bedingungen von Interventionen der internationalen Gemeinschaft in Konfliktkonstellationen oder der Prävention von Gewalt stellte

13 All diese Positionen finden sich in einzelnen Beiträgen des Heftes. Von dem "geistigen Roten Kreuz" spricht etwa Kathinka Dittrich van Weringh (S. 27f.); die Bindung von "Kultur" an "Werte" ist dem Beitrag von Monika Griefahn entnommen (S. 34).

14 | Siehe hierzu insbesondere den Beitrag des grünen MdB Winfried Nachtwei, ZfKA 2001, 2, S. 36.

15 Kofi Annan: Facing the humanitarian challenge: towards a culture of prevention. New York 1999. 
schließlich seit den I99oer Jahren ein zentrales Diskussionsfeld innerhalb der UNO dar. ${ }^{16}$

Auf Basis dieser Beispiele lässt sich somit die Entstehung einer neuen diskursiven Formation zur Legitimierung Auswärtiger Kulturpolitik festhalten, die zwar einen klar erkennbaren, aus vier Schlüsselbegriffen gebildeten Kern besitzt, zugleich aber über eine beachtliche Flexibilität in ihren möglichen Verknüpfungen mit anderen Positionen, Bedeutungen und Diskussionsfeldern verfügt.

Auch die dritte Argumentationsebene war in dem Zitat aus der Einleitung der Zeitschrift bereits angeklungen: »Was aber kann Kultur als ein möglicher Faktor internationaler Krisenprävention wirklich leisten?« Durchgängig fragen die Beiträge nach den Konsequenzen für die kulturpolitische Praxis und diskutieren mögliche Maßnahmen, über die eine kulturelle Konflikt- oder Krisenprävention umgesetzt werden könnte. Hier zeigen sich einerseits durchaus selbstbewusste Verweise auf die Vielfalt der etablierten Instrumente und die Vorteile des breiten Akteursfeldes aus Mittlerorganisationen: So unterschiedliche Projekte wie der Aufbau einer albanischsprachigen Universität in Mazedonien, die Förderung der Kooperation von Universitäten in Südosteuropa, die Eröffnung eines Goethe-Institutes in Sarajevo oder die Förderung einer kritischen Geschichtsvermittlung werden als mögliche Formen einer konfliktpräventiven Auswärtigen Kulturpolitik angeführt. Aber auch die direkte Unterstützung von Künstlerinnen und Intellektuellen in Diktaturen und Krisenregionen oder die Förderung klassischer Kunstprojekte ohne einer explizit präventiven Agenda werden als sinnvolle Umsetzungen genannt. Andererseits zieht sich eine gewisse Unsicherheit über die Wirksamkeit solcher Maßnahmen durch das Journal. Einige Beiträge verweisen auf das Problem, dass sich der Erfolg eines jeweiligen Formats im Hinblick auf die Prävention von Konflikten kaum evaluieren ließe; andere lehnen es ab, Kulturprojekte primär über ihren »politischen Ertrag « zu legitimieren. Dennoch wird deutlich, dass der Frage nach den Konsequenzen der neuen diskursiven Figur für die kulturpolitische Praxis auf dieser Tagung eine zentrale Rolle zukam.

Diese drei Ebenen - also die Mobilisierung politischer Narrative zur »Rolle Deutschlands in der Welt«; das Auftauchen und die Ausgestaltung einer neuen diskursiven Formation sowie die Frage nach der Beziehung zwischen Diskursen und Praxen innerhalb eines translokal vernetzten Politikbereichs - verweisen auf drei zentrale Felder, die auch im Rahmen dieser Studie untersucht werden.

16 | Siehe hierzu als zentrale Referenzpapiere exemplarisch: Boutros Boutros-Ghali: An Agenda for Peace. Preventive diplomacy, peacemaking and peace-keeping. United Nations, New York 1992 (= S/24111); Boutros Boutros-Ghali: Supplement to an Agenda for Peace. United Nations, New York 1995. (= S1995/1); Kofi Annan: Prevention of armed conflict. United Nations, New York 2001 (= S/2001/574). 


\section{Ethnografische Momente: ZugÄnge FüR die ANTHROPOLOGISCHE ERFORSCHUNG POLITISCHER FELDER}

Im Anschluss an gegenwärtige Überlegungen zu einer Anthropologie des Politischen betrachte ich diese diskursiven Verschiebungen als einen "genuin ethnografischen Moment«, der einen privilegierten »Einstieg in ein anthropologisches Forschungsfeld ermöglicht «. ${ }^{17} \mathrm{Im}$ Rahmen dieses Forschungszweiges wurde verschiedentlich vorgeschlagen, gerade »Brüche«, »Reibungen«, »Ausnahmesituationen « oder »Problematisierungen« als Ausgangspunkte für die ethnografische Untersuchung »politischer Felder« zu wählen. Denn zu solchen Anlässen, wenn ein routinierter politischer Prozess ins Stocken gerät, treten die sonst weitgehend naturalisierten Begründungsfiguren, Rationalitäten oder Akteurskonstellationen eines Politikbereichs besonders deutlich zutage. Vor diesem Hintergrund verstehe ich die Stuttgarter Konferenz als Ausdruck einer »Phase der Brüchigkeit« in den Aushandlungen eines Narrativs zur Legitimation Auswärtiger Kulturpolitik: Maßgebliche Angehörige einer »policy community « ${ }^{18}$ identifizieren einen Veränderungsdruck, »problematisieren« etablierte Strukturen, Sprechformen und Vorgehensweisen und tragen hierdurch dazu bei, sie »dem Status des Selbstverständlichen « zu entheben. ${ }^{19}$ Bundestagsabgeordnete der damals neuen rotgrünen Regierungskoalition, der Bundesaußenminister, Wissenschaftlerinnen, Politikberater und Kulturmittlerinnen unterziehen bisher kaum hinterfragte inhaltliche und organisatorische Arrangements einer Revision. Die offiziellen Zielsetzungen stehen in diesem Zusammenhang ebenso zur Debatte wie die regionalen Schwerpunkte und ins-

17 | Konzeptionelle Grundlagen und analytische Perspektiven einer Anthropologie des Politischen werden im Kapitel I.2 diskutiert. Siehe zur Einführung: Cris Shore \& Susan Wright (Hrsg.): Anthropology of Policy. Critical perspectives on governance and power. London, New York 1997; Cris Shore, Susan Wright \& Davide Però (Hrsg.): Policy Worlds. Anthropology and the Analysis of Contemporary Power. New York, Oxford 2011; "Ethnografische Momente « nach: Jens Adam \& Asta Vonderau: Formationen des Politischen. Überlegungen zu einer Anthropologie politischer Felder. In: Dies. (Hrsg.): Formationen des Politischen. Anthropologie politischer Felder. Bielefeld 2014, S. 7-32, S. 23f.; "Problematisieren « nach: Beate Binder: Troubling policies. Gender und queertheoretische Interventionen in die Anthropology of Policy. In: Ebd., S. 363-386, S. 365; „Phasen der ideologischen Brüchigkeit» nach: Stefan Wellgraf: Das Ende der Hauptschule in Berlin. Zur ideologischen Dimension von Bildungsmythen. In: Ebd., S. 35-65, S. 53ff. und S. 62; "Reibungen im Anschluss an: Anna Lowenhaupt Tsing: Friction. An Ethnography of Global Connection. Princeton, Oxford 2005.

18 | "Policy community“ im Anschluss an: Gregory Feldman: The Migration Apparatus. Security, Labor, and Policymaking in the European Union. Stanford 2012, S. 29.

19 | Binder: Troubling policies, S. 366. 
titutionellen Ordnungen, die sich im Kontext dieser Politik über die Jahrzehnte herausgebildet haben. Diese Diskussionen bleiben dabei nicht auf die internen Strukturen und konzeptionellen Zielvorstellungen innerhalb des Politikbereichs beschränkt, sondern beziehen die geopolitischen Rahmenbedingungen mit ein: Eine unübersichtliche Weltordnung nach Ende des Kalten Krieges, die bisherigen Formen und Mechanismen globaler Regierungsführung - die sich gerade im Umgang mit den Kriegen und Konflikten der I99oer Jahren als weitgehend unzureichend erwiesen hatten - sowie die Frage nach der Rolle des vereinten Deutschlands innerhalb dieser Welt sind aus Perspektive der außenpolitischen Eliten offensichtlich $\mathrm{zu} »$ Problemen« geworden und verlangen nach neuen Herangehensweisen. Zugleich zeigt sich, dass die bisherigen Leitvorstellungen, Infrastrukturen und Arbeitsformen des Politikbereichs nicht einfach als obsolet betrachtet und ersetzt, sondern im Rahmen von reflexiven Vorgehensweisen mit aktuellen Problemlagen, Themenfeldern und Rationalitäten neu zusammengefügt werden. Das Auftauchen der diskursiven Formation Auswärtige Kulturpolitik als Konfliktprävention lässt sich vor diesem Hintergrund auch als Ausdruck einer kollektiven Wissensarbeit verstehen, die durch die Hinterfragung bisheriger Selbstverständlichkeiten und die Suche nach einem »neuen Ansatz« erforderlich wurde. Die Stuttgarter Konferenz markiert entsprechend einen Moment, in dem sich Machtrelationen und Ordnungsvorstellungen, die dem Politikbereich eingelagert sind, aber auch die Effekte, die sich aus dem Aufeinandertreffen unterschiedlicher politischer Zielvorstellungen ergeben, ethnografisch nachzeichnen lassen sollten.

\section{FORSCHUNGSFRAGEN UND UNTERSUCHUNGSEBENEN: Formierungsprozesse einer Policy, Wissensarbeit, TRANSFORMATIONEN DES NATIONALEN}

Ausgehend von diesem Auftauchen einer neuen diskursiven Formation untersuche ich in diesem Buch Prozesse und Praxen des Zusammenfügens von Zielvorstellungen, Wissensbeständen, Ressourcen, infrastrukturellen Arrangements, lokalen und institutionellen Kontexten zu einer nationalstaatlichen Politik, die im globalen Rahmen operiert und auf translokale Vernetzungen ausgerichtet ist. Dieses Leitthema meiner Studie wird durch drei Frageperspektiven bearbeitet:

Erstens befasse ich mich mit den Bedingungen für die Initiierung von Wandlungsprozessen und die Entstehung von Neuheit innerhalb der etablierten Strukturen und Diskurse eines Politikbereichs - oder um es näher an den bisherigen Beobachtungen zu formulieren: ich interessiere mich für den Status oder die Nachhaltigkeit der diskursiven Verschiebungen und frage entsprechend nach ihren Auswirkungen auf das Selbstverständnis, das Narrativ, die 
Strukturen und die Praxis Auswärtiger Kulturpolitik. Idealtypisch gedacht, sind hierbei im Prinzip drei Alternativen denkbar: i) die diskursiven Veränderungen verweisen auf einen grundlegenden Paradigmenwechsel; ii) es handelt sich eher um die kurzfristige Konjunktur einiger neuer Schlüsselbegriffe, die nach einer zeitlich begrenzten Hochphase wieder in den Hintergrund treten oder sogar ganz verschwinden; iii) die neue diskursive Figur erlangt zwar eine gewisse Dauerhaftigkeit, wird aber als ein nachgeordnetes Element in einen weitgehend gleichbleibenden narrativen Rahmen, ein kaum verändertes grundlegendes Selbstverständnis des Politikbereichs inkorporiert.

Im Laufe meiner Forschung suchte ich entsprechend nach möglichen Verknüpfungen zwischen der neuen diskursiven Formation und der klassischen Begründungsfigur der Vermittlung von Deutschlandbildern; ich dokumentierte Situationen und Kontexte, in denen Kulturmittler oder Diplomatinnen Konfliktprävention als eine legitimierende Zielsetzung des Politikbereichs aufriefen, mobilisierten oder in Frage stellten; ich lotete aus, ob die neue Argumentationsfigur an die Stelle der etablierten Zielvorstellung einer kulturellen Selbstdarstellung Deutschlands trat oder ob sie sich eher als eine Möglichkeit erwies, um weiterhin kulturelle Selbstbilder - wenn auch durch neue Techniken und mit neuen Inhalten - zu mobilisieren. Die Ergebnisse dieser Suche werden in den empirischen Kapiteln dieser Arbeit (Kapitel II und III) vorgestellt und auf die Frage nach der Dauerhaftigkeit und den nachhaltigen Effekten einer neuen diskursiven Formation hin ausgerichtet.

Den zentralen konzeptionellen und methodischen Referenzrahmen für diese erste Frageperspektive - wie auch für mein gesamtes Buch - stellt das Programm einer Anthropologie des Politischen dar, deren Diskussionen hier in zumindest zwei Aspekten direkt aufgegriffen werden: zum einen in dem Verständnis von Policies als dynamischen Prozessen, die spätmoderne Gesellschaften machtvoll zu strukturieren vermögen, hierbei die unterschiedlichsten Ebenen, Kontexte, Personengruppen, Diskurse und Ressourcen miteinander verknüpfen und zugleich sowohl intendierte als auch nicht-intendierte Effekte hervorrufen; zum anderen in der Fokussierung auf Schlüsselbegriffe, die im Zuge solcher Prozesse ihre Position verändern, neue Bedeutungen akkumulieren oder in andere diskursive Formationen eingefügt werden können. Vor diesem Hintergrund werde ich das Forschungsprogramm einer Anthropology of Policy im Kapitel I.2 ausführlich diskutieren und hierauf aufbauend meine analytischen Perspektiven auf die Auswärtige Kulturpolitik genauer ausformulieren.

Zweitens frage ich nach der Beziehung zwischen Diskursen und Praxen oder - um es auf meinen Untersuchungsgegenstand hin zuzuspitzen - zwischen politisch formulierten Zielvorstellungen und dem Arbeitsalltag von Kulturmittlern in ihren Einsatzgebieten. Denn bereits zu Beginn meiner Forschung zeigte sich, dass meine Fragen nach der Bedeutung der neuen Zielsetzung Konfliktpräven- 
tion und ihrer Relevanz für die kulturpolitische Arbeitspraxis in dem Akteursfeld selbst nicht zu eindeutigen, allseits geteilten Positionen führten, sondern die unterschiedlichsten Reaktionen, Einschätzungen und Abgrenzungen hervorriefen (Kapitel I.I). Diese frühe Beobachtung hatte für meine Konzipierung des Feldes nachhaltige Konsequenzen: Hier traten die beiden Untersuchungsebenen hervor, die ich durch den gesamten Forschungsverlauf in den Blick nahm und die sich auch in der Struktur dieses Buches niedergeschlagen haben:

Zum einen erwies sich eine genaue Analyse der imaginären Dimensionen des Politikbereichs - im Kern also des politischen Narratives, das sich zur Begründung und Legitimierung Auswärtiger Kulturpolitik entwickelt hat und sich kontinuierlich fortschreibt - als erforderlich..$^{20}$ Denn erst durch ein solches Nachzeichnen der grundlegenden diskursiven Ordnungen und Bewegungen lassen sich die Position und die Bedeutung einer neuen Argumentationsfigur wirklich einordnen. Entsprechend arbeite ich in diesem Buch - anhand der Diskussionen auf Konferenzen sowie von offiziellen Papieren - die Schlüsselmetaphern, diskursiven Figuren und politischen Rationalitäten heraus, die im ersten Jahrzehnt nach der Jahrtausendwende das Gerüst dieses politischen Narrativs darstellen (Kapitel II.I-2) und gebe ihnen durch einen vergleichenden Blick in Regierungsdokumente aus den I970er Jahren historische Tiefenschärfe (Kapitel II.3). Sichtbar wird hierdurch, dass einige tatsächlich neue Argumentationsfiguren auf einem Reservoir weitgehend gleichbleibender Schlüsselbestände aufbauen - anders formuliert: der Eindruck von einem diskursiven Bruch, der sich anhand der Stuttgarter Konferenz gewinnen ließ, relativiert sich. Die Ausgestaltung der imaginären Dimension Auswärtiger Kulturpolitik zeigt sich auf dieser Basis als ein Zusammenspiel zirkulärer und linearer Bewegungen - also einer gleichzeitigen Aktivierung von etablierten Grundprinzipien und neuen Motiven (Kapitel II.I-3).

Zum anderen untersuche ich die Mobilisierungen solcher diskursiver Figuren innerhalb des translokalen Gefüges des Politikbereichs. Im Anschluss an das methodologische Programm einer »multi-sited ethnography ${ }^{21}$ verfolge ich hierzu die Schlüsselmetaphern des politischen Narrativs durch unterschiedliche Felder ihres Wirkens: etwa durch die so eben erwähnten Regierungsdokumente, durch Konferenzen des Auswärtigen Amtes oder durch die urbanen Bühnen, die im Zuge von Tagungen in Berlin entstehen (Kapitel II.4-5); vor allem aber durch die unterschiedlichen Kontexte der kulturpolitischen Projekt-

20 | "Imaginäre Dimension« im Anschluss an: Cris Shore \& Susan Wright: Introduction. Conceptualising Policy: Technologies of Governance and the Politics of Visibility. In: Cris Shore, Susan Wright \& Davide Però (Hrsg.): Policy Worlds, S. 1-25, S. 13.

21 | George Marcus: Ethnography in/of the World System. The Emergence of multi-sited Ethnography. Zuerst erschienen in: Annual Review of Anthropology 24 (1995), S. 95 117. Siehe zur methodischen Vorgehensweise in dieser Studie auch Kapitel I.2. 
und Repräsentationsarbeit in Sarajevo, Ramallah und Tel Aviv, welche die zweite Untersuchungsebene dieses Buches darstellen. Entsprechend beschäftige ich mich im gesamten Kapitel III mit der Frage, ob und auf welche Weise die offiziellen Zielsetzungen des Politikbereichs, dominante Argumentationsfiguren und Schlüsselbegriffe in die Arbeitspraxis der dortigen Goethe-Institute inkorporiert sind. Ich untersuche hierzu institutionelle Ordnungen, temporäre Projektformate und Sprechformen der Kulturmittler an diesen Einsatzorten; ich komme auf asymmetrische Relationen und normalisierte, nicht hinterfragte Ausgangspunkte in der Vermittlungsarbeit zu sprechen. In das Zentrum der Analyse rückt hierdurch die komplexe Wissensarbeit, die innerhalb und im Umfeld von Goethe-Instituten vonstattengeht.

Diese Wissensarbeit wird in diesem Buch primär als Übersetzungsarbeit untersucht, die durchgängig zwischen zwei Polen zu vermitteln hat: auf der einen Seite die Entscheidungszentren des Politikbereichs mit ihren Zielvorstellungen, Interessenkonjunkturen und Förderlogiken; auf der anderen Seite die lokalen Kontexte, in denen Kulturmittler effektvoll tätig werden sollen. Denn ganz unabhängig davon, ob eine Zielsetzung nun heißt, zeitgenössische Deutschlandbilder zu vermitteln, die deutsche Sprache zu fördern, Zivilgesellschaft zu stärken oder Konfliktprävention durch Wertedialog zu betreiben - jede dieser Formulierungen enthält den Anspruch, in Sarajevo, Ramallah oder an anderen Einsatzorten durch kulturpolitische Präsenzen und Praxen möglichst nachhaltige Wirkungen zu erzielen.

Ich werde im Rahmen dieser Studie aufzeigen, dass die Komplexität dieser Aufgabe dadurch zunimmt, dass die Kulturmittler regelmäßig mit den Grenzen ihres Wissens konfrontiert werden, wenn sie solche Zielsetzungen auf jeweilige lokale Handlungsräume zu beziehen versuchen: Was bedeutet es etwa, zeitgenössische Deutschlandbilder in Palästina einzubringen? Wie lässt sich Zivilgesellschaft in arabischen Ländern unterstützen und entwickeln? Welche Projektformate und Kooperationsformen vermögen tatsächlich einer Konfliktprävention in den komplexen politischen und sozialen Gefügen eines »Konfliktgebietes « zu dienen? Und handelt es sich hierbei überhaupt um einen realistischen und legitimen Zielhorizont? Da auf solche Fragen zumeist keine einfachen und direkten Antworten zu finden sind, lässt sich kulturelle Vermittlung auch als eine kontinuierliche Suche nach Bedeutungen, eine praxisnahe Erschließung neuer Expertisen und somit als eine alltägliche, auch improvisierende Arbeit an den Grenzen zwischen dem eigenen »Wissen « und »Nicht-Wissen« verstehen. Die Zentralität von Wissensarbeit in dieser Studie erklärt sich auch aus diesen Beobachtungen.

Zur Untersuchung dieser kulturpolitischen Wissensarbeit als einer translokalen Übersetzungsarbeit greife ich auf zwei analytische Modelle zurück: Zum einen analysiere ich im Anschluss an Richard Rottenburg die »Übersetzungsketten«, durch die Ideen, Ressourcen, Modelle oder Narrative inner- 
halb des Politikbereichs »translokal zirkulieren «. ${ }^{22}$ Hierdurch lassen sich die asymmetrischen Relationen herausarbeiten, die diese Fließmuster durchziehen und insbesondere auch das Verhältnis zwischen dem Auswärtigen Amt und den Kulturmittlern in den Regionen prägen (Kapitel II.6). Gleichzeitig kommen die Bedeutungsverschiebungen in den Blick, die einzelne Schlüsselbegriffe des politischen Narrativs durchlaufen, wenn sie zwischen institutionellen und lokalen Kontexten wandern und hierbei immer auch übersetzt werden müssen: Politische Diskurse und kulturpolitische Arbeitspraxis zeigen sich aus dieser Perspektive als »lose gekoppelt«. Zum anderen habe ich selbst das Modell der translokalen Praxis- und Übersetzungsfelder entwickelt, um Wissensarbeit zugleich situieren und differenzieren zu können (Kapitel III.2). Ich verstehe hierunter die temporären Handlungs- und Kommunikationskontexte, die in der kulturellen Vermittlungsarbeit etwa im Zuge eines Projektes entstehen und unterschiedliche Ressourcen, Wissensbestände, diskursive Figuren, Orte und Akteure in einen direkten, aber temporären Austausch bringen. Dieses Modell bietet den Rahmen, um insbesondere das Zusammenwirken der längerfristigen und relativ stabilen Diskurse und Infrastrukturen mit den flüchtigeren Veranstaltungsformaten, Begegnungen und Herangehensweisen im Alltag kultureller Vermittlung zu untersuchen. Auf dieser Basis werde ich im dritten Kapitel vier unterschiedliche Strategien kulturpolitischer Übersetzung aus meinem ethnografischen Material herausarbeiten: Strategien des Verortens, Strategien des Verankerns, Strategien zur Schaffung von Anschlüssen und Strategien des Verknüpfens setzen die beiden genannten Pole auf eine jeweils eigene Weise zueinander in Beziehung und geben hierdurch auch der flüchtigen alltäglichen Wissensarbeit Struktur und Konstanz (Kapitel III.3-5).

Drittens frage ich nach den Repräsentationstechniken und Positionierungen eines europäischen Nationalstaates aufglobalen Bühnen - oder um es auch an dieser Stelle direkt auf meine empirische Forschung zu beziehen: Ich untersuche die Ausgestaltung und Mobilisierung eines Narrativs vom Nationalen innerhalb einer Kulturpolitik, die auf weltweite Präsenzen und translokale Verflechtungen abzielt. Denn im Rahmen dieses Buches werde ich verdeutlichen, dass eben diesem Aspekt innerhalb der Auswärtigen Kulturpolitik auch weiterhin eine zentrale Rolle zukommt: eine Erzählung von »Deutschland« als einem kulturell beschreibbaren Gebilde, von seiner »nationalen Spezifik« und somit auch von »uns« als einem »kulturellen Wir« zu entwickeln und translokal zu mobilisieren. Entsprechend frage ich in dieser Untersuchung, was mit dem »Nationalen« - verstanden als einem historisch gewachsenen »symbolischen

22 | Richard Rottenburg: Weit hergeholte Fakten. Eine Parabel der Entwicklungshilfe. Stuttgart 2002, S. 16; auch der Ausdruck "lose gekoppelt" findet sich ebd., S. $97 \mathrm{ff}$. 
Raum « oder einem »System kultureller Repräsentationen « ${ }^{23}$ - passiert, wenn es den territorialen Rahmen seiner Entstehung verlässt und globale Bühnen und translokale Felder betritt. Durch welche Praxen, Strategien, Bilder und Repräsentationsformen wird es hier aktiviert und reproduziert? Welche Effekte lassen sich in diesen Kontexten durch eine solche Mobilisierung erzielen? Und welche Programmformate, Begegnungen und Diskussionen werden durch eine solche Zentrierung des Nationalen vielleicht ausgeschlossen?

Auch diese Fragestellung berührt offensichtlich die beiden Untersuchungsebenen dieser Studie und führt somit zu den Beziehungen, Übersetzungen, aber auch Differenzen zwischen imaginären Dimensionen und kulturpolitischen Praxisfeldern. So werde ich herausarbeiten, dass das politische Narrativ nicht nur immer wieder die Vermittlung eines modernen Deutschlandbildes als zentralen Arbeitsauftrag setzt, sondern auch die Grundfiguren des Nationalen als »nicht hinterfragbare Selbstverständlichkeiten « ${ }^{24}$ enthält: Neben der Vorstellung einer »nationalen Spezifik«, die sich kulturell darstellen lässt und der Idee eines »kulturellen Wir«, das sich weltweit mitteilen möchte, denke ich hierbei insbesondere an die elementare epistemologische Ordnung des Politikbereichs, die kontinuierlich das Bild einer Grenze zwischen einem »inneren« und einem »äußeren Raum der Kultur« reproduziert - auch und gerade in den Formulierungen, die zu ihrer Überschreitung und Relativierung im Zuge von kultureller Vermittlungsarbeit auffordern (Kapitel II.5). Im Hinblick auf die Praxisfelder der Vermittlungsarbeit lässt sich eine doppelte Bewegung festhalten: Zum einen greifen die Kulturmittler in ihrem Arbeitsalltag routiniert auf Figuren des Nationalen zurück, um den Zuschnitt ihrer Programme, ihre Position am Einsatzort, ihre Zuständigkeit oder auch die Hierarchien zwischen deutschen Entsandten und lokalen Mitarbeitern zu begründen (Kapitel III.3-4). Sie tragen hierdurch zu einer Plausibilisierung und Authentisierung des Nationalen als Ausgangspunkt des Politikbereichs bei. Zum anderen entwickeln sie gerade in der alltäglichen Projekt- und Vermittlungsarbeit Strategien der Flexibilisierung, die sich als Reaktionen auf die unterschiedlichen Erfordernisse und Rahmenbedingungen in jeweiligen lokalen Kontexten, aber auch auf Europäisierungsdynamiken (Kapitel III.4) oder die stärkere Orientierung des

23 | "Symbolischer Raum " nach: Beate Binder, Peter Niedermüller \& Wolfgang Kaschuba: Inszenierungen des Nationalen - einige einleitende Bemerkungen. In: Beate Binder, Wolfgang Kaschuba \& Peter Niedermüller (Hrsg.): Inszenierungen des Nationalen. Geschichte, Kultur und die Politik der Identitäten am Ende des 20. Jahrhunderts. Köln, Weimar, Wien 2001, S. 7-15; "System kultureller Repräsentationen " nach: Stuart Hall: Die Frage der kulturellen Identität. In: Ders.: Rassismus und kulturelle Identität (= Ausgewählte Schriften 2). Hamburg 1994, S. 180-222. Siehe zum "Nationalen" auch Kapitel I.3.

24 | So eine weitere Formulierung von Beate Binder in: Troubling Policies, S. 367. 
Politikbereichs an universalen politischen Projekten - also etwa Förderung der Menschenrechte oder Konfliktprävention erklären lassen. Die Bestände des Nationalen werden hierbei gewissermaßen zerlegt und einem Anwendungskontext entsprechend neu zusammengefügt - eine Dynamik, die sich im Prinzip auch auf den urbanen Bühnen des Politikbereichs in Berlin nachzeichnen lässt (Kapitel II.4).

Im Rahmen meiner Bemühungen, aus diesen Präsenzen und Bewegungen des Nationalen Sinn zu machen, führe ich im Kapitel I.3 zwei zentrale analytische Perspektiven aus zeitgenössischen anthropologischen, sozial- und kulturwissenschaftlichen Forschungen als Referenzrahmen ein: Zum einen diskutiere ich Beiträge, die nach den (neuartigen) Funktionslogiken, Bedeutungskontexten und Aktivierungsformen des Nationalen in spätmodernen europäischen Gesellschaften fragen; zum anderen beziehe ich mich auf Positionen, die Transformationen des Nationalen vor dem Hintergrund von Globalisierungsprozessen in den Blick nehmen. Ich möchte bereits an dieser Stelle drei wesentliche Konsequenzen dieser Auseinandersetzung für meine Forschung und dieses Buch besonders hervorheben:

(i) Im Rückgriff auf die europäisch-ethnologischen Arbeiten von Beate Binder, Wolfgang Kaschuba, Peter Niedermüller und Irene Götz ${ }^{25}$ fokussiere ich explizit Praxen und Herstellungszusammenhänge, durch die Schlüsselbestände des Nationalen innerhalb des Politikbereichs aktuell aufgerufen, inszeniert und mobilisiert werden. Mich interessiert hierbei insbesondere die Ausgestaltung von kulturpolitischen Strategien, die das Nationale auf eine flexible, mitunter improvisierende Weise einbinden, um in den unterschiedlichsten lokalen Feldern Anschlüsse und Effekte zu erzielen. So werde ich etwa am Beispiel der Vorbereitungen und Durchführung eines Oktoberfestes in Ramallah verdeutlichen, wie eine Erzählung von der kulturellen Besonderheit Deutschlands in der translokalen Praxis aus unterschiedlichen Elementen zusammengefügt wird (Kapitel III.4).

(ii) Ebenso untersuche ich Auswärtige Kulturpolitik - inspiriert durch die britische Sozialanthropologin Penelope Harvey - als eine »Technologie des Nationalen «. ${ }^{26}$ Durch diese Bezugnahme lässt sich verdeutlichen, dass die etablierten kulturpolitischen Infrastrukturen und Formate als Werkzeuge der Wissensproduktion - auch unabhängig vom Willen einer einzelnen Akteurin - bestimmte Vorstellungen von der epistemologischen Ordnung der Welt und insbesondere von der Bedeutung des nationalen Prinzips als struk-

25 | Siehe hierzu den in Fußnote 23 erwähnten, von Beate Binder et al. herausgegebenen Sammelband sowie: Irene Götz: Deutsche Identitäten. Die Wiederentdeckung des Nationalen nach 1989. Köln, Weimar, Wien 2011.

26 Penelope Harvey: Hybrids of Modernity. Anthropology, the nation state and the universal exhibition. London, New York 1996. 
turierendem Prinzip reproduzieren und auch sinnlich erfahrbar machen. Die Formulierung Technologie des Nationalen ermöglicht es mir somit, gerade die Beziehungen zwischen den infrastrukturellen Arrangements - also den Ressourcen, Orten, institutionellen Netzwerken und Fließmustern des Politikbereichs - und den Ordnungen des Wissens, die sie begleiten, zu untersuchen.

(iii) Gleichfalls greife ich Saskia Sassens Argument auf, dass das Nationale im Kontext von zeitgenössischen Globalisierungsdynamiken nicht verschwindet, sondern grundlegend transformiert wird und somit als eine relevante Kategorie erhalten bleibt. Sassens Vorschlag lautet, »Feldforschungen im Nationalen « zu betreiben, um nachzuzeichnen, wie Globalisierungs-, Transnationalisierungs- oder Kosmopolitisierungsprozesse eben innerhalb des Nationalen stattfinden und es von innen heraus verändern. ${ }^{27}$ Ich knüpfe an diese Aufforderung an und werde aufzeigen, dass das Nationale - trotz gewisser Dezentrierungsdynamiken zu Beginn des 2I. Jahrhunderts - als Dreh- und Angelpunkt der Auswärtigen Kulturpolitik nicht abhanden kommt, sondern in einer flexibilisierten Version fortbesteht: Insbesondere im Zuge der alltäglichen Wissens- und Übersetzungsarbeit deutscher Kulturmittler an Einsatzorten wie Ramallah, Sarajevo oder Tel Aviv erhält es ein translokales, partiell europäisiertes oder sogar kosmopolitisiertes Gewand.

Der Titel meines Buches greift diese Beobachtungen zu der bleibenden Wirkmächtigkeit des Nationalen als einem politischen, epistemologischen und alltagspraktischen Ordnungsprinzip auf. Gleichzeitig werde ich verdeutlichen, wie etablierte Konfigurationen des Nationalen durch das Auftauchen der Zielvorstellung Auswärtige Kulturpolitik als Konfliktprävention herausgefordert werden. Denn gerade mit ihren Aspirationen, sich explizit globalen Bruchlinien und Konfliktkonstellationen zuzuwenden, führt diese neue diskursive Formation zu einer unvermeidbaren Konfrontation mit einer Welt, die sich im doppelten Sinne als geteilt ${ }^{28}$ darstellt: Einerseits ist sie von vielfältigen Grenzziehungen, Exklusionen und Politiken der Differenz durchzogen und somit von einer zutiefst asymmetrischen Verteilung von Gesundheit, Zukunftschancen,

27 | Siehe hierzu: Saskia Sassen: Das Paradox des Nationalen. Territorium, Autorität und Rechte im globalen Zeitalter. Frankfurt 2008.

28 Hier im Anschluss an Shalini Randerias Bemerkungen zu der doppelten Bedeutung des deutschen Wortes "geteilt", das sich sowohl mit "divided" als auch mit "shared" ins Englische übersetzen lässt. Siehe hierzu: Shalini Randeria: Geteilte Geschichten und verwobene Modernen. In: Jörn Rüsen, Hanna Leitgeb \& Norbert Jegelka (Hrsg.): Zukunftsentwürfe: Ideen für eine Kultur der Veränderung. Frankfurt, New York 1999, S. 8796; Sebastian Conrad \& Shalini Randeria: Einleitung. Geteilte Geschichten - Europa in einer postkolonialen Welt. In: Dies. (Hrsg.): Jenseits des Eurozentrismus. Postkoloniale Perspektiven in den Geschichts- und Kulturwissenschaften. Frankfurt, New York 2002, S. 9-49. 
Wohlstand und Gewalt geprägt; andererseits bildet sie dennoch den einzig verfügbaren, gemeinsamen Kontext für globales Zusammenleben. Im Laufe dieses Buches wird deutlich werden, dass sich die Praxis Auswärtiger Kulturpolitik als Konfliktprävention in eben diesem Spannungsfeld zwischen den Ordnungen des Nationalen und der geteilten Welt bewegt. Dies zeigt sich etwa anhand von routinierten Bezugnahmen auf Bestände des Nationalen, um Konfliktkonstellationen an einem Einsatzort auch weiterhin als »externe Angelegenheiten « und »Probleme der Anderen « zu betrachten und somit zugleich eher klassische Vorstellungen von den Zuständigkeiten eines ausländischen Kulturinstituts an Einsatzorten wie Ramallah oder Sarajevo aufrechtzuerhalten. Dies zeigt sich aber auch anhand von Potenzialen der Kosmopolitisierung, die ich gegen Ende dieses Buches herausarbeiten werde und die in eine andere Richtung weisen (Kapitel IV). Hierunter verstehe ich Mikro-Dynamiken der Öffnung, Flexibilisierung oder Verflechtung von epistemologischen Grundfiguren des Politikbereichs, die als Effekte einer Konfrontation mit den lokal situierten Problemlagen, Themenstellungen und Erwartungen in »Konfliktgebieten « hervorgerufen werden - Konstellationen, die sich immer weniger innerhalb der etablierten Ordnungsmuster des Nationalen plausibel bearbeiten lassen. Bisher ereignen sich diese Potenziale der Kosmopolitisierung eher in der alläglichen Projektarbeit in den translokalen Praxis- und Übersetzungsfeldern, als dass sie wirklich beabsichtigt wären. Sollte die Konfliktträchtigkeit der gegenwärtigen Welt eine Bestätigung oder sogar eine Aktualisierung als Arbeitsfeld Auswärtiger Kulturpolitik erfahren, so wäre mein Plädoyer, eben diese Potenziale reflexiv voranzutreiben, stärker auch als intendierte Effekte zu betrachten und gleichfalls zu einer Neuausrichtung des politischen Narrativs heranzuziehen. Denn ein solcher Fokus würde es aus meiner Perspektive erforderlich machen, die symbolischen Räume des Nationalen als Refugien von Abgrenzung, Differenz und Selbstbespiegelung sukzessive hinter sich zu lassen und stattdessen die Erarbeitung von Positionen, welche die gegenwärtigen Bedingungen einer »gemeinsamen Zugehörigkeit zu ein und derselben Welt« - wie Achille Mbembe es ausdrückt ${ }^{29}$ - ausloten, als Zielhorizont kultureller Vermittlung zu setzen. So könnten innerhalb der etablierten Infrastrukturen Auswärtiger Kulturpolitik sukzessive kosmopolitische Wissens- und Handlungsräume entstehen, in denen die Konflikte, Brüche und Ungleichheiten einer geteilten Welt als integrative Bestandteile und nicht als äußerliche Probleme wahrgenommen und bearbeitet würden.

29 | Achille Mbembe: Kritik der schwarzen Vernunft. Frankfurt 2014, S. 11. 


\section{Rahmungen}

\section{Ethnografische Zugänge - theoretische Bezüge}

\section{Erste Einsichten: Auf der Suche NACH DER KONFLIKTPRÄVENTION}

Welche Relevanz besitzt der beschriebene diskursive Wandel für das Selbstverständnis und die Arbeitspraxis innerhalb des kulturpolitischen Akteursfeldes? Diese Frage markierte den Beginn der empirischen Arbeit für diese Studie und führte mich zu den Orten, an denen Auswärtige Kulturpolitik konzipiert, debattiert und umgesetzt wird: Ich nahm an Diskussionsveranstaltungen in Berlin teil, analysierte Materialien der Selbstdarstellung des Politikbereichs und traf mich mit Mitarbeiterinnen des Auswärtigen Amtes oder von Mittlerorganisationen zu ersten Gesprächen. Meine Fragen nach den Möglichkeiten einer konfliktpräventiven Auswärtigen Kulturpolitik riefen hier teils interessierte, mitunter aber auch reservierte oder sogar ablehnende Reaktionen hervor. Für meinen weiteren Forschungsverlauf ließen sich aus diesen frühen Beobachtungen zumindest zwei Schlussfolgerungen ziehen: Zum einen besaß Konfliktprävention für meine Gesprächspartner offenbar nicht die herausgehobene Bedeutung oder Dringlichkeit, die sich etwa auf der Stuttgarter Konferenz anzudeuten schien; zum anderen führten meine Fragen nicht zu allseits geteilten, eindeutigen Verständnisweisen, sondern eher zu unterschiedlichen Übersetzungsversuchen und Verknüpfungen mit etablierteren Zielvorstellungen. Meine »Suche nach der Konfliktprävention « stieß somit auf ein plurivokales Feld, in dem Begriffe, Bedeutungen und Arbeitsweisen in Bewegung geraten waren. Ich möchte nun anhand von sechs Positionen, die solche Reaktionen zu Beginn meiner Forschung prägnant zusammenfassen, die Perspektive auf dieses Akteursfeld, seine Dynamiken, Reibungen und Vielstimmigkeit eröffnen, um auf dieser Basis in den folgenden Abschnitten die konzeptionellen Grundlagen und methodischen Vorgehensweisen dieser Studie vorzustellen. 
"Wir stochern ziemlich im Nebel", äußert der Generalsekretär einer deutschen Mittlerorganisation, als ich inn zu Beginn meiner Forschung nach der Rolle der neuen Zielsetzung Konfliktprävention frage. Er zielt hierbei primär auf die Evaluierbarkeit der Wirkung einzelner Maßnahmen: „Es lässt sich nicht mit Gewissheit sagen, welche Instrumente im Hinblick auf die Krisenintervention erfolgreich sind."

Er reagiert somit ähnlich auf mein Forschungsvorhaben wie eine Mitarbeiterin des Auswärtigen Amtes wenige Wochen zuvor. Am Rande einer Veranstaltung in Berlin sagt sie: "Man kann es nicht überprüfen. Man müsste ja beweisen, dass setwas ${ }^{1}$ nicht stattgefunden hätte aufgrund unserer Projekte, Maßnahmen, Aktivitäten..." Beide formulieren entsprechend auch ein Interesse an den Ergebnissen meiner Forschung - in den Worten des Generalsekretärs: "Es wäre schon hilfreich, wenn wir wüssten, welche Maßnahmen welche Erfolge bringen. Auch dem Geldgeber gegenüber." In beiden Gesprächen begegne ich also einer ähnlichen Verbindung aus Interesse und Unsicherheit: Konfliktprävention gilt meinen Gesprächspartnern als eine relevante Zielsetzung; gleichzeitig wird deutlich, dass die deutsche Auswärtige Kulturpolitik hier ein ungesichertes Terrain betritt. Konfliktprävention gehört nicht zum fraglosen Kanon des Politikbereichs, sondern muss interpretiert, übersetzt und eingepasst werden. Ebenso zeigt sich, dass sich die Bedeutung dieser Zielsetzung aus ihren erhofften Effekten ergibt. Der Wunsch meiner Gesprächspartner, eine größere "Klarheit zu erzielen" - also einzelne Maßnahmen und ihre (politischen) Wirkungen eindeutig aufeinander beziehen zu können - wird vor diesem Hintergrund umso deutlicher sichtbar.

Im weiteren Gesprächsverlauf kommt der Generalsekretär zu einer Einschätzung, die mir im Vergleich zu dem Eingangs-Statement etwas widersprüchlich erscheint: Er evaluiert die Aktivitäten und Interventionen der „internationalen Gemeinschaft auf dem Balkan " positiv: "Ohne die ganzen Maßnahmen würde es da doch wieder knallen." Am Ende des Gesprächs bleibt eine Spannung zwischen dem eingestandenen nebulösen Wissen über die möglichen und tatsächlich erzielten Effekte, dem Wunsch nach evaluier- und beweisbaren Kausalitäten sowie einem intuitiven Glauben an die Wirksamkeit der kulturpolitischen Maßnahmen in konkreten Konfliktgebieten.

"Konfliktprävention ist zur Raison d'Être der Auswärtigen Kulturpolitik geworden", beschreibt ein leitender Beamter des Auswärtigen Amtes die Weiterentwicklung des Politikbereichs seit den späten 1990er Jahren: "Wir gehen bewusst in Konfliktregionen." Dieser Einstieg überrascht mich - als ich inn einige Wochen vorher zur Vorbereitung meiner Feldforschungen in Ramallah um ein Gespräch gebeten hatte, reagierte er ernüchtert: "Ach, die Konfliktprävention hat ja inzwischen nicht mehr so die Konjunktur."

Bei unserem Treffen begegnet mir die Perspektive des Geldgebers und zugleich der zentralen Steuerungsinstanz des weltumspannenden Netzwerkes aus Instituten,

1 Also ein gewalttätiger Konflikt oder eine Krise. 
Büros, Austauschprogrammen und Projekten, das durch die Förderung der Bundesregierung entstanden ist - eine Perspektive, die nach Effizienz und Wirkung der kulturpolitischen Aktivitäten schaut, insbesondere aber auch die Frage fokussiert, wie sich Handlungsspielräume für die deutsche Außenpolitik öffnen oder vergrößern lassen. Mein Gesprächspartner verweist auf die "schwierige Anfangsphase von Rotgrün" als Entstehungskontext der Zielsetzung Konfliktprävention: die Frage der Intervention im Kosovo, die Anschläge vom 11. September, Afghanistan, Irak - da kam die Frage auf: "Was kann man mit den Mitteln der Kultur hier erreichen?" Deutlich wird die Hoffnung auf - oder das Vertrauen in - "Kultur", die er in unterschiedlichen Äußerungen darstellt: "Kultur setzt da ein, wo Politik nicht mehr funktioniert"; oder "Kultur macht Dinge möglich, die politisch nicht laufen«; oder "Kultur kann da wirken, wo die Politik Mauern baut". Im Hinblick auf die gegenwärtige politische Situation in meinen Forschungsregionen sagt er: "Hätten wir die Kultur in Dayton oder Oslo dabei gehabt, dann wäre es vielleicht anders ausgegangen." Hier begegne ich also einem Diskursfeld, in dem entlang der neuen Zielsetzung auch die Beziehung zwischen "Politik" und "Kultur" in ihren unterschiedlichen Dimensionen für die deutsche Außenpolitik neu ausgelotet werden.

Mein Gesprächspartner belässt es aber nicht bei Grundsätzlichkeiten, sondern verweist auf praktische Suchbewegungen im Auswärtigen Amt: Er spricht von "Laboratorien" zur Entwicklung neuer "Querverbindungen" und "thematischer Überschneidungen«; er nennt Tagungen, auf denen sie mit neuen Themen experimentieren; er spricht von innovativen Ideen, die junge Mitarbeiterinnen verfolgen und von der Notwendigkeit des Ausprobierens neuer Gedankengänge, die mitunter auch im Nichts verlaufen. Deutlich wird die Suche nach neuen Formaten und inhaltlichen Verknüpfungen, um komplexe politische Problemstellungen im Modus der "Kultur" anders zu denken und zur Sprache zu bringen.

"Dialog führen und gleichzeitig missionieren wollen, ist ein Widerspruch, da will ich Sie drauf hinweisen", erklärt mir eine Diplomatin mit Blick auf das kulturpolitische Handlungsfeld Dialog mit der islamischen Welt, das als Folge der Anschläge von New York auf-, bzw. ausgebaut wurde und auf die Forderung "aus der Politik“ reagierte, "Maßnahmen zu entwickeln, die visibel sind ". Sie verweist in diesem Zusammenhang auf die Handlungszwänge, denen das Auswärtige Amt - anders als ein wissenschaftliches Forschungsprojekt - unterliege: Man müsse schnell auf die jeweilige Weltlage reagieren und die Öffentlichkeit erwarte dies auch.

In diesem Gespräch beeindruckt mich das Ringen um einen Ausgangspunkt, die Suche nach einer angemessenen Sprechposition für diesen Dialog - eine Suche, die durch eine grundlegende Spannung geprägt ist: "Man kann nicht von einem Dialog sprechen, wenn die eine Seite von ihrer Überlegenheit überzeugt ist und der anderen Seite den eigenen Weg absprechen möchte." 
Kulturpolitischen Herangehensweisen oder Fördermaßnahmen westlicher Akteure in Ländern des Nahen und Mittleren Ostens - so wird deutlich - ist häufig eine implizite Problematisierung des "Islams" oder eine Rückständigkeitsvermutung eingeschrieben: Verweise auf eine Modernisierungskrise der islamischen Welt, fehlende Demokratie, eine ausgebliebene Säkularisierung als Voraussetzung für Demokratisierung, die Unterdrückung der Frau oder die Gewaltbereitschaft konstituieren die Ausgangspunkte vieler Dialogprogramme und führen in einer gewissen Eigenlogik zur Übernahme einer missionarischen Rolle westlicher Akteure - eine Position, die meine Gesprächspartnerin als hierarchisierend und wenig zielführend erkennt: Sie spricht von "Übersetzungsfehlern", die entstehen, wenn "wir einen Bedarf, den wir empfinden, in die andere Seite hineinprojizieren“. Gleichzeitig hält sie es für unverzichtbar, im Rahmen solcher Dialogprogramme einen "festen Standpunkt" zu definieren und zu vertreten: "Man kann einen solchen Dialog nicht von einem wackligen Boot aus führen. Man braucht einen festen Boden." - Demokratie etwa, Menschenrechte oder Rechtsstaatlichkeit seien mögliche Ausgangspunkte. Aber befinden wir uns dann nicht wieder genau in der Sprechposition, von der sie sich zu Beginn unseres Gesprächs abgrenzen wollte, frage ich mich: Fest verankert in den eigenen Grundbegriffen, aber nicht hierarchisierend oder missionarisch - ist das machbar? Auch im Rahmen dieser Begegnung werden laborähnliche Arbeitsformen deutlich: das Bestreben, unterschiedliche Wissenstypen und Erfahrungen zu kombinieren, neue Zugänge zu finden, Vorzeigeprojekte zu konstruieren, kreativ zu sein und - eine angemessene eigene Sprechposition für den angestrebten Dialog zu definieren.

"Der Begriff ,Konfliktprävention، ist natürlich so breit, da kann man letztlich vieles oder alles drunter fassen ", erklärt mir eine deutsche Diplomatin in Ramallah, "und unser Ansatz, hier in Schulen eine qualitativ hochwertige Ausbildung mit zu unterstützen, hat meines Erachtens auch mit Konfliktprävention zu tun."

Die genaue Bedeutung der Zielsetzung Konfliktprävention für das Einsatzgebiet Palästinensische Gebiete - so zeigt mir dieses Gespräch - bleibt selbst Vertreterinnen des Auswärtigen Amtes eher unklar. Verständnisweisen und praktische Konsequenzen werden kaum durch die Zentrale vorgegeben, sondern müssen vor Ort entwickelt und ausgehandelt werden. Entsprechend ergeben sich für Diplomaten - und umso mehr für die entsandten Kulturmittler - Deutungs- und Handlungsspielräume, um eine solche Zielsetzung in die eigene Arbeitspraxis zu übersetzen. Bildungsprojekte stellen eine Möglichkeit dar, die offenbar sowohl in Berlin als auch in der deutschen Auslandsvertretung als plausibel erachtet wird; andere Übersetzungen wären denkbar. Die Beziehung zwischen politischen Zielen und lokalen Aktivitäten wird dabei laut meiner Gesprächspartnerin im Arbeitsalltag oft eher von der Praxis her gedacht: Es passiert demnach häufiger, dass man Projekte, die vor Ort entstehen, einer Zielsetzung zuordnet, als dass sie von vorneherein als eine systematische Umsetzung politischer Formulierungen angelegt und entwickelt würden. 
Gleichzeitig zeigt sich eine gewisse Unsicherheit, ob die gewählte Übersetzung tatsächlich "richtig" oder evaluierbar ist. Auf meine Frage, warum gerade Bildungsprojekte einen Beitrag zur Konfliktprävention darstellten, antwortet meine Gesprächspartnerin: "Also, einfach um Perspektiven zu geben, um den Leuten klar zu machen, wir bieten euch eine gute Bildung an und mit guter Bildung habt ihr euer Leben doch weitaus besser in der Hand und könnt vielleicht was erreichen, ohne euren Frust in Gewaltentladungen ausdrücken zu müssen. Ich weiß nicht, ob das soziologisch stimmt: Je besser man ausgebildet ist, desto weniger neigt man zu Gewalt. Vermutlich nicht (lachen). [...] Also, ja Bildung ist eine Art und Weise von rempowering, die vielleicht mittelfristig oder ganz mittelbar dazu führt, dass Konflikte abgebaut werden."

Im Hinblick auf die Frage nach der Übersetzung von Konfliktprävention erscheint mir hier die direkte Verknüpfung dieser Zielsetzung mit individuellen Lebenslagen und -entwürfen relevant: Offenbar sind es eher - potenziell gewalttätige - Individuen als gewaltdurchzogene politische Konstellationen, auf die man hofft, durch "Kultur» einwirken zu können.

"Das ist nicht der primäre Auftrag des Goethe-Instituts, dass man wirklich so Konfliktpräventionssachen, so Kulturentwicklungssachen macht, also diese ganzen Sachen, die wirklich Bedeutung haben für eine Postkonfliktgesellschaft", sagt mir ein bosnischer Mitarbeiter des Goethe-Instituts in Sarajevo. Die Effekte, die durch die Präsenzen und Aktivitäten deutscher Kulturmittler in Bosnien-Herzegowina möglicherweise erzielt werden können, schätzt er entsprechend nüchtern ein: "Das Land ist so kompliziert, diese ganzen Strukturen, Verhältnisse... Da kann ein Goethe-Institut einfach nicht viel machen, also mit den Kapazitäten, die es hat. Also da kann man so ein paar Sachen anreißen, aber nicht wirklich bewegen. Also diese Ideale braucht man auch nicht zu haben, meiner Ansicht nach." Hinzu kommt der andere Primärauftrag des Goethe-Instituts, den er folgendermaßen benennt: "deutsche Ideale oder deutsche Kultur - in welcher Form auch immer - sozusagen in das jeweilige Land zu bringen und zu präsentieren ".

Zu dieser inhaltlichen Distanzierung und den strukturellen Schwierigkeiten tritt eine ganze Reihe von praktischen Gründen, warum man von einem Goethe-Institut "auf keinen Fall" erwarten könne, "dass es noch dazu Konfliktprävention macht. Das geht einfach nicht! Dann musst du ein Institut mit 50 Mitarbeitern in jedem Land schaffen! Was will man denn mit sechs Mitarbeitern, die inhaltlich arbeiten? Mit den kleinen mickrigen Budgets? Das geht einfach nicht! Das Goethe-Institut hat auch inhaltlich nicht die Kenntnisse. Das Goethe-Institut hat die Strukturen nicht. Das Goethe-Institut hat auch nicht die politische Stärke, so was zu machen. Und wie soll man das, wie soll man auch durch Kultur Konfliktprävention machen?" In dieser umfassenden Abgrenzung verweist mein Gesprächspartner also erstens auf ein Spannungsverhältnis in dem institutionellen Selbstverständnis zwischen 
eigentlichem Kernauftrag und der Zielsetzung Konfliktprävention; er betont zweitens die gesellschaftliche und politische Komplexität einer Postkonfliktgesellschaft sowie drittens die mangelhafte Ausstattung eines deutschen Kulturinstitutes - mit Mitarbeiterinnen, Ressourcen, Kompetenzen, politischem Einfluss -, um ernsthaft konfliktpräventive Effekte erzielen zu können; und viertens bereitet es inm Schwierigkeiten, sich konzeptionell eine Konfliktprävention durch Kultur vorstellen zu können - dieser letzte Aspekt relativiert sich im Laufe unseres Gespräches als er, ähnlich wie andere Kulturmittler auch, dennoch konkrete Projekte und Programme seines Instituts benennt, die vielleicht doch zur Konfliktbearbeitung und zur "Entwicklungsarbeit im Kulturbereich" beitragen könnten.

"Ihre Frage ist eigentlich falsch gestellt", antwortet mir die Leiterin des GoetheInstituts in Sarajevo als ich sie frage, ob sich ein bestimmtes Projekt der Zielsetzung Konfliktprävention zuordnen lassen und sich daher für meine ethnografischen Beobachtungen eignen könnte. Aus Perspektive des Auswärtigen Amtes - so fährt sie fort - mache es vielleicht Sinn, das Goethe-Institut als ein Instrument der Konfliktprävention zu betrachten, aber ihre Perspektive sei eine andere: "Man kann die Arbeit des Goethe-Instituts nicht daran messen, ob sie Konflikte verhindert."

Eine ähnliche Haltung nimmt inr Kollege in Tel Aviv ein. Auf meine Frage "Was wäre aus Ihrer Perspektive eine sinnvolle Übersetzung der Zielsetzung Konfliktprävention durch Wertedialog?" antwortet er: "Ich habe mir über diese Zielsetzung noch nie wirklich Gedanken gemacht, muss ich Ihnen sagen. Nein, ich finde das deutlich zu hoch gestochen. Wir arbeiten im Kleinen. Also ich möchte an der Basis arbeiten. Und dass ich damit potenziell Konfliktprävention betreiben kann, will ich mal akzeptieren. Aber ich kann nicht sagen- Das ist einfach viel zu viel. Das kann das Auswärtige Amt sagen. Die müssen das sagen. Und die wollen das auch. Wenn ich mir das aber als Leitmotiv setze, kann ich nur scheitern. Das ist viel zu sehr zielorientiert, oder zielfixiert und nicht prozessorientiert. Ich denke, diese Prozesse, die wir versuchen in Gang zu setzen, tun sicher im Endeffekt genau das: Räume schaffen, geschützte Räume und offene Plattformen schaffen. Nichts anderes."

Meine Fragen - so wird hier deutlich - gingen mitunter von einer direkten Beziehung zwischen den politischen Zielsetzungen und dem Arbeitsalltag von Kulturmittlern aus, die für meine Gesprächspartner in dieser Form nicht gegeben war. Häufig reagierten sie mit einer doppelten Positionierung: Eine fast impulshafte Abwehr gegen die zu zielorientierte politische Formel wurde kombiniert mit Verweisen auf die eigene konkrete Arbeit an der Basis, die sie - zumindest mittelbar - doch mit dieser Zielsetzung in Verbindung bringen konnten: Projekte, Prozesse, Räume, Plattformen, die in Ramallah, Sarajevo, Jerusalem oder Tel Aviv gewissermaßen zwangsläufig im Kontext eines Konflikts operieren, an diese spezifische Konstellation auf unterschiedliche Weise anknüpfen und hierdurch eventuell eine intuitiv spürbare, aber nicht eindeutig messbare Wirkung erzielen. 
Diese sechs Positionen verdeutlichen nicht nur unterschiedliche Haltungen und Erfahrungen innerhalb eines vielstimmigen Akteursfeldes, sondern sie führen zu Fragen nach Machtrelationen und der Verteilung von Ressourcen, nach den inhaltlichen Verknüpfungen neuer politischer Schlüsselbegriffe und den möglichen Effekten einzelner kulturpolitischer Maßnahmen, nach der Ausgestaltung einer translokalen Wissensarbeit und generell nach den Beziehungen zwischen Zielsetzungen und Arbeitspraxis in einem institutionell und lokal weit verzweigten Politikbereich. Diese Fragen, die das gesamte Buch durchziehen, lassen sich mit dem theoretischen und methodischen Instrumentarium einer Anthropology of Policy bearbeiten. Entsprechend wird dieser Ansatz und seine Relevanz für diese Studie im folgenden Abschnitt diskutiert.

\section{Konzeptionelle Grundlagen: Politiken als anthropologische Forschungsfelder}

Dieses Buch ist als Beitrag zu einer Anthropologie des Politischen angelegt. Denn im Zentrum stehen hier die Beobachtung und Analyse der Dynamiken, Relationen und Effekte, die durch eine spezifische Politik ${ }^{2}$ hervorgerufen werden. Aber wie lässt sich ein Interesse an Auswärtiger Kulturpolitik als Konfliktprävention in ein explizit anthropologisches Forschungsprogramm überführen? Die Überlegungen zu einer Anthropology of Policy stellen hierzu den zentralen Bezugsrahmen dar. In diesem Ansatz geht es nicht alleine um die Anerkennung, dass »Kultur« immer auch eine politische Dimension besitzt oder um die Reflektion der politischen Implikationen anthropologischer Studien und Begrifflichkeiten, sondern eben um die Definition von »Policies« als elementare und eigenständige Felder anthropologischer Forschung. Den wichtigsten Referenzpunkt hierzu bilden zwei einflussreiche Sammelbände und insbesondere die beiden programmatisch gehaltenen Einleitungen der Herausgeber: ${ }^{3}$ Cris Shore und Susan Wright begründen hier ihre Forschungsperspektive gerade in Abgrenzung zu politikwissenschaftlichen Herangehensweisen, die - zumindest in ihrer Lesart - Politiken zumeist als »objektive Einheiten« und logisches »Ergebnis von den Entscheidungen rationaler Autoritäten « und

2 | Hier im Sinne des englischen Begriffes "policy", wie etwa in "Gesundheits- oder Wohnungsbaupolitik".

3 | Cris Shore \& Susan Wright: Policy: A new field of anthropology. In: Dies. (Hrsg.): Anthropology of Policy. Critical perspectives on governance and power. London, New York 1997, S. 3-40; sowie: Cris Shore \& Susan Wright: Introduction. Conceptualising Policy: Technologies of Governance and the Politics of Visibility. In: Cris Shore, Susan Wright \& Davide Però (Hrsg.): Policy Worlds. Anthropology and Analysis of Contemporary Power. New York, Oxford 2011, S. 1-25. 
Regierungseliten zur Bearbeitung »realer Problemlagen « konzipieren. ${ }^{4}$ Im Unterschied hierzu betrachten Shore und Wright »Policy« als ein grundlegendes »Organisationsprinzip« zur Gestaltung gegenwärtiger Gesellschaften, das unterschiedliche Kontexte und Gemeinschaften durchzieht, verbindet, verändert oder überhaupt erst erschafft und in diesem Rahmen immer auch umstrittene kulturelle Bedeutungen mobilisiert. Die gewachsene Bedeutung von Politiken ergibt sich demnach zunächst aus ihrem Vermögen, Individuen, Gruppen und soziale Räume zu klassifizieren, zu kategorisieren sowie zu kontrollieren und somit spätmoderne Gesellschaften grundlegend zu strukturieren: »Policy is a fundamental >organising principle $<$ of society which, like >family<, >nation<, >class< or >citizenship < provides a way of conceptualising and symbolising social relations, and around which people live their lives and structure their realities. $\ll^{5}$

Politiken durchdringen also zunehmend sämtliche Lebensbereiche der Menschen und bestimmen hierdurch zugleich die Formen, in denen sich Individuen als Subjekte wahrnehmen und konstruieren oder Kollektiven zuordnen: »From the cradle to the grave, people are classified, shaped and ordered according to policies, but they may have little consciousness of or control over the processes at work. $\aleph^{6}$

Aufgrund dieser vielfältigen Präsenzen und ihrer Gestaltungskraft betrachten Shore und Wright Politiken explizit als »anthropologische Phänomene«, deren Untersuchung zu zentralen Fragestellungen und etablierten Begrifflichkeiten des Faches führt: »The study of policy therefore leads straight into issues at the heart of anthropology: norms and institutions; ideology and consciousness; knowledge and power; rhetoric and discourse; meaning and interpretation; the global and the local.«

Politiken lassen sich demnach also als vielschichtige Beziehungsnetze oder auch räumlich und zeitlich zunächst schwer eingrenzbare Fließmuster verstehen. Sie bilden aber zugleich dynamische Machtfelder, in denen um Positionen, Einfluss, Ressourcen, gesellschaftliche Grundbegriffe und die Gestaltung von Wirklichkeit gerungen wird. Diese Dynamiken bleiben dabei nicht auf das engere Akteursfeld der policymakers begrenzt. Denn im Zuge eines politischen Prozesses entsteht eine vielstimmige Formation, die - je nach Politikbereich Interessenverbände, Universitäten, NGOs, Wirtschaftsunternehmen, Behörden, Medien, Politikerinnen und Bürger miteinander verbindet. Politiken wandern gewissermaßen durch unterschiedliche institutionelle und lokale Kontexte und können hier aufgegriffen, umgestaltet oder auch umgangen werden.

4| Cris Shore \& Susan Wright: Introduction. Conceptualising Policy, S. 3ff.

5 | Ebd., S. 2.

6 | Cris Shore \& Susan Wright: Policy: A new field of anthropology, S. 4; auch das folgende Zitat. 
Die Relevanz und Wirkmächtigkeit einer Politik lässt sich entsprechend nicht alleine durch eine Forschung in den offiziellen Foren und Entscheidungszentren erfassen. Die Adressatinnen, Kooperationspartner und Vermittlungsinstanzen sind Teil des Feldes, in dem eine Politik ihre Bedeutung und ihre Gestalt erhält.7 Folgerichtig verstehen Shore und Wright Politiken auch nicht als exklusive Instrumente staatlicher Akteure: Internationale Organisationen, NGOs, Unternehmen oder Agenturen entwickeln und nutzen gleichfalls Policies, um Räume, Individuen und Gruppen zu regulieren, Ressourcen zu verteilen, Loyalitäten einzufordern oder Diskurse zu verändern. Die anthropologische Analyse von Politiken führt entsprechend in die unübersichtlichen Felder spätmoderner, gerade auch transnationaler Machttechniken. Eine Anthropology of Policy verfolgt nicht alleine das Ziel, eine jeweilige Politik zu erforschen, sondern beabsichtigt zugleich »elementare und die gegenwärtige Welt prägende Prozesse der Regierungsführung, der Machtausübung und des sozialen Wandels aufzudecken «. 8

Im Anschluss an diese grundlegenden Überlegungen möchte ich fünf Aspekte dieses Forschungsprogramms herausstellen, die für die konzeptionelle Rahmung meiner Studie von besonderer Bedeutung sind.

\section{(i) Politiken führen soziale Leben}

Zunächst betonen Shore und Wright die Produktivität und Prozesshaftigkeit einer Policy. Eine Politik »arbeitet« demnach und stellt permanent neue Verbindungen zwischen Bürgerinnen, Institutionen, Ressourcen und staatlichen Akteuren her. Ihre Kontur und ihre Beschaffenheit verändern sich kontinuierlich sowohl im Rahmen der politischen Auseinandersetzungen, die ihre Konzipierung umgeben, als auch in ihrer Umsetzung. Im Anschluss an Arjun Appadurai sprechen Shore und Wright von den »komplexen sozialen Leben«, die Politiken führen: Sie gestalten ihre Umgebung, gehen Beziehungen mit Institutionen und Akteursgruppen ein, verändern sich im Laufe der Zeit, produzieren unerwartete Effekte und entfalten häufig eine längere Wirkung als von ihren Initiatorinnen erdacht. Unter Bezugnahme auf Bruno Latour benennen sie Politiken als »Aktanten « und schreiben ihnen somit ein eigenes Handlungsvermögen $\mathrm{zu}$ - ein Handlungsvermögen, das sich etwa in ihren Interaktionen und Beziehungsgeflechten zeigt: »Policies have agency; they shift action, and, like machines, they perform tasks and are endowed with certain competencies. Importantly, actants typically interact with other social agents in processes that are dynamic and contingent, and therefore have unpredictable effects. «

7 | Cris Shore \& Susan Wright: Introduction. Conceptualising Policy, S. 12.

8 | Ebd., S. 1. Übersetzungen - auch folgende - durch den Autor.

9 | Cris Shore \& Susan Wright: Introduction. Conceptualising Policy, S. 3. Im Falle von "sozialen Leben" beziehen sie sich primär auf: Arjun Appadurai (Hrsg.): The Social Life 


\section{(ii) Politiken operieren durch Sprache}

Zweitens arbeiten Shore und Wright die Bedeutung von Sprache und insbesondere von geschriebenen Dokumenten für die Konstituierung einer Politik heraus: Strategiepapiere, Reden, Protokolle von parlamentarischen Debatten, Regierungsberichte oder auch mediale Erzeugnisse betrachten sie als »kulturelle Texte«, durch die Bedeutungen produziert und die »Problematik« eines Politikbereichs formuliert, diskutiert oder in Frage gestellt werden. ${ }^{10}$

Policies operieren demnach durch Diskurse, ${ }^{11}$ besitzen aber gleichzeitig das Vermögen, diese zu verändern. So kann ein Regierungsdokument einen Politikbereich mit einem alternativen Diskurs verknüpfen, bisher unbekannte diskursive Figuren einführen oder neue Begriffe setzen. Von Interesse sind hierbei nicht alleine die Bedeutungen und Verbindungen, die sich aus dem Dokument herauslesen lassen, sondern gerade auch die »diskursiven Praxen«, durch die etwa Probleme aktiv definiert oder Gruppen kategorisiert werden. Die Beschäftigung mit solchen Texten führt also zu konkreten Akteuren, die über die Macht verfügen, eine Problemstellung oder einen Handlungsbedarf festzulegen, Richtlinien für die politische Auseinandersetzung zu bestimmen und hierüber auch Alternativen auszuschließen. ${ }^{12}$ Das Verfassen von politischen Dokumenten zeigt sich aus dieser Perspektive als eine aktive, machtdurchzogene Wissenspraxis. Eine Analyse der Diskurse und Rhetoriken führt aber nicht nur zu diesen Akteuren, sondern zugleich zu den Rationalitäten, Ideologien oder kulturellen Logiken, die innerhalb des untersuchten Feldes dominieren. Der Erkenntnisgewinn soll dabei über den jeweiligen Politikbereich hinausgehen: Die Erforschung der Diskurse einer Policy »bietet den Schlüssel zur Analyse der Architektur gegenwärtiger Machtbeziehungen «. ${ }^{13}$

Shore und Wright schlagen zur Erforschung solcher diskursiven Felder die Konzentration auf »Schlüsselbegriffe«, »Kern-Symbole« oder »Metaphern« vor. Die Entwicklung einer neuen politischen Argumentation, die Verflechtungen zwischen diskursiven Figuren und einem bestimmten Politikbereich oder grundlegende Veränderungen eines Diskurses sollten sich anhand des Bedeutungs- und Positionswandels solcher Schlüsselbegriffe nachzeichnen

of Things: Commodities in Cultural Perspective. Cambridge, New York 1986; im Falle von "Aktanten" auf die Arbeiten Bruno Latours, etwa: Aramis or the Love of Technology. Cambridge, MA 1996.

10 | Cris Shore \& Susan Wright: Introduction. Conceptualising Policy, S. 15.

11 | "Diskurse" definieren sie als "Konfigurationen von Ideen, die die Fäden zur Verfügung stellen, aus denen Ideologien gewoben werden «. In: Cris Shore \& Susan Wright: Policy: A new field of anthropology, S. 18.

12 Cris Shore \& Susan Wright: Policy: A new field of anthropology, S. 18.

13 | Ebd., S. 12. 
lassen. ${ }^{14}$ Gelegentlich führen politische Dokumente völlig neue Metaphern in ein jeweiliges politisches Feld ein; häufig werden aber auch ältere Schlüsselbegriffe in neue Gruppierungen von Bedeutungen integriert: Ein bekannter Begriff erscheint in einer neuen Kombination und erhält hierdurch eine andere Aussagekraft. Die Relevanz solcher Metaphern für die Analyse politischer Felder ergibt sich auch daraus, dass sie »historische Bedeutungen akkumulieren« können. Vergangene Konnotationen bleiben ihnen demnach eingeschrieben und sind potenziell reaktivierbar, auch wenn diesen in einem aktuellen politischen Diskurs ein neuer Sinngehalt zugewiesen wird: »The important point, however, is that keywords accumulate meanings historically and that whereas one meaning may dominate at any particular moment, previous meanings, although eclipsed, can always be resurrected. $\ll^{15}$

Erlangen einzelne Begriffe eine hervorstechende Dominanz, so lassen sie sich als »mobilizing metaphors « untersuchen. ${ }^{16}$ Die Zugkraft, die solche besonders flexiblen und wirkmächtigen Schlüsselbegriffe erzielen, erklärt sich demnach durch zwei Eigenschaften: Einerseits besitzen sie das Vermögen, eine Vielzahl von Verknüpfungen mit anderen Konzepten oder Symbolen einzugehen und somit Bedeutungen zu akkumulieren. Sie nehmen also in dem diskursiven Feld, das einen Politikbereich strukturiert, eine zentrale Position ein. Andererseits lassen sich über diese Metaphern Zustimmung und Loyalitäten in einer Bevölkerung oder einer sozialen Gruppe herstellen. Diese Kategorie von Schlüsselbegriffen zeichnet sich also dadurch aus, dass sie sowohl auf diskursiver Ebene als auch in sozialen und politischen Praxisfeldern weitreichende Effekte erzielt. Die Produktivität und die wandelnden Konfigurationen einer Policy sollten sich demnach anhand der Bewegungen und Anschlüsse solcher »mobilizing metaphors« empirisch nachzeichnen lassen.

\section{(iii) Politiken besitzen eine imaginäre Dimension}

Drittens verweisen Shore und Wright auf die imaginäre Dimension politischer Felder. Eine Policy ruft demnach Vorstellungen von sich selbst und ihrem Wirken hervor - Vorstellungen, die sich zu »umstrittenen Narrativen« zusammenfügen und so durch Raum und Zeit wandern. ${ }^{17}$ In der Tat produzieren viele Politiken Erzählungen, etwa vom guten und richtigen Leben, von Gerechtigkeit, einer besseren Zukunft, von notwendigen Veränderungen oder vom Glück und möglichen Scheitern. Erfolgreiche Politiken etablieren ein offizielles Narrativ von dem zentralen Problem sowie seiner Lösung in einem jeweiligen Feld. Eine Anthropology of Policy untersucht, wie solche Narrative entstehen,

$14 \mid$ Ebd., S. 18.

$15 \mid$ Ebd., S. 19.

16 | Ebd., S. 20.

17 | Cris Shore \& Susan Wright: Introduction. Conceptualising Policy, S. 13. 
insbesondere aber wie sie sich in neue soziale und politische Räume hineinbewegen und hierbei immer wieder aufs Neue übersetzt werden müssen. Shore und Wright betonen zugleich, dass solche Erzählungen nicht zwingend in politischen Macht- und Entscheidungszentren ihren Ausgang nehmen müssen:

"Sometimes, this movement is initiated from the top, with a narrative translated from a political speech to newspaper articles, to a law text, to guidance notes intended to reform bureaucratic action, to technical procedures to follow or forms to fill in on paper or on the computer. At other times, this movement starts among user groups or professionals who provide a service. Each move necessarily entails a re-translation from one genre to another, opening up space for further contestation. At every moment of translation, new voices enter with new ways of seeing the problem, reinforcing or contesting the concepts and assumptions written into the policy texts. “ ${ }^{18}$

Durch die ethnografische Verfolgung von umstrittenen Narrativen können also sowohl ihr Wandern zwischen unterschiedlichen lokalen und institutionellen Kontexten als auch ihre Wechselbewegungen zwischen verschiedenen Medien, Genres und Formaten nachgezeichnet werden. Als Konsequenz lassen sich die Konstellationen und Praxen, in denen politische Narrative übersetzt werden, als privilegierte Orte für die anthropologische Erforschung eines Politikbereiches betrachten. Denn in solchen Situationen werden Verbindungen und Bedeutungen konkret ausgehandelt und hergestellt; eine Policy zeigt sich hier als »a political process involving many actors all proposing how people should relate to each other, conduct themselves and be governed «. ${ }^{19}$

\section{(iv) Politiken und Handlungsvermögen}

Politiken sind also produktiv, entfalten vielfältige Effekte, prägen Diskussionen und dringen in die unterschiedlichsten gesellschaftlichen Räume ein aber wie steht es mit dem Handlungsvermögen von Individuen oder Gruppen, die im Kontext solcher Konfigurationen agieren? Hier lässt sich innerhalb des Ansatzes eine Spannung erkennen:

Einerseits betonen Shore und Wright die Macht von Politiken, die Diskurse und Kategorien zu setzen, über die gesellschaftliche Wirklichkeit debattiert, strukturiert und wahrgenommen wird. Durch Policies lassen sich gleichzeitig Regierungslogiken und Machttechniken etablieren, die eine Bürgerin mit neuen Verhaltensanforderungen, Wertigkeiten und »Idealen vom Selbst« konfrontieren. So lässt sich entlang von Politiken etwa die Durchsetzung von neoliberalen Rationalitäten und Subjektivitätsvorstellungen in lokalen Ge-

18 | Ebd., S. 14.

19 | Ebd., S. 14. 
meinschaften und Räumen nachzeichnen..$^{20}$ Insbesondere in dem ersten Band entsteht das Bild von Politiken, die in alle Lebensbereiche eindringen und deren Kategorisierungen, Logiken und Technologien sich die Individuen kaum entziehen können.

Andererseits wird im zweiten Band deutlich stärker ein kritisches Potenzial von Bürgern und Bürgerinnen betont, sich eben solchen dominanten Diskursen, politischen Ordnungsprinzipien und den damit verbundenen Vorstellungen guter Lebensführung zu widersetzen. Individuen erscheinen hier nicht einfach als »durch die Mikro-Physik der Disziplinierungsmacht produzierte fügsame Körper « ${ }^{21}$, sondern sie werden als »skeptische« und »reflexive Subjekte« fokussiert, die unter bestimmten Bedingungen die Machtlogiken und Deutungsansprüche einer Politik durchschauen und oppositionelle Haltungen zu entwickeln vermögen. Unter welchen Umständen dieses kritische Potenzial von Individuen die Oberhand gewinnt und eine Politik ihre hegemoniale Macht verliert, wird nicht abschließend geklärt und bleibt somit eine empirische Fragestellung. Entsprechend zeigen einige der Beiträge, wie im Zuge eines politischen Prozesses (ungeplante) Handlungsspielräume für unterschiedliche Formen der Einmischung und des Protests, für oppositionelle Allianzen, kritische Haltungen und widerständige Praxen entstehen. ${ }^{22}$

\section{(v) Forschungsstrategien einer anthropologischen Policy-Forschung}

Eine Politik wird hier also nicht als ein primär durch Regierungshandeln bestimmter »objektiver Sachverhalt« begriffen, sondern als ein »sozialer und politischer Raum, der durch Machtbeziehungen und Systeme der Regierungsführung gegliedert wird «. ${ }^{23}$ Policies verbinden Orte, Akteursgruppen, Ressourcen und Wissensbestände und durchfließen lokale, nationale und globale Ebenen. Konzipiert man den Untersuchungsgegenstand entsprechend trans-

20 | Siehe exemplarisch: Susan Brin Hyatt: Poverty in a 'post-welfare, landscape: Tenant management policies, self-governance and the democratization of knowledge in Great Britain. In: Cris Shore \& Susan Wright (Hrsg.): Anthropology of Policy, S. 217-238.

21 Cris Shore \& Susan Wright: Introduction. Conceptualising Policy, S. $17 f$.

22 | Siehe folgende Beiträge in dem Band «Policy Worlds": Gritt B. Nielsen: Peopling Policy: On Conflicting Subjectivities of Fee-Paying Students, S. 68-85; Shalini Randeria \& Ciara Grunder: The (Un)Making of Policy in the Shadow of the World Bank: Infrastructure Development, Urban Resettlement and the Cunning State in India, S. 187-204; Dorothy Louise Zinn: The Case of Scanzano: Raison d'État and the Reason for a Rebellion, S. 227-243; Davide Però: Migrants' Practices of Citizenship and Policy Change, S. 244263; Birgit Müller: The Elephant in the Room: Multistakeholder Dialogue on Agricultural Biotechnology in the Food and Agriculture Organization, S. 282-299.

23 | Cris Shore \& Susan Wright: Policy: A new field of anthropology, S. 14. 
lokal, kaum begrenzbar und vielfältig verflochten, so stellt sich die Frage nach seiner ethnografischen Erforschbarkeit: Welche methodischen Vorschläge entwickeln die Protagonisten einer Anthropology of Policy, um solche Beziehungsnetze und Machtfelder zu untersuchen?

Grundsätzlich greifen Shore und Wright auf akteurszentrierte Vorgehensweisen zurück, wie sie auch innerhalb der Europäischen Ethnologie etabliert sind. Von Interesse sind demnach die Bedeutungen und Funktionen, die eine jeweilige Policy für unterschiedliche Personengruppen besitzt. Die Stärke ethnografischer Methoden liegt dabei in »its sensitivity towards the way in which policies work as instruments of governance, and its concern to explore how policies are understood by differently situated actors « ${ }^{24}$ Allerdings stellen diese Erkundungen der Bedeutungen und Konsequenzen einer Politik für einzelne Personen, lokale Gemeinschaften oder Akteursgruppen nicht das eigentliche Ziel dar; sie bilden vielmehr Ausgangspunkte, um die im Rahmen einer Policy entstehenden Fließmuster und Verflechtungen zwischen unterschiedlichen Akteuren, Diskursen, Orten, Ebenen, sozialen Prozessen sowie deren Auswirkungen auf Lokalitäten oder Gemeinschaften in den Blick zu nehmen. ${ }^{25}$ Zum zentralen Kriterium zur Bestimmung der »Topographie des Feldes« wird daher die Frage nach dem Spektrum von »Organisationen oder Personengruppen, die sich in irgendeiner Weise an dem Prozess der Auseinandersetzung über eine Politik beteiligen können «. ${ }^{26}$ In Anknüpfung an Pierre Bourdieus Überlegungen zur Begrenzung eines Feldes ${ }^{27}$ ließe sich formulieren, dass es die Gesamtheit der Akteure einzubeziehen gilt, die über Potenziale oder Kapitalien verfügen, im Rahmen einer Policy Effekte auszuüben.

Der Anspruch lautet, Herangehensweisen zu entwickeln, um »die Wege nachzuzeichnen, durch die >Macht< Netzwerke und Beziehungen zwischen Akteuren, Institutionen und Diskursen durch Zeit und Raum schafft«. ${ }^{28}$ Für die methodische Umsetzung bedeutet dies, akteurszentrierte Forschungspraxen mit Forschungsstrategien einer multi-sited ethnography sowie mit Diskursanalysen zu verbinden. Insbesondere im neueren Sammelband werden zumindest zwei Vorgehensweisen vorgeschlagen:

24 | Cris Shore \& Susan Wright: Introduction. Conceptualising Policy, S. 20.

25 | Cris Shore \& Susan Wright: Policy: A new field of anthropology, S. 14.

26 | Cris Shore \& Susan Wright: Introduction. Conceptualising Policy, S. 11.

27 | "[...] die Grenze eines Feldes ist die Grenze seiner Effekte oder, andersherum, ein Akteur oder eine Institution gehören insoweit zu einem Feld, als sie in inm Effekten unterliegen und Effekte produzieren. In: Pierre Bourdieu: Die Praxis der reflexiven Anthropologie." In: Pierre Bourdieu \& Loïc Wacquant: Reflexive Anthropologie. Frankfurt 2006; S. 251-294, S. 266.

28 | Cris Shore \& Susan Wright: Policy: A new field of anthropology, S. 14. 
Erstens diskutieren Susan Wright und Sue Reinhold die Strategie eines »studying through«, die den Verlauf öffentlicher Diskussionen und Konflikte in das Zentrum einer ethnografischen Forschung rückt: »)Studying through< follows a discussion or a conflict as it ranges back and forth and back again between protagonists, and up and down and up again between a range of local and national sites. ${ }^{29}$

Der Vorschlag lautet also, einen Prozess der politischen Auseinandersetzung in seinen historischen Entwicklungslinien und zirkulären Verflechtungen durch unterschiedliche lokale Räume, Institutionen, Medien und Akteurskonstellationen zu verfolgen, um anhand des Bedeutungswandels von Schlüsselbegriffen sowie der Entstehung neuer diskursiver Formationen den Ablauf politischer Transformationen analysieren zu können. Die Konzentration auf Metaphern und Narrative soll demnach nicht nur den Einstieg zur Erforschung von Machtfeldern bieten, sondern gleichfalls zum Verständnis längerfristiger und grundsätzlicher gesellschaftlicher Wandlungsprozesse führen.

Zweitens finden sich in dem Sammelband Beiträge, die Politiken und ihre Machtfelder durch Bezugnahmen auf Michel Foucaults Konzept von »Dispositiven« oder zeitgenössische Diskussionen $\mathrm{zu} »$ Assemblagen« $\mathrm{zu}$ analysieren versuchen. ${ }^{30}$ Hier steht der Gedanke im Zentrum, dass zur Regulierung von politischen oder ökonomischen »Problemen« temporäre Ensembles aus so unterschiedlichen Elementen wie Institutionen, Gesetzen, administrativen Maßnahmen, politischen Logiken, materiellen Ressourcen, Praxen, architektonischen Arrangements usw. entstehen, die sich als Dispositive oder noch nicht in gleicher Weise verstetigte Assemblagen begreifen lassen - in beiden Fällen Felder der Machtausübung, für die kein klares Zentrum, keine eindeutige Autorin identifiziert werden kann. Gleichzeitig zeigen einige der Buchbeiträge, dass sich gerade in den Zwischenräumen oder Kontaktzonen eines solchen Gefüges Handlungsspielräume eröffnen können, um Regulierungsansprüche zu umgehen und somit auf politische Felder einzuwirken. Eine solche Forschungsstrategie kann daher sowohl zu einem Nachzeichnen der wandelbaren Ansammlungen heterogener Elemente als auch zu einer Zentrierung auf Ak-

29 | Susan Wright \& Sue Reinhold: ,Studying through: A Strategy for Studying Political Transformation. Or Sex, Lies and British Politics. In: Cris Shore, Susan Wright \& Davide Però (Hrsg.): Policy Worlds, S. 86-104, S. 101. Siehe hierzu auch: Cris Shore \& Susan Wright: Policy: A new field of anthropology, S. 14.

30 | Siehe: Michel Foucault: "Le jeu de Michel Foucault". In: Ders.: Dits et écrits II, Paris 1994 (1977), S. 298-329; Hubert Dreyfus \& Paul Rabinow: Michel Foucault. Jenseits von Strukturalismus und Hermeneutik. Weinheim 1994; Paul Rabinow: Anthropologie der Vernunft. Studien zu Wissenschaft und Lebensführung. Frankfurt 1994; Aihwa Ong \& Stephen J. Collier (Hrsg.): Global Assemblages. Technology, Politics, and Ethics as Anthropological Problems. Malden, Oxford, Carlton 2005. 
teure, ihre Strategien und Handlungsspielräume innerhalb dieser temporären Ensembles führen. ${ }^{31}$ Eine Policy kann hierbei entweder als eines der Elemente oder als Ausgangspunkt für die Entwicklung einer Assemblage oder eines Dispositives untersucht werden.

In all diesen Forschungsstrategien wird eine Politik also als eine Konfiguration fokussiert, die sich nicht alleine durch eine klassische ortsgebundene teilnehmende Beobachtung untersuchen lässt: ${ }^{32}$

"The key is to grasp the interactions (and disjunctions) between different sites or levels in policy processes. Thus, studying through entails multi-site ethnographies which trace policy connections between different organizational and everyday worlds, even where actors in different sites do not know each other or share a moral universe." ${ }^{33}$

Die Herausforderung besteht darin, »Aussichtspunkte « zu identifizieren und miteinander zu kombinieren, von denen aus sich diese Verflechtungen, Netzwerke, diskursiven Verschiebungen und Regierungstechniken ethnografisch beobachten lassen. Im Prinzip können hierzu sehr unterschiedliche Mikro-Felder herangezogen werden: soziale Konstellationen, in denen unterschiedliche Akteurs- und Interessensgruppen interagieren; Situationen, in denen ein politisches Narrativ übersetzt wird; Kontaktzonen, in denen ein dominanter Diskurs hinterfragt wird; Institutionen, in denen Diskurse und Politikbereiche zusammenfließen; Behörden, in denen soziale Gruppen als Adressatinnen einer Politik definiert werden; oder Dokumente und Reden, die ein »politisches Problem« formulieren.

31 | Siehe hierzu etwa die bereits angeführten Beiträge im Buch "Policy Worlds" von: Gritt B. Nielsen, Dorothy Zinn, Davide Però, Clarissa Kugelberg und Birgit Müller sowie den Artikel von Gregory Feldman: Illuminating the Apparatus: Steps toward a Nonlocal Ethnography of Global Governance. In: Ebd., S. 32-49.

32 Gregory Feldman entwickelt in seinem Beitrag den Vorschlag zu einer "nonlocal ethnography" und stellt hierdurch etablierte ortsgebundene Vorgehensweisen der Ethnografie grundlegend in Frage: Ein Ort oder eine Institution bilden demnach nur den Ausgangspunkt, um das Zusammenfließen von Diskursen, politischen Rationalitäten, Wissenspraxen und Machtfeldern zu wirkmächtigen Dispositiven zur Regulierung eines politischen oder ökonomischen "Problems" zu untersuchen.

33 | Cris Shore \& Susan Wright: Policy: A new field of anthropology, S. 14. 


\section{Perspektivierung: Imaginäre Dimensionen, Mobilisierungen und Wissensarbeit in der Auswärtigen Kulturpolitik}

Aus den diskutierten Überlegungen lassen sich zumindest zwei Weisen herauslesen, eine Politik als ein anthropologisches Feld zu konzipieren und ethnografisch zu erforschen: Einerseits als einen Prozess der Auseinandersetzung, der sich durch unterschiedliche Arenen, Ebenen, Medien, lokale und institutionelle Kontexte entfaltet; andererseits als ein Gefüge aus heterogenen Elementen mit einer jeweiligen Emergenz, Dynamik und Beschaffenheit. Beide Herangehensweisen schließen sich nicht aus. Entsprechend kombiniere ich sie in dieser Studie, indem ich sowohl die Mobilisierung eines politischen Narrativs durch die unterschiedlichen Kontexte seiner Entstehung und Wirkung verfolge als auch Prozesse des Zusammenfügens von diskursiven Formationen, Argumentationsfiguren, Akteuren, Ressourcen, Institutionen und Orten zu einer kohärent erscheinenden Politik in den Blick nehme. Ich habe hierzu drei analytische Perspektiven entwickelt, die ich nun als Beitrag zu einer Anthropo$\log$ of Policy ausformulieren möchte:

Erstens frage ich nach den imaginären Dimensionen Auswärtiger Kulturpolitik. Ich untersuche hierzu sowohl den Verlauf der Diskussionen auf Tagungen des Politikbereichs als auch die diskursiven Bewegungen, die sich aus politischen Papieren und Regierungsdokumenten herausarbeiten lassen. Ich identifiziere die dominanten Schlüsselbegriffe und zeichne nach, welche Bedeutungen sie über die Jahrzehnte akkumulieren konnten sowie in welch unterschiedlichen Konfigurationen sie auftreten. Auf dieser Basis lassen sich die zentralen Rationalitäten und epistemologischen Ordnungen herausstellen, welche die imaginären Dimensionen des Politikbereichs strukturieren. Zugleich werden Entstehungskontexte, Akteurskonstellationen und Dynamiken der Ausgestaltung des offiziellen Narrativs zur Legitimierung und Erklärung Auswärtiger Kulturpolitik sichtbar.

Hierauf aufbauend analysiere ich zweitens die Mobilisierungen, die im Rahmen dieser Policy hervorgerufen werden sowie die Bahnen, über die diese Mobilisierungen verlaufen. Ganz im Sinne einer multi-sited ethnography verfolge ich das »Narrativ« und die zentralen »Metaphern«, aber auch die Kulturmittler durch die unterschiedlichen Foren und Arbeitsfelder des Politikbereichs. ${ }^{34}$ Ich frage zum einen, was mit der offiziellen Erzählung passiert, wenn sie translokale Übersetzungsketten durchläuft und an Orten wie Ramallah, Sarajevo oder Tel Aviv den Boden berührt; zum anderen interessieren mich die Effekte, die solche Mobilisierungen in diesen lokalen Kontexte hervorrufen. Diese Fra-

34 | Siehe zu den Forschungsstrategien "Follow the People", "Follow the Metaphor" und "Follow the Plot, Story, or Allegory": George Marcus: Ethnography in/of the World System, S. $106 \mathrm{ff}$. 
gen führen direkt zu den Machtrelationen und institutionellen Arrangements, die den Politikbereich gegenwärtig auszeichnen und zugleich die Bahnen konfigurieren, über die Metaphern, Narrative, Personen oder Ressourcen mobilisiert werden.

Drittens untersuche ich die Wissensarbeit, die in den translokalen Praxisund Übersetzungsfeldern Auswärtiger Kulturpolitik vonstattengeht - anders formuliert: Ich fokussiere die Kontexte, in denen aus den materiellen Ressourcen und den mobilisierten Beständen des politischen Narrativs kulturpolitische Praxis wird. Von Interesse ist hierbei primär die Übersetzungsarbeit, die Kulturmittler zwischen den Zielvorstellungen und Rationalitäten des Politikbereichs einerseits und den Rahmenbedingungen und Bedürfnislagen in den jeweiligen lokalen Arbeitskontexten andererseits realisieren. Eine solche praxeografische Perspektive - die innerhalb der Anthropology of Policy angelegt, bisher aber nicht hinreichend ausformuliert worden ist - lenkt den Blick nicht nur auf vier unterschiedliche kulturpolitische Strategien (Kapitel III), die diese Übersetzungsarbeit auf eine jeweilige Weise »lösen«, sondern zugleich auf die Notwendigkeit, in einem global verzweigten Politikbereich durch verschiedene Verfahren Kohärenz herzustellen. Die translokale Übersetzungsarbeit der Kulturmittler leistet hierzu einen wesentlichen Beitrag. Vor diesem Hintergrund lässt sich auch die Frage aufgreifen, ob sich die Position von Kulturmittlern in diesen Praxis- und Übersetzungsfeldern eher als »fügsame Körper« oder als »reflexive Subjekte« verstehen lässt. Orientieren sie sich also in der Vermittlungsarbeit an ihren Einsatzorten eng an den Vorgaben, Rationalitäten und Regierungslogiken der politischen Zentren? Oder entwickeln sie kritische Perspektiven auf die Deutungsansprüche des politischen Narrativs, die ihnen erweiterte Handlungsspielräume für die eigene translokale Arbeitspraxis eröffnen?

Für die methodische Umsetzung dieses Forschungsprogramms habe ich längerfristig in Diskussionsräumen, Kontaktzonen, Praxis- und Übersetzungsfeldern des Politikbereichs in Berlin, Ramallah und Sarajevo geforscht und zusätzlich kürzere Einblicke in die Arbeit der Goethe-Institute in Tel Aviv und (West-)Jerusalem genommen. Ich betrachte diese Kontexte als Aussichtspunkte, um den Prozess der Auseinandersetzung um die Möglichkeiten einer Auswärtigen Kulturpolitik als Konfliktprävention und somit das translokale Gefüge dieses Politikbereiches beobachten und analysieren zu können. Das komplexe soziale Leben dieser Policy wird in den Kapiteln II und III von eben diesen Aussichtspunkten her beleuchtet. Bevor ich mich aber dort der Analyse der imaginären Dimensionen sowie der translokalen Übersetzungsarbeit Auswärtiger Kulturpolitik zuwende, möchte ich zeitgenössische Positionen zur Erforschung des »Nationalen« als den zweiten zentralen theoretischen Bezugsrahmen meiner Arbeit diskutieren: Denn im Zuge meiner Forschung wurde immer deutlicher, wie stark Schlüsselmetaphern und Narrative des Politikbereichs auf Re- 
präsentationen des Nationalen rekurrieren. Die ethnografische Untersuchung Auswärtiger Kulturpolitik bekam somit den Charakter einer Feldforschung im Nationalen.

\section{Leitmotiv: Feldforschungen im Nationalen}

In ihrer Studie zu den materiellen und symbolischen Ordnungen von Weltausstellungen hat Penelope Harvey den Begriff »Technologies of Nationhood « entwickelt. ${ }^{35}$ Im Anschluss an Michel Foucault versteht sie »soziale Technologien« als mächtige »Werkzeuge der Wissensproduktion«, die an der epistemologischen und politischen Produktion und Durchsetzung bestimmter Vorstellungen, Konzepte und Ordnungsmuster mitwirken. Weltausstellungen trugen demnach - ähnlich wie etwa Nationalmuseen - seit dem späten I9. Jahrhundert dazu bei, die eigentlich junge Idee des modernen europäischen Nationalstaates als ein selbstverständliches und dauerhaftes soziales Gebilde sowie als grundlegende Einheit des internationalen Systems zu etablieren. Denn Weltausstellungen organisieren sich räumlich und konzeptionell entlang von Orten und Formen nationalstaatlicher Selbstdarstellungen, so dass Besucher hier die Vorstellung der Welt als ein Mosaik distinkter, prinzipiell gleichwertiger nationaler Einheiten mental und körperlich erfahren können. Sie wirken als »Technologien des Nationalen«, indem sie Organisationsformate und Infrastrukturen bereitstellen, welche die Entwicklung und Verbreitung von Narrativen des Nationalen nahelegen.

Durch ihre Analyse der Darstellungsformen in den Länderpavillons auf der Expo 1992 in Sevilla verdeutlicht Harvey zumindest drei Aspekte, die für ein Verständnis der Repräsentationstechniken und Positionierungen von Nationalstaaten auf globalen Bühnen von Bedeutung sind: Demnach erweist es sich erstens als zweitrangig, ob sich Staaten eher als ahistorische, quasi naturgegebene oder aber als politisch und gesellschaftlich konstruierte und somit veränderbare Einheiten präsentieren. Am Beispiel des Schweizer Pavillons demonstriert sie, dass selbst die hier vollzogene gezielte und radikale Dekonstruktion der Idee einer abgrenzbaren, substanziellen und distinkten Nationalkultur den Eindruck einer nationalen Spezifik hervorruft. Denn gerade im Vergleich zu den anderen Pavillons entsteht auch im Rahmen einer solchen Repräsentationsstrategie der Eindruck von einer Typik, die sich eben in diesem - als »schweizerisch « ausgewiesenen - reflexiven Umgang mit »Nationalkultur« zeigt. Zweitens unterstreicht Harvey, dass nationale Narrative - trotz ihres ho-

35 Penelope Harvey: Hybrids of Modernity. Anthropology, the nation state and the universal exhibition. London, New York 1996; siehe für die folgenden Bezugnahmen S. $50 \mathrm{ff}$. sowie das gesamte dritte Kapitel. 
mogenisierenden und standardisierenden Charakters - nicht zwangsläufig ein Vorhandensein von Differenz negieren, sondern durchaus Bilder von Verschiedenheit aufgreifen. Die Betonung einer spezifischen Ausformung interner kultureller Vielfalt oder auch eines ganz bestimmten Umgangs mit Differenz können in Repräsentationsstrategien zur Abgrenzung von anderen Nationalstaaten integriert werden. Und drittens zeigt Harvey, dass sich die Selbstdarstellungen in den Länderpavillons zunehmend an den Erfordernissen und Logiken eines flexiblen, globalen Kapitalismus orientieren: »Nationale Spezifik« und »Differenz« werden nicht einfach repräsentiert, sondern sie müssen zunehmend als Kern einer Markenidentität »simuliert« werden, um Staaten und ihre ökonomische Potenz auf globalen Bühnen zu positionieren.

Im Anschluss an Penelope Harvey betrachte ich Auswärtige Kulturpolitik in dieser Studie als eine Technologie des Nationalen: Die Repräsentationstechniken, inhaltlichen Schwerpunkte oder der Umgang mit »Differenz« mögen durch die Jahrzehnte und innerhalb des Politikbereichs variieren - dennoch entfaltet sich auch hier im Zusammenwirken von infrastrukturellen, organisatorischen und diskursiven Formaten eine kulturelle Logik, welche Grundfiguren des Nationalen - auch unabhängig vom Willen oder den Positionen einzelner Akteure - hervorruft, reproduziert und mobilisiert.

\section{Explizite und implizite Dimensionen der kulturellen Selbstdarstellung}

Den Ausgangspunkt dieses Abschnitts bildet also die Beobachtung, dass Grundfiguren des Nationalen - auch nach den diskursiven Veränderungen um die Jahrtausendwende - weitgehend naturalisierte Bezugspunkte in den Diskussions- und Praxisfeldern Auswärtiger Kulturpolitik darstellen. Denn in diesen Kontexten wird ein Narrativ zur kulturellen Selbstdarstellung erarbeitet, das Vorstellungen von der Existenz einer »nationalen Spezifik« oder eines »kulturellen Wir«, das sich mitteilen möchte, aufgreift und translokal mobilisiert. Ich werde diesen Tatbestand in den empirischen Kapiteln ausführlich untersuchen; an dieser Stelle beschränke ich mich auf einige grundlegende Beobachtungen, um auf dieser Basis eine analytische Perspektive auf die Funktionslogiken und Herstellungszusammenhänge des Nationalen innerhalb dieses Politikbereichs zu entwickeln.

So findet sich etwa die Zielsetzung Sympathiewerbung für Deutschland und die Vermittlung eines zeitgenössischen Deutschlandbildes in leicht variierendem Wortlaut regelmäßig in den Jahresberichten der Bundesregierung zur Auswärtigen Kulturpolitik an einer vorderen Position. Auch in öffentlichen Äußerungen zeigt sich kulturelle Selbstdarstellung als der eigentliche Dreh- und Angelpunkt des Politikbereichs: Im Jahr 2009 spricht etwa Außenminister Steinmeier davon, »Kultur- und Bildungspolitik wieder ins Zentrum unserer 
Außendarstellung zu rücken « ${ }^{36}$ und formuliert als Reaktion auf gegenwärtige geopolitische Herausforderungen: »Wir müssen uns daher mit ganzer Energie dafür einsetzen, für unser Land, unsere Kultur und unsere Gesellschaft zu werben und Interesse daran zu wecken. «"

Im Laufe meiner Forschung konnte ich beobachten, dass dieses Leitmotiv der kulturellen Selbstdarstellung sowohl eine implizite als auch eine explizite Dimension besitzt:

Gerade aus einer historischen Perspektive ${ }^{38}$ lässt sich aufzeigen, dass der strategische Einsatz kultureller Selbstbilder zum internationalen Reputationsgewinn und zur Flankierung nationalstaatlicher Interessenspolitik einen der Ursprungskerne und eine wirkmächtige explizite Zielsetzung dieses Politikbereichs darstellt. Insbesondere nach den beiden Weltkriegen wurde auf eine grenzüberschreitende Strahlkraft »deutscher« Kultur und Wissenschaft gehofft. Demnach ging es etwa in der Auswärtigen Kulturpolitik der Weimarer Republik

"vor allem um die Wiederaufnahme Deutschlands in die internationale Völkergemeinschaft, die Gewinnung von neuen Freunden und Partnern, um Unterstützung für deutsche Sichtweisen und Positionen, um das Wiedererlangen von Ansehen, Vertrauen und Sympathie. Natürlich sollte mit der Ausstrahlung deutscher Kultur und Geistestradition auch die Legitimation Deutschlands als Kulturstaat unterstützt werden, ebenso wie ein positives Deutschlandbild." 39

Eine ähnliche Logik bestimmte die Entwicklung des Politikbereichs auch in den Anfangsjahren der Bundesrepublik. Nach verlorenem Krieg, Völkermord und Diktatur sollte die Selbstdarstellung im Modus der Kultur »die Wiedereingliederung des deutschen Volkes in die internationale Gemeinschaft ${ }^{40}$ un-

36 | Auswärtiges Amt: Konferenzdokumentation "Menschen bewegen - Kulturpolitik im Zeitalter der Globalisierung“. Berlin, 18.-26. April 2009, S. 9.

37 | Auswärtiges Amt: Wo die Musik spielt. Kultur und Kommunikation in der deutschen Außenpolitik (o. J.), S. 3.

38 | Siehe hierzu etwa: Kurt Düwell: Zwischen Propaganda und Friedensarbeit - 100 Jahre Geschichte der deutschen Auswärtigen Kulturpolitik. In: Kurt-Jürgen Maaß (Hrsg.): Kultur und Außenpolitik. Handbuch für Studium und Praxis. Baden-Baden 2009 (2. vollständig überarbeitete und erweiterte Auflage), S. 61-111.

39 | Kurt-Jürgen Maaß: Überblick: Ziele und Instrumente der Auswärtigen Kulturpolitik. In: Ders. (Hrsg.): Kultur und Außenpolitik, S. 25-32, S. 25.

40 | Deutscher Bundestag (Hrsg.): Bericht der Enquete-Kommission Auswärtige Kulturpolitik gemäß Beschluß des Deutschen Bundestages vom 23. Februar 1973, Bundesdrucksache 7/4121. Bonn 1975. 
terstützen, wenn sich auch die Modi und Argumentationslinien der Vermittlungsarbeiten partiell wandelten:

"Nach dem Jahrhundertverbrechen an den europäischen Juden war der Neuanfang ungleich schwieriger. So rückte die Vermittlung eines realistischen, offenen und zukunftsgerichteten Bildes von der neuen deutschen Demokratie und ihrem Umgang mit der eigenen schwierigen Vergangenheit für mehrere Jahrzehnte in den Mittelpunkt der auswärtigen Kulturarbeit. " ${ }^{41}$

Bis in die Gegenwart erkennen Beobachter des Politikbereichs die Fortsetzung dieser historischen Entwicklungslinie in der »Leitfrage der Auswärtigen Kulturpolitik [...], die die Vermittlung eines Bildes unseres Landes in den Mittelpunkt stellt: >Mit welchen Kernbotschaften soll sich die Kulturnation Deutschland im Ausland präsentieren?««2

Darüber hinaus lässt sich auch eine dem Politikbereich eingelagerte implizite Dimension der kulturellen Selbstdarstellung herausarbeiten. Denn auch Zielvorstellungen, die in ihrer Ausformulierung andere Schwerpunkte setzen - etwa Konfliktprävention, Förderung von Zivilgesellschaft, von Demokratisierungsprozessen oder der Europäischen Integration ${ }^{43}$ - tragen zu einer Produktion und Mobilisierung von Deutschlandbildern bei: Einerseits, da sie zumeist innerhalb der institutionellen Strukturen der deutschen Auswärtigen Kulturpolitik bearbeitet, in Projektformate übersetzt und hierdurch mit Repräsentationstechniken und -modi der kulturellen Selbstdarstellung verknüpft werden. Andererseits, da auch solche universelleren, ursprünglich nicht nationalkulturell begründeten Zielsetzungen häufig konzeptionell mit »deutschen Erfahrungen« und Entwicklungen verbunden werden, wie sich exemplarisch an einer Stellungnahme von Joschka Fischer verdeutlichen lässt:

„Die besonderen Möglichkeiten der Auswärtigen Kulturpolitik, abseits offizieller Positionen durch Projekte und Veranstaltungen, Menschenrechtsdialoge und Austauschprogramme gezielt zur Stärkung von Zivilgesellschaften beizutragen, prädestinieren sie unter den neuen Bedingungen für eine prominentere und stärkere Rolle. Dies verbindet

41 | Kurt-Jürgen Maaß: Überblick: Ziele und Instrumente der Auswärtigen Kulturpolitik, S. 25.

42 | Wolfgang Schneider: Vom Export zum Netzwerk, vom Event zur Intervention. Zum Wandel Auswärtiger Kulturpolitik. In: Ders. (Hrsg.): Auswärtige Kulturpolitik. Dialog als Auftrag - Partnerschaft als Prinzip. Bonn 2008, S. 13-31, S. 26.

43 | So einige der offiziellen Zielsetzungen des Politikbereichs, die sich in unterschiedlichen Regierungsdokumenten oder Reden des Bundesaußenministers finden Iassen. Eine systematische Beschäftigung mit solchen offiziellen Papieren und Zielhorizonten erfolgt im Kapitel II dieser Studie. 
sich mit den wachsenden internationalen Erwartungen an das wiedervereinte Deutschland. Wir sollten unsere Erfahrungen mit der nach dem Krieg gewonnen Demokratie, mit dem friedensstiftenden Projekt der europäischen Einigung weitergeben - nicht als Staatsbürgerkundeunterricht und schon gar nicht als vorgestrige machtpolitische Selbstdarstellung des vereinten deutschen Nationalstaates, sondern so offen wie möglich und auf Dialog ausgerichtet: Sensibel in der Form, fest in der Sache. " ${ }^{44}$

Die Formel Vermittlung von Deutschlandbildern besitzt innerhalb der Auswärtigen Kulturpolitik also nicht nur den Status einer expliziten Zielsetzung, sondern führt gleichfalls zu impliziten Effekten, die sich sowohl aus der Existenz einer weltumspannenden institutionellen Struktur als auch aus der Möglichkeit, »eigene Erfahrungen« mit sehr verschiedenen Diskursfeldern und Fragestellungen zu verknüpfen, ergeben - anders formuliert: Der Politikbereich produziert einen infrastrukturellen und diskursiven Rahmen, in dem die unterschiedlichsten Ziele, Inhalte, Themenfelder und Formate positioniert werden können und hierdurch auch implizit zu einer Selbstdarstellung im Modus der Kultur beitragen. Entscheidend ist, dass in diesen Kontexten Grundkategorien des Nationalen global mobilisiert werden und hierbei zugleich eine Bestätigung in ihrer »nicht hinterfragbaren Selbstverständlichkeit ${ }^{45}$ als Ausgangspunkte des Politikbereichs erfahren. Neben den soeben genannten Figuren einer »nationalen Spezifik« und eines »kulturellen Wir« denke ich hierbei insbesondere auch an die Vorstellung von der Existenz distinkter Nationalstaaten als maßgebliche Grundeinheiten der Welt und internationaler kultureller Begegnung.

\section{Zweifel an der Selbstverständlichkeit: Dezentrierungen und Dekonstruktionen des Nationalen}

Vor dem Hintergrund wissenschaftlicher Diskussionen, die eher Tendenzen einer Dezentrierung des Nationalen konstatieren, vermögen diese Befunde etwas zu überraschen. Zwei kritische Interventionen drängen sich auf:

Zum einen wurde vielfach herausgearbeitet, dass sich solche Grundfiguren des Nationalen vor dem Hintergrund von Globalisierungsprozessen zunehmend als brüchig erweisen. Als Konsequenz einer wachsenden transnationalen Mobilität von Menschen, Bildern, Informationen, Gütern und Kapitalien verliert die Vorstellung von »nationalstaatlichen Containern $\aleph^{46}$ als gewissermaßen gegebenen Umgrenzungen von Identitäten, Gemeinschaften oder Kulturen an

44 I Joschka Fischer: Sensibel in der Form, fest in der Sache. In: Zeitschrift für Kulturaustausch 2002/01, S. 24-26, S. $24 f$.

45 | Beate Binder: Troubling policies, S. 367.

46 | Ulrich Beck: Was ist Globalisierung? Frankfurt 1998, S. $48 \mathrm{ff}$. 
Überzeugungskraft. Die Beziehungen zwischen Gruppenzugehörigkeiten und Territorien flexibilisieren sich; Identitäten gestalten sich »positionaler, politischer, pluraler und vielfältiger sowie weniger fixiert, einheitlich und transhistorisch ${ }^{47}{ }^{47}$ Entsprechend verortet Stuart Hall in solchen Dynamiken die Kraft, »nationale kulturelle Identitäten zu zerstreuen « und ihnen hierdurch ihre bisherige Fraglosigkeit zu nehmen: »Nationale Identitäten (waren) solange einheitlich und homogen, wie es ihnen gelang, sich als solche zu repräsentieren.« ${ }^{48}$

Auf dieser Basis möchte ich eine erste Spannung festhalten: Wenn nationale Identitäten gegenwärtig kraftvoll zerstreut werden, wie erklärt sich dann dieses Festhalten an Grundfiguren des Nationalen innerhalb der Auswärtigen Kulturpolitik? Unter welchen Bedingungen und mittels welcher Sprechweisen oder Arbeitsformen kann es dennoch gelingen, ein kohärentes Narrativ zur kulturellen Selbstdarstellung herzustellen? Und sollte man den Politikbereich als einen staatlich finanzierten Versuch zur Rezentrierung des Nationalen vor dem Hintergrund wirkmächtiger Tendenzen zu seiner Dezentrierung verstehen?

Zum anderen möchte ich an Positionen erinnern, die das politische Ordnungsprinzip des Nationalstaates als einen wesentlichen Beitrag zur Reproduktion globaler Asymmetrien diskutieren. In einem anderen inhaltlichen Zusammenhang hat etwa Ulrich Beck darauf hingewiesen, dass das Nationalstaatsprinzip globale Ungleichheiten legitimiert, indem nationale Grenzen sowie die Konservierung des »nationalen Blicks« politischen Öffentlichkeiten und soziologischen Forschungen die Möglichkeit geben, »scharf zwischen politisch relevanter und irrelevanter Ungleichheit« zu trennen: »Ungleichheiten innerhalb nationaler Gesellschaften werden in der Wahrnehmung enorm vergrößert; gleichzeitig werden Ungleichheiten zwischen nationalen Gesellschaften ausgeblendet. $^{49}$

Insbesondere vor dem Hintergrund der diskursiven Figur einer Auswärtigen Kulturpolitik als Konfliktprävention lässt sich eine zweite Spannung festhalten: Kann ein Politikbereich, der so deutlich an Grundfiguren des Nationalen festhält, tatsächlich Potenziale zu einer produktiven Bearbeitung der Konfliktträchtigkeit der gegenwärtigen Welt entfalten? Gälte es hierzu nicht stärker einzubeziehen, dass ein jedwedes Narrativ vom Nationalen kein »unschuldiges« kulturelles Produkt darstellt, sondern zutiefst politische Botschaften von Zugehörigkeiten und Grenzen, von Zuständigkeiten und Verantwortlichkeiten für soziale Ungleichheiten und Problemlagen in globalen Feldern

47 | Ebd., S. 217.

48 | Stuart Hall: Die Frage der kulturellen Identität, S. 208.

49 | Ulrich Beck: Die Neuvermessung der Ungleichheit unter den Menschen: Soziologische Aufklärung im 21. Jahrhundert. Frankfurt 2008, S. 13. 
enthält und genau hierdurch einen Beitrag leistet zur symbolischen und politischen Ordnung der Welt, die eine hierarchische ist?

Ich werde auf diese beiden Spannungen und ihre Bearbeitung in den Praxisund Übersetzungsfeldern Auswärtiger Kulturpolitik in meinen empirischen Kapiteln zurückkommen. Zur Vorbereitung möchte ich auf den verbleibenden Seiten dieses Abschnitts auf jüngere Diskussionen und Arbeiten aus der Europäischen Ethnologie und ihren Nachbardisziplinen zurückgreifen, um eine analytische Perspektive auf die Funktionslogiken und Verwendungskontexte des Nationalen in dieser Policy zu entwickeln. Ziel ist es, die Möglichkeiten einer »Feldforschung im Nationalen $\aleph^{50}$ auszuloten, um hierdurch ein Fenster zur Untersuchung der Repräsentationstechniken und Positionierungen eines europäischen Nationalstaates auf globalen Bühnen zu öffnen.

\section{Das Narrativ vom Nationalen: Soziale Imagination und historische Konstruktionsbedingungen}

Die Betrachtung von Nationen oder Nationalkulturen als »historische Konstrukte« oder »soziale Imaginationen « hat sich innerhalb den Geschichtsund Kulturwissenschaften weitgehend als Konsens etabliert. So wurde vielfach herausgearbeitet, dass die Vorstellung von dem Übereinanderfallen von Territorium, Staat, Nation und Kultur - wie sie als ideale Grundlage für den europäischen Nationalstaat gedacht wird - eine verhältnismäßig junge politische Konstellation ist, die sich etwa seit dem späten I8. Jahrhundert in Europa allmählich gegenüber anderen politischen Organisationsformen durchgesetzt hat. ${ }^{51}$ Die zeitgenössische Nationalismusforschung betrachtet Nationen entsprechend nicht als ahistorische, (natur-)gegebene und klar voneinander abgegrenzte Einheiten, sondern nimmt eher eine relationale und prozessuale Perspektive ein, um die Entstehung und Entwicklung von nationalen Bewegungen und nationalstaatlichen Einheiten zu erklären: Die Durchsetzung des Nationalstaates als »politischer Normalfall« lässt sich demnach gerade aus dem Zusammenspiel und Ineinanderwirken konkurrierender nationaler Projekte verstehen. Die Vorstellung von der »eigenständigen und authentischen Nationalkultur« wurde hierbei zu einem zentralen Modus, um die Macht- und Ge-

50 | Saskia Sassen: Das Paradox des Nationalen, S. 9.

51 Siehe hierzu beispielsweise: Benedict Anderson: Die Erfindung der Nation. Zur Karriere eines folgenreichen Konzeptes. Berlin 1988; Ernest Gellner: Nationalismus und Moderne. Berlin 1991; Eric Hobsbawm: Nationen und Nationalismus. Mythos und Realität seit 1780. München 1996; Jürgen Osterhammel: Die Verwandlung der Welt. Eine Geschichte des 19. Jahrhunderts. München 2011 (Sonderausgabe, Erstausgabe 2009). 
staltungsansprüche einer jeweiligen nationalen Bewegung in Abgrenzung zu anderen zu legitimieren. ${ }^{52}$

Kultur wurde somit im Zuge der europäischen Nationalstaatsbildungen zu einem der entscheidenden Felder, in dem die neuen nationalen Kollektive ihre Zusammengehörigkeit und »jene spezifische Form der (vorgestellten) Vertrautheit« konstruierten und erlebten: »Entscheidend ist dabei, dass die Identitätspolitik der nationalen Kultur selbstverständlich nicht ein bereits vorhandenes kulturelles Motiv aufnimmt, sondern dass das kulturelle Muster selbst erst Resultat der Suche nach ihm ist. Nationen werden nicht gefunden, sondern erfunden. ${ }^{53}$ Kulturelle Gemeinsamkeiten mussten demnach zunächst politisch erschaffen und repräsentierbar werden, indem Konfliktkonstellationen und Differenzen innerhalb eines Territoriums mittels der Vorstellung einer Nationalkultur »zu einer Identität vernäht « ${ }^{54}$ wurden. Die Einebnung interner Unterschiede bedeutete dabei automatisch eine Abgrenzung von - sowie die Dramatisierung der Differenzen zu - anderen Nationalkulturen.

Stuart Hall spricht von einem »System kultureller Repräsentationen « ${ }^{55}$, das sich in der Idee von der Nationalkultur konstituierte und das durch »eine Erzählung von der Nation« erfahrbar und mit dem Alltag der Menschen verwoben wurde:

"Es gibt eine Erzählung der Nation, die in Nationalgeschichten, in der Literatur, den Medien und der Alltagskultur immer wieder vorgetragen wird. Diese stellt einen Zusammenhang von Geschichten, Vorstellungen, Landschaften, Szenarien, geschichtlichen Ereignissen, nationalen Symbolen und Ritualen her, die die geteilten Erfahrungen und Sorgen, Triumphe und vernichtenden Niederlagen repräsentieren, die einer Nation Bedeutung verleihen. " ${ }^{56}$

Hall verdeutlicht am Beispiel Englands, welche unterschiedlichen Motive in einem solchen Narrativ miteinander verknüpft werden:

52 Siehe zur Anwendung einer relationalen Perspektive zur Analyse der Entstehung und Funktionsweisen von multi-nationalen Politikfeldern: Rogers Brubaker et al.: Nationalist Politics and Everyday Ethnicity in a Transylvanian Town. Princeton, Oxford 2006. Grundlegend für ein relationales Verständnis von Gruppenidentitäten in der Anthropologie: Fredrik Barth: Introduction. In: Ders. (Hrsg.): Ethnic Groups and Boundaries. The Social Organization of Culture Difference. Boston, Oslo et al. 1969, S. 9-38.

53 | Armin Nassehi: Das stahlharte Gehäuse der Zugehörigkeit. Unschärfen im Diskurs um die "multikulturelle Gesellschaft". In: Ders. (Hrsg.): Nation, Ethnie, Minderheit. Beiträge zur Aktualität ethnischer Konflikte. Köln, Weimar, Wien 1997, S. 177-208.

54 | Stuart Hall: Die Frage der kulturellen Identität, S. 208.

55 | Ebd., S. 200.

56 | Ebd., S. 202; auch das folgende Zitat. 
"Von Englands grünen und freundlichen Landen, seinen sanften hügeligen Landstrichen, rosenbestockten Cottages und Landhausgärten - Shakespeares ,königliche Insel - bis zu öffentlichen Zeremonien wie dem Abnehmen der Fahnenparade und dem Poppy Day (Gedenktag des englischen Sieges im Ersten Weltkrieg) repräsentieren die Diskurse des 'Englischseins`, was 'England، ist, geben der Identität, ,englisch zu sein, eine Bedeutung und fixieren 'Englandı als Zentrum der Identifikation für englische (und anglophile) Herzen."

Die Rede von »Imaginationen«, »Konstruktionen« oder »Narrativen« bedeutet dabei nicht, dass Nationalkulturen keine politischen oder sozialen Wirkungen entfalteten. Ganz im Gegenteil waren sie bereits in ihrer Entstehung an Felder politischer Auseinandersetzung geknüpft, in denen soziale Gruppen, Parteien oder Akteure sehr unterschiedliche Antworten auf die Frage, »was Nationalkultur sei« gegeben haben. ${ }^{57}$ Durch die Diskussionen zur Etablierung und Abgrenzung einer Nationalkultur entwickelte sich - gerade innerhalb der Nationalbewegungen und der neu entstehenden Staaten in Mittel- und Osteuropa sowie später in den kolonialen Befreiungsbewegungen auch im globalen Rahmen - »ein symbolischer Raum [...] innerhalb dessen politische und/ oder soziale Ziele gesetzt und eben realisiert werden konnten«. Seine Sicht von den Schlüsselbegriffen, Inhalten, Grenzen und historischen Bedingungen einer Nationalkultur durchzusetzen, bedeutete für einen politischen Akteur oder eine soziale Gruppe, ein wesentliches symbolisches Kapital zu erwerben, um an der Definition und Gestaltung der sozialen und politischen Gegenwart mitwirken zu können. Insofern sind in den Vorstellungen, Bildern und Narrativen, die sich innerhalb einer Nationalkultur durchsetzen, die Verläufe solcher Konflikte eingeschrieben: »Die Nationalkultur ist deshalb immer die symbolische Repräsentation sozialer Hegemonie und politischer Macht und kann nur im Kontext der Machtverhältnisse einer Gesellschaft interpretiert werden. ${ }^{58}$

Auch Stuart Hall weist darauf hin, dass Nationalkulturen immer auch »eine Struktur kultureller Macht« darstellten, die sich beispielsweise in der »gewaltsamen Unterdrückung kultureller Differenzen«, der Normierung von Lebensformen oder der Legitimierung kolonialer Machtausübung zeigte. ${ }^{59}$

57 | Peter Niedermüller: Diskurs, Kultur, Politik. Zur Herausbildung der Nationalkultur in Ungarn. In: Beate Binder et al.: Inszenierungen des Nationalen, S. 167-185, S. 171; das folgende Zitat ebenda.

58 | Ebd., S. 173.

59 | Stuart Hall: Die Frage der kulturellen Identität, S. 205. 


\section{Inszenierungen des Nationalen: Bedeutungen und Verwendungskontexte des Nationalen in der europäischen Gegenwart}

Die Vorstellung von nationalen Kulturen und Identitäten zeigt sich aber auch in der Gegenwart als eine wirkmächtige Bezugsgröße, die zur Begründung der symbolischen Ordnung einer Gesellschaft oder zur politischen Mobilisierung genutzt werden kann. Innerhalb der Europäischen Ethnologie wurde das »Nationale«als eine Kategorie entwickelt, die eine empirische und analytische Perspektive auf unterschiedliche Formen der Diskussion, Konstituierung oder Politisierung nationaler Symboliken, Identitätsmuster und Bewegungen in der europäischen Gegenwart eröffnet. Es wird dabei verstanden als ein »symbolischer Raum [...] aus sprachlichen Formen, aus Texten, Begriffen, Konzeptionen, aus kulturellem Wissen, symbolischen Handlungen und Aktionen [...], der jene Vertrautheit erzeugt, die wiederum die Basis nationaler Wir-Gefühle bildet. ${ }^{60}$ Als Ausgangspunkte für die Untersuchungen des Nationalen werden hier einerseits seine »symbolischen Dimensionen « und andererseits die politischen, sozialen und kulturellen Zusammenhänge, in denen es hergestellt und inszeniert wird, vorgeschlagen.

Die verstärkte Thematisierung des Nationalen, die sich in vielen europäischen Gesellschaften Ende des 20. Jahrhunderts beobachten ließ, wird in dieser Forschungsrichtung nicht als ein irrationales Festhalten an eigentlich überholten Bildern nationaler Selbstwahrnehmung oder als eine - mit Blick auf Osteuropa - »>natürliche< Konsequenz des ideologischen Vakuums bzw. als Reaktion auf die vorangegangene sozialistische Zeit « ${ }^{61}$ betrachtet. Solche gegenwärtigen Bezugnahmen werden stattdessen als »qualitativ neu« klassifiziert - das »Neue« liegt dabei weniger in den »Argumenten und Bildern selbst $«$, sondern in dem »Bedeutungs- und Verwendungskontext ${ }^{62}$, in dem das Nationale positioniert und aktiviert wird. Diese neue Rolle und Funktion des Nationalen zeigt sich demnach insbesondere in dem Rückgriff auf Geschichte vor dem Hintergrund zunehmend heterogener Identitätskonstellationen in spätmodernen Gesellschaften. Fokussiert wird hierdurch das weite Feld von Geschichts- und Erinnerungspolitiken, in denen zunehmend auch nicht-staatliche Akteure Bilder von der Vergangenheit und von kollektiven Identitäten produzieren, um gesellschaftliche Loyalitäten einzufordern. In den

60 | Beate Binder, Peter Niedermüller \& Wolfgang Kaschuba: Inszenierungen des Nationalen - einige einleitende Bemerkungen, S. 8.

$61 \mid$ Ebd., S. 7.

62 | Wolfgang Kaschuba: Geschichtspolitik und Identitätspolitik. Nationale und ethnische Diskurse im Kulturvergleich. In: Beate Binder et al. (Hrsg.): Inszenierungen des Nationalen, S. 19-42, S. 27. 
Blick geraten somit etwa die medialen Thematisierungen und politischen Inszenierungen von Jahrestagen, Denkmalen und anderen Erinnerungsorten, in deren Rahmen die Vorstellung von der »Nation« als gewissermaßen gegebener Schicksals- und Erinnerungsgemeinschaft gleichzeitig reaktiviert und verfestigt wird. ${ }^{63}$

Wolfgang Kaschuba betont - als weiteren qualitativ neuen Aspekt - die grundlegend gewandelte Beziehung zwischen »Kultur« und dem »Nationalen« in spätmodernen Gesellschaften: In der Phase der europäischen Nationalstaatsbildung sei Kultur insbesondere von staatlichen Akteuren als ein nationales Integrationsmedium genutzt worden, also als eine Ressource, um das Ideal von dem integrierten und homogenen Nationalstaat in die Realität umzusetzen. Heute hingegen habe sich dieses Verhältnis gewissermaßen umgedreht: Das Nationale zeige sich als ein »kulturelles Integrationskonzept « ${ }^{64}$ als eine »kulturelle Strategie«, die durch die unterschiedlichsten Akteure und Gruppierungen genutzt wird, um im Rahmen von gesellschaftlichen und politischen Diskursen »Wir-Bilder« zu positionieren und hierdurch auch Grenzlinien der Zugehörigkeit zu verlegen. Die Aktivierung und die Funktionslogik dieser Strategie lässt sich anhand von Debatten beobachten, in denen mit Geschichte, Herkunft oder Identität argumentiert wird und die sich etwa aus Anlass von Gedenkfeierlichkeiten, Straßenumbenennungen oder Planungen von Denkmalen entzünden.

Die Wirkmächtigkeit und gesellschaftliche Plausibilität solcher kultureller Strategien ergeben sich aus ihrem Rückgriff auf etablierte Narrative, Symbole und ästhetische Formate, die sich auf das Nationale als Raum »kultureller Authentizität« beziehen:

"Nationale und ethnische Zuschreibungen [...] sind in der Lage, in wenigen Zeichen und Signalen komplexe gesellschaftliche Semantiken und Gruppenhorizonte aufzurufen und zu mobilisieren. Dies kann nur gelingen, weil sie an historisch eingeübten, kollektiv verfügbaren und zugleich aktuell gültigen Denkweisen und Symbolen des Nationalen anknüpfen." 65

Das Nationale wird also aufgrund etablierter kultureller Codierungen gesellschaftlich als authentisch verstanden und ist daher in den unterschiedlichsten politischen Kontexten und Debatten anschlussfähig - gerade auch, da es sich

63 | Siehe hierzu insbesondere: Wolfgang Kaschuba: Geschichtspolitik und Identitätspolitik; Beate Binder: 50. Wiederkehr des Kriegsendes: Zur Konstruktion der Gedächtnislandschaft Berlin. In: Beate Binder et al.: Inszenierungen des Nationalen, S. 285-308; Margit Feischmidt: Symbolische Kämpfe der Nationalisierung. In: Ebd., S. 263-284.

64 | Wolfgang Kaschuba: Geschichtspolitik und Identitätspolitik, S. 20.

$65 \mid$ Ebd., S. 23. 
offenbar besonders gut von einer Ebene des Sprechens in solche Praxen, Ereignisse und Rituale übersetzen lässt, die Menschen zu mobilisieren vermögen.

Solche innergesellschaftlichen Authentisierungsstrategien wirken dabei immer auch als Inklusions- und Exklusionsmechanismen und genau hieraus ergibt sich ihre Problematik in zunehmend heterogenen Gesellschaften, in denen die unterschiedlichsten biografischen Erfahrungen, Erinnerungen und Gruppengedächtnisse aufeinander treffen. Entsprechend bemerkt Wolfgang Kaschuba im Hinblick auf die kontinuierlichen medialen und politischen Reproduktionen der deutschen Nation als Erinnerungs- und Gedächtnisgemeinschaft:

"Man mag sich dabei fragen, wie andere, Zugewanderte und Eingeheiratete, überhaupt Zugang in diese nationale deutsche Diskursgemeinschaft finden sollen, die durch den Konsens des 'Gedenkensı und die Kontroversen des 'Gedächtnissesı so fest zusammengehalten wird. Wahrscheinlich überhaupt nicht, weil der Modus, nach dem der Erinnerungsdiskurs geregelt und ritualisiert ist, dies systematisch verhindert. " 66

Dieser Ansatz für eine ethnologisch ausgerichtete Nationalismusforschung betrachtet das Nationale somit nicht nur als einen »symbolischen Raum«, sondern etabliert es auch als eine analytische Kategorie, welche anhand der Untersuchung von strategischen Bezugnahmen auf historisch gesättigte Bilder, Narrative und Identitätsmuster den Zugriff auf gegenwärtige politische Diskurse, Handlungslogiken und Machtfelder ermöglicht. Gedenkfeiern, Erinnerungsrituale oder Geschichtsdebatten werden als ethnografische Felder betrachtet, an denen sich das Ineinanderwirken von nationalen Argumentationslogiken und politischen Mobilisierungen, von »Diskurs« und »Praxis« untersuchen lässt. Das übergeordnete Ziel ist es, hierdurch die grundlegenden Funktionsweisen, Konstruktionsprinzipien und kulturellen Logiken herauszuarbeiten, »durch die mittels des Diskurses des Nationalen Wirklichkeit machtvoll strukturiert wird «. ${ }^{67}$

Die Beiträge des Sammelbandes Inszenierungen des Nationalen konzentrieren sich dabei auf aktuelle innergesellschaftliche Konstellationen in europäischen Staaten. Die Frage, was mit dem Nationalen passiert, wenn es globale Bühnen betritt und hier in Verbindung mit - oder auch in Konkurrenz zu anderen Narrativen und Identitätskonzepten tritt, wurde hier hingegen kaum berücksichtigt.

$66 \mid$ Ebd., S. 38.

67 Beate Binder, Peter Niedermüller \& Wolfgang Kaschuba: Inszenierungen des Nationalen, S. 8. 


\section{Das Nationale auf globalen Bühnen: Brüche, Transformationen, Assemblagen}

Diese Frage ist dabei zur Untersuchung der nationalstaatlich initiierten Auswärtigen Kulturpolitik von zentraler Relevanz. Schließlich ist es ihr erklärtes Ziel, sich mit Bildern und einem Narrativ vom Nationalen in globale Dynamiken einzuschalten, die sich in einer zunehmend unübersichtlichen Weltordnung widerspiegeln: Das Auswärtige Amt selbst verweist auf neue kulturelle Gravitationszentren, Identitätskonstellationen und Konfliktlinien als veränderte Rahmenbedingungen, in denen Wirkung erzielt werden soll. ${ }^{68}$

Auswärtige Kulturpolitik setzt somit das »Nationale« und das »Globale« zueinander in Beziehung und wird daher gerade auch vor dem Hintergrund aktueller sozial- und kulturwissenschaftlicher Debatten zu Globalisierungsprozessen zu einem interessanten Feld. »Globalisierung« verweist hierbei grundsätzlich auf ein Bündel von Prozessen wachsender Interdependenzen vormals souverän gedachter staatlicher Einheiten, beschleunigter Mobilitäten von Personen, Gütern, Bildern und Kapitalien und der zunehmenden Bedeutung global wirksamer Wissensbestände sowie rechtlicher und politischer Regulierungssysteme. Die Frage nach der Zukunft des Nationalstaates, seiner Souveränität und politischen Gestaltungsmacht spielt in diesen Debatten eine herausgehobene, sogar strukturierende Rolle. Insbesondere in den I99oer Jahren überwogen Stimmen, die aufgrund der globalen ökonomischen und politischen Dynamiken den »Niedergang des alten Nationalstaates als eines funktionsfähigen politischen Gebildes« erwarteten: »Nationen und Nationalismus wird es auch in Zukunft geben, aber in der Geschichte werden sie von untergeordneter und vielfach sehr geringer Bedeutung sein«, schrieb etwa Eric Hobsbawm zu Beginn des Jahrzehnts und sagte die Entstehung eines neuen, multilateralen internationalen Systems voraus, dessen Hauptakteure »weit größere Gebilde « als Nationalstaaten sein werden. ${ }^{69}$ Zygmunt Bauman sprach einige Jahre später vom »allmählichen Verschwinden des Staates« und damit auch des Rahmens, in dem soziale Fragen zu politischen Handlungen geformt werden können. In Konfrontation mit dem global mobilen, extraterritorialen und daher unkontrollierbaren Kapital verliere der Staat zunehmend seine ökonomische, militärische und kulturelle Selbständigkeit und somit seine politische Souveränität. ${ }^{70}$ Jürgen Habermas wiederum rief zum verstärkten Aufbau von supranationalen Institutionen und grenzüberschreitenden Öffentlichkei-

68 | Siehe hierzu etwa: Auswärtiges Amt: Auswärtige Kulturpolitik - Konzeption 2000. Berlin 2000.

69 | Eric Hobsbawm: Nationen und Nationalismus, S. 211 und S. 220.

70 | Zygmunt Bauman: After the Nation State - What? In: Ders.: Globalization. The Human Consequences. Cambridge 1998, S. 55-76, S. 64 und S. 69. 
ten auf, um den rapiden Verlust politischer Gestaltungsmacht der Nationalstaaten auf einer höheren Ebene auszugleichen und somit Grundlagen für eine notwendige »transnationale Weltinnenpolitik « zu schaffen. ${ }^{71}$

Im Hinblick auf das kulturelle Selbstverständnis von Nationalstaaten haben zeitgleich Kulturanthropologen wie etwa Ulf Hannerz oder Arjun Appadurai begonnen, die Konsequenzen der Globalisierung zu diskutieren und ein Vokabular zu entwickeln, um zunehmend grenzüberschreitende kulturelle Formationen zu untersuchen. ${ }^{72}$ So hat Appadurai aufgezeigt, wie gerade aus dem Zusammenspiel von transnationalen Migrationen und global mobilen Bildern ein neues Potenzial für menschliche Vorstellungskraft als einer wirkmächtigen sozialen Praxis entsteht: Demnach können Individuen nunmehr weltweit auf medial verbreitete Bilder, Narrative und Idealvorstellungen von möglichen Leben zurückgreifen, um sich selbst kulturell zu verorten. Identitäten und transnationale Beziehungsgeflechte entstehen zunehmend im Rahmen solcher globaler Zirkulationen ohne durch Örtlichkeit oder räumliche Bindung nachhaltig begrenzt zu werden. Der Nationalstaat verliert demnach mehr und mehr sein Vermögen, Gruppenzugehörigkeiten und kulturelles Selbstverständnis zu strukturieren. Für eine wissenschaftliche Analyse ergibt sich hieraus die Herausforderung, kulturelle Formen der Gegenwart nicht länger nationalstaatlich geordnet sondern »fraktal« zu denken - also sich transnational überlappend, ohne klare Grenzen, Regeln und räumliche Strukturen. ${ }^{73}$

Auch Appadurai kommt zu der Schlussfolgerung, dass die Epoche des Nationalstaates ihrem Ende entgegengeht. Die »terminal crisis« des Nationalstaates $^{74}$ verweist dabei auf die Krise des internationalen Staatensystems, das immer weniger in der Lage ist, mit den grenzüberschreitenden Fließmustern, welche die kulturelle Dynamik der Gegenwart ausmachen, umzugehen. Neben dieser alten Ordnung prinzipiell gleichwertiger staatlicher Einheiten werden zunehmend die Konturen eines neuen globalen Systems heterogener Formationen erkennbar, das sich aus so diversen, räumlich flexibleren und von staatlichen Vorgaben unabhängigeren Akteuren wie sozialen Bewegungen, Diaspora-Öffentlichkeiten, Interessensverbänden, Nichtregierungsorganisationen,

71 Jürgen Habermas: Jenseits des Nationalstaates? Bemerkungen zu Folgeproblemen der wirtschaftlichen Globalisierung. In: Ulrich Beck (Hrsg.): Politik der Globalisierung. Frankfurt 1998, S. 67-84, S. 78.

72 Arjun Appadurai: Modernity at Large. Cultural Dimensions of Globalization. Minneapolis 1996. Ulf Hannerz: Transnational Connections. Culture, people, places. London, New York 1996.

73 | Arjun Appadurai: Disjuncture and Difference in the Global Cultural Economy. In: Ders.: Modernity at Large, S. 27-47, S. 46.

74 | Arjun Appadurai: Here and Now. In: Ders.: Modernity at Large, S. 1-23, S. 19. 
bewaffneten Gruppen oder Organen der Rechtsprechung zusammensetzt. ${ }^{75}$ Die Krise und Brüche des Nationalstaates zeigen sich demnach eben dort, wo sich die Dynamiken und globalen Verknüpfungen dieser heterogenen Einheiten nicht mehr durch staatliche Logiken und Regulierungen beherrschen lassen: Moderne Nationalstaaten wissen, dass sie zum alten System gehören »und dass sie darin wie die letzten Dinosaurier um ihr Überleben kämpfen «. ${ }^{76}$

Saskia Sassen betont im Vergleich hierzu stärker die vielfältigen Verknüpfungen, die das Nationale und das Globale miteinander eingehen und versucht somit eine dichotomische Perspektive, die beides zueinander in Opposition setzt, zu überwinden. ${ }^{77}$ Globalisierung führt demnach nicht einfach zum Bedeutungsverlust des Nationalstaates, sondern sie findet im großen Umfang gerade im Nationalen statt. So stellt Sassen einerseits heraus, dass Nationalstaaten aktiv an der Gestaltung der Globalisierung beteiligt sind, indem sie etwa Politiken der Deregulierung und Privatisierung initiieren oder zunehmend Kompetenzen und Autorität an nicht-staatliche Akteure übertragen. Andererseits zeigt sie auf, dass sich Teilbereiche des Nationalstaates - beispielsweise einzelne Ministerien, Regulierungsbehörden oder Organe der Rechtsprechung - mit supranationalen, privatwirtschaftlichen oder zivilgesellschaftlichen Akteuren zu neuartigen Assemblagen verknüpfen. Solche grenzüberschreitenden Gefüge entwickeln etwa im Bereich der globalen Finanzwirtschaft, der Regulierung von Umweltrisiken oder der Durchsetzung von Menschenrechten eine Gestaltungsmacht, die sich nicht mehr durch eine nationalstaatliche Logik dominieren lässt. Sassen unterscheidet entsprechend zwischen der nationalstaatlichen Epoche, in der internationale Felder der Ökonomie, der Politik, der Rechtsprechung oder Kultur durch die zentripetale Logik der Staaten geordnet wurden und der gegenwärtigen globalen Epoche, in der eine Vielzahl von Assemblagen und Akteurskonstellationen nach jeweils eigenen Logiken nebeneinander operieren. ${ }^{78}$ Nationalstaaten agieren demnach in einer $»$ neuen Geographie der Macht«, in der zentrifugale Tendenzen vorherrschen und die deutlich vielfältiger ist als das herkömmliche internationale Staatensystem. ${ }^{79}$ In der Konsequenz erwartet Sassen nicht einfach das »Verschwinden des Nationalstaates als einer wichtigen Form«, sondern betrachtet ihn stattdessen als

75 | Ebd., S. 23. Siehe hierzu auch die Weiterentwicklung dieses Gedankens in: Arjun Appadurai: Die Geographie des Zorns. Frankfurt 2009, S. $35 f f$.

76 | Arjun Appadurai: Die Geographie des Zorns, S. 35.

77 | Saskia Sassen: Das Paradox des Nationalen; Saskia Sassen: The global inside the national. A research agenda for sociology. Veröffentlicht auf: Sociopedia.isa 2010,1, S. 3-10.

78 | Saskia Sassen: Das Paradox des Nationalen, S. $676 \mathrm{ff}$.

79 | Ebd., S. 256. 
einen »Schauplatz fundamentaler Transformationen«, der als »ein tiefgreifend gewandeltes Gebilde « erhalten bleiben wird. ${ }^{80}$

Hierdurch lenkt sie den Blick auf die nicht geradlinig verlaufenden Neu-Konfigurierungen des Nationalen im Rahmen dieser Prozesse: Demnach können gewisse Teilbereiche des Nationalen eine Entnationalisierung erfahren, wenn etwa nationale Rechts- und Wirtschaftsordnungen oder Kompetenzverteilungen im Zusammenspiel mit globalen Akteuren oder Regelungssystemen von innen heraus verändert werden. Andere Bereiche - etwa politische Rhetoriken oder die Regulierung von Zugehörigkeit - können hingegen als Reaktion auf Globalisierungsdynamiken temporär renationalisiert werden.

Globalisierung findet demnach im Nationalen statt und verändert die Machtverteilungen, Handlungslogiken sowie das Selbstverständnis einzelner Akteure im Inneren des Nationalstaates grundlegend. Im Anschluss lässt sich vermuten, dass auch Vorstellungen von nationaler Identität und Kultur durch ihre Konfrontation mit globalen Prozessen nicht einfach an Bedeutung verlieren oder verschwinden, sondern stattdessen in ihrem Inneren grundlegend transformiert werden.

\section{Perspektivierung: Transformationen des Nationalen in den Praxis- und Übersetzungsfeldern Auswärtiger Kulturpolitik}

Saskia Sassens Vorschlag »Feldforschungen im Nationalen ${ }^{81} \mathrm{zu}$ betreiben auch um hier den »Ursprung des Globalen ${ }^{82}$ zu untersuchen - eröffnet für die Erforschung Auswärtiger Kulturpolitik interessante Perspektiven. Ich habe in diesem Abschnitt zumindest zwei Spannungen identifiziert, die sich aus den Präsenzen und Aktivierungen von Grundfiguren des Nationalen in diesem Politikbereich ergeben: zum einen die Frage nach den Sprechweisen, Arbeitstechniken und Projektformaten, durch die sich trotz wirkmächtiger Tendenzen zur Dezentrierung des Nationalen ein kohärentes Narrativ zur kulturellen Selbstdarstellung herstellen lässt; zum anderen den Zweifel an den konfliktpräventiven Potenzialen einer Auswärtigen Kulturpolitik, die so deutlich an Grundfiguren des Nationalen festhält. Beide Spannungen lassen sich im Rückgriff auf Sassens Überlegungen neu perspektivieren. So gerät einerseits in den Blick, ob und in welchen konkreten Ausformungen Schlüsselbestände des Nationalen im Rahmen dieser Policy nicht einfach reproduziert, sondern von innen heraus neu konfiguriert werden: Zeigen sich vielleicht Tendenzen zu einer Denationalisierung und kosmopolitischen Öffnung ihrer Kernbegriffe? Oder werden hier - allen gegenläufigen Tendenzen zum Trotz - Bilder von kulturellen

\footnotetext{
80 | Ebd., S. 680.

81 Ebd., S. 9.

$82 \mid$ Ebd., S. 7.
} 
Grenzen und Differenzen, vom »historischen Erbe« und der »nationalen Spezifik« rezentriert? Andererseits lässt sich untersuchen, ob die Orientierung an Grundfiguren des Nationalen tatsächlich einer produktiven Bearbeitung von Konfliktkonstellationen im Wege steht oder ob die Mobilisierung eines entsprechend transformierten Narrativs nicht eher zu neuartigen Konstellationen und Assemblagen führt: Eignet sich eine flexibilisierte oder kosmopolitisierte Version des Nationalen vielleicht besonders gut, um auf globalen Bühnen Positionen zu beziehen und Wirkung zu entfalten?

In den folgenden Kapiteln werde ich diese Frage anhand von Konferenzen und Tagungen des Politikbereichs in Berlin sowie anhand der translokalen Praxis- und Übersetzungsfelder Auswärtiger Kulturpolitik in Ramallah, Sarajevo und Tel Aviv diskutieren. Ich greife hierzu auf die diskutierten europäisch-ethnologischen Beiträge zurück, indem ich die Mobilisierung von Grundfiguren des Nationalen als Bestandteile von kulturpolitischen Strategien untersuche - Strategien, die in diesen Kontexten erarbeitet werden, nicht um in innergesellschaftlichen Debatten Loyalitäten einzufordern, sondern um auf globalen Bühnen und in translokalen Kontaktzonen Anschlüsse, Präsenzen und Effekte zu erzielen. Ich orientiere mich hierbei zugleich an Harveys Begriff »Technologie des Nationalen«, indem ich gerade das Zusammenwirken von diskursiven, infrastrukturellen und organisatorischen Formaten als eine zentrale Rahmenbedingung für die Entwicklung solcher Repräsentationsweisen sowie insgesamt für die Wissens- und Übersetzungsarbeit, die in diesem Politikbereich vonstattengeht, untersuche. 



\section{Ein politisches Feld \\ Imaginäre Dimensionen, policy community, Übersetzungsketten}

In diesem Kapitel wende ich mich nun den offiziellen Zentren, Diskussionsforen und urbanen Bühnen Auswärtiger Kulturpolitik zu. Neben meinen ethnografischen Beobachtungen auf Konferenzen, Tagungen und Veranstaltungen in Berlin stellen politische Dokumente die empirische Basis dar, um Narrative, Rationalitäten und Akteursfelder dieser Policy genauer herauszuarbeiten. Hierbei verfolge ich im Kern drei analytische Perspektiven:

Erstens untersuche ich die imaginäre Dimension Auswärtiger Kulturpolitik, wie sie sich im Umfeld ihrer Steuerungs- und Planungszentren darstellt. Ich frage also nach dem offiziellen Narrativ von dem Auftrag, den zentralen Problemen und der Legitimität, das der Politikbereich von sich selbst produziert; ich untersuche die Vorstellungen von den geopolitischen Rahmenbedingungen, den relevanten Akteuren und kulturpolitischen Herangehensweisen, die sich hier manifestieren; ich fokussiere Konstanz und Veränderungen, Dominanz und Ambivalenz in den zentralen Begriffen, epistemologischen Ordnungen und Erzählsträngen; und ich zeichne nach, wie dieses Narrativ im Rahmen von öffentlichen Veranstaltungen mit städtischen Kontexten verwoben und somit im urbanen Raum manifest und greifbar wird.

Zweitens analysiere ich kulturpolitische Konferenzen und Veranstaltungen als Orte, an denen die Formierung einer policy community sichtbar wird. ${ }^{1}$ Hierunter verstehe ich ein - zumindest in seinem Kern - verdichtetes Akteursfeld, dessen Herstellung gerade in einem Politikbereich, der sich durch ein global verzweigtes Netzwerk auszeichnet, ebenso herausfordernd wie unverzichtbar ist. Vor diesem Hintergrund betrachte ich Problemdefinitionen, Schlüsselbegriffe oder diskursive Formationen nicht nur als Elemente der imaginären Dimension Auswärtiger Kulturpolitik, sondern zugleich als Hinweise auf die

1 | "Policy community“ im Anschluss an: Gregory Feldman: The Migration Apparatus, S. $29 \mathrm{ff}$. 
impliziten Wissensbestände, die eine Akteurin zumindest grundsätzlich teilen muss, um zu dieser policy community zu gehören; ich fokussiere Tagungen als soziale und politische Kontexte, an denen diese Wissensbestände produziert und zugleich die zentralen diskursiven Logiken des Politikbereichs stabilisiert werden; ich zeichne die Etablierung bestimmter Argumentationsfiguren und politischer Rationalitäten als »Leitplanken« nach, die sowohl Debatten zur Auswärtigen Kulturpolitik als auch kulturpolitische Handlungsfelder maßgeblich strukturieren und limitieren; und ich frage nach der Formierung eines Habitus kultureller Repräsentation als einem Beitrag zur Regulierung von Zugehörigkeit und Abgrenzung innerhalb dieser policy community.

Drittens beschäftige ich mich mit Infrastrukturen und institutionellen Ordnungen als einer zentralen Rahmenbedingung für die globale Mobilisierung von politischen Narrativen, Metaphern und Rationalitäten. Auch wenn sich auf den Konferenzen das Bemühen des Auswärtigen Amtes zeigt, das etablierte Netzwerk um private Geldgeber und Initiativen zu erweitern, so wird hier dennoch zugleich das elementare Beziehungsmuster des Politikbereichs sichtbar: In dessen Zentrum stehen weiterhin asymmetrische Relationen zwischen Auswärtigem Amt und den Mittlerorganisationen, die nicht nur den Löwenanteil der staatlichen Gelder erhalten, sondern gleichfalls in die Entwicklung kulturpolitischer Diskurse eingebunden sind. Entsprechend analysiere ich strukturelle Grundlagen für diese Asymmetrien als eine wesentliche Rahmenbedingung für das translokale Wandern von politischen Zielvorstellungen und Schlüsselbegriffen; ich zeichne Übersetzungsketten zwischen den politischen Zentren und den Praxisfeldern Auswärtiger Kulturpolitik in den Zielregionen nach; ich verdeutliche Handlungsspielräume, die sich - trotz dieser Abhängigkeiten - für Kulturmittler insbesondere aus ihren Präsenzen vor Ort, ihren (trans-) lokalen Kontakten und Erfahrungen ergeben.

Diese drei Perspektiven werden den Charakter Auswärtiger Kulturpolitik sowohl als einer Technologie des Nationalen als auch als einem zutiefst politischen Feld unterstreichen: Denn hier werden Bedeutungen gesetzt, Diskurse geprägt, Alternativen ausgeschlossen, allegorische Pakete gepackt und implizite Regeln der Zugehörigkeit definiert; hier entstehen die Grundlagen für die Formierung eines Narrativs des Nationalen, das globale Bühnen und translokale Kontaktzonen betritt. 


\section{Menschen bewegen: Akteursfeld und politisches Narrativ auf ZWEI Berliner Konferenzen}

Menschen bewegen lautet der Titel zweier Konferenzen, zu denen das Auswärtige Amt nach Berlin einlud, um im Jahr 2006 zunächst »über die zukünftige Ausrichtung der Auswärtigen Kultur- und Bildungspolitik « ${ }^{2}$ zu diskutieren und drei Jahre später Bilanz zu ziehen, »was wir bislang gemeinsam bewegt haben «. ${ }^{3}$ Die Konferenzen adressieren ein breites Spektrum von Individuen und Institutionen und lassen somit das Akteursfeld sichtbar werden, das aus Perspektive des Auswärtigen Amtes - an der Konzipierung, Planung und Umsetzung Auswärtiger Kulturpolitik zu beteiligen ist. Der Teilnehmerkreis setzt sich jeweils aus mehreren hundert Personen zusammen: Vertreterinnen von Stiftungen, Unternehmen, städtischen Kulturbehörden, namhaften Kultureinrichtungen und Vereinen treffen hier auf Mitarbeiter des Auswärtigen Amtes und der kulturpolitischen Mittlerorganisationen sowie auf Bundestagsabgeordnete, Wissenschaftlerinnen, Journalisten und Künstlerinnen. Neben einer jeweiligen Grundsatzrede des Bundesaußenministers stehen Diskussionsrunden, inhaltliche Gesprächskreise und Foren informellen Austauschs im Zentrum der Veranstaltungen.

Die Konferenztitel, die Größe der Veranstaltungen, die Breite des Teilnehmerkreises sowie die Themenvielfalt produzieren das Bild von einem dynamischen Politikfeld, das Verbindungen und Austausch sucht. Größe und Anspruch der Tagungen lassen sich als Ausdruck einer expansiven Phase des Politikbereichs verstehen, die zwischen den Jahren 2006 und $2009 \mathrm{zu}$ einem Mittelzuwachs um etwa 30\% führt. ${ }^{4}$ Nach einer Phase der finanziellen Kürzungen in der vorhergehenden Dekade ${ }^{5}$ bietet sich nun die Möglichkeit,

2 | So die Formulierung im Grußwort zu der Tagungsdokumentation 2006. Die Konferenz Menschen bewegen - Kultur und Bildung in der deutschen Außenpolitik fand am 25./26. Oktober 2006 statt. Die zweite Konferenz wurde am 25./26. April 2009 unter dem Titel Menschen bewegen - Kulturpolitik im Zeitalter der Globalisierung organisiert. Die Reden und Diskussionen beider Tagungen sind in der Publikationsreihe des Auswärtigen Amtes Edition Diplomatie unter dem jeweiligen Konferenztitel dokumentiert.

3 | So Frank-Walter Steinmeier in seinem Grußwort zur Konferenzdokumentation 2009. In: Menschen bewegen, Konferenzdokumentation 2009, S. 3.

4 | Diese Steigerungsrate führt Bundesminister Steinmeier selbst in seiner Rede auf der Konferenz im Jahre 2009 an: Menschen bewegen, Konferenzdokumentation 2009, S. 12.

5 | Die Gesamtausgaben des Bundes für AKBP fielen demnach zwischen 1996 und 2005 von 1,171 Milliarden Euro auf 1,077 Milliarden Euro. Die durch das Auswärtige Amt verantworteten Ausgaben wurden in diesem Zeitraum von etwa 667 Millionen Euro auf etwa 601 Millionen Euro reduziert. Siehe hierzu: Deutscher Bundestag (Hrsg.): Ant- 
Strukturen und Aktivitäten der Auswärtigen Kulturpolitik auszubauen: Das Bild von einem »Politikfeld in Bewegung « besitzt eine materielle Grundlage und gewinnt hierdurch an Überzeugungskraft.

Menschen bewegen lässt sich im Anschluss an die Überlegungen zu einer Anthropology of Policy als eine »mobilizing metaphor« verstehen, die Zugang zu den sozialen Dynamiken, Machttechniken und imaginären Dimensionen eines Politikbereichs bietet. Entsprechend möchte ich nun diese Metapher durch unterschiedliche Ebenen und Foren verfolgen, um folgende Fragen $\mathrm{zu}$ bearbeiten: Welches Akteursfeld wird hier adressiert? Welche politischen Organisationsformen und Regierungslogiken deuten sich an? Welche Narrative vom Charakter und dem Zweck des Politikbereichs werden hierdurch aktiviert?

Die Konferenztitel lassen sich zunächst mit dem Rahmennarrativ der Tagungen in Zusammenhang bringen: Weitreichende geopolitische Dynamiken machen - nach offizieller Lesart - grundlegende Diskussionen und Anpassungen Auswärtiger Kulturpolitik erforderlich. Der Minister formuliert diesen Ausgangspunkt der Konferenzen etwa folgendermaßen:

"Die Zeit drängt. Die Welt verändert sich in rasender Geschwindigkeit; und mit ihr die Möglichkeiten von Politik, insbesondere Außenpolitik! Die zynischen Gewissheiten des Kalten Krieges sind weg. Neue Unübersichtlichkeiten sind an ihre Stelle, neue Player sind auf die Bühne getreten. Krisen und Konflikte sind in diesem 21. Jahrhundert nicht weniger geworden. Außenpolitik war [in den letzten Jahren, J. A.] als Krisenmanagement gefragt wie nie zuvor! « 6

Diese Wahrnehmung von grundlegenden Veränderungen globaler Ordnungen führt demnach zur Notwendigkeit, über neue Themenfelder, Herangehensweisen und Akteurskonstellationen nachzudenken: So mobilisiert der Minister das Bild von einem offenen, flexiblen Netzwerk, das sich - auch wenn die etablierten Mittlerorganisationen weiterhin sein Zentrum bilden - nicht auf staatliche oder staatlich finanzierte Akteure beschränkt, sondern vielfältige Allianzen mit zivilgesellschaftlichen oder privatwirtschaftlichen Akteuren beinhalten soll:

"Auswärtige Kulturpolitik findet längst in einem sehr viel größeren Netzwerk statt. [...] viele Künstlerinnen und Künstler, Unternehmen der Kulturindustrie, Stiftungen und unabhängige Organisationen der Zivilgesellschaft, Vertreter anderer Bundesministerien und nicht zuletzt die Kulturstiftung des Bundes [...] sind Teil des Netzwerkes. Wir wollen

wort der Bundesregierung auf die Große Anfrage der Abgeordneten Dr. Uschi Eid, Marieluise Beck (Bremen), Birgitt Bender, weiterer Abgeordneter und der Fraktion Bündnis 90/ Die Grünen, Drucksache 16/4024. Berlin 2007, S. $23 \mathrm{ff}$.

6 | Menschen bewegen, Konferenzdokumentation 2009, S. 9. 
mit der heutigen Konferenz ein Startsignal geben, eine breiter werdende Zusammenarbeit in diesem Netzwerk zu erreichen. ${ }^{7}$

Gleichzeitig setzt er das Bild vom Auswärtigen Amt als einer Art Steuerungsund Dienstleistungszentrum für dieses heterogene, weltumspannende Netzwerk:

"Unser Ziel ist es, Innen eine Plattform anzubieten. Sie sind als Künstler, Kulturschaffende und Kulturvermittler die Experten - wir bieten Ihnen sozusagen außenpolitische Expertise, das Netz unserer Auslandsvertretungen, unsere diplomatischen und außenwirtschaftlichen Aktivitäten die Plattform, an die es sich anzudocken lohnt. " ${ }^{8}$

Der Konferenztitel Menschen bewegen führt hier somit zu der Vorstellung von einem sich ausdehnenden Akteursfeld, das immer neue kultur- und bildungspolitische Verknüpfungen Deutschlands mit der Welt herstellt:

"Wir hatten uns während der Konferenz 'Menschen bewegen، im Jahr 2006 vorgenommen, das Auswärtige Amt zu einem Knotenpunkt in weltweiten Netzwerken zu entwickeln und neue Netzwerke zu knüpfen. [...] Wir haben tausende neuer Kontakte in aller Welt gewonnen - als Ansprechpartner für Wirtschaft, Politik und Kultur." ${ }^{9}$

Diese Rede von Netzwerken, Knotenpunkten, Plattformen und Bewegung lässt sich als Referenz an neoliberale Regierungslogiken verstehen, die darauf abzielen, staatliche Politikfelder zugleich zu flexibilisieren und zu dynamisieren sowie durch die Mobilisierung und Einbindung privater Akteure neu zu konfigurieren. Auch die Absicht, Auswärtige Kulturpolitik im Rahmen dieser Konferenzen inhaltlich und konzeptionell mit neuen Themenfeldern zu verknüpfen und hierzu Problemstellungen und Schlagworte etwa aus wissenschaftlichen Debatten oder aus anderen Politikbereichen aufzugreifen, weist in die gleiche Richtung: Die Tagungen produzieren das Bild von einem offenen Politikstil und partizipativen Foren, in denen Inhalte, Grenzen und Zuständigkeiten einer (staatlichen) Kulturpolitik durch breite Diskussionen neu bestimmt werden. Insbesondere die thematischen Panels werden dabei nicht nur als Orte einer produktiven Wissensarbeit präsentiert, sondern gleichfalls als Foren, in denen sehr unterschiedliche Visionen von den Aufgaben und der zukünftigen Entwicklung des Politikbereichs positioniert werden können. Hier werden laborhafte Arbeits- und Organisationsformen sichtbar, deren Praxis ich an drei Beispielen kurz andeuten möchte:

7 | Menschen bewegen, Konferenzdokumentation 2006, S. 11.

$8 \mid$ Ebd., S. 13.

9 | Menschen bewegen, Konferenzdokumentation 2009, S. 71. 
In dem Panel zur Rolle der Kreativindustrie weist etwa ein Referent den deutschen Kulturinstituten die Rolle von »Außenwirtschaftsvertretungen« zu, die den expandierenden Sektor in der globalen Vermarktung seiner Produkte unterstützen sollten. Auswärtige Kulturpolitik wird hier als ein »knallharter Wirtschaftsfaktor« verstanden, der - durch die Unterstützung der Kreativindustrien - zur Zukunftsfähigkeit des ressourcenarmen Deutschlands beiträgt. Kurz: Auswärtige Kulturpolitik wird in diesem Panel über eine Sprache und Logik der Ökonomie debattiert. ${ }^{10}$

In dem Panel Erste Schritte wird Kulturpolitik - anhand von konkreten, thematisch sehr unterschiedlichen Projektbeispielen aus Afghanistan, Palästina oder Indien/Pakistan - als ein Instrument zur Verständigung und Stabilisierung diskutiert. Referentinnen und Diskussionsteilnehmer loten hierbei Verknüpfungsmöglichkeiten mit Herangehensweisen der Sicherheits- und Entwicklungspolitik sowie der zivilen Konfliktbearbeitung aus und debattieren den möglichen Beitrag deutscher Auswärtiger Kulturpolitik zu internationalen Missionen in Konfliktgebieten. Die Leitfragen, welche die Organisatoren formuliert hatten, um die Diskussionen zu strukturieren, unterstützen das Bild von dem Panel als einer Art Wissenslabor: »Welchen konkreten (politischen) Mehrwert schafft Kulturarbeit in Konflikten? Wo sind die Grenzen? Wie ist das Verhältnis zu politischen Entwicklungen?«11

In dem Panel KulturKlimaWandel stehen Möglichkeiten einer Zusammenführung Auswärtiger Kulturpolitik mit dem »außenpolitischen Ziel Klimaschutz« im Zentrum: »Die Idee geht dabei in beide Richtungen: Kultur- und Bildungsarbeit als klimapolitische Instrumente - aber auch: den Klimawandel als kulturelles Phänomen aufzugreifen. « ${ }^{12}$ Hier wird etwa das Potenzial diskutiert, die Dynamik »grüner Industrien« für die Zielsetzung der Vermittlung eines attraktiven und zeitgemäßen Deutschlandbildes nutzbar zu machen. Umweltbewusstsein wird gleichfalls als ein Faktor benannt, an dem sich »kulturelle Unterschiede« zwischen den USA und Deutschland aufzeigen und durch kulturpolitische Programme bearbeiten lassen.

Diese Beispiele ließen sich fortsetzen. Wichtig ist die Beobachtung, dass Menschen bewegen sich hier auch mit dem Bild eines dynamischen Diskussionsfeldes in Verbindung bringen lässt, das auf neue Verbindungen mit anderen Wissens- und Politikfeldern ausgerichtet ist und zugleich unterschiedlichen Akteuren die Möglichkeit bietet, - zumindest situativ - zu Teilhabern dieser policy community zu werden. Menschen bewegen deutet aber auch auf ein Selbstverständnis von den grundsätzlichen Herangehensweisen des Politikbereichs. Schließlich sind seine Zielsetzungen und Maßnahmen im großen

10| Menschen bewegen, Konferenzdokumentation 2006, S. $36 \mathrm{ff}$.

11 Menschen bewegen, Konferenzdokumentation 2009, S. 50.

$12 \mid$ Ebd., S. 89. 
Umfang auf die globale Mobilisierung von Menschen, Gütern und Wissensbeständen ausgerichtet:

Die Formel Menschen bewegen führt hierbei zunächst zu den Aktivitäten innerhalb der institutionellen Struktur, in der sich Auswärtige Kulturpolitik in der Praxis realisiert: Goethe-Institute, Auslandsschulen, Wissenschaftszentren, Stipendiaten-, Austausch- und Förderprogramme, Einladungen, Konferenzen und andere Veranstaltungen teilen - bei aller Unterschiedlichkeit in den konkreten Arbeitsweisen - das Ziel, Menschen in einem globalen Rahmen $\mathrm{zu}$ mobilisieren und hierdurch in Beziehung und Austausch zu bringen.

Zugleich verweisen die Konferenztitel auf das Anliegen, durch kulturpolitische Maßnahmen und Programme innerhalb von Menschen etwas in Bewegung zu setzen: Durch die Mobilisierung von Individuen, Inhalten und Gütern verfolgt man die Absicht, »Menschen in aller Welt zu erreichen « und Wirkungen auf deren kulturelle Interessen, Einstellungen oder sogar auf deren Verhalten zu erzielen. So möchte man zum einen »weltweit das Interesse an der deutschen Sprache und Kultur, aber auch an der deutschen Technologie, Innovation und Kreativität« fördern. Junge Menschen sollen davon überzeugt werden, »ihr Studium in Deutschland aufzunehmen «, ${ }^{13}$ um hierdurch den Studienstandort Deutschland zu stärken und zugleich ein weltweites Netzwerk von »Ansprechpartnern« und »Freunden« aufzubauen. Zum anderen geht es aber auch um die globale Verbreitung bestimmter »Werte« wie »Toleranz«, »Achtung« und »gegenseitiges Verständnis« als Grundlagen menschlichen Zusammenlebens, die durch Aktivitäten im Rahmen der Auswärtige Kulturpolitik gefördert werden sollen: »Mit ihr gelingt es, Brücken zwischen den Menschen über Grenzen hinweg zu bauen. « ${ }^{14}$ Der Konferenztitel Menschen bewegen deutet somit auch auf die intendierte Mobilisierung von Werten, politischen Begriffen und Idealvorstellungen von Lebensweisen, um hierdurch Individuen und Gruppen in den unterschiedlichsten Kontaktzonen und Handlungsfeldern Auswärtiger Kulturpolitik zu erreichen.

Um es zusammenzufassen: Entlang des Konferenztitels und der zentralen Metapher Bewegung deutet sich ein politisches Narrativ an, das von geopolitischen Veränderungen, partizipativen Politikformen, dynamischen weltumspannenden Netzwerken und laborartigen Arbeitsweisen ebenso erzählt wie von den Intentionen und Adressatinnen Auswärtiger Kulturpolitik. Das globale Zielpublikum, das es dabei zu bewegen gilt, sowie die Partner aus den Einsatzgebieten sind auf den Konferenzen praktisch nicht vertreten: Das Akteursfeld, das sich zur Diskussion und Konzipierung dieser auf weltweite Präsenzen und Wirkung ausgelegten Policy konstituiert, setzt sich bis auf wenige Ausnahmen aus Deutschen zusammen - ein erstaunlicher Befund, wenn

13 | Menschen bewegen, Konferenzdokumentation 2006, S. 16.

14 | Menschen bewegen, Konferenzdokumentation 2009, S. 3. 
man bedenkt, dass es im Selbstverständnis dieses Politikbereichs eben um das »Bauen von Brücken«, das »Knüpfen von Kontakten« oder um den Aufbau von weltweiten »Dialogbeziehungen « gehen soll. ${ }^{15}$

\section{Diskursive Formationen: SCHLÜSSELBEgRIfFe, Konfigurationen KULtURELler Bedeutungen, Politische Rationalitäten}

In diesem Abschnitt untersuche ich nun explizit den inhaltlichen Kern des politischen Narrativs Auswärtiger Kulturpolitik. Ich fokussiere hierzu seine elementaren diskursiven Formationen, deren innere Struktur, Dynamiken und zentralen Bestandteile ich auf Basis der Redebeiträge und Diskussionen auf den beiden Konferenzen über einen analytischen Dreischritt herausarbeiten möchte: Zunächst identifiziere ich mit »Deutschlandbilder vermitteln « und »Dialog« zwei Schlüsselbegriffe, die nicht nur die Debatten strukturieren, sondern zugleich in einer Art kollektiven Selbstverständigung die grundlegenden Annahmen vom Sinn und Zweck des Politikbereichs festigen. Zweitens zeichne ich die Konfigurationen kultureller Bedeutungen nach, die diese Metaphern umgeben. Hierunter verstehe ich das jeweilige Geflecht von Argumentationsketten, das sich um einen zentralen Begriff gruppiert und Begründungen für die thematischen Schwerpunkte und Aktivitäten Auswärtiger Kulturpolitik anbietet. Diese Konfigurationen bilden die Basis, um drittens die politischen Rationalitäten herauszuarbeiten, welche die Diskussionen durchziehen, zugleich in Regierungstechniken und Alltagspraxen zur Anwendung kommen und somit zentrale Verbindungslinien zwischen Diskurs- und Praxisfeldern darstellen. Bei der Untersuchung solcher Rationalitäten geht es nicht um die Frage, ob sich ein Politikbereich anhand irgendwelcher Kriterien als »rational« beschreiben lässt, sondern um die Analyse der jeweiligen Typen von Rationalität, die sich im Rahmen dieser Politik konstituieren: »Eine politische Rationalität ist eine Rationalität der Politik und nicht eine Reflektion über Politik. Das Interesse richtet sich auf das den Praktiken immanente Wissen, die Systematisierung und >Rationalisierung< einer Pragmatik der Führung. « $^{16}$

15 | Diese Zusammensetzung des Teilnehmerkreises lässt sich anhand der Teilnehmerlisten in den beiden Konferenzdokumentationen nachvollziehen: Menschen bewegen, Konferenzdokumentation 2006, S. 191-209; Menschen bewegen, Konferenzdokumentation 2009, S. 159-175.

16 | Thomas Lemke, Susanne Krasmann \& Ulrich Bröckling: Gouvernementalität, Neoliberalismus und Selbsttechnologien. Eine Einleitung. In: Ulrich Bröckling, Susanne Krasmann und Thomas Lemke (Hrsg.): Gouvernementalität der Gegenwart. Studien zur Ökonomisierung des Sozialen. Frankfurt 2000, S. 7-40, S. 20. Siehe auch: Michel 
Hieran anknüpfend verstehe ich politische Rationalitäten als steuernde Logiken oder - konkreter ausgedrückt - als Regulierungs-, Gestaltungs- und Ordnungsansprüche, die einem Politikbereich eingelagert sind.

Die Metaphern, Konfigurationen und Rationalitäten führen zugleich zu den Wissensbeständen und Auffassungen, die eine Person oder Institution teilen muss, um innerhalb des Feldes Auswärtige Kulturpolitik »mitspielen« zu können. Sie bieten somit Zugang zur Erforschung der Machtrelationen sowie der Regeln der Zugehörigkeit, die den Politikbereich durchziehen. Pierre Bourdieu hat daran erinnert, dass Status, Teilhabe und Positionen in vielen sozialen, politischen oder kulturellen Feldern gerade auch auf einem »impliziten Wissen « und der Akzeptanz gewisser Grundannahmen beruhen. Im Kern geht es dabei um Wissensbestände, Fertigkeiten oder Haltungen, über die ein Akteur verfügen muss, um sich in einem jeweiligen Feld »normal« verhalten zu können. Eine Person oder eine Institution, die dieses Wissen nicht teilt, wird sich demnach in ihm bewegen, »wie ein Hund in einem Kegelspiel, einem Spiel, dessen implizite Regeln er nicht kennt «. ${ }^{17}$ Die Analyse diskursiver Formationen führt daher nicht nur zu den imaginären Dimensionen eines Politikbereichs; sie leistet zugleich einen Beitrag zu der Ethnografie der Formierung einer policy community.

\subsection{Schlüsselbegriff und Konfiguration kultureller Bedeutungen I: Deutschlandbilder vermitteln oder "Womit soll Deutschland kulturell leuchten?"}

Deutschlandbilder vermitteln lässt sich als eine erste Metapher identifizieren, welche die Konferenzen durchzieht und zugleich eine zentrale Figur zur Legitimierung des Politikbereichs bildet. Der Wunsch, Deutschlandbilder in internationale Kontexte einzubringen und somit also eine Imagologie und ein Narrativ vom Nationalen für globale Bühnen zu entwickeln, wird als Dreh- und Angelpunkt der Debatten erkennbar: »Deutschland ist ein facettenreiches, innovatives und kreatives Land. Aber wie zeigen wir das unseren Partnern in der Welt? ${ }^{18}$ heißt es etwa im Jahr 2009 zur Einleitung des Panels Deutschlandmosaik. Deutlich wird hierbei, dass es um eine spezifische Form der Außendarstellung geht: Deutschland wird mit positiv konnotierten Eigenschaften verknüpft; Kultur bietet hierzu wesentliche Potenziale. Diese Denkfigur lässt sich anhand eines Redebeitrags des Außenministers verdeutlichen:

Foucault: Für eine Kritik der politischen Vernunft, in: Lettre International, 1 (1988), S. 58-66.

17 | Siehe hierzu: Pierre Bourdieu: Das politische Feld. Zur Kritik der politischen Vernunft. Konstanz 2001, S. 47.

18 | Menschen bewegen, Konferenzdokumentation 2009, S. 105. 
"Und ohne den spektakulären Erfolg der Fußball-WM für unser Bild im Ausland unterschätzen zu wollen: Wenn Deutschland zunehmend als fröhliches, innovatives, offenes und tolerantes Land wahrgenommen wird mit einer eigenen und aussagekräftigen Kreativität, dann ist das auch, wenn nicht zuförderst das Verdienst der Kulturschaffenden in Deutschland! «19

Die Besucher begegnen dem Selbstbild von dem »fröhlichen, innovativen, offenen Land « sowie dem Auftrag zur Vermittlung von Deutschlandbildern nicht nur in Diskussionen und Redebeiträgen, sondern gleichfalls in der Ausgestaltung der Räumlichkeiten: Im Jahr 2009 ist im Eingangsbereich der Konferenz die Deutschlandkollektion ausgestellt - »zeitgemäße Kontaktpflegegeschenke für das Auswärtige Amt«, die in einem Kooperationsprojekt von Studierenden vier deutscher Kunst- und Designhochschulen entworfen worden waren. »Praktisch und unterhaltsam sollten diese sein, von einem weltoffenen Deutschland künden und nicht als Staubfänger in Schrankwänden verschwinden«, heißt es zur Erklärung. ${ }^{20}$ Als Ergebnis entstanden etwa Minidrehorgeln mit »weltbekannten deutschen Hits«, ein Zauberwürfel mit Umlauten und »ß«, eine schwarz-rot-goldene Tragetasche namens BotschaftsBEUTEL, oder ein Tischmagnet in Gestalt des Brandenburger Tors zum Sammeln von Büroklammern: »Charmant, witzig und mit einem verschmitzten Blick auf die eigene Kultur«, charakterisieren die Projektteilnehmer ihre Produkte. ${ }^{21}$

Die Figur Deutschlandbilder vermitteln führt hier also zu einer Ästhetisierung der Vorstellungen von nationalen Klischees, Stereotypen und kulturellen Besonderheiten sowie zu deren Übersetzung in materialisierte Formen, die als Geschenke durch das globale Netzwerk deutscher Außenpolitik wandern können. Die Projektgruppe nutzt ähnliche Adjektive wie der Minister zu der Beschreibung des Landes und positioniert ihre Objekte somit als Beiträge zu einem mobilisierbaren Narrativ vom Nationalen: »Die Deutschlandkollektion zeigt sich jetzt vielfältig. Sie ist unkonventionell, zeitgemäß, persönlich, heiter, spielerisch, mehrdeutig, schwarz, rot, gold, herzlich, zweckmäßig und natürlich sehr deutsch. ${ }^{22}$

Im Jahr 2006 werden die Konferenzteilnehmer aufgefordert, die Frage »Womit soll Deutschland im Ausland kulturell leuchten?« auf Kärtchen zu beantworten und ihre Vorschläge auf einer Pinnwand zu präsentieren. Die Zielvorstellung einer positiven Außendarstellung wird somit für Assoziationen geöffnet und gleichzeitig in ihrer Zentralität bestätigt. Anhand einer Auswahl von Antworten lassen sich die vielfältigen Verknüpfungsmöglichkeiten erkennen:

19 | Menschen bewegen, Konferenzdokumentation 2006, S. 18.

20 | Menschen bewegen, Konferenzdokumentation 2009, S. 21.

21 | http://deutschlandkollektion.de (letzter Zugriff am 11. August 2017).

22 | Menschen bewegen, Konferenzdokumentation 2009, S. $20 \mathrm{ff}$. 
"Durch die Vermittlung seines kulturellen Erbes als wichtigem Bestandteil unserer europäischen und global(isiert)en Gegenwart" "Selbstkritisch und selbstreflektiert als ein positives Beispiel für eine lebendige Demokratie"

"Als sprachliches und kulturelles Herz Europas"

"Mit uns Menschen mit Migrations-Hintergrund"

"Mit Friedenspolitik gegen ABC-Waffen"

"Warum sollte Deutschland leuchten? Es reicht doch, wenn wir mit allem Licht und Schatten im Ausland fair wahrgenommen werden" "Es leuchtet bereits mit Fußball, Hip-Hop, Rap und Berlin «. ${ }^{23}$

»Deutschland« bildet auf beiden Konferenzen auch den Schlüsselbegriff der jeweiligen Abschlussdiskussion: Im Jahr 2009 trägt sie etwa den Titel MY Space Deutschland - Über Deutschland im 21. Jahrhundert und lädt ein zu einer reflexiven Positionsbestimmung anhand der Fragen: »Welche Identität haben die Deutschen? Was ist typisch deutsch? Welches Image hat die Bundesrepublik im Ausland? $^{24}$

Auf den Konferenzen entstehen also gewissermaßen Panoramen des Nationalen, die einen offiziellen Konsens von der Kernaufgabe des Politikbereichs in diskursiven und materiellen Formen erfahrbar machen: Es geht um eine kulturell unterfütterte Selbstdarstellung, die in einer Verknüpfung des Landes mit positiv konnotierten Begriffen und Bildern gründet. Die Metapher Vermittlung von Deutschlandbildern führt somit nicht nur zu unterschiedlichen Varianten der Ästhetisierung und Reflexivierung des Nationalen, sondern zugleich zu zentralen Wissensbeständen und Grundfiguren des Politikbereichs, die im Rahmen solcher Konferenzen reproduziert werden: Die Existenz einer »nationalen Spezifik«, die sich auch kulturell repräsentieren lässt, wird hier ebenso vorausgesetzt wie die Realität eines »kulturellen Wir« als Ausgangspunkt globaler Vermittlungsprojekte.

Von Interesse ist insbesondere die Vielfalt der Verknüpfungsmöglichkeiten dieses Schlüsselbegriffs. Ich möchte seine Konfiguration kultureller Bedeutungen nun anhand von drei Argumentationsketten erschließen, die auf den Konferenzen besonders in den Vordergrund treten.

\section{Argumentationskette I: "...wo die Kreativität zu Hause ist" - Deutschland als globale Marke?}

Im Jahr 2006 besitzen Bezüge zu ökonomischen Begrifflichkeiten und Denkformen eine herausgehobene Präsenz. Diese zeigen sich etwa in den Eröffnungsreden des Ministers und vor allem des Vorstandsvorsitzenden der global

23 | Konferenzdokumentation 2006, S.185-189.

24 | Konferenzdokumentation 2009, S. 129. 
erfolgreichen Firma SAP, aber auch in dem Panel Modernes Profil: Die Rolle der Kreativindustrie: Hier wird die »wirtschaftliche Bedeutung des Kultursektors für Arbeitsmarkt, Wachstum und technologischen Fortschritt« herausgestellt und Auswärtige Kulturpolitik vor diesem Hintergrund als eine »Investition in die Zukunft« bezeichnet. ${ }^{25}$

Aber auch auf »Deutschland « selbst wird eine unternehmerische Logik angewandt: In Rede- und Diskussionsbeiträgen scheint die Denkfigur hindurch, das Land gewissermaßen zu einer »globalen Marke« zu entwickeln, deren Kern aus Begriffen wie »Toleranz«, »Offenheit«, »Innovation« und »Kreativität« besteht. Kultur und Kulturpolitik erscheinen aus dieser Perspektive als Beitrag zur Produktion eines weltweit abrufbaren Images, um die Aufmerksamkeit junger Eliten auf das Land zu ziehen. Deutschlandbilder vermitteln erfährt hierbei eine Verknüpfung mit dem Konferenztitel Menschen bewegen - eine attraktive, kulturell ausgestaltetes Außendarstellung soll die »besten Köpfe« der Welt für deutsche Universitäten und Unternehmen interessieren und somit aus dem Land ein führendes »Gravitationszentrum von Innovationsnetzwerken« machen. ${ }^{26}$ In dieser Argumentationslinie erscheinen kulturpolitische Formate somit als Bausteine zur Entwicklung eines kommodifizierbaren Bildes von der »Innovationsnation Deutschland $\aleph^{27}$, »wo die Kreativität zuhause ist «. ${ }^{28}$ Eine fehlende Flexibilität - etwa bei der »gezielten Förderung von Existenzgründungen« - oder »Ausländerhass« in Deutschland werden hierbei als Standortnachteile im Kampf um die global mobilen Wissenseliten zum Problem. ${ }^{29}$

Deutlich sind allerdings Unterschiede zwischen beiden Konferenzen: Die Verbindung zwischen Deutschlandbildern, Ökonomie, Kulturpolitik und Zukunftsfähigkeit stellt auf der ersten Konferenz eine herausgehobene Argumentationslinie dar, um drei Jahre später - schon unter dem Eindruck der globalen Finanz- und Wirtschaftskrise - zumindest aus den Diskussionen weitgehend zu verschwinden. Solche Unterschiede verweisen grundsätzlich auf die allegorischen Potenziale von Schlüsselbegriffen, die sich realisieren können, aber nicht müssen. Zugleich hat die Vorstellung der Entwicklung einer Markenidentität für »Deutschland « in anderen Formen, etwa durch die vorgestellte Deutschlandkollektion, auch auf der zweiten Konferenz Eingang gefunden und bleibt somit als Bedeutungskette präsent.

25 So Frank-Walter Steinmeier, in: Menschen bewegen, Konferenzdokumentation 2006, S. $16 \mathrm{f}$.

26 | So Henning Kagermann, in: Ebd., S. 29.

27 | Ebd., S. 30.

28 | So ein Diskussionsbeitrag, in: Ebd., S. 57.

29 | Ebd., S. 29. 


\section{Argumentationskette II: "Nationale Leitkultur" versus "Abschied vom Länderspiel«? - konzeptionelle Ausgangspunkte der kulturellen Selbstdarstellung}

Entlang einer zweiten Argumentationskette lassen sich Debatten über die Grundlagen und Ausgangspunkte der Selbstdarstellung im Modus der Kultur nachzeichnen. Hier finden sich einerseits Positionen, die einen nationalkulturellen »Kanon« oder eine Selbstvergewisserung über »eigene Kultur« und »eigene Werte« als notwendige Basis einfordern. So formuliert etwa die Bundestagsabgeordnete Monika Grütters ihr Verständnis von kollektiv bindenden »Kulturen«, das auf Vorstellungen von Begrenzung, »Fundament« und Subs$\operatorname{tanz}$ aufbaut:

"Um Brücken zwischen den Kulturen zu bauen und neue Einflüsse aufzunehmen, bedarf es meiner Ansicht nach eines eigenen Grundverständnisses. Damit stoßen wir in die Diskussion über eine nationale Leitkultur. Ohne eigenes Fundament ethischer, kultureller oder religiöser Werte werden die Vorstellungen anderer Kulturen nicht greifbar, nicht verstehbar. ${ }^{30}$

Hieran anknüpfend fordert ein Teilnehmer in der folgenden Diskussion, »Deutschland müsse sich seiner eigenen Identität bewusst werden und einen Kanon ausbilden, der im Ausland vorgestellt werden solle. ${ }^{31}$

Andererseits zeigen sich Gegenpositionen, die aufgrund der sichtbaren Diversität und globaler Verflechtungen in Gegenwartsgesellschaften einem »statischen Begriff von Kultur« kritisch gegenüber stehen. So formuliert etwa der Minister: »Deswegen kann Auswärtige Kulturpolitik auch nicht mit Kultur als einem homogenen, nationalstaatlich eingegrenzten, fest stehenden Block oder Kanon von Werken, Werten oder kulturellen Waren arbeiten. «2 $^{32}$

Verweise auf die Flexibilisierung nationaler Identitäten, die Bedeutung von Migrationsbewegungen oder auf die Normalität grenzüberschreitender kultureller Produktionen führen auch zu der Forderung nach einem »Abschied vom Länderspiel ${ }^{33}$ als der bisherigen Leitvorstellung Auswärtiger Kulturpolitik. Aus der Perspektive der kulturpolitischen Praxis werden Präsenzen und Strategien in Feldern diskutiert, in denen ein nationalkultureller Ansatz an Grenzen stößt: »Ist in Metropolen Platz für eine >klassische nationale< Kulturpolitik? Wenn ja: wie müsste sie aussehen? Kommen wir unter Umständen mit einem projektbezogenen Ansatz weiter? $^{34}$

30 | Ebd., S. 66.

31 | Ebd., S. $73 f$.

$32 \mid$ Ebd., S. 13.

33 | So Zafer Senocak, in: Ebd., S. $70 f$.

34 | Menschen bewegen, Konferenzdokumentation 2009, S. 29. 
Auf den Konferenzen können also recht unterschiedliche Positionen zu kulturpolitischen Grundbegriffen, kulturellen Grenzen und Dynamiken auftauchen, sich mit etablierten Argumentationsfiguren verbinden, aber auch ohne größere Spuren wieder verschwinden. Hierbei werden Bezüge zu zeitgenössischen Diskussionen $\mathrm{zu} » K u l t u r «$ jenseits des Politikbereichs sichtbar. Aber gerade anhand der eher zaghaften Versuche, die Grundvorstellungen einer nationalkulturellen Repräsentation infrage zu stellen, zeigt sich nochmals, dass die Vermittlung einer kulturell darstellbaren »nationalen Spezifik« bisher den weitgehend gesetzten Kernauftrag des Politikbereichs ausmachte.

\section{Argumentationskette III: "Kultur der Demokratie" - Projekte zur Universalisierung nationaler Spezifik?}

Drittens treten Bedeutungsketten hervor, die Deutschlandbilder vermitteln mit Kategorien verknüpfen, die keine klassischen Bestandteile des Kanons einer »deutschen Kulturnation ${ }^{35}$ darstellen, sondern eher den Vorstellungen einer Zugehörigkeit zu einer »westlichen Zivilisation«, einer »europäischen Kultur« oder auch zeitgenössischen Diskussionen im Kontext von internationalen Organisationen entstammen. So tauchen Demokratie, Zivilgesellschaft oder Rechtsstaatlichkeit als Elemente der Selbstbeschreibung auf, wie sich anhand der Einleitung zu dem Panel Erste Schritte zeigt: $Z$ Zu unserer Kultur zählt ohne Zweifel auch eine >Kultur der Demokratie $<$ und der Zivilität. ${ }^{36}$ Auch die Zielvorstellung Konfliktprävention führt zu solchen neuen Verbindungen, deren Ausgestaltung in den folgenden Abschnitten vertiefend untersucht wird. Fraglos zeigen sich hier Bemühungen, einen nationalstaatlichen Politikbereich auf neue Problemlagen und Arbeitsfelder hin auszurichten; Formulierungen wie »unsere Kultur« oder »unsere Werte« verweisen aber zugleich auf ein Festhalten an den etablierten Grundfiguren der kulturellen Selbstdarstellung.

Die Konfiguration kultureller Bedeutungen, die sich auf den beiden Konferenzen um die Metapher Deutschlandbilder vermitteln gruppiert, lässt sich zusammenfassend durch vier Diskussionsfelder darstellen, die auf jeweils komplexe Argumentationslinien verweisen und in der folgenden Illustration durch vier Schlagworte angedeutet werden:

35 | Siehe zum Begriff der "Kulturnation «: Irene Götz: Deutsche Identitäten, insbesondere S. $119 \mathrm{ff}$.

36 | Menschen bewegen, Konferenzdokumentation 2009, S. 51. 


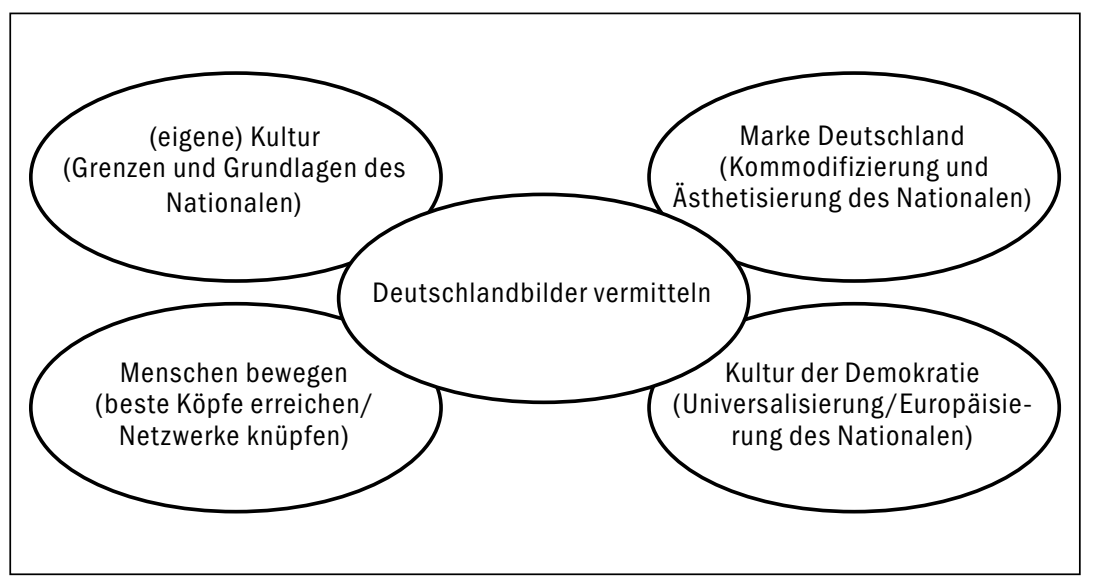

\subsection{Schlüsselbegriff und Konfiguration kultureller Bedeutungen II: Dialog oder "das oberste Prinzip Auswärtiger Kulturpolitik“}

Dialog bildet den zweiten Schlüsselbegriff, der die Diskussionen auf den Konferenzen durchdringt und durch den Minister sogar als Leitgedanke des Politikbereichs angeführt wird: »Dialog ist das oberste Prinzip der Außenpolitik und auch der Auswärtigen Kulturpolitik.«37 Die Metapher Dialog lenkt zunächst den Blick auf das offizielle und öffentlich gemachte Selbstverständnis deutscher Außenpolitik, das sich um Zielvorstellungen wie Multilateralismus, Kooperation, Ausgleich und friedliche Konfliktlösung gruppiert ${ }^{38}$ und lässt sich zugleich mit dem Motiv der Vermittlung von Deutschlandbildern verbinden: Denn auch wenn Dialog nicht direkt auf die etablierten Bilder der »Kulturnation« verweist, so führt der Begriff dennoch zu den Formen einer impliziten Selbstdarstellung. Dialog bezeichnet ein »Prinzip« oder eine »Haltung « und steht somit für ein Idealbild von den eigenen Herangehensweisen, das - unabhängig von jeweiligen Inhalten - in die Repräsentationen Deutschlands auf globalen Bühnen einfließen soll.

Auch die Metapher Dialog besitzt auf den Tagungen eine spürbare Präsenz - ein dialogisches Prinzip findet sich bereits in Organisationsformen wieder und ist somit im Programmablauf erfahrbar: Arbeitsgruppen bilden hier den Rahmen zur Diskussion thematischer Schwerpunkte, zur Einbindung der Perspektiven unterschiedlicher Akteure sowie zur Erarbeitung von Ergebnissen, die hinterher zur weiteren Diskussion gestellt werden. Dialog führt hier also

37 | Menschen bewegen, Konferenzdokumentation 2006, S. 15.

38 | So in der Selbstdarstellung auf der Homepage des Auswärtigen Amtes zu den "Grundsätzen deutscher Außenpolitik“ im Jahr 2009. 
auch zu spezifischen Regierungstechniken, die demonstrativ einbinden, zur Partizipation einladen und sich um das Knüpfen von Netzwerken bemühen. Die Metapher findet hierdurch Anschluss an den Konferenztitel Menschen bewegen, wie sich etwa anhand folgender Stellungnahme des Ministers zeigt:

"[...] wir wollen regelmäßig Verantwortliche aus vielen Bereichen zum Gespräch einladen. Eine Kultur des Dialogs auch zwischen uns und dem hier vertretenen Netzwerk etablieren. Um mit Innen gemeinsam Ideen zu sammeln und zu besprechen, neue Entwicklungen und Anforderungen zu diskutieren und gemeinsam in die Praxis umzusetzen. ${ }^{39}$

Die Metapher Dialog verweist aber zugleich auf inhaltliche Überlegungen zu den Arbeitsformen, Ausgangspunkten und dem Selbstverständnis kultureller Vermittlung - für die Erarbeitung der Konfiguration kultureller Bedeutungen, die diesen zweiten Schlüsselbegriff umgibt, sind zwei Argumentationslinien von besonderer Relevanz.

\section{Argumentationskette I: Variationen zum Thema Wechselseitigkeit - Was bedeutet Dialog in der kulturpolitischen Praxis?}

So treten zunächst unterschiedliche Positionen zutage, was das Ideal von einem »dialogischen Prinzip« für die kulturpolitische Praxis bedeuten kann. In vielen Redebeiträgen finden sich Motive von einer Wechselseitigkeit oder vom Kulturaustausch als Zweibahnstraße, um erstrebenswerte Formen der kulturellen Vermittlung zu beschreiben. Entlang des Schlüsselbegriffs Dialog werden somit Ideal- und Selbstbilder einer policy community deutlich, der es erklärtermaßen nicht alleine um eine einseitige Präsentation von Deutschlandbildern im Ausland geht, sondern gleichfalls um die parallele Vermittlung kultureller Bilder, Perspektiven und Positionen nach Deutschland - nicht zuletzt um hierdurch das Land, seine kulturelle Szene und Identität zu internationalisieren. Dieser Zusammenhang zeigt sich in dem Verweis des Ministers auf das in Berlins Mitte entstehende Humboldt-Forum als einen Ort,

"wo sich nationales Kulturerbe, die Kulturen der Welt und Gäste aus aller Welt treffen. Wo wir den Weltkulturen einen gleichberechtigten Platz einräumen, in einen neuartigen Dialog mit den Kulturen der Welt eintreten wollen und auch für unsere eigene Gesellschaft klar machen können: unterschiedliche Herkunft und gemeinsame Zukunft, das sind keine Gegensätze. « ${ }^{40}$

Die Betonung von Wechselseitigkeit führt auch zu Überlegungen, wie mit den globalen politischen oder ökonomischen Asymmetrien, die kulturpolitische

39 | Menschen bewegen, Konferenzdokumentation 2006, S. 13.

40 | Menschen bewegen, Konferenzdokumentation 2009, S. 18. 
Handlungsfelder offensichtlich durchziehen, umzugehen ist: Eine Bundestagsabgeordnete betont etwa, »Kulturdialog müsse auf Augenhöhe stattfinden und die Kultur der Zielländer unterstützen ${ }^{41}$ In anderen Positionen wird Dialog noch weitergehend als ein mögliches Verfahren verstanden, um in einer heterogenen, konfliktreichen und hierarchischen Welt Gleichwertigkeit herzustellen. ${ }^{42}$ In beiden Fällen bleibt allerdings offen, was dies für die praktische Umsetzung kultureller Vermittlung bedeuten kann.

An solche Überlegungen schließt sich direkt die Frage nach den jeweiligen Vorstellungen von den Dialogpartnern an. Einerseits zielt Dialog häufig auf Formen der Begegnung zwischen nationalstaatlichen Einheiten und leistet somit einen Beitrag zur Naturalisierung der etablierten Ideen vom »Normalzustand « des Kulturaustauschs im internationalen Rahmen: So benennt etwa der Minister die mit seinem türkischen Amtskollegen vereinbarte deutsch-türkische Ernst-Reuter-Initiative als ein Beispiel für die »dialogische Haltung« innerhalb der Auswärtigen Kulturpolitik. ${ }^{43}$ Andererseits finden sich Argumentationsketten, die Dialog als einen Beitrag zur Veränderung solcher Vorstellungen von den Grundelementen des internationalen Kulturaustauschs betrachten. Das Ideal von der kulturpolitischen Wechselseitigkeit kann demnach zu der Relativierung des Bildes von der Existenz eines eindeutig abgrenzbaren »kulturellen Innen und Außen« führen, wie sich wiederum an einem Redebeitrag des Außenministers zeigen lässt:

"In einer globalisierten Welt sind Innen- und Außensicht kaum noch zu trennen. Wir brauchen die kreative Auseinandersetzung mit dem scheinbar Fremden. Nicht nur um scheinbar Fremdes besser zu verstehen. Sondern vor allem: um das je Eigene besser zu begreifen. Kreativität als Voraussetzung kulturellen - und wirtschaftlichen - Handelns braucht den Austausch, die Diskussion und die Differenzierung. Deswegen habe ich seit Beginn meiner Amtszeit einen Schwerpunkt gelegt auf Projekte, die eine Kultur des Dialogs unterstützen." ${ }^{44}$

Entlang der Metapher Dialog werden somit gewisse Ambivalenzen einer Kulturpolitik sichtbar, die ursprünglich um die Zielsetzung der kulturellen Repräsentation eines Nationalstaates organisiert war, aber zugleich zunehmend mit politischen, kulturellen und gesellschaftlichen Tendenzen zur Flexibilisierung solcher Vorstellungen konfrontiert wird.

$41 \mid$ Ebd., S. 52.

42 So im Prinzip: Naika Foroutan, in: Menschen bewegen, Konferenzdokumentation 2006, S. 149.

43 | Ebd., S. 15.

44 | Ebd., S. 14. 


\section{Argumentationskette II: Dialog, Kultur, Konflikt - Wissensarbeit einer policy community}

Ein zweites Diskussionsfeld verknüpft Dialog mit den Begriffen Kultur und Konflikt und weist somit in die Richtung der in dieser Studie zentralen diskursiven Formation einer Auswärtigen Kulturpolitik als Konfliktprävention. In verschiedenen Stellungnahmen werden die Potenziale von Kultur und Dialog zur Konfliktbearbeitung betont und hieraus ein besonderer außenpolitischer Wert abgeleitet, wie sich anhand eines weiteren Redebeitrags des Ministers verdeutlichen lässt:

"Ich will Kultur nicht instrumentalisieren, nicht politisieren und insbesondere nicht überfordern. Aber ich weiß, dass Kultur etwas kann, was Politik nicht kann: Sie kann abseits der Alltagskonflikte und politischen Interessen die Steine sammeln für die Brückenpfeiler, über die Verständigung läuft, wenn sie stark genug sind. Das verstehe ich unter kulturellem Dialog: Ein Dialog, der fordert, aber auch hinhört. «45

Deutlich wird hier die Verfestigung der Hoffnung auf kulturelle Effekte in politisch schwierigen Konstellationen und somit auch der Vorstellung, dass kulturpolitische Maßnahmen zu einer positiven Bearbeitung von Konflikten eingesetzt werden können. Dieser Prozess der Verfestigung - etwa im Vergleich zu der in der Einleitung erwähnten Stuttgarter Konferenz im Jahr 200I - zeigt sich auch anhand der unterschiedlichen Schwerpunkte der beiden Panels, die sich in den Jahren 2006 und 2009 dieser Verbindung von Dialog, Kultur und Konflikt widmen: Auf der ersten Konferenz stehen grundsätzliche Diskussionen zu der Bedeutung und möglichen Programmatik einer Kultur des Dialoges in einer globalisierten Welt ${ }^{46}$ im Zentrum; im Jahr 2009 tritt hingegen ein eher praxisorientiertes Interesse in den Vordergrund - also ein Fokus auf sehr unterschiedliche, laborartig nebeneinandergestellte kulturpolitische Aktivitäten, Projektformen und institutionelle Arrangements sowie auf deren mögliche Wirkungen in verschiedenen Konfliktgebieten.

Der Gedanke einer Auswärtigen Kulturpolitik als Konfliktprävention hat sich somit etabliert, stellt sich aber zugleich nicht als das neue Leitmotiv des gesamten Politikbereichs dar, sondern als eines von mehreren wichtigen Themen, die jeweils in eine eigene Sektion verwiesen werden. Dabei wird anhand des Namens und der Beschreibung des Panels Erste Schritte deutlich, dass es - aus Perspektive des politischen Zentrums - weiterhin darum geht, Informationen zu sammeln, Erfahrungen auszutauschen und sinnvolle Maßnahmen zu eva-

45 | Menschen bewegen, Konferenzdokumentation 2009, S. 10.

46 | So der Titel der Arbeitsgruppe im Jahr 2006; siehe hierzu die Dokumentation des Panels in: Menschen bewegen, Konferenzdokumentation 2006, S. $142 \mathrm{ff}$. 
luieren. Der Prozess der Etablierung der kulturpolitischen Zielsetzung Konfliktprävention zeigt sich demnach als nicht abgeschlossen:

"Wo Politik trennt, kann Kultur einen Raum zumindest der Begegnung, vielleicht auch der Verständigung schaffen. Deshalb haben wir in den vergangenen Jahren die Instrumente der Auswärtigen Kultur- und Bildungspolitik verstärkt in Regionen eingesetzt, die von inner- oder zwischenstaatlichen Konflikten und Spannungen geprägt sind: um Sprachlosigkeit zu überwinden und Dialoge zu beginnen bzw. fortzusetzen. [...] Über diese ersten Schritte haben wir gesprochen, über die daraus gewonnenen Erfahrungen, aber auch über neu einzuschlagende Wege der Kultur- und Bildungsarbeit in Krisen- und Konfliktregionen." ${ }^{47}$

Dialog wird in diesen Panels somit auch als eine Vorgehensweise genutzt, um innerhalb der policy community neues Wissen zu erarbeiten: etwa zu dem Charakter unterschiedlicher Konfliktkonstellationen und den jeweiligen kulturpolitischen Interventionsmöglichkeiten; zu Formen der Zusammenarbeit zwischen verschiedenen Politik- und Wissensfeldern wie etwa Auswärtige Kulturpolitik, Entwicklungszusammenarbeit und zivile Konfliktbearbeitung; aber auch zu passenden Projektformaten in der konkreten Arbeitspraxis. Das Bild einer dialogischen Wissensproduktion innerhalb der Auswärtigen Kulturpolitik und ihr möglicher Beitrag zu globalen Politikfeldern und Austauschprozessen lässt sich anhand der Position eines Diskussionsteilnehmers verdeutlichen:

"Unsere 'globalisierte Weltı ist aber (noch) keine Welt der gegenseitigen Kenntnis. In dieser Welt erleben wir heute weniger einen Zusammenstoß der Kulturen oder der Zivilisationen, der ,den Westen ' gegen die rarabisch-muslimische Welt in Stellung bringen würde - ein Kulturkampf findet eher innerhalb der arabischen und muslimischen Gesellschaften statt -, als vielmehr eine gegenseitige kulturelle Ignoranz (wenig echtes Wissen, viele 'Eindrücke،) und Indifferenz gegenüber den anderen Kulturen, ihren Leistungen, Werten, Ängsten, Leiden oder Problemen. Kulturdialog bedeutet tatsächlich eine wichtige Möglichkeit, diesen Missstand zu beheben. Aber: Echte Konflikte sind kaum durch Kulturaustausch oder -dialog lösbar. " ${ }^{48}$

Dialog wird in dieser Stellungnahme weniger als eine statische Gesprächskonstellation zwischen abgrenzbaren Kulturen verstanden, sondern als eine dynamische epistemologische Unternehmung, die ein zentrales Problem - »Unwissen « - zu bearbeiten hilft. In anderen Stellungnahmen wird hingegen die Position von der Notwendigkeit eines »Dialogs mit der islamisch geprägten

47 | Menschen bewegen, Konferenzdokumentation 2009, S. 51.

48 | Volker Perthes, in: Menschen bewegen, Konferenzdokumentation 2006, S. 144. 
Welt als eine der größten Herausforderung für die Auswärtige Kulturpolitik « ${ }^{49}$ vertreten und somit das Gegenbild von sich gegenüberstehenden Kulturen oder Zivilisationen als relevantem globalen Ordnungsprinzip aufgerufen.

Gerade entlang der diskursiven Verknüpfungen von Dialog, Kultur und Konflikt zeigt sich Auswärtige Kulturpolitik als ein produktives Feld: Auch wenn diese Argumentationsfigur nicht zu einem klar ausformulierten Aktionsprogramm führt, so werden Tendenzen der Ausdehnung des Zuständigkeitsbereichs, der Verflechtung mit grundlegenden politischen Problemstellungen, zum Austesten von Herangehensweisen, Techniken und Instrumenten und gleichfalls die Suche nach Möglichkeiten zur Erweiterung außenpolitischer Handlungsspielräume sichtbar.

Auch die Konfiguration kultureller Bedeutungen, die sich auf diesen Tagungen um die Metapher Dialog entwickelt, lässt sich durch vier Diskussionsfelder andeuten, die wiederum auf sich anschließende Argumentationslinien verweisen:

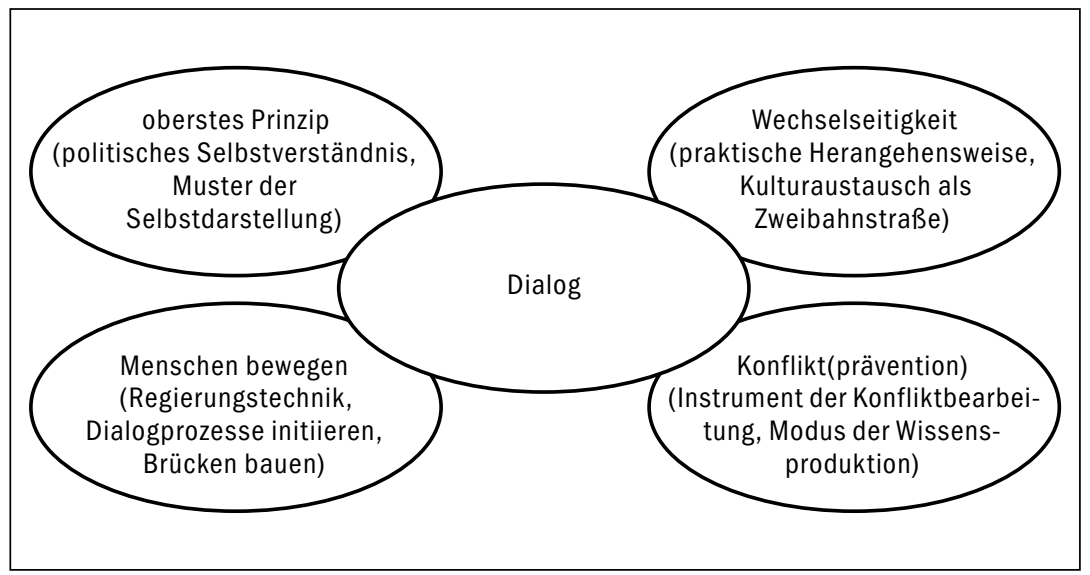

49 | So der Generalsekretär einer Mittlerorganisation, in: Ebd., S. 153. 


\subsection{Politische Rationalitäten Auswärtiger Kulturpolitik}

Zum Verständnis der imaginären Dimensionen Auswärtiger Kulturpolitik sind insbesondere auch die bereits herausgestellten Verbindungslinien zwischen den beiden Metaphern und Konfigurationen von Relevanz. So zeigte sich einerseits, dass Dialog auch als ein Modus der kulturellen Selbstdarstellung gelesen werden kann und sich somit mit der Zielvorstellung Vermittlung von Deutschlandbildern verbinden lässt. Andererseits wurde deutlich, dass Idealvorstellungen von einer Kultur des Dialogs oder einer Kultur der Demokratie und der Zivilität in solche Muster der Selbstdarstellung einfließen und den etablierten Kanon der »Kulturnation« zu flexibilisieren vermögen. Die Figuren Konfliktprävention oder Förderung von Dialogprozessen führen daher nicht zu einer Abkehr von der etablierten Absicht einer Vermittlung von Deutschlandbildern, sondern sie lassen sich mit ihr verweben und tragen letztlich zu einer Erweiterung ihrer allegorischen Möglichkeiten - also ihrer potenziellen Anschlüsse in unterschiedlichen Diskurs- oder Praxisfeldern - bei.

Auf dieser Basis lassen sich die beiden Konfigurationen kultureller Bedeutungen zu einer Skizze des offiziellen Narrativs Auswärtiger Kulturpolitik zusammenfügen, die zentrale Argumentationsketten zu Auftrag, Legitimität und Handlungsfeldern des Politikbereichs illustriert. Hierbei ist die Bemerkung wichtig, dass Kultur als zentrale Größe im mittleren Feld enthalten ist und somit in alle sich anschließenden Argumentationsketten hineinreicht:

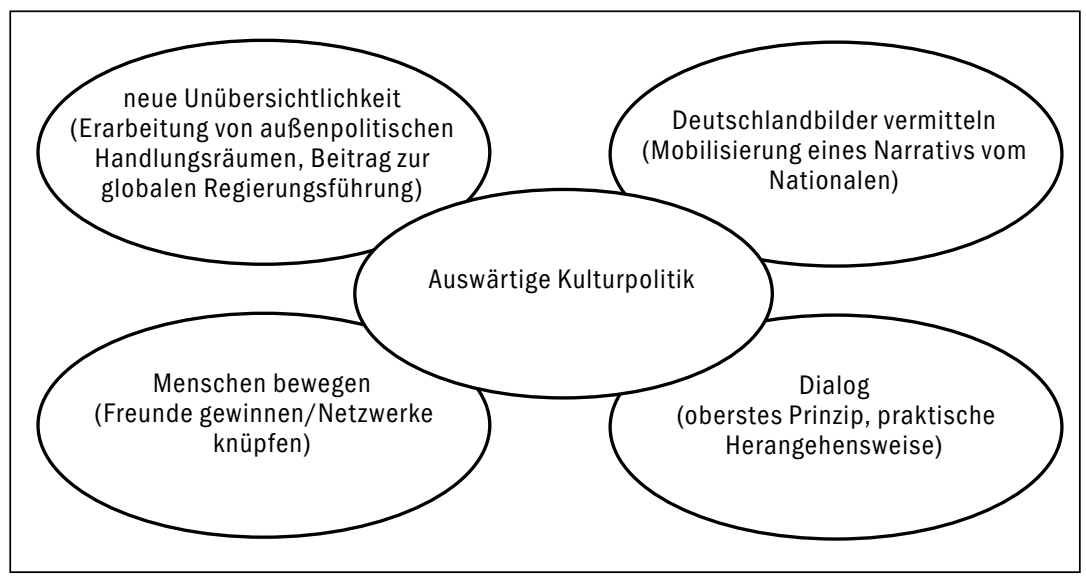


Vor diesem Hintergrund möchte ich nun die politischen Rationalitäten herausarbeiten, die anhand dieser Konfigurationen sichtbar werden. Mir geht es hierbei um Regulierungs-, Gestaltungs- und Ordnungsansprüche, die sich in dem Politikbereich formieren und im Rahmen seiner imaginären Dimension rationalisiert werden - anders ausgedrückt: um Formen der Machtausübung, die innerhalb des politischen Narrativs als legitim, vernünftig, logisch oder angemessen erscheinen. Auf Basis der Diskussionen auf den beiden Berliner Konferenzen lassen sich drei Rationalitätstypen identifizieren.

\section{Rationalitätstyp I: \\ Deutschland global repräsentieren und positionieren}

Ein erster Rationalitätstyp besteht in dem Anliegen, die Präsenzen »Deutschlands « in globalen, internationalen oder lokalen Kontexten durch kulturelle Repräsentationsformen zu vergrößern und aktiv zu steuern. Dieser Regulierungsanspruch bewegt sich dabei in einer Dynamik zwischen weitgehend naturalisierten Ausgangspunkten - etwa der Vorstellung von einer darstellbaren »nationalen Spezifik« und der Existenz eines »kulturellen Wirs« - und einer Suche nach neuen Anschlüssen oder Bezugnahmen, die auch Potenziale zur Öffnung und Flexibilisierung etablierter Grundfiguren mit sich bringt.

Dieser Rationalitätstyp manifestiert sich einerseits in der fortwährenden Ausgestaltung eines Narrativs vom Nationalen, das aus Bildern, kulturellen Positionen, Erfahrungen, Geschichtsbezügen, ästhetischen Arrangements, Herangehensweisen oder Erwartungen zusammengefügt sowie global mobilisiert wird und hierdurch symbolische Präsenzen schafft; er manifestiert sich andererseits in der kontinuierlichen Finanzierung und Entwicklung einer Infrastruktur aus Kulturinstituten, akademischen Repräsentanzen, Austauschprogrammen oder Ansprechpartnern, um in den unterschiedlichsten Ländern, in lokalen und institutionellen Kontexten greifbar zu sein. Gerade diese Verflechtungen aus symbolischen und materiellen Präsenzen stellen Grundbestandteile kulturpolitischer Strategien dar, die auf den Ausbau von weltweiten Verbindungen und hierdurch zugleich auf die Positionierung und Repräsentation Deutschlands im Modus der Kultur abzielen.

Die Wirkmächtigkeit dieser Rationalität zeigte sich auf den Konferenzen insbesondere in den Bemühungen um neue Anschlussmöglichkeiten - zur Illustration sei an drei Beobachtungen erinnert: das aktive Werben um neue Partner zur Erweiterung des kulturpolitischen Netzwerkes; die Versuche, »Kultur« für die Produktion einer positiven, ästhetisierten und kommodifizierbaren »Markenidentität Deutschlands « nutzbar zu machen, um internationale Unternehmen und mobile Wissenseliten anzuziehen; und die Tendenzen, Vorstellungen von »unserer Kultur« mit »westlichen« oder »universalen« Werten zu verknüpfen, auch um hierdurch flexiblere Positionierungen auf globalen Bühnen zu ermöglichen. 


\section{Rationalitätstyp II:}

\section{Außenpolitische Handlungsspielräume erweitern}

Ein zweiter Rationalitätstyp wird anhand der Verweise auf gegenwärtige geopolitische Transformationen sichtbar - also etwa anhand der Sprechformen von der »neuen Unübersichtlichkeit« in einer »multipolaren Welt«, in der »Krisen und Konflikte [...] nicht weniger geworden sind« oder von den »wachsenden internationalen Erwartungen an das wiedervereinte Deutschland.« In solchen Argumentationsfiguren manifestiert sich das Anliegen deutscher außenpolitischer Eliten, stärker an der Diskussion und Regulierung globaler Problem- und Konfliktfelder mitzuwirken und hierdurch Handlungsspielräume in internationalen Kontexten zu gewinnen. Die Strukturen, Netzwerke, Herangehensweisen und Instrumente eines ursprünglich auf nationalkulturelle Repräsentationen ausgerichteten Politikbereichs werden vor diesem Hintergrund neu perspektiviert und auf ihr Potenzial zur Teilnahme an Diskursen, Mechanismen und Handlungsfeldern einer entstehenden globalen Regierungsführung hin befragt. Die Äußerung eines leitenden Mitarbeiters des Auswärtigen Amtes im Hinblick auf aktuelle Konfliktherde in Afghanistan und im Irak illustriert diesen Regulierungs- und Gestaltungsanspruch besonders gut: »Was kann man mit den Mitteln der Kultur hier erreichen?«

Entsprechend zeigte sich dieser Rationalitätstyp auf den Konferenzen am deutlichsten an den Diskussionen zu den Möglichkeiten einer konfliktpräventiven Auswärtigen Kulturpolitik: Der Politikbereich berührt hier besonders spürbar Grenzen seiner ursprünglichen konzeptionellen Ausrichtung; deutsche Außenpolitik kann genau hierdurch neue inhaltliche und praktische Anschlussmöglichkeiten an internationale Missionen in »Krisen- und Konfliktgebieten« gewinnen. Gleichzeitig lassen sich innerhalb dieser Rationalität Argumentationen entwickeln, um (kultur-)politische Interventionen Deutschlands in instabilen Regionen plausibel zu machen. Sichtbar wurde auch, dass es bei solchen Überlegungen ebenfalls um einen möglichen Reputationsgewinn auf internationalen Bühnen geht, der hier allerdings eher durch die Demonstration von Kooperationsbereitschaft und globalem Verantwortungsbewusstsein und weniger durch Verweise auf einen nationalkulturellen Kanon erzielt werden soll.

\section{Rationalitätstyp III:}

\section{Menschen erreichen, mobilisieren und verändern}

Drittens zeigt sich ein Rationalitätstyp, der im Prinzip weltweit Individuen und Gruppen in sehr unterschiedlichen lokalen, regionalen oder institutionellen Kontexten fokussieren kann: Kulturpolitische Maßnahmen und Programme erscheinen hierbei als Möglichkeiten, um Perspektiven, Einstellungen oder sogar Verhaltensweisen zu verändern. Diese Rationalität manifestiert sich etwa in Zielvorstellungen und Argumentationslinien, die in diesem Kapitel bereits 
entlang des Konferenztitels Menschen bewegen und den beiden Schlüsselbegriffen vorgestellt worden sind: So soll das Interesse von spezifischen Personen an Deutschland, deutscher Sprache oder Kultur geweckt werden; (zukünftige) Eliten sollen zur Aufnahme eines Studiums oder einer Beschäftigung in Deutschland ermutigt werden; es gilt, »Freunde für Deutschland « weltweit zu gewinnen oder Angehörige von Konfliktparteien zur Teilnahme an Dialogprozessen zu bewegen; »Werte« wie »Offenheit«, »Toleranz«, »Menschenrechte« oder Formen friedlicher Konfliktlösung sollen im globalen Rahmen verbreitet und »Brücken errichtet« werden, über die Individuen und Kollektive »Verständigung« suchen können.

Von Interesse ist zunächst, dass in solchen Formulierungen sehr unterschiedliche Subjektpositionen hervorgerufen werden: So tauchten in einzelnen Redebeiträgen etwa Schülerinnen und Schüler, Studierende, »zukünftige Entscheidungsträger«, Bewohner von Konfliktgebieten, ehemals verfeindete Kollektive oder »Eliten« als potenzielle Zielgruppen auf. Zwei weitere Aspekte sind bemerkenswert: Einerseits kann das Ausmaß einer möglichen Mobilisierung von »Interesse wecken« über »Freunde gewinnen« bis zu»Verhaltensweisen ändern« sehr unterschiedlich gedacht werden; andererseits adressiert die Kulturpolitik eines europäischen Nationalstaates hier explizit Perspektiven, Einstellungen und Verhaltensweisen von Akteursgruppen jenseits seiner eigenen Grenzen. Diese Rationalitätsform lässt somit Interventionen im Modus der Kultur im internationalen Rahmen und in lokalen Kontexten legitim erscheinen.

\section{Perspektivierung: Historische Entwicklungslinien, urbane Bühnen und Übersetzungsketten des politischen Narrativs}

So etwa stellen sich also die imaginären Dimensionen Auswärtiger Kulturpolitik auf zwei Berliner Konferenzen dar. Im Zentrum stehen zwei diskursive Formationen, die sich um die beiden Schlüsselbegriffe Deutschlandbilder vermitteln und Dialog gruppieren und weitreichende Regulierungs-, Gestaltungsund Ordnungsansprüche plausibel erscheinen lassen. Auf dieser Basis lässt sich bereits an dieser Stelle formulieren, dass die Figur Auswärtige Kulturpolitik als Konfliktprävention - deren Auftauchen um die Jahrtausendwende den Ausgangspunkt dieser Studie bildet - offenbar nicht zu einem grundlegenden Paradigmenwechsel in den Diskussions- und Begründungszusammenhängen des Politikbereichs geführt hat. Sie wird vielmehr sukzessive als ein dynamisches Element in ein politisches Narrativ inkorporiert, dem weiterhin durch längerfristig etablierte Schlüsselbegriffe die maßgebliche Struktur gegeben wird. Dennoch wurde sichtbar, wie gerade Bezugnahmen auf Konfliktprävention, Demokratie oder Förderung von Menschenrechten als neue kulturpolitische Zielhorizonte die allegorischen Möglichkeiten dieses Narrativs vergrößern: zum 
einen im Hinblick auf die Formen und Techniken der kulturellen Selbstdarstellung; zum anderen im Hinblick auf die Verknüpfung der etablierten kulturpolitischen Infrastruktur mit Problemstellungen und Handlungsfeldern einer globalen Regierungsführung, die sich gegenwärtig insbesondere im Umgang mit »instabilen Regionen« oder »Konfliktgebieten« entwickelt. Entlang dieser Suche der policy community nach neuen thematischen Verbindungen, Herangehensweisen und Tätigkeitsfeldern sowie den damit verbundenen diskursiven Bewegungen lassen sich somit auch die Prozesshaftigkeit und Produktivität dieses Politikbereichs illustrieren.

Bevor ich mich im dritten Kapitel dieses Buches der Frage nach der Bedeutung solcher Schlüsselbegriffe, Konfigurationen kultureller Bedeutungen und politischer Rationalitäten für die Arbeitspraxis von Kulturmittlern in Ramallah, Tel Aviv und Sarajevo zuwende, verfolge ich diese diskursiven Formationen in den verbleibenden Abschnitten dieses Kapitel durch drei weitere Arenen des Politikbereichs: Im nächsten Textteil statte ich sie mit einer historischen Tiefenschärfe aus, indem ich anhand von Regierungsdokumenten seit den I97oer Jahren kontinuierliche Entwicklungslinien und einzelne Brüche des politischen Narrativs aufzeige; im Abschnitt II.4. zeichne ich am Beispiel einer hochkarätigen Konferenz des Goethe-Instituts in Berlin Übersetzungen und Effekte des politischen Narrativs auf urbanen Bühnen nach; auf dieser Basis arbeite ich im Abschnitt II.5 die grundlegende epistemologische Ordnung des politischen Narrativs - nämlich die Unterteilung von »Kultur« in einen »inneren« und einen »äußeren Raum« - heraus und frage nach deren Beziehung zu den Infrastrukturen des Politikbereichs. Im letzten Abschnitt des Kapitels (II.6) wende ich mich dann den translokalen Übersetzungsketten zu, über die Schlüsselbegriffe und Zielvorstellungen, aber auch Ressourcen und Menschen in die Einsatzgebiete Auswärtiger Kulturpolitik wandern.

\section{ENTWICKLUNGSLINIEN: BEGRÜNDUNGSFIGUREN AusWÄrtiger Kulturpolitik in RegierungsdokUmenten}

Auch in diesem Abschnitt knüpfe ich an die Überlegungen zu dem Forschungsprogramm einer Anthropology of Policy (Kapitel I.2) an, indem ich diskursive Verschiebungen und Kontinuitäten in der imaginären Dimension Auswärtiger Kulturpolitik nunmehr anhand von Regierungsdokumenten untersuche. Hierbei gehe ich davon aus, dass gerade in einem Politikbereich, der primär durch staatliche Gelder ermöglicht wird, solchen offiziellen Papieren nochmals eine besondere Bedeutung zukommt: Denn hier werden die »staatlich anerkannten Probleme ${ }^{50}$, um deren Bearbeitung es gehen soll, gewissermaßen als verbind-

50 | Pierre Bourdieu: Die Praxis der reflexiven Anthropologie, S. $271 \mathrm{f}$. 
liche Referenzpunkte und »Tatsachen« gesetzt. Im Zentrum der folgenden Analyse stehen vier Regierungsdokumente, von denen die zwei älteren in der Bonner Republik und somit zur Zeit des Kalten Krieges entstanden sind: einerseits die im Jahre 1970 durch das Auswärtige Amt formulierten Leitsätze für die auswärtige Kulturpolitik, ${ }^{1}$ andererseits die 1977 verfasste Stellungnahme der Bundesregierung zu dem Bericht der Enquete-Kommission »Auswärtige Kulturpolitik des Deutschen Bundestages, die zwischen I970 und I975 getagt und umfangreiche Empfehlungen zur Entwicklung des Politikbereichs formuliert hatte. ${ }^{52}$ Die beiden jüngeren Dokumente sind hingegen nach der deutschen Vereinigung in der Berliner Republik entstanden: zum einen das während der rotgrünen Regierungszeit im Jahr 1999 durch das Auswärtige Amt verfasste Papier Auswärtige Kulturpolitik - Konzeption 2000;53 zum anderen die 2007 dem Parlament übermittelte Antwort der Bundesregierung auf die Große Anfrage der Bundestagsfraktion von Bündnis 9o/Die Grünen zur Auswärtigen Kulturpolitik aus der Regierungszeit der damaligen großen Koalition. ${ }^{54}$

Es handelt sich also um zwei Dokumente, die das Auswärtige Amt erklärtermaßen als Grundsatzpapiere veröffentlicht hat, sowie um zwei weitere Papiere, die durch die jeweilige Bundesregierung als Reaktion auf Anfragen oder Vorschläge parlamentarischer Gremien verfasst wurden. Zur weiteren Bestimmung der Bedeutung dieser Dokumente ist der Hinweis wichtig, dass die Ausarbeitung eines den gesamten Politikbereich fokussierenden konzeptionellen Grundlagenpapiers ein eher seltenes Projekt ist. So veröffentlichte das Auswärtige Amt zwischen den Leitsätzen und der Konzeption 2000 über einen Zeitraum von 30 Jahren kein entsprechendes Dokument. ${ }^{55}$

In der Beschäftigung mit diesen vier Papieren orientiere ich mich an drei Frageperspektiven: zunächst suche ich nach den zentralen Erzählsträngen des politischen Narrativs und frage nach deren Konstanz und Veränderung

51 | Auswärtiges Amt: Leitsätze für die auswärtige Kulturpolitik. Bonn 1970.

52 | Deutscher Bundestag (Hrsg.): Stellungnahme der Bundesregierung zu dem Bericht der Enquete-Kommission "Auswärtige Kulturpolitik“ des Deutschen Bundestages. Drucksache 8/927. Bonn 1977.

53 | Auswärtiges Amt: Auswärtige Kulturpolitik - Konzeption 2000. Berlin 2000.

54 | Deutscher Bundestag (Hrsg.): Antwort der Bundesregierung auf die Große Anfrage der Abgeordneten Dr. Uschi Eid, Marieluise Beck (Bremen), Birgitt Bender, weiterer Abgeordneter und der Fraktion Bündnis 90/Die Grünen, Drucksache 16/4024. Berlin 2007.

55 | Allerdings hat sich diese Situation dadurch verändert, dass das Auswärtige Amt seit der Jahrtausendwende Jahresberichte zur Auswärtigen Kulturpolitik publiziert hat, die zwar keinen grundlegend konzeptionellen Anspruch verfolgen, aber Zielsetzungen und Praxis des Politikbereichs öffentlich nachvollziehbar darstellen und somit die Diskurse "amtlicherseits" laufend fortschreiben. 
durch die Jahrzehnte; zweitens analysiere ich Genealogien, Ausmaß und Konsequenzen der diskursiven Verschiebungen hin zu einer konfliktpräventiven Auswärtigen Kulturpolitik; und drittens dokumentiere ich Bedeutungen und Argumentationslinien, welche zentrale Metaphern des Politikbereichs über die Jahre akkumuliert haben. Hierzu verfolge ich insbesondere die bereits bekannten Schlüsselbegriffe Deutschlandbilder vermitteln und Dialog durch die vier Dokumente. Bevor ich mich mit diesen Metaphern, Leitmotiven und narrativen Veränderungen beschäftige, wende ich mich aber zunächst der Frage zu, wie innerhalb dieser Papiere Auswärtige Kulturpolitik in Beziehung zu den übergreifenden Zielsetzungen einer nationalstaatlichen Außenpolitik gesetzt wird. Diese Perspektive führt automatisch zu den jeweiligen Wahrnehmungsmustern von der politischen und kulturellen Ordnung der Welt, von der internationalen Stellung der Bundesrepublik Deutschland und somit von den geopolitischen Rahmenbedingungen deutscher Außenpolitik. Deutliche Veränderungen in diesen Wahrnehmungsmustern erklären auch gewisse Schwerpunktverlagerungen in dem politischen Narrativ von dem Auftrag und den Potenzialen Auswärtiger Kulturpolitik.

\subsection{Ausgangspunkte: Außenpolitische Zielsetzungen und geopolitische Wahrnehmungsmuster}

Aus Perspektive des Auswärtigen Amtes wird Auswärtige Kulturpolitik durchgängig als ein integraler Bestandteil deutscher Außenpolitik und somit auch als ein Instrument zur Durchsetzung deutscher Interessen verstanden. Diese Einordnung wird in ähnlichen Formulierungen in allen Papieren herausgestellt. In den Leitsätzen aus dem Jahr I970 heißt es hierzu etwa: »Unsere auswärtige Kulturpolitik ist internationale Zusammenarbeit im kulturellen Bereich. Sie ist Teil unserer Außenpolitik, einer Außenpolitik, die der Sicherung des Friedens in der Welt dienen will. « ${ }^{56}$

Grundlegende außenpolitische Zielsetzungen wie die »internationale Verständigung «,»das politischeZusammenwachsen Europas«,»Friedenssicherung und eine realistische Entspannungspolitik« oder der »Interessensausgleich zwischen Industrie- und Entwicklungsländern« werden sieben Jahre später gleichfalls als Orientierungspunkte für »die Konzeption der auswärtigen Kulturpolitik und ihre Gestaltung im einzelnen « angeführt. ${ }^{57}$

Auch um die Jahrtausendwende werden Ausgangspunkt und Zielhorizont des Politikbereichs in der Konzeption 2000 sehr ähnlich definiert - allerdings stechen explizite Verweise auf deutsche Interessen nun deutlicher hervor:

56 | Leitsätze, 1970, S. 5.

57 | Stellungnahme der Bundesregierung, 1977, S. 4f. 
"Die Auswärtige Kulturpolitik ist integraler Teil unserer Außenpolitik. Sie ist an den allgemeinen Zielen und Interessen der deutschen Außenpolitik - Sicherung des Friedens, Konfliktverhütung, Verwirklichung der Menschenrechte, partnerschaftliche Zusammenarbeit - ausgerichtet und unterstützt sie.“58

Wenn auch zum Teil leicht veränderte Formulierungen gewählt werden, so bleiben die grundlegenden außenpolitischen Zielhorizonte sehr ähnlich: Frieden, Ausgleich, Verständigung, Kooperation und Europäische Integration bilden durch die Jahrzehnte die zentralen Begriffe, an denen sich auch die Konzipierung und Umsetzung Auswärtiger Kulturpolitik zu orientieren hat. Die Zielsetzung Förderung der Menschenrechte taucht in den I970er Jahren bereits vereinzelt auf, rückt aber in den jüngeren Dokumenten, gemeinsam etwa mit Demokratieförderung oder Konfliktprävention, spürbar in den Vordergrund - ein Zusammenhang der im weiteren Verlauf dieses Abschnitts größere Beachtung finden wird.

Entsprechend setzen Regierungsvertreter und Ministerialbeamte also den politischen Rahmen und beanspruchen die Verantwortung für die Übereinstimmung Auswärtiger Kulturpolitik mit den außenpolitischen Zielen der Bundesrepublik. Die steuernde Rolle des Auswärtigen Amtes wird im Jahr 2007 über seinen »verfassungsmäßigen Auftrag insgesamt für die Sicherstellung einer kohärenten Außenpolitik Sorge zu tragen « begründet und als Legitimationsbasis des Politikbereichs festgeschrieben. ${ }^{59}$ In ähnlicher Weise wird I977 formuliert: »Die Bundesregierung muß, auch wenn sie die Durchführung der auswärtigen Kulturpolitik teilweise anderen Stellen übertragen hat, stets die Übereinstimmung dieser Maßnahmen mit der Außenpolitik garantieren können. ${ }^{60}$

Gleichzeitig finden sich durchgängig deutliche Relativierungen einer anklingenden Unterordnung von »Kultur« unter (außen-)politische Interessen - auch hier zeigen sich spürbare Kontinuitäten, so heißt es I977: »Diese Orientierung der auswärtigen Kulturpolitik an den außenpolitischen Zielen darf nicht als Absicht der Bundesregierung mißverstanden werden, sie wolle die Kultur zur >Magd $<$ des Politischen oder gar ihrer Außenpolitik machen. ${ }^{61}$

Die Unabhängigkeit der »Mittlerorganisationen « in ihrer Programmgestaltung wird durch die Jahrzehnte hinweg als Beitrag zur Sicherung der Autonomie der Kultur trotz übergeordneter politischer Zielsetzungen genannt. In der Konzeption 2000 ist hierzu zu lesen:

58 | Konzeption 2000, S. 1.

59 | Antwort der Bundesregierung, 2007, S. 15.

60 | Stellungnahme der Bundesregierung, 1977, S. 6.

61| Ebd., S. 5. 
"In Deutschland herrscht Kulturfreiheit; es gibt keine Staatskultur. Pluralismus und regierungsferne Organisation der Mittler garantieren Vielfalt und Unabhängigkeit der Kulturarbeit im Ausland. Die Mittler setzen die Leitlinien der Auswärtigen Kulturpolitik der Bundesregierung differenziert nach inren jeweiligen Aufgabenprofilen und Programmstrukturen um." 62

Deutlich wird hier somit ein durch die Jahrzehnte etabliertes, kontinuierliches Motiv des politischen Narrativs - eine Denklogik, die Auswärtige Kulturpolitik über ihren Beitrag zu übergreifenden außenpolitischen Zielsetzungen und Interessen legitimiert, gleichzeitig aber die Unabhängigkeit der »Kultur« von staatlicher Dominanz und Einflussnahme betont - die institutionelle Eigenständigkeit der Mittlerorganisationen wird als zentraler Beitrag angeführt, um diese Ambivalenz in der Praxis durchzusetzen.

Deutlich wird aber auch der Zusammenhang zwischen den Begründungen der zentralen außenpolitischen Zielsetzungen und den Wahrnehmungsmustern von geopolitischen Rahmenbedingungen sowie der internationalen Position Deutschlands. Anhand der Dokumente lässt sich hier eine entscheidende Transformation ablesen: In den ersten beiden Papieren erscheint die Welt zwar durch den Kalten Krieg prinzipiell gespalten, aber dennoch durch eine relativ stabile Struktur geordnet. Dies manifestiert sich etwa in dem Aufbau der Stellungnahme aus dem Jahr I977, die »Schwerpunkte der auswärtigen Kulturpolitik« anhand von drei voneinander abgegrenzten geopolitischen Räumen diskutiert: »Westliche Industriestaaten«, »Osteuropa und Sowjetunion« sowie »Entwicklungsländer «. ${ }^{63}$

In den jüngeren Dokumenten erscheint hingegen das - auch auf den beiden Konferenzen gezeichnete - Bild einer grundlegend neuen Konstellation, deren Konturen erst allmählich erkennbar werden: Das »Ende der Blockkonfrontation«, zunehmende »internationale Interdependenzen«, »Globalisierung«, die Entstehung »neuer politischer, wirtschaftlicher, aber auch kultureller Gravitationszentren « sind einige der Schlagworte, durch welche die »neuen Unübersichtlichkeiten« der Weltordnung nun beschrieben werden. Diese Veränderungen gelten als Gründe für die Notwendigkeit neuer konzeptioneller Überlegungen zur Auswärtigen Kulturpolitik:

"Die für die deutsche Außenpolitik spezifischen Bedingungen der Nachkriegszeit sind nicht mehr bestimmend. Die besondere Herausforderung an unsere Auswärtige Kulturpolitik, die sich aus der Existenz zweier deutscher Staaten und der Konfrontation der Blöcke ergab, ist Geschichte. Das wiedervereinigte Deutschland sieht sich weiter wach-

62 | Konzeption 2000, S. 2.

63 | Stellungnahme der Bundesregierung, 1977, S. $15 f f$. 
senden internationalen Erwartungen gegenüber und ist bereit, eine gestaltende Rolle zu übernehmen." ${ }^{64}$

Aus Perspektive des politischen Zentrums gilt es, den Politikbereich also an die neue globale (Un-)Ordnung sowie an die sich vergrößernden internationalen Erwartungen an das vereinte Deutschland anzupassen. Diese unübersichtliche Weltlage wird im folgenden Zitat noch deutlicher als neue Rahmenhandlung des Politikbereichs sichtbar:

"Die mit der Globalisierung einhergehenden Veränderungen bieten viele neue Chancen, lösen aber auch Verunsicherung und Abwehrreaktionen aus, vor allem jener Staaten und Bevölkerungsgruppen, die durch diese Entwicklung marginalisiert zu werden drohen. Die globale Angleichung der Alltagskultur führt zum Verblassen zahlreicher Regionalkulturen und als Gegenreaktion zur Besinnung auf tradierte Kulturformen bis hin zu religiös und kulturell begründeten Konflikten." 65

Vor diesem Hintergrund tritt in der Antwort der Bundesregierung aus dem Jahr 2007 deutlich eine neue geopolitische Bruchlinie hervor, die in den Dokumenten der I970er Jahre, bezeichnenderweise aber auch in der um die Jahrtausendwende verfassten Konzeption 2000, nicht existierte: Der »Islam« wird nun als ein »kulturelles Phänomen«, als ein »kultureller Raum« und auch als ein (kultur-)politisches »Problem« sichtbar. ${ }^{66}$ Innerhalb weniger Jahre wurde somit nicht nur die Vorstellung von den »islamisch geprägten Ländern« etabliert, sondern auch eine diskursive Verknüpfung zwischen »Konflikten«, »Terrorismus« und »Islam«, die einer besonderen kulturpolitischen Reaktion bedürfe, als neues Motiv des politischen Narrativs entwickelt. Drastischer als in den Formulierungen der Bundesregierung selbst, zeigt sich diese Verbindung in der Anfrage von Bündnis 90/Die Grünen: »Erforderlich wurde in jüngster Zeit auch - insbesondere mit dem weltweiten Auftreten des islamischen Terrors - eine intensivierte Kommunikation zwischen den Kulturen und der Aufbau von kulturpolitischen Dialogstrukturen auf internationaler Ebene. ${ }^{67}$

Die Bundesregierung verweist in ihrer Antwort auf die »im Gefolge des II. September 200I« erfolgte Einrichtung des »Politikschwerpunkt >Dialog mit der islamischen Welt««, dessen Ziel es sei, »durch spezifische Projekte das gegenseitige Verständnis zwischen westlicher und islamisch geprägter Welt zu

64 Konzeption 2000, S. 2 f.

$65 \mid$ Ebd., S. 3.

66 | In der Stellungnahme der Bundesregierung aus dem Jahr 1977 ist stattdessen etwa vom "Europäisch-Arabischen Dialog" die Rede. Siehe: Ebd., S. 25.

67 | Vorbemerkung der Fragesteller, in: Antwort der Bundesregierung, 2007, S. 1. 
verbessern $\aleph^{68}$ und verdeutlicht somit die Entwicklung neuer kulturpolitischer Handlungsfelder, die aus einer veränderten Problemwahrnehmung entstanden sind. Gleichfalls werden die Möglichkeiten einer Auswärtigen Kulturpolitik als Konfliktprävention in diesem Dokument fast ausschließlich im Hinblick auf die »islamische Welt« diskutiert. Die um die Jahrtausendwende entstandene, damals geografisch noch nicht spezifizierte diskursive Formation scheint somit ihre primäre geopolitische Zielregion gefunden zu haben.

\subsection{Leitmotive: Kontinuitäten und Erweiterungen der Grundprinzipien Auswärtiger Kulturpolitik}

Die zwei Schlüsselbegriffe Deutschlandbilder vermitteln und Dialog führen in allen vier Dokumenten zu den zwei Grundprinzipien, die durch die Jahrzehnte hindurch die zentralen Achsen zur Legitimierung Auswärtiger Kulturpolitik bilden und somit historisch etablierte Leitmotive ihrer imaginären Dimension darstellen: das Prinzip der kulturellen Selbstdarstellung und das Prinzip der Wechselseitigkeit. In den konkreten Verknüpfungen, der Ausgestaltung und dem Stellenwert dieser Prinzipien sind allerdings Variationen erkennbar.

\section{Leitmotiv I: Das Prinzip der kulturellen Selbstdarstellung}

Das Prinzip der kulturellen Selbstdarstellung zeigt sich zunächst in zwei miteinander verknüpften Argumentationsfiguren, die in allen Dokumenten das Gerüst zur Legitimation des Politikbereichs bilden: einerseits die Vorstellung, dass sich das staatliche Gebilde »Deutschland« durch eine Spezifik auszeichne, die sich kulturell repräsentieren lasse; und andererseits der Glaube, dass durch Formen kultureller Selbstdarstellung ein nachhaltiger Reputationsgewinn zu erzielen sei, der sich auch in anderen Politikfeldern positiv einsetzen lasse. Hierzu wird durchgängig das Bild einer »Kulturnation« oder eines »Kulturstaates« als Rahmen gesetzt: Im Jahr I977 wird etwa zur elementaren Begründung des Politikbereichs »die Legitimation der Bundesrepublik als Kulturstaat in einer sich wandelnden Welt« als »übergreifende Aufgabe und als ständiges Ziel der auswärtigen Kulturpolitik« angeführt. ${ }^{69}$ In der Antwort der Bundesregierung aus dem Jahr 2007 ist zu lesen, es entspräche »dem allgemeinen Selbstverständnis Deutschlands als Kulturnation, die Qualität und Vielfalt der deutschen Kulturlandschaft auch unseren Partnern im Ausland umfassend zu präsentieren «. ${ }^{70}$ Gleichfalls findet sich bereits in den I970er Jahren die Vorstellung, eine kulturelle Selbstdarstellung wirke sich positiv auf die Reputation und internationale Stellung Deutschlands aus. So heißt es in den Leitsätzen:

$68 \mid$ Ebd., S. 35.

69 | Stellungnahme der Bundesregierung, 1977, S. 4.

70 | Antwort der Bundesregierung, 2007, S. 6. 
»Im internationalen Konzert ist das politische Gewicht der Bundesrepublik neben ihren wirtschaftlichen vornehmlich von ihren geistigen Leistungen abhängig. «11

Sieben Jahre später wird in ähnlicher Weise eine enge Beziehung zwischen der »Darstellung ihrer kulturellen Leistungen « und dem »Ansehen der Bundesrepublik Deutschland « vorausgesetzt. ${ }^{72}$ Diese angenommene Beziehung wird noch deutlicher, wenn 1977 ein aktueller Handlungsbedarf angeführt wird, um auf die »Trübung des Bildes unseres Landes« und der »Wiederbelebung von [...] Vorurteilen ${ }^{73} \mathrm{zu}$ reagieren: »Der in den letzten Jahren gelegentlich feststellbaren Verzerrung des Deutschlandbildes muß durch eine ausgewogene Selbstdarstellung entgegengewirkt werden. ${ }^{74}$

Sichtbar wird in solchen Formulierungen somit eine durch die Jahrzehnte naturalisierte Denk- und Begründungsfigur, die auch in der Gegenwart in großer Selbstverständlichkeit genutzt wird. In diesem Zusammenhang lohnt der Blick in den 10. Bericht der Bundesregierung zur Auswärtigen Kultur- und Bildungspolitik - ein Papier, auf das die Bundesregierung in ihrer Antwort aus dem Jahr 2007 selbst zur weiteren Erläuterung »der Leitlinien, konzeptionellen Ansätze und inhaltlichen Schwerpunkte der AKBP« verweist. ${ }^{75}$ Der gefestigte Charakter dieser Denkfigur zeigt sich hier noch deutlicher: Sympathiewerbung für Deutschland/Vermittlung eines zeitgemäßen Deutschlandbildes wird als zweite von insgesamt vier Zielsetzungen des Politikbereichs aufgeführt und anhand der Beispiele des Deutschlandjahres in Japan 2005/2006 und des Deutsch-Polnischen Jahres 2005/2006 mit jeweils über I.000 Aktivitäten illustriert. Zur Begründung dieser Zielsetzung ist hier zu lesen:

"Mit Mitteln der AKBP präsentiert sich Deutschland als ein Partner, der für klassische und moderne Kultur steht, der an gleichberechtigtem Dialog und Austausch interessiert ist und sich seiner Vergangenheit glaubwürdig in offener Auseinandersetzung stellt. Ein wichtiges kulturpolitisches Instrument ist [...] die Veranstaltung von Kulturtagen, -wochen oder -jahren. Sie sind im besten Sinne Visitenkarten der Kulturnation Deutschland." ${ }^{76}$

Allerdings lässt sich der Rahmen der »Kulturnation« bereits in den I97oer Jahren auf verschiedene Weise mit Bedeutungen und Inhalten füllen. In den

71 | Leitsätze, 1970, S. 6.

72 | Stellungnahme der Bundesregierung, 1977, S. 5.

73 | Ebd., S. 16.

74 | Ebd., S. 5.

75 | Antwort der Bundesregierung, 2007, S. 7.

76 | Bericht der Bundesregierung zur Auswärtigen Kulturpolitik 2005/2006. Berlin 2006, S. 6. 
Leitsätzen heißt es etwa: »In unserem Beitrag zum internationalen kulturellen Austausch müssen wir [...] unseren Partnern auch ein Bild dessen vermitteln, >was in dieser Zeit des Übergangs auch in Deutschland an geistiger Auseinandersetzung und fruchtbarer Unruhe tägliche Wirklichkeit ist.« «77

»Kultur« - als Basis der Selbstdarstellung - wird hier eher als ein gegenwärtiger und dynamischer Prozess verstanden, der möglichst alle gesellschaftlichen Gruppen einbezieht, gerade auch im Alltag stattfindet, zu permanenten Veränderungen führt und Weltoffenheit mit sich bringen soll. ${ }^{78}$ In der Stellungnahme der Bundesregierung sieben Jahre später tritt hingegen stärker ein substanzielles und ahistorisches Verständnis von »deutscher Kultur« hervor, das auf einer dauerhaften Bindung zwischen »Volk« und »Kultur« aufbaut und eine jeweilige staatliche Organisation überdauert:

"Die auswärtige Kulturpolitik der Bundesrepublik Deutschland [...] geht trotz der staatlichen Trennung der Deutschen von der gemeinsamen deutschen Kultur aus. Wir halten daran fest, daß gemeinsame Geschichte, Kultur und Sprache schon immer die entscheidenden Klammern der Einheit unseres Volkes waren und es auch bleiben werden. " ${ }^{79}$ Und später: "Die Substanz der Kulturpolitik ist die Summe aller kulturellen Leistungen unseres Volkes in Vergangenheit und Gegenwart. Diese Substanz kann und darf von der Bundesregierung nicht bestimmt werden. ${ }^{80}$

Die Orientierung an dem Bild einer quasi ahistorischen Substanz als Ausgangspunkt Auswärtiger Kulturpolitik ermöglicht hier das politisch gewollte Festhalten an dem Konzept »einer gemeinsamen Kultur«, die trotz Teilung die Nation »zeitlos« eint. Deutlich scheint hier das in Deutschland wirkmächtige Motiv von der überstaatlichen »Kulturnation« mit der Vorstellung einer organischen Beziehung zwischen deutschem Volk und deutscher Kultur hindurch, wie es sich im territorial zersplitterten Mitteleuropa seit dem späten I8. Jahrhundert entwickelt hat.

So gilt es festzuhalten, dass in den Dokumenten der I970er Jahre sowohl ein eher prozessualer Kulturbegriff, der Gegenwartsentwicklungen, Veränderungen und Offenheit betont, als auch ein substanzieller Kulturbegriff, der stärker auf der Behauptung einer ahistorischen ethnokulturellen Essenz aufbaut, als Grundlage der kulturellen Selbstdarstellung angeführt werden konnte anders formuliert: zwei eigentlich sehr unterschiedliche Konzepte wirken hier in der Legitimierung des Politikbereiches nebeneinander.

77 | Leitsätze, 1970, S. 15.

78 | Siehe hierzu auch den Abschnitt "Erweiterung des Kulturbegriffs", ebd., S. 5 f.

79 | Stellungnahme der Bundesregierung, 1977, S. 4.

80 | Ebd., S. 6. 
In den beiden jüngeren Dokumenten treten substanzielle Vorstellungen von »deutscher Kultur« in den Hintergrund - sie verschwinden aber nicht ganz, bilden zum Teil naturalisierte Grundlagen der hier formulierten kulturpolitischen Zielsetzungen und bleiben somit im Prinzip reaktivierbar. »Kultur« zeigt sich als ein Schlüsselbegriff des Politikbereichs, der stärker zirkulär Bedeutungen akkumuliert als sich eindeutig linear zu verändern. So finden sich auch in den neueren Papieren noch Bezugnahmen auf Vorstellungen von »unserer Kultur« oder auf den durch die Jahrzehnte hinweg konstanten Schwerpunkt der »Förderung der deutschen Sprache«, der »Zugang zur deutschen Kultur« bieten soll. Gleichzeitig rückt in diesen Dokumenten aber ein Verständnis in den Vordergrund, das stärker Heterogenität und internationale Verwebungen einzubeziehen versucht: »Auswärtige Kulturpolitik vermittelt Kultur aus Deutschland als Teil der europäischen Kultur«, heißt es etwa in der Konzeption 2000. ${ }^{81}$ Bezugsgröße ist hier offenbar nicht das »Volk« als Träger einer kulturellen Substanz, sondern die territoriale Einheit »Deutschland« im Prinzip mit aller Vielfalt, allen Positionen und Verknüpfungen, die sich in diesem Raum finden lassen. Auch der deutliche Verweis auf den europäischen Kontext trägt zu einer gewissen Flexibilisierung der Vorstellung von der Basis des Politikbereichs bei. Entscheidend ist allerdings, dass sowohl ein eher substanzielles als auch ein eher prozessuales Verständnis von Kultur - bei allen inhaltlichen Unterschieden - die beiden konstanten Argumentationsfiguren von der repräsentierbaren nationalen Spezifik sowie von dem erwarteten Reputationsgewinn durch kulturelle Selbstdarstellung unterstützt.

Gleichzeitig werden in den jüngeren Dokumenten zwei neue Erzählstränge sichtbar, die direkt an das Prinzip der kulturellen Selbstdarstellung anschließen - zwei Verknüpfungen, die auch schon auf den Berliner Konferenzen deutlich hervorgetreten sind:

Zum einen zeigen sich auch in diesen Regierungsdokumenten Hinweise auf die neoliberalen Zielvorstellungen einer Standortpolitik und somit auf die ökonomischen Potenziale der kulturellen Selbstdarstellung als komplett neue Argumentationsfigur. Besonders deutlich manifestiert sich diese Verbindung am Beispiel der Hochschulpolitik. In der Konzeption 2000 ist hierzu zu lesen:

"Im Zeitalter der Globalisierung ist die internationale Wettbewerbsfähigkeit des Wirtschaftsstandorts Deutschland mehr denn je nur dadurch zu sichern, dass Deutschland seine Spitzenstellung als Wirtschafts- und Studienstandort im Ausland unter Beweis stellt. Deshalb kommt der Öffnung und Zusammenarbeit im Bereich Wissenschaft und Hochschulen künftig noch größere Bedeutung zu." 82

81| Konzeption 2000, S. 1.

$82 \mid$ Ebd., S. 13. 
Eine entsprechende Verknüpfung kulturpolitischer Handlungsfelder mit ökonomischen Zielvorstellungen ist in den Dokumenten der I970er Jahre nicht zu finden. Zum anderen werden »Werte« als eine Basis der kulturellen Selbstdarstellung angeführt. So wird etwa in der Konzeption 2000 formuliert:

"Unsere Kulturarbeit im Ausland ist nicht einfach neutral, sondern orientiert sich an Werten. In Fragen der Demokratieförderung, Verwirklichung der Menschenrechte, Nachhaltigkeit des Wachstums, Teilhabe am wissenschaftlich-technologischen Fortschritt, Armutsbekämpfung oder Schutz der natürlichen Ressourcen bezieht sie eindeutig Position." 83

Grundsätzlich ist der Hinweis wichtig, dass in den Dokumenten der I97oer Jahre auch eine solche Argumentationslinie kaum auftaucht. Hier handelt es sich gleichfalls um eine neue diskursive Figur, die mit dem etablierten Prinzip der kulturellen Selbstdarstellung verwoben wird, aber zugleich ein Potenzial zur Flexibilisierung etablierter Vorstellungen von »unserer Kultur« und »nationaler Spezifik « mit sich bringt. »Werte« werden gleich bei der Verfolgung der Metapher Dialog durch die Regierungsdokumente nochmals eine Rolle spielen.

\section{Leitmotiv II: Das Prinzip der Wechselseitigkeit}

Die Metapher Dialog führt zu dem zweiten Leitmotiv Auswärtiger Kulturpolitik, das ich als Prinzip der Wechselseitigkeit bezeichnen möchte. Zwar taucht dieser Begriff selbst in den I970er Jahren selten auf und rückt erst in den jüngeren Dokumenten als vielseitig anschlussfähige Metapher in den Vordergrund - die Bedeutung dieses zweiten Prinzips zur Legitimierung des Politikbereichs zeigt sich in den älteren Papieren aber an dem Gebrauch verwandter Begriffe, die ähnliche Vorstellungen transportieren: so etwa Austausch, wechselseitiges Verständnis, Gegenseitigkeit oder Zusammenarbeit.

Bereits in den Dokumenten aus den siebziger Jahren verweisen diese Begriffe auf zwei elementare Argumentationsfiguren: Auf der einen Seite findet sich in allen Papieren das Bild von einem bilateralen Dialog, der zwischen nationalstaatlichen Einheiten zu realisieren ist und gewissermaßen den über die Jahrzehnte etablierten Normalfall kultureller Vermittlung darstellt. Auf der anderen Seite steht die Vorstellung vom Dialog, der Frieden und internationalem Ausgleich dient und sich somit als eine Form der kulturpolitischen Bearbeitung globaler Themenfelder und Problemlagen anbietet - eine Figur, die sich als multilateraler oder in der Weiterentwicklung auch als konfliktbearbeitender Dialog benennen lässt.

Die erste Argumentationsfigur wird häufig genutzt, um Herangehensweisen der kulturellen Vermittlungsarbeit zu präzisieren, wie sich anhand einer

83 | Konzeption 2000, S. 1. 
griffigen Formulierung aus dem Jahr I970 verdeutlichen lässt: »Auswärtige Kulturpolitik ist nicht nur Information über unsere Kultur, sondern auch Austausch und Zusammenarbeit. [...] Was wir geben, ist nur so viel wert, wie unsere Bereitschaft zu nehmen. Offenheit für das andere ist daher ein Prinzip unserer auswärtigen Kulturpolitik. « ${ }^{84}$

Sieben Jahre später wird dieses Ideal der Reziprozität deutlicher auf mögliche Inhalte der kulturpolitischen Vermittlung bezogen:

"Die internationalen Kulturbeziehungen können daher nicht mehr auf einseitigen ,Kulturexport (von Sprache, Wissenschaft, Kultur, Kunst etc.) beschränkt bleiben. Notwendig ist vielmehr eine auswärtige Kulturpolitik, die sich den kulturellen Wechselbeziehungen und der partnerschaftlichen Zusammenarbeit öffnet. Dabei muß dem seit 1970 entwickelten auf Gegenseitigkeit angelegten erweiterten Kulturbegriff Rechnung getragen werden. ${ }^{85}$

Wechselseitigkeit wird also bereits in diesen frühen Papieren deutlich mit dem Prinzip der kulturellen Selbstdarstellung verwoben, um den möglichen Vorwurf eines einseitigen Kulturexports einer wohlhabenden europäischen Industrienation zu entkräften. Entsprechend wird Dialog zweieinhalb Jahrzehnte später sowohl als Zielsetzung als auch als zentrale Technik Auswärtiger Kulturpolitik angeführt: »(Auswärtige Kulturpolitik) kennzeichnet Deutschland als Kulturstaat im Dialog mit der internationalen Gemeinschaft der Staaten. [...] Auswärtige Kulturpolitik beschränkt sich jedoch nicht auf Kulturvermittlung, sondern fördert Dialog, Austausch und Zusammenarbeit zwischen Menschen und Kulturen.« ${ }^{86}$

Redeweisen vom »lebendigen Austausch zwischen den Völkern« oder von dem »wechselseitigen Verständnis für die innere Entwicklung der Völker « ${ }^{87}$ verweisen zugleich auf eine historisch etablierte Grundkonstellation des Politikbereichs: »Völker« oder »Nationen«, die jeweils über einen abgrenzbaren »kulturellen Raum« verfügen, werden durch kulturpolitische Aktivitäten in einen bilateralen Austausch gebracht. Dieses Verständnis von Wechselseitigkeit kann auch zu kulturpolitischen Feldern im Inland führen, die - dem Idealbild nach - in die dialogische Praxis einbezogen werden sollen. In der Konzeption 2000 wird formuliert: »Auswärtige Kulturpolitik findet also in zwei Richtungen statt: Gleichberechtigt neben die Beteiligung Deutschlands am Kulturdi-

84 | Leitsätze, 1970, S. 7.

85 | Stellungnahme der Bundesregierung, 1977, S. 5.

86 | Konzeption 2000, S. 1.

87 | Diese beiden Formulierungen aus den Leitsätzen werden bezeichnenderweise in der Stellungnahme der Bundesregierung als weiterhin gültig zitiert. Siehe hierzu: Ebd., S. 4 und S. 13. 
alog im Ausland tritt [...] die Förderung des Kulturdialogs im Inland. Die Vorstellungen der Partnerländer, ihre Erwartungen und Konzeptionen sind dabei zu berücksichtigen. $^{88}$

Die zweite Argumentationsfigur verweist auf andere politische Konstellationen: Im Zentrum steht hier die Vorstellung, wechselseitiges Verständnis könne zur globalen Friedenssicherung und Konfliktlösung beitragen - ein Gedanke, der sich in unterschiedlichen Variationen und Verbindungen ebenfalls durch alle Dokumente zieht und sich somit als ein kontinuierlicher Wissensbestand des Politikbereichs zeigt. So heißt es im Schlusswort der Leitsätze von I970: »Vor allem kommt es darauf an, das Ziel der auswärtigen Kulturpolitik, der Verständigung zwischen den Menschen und so der Sicherung des Friedens zu dienen, nie aus den Augen zu verlieren. ${ }^{89}$

Die zweite Argumentationsfigur, die also kulturellen Austausch, Begegnung und Kommunikation als wirksame Beiträge $\mathrm{zu} »$ Frieden« und »internationalem Ausgleich« versteht, führt zugleich bereits in den siebziger Jahren zu Diskussionen internationaler Problemstellungen im Umfeld von multilateralen Organisationen. Für eine nationalstaatliche Politik eröffnen sich hierdurch Handlungsspielräume, die über eine bilateral angelegte kulturelle Selbstdarstellung alleine kaum erschlossen werden könnten. Dieser Zusammenhang lässt sich durch ein Zitat aus dem Jahr I977 verdeutlichen:

"Unter diesen Organisationen mißt die Bundesregierung der UNESCO besondere Bedeutung zu. In ihrem Rahmen wirkt sie aktiv bei dem großen Dialog mit, der auch dort zwischen Völkern verschiedener politischer Systeme, kultureller Traditionen und Entwicklungsstufen über die zukünftige Wirtschafts- und Sozialordnung der Welt geführt wird. Förderung der Menschenrechte, gleicher Zugang zu modernen Informationsmitteln, Informationsfreiheit, Recht auf Bildung sind die Hauptthemen dieser Dialoge. ${ }^{90}$

Hier klingen bereits multilaterale kulturpolitische Foren und Herangehensweisen an, die später in der Konzeption 2000 an Bedeutung gewinnen. Aus dieser Perspektive lässt sich der Gedanke einer Auswärtigen Kulturpolitik als Konfliktprävention auch als eine Konsequenz des bereits in den I970er Jahren in Grundzügen etablierten Prinzips der Wechselseitigkeit verstehen und weniger als der komplett neue Politikansatz als der er häufig dargestellt wird. ${ }^{91}$

88 | Konzeption 2000, S. 2.

89 | Leitsätze, 1970, S. 15.

90 | Stellungnahme, 1977, S. 15.

91 So etwa durch die Bundesregierung selbst in: Antwort der Bundesregierung, 2007, S. 6; aber auch in Publikationen zur Auswärtigen Kulturpolitik: Kurt-Jürgen Maaß: Überblick: Ziele und Instrumente der Auswärtigen Kulturpolitik. In: Ders. (Hrsg.): Kultur und Außenpolitik, S. 28. 
Die Zielhorizonte einer Konfliktprävention durch Dialog, Förderung demokratischer Entwicklungsprozesse und Verwirklichung der Menschenrechte rücken in den jüngeren Papieren allerdings in den Vordergrund; die Argumentationsfigur eines konfliktbearbeitenden Dialogs gewinnt somit deutlich an Präsenz. Gleichzeitig bleibt ihre Anwendung nicht auf multilaterale Organisationen beschränkt - die komplexen, netzwerkartigen und zum Teil temporären Akteursfelder, die sich zunehmend zur Bearbeitung von Konfliktkonstellationen konstituieren, klingen in folgender Formulierung an:

"Zentrale Aufgabe der kommenden Jahre wird es sein, über politische und kulturelle Grenzen hinweg mehr Foren des Dialogs und globale Netzwerke aufzubauen und mitzuhelfen, auf der Basis eigener Interessen Beiträge zur Lösung gemeinsamer Probleme zu finden. In solchen 'Lerngemeinschaften، sollte es am ehesten gelingen, Konflikten durch besseres Wissen voneinander und mehr Verständnis füreinander vorzubeugen. “92

Was »Foren des Dialogs«, »globale Netzwerke« oder »Lerngemeinschaften« konkret für kulturpolitische Formate und Einsatzgebiete bedeuten, wird hier nicht weiter ausformuliert. Dennoch lässt sich festhalten, dass gerade die zweite Argumentationsfigur eine spürbare Ausdehnung erfährt und somit auch die jüngere Dynamik in diesem Politikbereich verdeutlicht. Sie gewinnt dabei nicht nur grundsätzlich an Präsenz, sondern führt zu neuen diskursiven Anschlüssen und Praxisfeldern, die sich entlang des Begriffs Dialog gut nachzeichnen lassen: Dieser findet sich nicht nur in dem Ansatz einer Auswärtigen Kulturpolitik als Konfliktprävention, sondern er avanciert nach der Jahrtausendwende zu einem ebenso elementaren wie flexibel einsetzbaren Passepartout zur offiziellen Erklärung des Politikbereichs. Diese herausgehobene Position zeigt sich etwa in den Formulierungen zu den konzeptionellen Grundlagen des Politikbereichs aus dem Jahr 2007: »Auswärtige Kultur- und Bildungspolitik (AKBP) ist auf langfristige Wirkung angelegt. Ihre übergreifende Aufgabe ist die Förderung des Dialogs zwischen Menschen unterschiedlicher kultureller Hintergründe und die Schaffung von Vertrauen und dauerhaften weltweiten Partnerschaften mit unserem Land. «"3

Dialog tritt an dieser Stelle also zunächst als grundlegendes Leitmotiv zur Legitimierung des gesamten Politikbereichs hervor. Bemerkenswert ist weiterhin, dass hier nun »Menschen « - und nicht primär Staaten - als Dialogpartner angeführt werden. In dem gleichen Papier findet sich zugleich eine Vielzahl von Formulierungen, die direkt auf operative Aufgaben und konkrete Maßnahmen verweisen. Dialog erscheint hier als das angemessene Format, um auf die beschriebenen Herausforderungen einer »unübersichtlichen Welt« zu

92 | Konzeption 2000, S. 4.

93 | Antwort der Bundesregierung, 2007, S. 6. 
reagieren: So werden die Dialogpunkte des Goethe-Instituts im Nahen Osten als gelungene Umsetzung des Politikschwerpunktes Dialog mit der islamischen Welt angeführt; Dialogforen, Dialogseminare, bewusste Dialogpolitik, das Konzept vom Dialog der Kulturen, Europäisch-Islamischer Kulturdialog, Deutsch-Arabischer Mediendialog oder interkultureller Dialog mit dem Islam ${ }^{94}$ sind weitere Beispiele für die bemerkenswerte Anschlussfähigkeit des Begriffs in den jüngeren Regierungspapieren.

Aber zurück zur diskursiven Figur einer Auswärtigen Kulturpolitik als Konfliktprävention: Diese gewinnt gerade durch die vielfältigen Verknüpfungen von Dialog deutlich an Präsenz und avanciert in den Berichten der Bundesregierung sogar zu einer der vier offiziellen Zielsetzungen des Politikbereichs: $z$ ur Konfliktprävention durch Wertedialog beitragen. ${ }^{95}$ Wie bereits auf den Berliner Konferenzen beobachtet, gehen Konfliktprävention und Dialog nun also eine enge Verbindung mit »Werten« ein. Diese Verknüpfung lässt sich als Konkretisierung, zugleich aber als Verengung der zunächst - etwa auf der in der Einleitung vorgestellten Stuttgarter Tagung - breit diskutierten Möglichkeiten einer konfliktpräventiven Kulturpolitik verstehen: War zu Beginn der Anspruch spürbar, Konfliktlinien zu bearbeiten, die sich aus einer ungerechten Weltordnung oder den Verwerfungen der Globalisierung ergeben, so scheint im jüngsten Dokument die Zuständigkeit auf solche Konflikte beschränkt, die sich aus »unterschiedlichen Werten« ergeben, »kulturelle Ursachen« haben und sich durch einen »Dialog über Werte« bearbeiten lassen:

"Es bleibt die Auffassung der Bundesregierung, dass die Förderung von interkulturellem Verständnis, der Dialog über Werte und die Unterstützung beim Aufbau moderner, freiheitlich orientierter Bildungssysteme konfliktmindernd wirken und verhindern helfen kann, dass aus kulturellen Unterschieden Konfrontation und Gewalt erwachsen. “96

Diese Verengung lässt sich dabei über zwei Perspektiven interpretieren, die auf den ersten Blick in unterschiedliche Richtungen weisen: Sie kann sowohl als Tendenz zu einer Kulturalisierung der Ursachen globaler Konflikte verstanden werden als auch als eine bewusste Zurückhaltung, politische Konflikte kulturell bearbeiten und lösen zu wollen. So finden sich etwa in der Antwort der Bundesregierung Passagen, die eher eine solche Ernüchterung vermuten lassen:

"Dies schließt eine nüchterne Sicht von den Grenzen der kulturpolitischen Möglichkeiten ein. Krisen und Konflikte lassen sich mit Mitteln der Kultur und Bildung allein nicht

94 | All diese Formulierungen sind zu finden in: Ebd., S. 34-37 und S. 40.

95 | Bericht zur Auswärtigen Kulturpolitik 2005/2006, S. 5.

96 | Antwort der Bundesregierung, 2007, S. 8. 
Iösen. Umgekehrt gilt: Ohne Begegnungs- und Bildungsprojekte, ohne eine Unterstützung des innergesellschaftlichen Dialogs, der Einbeziehung von Minderheiten, Frauen und Jugendlichen, ohne Schaffung eines gemeinsamen kulturellen Verständnisses wird es erst recht nicht gelingen, Feindbilder und Gewaltbereitschaft abzubauen und die Voraussetzungen für politische Lösungen von Konflikten zu verbessern. ${ }^{97}$

In die andere Richtung - hin zu einer Kulturalisierung politischer Konfliktlinien - weist der Politikbereich Dialog mit dem Islam $^{98}$, der nach den Attentaten vom II. September 200I aufgebaut wurde und in der Antwort der Bundesregierung als konkrete und erfolgreiche Umsetzung einer konfliktpräventiven Auswärtigen Kulturpolitik angeführt wird. Hier werden Bilder von unterschiedlichen »Zivilisationsräumen« und geopolitischen Trennlinien entlang von »Kultur« und »Werten« gezeichnet:

„Die anlässlich der Veröffentlichung von Mohammed-Karikaturen Ende 2005 und der nachfolgenden, zum Teil gewalttätigen Proteste in der islamischen Welt entbrannte Kultur- und Wertedebatte zeigt, dass die kulturelle Kluft zwischen dem Westen und der islamischen Welt sich zu vertiefen droht. Ziel des Europäisch-Islamischen Kulturdialogs ist es, durch praktische Maßnahmen der Kultur- und Bildungszusammenarbeit das gegenseitige Verständnis zwischen westlicher und islamischer Welt zu verbessern, Missverständnisse aufzulösen und die Ablehnung, die zahlreiche Akteure der Region unseren Werten entgegenbringen, abzubauen. “ ${ }^{99}$

Somit wird sichtbar, dass die diskursive Figur einer Auswärtigen Kulturpolitik als Konfliktprävention weiterhin zu unterschiedlichen Übersetzungen und Anschlüssen führen kann. Auch anhand der Regierungsdokumente lässt sich somit die tastende Wissensarbeit von Angehörigen der policy community erahnen. Dennoch wird eine Entwicklungstendenz erkennbar: Die zunächst eher breit diskutierte Idee einer konfliktpräventiven Auswärtigen Kulturpolitik verengt sich auf das politische Programm eines »Wertedialogs« zwischen dem »Westen« und der »islamisch geprägten Welt«, der über »Missverständnisse« geführt werden soll, um hierdurch Brücken über die »kulturelle Kluft« zu bauen.

97 | Ebd., S. 8 f.

98 | Siehe hierzu vertiefend: Marcel Ernst: Der deutsche "Dialog mit der islamischen Welt". Diskurse deutscher Auswärtiger Kultur- und Bildungspolitik im Maghreb. Bielefeld 2014.

99 | Bericht der Bundesregierung zur Auswärtigen Kulturpolitik 2005/2006, S. 8. 


\subsection{Erweiterungen: Regulierungs- und Gestaltungsansprüche eines "Nationalstaates im Umbau"}

Durch eine solche vergleichende Analyse von Regierungsdokumenten lässt sich also ein Wechselspiel zwischen zirkulären Bezugnahmen auf stabile Bestände und linearen Veränderungen in der Ausgestaltung der imaginären Dimension des Politikbereichs aufzeigen. Zu den kontinuierlichen Elementen gehören zwei Leitmotive zur Legitimierung Auswärtiger Kulturpolitik - das Prinzip der kulturellen Selbstdarstellung und das Prinzip der Wechselseitigkeit - sowie einige weitgehend naturalisierte Grundfiguren: der Glaube an die Existenz einer kollektiv geteilten nationalen Spezifik etwa, die Hoffnung auf Reputationsgewinne durch kulturelle Vermittlung oder die Überzeugung, dass kultureller Austausch sich positiv auf »internationale Verständigung u und »Frieden« auswirkt. Zugleich zeigen sich nach der Jahrtausendwende einige lineare Verschiebungen, die tatsächlich zu neuen Argumentationsfiguren führen: Besonders bedeutungsvoll erscheinen hierbei die Entwicklung eines komplett modifizierten Rahmennarrativs von der »unübersichtlichen Welt« nach Ende des Kalten Krieges, eine modifizierte Wahrnehmung von der geopolitischen Rolle Deutschlands, die völlig neue Betonung einer Konfliktlinie zwischen dem »Westen« und der »islamischen Welt« als zentralem kulturpolitischen Arbeitsfeld sowie die gleichfalls neuen Verknüpfungen etablierter Zielvorstellungen mit ökonomischen Denklogiken einerseits und mit universalen Werten andererseits. Darüber hinaus zeigt sich nach der Jahrtausendwende eine Tendenz zur Erweiterung der Zuständigkeit des Politikbereichs innerhalb seines offiziellen Narrativs, die sich anhand der im letzten Abschnitt diskutierten politischen Rationalitäten aufzeigen lässt. Die drei Regulierungs-, Gestaltungs- und Ordnungsansprüche sind zwar im Prinzip bereits in den I970er Jahren vorhanden, sie erfahren in den jüngeren Papieren aber zum Teil erhebliche Erweiterungen: Etwa wenn die globale Positionierung Deutschlands im Modus der Kultur nun auch »die besten Köpfe« für Deutschlands Hochschulen und Industrie gewinnen soll oder auf universale Projekte - »Krisenprävention« oder »Förderung von Menschenrechten« - zurückgreift. In der zunehmenden Bedeutung von Verknüpfungen des Politikbereichs mit solchen Begriffen, die auf Felder multilateraler Konfliktbearbeitung und globaler Regierungsführung verweisen, manifestiert sich gleichfalls die immer wichtiger werdende Suche nach einer Erweiterung außenpolitischer Handlungsspielräume durch kulturpolitische Instrumente. Besonders relevant erscheinen mir aber die Ausdehnungen im Hinblick auf den dritten Rationalitätstyp: Eine markante Erweiterung des Anspruchs, Perspektiven, Einstellungen und Verhaltensweisen zu verändern zeigt sich in den jüngeren Dokumenten in Formulierungen, die explizit die Lebensführung von Individuen oder Kollektiven fokussieren. So heißt es etwa in der Antwort der Bundesregierung aus dem Jahr 2007 im Abschnitt Dialog zwischen 
den Kulturen: »Der Erfolg eines interkulturellen Dialogs besteht in der Einstellungs- und Verhaltensänderung von Individuen. ${ }^{100}$ Ein ähnliches Erfolgskriterium wird im Kapitel Auswärtige Kulturpolitik als Mittel der Krisen- und Konfliktverhütung angeführt:

"Konfliktprävention ist jedoch ebenso wie andere Aufgaben der Auswärtigen Kultur- und Bildungspolitik langfristig angelegt; die Ergebnisse lassen sich kaum in Form von Entschärfung einer konkreten Konfliktsituation messen. Maßnahmen der Auswärtigen Kultur- und Bildungspolitik zielen vielmehr ab auf nachhaltige Verhaltensänderungen. «101

Hier wird ein neuartiger Regulierungs- und Gestaltungsanspruch greifbar: Es geht offensichtlich nicht mehr alleine um die Vermittlung deutscher Sprache und Kultur oder um den Erwerb von Sympathie, sondern gleichfalls um ein Einwirken auf Einstellungen, Verhaltensweisen und Wissensbestände von Individuen und Gruppen, die nicht Staatsbürger der Bundesrepublik sind. In der Konsequenz deutet sich hier eine gewisse Flexibilisierung der Vorstellung von staatlichen Zuständigkeiten an: Politische Instabilitäten oder Konfliktkonstellationen - gerade wenn sich aus ihnen ein Sicherheitsrisiko für Deutschland ergibt - lassen (kultur-)politische Interventionen legitim erscheinen. Diese Veränderungen werden noch deutlicher, wenn man sie mit Formulierungen aus dem Jahr I970 konfrontiert: »Dabei ist darauf zu achten, daß die Aufgabe Verständigung und Zusammenarbeit lautet, daß also alles zu vermeiden ist, was als Einmischung in die inneren Angelegenheiten anderer Länder erscheinen könnte. $\ll^{102}$

Nun lautet meine Schlussfolgerung nicht, dass außenpolitische Eliten des vereinten Deutschlands etablierte Vorstellungen von staatlicher Souveränität grundlegend in Frage stellen oder auf umfassende politische Interventionen in »Konfliktgebieten« hinarbeiten. Vielmehr geht es mir darum, spürbare diskursive Verschiebungen festzuhalten, die neue Formen der Präsenz oder Einflussnahme in Krisen- oder Transformationsregionen plausibel erscheinen lassen. Es ist naheliegend, diese Verschiebungen zum einen mit internationalen Diskussionen zur Legitimität von politischen oder militärischen Interventionen - wie sie seit den I990er Jahren geführt werden ${ }^{103}$ - und zum anderen mit der Suche der außenpolitischen Eliten eines »Nationalstaats im Umbau ${ }^{104}$

100 | Antwort der Bundesregierung, 2007, S. 37.

101 | Ebd., S. 39.

102 | Leitsätze, 1979, S. 6.

103 | Siehe hierzu etwa: Didier Fassin \& Mariella Pandolfi (Hrsg.): Contemporary States of Emergency. The Politics of Military and Humanitarian Interventions. New York 2013.

104 | Irene Götz: Deutsche Identitäten, S. 348. 
nach neuen politischen Positionierungen und kulturellen Repräsentationen in einer unübersichtlichen globalen Ordnung in Zusammenhang zu bringen. Diese diskursiven Bewegungen verweisen zugleich auf Dynamiken im Narrativ vom Nationalen, in denen sich solche veränderten geopolitischen Rahmenbedingungen, globalen Diskurse oder transnationalen Problemstellungen widerspiegeln. Wie sich diese Dynamiken auf urbanen Bühnen Berlins darstellen, werde ich im folgenden Kapitel untersuchen.

\section{Policy takes place: Urbane Bühnen, Politische Materialitäten, Formierungsmacht}

In diesem Abschnitt setze ich die Beschäftigung mit den imaginären Dimensionen Auswärtiger Kulturpolitik sowie mit der Wissensarbeit ihrer policy community fort. Allerdings fokussiere ich nun explizit den urbanen Raum Berlin und insbesondere Prozesse der Materialisierung des politischen Narrativs, die in ihm sichtbar werden. Den empirischen Ausgangspunkt bilden wiederum Konferenzen oder Kulturveranstaltungen, die das Auswärtige Amt oder Mittlerorganisationen zu kulturpolitischen Fragestellungen in der Hauptstadt realisieren. ${ }^{105}$ Von Interesse sind nun Formen des Raumgreifens dieser Politik - also die temporären Präsenzen Auswärtiger Kulturpolitik und ihrer Schlüsselbegriffe an ausgewählten Orten in Berlin, die Verwebungen ihres politischen Narrativs mit städtischen Kontexten und die Produktion urbaner Bühnen, auf denen kulturpolitische Themen und Akteure einem breiteren Publikum vorgestellt werden können. Im Zuge der Verknüpfungen von politischen Zielsetzungen, Schlüsselbegriffen, Programmen, Orten und Akteuren, die zu solchen Anlässen hergestellt werden, zeichnet der Politikbereich temporäre Formationen in den städtischen Raum und wird hierdurch sicht- und greifbar.

105 | Ich beziehe mich in diesem Abschnitt primär auf meine ethnografischen Beobachtungen auf folgenden Veranstaltungen: Menschen bewegen - Kultur und Bildung in der deutschen Außenpolitik, Berlin, Auswärtiges Amt, 25.-26. Oktober 2006; Menschen bewegen - Kulturpolitik im Zeitalter der Globalisierung, Berlin, Auswärtiges Amt, 25.26. April 2009; Perspektive Europa, eine Veranstaltung der Akademie der Künste in Zusammenarbeit mit dem Auswärtigen Amt in der Akademie der Künste, 1.-2. Juni 2007; Wiedervorlage: Nationalkultur, eine Veranstaltung des Goethe-Instituts in Partnerschaft mit den Staatlichen Museen zu Berlin, in Zusammenarbeit mit der Japan Foundation, der Allianz Kulturstiftung und dem Auswärtigen Amt auf der Museumsinsel Berlin, 24.-26. April 2008 und Illusion der Nähe? Ausblicke auf die europäische Nachbarschaft von Morgen, ein Forum des Goethe-Instituts in Partnerschaft mit dem Polnischen Institut Berlin und dem Deutsch-Französischen Institut Berlin im ehemaligen Flughafen Tempelhof, Berlin, 27.-29. Oktober 2010. 


\section{Rituelle Abläufe und besondere Orte: Urbane Präsenzen und Performanzen einer policy community}

Der Auftakt solcher Tagungen verläuft zumeist nach einem fast rituellen Schema: Man trifft sich zu einem feierlichen Eröffnungsabend an einem »besonderen Ort« in Berlin - eine junge Kultureinrichtung mit postindustriellem Charme etwa, ein überregional bekanntes Bauwerk mit historischen Bezügen oder auch eine renommierte, im Kulturleben etablierte Institution können den Rahmen für eine zumeist halb-öffentliche Veranstaltung bilden. »Auf gesonderte Einladung « heißt es dann in dem Tagungsprogramm und entsprechend überprüfen Mitarbeiter der Organisatoren am Eingang die Berechtigung der Teilnahme einer Besucherin. Sofern ein Name identifiziert werden konnte, bekommt man ein Bändchen um das Handgelenk gebunden oder auch ein Namensschild und kann die inneren Räumlichkeiten betreten. Den Auftakt bildet eine Rede des Ministers, des Präsidenten des Goethe-Instituts oder auch eine Podiumsdiskussion mit einem eher vage definierten Titel, die in das Themenfeld der Konferenz einführen soll. Auf dem Podium sitzen in diesem Fall ranghohe Politikerinnen, führende Vertreter des Goethe-Institutes, zuweilen auch Diplomatinnen, häufig ergänzt durch Kulturschaffende oder Wissenschaftler. Diese Wortbeiträge werden begleitet durch ein leicht verdauliches - also kurz gehaltenes - kulturelles Rahmenprogramm, das in den informellen Teil des Abends überleitet: Gespräche in wechselnden Kleingruppen an Cocktailtischen, die in Reichweite eines Buffets platziert sind. Hier begegnet sich die policy community in einer Zusammensetzung, die vom konkreten thematischen Zuschnitt und der Größe der Tagung abhängig ist. Das regelmäßige Zentrum bilden die Mitarbeiterinnen der Mittlerorganisationen, der Abteilung Kultur und Kommunikation des Auswärtigen Amtes sowie der wichtigen Partnerorganisationen. Hinzu kommen - je nach Art der Veranstaltung - Politiker, ausländische Diplomaten, Künstler, Wissenschaftlerinnen, Journalistinnen und ein interessiertes Fachpublikum. Solche Tagungen bieten also die Möglichkeit, in das Akteurs- und Diskussionsfeld Auswärtige Kulturpolitik einzutauchen und eine policy community in ihrer Struktur - mit ihren Zentren und Verflechtungen - zu erfahren: Die spezifische Konstellation, die den Politikbereich prägt, findet hier ihre performative Bestätigung.

Einen bereits angedeuteten Aspekt dieser urbanen Inszenierungen möchte ich nun vertiefen: Auffällig ist der Rückgriff auf »besondere Orte« als räumlichen Rahmen für solche Veranstaltungen. Die beiden in den letzten Abschnitten diskutierten Konferenzen Menschen bewegen werden jeweils mit einem »Kulturabend « in einer aufstrebenden, kürzlich etablierten Kultureinrichtung eröffnet. Im Jahr 2006 werden die Besucher in das in einem ehemaligen Pumpwerk am Spreeufer in Berlin-Mitte neu gegründete Radialsystem $V$ geführt, das sich als ein unabhängiger »offener Raum für den Dialog 
der Künste « und als Ort »innovativer und hochwertiger Konzerte, Tanz- und Musiktheateraufführungen « präsentiert. ${ }^{106}$ Drei Jahre später findet die Eröffnungsveranstaltung in den Uferhallen im Wedding statt - ein »Kulturzentrum in privater Trägerschaft«, das zu diesem Zeitpunkt gerade in der ehemaligen Zentralwerkstatt der Berliner Verkehrsbetriebe entstand. Der Komplex wurde hierzu im Jahr 2007 von einer Uferhallen AG erworben und »bietet heute eine gemeinsame Plattform für Bildende Künstler, Musiker, Tanzprojekte, Theaterinszenierungen, Ausstellungen, Wohnexperimente, Tonstudios und Proberäume. Dieses kreative Nebeneinander verschiedener Kunstrichtungen schafft Synergien und fördert übergreifende Dialoge.« ${ }^{107}$

In beiden Fällen werden also Orte gewählt, an denen kulturpolitische Metaphern und Narrative, Zielvorstellungen und Erwartungen eine materielle und performative Form erhalten und somit auch greifbar werden: etwa die Möglichkeit einer Umnutzung ehemaliger industrieller oder gewerblicher Flächen und Gebäudeensembles durch »Kultur« in der postindustriellen Stadt; oder die Suche nach privaten oder flexiblen Trägerschaften und Förderstrukturen, die nicht primär auf öffentliche Gelder abzielen; oder die Orientierung einer Kultureinrichtung an gegenwärtigen Schlüsselbegriffen wie Dialog, Innovation und Kreativität; und auch die Offenheit einer staatlich finanzierten kulturpolitischen Struktur für gegenwärtige Dynamiken und Knotenpunkte des urbanen kulturellen Lebens - kurz: durch eine solche Ortswahl lassen sich Assoziationsketten transportieren, die anschlussfähig sind für zeitgenössische Diskussionen und Positionierungen des Politikbereichs.

Auch durch weitere Beispiele ließe sich diese spezifische Form des Raumgreifens Auswärtiger Kulturpolitik verdeutlichen: Zur öffentlichen Präsenz und Selbstdarstellung schafft sich der Politikbereich urbane Bühnen oder Kontexte und bindet hierzu die Reputation oder die Aura »besonderer Orte« ein - eine Reputation, die durch die Orientierung an zeitgenössischen kulturpolitischen Schlüsselbegriffen, durch eine herausgehobene öffentliche Aufmerksamkeit, ${ }^{108}$ durch historische Bezüge oder auch - wie noch zu zeigen sein wird - durch die Versammlung seltener Güter begründet sein kann. Für die Sichtbarkeit Auswärtiger Kulturpolitik erfüllen diese »besonderen Orte« eine

106 | So Formulierungen in der Selbstbeschreibung: www.radialsystem.de (letzter Zugriff am 11. August 2017).

107 So im Dezember 2011 zu lesen auf: http://www.uferhallen.de/index.php? option=com_content $\&$ task=view \&id=1\&Itemid=43.

108 So veranstaltete das Goethe-Institut im Jahr 2010 die Tagung Illusion der Nähe? Ausblicke auf die europäische Nachbarschaft von Morgen in dem Empfangsgebäude des kurz zuvor stillgelegten Flughafens Berlin-Tempelhof, dessen multiple historische Bezüge und zukünftige Nutzungsoptionen zu diesem Zeitpunkt umfangreich politisch und medial diskutiert worden waren. 
wichtige Funktion, denn sie - so möchte ich im Anschluss an Pierre Bourdieu formulieren - »weihen« den Politikbereich und seine policy community durch das »symbolische Kapitak«, das sie akkumuliert haben. ${ }^{109}$

\section{Ortseffekte: Die symbolische und gesellschaftspolitische Dimension von Räumen}

In seinem Artikel Ortseffekte erinnert Bourdieu an eine solche Wechselwirkung zwischen den symbolischen Dimensionen des Raums und seinen Bewohnerinnen oder regelmäßigen Nutzern. Den Ausgangspunkt bildet hierbei die Überlegung, dass in einer Gesellschaft, die von sozialer Ungleichheit geprägt ist, auch die Räume hierarchisiert sind und somit »soziale Abstände zum Ausdruck « bringen - anders formuliert: der bewohnte und angeeignete Raum lässt sich zugleich als Materialisierung sowie als Symbolisierung sozialer Relationen lesen. ${ }^{110}$ Die Verknüpfung von Räumen mit Wertigkeiten verweist demnach also auf die grundlegenden Beziehungsstrukturen einer Gesellschaft; eine solche räumliche Rangordnung kann zugleich durch politische Entscheidungen und mediale Berichte verstärkt werden und zeigt sich etwa anhand der Gerüchte, Narrative, Reputationen oder Stigmata, die Orte umgeben und repräsentieren. Die »Ortseffekte«, die im Rahmen dieser Wechselbeziehung zwischen den symbolischen Bedeutungen des Raums und der sozialen Position seiner Bewohner entstehen, lassen sich am Beispiel urbaner Armutsquartiere nachzeichnen: Der negative Ruf, den ein solches Viertel häufig besitzt, bestätigt sich demnach im Zuge von sozialen Prozessen, da eine Bevölkerung, die sich durch eine geringe Verfügung über Geld, einen Mangel an sozialen Beziehungen zu den gesellschaftlichen Eliten sowie an angesehenen Bildungsabschlüssen auszeichnet, sich tatsächlich hier ansiedelt oder - aufgrund von politischen und ökonomischen Zwängen - hier ansiedeln muss. Das Stigma des »Slums« oder des »Problemquartiers« geht auf die Bewohner über und wird somit zu einem relevanten Effekt in der Hierarchisierung einer Gesellschaft. ${ }^{111}$ Eine ähnliche Wechselwirkung beobachtet Bourdieu im Hinblick auf die Wohngebiete der Reichen oder auf die Zentren politischer und ökonomischer Macht, in denen sich »seltene Güter und ihre Besitzer« konzentrieren: Die materiellen Ressourcen, sozialen Beziehungen und das hohe Bildungsni-

109 | Pierre Bourdieu: Ortseffekte. In: Pierre Bourdieu et al.: Das Elend der Welt. Zeugnisse und Diagnosen alltäglichen Leidens an der Gesellschaft. Konstanz 1997, S. 159-167.

110 | Ebd., S. 160.

111 | Siehe hierzu: Jens Adam: "Kaum noch normale Berliner". Stadtethnologische Erkundungen in einem "sozialen Problemquartier". Münster 2005. 
veau der hier versammelten Akteure übertragen sich als »symbolisches Kapital« auf den Ort und die hier Anwesenden. In der Konsequenz

"weiht das schicke Wohnviertel jeden einzelnen seiner Bewohner symbolisch, indem es innen erlaubt, an der Gesamtheit des akkumulierten Kapitals aller Bewohner Anteil zu haben. Umgekehrt degradiert das stigmatisierte Viertel symbolisch jeden einzelnen seiner Bewohner, der das Viertel degradiert, denn er erfüllt die von den verschiedenen gesellschaftlichen Spielen geforderten Voraussetzungen ja nicht. “112

\section{Die Greifbarkeit kulturpolitischer Diskurse im urbanen Raum: Potenziale der Sichtbarkeit und Formierungsmacht}

Im Anschluss an Bourdieu lässt sich somit nach den symbolischen und gesellschaftspolitischen Dimensionen der beschriebenen Formen des Raumgreifens Auswärtiger Kulturpolitik fragen: Welche Schlussfolgerungen zu dem Selbstverständnis des Politikbereichs lassen sich aus diesen Strategien der Ortswahl ziehen? Welche Wechselwirkungen zeigen sich zwischen einer policy community und den urbanen Kontexten, an denen sie sich trifft und bildet? Und welche Effekte ergeben sich aus der Produktion solcher urbaner Bühnen für die öffentliche Präsenz und Positionierung Auswärtiger Kulturpolitik und ihres Narrativs?

Zwei Konsequenzen stechen dabei hervor, von denen die erste bereits angeklungen ist: Die »besonderen Orte « bieten für die policy community Potenziale der Sichtbarkeit - ein Politikbereich, der im großen Maße außerhalb Deutschlands operiert, wird in der Hauptstadt greifbar. Im urbanen Raum eröffnen sich temporäre Foren, in denen sich nicht nur die policy community begegnen und formieren kann, sondern sich zugleich durch die Präsenz hochrangiger Politiker die exklusive Nähe der Mittlerorganisationen zum »politischen Feld « ${ }^{113}$ zur Schau stellen lässt - denn schließlich besitzt man einen »offiziellen Auftrag« zur kulturellen Vermittlung und setzt sich hierdurch von anderen Akteuren ab. Zugleich tragen das Renommee der Kultureinrichtungen und die Demonstration der Verflechtungen der policy community mit den Dynamiken und Innovationen des gegenwärtigen kulturellen Lebens in Deutschland zur Steigerung der Legitimität des Politikbereichs bei - kurz: die spezifische

112 | Pierre Bourdieu: Ortseffekte, S. 166.

113 | Im Anschluss an Bourdieu meint "politisches Feld " hier den "Mikrokosmos", in dem führende Politiker, Regierungsvertreter und Abgeordnete, aber auch einflussreiche Journalisten, Meinungsforscher, Denkfabriken usw. die "politischen Produkte" - also etwa "Probleme, Programme, Analysen, Kommentare, Konzepte, Ereignisse" - erzeugen, die den öffentlichen Diskurs bestimmen. Siehe hierzu: Pierre Bourdieu: Das politische Feld, S. $13 \mathrm{ff}$. 
Struktur Auswärtiger Kulturpolitik an der Schnittstelle von Politik und Kultur und das Selbstverständnis ihrer Akteure werden auf urbanen Bühnen sichtbar.

Der zweite Effekt baut auf diesen Potenzialen auf, geht aber in seinen politischen Dimensionen über sie hinaus: In den beschriebenen Prozessen des Raumgreifens tritt ein Vermögen zutage, politische Narrative, Schlüsselbegriffe, Wissensbestände, Orte, Akteure und Ressourcen zu temporären Formationen zusammenzufügen und hierdurch auch kulturpolitische Diskurse wirkmächtig zu strukturieren - anders formuliert: durch die Materialisierung bestimmter Schlüsselbegriffe in urbanen Kontexten wird das offizielle Narrativ Auswärtiger Kulturpolitik nicht nur greifbar, sondern es lässt sich gewissermaßen ein »Standpunkt« schaffen, der Kernaussagen und Argumentationsfiguren institutionalisiert und als Ausgangspunkte für die weitere kulturpolitische Diskussion und Praxis etabliert. Dieses Vermögen, das ich als Formierungsmacht bezeichne, setzt sich aus zumindest drei Bestandteilen zusammen: zunächst aus dem Potenzial, ausgewählte Schlüsselbegriffe etwa im Rahmen von Tagungen und Veranstaltungen zum Kristallisationspunkt kulturpolitischer Öffentlichkeiten zu machen; zweitens aus der Verfügung über einen »offiziellen Auftrag« und somit der Nähe zum »politischen Feld«, durch die solche Begriffe eine »objektivierte, unmittelbar für alle sichtbare, öffentliche, offizielle und damit autorisierte Existenz« erlangen können; ${ }^{114}$ und drittens aus den finanziellen und institutionellen Ressourcen eines staatlich initiierten Politikbereichs, die es ermöglichen, solche bestätigten oder neu-konfigurierten Schlüsselbegriffe durch ein weltweites Netzwerk zu mobilisieren und zu Ausgangspunkten der Programmarbeit in den unterschiedlichsten lokalen Kontexten zu machen. Ob und in welchem Umfang sich diese Potenziale realisieren, wäre im Hinblick auf ein jeweiliges Tagungsthema zu prüfen; sicherlich laufen auch manche temporäre Formationen und ihre urbanen Inszenierungen ins Leere. Im Prinzip kann aber das Wirken der Formierungsmacht zu Konsequenzen führen, die über die Strukturierung aktueller Diskussions- und Handlungsfelder hinausgehen. Ihr mächtigster Effekt besteht in der Etablierung, Veränderung oder Bestätigung von grundlegenden Ordnungsmustern und Denkmodellen, über die internationale Kulturbeziehungen gedacht und praktiziert werden können. Diese Zusammenhänge möchte ich nun an einem weiteren Beispiel genauer erläutern.

114 | Ebd., S. 82. 


\section{"Wiedervorlage: Nationalkultur": Reflexivierung und Rezentrierung eines kulturpolitischen Schlüsselbegriffs}

Im April 2008 organisiert das Goethe-Institut gemeinsam mit den Staatlichen Museen zu Berlin ein Symposium unter dem Titel Wiedervorlage: Nationalkultur. Zu diesem Anlass bietet die Museumsinsel im Zentrum Berlins die urbane Bühne zur Sichtbarmachung und Materialisierung Auswärtiger Kulturpolitik in der deutschen Hauptstadt. Der Ortseffekt ergibt sich in diesem Fall aus der Versammlung seltener und wertvoller Güter an einem zentralen Platz der Republik sowie aus dem historisch gewachsenen Renommee von Museen als Institutionen von nationalem und globalem Rang. Am Beispiel dieses Symposiums lassen sich nicht nur die bereits beschriebenen Prozesse des Raumgreifens eines Politikbereichs weiter verdeutlichen, sondern zugleich unterschiedliche Strategien der Rezentrierung eines Schlüsselbegriffs und elementaren Ordnungsmusters Auswärtiger Kulturpolitik durch seine Inszenierung auf urbanen Bühnen analysieren.

»Darf man in Deutschland wieder von Nationalkultur sprechen?«, fragt der Generalsekretär des Goethe-Instituts in seiner Einleitung im Programmheft und begründet den thematischen Zuschnitt des Symposiums mit einem gegenwärtig zunehmenden »Bedürfnis [...], sich über wirtschaftliche und politische Aspekte hinaus mit dem eigenen Land zu identifizieren «. ${ }^{115}$ Die europäischen Hoffnungen gerade der Deutschen im Hinblick auf eine Auflösung des »nationalen Bewusstseins« hätten sich nicht erfüllt:

"Nationalstaaten werden hier noch lange die Bezugspunkte kultureller Identität bleiben. 9/11 oder der Karikaturenstreit verstärken kulturelle Abgrenzungssehnsüchte. Und schließlich sorgt die Globalisierung auch bei uns dafür, dass die Menschen der zunehmenden grenzüberschreitenden Lebensgewohnheiten weltweit vereinheitlichenden Dynamik ortsbezogene kulturelle Identifikationen entgegensetzen. Hierhin gehört auch die teilweise ausgeprägte Hinwendung von Migranten zu ihrer Herkunftskultur womit wiederum die Gesellschaften in den Zielländern der Migration umzugehen lernen müssen."

Ziel des Symposiums sei es, »diese Entwicklungen zu diskutieren und zu überprüfen, inwieweit die Rückbesinnung auf nationale Orientierungen tatsächlich zeitgemäß und zukunftsfähig ist, insbesondere in Hinblick auf eine moderne internationale Kulturarbeit.«

115 | Dieses und die beiden folgenden Zitate des Generalsekretärs: Hans-Georg Knopp: Zur Sache. In: Goethe-Institut \& Staatliche Museen zu Berlin: Wiedervorlage: Nationalkultur. 24. - 26. April 2008 auf der Museumsinsel Berlin. Programmablauf, S. 5. 
In diesen Formulierungen zeigt sich ein Wechselspiel zwischen Reflexivierung und Rezentrierung von Nationalkultur als einem Schlüsselbegriff des Politikbereichs, das ich anhand dieser Tagung genauer untersuchen möchte. Hierzu werde ich erstens die bereits herausgearbeiteten Wirkungsweisen der Formierungsmacht an diesem besonders illustrativen Beispiel weiter erläutern. Ich greife dabei auf Pierre Bourdieu zurück, der darauf hingewiesen hat, dass die legitimen Themen des öffentlichen Diskurses nicht gegeben sind, sondern in politischen, medialen oder gesellschaftlichen Konstellationen produziert werden: »Jede Gesellschaft entwickelt unablässig einen Komplex von sozialen Problemen, die als legitim gelten, als diskussionswürdige, öffentliche, manchmal zu offiziellen erhobene und gewissermaßen staatlich anerkannte Probleme. «116Vor diesem Hintergrund untersuche ich, wie Nationalkultur durch eine temporäre Materialisierung im urbanen Raum und im Zusammenspiel von Kulturmittlern und maßgeblichen Akteuren des »politischen Feldes« eine erneute offizielle Anerkennung als elementares Ordnungsmuster und legitimer Ausgangspunkt Auswärtiger Kulturpolitik erfährt. Zweitens geht es mir um die inhaltlichen Dimensionen dieser Rezentrierung - ich frage also nach der konkreten Ausgestaltung des Konzeptes von Nationalkultur, die auf diesem Symposium sichtbar wird: Mit welchen Begriffen und thematischen Bezügen wird es verbunden? Welche diskursiven Formationen werden im Rahmen dieser Debatten gestärkt? Und zeigen sich offensichtliche Auslassungen, also naheliegende Problematisierungen oder Verknüpfungen von Nationalkultur, die nicht thematisiert werden? In einem dritten Schritt gehe ich davon aus, dass auch hier implizite Wissensbestände der policy community sichtbar werden. Von den Formen des Raumgreifens Auswärtiger Kulturpolitik und den hierdurch gestärkten Wissensordnungen schließe ich auf die Formierung eines Habitus kultureller Repräsentation. Hierunter verstehe ich ein kollektiv entwickeltes Wissens- und Erfahrungssystem, das sowohl öffentliches Auftreten, Äußerungen und Haltungen innerhalb der policy community maßgeblich strukturiert als auch der Distinktion gegenüber anderen Akteursgruppen dient.

\section{Nationalkultur als räumliche Erfahrung: Symbolisches Kapital und materielle Botschaften der Museumsinsel in Berlin}

Für dieses Symposium wurde mit der Museumsinsel also wiederum ein Ort ausgewählt, dessen topografische Beschaffenheit, Vergangenheit und Reputation eine plausible materielle Basis für das temporäre Raumgreifen Auswärtiger Kulturpolitik darstellen: Die Basilika und der Große Kuppelsaal des Bode-Museums, die Rotunde des Alten Museums oder der Theodor-Wiegand-Saal im Pergamonmuseum bilden den Rahmen für die Reden, Foren und Diskussions-

116 | Pierre Bourdieu: Die Praxis der reflexiven Anthropologie, S. 271. 
runden, zu denen das Symposium an drei Tagen einlädt. Im Begleitprogramm werden Führungen durch unterschiedliche Sammlungen der Staatlichen Museen organisiert. Die Museumsinsel bietet nicht nur einen besonderen Rahmen für die Diskussionen zum Konferenzthema Nationalkultur, sondern gleichzeitig die Möglichkeit zu einer einprägsamen räumlichen und kulturellen Erfahrung für die Teilnehmer, die auf ihrem Weg zum nächsten Panel an Statuen, Gemälden und archäologischen Objekten vorbeilaufen. ${ }^{117}$

Mit diesem Ort lassen sich zumindest drei historisch begründete und zugleich materiell greifbare Botschaften verbinden, die wesentlich zu seinem symbolischen Kapital beitragen und sich zugleich auf einer inhaltlichen Ebene für dieses Symposium als anschlussfähig erweisen:

Zunächst zieht die Museumsinsel seit der Vereinigung der beiden deutschen Staaten als ein zentrales, langwieriges und ambitioniertes kulturpolitisches Vorhaben die Aufmerksamkeit auf sich. Im Kern geht es dabei um die Renovierung und bauliche Umgestaltung sowie die Restrukturierung der Sammlungen von insgesamt fünf Museumsbauten. Die Gebäude und Exponate wurden nach Mauerfall in die Obhut der Staatlichen Museen zu Berlin übergeben und somit mit deren in West-Berlin untergebrachten Sammlungen institutionell vereinigt. Die Entwicklung und Diskussion eines städtebaulichen Masterplans ${ }^{118}$ für die gesamte Museumsinsel sowie der Beginn oder Abschluss einzelner Bauabschnitte erfuhren jeweils eine prominente mediale Begleitung. Dieses Interesse bleibt dabei nicht auf die urbane Öffentlichkeit begrenzt: Die Wiedereröffnungen der Alten Nationalgalerie, des Bode-Museums oder des Neuen Museums wurden jeweils als nationale Ereignisse markiert. Hochrangige Vertreter des politischen Feldes hielten auf den Festakten die zentralen Reden, ${ }^{119}$ die großen deutsch(sprachig)en Zeitungen und Fernsehstationen berichteten von den Feierlichkeiten und stellten die renovierten Museen und ihre Sammlungen in Bildgalerien vor. Ein Foto der Bundeskanzlerin neben der im Neuen Museum ausgestellten Büste der Nofretete wurde auf den Titelseiten einer Vielzahl großer Tageszeitungen abgedruckt. ${ }^{120}$

117 | Feldnotizen, 26. April 2008.

118 | Siehe hierzu: www.museumsinsel-berlin.de (letzter Zugriff am 11. August 2017).

119 | Zur Eröffnung der Alten Nationalgalerie am 2. Dezember 2002 sprachen Kulturstaatssekretär Julian Nida-Rümelin und der Regierende Bürgermeister von Berlin Klaus Wowereit; an der Eröffnung des Bode-Museums am 17. Oktober 2006 nahmen der Bundestagspräsident Norbert Lammert und Kulturstaatsminister Bernd Neumann teil; die zentrale Rede zur Eröffnung des Neuen Museums am 16. Oktober 2009 hielt die Bundeskanzlerin Angela Merkel.

120 | So in der FAZ, dem Handelsblatt, der Neuen Zürcher Zeitung, dem Tagesspiegel und der BZ, zumeist in ihren Ausgaben vom 17. Oktober 2009. In ihrer Rede »bei der Eröffnung des Neuen Museums" bezeichnete die Bundeskanzlerin die Museumsinsel als "ei- 
Die zweite Botschaft ergibt sich aus den kulturpolitischen Projekten unterschiedlicher Epochen deutscher Geschichte, die sich hier im urbanen Raum materialisiert haben: Die Ursprünge des Konzepts und der baulichen Gestaltung des Areals liegen im frühen I9. Jahrhundert und verweisen auf den Plan, einen ausgewiesenen Ort für Kunst und Wissenschaft in der Nähe des politischen Zentrums Preußens zu schaffen. Den Anfang machten das Alte Museum und das Neue Museum, die in den Jahren I830 und I855 eröffnet wurden. Während des Deutschen Kaiserreichs folgten die Alte Nationalgalerie (I876) und das Bode-Museum (I904). Aus dieser Zeit stammt auch der Name Museumsinsel für den Gesamtkomplex, durch den der Anspruch formuliert wurde, mit ähnlichen Museen westeuropäischer Staaten - insbesondere dem Louvre und dem Britischen Museum - an Relevanz gleichzuziehen und somit die gesteigerte Bedeutung des geeinten Deutschen Reichs kulturpolitisch zu untermauern. ${ }^{121}$ Dieses neue nationalkulturelle Selbstbewusstsein wurde durch die Inschrift »Der Deutschen Kunst« auf der Fassade der Nationalgalerie öffentlich sichtbar demonstriert - ein Motto, das sich zugleich als Referenz an das in Deutschland wirkmächtige Konzept der »Kulturnation« als Basis politischer Einigungsbestrebungen lesen lässt. ${ }^{122}$

Neben dieser Konkurrenzkonstellation europäischer Staaten und Imperien ist der Museumsinsel aber auch der geopolitische Kontext ihrer Entstehung als eine dritte Bedeutungsebene eingeschrieben: Im großen Umfang werden in den Museen archäologische und künstlerische Objekte ausgestellt, die im Zuge europäischer Hegemonie und kolonialer Dominanz im Nahen und Mittleren Osten oder in Nordafrika ausgegraben oder erworben wurden. Die archäologischen Sammlungen stellen bis heute - bisher meist ohne diesen Aspekt ihrer

nen der schönsten und interessantesten Orte in Berlin«. Die Wiedereröffnung des Neuen Museums beschreibt für sie "ein Stück des Weges, den wir in diesem Deutschland seit 1990 nunmehr gemeinsam gegangen sind «. Im Zusammenhang dieser Arbeit interessiert aber insbesondere die Verknüpfung des Ortes mit dem Bild der "Kulturnation “, deren "Einheit [...] immer als das entscheidende Bindeglied für die künftige Einheit Deutschlands dargestellt worden “ sei. Die Förderung der Umsetzung des Masterplans für die Museumsinsel bezeichnete sie als "eine gute Investition in unsere Kulturnation, in unsere Bundesrepublik Deutschland “. In: Bulletin der Bundesregierung Nr. 104-2 vom 16. Oktober 2009; online abrufbar unter: https://www.bundesregierung.de/Content/DE/Bulletin/ 2009/10/104-2-bk-neues-museum.html (letzter Zugriff am 11. August 2017).

121 | Das Pergamonmuseum wurde als letztes der fünf Museumsbauten erst während der Weimarer Republik im Jahre 1930 eröffnet.

122 | Siehe zur Etablierung und Wirkungsgeschichte des Konzepts der "Kulturnation «: Irene Götz: Deutsche Identitäten, insbesondere S. $119 \mathrm{ff}$. 
Entstehungsgeschichte zu problematisieren ${ }^{123}$ - einen zentralen Baustein in der Außendarstellung der Museumsinsel dar: So sieht der Masterplan die Gestaltung einer archäologischen Promenade vor, welche die verschiedenen Museen räumlich und inhaltlich miteinander verknüpfen soll.

Eine wesentliche Bedeutungsebene dieses Ortes besteht somit in der Positionierung eines nationalstaatlichen kulturpolitischen Projekts und nationalkultureller Wissensordnungen in einen universalen Kontext: Ein einstmals patriotisches Konzept von »Deutscher Kunst« sowie der Anspruch des Deutschen Reichs auf »Weltgeltung « wurden hier in eine enge räumliche und inhaltliche Beziehung zu Vorstellungen und Objekten eines »universalen kulturellen Erbe« gesetzt. Diese Verbindung zwischen National- und Universalkultur wurde in aktualisierter Form durch die Aufnahme der Museumsinsel auf die UNESCO-Liste des Weltkulturerbes im Jahre I999 öffentlichkeitswirksam aktiviert und endgültig in ein symbolisches Kapital des Ortes transferiert. ${ }^{124}$ Somit lässt sich formulieren, dass mit der Museumsinsel ein Ort für das Symposium gewählt wurde, an dem eine konzeptionelle Grundspannung, die auch der Auswärtigen Kulturpolitik weiterhin eingeschrieben ist, im urbanen Raum der deutschen Hauptstadt eine materielle Form gefunden hat nämlich die Ausgestaltung der Beziehungen zwischen der Vorstellung eines begrenzbaren, distinkten nationalkulturellen Raums einerseits sowie seines globalen Kontexts und Bezugsrahmens andererseits.

\section{Nationalkultur als diskursive Formation: Strategien der Rezentrierung und inhaltliche Dimensionen eines Schlüsselbegriffs}

Sowohl in den Redebeiträgen als auch in den Druckprodukten des Symposiums wird mehrfach auf solche Bedeutungsebenen Bezug genommen: So ist etwa im Vorwort zum Konferenzband zu lesen, dass diese Tagung an einem Ort stattgefunden habe, »der das >Patrimonium der Deutschen< versammelt und zugleich $>$ Weltkulturerbe $<$ ist $\ll .{ }^{125}$ Der Außenminister betont gleichfalls in seiner Eröffnungsrede: »Es gibt keinen besseren Ort als die Museumsinsel, um sich für einen Moment die kulturellen Leistungen unseres Landes zu verge-

123 | So stellte es sich zumindest zum Zeitpunkt meiner teilnehmenden Beobachtung auf dieser Konferenz im Jahr 2008 dar.

124 Siehe hierzu: http://www.unesco.de/kultur/welterbe/welterbe-deutschland/ museumsinsel-berlin.html (letzter Zugriff am 11. August 2017).

125 | Christoph Bartmann \& Hans-Georg Knopp: Vorwort. In: Christoph Bartmann, Carola Dürr \& Hans-Georg Knopp (Hrsg.): Wiedervorlage: Nationalkultur. Variationen über ein neuralgisches Thema. Göttingen 2010, S. 7-10, S. 7. 
genwärtigen. ${ }^{126}$ Das symbolische Kapital der Museumsinsel wird also von zentralen Akteuren bewusst zur öffentlichen Positionierung des Tagungsthemas genutzt und unterstützt somit einen Prozess der Rezentrierung elementarer Ordnungsmuster des Politikbereichs, die als räumlich erfahrbare Konzepte - um es noch einmal mit Bourdieu auszudrücken - eine »offizielle und damit autorisierte Existenz« gewinnen.

Vor diesem Hintergrund möchte ich mich nun den inhaltlichen Dimensionen des somit autorisierten Konzepts zuwenden. Bereits eine Beschäftigung mit dem Aufbau des Tagungsprogramms führt zu den inhaltlichen Schwerpunkten und Strukturierungsprinzipien: So sticht zunächst die Verknüpfung von politischen Positionen, publizistischen Debatten, wissenschaftlichen Diskussionen, populärkulturellen Phänomenen und kultureller Vermittlungspraxis hervor. Das Symposium eröffnet somit ein Übersetzungsfeld, das gerade durch diese Verbindungen zwischen unterschiedlichen Diskussions- und Handlungszusammenhängen seine Spezifik erhält. Hierdurch entsteht eine temporäre »Arena kultureller Produktion « ${ }^{127}$, in der eine kulturpolitische Elite einen Schlüsselbegriff ihrer Arbeit in seinen unterschiedlichen Bedeutungsebenen und Verbindungen auslotet und neu positioniert.

Konkret folgt auf den Eröffnungsabend mit einer Rede des Bundesaußenministers und einem Vortrag der Journalistin Mely Kiyak zu dem Thema Deutsche Nationalkultur mit Migrationshintergrund? das eigentliche Tagungsprogramm mit einer Aneinanderreihung von Vorträgen, Diskussionsrunden und Panels:

„Plenarvortrag Saskia Sassen Das Paradox des Nationalen

Podiumsdiskussion Wie viel Nationalkultur braucht der Mensch?

Forum $1 \quad$ Zurück in die Zukunft? Nation und Kultur im östlichen Europa

Forum $2 \quad$ Von der Nationalkultur zur Kulturnation? Tendenzen im westlichen Europa

Forum $3 \quad$ Nationalkultur und regionale Integration in Asien

Forum $4 \quad$ Postnationale Geschichtsbilder? Zum Wandel unserer Geschichtswahrnehmung

Forum $5 \quad$ Vom 'Deutschen Klang: Wie national ist die Musik?

Forum 6 Bühne der Nation? Zum Selbstverständnis des Gegenwartstheaters

126 | Frank-Walter Steinmeier: "Nie geraten die Deutschen so außer sich, wie wenn sie zu sich kommen wollen " - Was heißt Nationalkultur im 21. Jahrhundert? In: Christoph Bartmann et al. (Hrsg.): Wiedervorlage: Nationalkultur, S. 17-25, S. 17.

127 | Anna Lowenhaupt Tsing: Friction, S. 51. 
Forum 7

Fernsehen: Das Wohnzimmer der Nation? Zum Verhältnis von Medien und kultureller Identität

Forum 8 Nationale Sammlungen und universale Kulturgüter: Wohin bewegt sich das Nationalmuseum “128

Nationalkultur besitzt hier gewissermaßen eine Omnipräsenz: Museen, Fernsehen, Theater, Musik, Geschichtsbilder, Europa oder menschliches Bedürfnis - überall stellt sie sich aus Sicht der Veranstalter als eine relevante und erklärungsmächtige Kategorie dar. Wenn man das Symposium als ein Projekt zur Rezentrierung von Nationalkultur als einem kulturpolitischen Schlüsselbegriff versteht, so wird hier eine erste Strategie sichtbar: Das Nationale wird durch diese Vielzahl an inhaltlichen und sektoralen Verknüpfungsmöglichkeiten als eine Art »Zentralperspektive« (re-)etabliert, um die unterschiedlichsten kulturellen Felder, Fragestellungen und Dynamiken zu diskutieren. ${ }^{129}$

Eine zweite Strategie wird deutlicher, wenn man zentrale Redebeiträge auf der Tagung sowie die einleitenden Texte ihrer Initiatoren in die Analyse mit einbezieht. Hier zeigt sich eine durchgängige Wechselbewegung zwischen Rezentrierung und Reflexivierung von Nationalkultur als das eigentliche Kernelement der diskursiven Formation, die im Rahmen der Konferenz entsteht: Einerseits wird die Vorstellung einer grundlegenden Bindung zwischen dem »Nationalen«, seinem »Territorium« und »Kultur« hervorgerufen und reproduziert; andererseits werden die genaue Gestalt und die aktuelle Relevanz dieser Beziehung aus unterschiedlichen Perspektiven diskutiert, problematisiert und kommentiert. Dieser reflexive Modus, also die routinierte Reflexivierung des Begriffs Nationalkultur auf dieser Konferenz und innerhalb der policy community, führt letztlich - so die These - zu seiner Rezentrierung. Diesen Zusammenhang möchte ich anhand von einigen Beispielen erläutern:

In ihren einleitenden Beiträgen knüpfen die Initiatoren an verbreitete Beobachtungen und wissenschaftliche Debatten an, die einen Bedeutungsverlust nationalstaatlicher Bindungen festhalten. So ist die Rede von Prozessen der Entnationalisierung, von der selbstverständlichen Mobilität im internationalen Kulturbetrieb, von grenzüberschreitenden Bildungsbiografien im Zeitalter von Erasmus oder von der grundsätzlich zunehmenden Durchlässigkeit nationaler Grenzen innerhalb der erweiterten Europäischen Union: »Dass die Nation - in

128 | Goethe-Institut \& Staatliche Museen zu Berlin: Wiedervorlage: Nationalkultur, Programmablauf, S. 3.

129 | Irene Götz nutzt den Begriff "Zentralperspektive", um die Etablierung der Nation als grundlegendes politisches, wissenschaftliches, gesellschaftliches Ordnungsprinzip im 19. Jahrhundert zu benennen. Siehe: Irene Götz: Deutsche Identitäten, S. 106. "Strategie" im Anschluss an Wolfgang Kaschuba, Pierre Bourdieu sowie auf Basis meiner eigenen Überlegungen; siehe hierzu die Kapitel I.3 und III.2. 
Deutschland hat sich auch der Begriff >Kulturnation< etabliert - eine weiterhin gültige Zurechnungsgröße für kulturelle Phänomene und Produkte darstellen könne, gilt vielen als überholte Vorstellung.«130

Diese Entwicklungen bilden aus Perspektive der Veranstalter allerdings nur die eine Tendenz, die Gegenbewegungen hervorruft: Kontroversen über den Neubau von Moscheen in Mitteleuropa, staatlich initiierte Reflexionen über die »nationale Identität« im 2I. Jahrhundert, ein Revival nationaler Geschichtsprogramme im Fernsehen oder die Popularität der Fußballnationalmannschaft werden als Beispiele angeführt, die auf die bleibende oder wiederkehrende Bedeutung des nationalen Narrativs sowie auf die Normalität territorialer Bindungen von Menschen und Kultur verweisen:

"Auch wenn man das alte Narrativ der Nationalkultur für erschöpft hält, sollte man freilich bedenken, dass die Mehrheit der Bevölkerung und Kulturschaffenden weiterhin nicht in transnationalen und global vernetzten Kulturwelten zu Hause ist. Zwar gibt es ein modernes Nomadentum, das Kuratoren und Künstler mit anderen Arbeitsmigranten teilen, aber diese instabilitas loci bleibt selbst unter heutigen Bedingungen die Ausnahme. Der überwiegende Teil der Bevölkerung erweist sich als ausgesprochen ortsfest. Die Beharrungskräfte in der Gesellschaft entwickeln sich proportional zum Anstieg der Mobilität. "131

Es sind gerade diese Gegenbewegungen, diese Beharrungskräfte und nachhaltigen Ortsbindungen, aus denen sich - in der Einschätzung der Veranstalterinnen - die Relevanz und Legitimität eines Symposiums zu den nationalen Bindungen von Kultur in der Gegenwart ergibt:

"Diese gegenläufigen Bewegungen transnationaler Mobilität und nationaler Selbstvergewisserung machen eines deutlich: So sehr sich viele vor allem in Deutschland wünschen, das Kapitel der nationalen Verankerung von Kultur zu schließen und die Akte ins Archiv zu tragen - es verlangt heute nach abermaliger Diskussion und ist unter den aktuellen Gegebenheiten neu zu beleuchten, wie empfindlich der damit stimulierte Nerv auch sei. ${ }^{132}$

130 | Christoph Bartmann \& Hans-Georg Knopp: Vorwort, S. 7.

131 | Ebd., S. 8.

132 | Carola Dürr: Nationalkultur - schon wieder oder immer noch? Eine Einführung. In: Christoph Bartmann et al. (Hrsg.): Wiedervorlage: Nationalkultur, S. 11-16, S. 11. 


\section{Elemente eines Habitus kultureller Repräsentation}

Dieses letzte Zitat führt zugleich zu einer Position, die mir für die Erarbeitung der impliziten Wissensbestände der policy community und somit auch eines Habitus kultureller Repräsentation zentral erscheint: Sichtbar wird hier die Argumentationsfigur, dass es in Deutschland eine besondere Zurückhaltung in der Thematisierung und Wahrnehmung nationaler Bindungen von Kultur und Identität gäbe. Solche Vorstellungen von einem spezifisch deutschen Unwillen, sich gewissen Diskussionen zu stellen oder sogar von der Tabuisierung bestimmter Herangehensweisen an das Nationale, bilden Grundmotive sowohl der Tagung als auch anderer Foren deutscher kulturpolitischer Eliten. Zugleich zeigt sich, dass genau diese Feststellung einer angeblichen Zurückhaltung eine erneuerte und explizite Beschäftigung mit dem Nationalen und der Nationalkultur in Deutschland ermöglicht. Entsprechend werden diese Argumentationsfiguren auch für die Diskussion einer zeitgemäßen Auswärtigen Kulturpolitik mobilisiert:

"Als Leitbegriff der deutschen auswärtigen Kulturpolitik weithin außer Gebrauch, ist sie doch weiterhin latent gegenwärtig, [...] Worum sollen sich nationale Kulturinstitute eigentlich kümmern, wenn nicht um die jeweils nationale Kultur - die sie andernorts in neue und produktive Diskussions- und Produktionszusammenhänge einbringen? [...] Nationale Kulturinstitute bekommen inr Geld vom Parlament und letztlich vom Steuerzahler, damit sie im Ausland etwas von der grandeur der eigenen Kultur vermitteln. Ein wenig anders stellt sich die Situation in Deutschland dar: Das Nationale in und an der , deutschen Kultur - und vor allem der Gegenwartskultur - bleibt ein neuralgisches Thema. ${ }^{133}$

Der ambivalente Charakter, den die Kategorie Nationalkultur ${ }^{134}$ aus Perspektive der Initiatorinnen besitzt, der reflexive Modus, über den sich kulturpolitische Eliten ihr nähern sowie die Überzeugung von einer spezifischen deutschen Konstellation werden auch in diesem Zitat deutlich. Für die Erarbeitung impliziter Wissensbestände der policy community und ihres Habitus kultureller Repräsentation erscheinen mir zwei weitere Argumentationslinien zentral:

Einerseits behaupten die Initiatoren ein Fortwirken nationalstaatlicher Konkurrenzen und betonen hierdurch die Rolle von Nationalkultur als einem Schlüsselkonzept:

133 | Christoph Bartmann \& Hans-Georg Knopp: Vorwort, S. 7.

134 | "Es gibt also die Nationalkultur, und es gibt sie nicht.", heißt es an anderer Stelle: Ebd., S. 9. 
"Ist die Auswärtige Kulturpolitik eine Kulturpolitik nach der Nationalkultur? [...] Das Zeitalter der nationalen Konkurrenzen ist nicht vorbei, es erfindet sich unter aktuellen Auspizien jeweils neu, und die nationalen Kulturen definieren oder branden sich als Kultur-, Wirtschafts- und Studienstandorte unablässig neu. Die Rede vom internationalen Kulturaustausch hilft lediglich, das ungebrochene Konkurrenzgebaren der Nationen zu camouflieren. ${ }^{135}$

Andererseits zeigen sich Wunsch und Anspruch, Nationalkultur mit neuen Bedeutungsgehalten zu verknüpfen. Gerade die Thematisierung von »Einwanderung « und ihren Folgen führt auf dieser Tagung weniger zu der Suche nach neuen Grundbegriffen, um eine sich verändernde Wirklichkeit adäquat beschreiben zu können, sondern zu dem Bemühen, eine sich rasant modifizierende Gegenwart in die etablierten Ordnungsmuster einzupassen. Den Anknüpfungspunkt bilden hierzu die integrativen und emanzipatorischen Potenziale, die das Konzept Nationalkultur ursprünglich einmal besessen habe:

"Warum ist Nationalkultur heute in Deutschland eine Provokation? Weil die früher einmal inklusive Dimension des Begriffs gänzlich von der exklusiven verdrängt worden ist. Auf Nationalkultur zu pochen, heißt offenbar: Bürger im eigenen Land und den Rest der Welt von ihr auszuschließen. Wer so redet, verkennt den Umstand, dass Nationalkultur in ihrer progressiven Idee ein Angebot zur Teilhabe war. Jeder Bürger, auch die Vertreter von Minderheiten, sollte einen Beitrag zu diesem Projekt leisten können, und den anderen Nationen konnte unsere Nationalkultur dienen als eine Erzählung von unserer Art zu sein. Wer heute die ,Erste Person Pluralı, die kollektiven Erzählungen und das 'Wir-Gefühlı beschwört, könnte an solche Erzählungen anschließen. “136

Dieser Versuch, neue Erzählungen zu konstruieren, führt also zunächst zu dem Festhalten an einer Reihe von Vorstellungen, die in der Phase der Nationenbildung im I9. Jahrhundert entwickelt und etabliert worden waren: etwa von der Integration eines Kollektivs über die gemeinsame Kultur; von der Existenz eines »kulturellen Wir«; von dem Übereinanderfallen von Kultur, Nation und Territorium als weiterhin anzustrebenden Normal- und Idealfall oder auch von der Markierung von Grenzen durch Kultur. Dieser Versuch führt aber auch zu Verweisen auf die historischen Begrenzungen des Konzepts; zu einer expliziten Orientierung an prozessualen und dynamischen Vorstellungen von »Kultur« sowie zu einem Plädoyer für eine bewusste Öffnung der $\mathrm{Na}$ tionalkultur für Differenz:

135 | Ebd., S. 9.

136 | Ebd., S. 9. 
"Warum nicht eine inklusive Nationalkultur befördern, die den Vielen und Verschiedenen eine Chance zur Partizipation einräumt, die sich der Tatsache bewusst ist, dass Nationalkultur kein Museum, sondern vielmehr dessen ewige Baustelle ist, und die entsprechend handelt? Wäre nicht das progressive Moment einer solchen Nationalkultur, dass sie Grenzen anerkennt, in denen sich leben lässt, wohl wissend, dass die europäische kulturelle Identität ein Projekt für Generationen ist und noch auf sich warten lässt. Was wäre falsch an einer integrativen `Nationalkultur für alle`? «137

Aus der Organisation und inhaltlichen Ausgestaltung dieses Symposiums lässt sich somit auch der Anspruch kulturpolitischer Eliten ablesen, Diskurse mitzugestalten und hierbei Grundbegriffe zu setzen oder zu verschieben. In diesem Fall führt dies zu einer Rezentrierung von Nationalkultur als dem Ordnungsmodell, über die »Kultur« oder »internationale Kulturbeziehungen« gedacht und diskutiert werden können: Das Bild von abgrenzbaren nationalstaatlichen Einheiten, die durch Auswärtige Kulturpolitiken in Dialog treten, erfährt ebenso eine Bestätigung wie die Vorstellung von deren innerer Integration über eine gemeinsame Kultur.

\section{Auslassungen: Politische Aushandlungen und die "pathologischen Elemente" des Nationalen}

Hieraus ergibt sich eine erste Auslassung in der diskursiven Formation, die dieses Symposium strukturiert - eine Auslassung, die bereits Erwähnung gefunden hat: Es zeigen sich kaum Ambitionen, nach Begriffen und Ordnungsmustern zu suchen, die vielleicht besser geeignet wären, kulturelle Dynamiken im Kontext von vielfältigen Mobilitäten, von Globalisierungs- und Europäisierungsprozessen einzufangen als diese Betonungen einer territorialen Verankerung von »Kultur« und der angenommenen Normalität nationalkultureller Identitäten. Anstatt also die enge Bindung von Kultur, Territorium und Nation grundlegend in Frage zu stellen und Deutschland etwa als einen kosmopolitischen Kreuzungspunkt unterschiedlicher Migrationsbewegungen und transnationaler Mobilitäten ${ }^{138}$ auch konzeptionell neu zu denken, führt das Symposium zu einer Bestätigung etablierter Ordnungsmodelle, die eine nationale Bindung von Kultur als Ideal- und Normalfall betrachten. Hierbei ist entscheidend, dass die damit verbundenen Vorstellungen von »unserer Art $\mathrm{zu}$ sein« und einer verbindenden »eigenen Kultur« im Kontext von globalen

137 | Ebd., S. 10.

138 | Hier im Anschluss an: Regina Römhild: Transnationale Migration und soziokulturelle Transformation: Die Kosmopolitisierung der Gesellschaft; siehe: http://heimatkunde.boell.de/2011/05/18/transnationale-migration-und-soziokulturelle-transformation-die-kosmopolitisierung-der (letzter Zugriff am 24. November 2017). 
Verflechtungen, Mobilitäten, politischen und medialen Entgrenzungen, aber auch von innergesellschaftlichen Individualisierungsprozessen zunehmend ihre Fraglosigkeit verlieren. An ihre Stelle treten Verhandlungen, in denen im Kern um Zugehörigkeiten, die Sichtbarkeit unterschiedlicher Gruppen und Partizipationsrechte gerungen wird - anders formuliert: die Fragen nach »Zusammengehörigkeit«, gesellschaftlicher »Integration«, aber auch nach »kultureller Außendarstellung « sind zutiefst politische, wie sich anhand eines Zitates verdeutlichen lässt:

"Whose culture shall be the official one and whose shall be subordinated? What culture shall be regarded as worthy of display and which shall be hidden? Whose history shall be remembered and whose forgotten? What images of social life shall be projected and which shall be marginalized? What voices shall be heard and which silenced? Who is representing whom and on what basis? „139

Bemerkenswert ist, dass dieses Symposium solche politischen Aushandlungen um Partizipation und Sichtbarkeit kaum als Grundlage spätmoderner Gesellschaften einbezieht und sich stattdessen darauf konzentriert, Zugehörigkeit zu rekulturalisieren. In den Hintergrund gerät hierbei, dass sich Vorstellungen von einer verbindenden Nationalkultur oder einer geteilten nationalen Identität - in Anbetracht der bereits im Abschnitt I.3 diskutierten Dynamiken - nur durch politische, kulturelle und mediale Verfahren oder »Technologien« zur Rezentrierung aufrecht erhalten lassen. Das Beispiel dieses Symposiums verdeutlicht, wie aktiv Angehörige der policy community an solchen Konstruktionsprozessen beteiligt sind. Sie zeigen sich hier gewissermaßen als »Technologen des Nationalen«, die an der Anpassung und Bestätigung etablierter Begriffe mitwirken und zumindest in diesem Rahmen die Suche nach neuen Beschreibungsmustern eher vernachlässigen.

Eine zweite Auslassung bezieht sich stärker auf systemische Faktoren, aufgrund derer die Kategorien des Nationalen überhaupt erst zum »Problem« geworden sind. Arjun Appadurai hat darauf hingewiesen, dass »hinter dem modernen Nationalstaat eine fundamentale und gefährliche Idee steckt: die des >nationalen Ethnos «. Die ausschließenden und ausgrenzenden Effekte der nationalen Idee sind demnach weniger Ausdruck eines seltenen Extremfalls, sondern ihr gewissermaßen systemisch mitgegeben:

"Keine moderne Nation, so freundlich bzw. gutmütig ihre Regierung auch sein mag und so beredt ihre Repräsentanten in der Öffentlichkeit die Werte von Toleranz, Multikultu-

139 | Glen Jordan \& Chris Weedon: Cultural Politics: Class, Gender, Race and the Postmodern World. Oxford 1995, S. 4. 
ralismus und Inklusion auch beschwören mögen, kommt ohne die Vorstellung aus, ihre nationale Souveränität gründe sich auf eine Art ethnischen Geist. “140

Im Anschluss an maßgebliche Theoretiker des Nationalstaates betont Appadurai, dass die »Idee eines einzigartigen nationalen Ethnos« keineswegs »natürlich« entstanden sei, sondern durch die »Rhetorik des Krieges und des Opfers« sowie durch repressive kultur- und sprachpolitische Maßnahmen »konstruiert und naturalisiert wurde «. ${ }^{141}$ Die Globalisierung - so seine ernüchternde These - fördert die »pathologischen Elemente im Kern der heiligen Ideologie der Nation und der nationalen Identität « ${ }^{142}$ besonders deutlich zutage, da sich im Kontext von mobilen Bildern, Kapitalien und Menschen die Illusionen einer Kongruenz von Territorium, Nation und Bevölkerung, von einem homogenen Kollektiv oder einer »reinen Nationalkultur« letztlich nur durch Gewalt und Politiken der Exklusion pflegen lassen.

Sicher macht es einen Unterschied, ob man Nationalkultur »inklusiv« oder »exklusiv« versteht und welche Bestände des tradierten nationalen Diskurses man in der Gegenwart aufgreifen möchte - aber in jedem Fall handelt es sich um ein Konzept, das irgendwo einen Anfang hat, anhand bestimmter Kriterien begrenzt werden muss und ein distinktes »kulturelles Wir« hervorruft. Vor dem Hintergrund rassifizierender Debatten um Zugehörigkeit und unterschiedlicher Ausformungen von Mehrheitschauvinismen in vielen europäischen Staaten, der weitgehenden Hinnahme der Marginalisierung oder Exklusion großer Bevölkerungsteile und der zunehmenden Kategorisierung von Migranten entlang ihrer »Brauchbarkeit« scheint eine gewisse Skepsis gegenüber der Intention der Organisatorinnen, durch das Symposium ein »inklusives« Verständnis zu stärken, angemessen - oder um es expliziter zu formulieren: die Gewalt- und Ausgrenzungsgeschichte ist dem Konzept der Nationalkultur so elementar eingeschrieben, dass sie, aus meiner Perspektive, den zentralen Ausgangspunkt der Reflexionen über zeitgemäße Beschreibungsmuster für Zugehörigkeiten und Ordnungsmodelle für internationalen Kulturaustausch darstellen müsste.

140 | Arjun Appadurai: Die Geographie des Zorns, S. $15 f$.

141 | Ebd., S. 16.

142 | Ebd., S. 13. 


\section{Zeithistorische Kontexte: Nation Rebuilding in der Berliner Republik}

Abschließend möchte ich das Symposium vor dem Hintergrund der Beobachtungen von Irene Götz zu einer Wiederentdeckung des Nationalen nach $1989^{143}$ in einen breiteren zeithistorischen Rahmen einordnen. Götz entwickelt den Begriff des »Nation Rebuilding«, um eine neue Konjunktur des Nationalen nach der deutschen Vereinigung, sein vermehrtes Auftauchen in den Feuilletons, in Ausstellungen, erinnerungspolitischen Debatten, Fernsehsendungen oder Symposien zu interpretieren. Im Kern erkennt sie ein »Wechselspiel von Deund Renationalisierungsprozessen, das die Konjunktur nationaler Diskurse und entsprechender Praktiken seit den I990er Jahren bestimmt $\ll^{114}$ und letztlich auf grundlegende Transformationsprozesse des deutschen Nationalstaates verweist. Auf der einen Seite stehen demnach also umfassende Prozesse der Denationalisierung vormals staatlicher Aufgaben und Souveränitäten, politischer Zuständigkeiten und biografischer Bezugssysteme. Auf der anderen Seite beobachtet sie »die mit diesen post- und transnationalen Strukturveränderungen auch verbundenen Gegen- oder Rückwärtsbewegungen: die Tendenzen einer Renationalisierung « ${ }^{145}$ und somit den verstärkten »Rückbezug auf tradierte nationale Leitbilder und Semantiken gerade im politischen Alltag «. ${ }^{146}$

Diese Vorstellung eines Wechselspiels - welche die Initiatoren des Symposiums in ähnlicher Weise bedienen - ergänzt Irene Götz durch eine dritte Dynamik, die zur Analyse dieser Tagung und auch zur Identifizierung eines Habitus kultureller Repräsentation besonders interessant erscheint: Die zunehmende Aktivierung des Nationalen als einer »reflexiven Größe«, die sich etwa in der Gründung oder Etablierung neuer staatlicher Institutionen, politischer Rituale und Mythen, neuer Geschichtsbilder und Symbole zeigt und auf das Ziel verweist,

"dem nach 1990 gewissermaßen neu gegründeten Nationalstaat veränderte Konturen und gewandelte identitätspolitische Semantiken zu verleihen sowie inn transnational zu positionieren, wozu die verschiedenen Akteursgruppen von Politik, Medien, Kunst und Wirtschaft alte und neue Bilder der Nation, je nach Kontext in unterschiedlicher Gewichtung und Zusammenstellung zusammenfügen. Diese hybriden Formen des Nation Branding sind dabei eng mit dem Prozess eines Building Europe verknüpft. “147

143 | So der Untertitel ihrer bereits mehrfach zitierten Studie: Irene Götz: Deutsche Identitäten.

144 | Ebd., S. 89. Auch der Begriff "Nation Rebuilding" findet sich auf dieser Seite.

$145 \mid$ Ebd., S. 89.

146 | Ebd., S. 90.

147 | Ebd., S. 92. 
Der diskursive Raum, der im Rahmen des Symposiums Wiedervorlage: Nationalkultur entsteht, wird maßgeblich durch diese drei Entwicklungen durchzogen und bestimmt: Der Verweis auf Gegenreaktionen zu aktuell wirkmächtigen Denationalisierungsprozessen stellt eine wesentliche Argumentationsfigur dar, über welche die Initiatorinnen den inhaltlichen Fokus ihrer Tagung begründen. Hinzu treten in den Diskussionen und Redebeiträgen routinierte Bezugnahmen auf Leitbilder und Semantiken des Nationalen, die zumeist im I9. Jahrhundert entwickelt worden sind: etwa auf unsere gemeinsame Muttersprache Deutsch als selbstverständlichen Kernbestand der verbindenden Nationalkultur, auf das Idealbild von der Kulturnation oder auf die Vorstellung von einer besonderen geschichtlichen Erfahrung, welche die Deutschen als Kollektiv identitär und kulturell präge. ${ }^{148}$ Zugleich ließ sich ein reflexives Moment in zumindest zweierlei Hinsicht erkennen: Einerseits als ein Modus, um sich dem Nationalen mit einer demonstrativen Distanz und Diskussionsbereitschaft zu nähern; andererseits als eine Praxisform, die bestimmte Elemente des tradierten Diskurs vom Nationalen entdeckt, betont oder herausstellt und zu einem erneuerten Narrativ von der integrativen Nationalkultur zusammenfügt. Sichtbar wird somit, in welch grundlegendem Umfang das Akteursfeld Auswärtiger Kulturpolitik an den Prozessen einer reflexiven Produktion von identitätspolitischen Semantiken und symbolischen Räumen des Nationalen in der Berliner Republik beteiligt ist.

Reflexiver Modus und reflexive Praxisformen im Umgang mit dem Nationalen lassen sich somit auch als Schlüsselbestand eines Habitus kultureller Repräsentation - wie er sich auf dem Symposium darstellte - festhalten: also des Ensembles von Positionen, Haltungen und Wissensbeständen, mit denen auch eine Kulturmittlerin in Sarajevo oder Ramallah umgehen können muss, um zwischen kulturpolitischen Diskursen und Förderinstanzen einerseits und ihren lokalen Arbeitskontexten andererseits plausible Verbindungen herzustellen. Grundlegend erscheint mir hierbei die routinierte Bewegung zwischen Reflexion und Rezentrierung, zwischen Flexibilisierung und Authentisierung im Umgang mit »Kultur« und ihren nationalstaatlichen Bindungen. In dieser Bewegung lässt sich ein Narrativ entwickeln, das durch Bezugnahmen auf klassische nationale Leitbilder - gemeinsame Sprache und Kultur, geteilte Geschichte und Identität - eine Authentisierung erfährt und sich durch Verweise auf die Weltoffenheit Deutschlands, die innere Vielfalt oder den europäischen Rahmen der deutschen Nationalkultur flexibilisieren lässt. Diese reflexive Verknüpfung lässt sich anhand eines Zitates aus der Rede des Bundesaußenministers verdeutlichen:

148 | Siehe hierzu etwa den Beitrag des Präsidenten des Goethe-Institutes: Klaus-Dieter Lehmann: Selbstvergewisserung und Integration durch praktisches HandeIn. In: Christoph Bartmann et al. (Hrsg.): Wiedervorlage: Nationalkultur, S. 27-33. 
"Weltoffenheit, Weltzugewandtheit, aber auch: Eigenständigkeit, das Ganze gepaart mit vernünftigen und modernen Strukturen und einer Prise Ironie, einer Identität im Zweifelı wie das der Historiker Hans Belting genannt hat, scheinen mir für beides, für äußeres Ansehen und für eine aktuelle kulturelle Blüte, eine Rolle zu spielen. " ${ }^{149}$

Entscheidend ist hier das Festhalten an der Idee von der Eigenständigkeit - anders ausgedrückt: Reflexivität, Zweifel oder Weltoffenheit bestimmen vielleicht die konkreten Inhalte und Erzähltechniken des Narrativs vom Nationalen, das im Rahmen eines solchen Symposiums zusammengesetzt und mobilisiert wird; zugleich erfahren aber elementare Grundmuster eine Naturalisierung als »Geschäftsgrundlage« des Akteursfeldes Auswärtiger Kulturpolitik: etwa von der zu bewahrenden Eigenständigkeit einer geteilten Kultur und Identität oder von den gemeinsamen Erfahrungen eines »kulturellen Wirs«.

Diese Überlegungen bieten zugleich die Möglichkeit, die Veranstaltung eines Symposiums mit dem Titel Wiedervorlage: Nationalkultur mit dem Fokus dieser Studie in Zusammenhang zu bringen: Denn die Definition von Konfliktprävention oder der Förderung von Demokratie und Menschenrechten als Zielsetzungen Auswärtiger Kulturpolitik um die Jahrausendwende lässt sich als eine Bewegung zur Denationalisierung und kosmopolitischen Öffnung des Politikbereichs verstehen. Sicherlich ist es nicht angemessen, eine direkte Abhängigkeit zwischen politischen Papieren - wie der Konzeption 2000 - und einem solchen Symposium zu behaupten. Aus einer diskursanalytischen Sicht liegt es aber doch nahe, die Organisation einer so hochkarätigen Tagung im Zentrum Berlins als eine Art renationalisierende Gegenbewegung zu den entnationalisierenden Tendenzen in der diskursiven Formation einer Auswärtigen Kulturpolitik als Konfliktprävention zu interpretieren.

\section{Dichotomien: Der inNere UND DER ÄUSSERE RAUM DER KULTUR}

In diesem Abschnitt führe ich die bisherigen Analysen zur imaginären Dimension Auswärtiger Kulturpolitik zusammen, indem ich abschließend die epistemologische Ordnung, welche die Diskurse, Sprechpositionen und Wissensbestände in diesem Politikbereich auf einer elementaren Ebene strukturiert, herausarbeite. Die These lautet, dass sich diese epistemologische Ordnung durch eine dichotomische Logik im Umgang mit »Kultur« auszeichnet. Diese Logik führt zu einem bemerkenswerten doppelten Effekt: Einerseits wird

149 | Frank-Walter Steinmeier: "Nie geraten die Deutschen so außer sich, wie wenn sie zu sich kommen wollen " - Was heißt Nationalkultur im 21. Jahrhundert? In: Christoph Bartmann et al. (Hrsg.): Wiedervorlage: Nationalkultur, S. 17-25, S. 20. 
durchgängig das Bild einer Grenze zwischen einem inneren und einem äußeren Raum der Kultur reproduziert und andererseits zugleich kontinuierlich zu ihrer Bearbeitung, Überschreitung und Relativierung aufgefordert. Das Vermögen, mit dieser Logik umzugehen, also routiniert entlang dieser imaginären Grenze zu operieren, zähle ich wiederum zu den impliziten Wissensbeständen, über die ein Akteur verfügen muss, um sich dauerhaft innerhalb der policy community bewegen zu können.

\section{"Unsere Geschichte, Traditionen, Werte und Haltungen «: Die epistemologische Grundfigur Auswärtiger Kulturpolitik}

Die Grundfigur zur Ordnung von Wissen in diesem Politikbereich lässt sich anhand einer Äußerung von Außenminister Steinmeier illustrieren:

"Die neue unübersichtliche, oder in diplomatischer Sprache multipolare Welt, das Wachsen neuer Zentren mit wirtschaftlichem und politischem Einfluss führt auch zu neuem kulturellen Selbstbewusstsein in Ostasien, Afrika und der arabischen Welt. Weniger denn je können wir davon ausgehen, dass europäische Werte und Traditionen selbstverständlich Ziel und Orientierungspunkt der gesellschaftlichen Entwicklung in anderen Regionen der Erde sind. [...] Wir haben keine Garantie, dass wir gehört werden. Aber wir müssen mehr investieren: kulturell, politisch, finanziell, um uns im besten Sinne des Wortes iverständlich، zu machen, uns zu erklären, draußen in der Welt, unsere Geschichte, Traditionen, Werte und Haltungen, um Gefahr von Missverständnissen zu reduzieren. ${ }^{150}$

Hier fällt auf, dass in einer Äußerung, die eine zunehmend »unübersichtliche Welt« zum Ausgangspunkt nimmt und eine Reflexion über die eigenen kulturpolitischen Herangehensweisen einfordert, die Existenz eines »Wirs«, das über eine gemeinsame »Geschichte«, über geteilte »Traditionen, Werte und Haltungen « verfügt und sich »draußen in der Welt«»verständlich machen will«, nicht zur Debatte steht. Entscheidend ist, dass sich die Logik der Grenzziehung innerhalb der Selbstverständigungen der policy community als konstanter erweist als die konkreten Motive, die zur Begründung der Differenz zwischen »Innen« und »Außen« angeführt werden. In der Kombination von Inhalten zur Illustration der Vorstellung von »unserer Kultur« tritt eine gewisse Flexibilität zutage, auch wenn Bezugnahmen auf die etablierten oder auch jüngeren Bestände des Narrativs vom Nationalen zumeist eine zentrale Rolle spielen: etwa auf das Bild von der geteilten deutschen Sprache; auf die Vorstellung einer durch die kollektiven Erfahrungen von Krieg und Schuld, Teilung und Wiedervereinigung historisch gewachsenen Schicksalsgemeinschaft; auf den öffentlich

150 | Menschen bewegen, Konferenzdokumentation 2009, S. 10. 
gemachten Stolz auf die großartigen globalen Erfolge deutscher Kunst; auf die Betonung einer besonderen Ästhetik der zeitgenössischen deutschen Architektur; oder auf die globale Anziehungskraft deutscher Bildungstraditionen. Die Motive, die in der Phase der Nationalstaatsbildung zur Begründung der Vorstellung einer "geteilten Kultur « etabliert worden sind, können flexibel kombiniert und situativ mit neueren Figuren verknüpft werden. So lässt sich etwa die altbekannte Vorstellung einer nationalen Schicksalsgemeinschaft durch Verweise auf die besondere Erfahrung der Deutschen in der Aufarbeitung und Überwindung von Diktaturen aktualisieren und als Ausgangspunkt gegenwärtiger kulturpolitischer Vermittlungsarbeit in Transformationsländern repositionieren. ${ }^{151}$

\section{Authentisierung und Flexibilisierung des inneren Raums: Das Wechselspiel zwischen Betonung und Relativierung von kultureller Differenz}

Im Anschluss an die Befunde der letzten Abschnitte lässt sich somit auch in der inhaltlichen Füllung der epistemologischen Grundfigur des »inneren Raums « ein Wechselspiel zwischen Rezentrierung und Reflexivierung des Nationalen, zwischen Strategien der Authentisierung und Flexibilisierung nachzeichnen: Denn trotz der anpassungsfähigen Kombination von Motiven und Bezugnahmen wird die Vorstellung von einem begrenzbaren »inneren Raum« in all diesen Fällen kulturalisiert und zugleich authentisiert. Die zentrale Strategie der Authentisierung stellt hier die kontinuierliche Reproduktion des Bildes von einer Grenze dar, das die Grundfigur des Politikbereichs von einem »kulturellen Wir«, das sich verständlich machen will, immer wieder aufs Neue bestätigt. Die relative Flexibilität im Umgang mit Inhalten lässt sich hingegen als eine Konsequenz der besonderen Verwendungskontexte von Kultur in diesem Politikbereich verstehen: Denn hier geht es weder darum, das »Innere « gegen das »Äußere« undurchlässig abzuschließen, noch um eine einseitige Präsentation gleichbleibender Inhalte einer »eigenen Kultur« jenseits ihrer Grenzen. Im Zentrum steht stattdessen die Absicht, Beziehungen zwischen dem inneren und dem äußeren Raum herzustellen und somit kontinuierlich entlang dieser imaginären Grenze kulturpolitische Praxisfelder zu eröffnen - um Bilder und Positionen aus dem »Innen « nach »Außen« zu vermitteln; um Dialogprozesse zwischen dem inneren und dem äußeren Raum zu initiieren; aber auch um etwa im Zuge einer Standortpolitik zur positiven Entwicklung des inneren Bereichs durch die Einbeziehung von Kreativität und wissenschaftlicher Exzellenz aus dem »Außen« beizutragen. Gemäß dem politischen Narrativ geht es im Kern darum, Anschlüsse zu produzieren, Verknüpfungen herzustellen und somit die Grenze in der kulturpolitischen Praxis routiniert zu überschrei-

151 | Alle diese Positionen wurden auf den Berliner Konferenzen vorgebracht. 
ten. Auswärtige Kulturpolitik ist also durch eine epistemologische Grundfigur geprägt, die einerseits durchgängig die Vorstellung einer Grenze reproduziert und andererseits kontinuierlich zur Relativierung dieser Grenze durch Dialog, Begegnung und Austausch auffordert. Wie erklärt sich dieses auf den ersten Blick etwas ambivalente Wechselspiel?

Diese Fragestellung lässt sich durch einen Rückgriff auf Herangehensweisen einer anthropologischen Ethnizitäts- und Nationalismusforschung bearbeiten, in denen dem Problem der Beziehung zwischen Grenzen und kultureller Differenz eine besondere Beachtung geschenkt wird. Fredrik Barth hat dieses Forschungsfeld Ende der ig6oer Jahre grundlegend verändert, indem er aufzeigte, dass sich Vorstellungen von kulturellen Unterschieden häufig gerade durch grenzüberschreitende Austauschs- und Interaktionsprozesse zwischen sozialen und ethnischen Gruppen immer wieder aufs Neue konstituieren. ${ }^{152}$ Die analytische Perspektive auf sozial wirkmächtige Bilder von »kultureller Differenz« oder von der »eigenen Kultur« hat sich durch diese Beobachtung gewissermaßen umgedreht: Anstatt kulturelle Unterschiede zwischen sozialen Gruppen oder staatlichen Einheiten als substanziell gegeben und Abgrenzungen als deren natürliche Folge zu betrachten, rückten Prozesse, Mechanismen und Techniken der Grenzziehung in den Fokus. Durch eine solche Herangehensweise lässt sich aufzeigen, dass die Aufrechterhaltung von Grenzen in vielen Feldern gewissermaßen das eigentliche »Problem « darstellt, da ohne sie die Vorstellung von nach außen abgrenzbaren und nach innen kohärenten Gruppen kaum am Leben zu halten wäre. »Kultur« wird vor diesem Hintergrund zu einem zentralen Modus, um Bilder von der Existenz distinkter Kollektive immer wieder hervorzurufen. Grenzüberschreitende Austauschprozesse führen demnach nicht zwingend zur Verflüchtigung der Vorstellungen von kulturellen Unterschieden, sondern häufig ganz im Gegenteil zur Verfestigung und Inszenierung von »Differenz«, die anhand bestimmter Symbole, Praxen oder Rituale markiert und sichtbar gehalten wird.

Wendet man diese Erkenntnisse auf die Analyse der epistemologischen Grundfigur Auswärtiger Kulturpolitik an, so wird deutlich, dass die policy community gewissermaßen systemisch zur Reproduktion des Bildes einer Grenze angehalten ist, um elementare Ausgangspunkte des politischen Narrativs aufrechtzuerhalten: Eine dichotomische Logik durchzieht die Akteursund Diskursfelder, ordnet die Wissensbestände, kulturellen Positionen und Personen einem »Innen« oder »Außen« zu und führt zugleich zu dem Auftrag, diese beiden Sphären in einen Austausch zu bringen. Bereits in Vorbereitung auf das folgende Kapitel lässt sich festhalten, dass die kontinuierliche

152 Siehe hierzu: Fredrik Barth: Introduction. In: Ders. (Hrsg.): Ethnic Groups and Boundaries: The Social Organization of Culture Difference. Boston, Oslo et al. 1969, S. 9-38. 
Betonung von Dialog und Begegnung nicht nur auf einer diskursiven Ebene zu einer Wechselwirkung zwischen Reproduktion und Relativierung der Vorstellungen von »Grenzen« und »kulturellen Unterschieden« führt, sondern zugleich im Hinblick auf die kulturpolitische Praxis in den Zielregionen einen doppelten Effekt hervorruft: Die dichotomische Logik verlangt einerseits nach Bühnen zur Repräsentation und Inszenierung der distinkten »eigenen Kultur« und »nationalen Spezifik«; erforderlich sind andererseits Praxis- und Übersetzungsfelder, in denen grenzüberschreitende Kooperationen, Interaktionen und Austauschprozesse zwischen Individuen und Gruppen konkret stattfinden können. Goethe-Institute im Ausland müssen beide Funktionen erfüllen, um den Vorgaben des offiziellen Narrativs und seiner epistemologischen Grundfigur gerecht zu werden.

\section{Materielle Effekte der epistemologischen Grundfigur: Goethe-Institute im Ausland}

Ein Goethe-Institut selbst lässt sich dabei als eine Materialisierung dieser epistemologischen Grundfigur verstehen. Hier findet das Bild von einem »inneren Raum«, der sich durch kulturelle Differenz von seiner Umgebung unterscheidet und zugleich den Ausgangspunkt für Dialog und Austausch mit dem »äußeren Raum« darstellt, eine physische Form: Betritt eine Besucherin ein Goethe-Institut in Sarajevo, Ramallah oder Tel Aviv, so überschreitet sie eine wahrnehmbare Grenze und findet sich in einem ästhetisch und symbolisch markierten Raum wieder. Goethe-Institute vermitteln auf einer sinnlich erfahrbaren Ebene den Unterschied zwischen einem inneren und einem äußeren Raum der Kultur: die Beschriftungen am Gebäude und Wegweisern im Inneren sind zweisprachig auf Deutsch und in der Landessprache gehalten; ein Deutsche-Welle-Punkt - im Prinzip ein etwas höher montierter, von Stellwänden eingefasster Fernsehapparat - breitet ganztägig einen Klangteppich mit dem Programm des deutschen Auslandssenders aus; in Ständern sind Werbematerialien mit Deutschlandbezug und zu Kulturveranstaltungen untergebracht; die Wände sind durch Plakate mit deutschen Sehenswürdigkeiten, Verbkonjugationen oder Hinweisen auf Sprachkurse geschmückt; ein Bereich des Hauses enthält in der Regel eine Bibliothek, in der Bücher auf Deutsch oder zu Deutschland bereitstehen; in Klassenräumen findet deutscher Sprachunterricht statt; eine Programmabteilung setzt »deutsche Impulse« im Kulturleben des »Gastlandes«; aus Deutschland eingeladene Künstler, Schriftstellerinnen oder Musikerinnen werden durch ihren Auftritt an derart codierten Orten zu Repräsentanten »deutscher Kultur«; ein deutsches Leitungspersonal demonstriert »Deutschsein« bereits durch seine körperliche Präsenz und einen inkorporierten Habitus kultureller Repräsentation. Zugleich ist die Arbeit eines solchen Instituts darauf ausgerichtet, vielfältige Räume und Situationen 
des Austauschs, der Begegnung und des praktischen Dialogs zu eröffnen kurz: die epistemologische Grundfigur des politischen Narrativs findet sich auch in räumlichen Ordnungen und institutionellen Arrangements des Politikbereichs wieder.

\section{Skalierungen des inneren Raums: Europa und der Westen als kulturpolitische Sprechpositionen}

Anhand einzelner öffentlicher Sprechakte, aber auch anhand von Projekt- und Veranstaltungsformaten lässt sich zeigen, dass diese epistemologische Grundfigur skalierbar ist: In der Regel wird sie durch Bezugnahmen auf die Bestände des symbolischen Raums des Nationalen begründet; in einigen Fällen führt sie aber zu Bildern von der Existenz einer umgrenzbaren »europäischen Kultur« oder vom »Westen« als einer zivilisatorischen Einheit. Der Verlauf der Grenze lässt sich also in einem gewissen Umfang flexibel ziehen - das konkrete Ausmaß des kulturellen Kollektivs, das sie umschließt, ist partiell variabel. Die systemische Wirkmächtigkeit der dichotomischen Logik zeigt sich auch hier, da die epistemologische Grundfigur, um »Kultur« zu denken und die eigene Sprechposition zu definieren, weitgehend unhinterfragt bleibt. Dies möchte ich an zwei Beispielen erläutern:

Im Rahmen einer Konferenz, die das Auswärtige Amt gemeinsam mit der Akademie der Künste in Berlin im Jahr 2007 unter dem Titel Perspektive Europa veranstaltet, wird die Skalierung des »inneren Raums« auf eine europäische Dimension deutlich. ${ }^{153}$ Im Zentrum der Veranstaltung stehen - neben einer Rede des Außenministers - zwei Panels, in denen »europäische« und »nicht-europäische« Intellektuelle und Schriftsteller getrennt voneinander diskutieren. Im Falle der Rede Steinmeiers ist die Tendenz zur Skalierung der Vorstellung von einem »inneren Raum« auf eine europäische Ebene bereits im Titel Europa und der Blick von außen enthalten. Auch die beiden Diskussionsrunden reproduzieren diese Logik: Hier äußern sich zunächst die als »Außereuropäer« eingeladenen Intellektuellen Assia Djebar, Wang Hui, Elias Khoury und Wole Soyinka zu dem vorgegebenen Thema Der Blick von außen. Darauf folgt eine Runde mitteleuropäischer Autoren und Kulturschaffender, die zu der Frage Wer ist Europäer? diskutieren. ${ }^{154}$ Ein öffentlicher Austausch zwischen den Teilnehmern und Positionen der beiden Panels ist nicht vorge-

153 | Feldnotizen zu der - auch medial viel beachteten - Veranstaltung Perspektive Europa, Akademie der Künste Berlin, 1.-2. Juni 2007. Siehe hierzu das Programmheft der Akademie der Künste oder den Eintrag auf der Homepage: http://projekte.adk.de/ europa/home.html (letzter Zugriff am 11. August 2017).

154 | In diesem Panel diskutierten Mario Adorf, György Konrád, Andrzej Stasiuk und Ilija Trojanow. 
sehen, so dass das Bild von einem abgrenzbaren »Europa«, das sich von außen beobachten und kommentieren lässt, entsteht. Die Problematik dieser Ordnungslogik zeigt sich besonders deutlich anhand des Panels der »Außereuropäer«: Die Diskussionsteilnehmer werden durch die Fragen der Moderatorin durchgängig dazu aufgefordert, eine externe Position einzunehmen, obwohl ihr intellektuelles Schaffen aufgrund ihrer (post-)kolonialen Bildungs- und Arbeitsbiographien vielfältig mit Europa, seiner Geschichte, seinen Diskursen und Sprachen verwoben ist - anders formuliert: durch die dichotomische Grundfigur wird Intellektuellen, die fraglos Teil eines global verflochtenen Europa sind, eine Außenposition zugewiesen. Die historisch gewachsenen, weltweiten kulturellen Verwebungen, aus denen Europa in seiner gegenwärtigen Form überhaupt entstanden ist und welche eine zentrale Rahmenbedingung der künstlerischen Arbeit der Diskutanten darstellen, verschwinden durch die Organisationslogik der Veranstaltung - die auf die epistemologische Ordnung des Politikbereichs verweist - zugunsten der Vorstellung eines umgrenzbaren »inneren europäischen Raums«, der sich von außen betrachten lässt.

Das zweite Beispiel betrifft den Politikschwerpunkt Dialog mit der islamischen Welt, der als Reaktion auf die Anschläge vom II. September 200I in New York geschaffen wurde. Hierzu wurde die Position eines Beauftragten für den Dialog zwischen den Kulturen und ein Referat Dialog mit der islamischen Welt innerhalb des Auswärtigen Amtes eingerichtet sowie ein Netzwerk aus sprachund fachkundigen Referenten an den deutschen Auslandsvertretungen in der Zielregion, die »über die Bedeutung von Islam und Islamismus in ihren Gastländern« berichten, aufgebaut. ${ }^{155}$ Weiterhin stehen seit 2002 jährlich zwischen 4 und 5 Millionen Euro als Sondermittel für Projekte und Austauschprogramme zur Verfügung. Die dichotomische Logik ist hier bereits in die Benennung des Förderprogramms eingeschrieben: Europäisch-Islamischer Kulturdialog. Der »innere Raum« bezieht sich in diesem Politikbereich zum Teil auf »Europa«, häufig aber auch auf die Vorstellung vom »Westen« als einer kulturell oder zivilisatorisch kohärenten Größe, die gerade in dieser Gegenüberstellung zu dem Bild einer »islamischen Welt« ihre Konturen erhält. Dieser Zusammenhang zeigt sich etwa in der Selbstdarstellung des Politikbereichs auf der Homepage des Auswärtigen Amtes:

"Das Verhältnis zwischen westlichen und islamisch geprägten Gesellschaften ist vielfach von Missverständnissen und stereotypen Wahrnehmungen geprägt. In den meisten islamisch geprägten Staaten ist zudem in den vergangenen Jahren die Popularität des Islamismus gestiegen - einer Ideologie, die darauf zielt, Staat und Gesellschaft nach den Vorschriften des Islam einzurichten. Die politischen Konzepte von Demokratie,

155 | So hieß es im Jahr 2008 auf: http://www.auswaertiges-amt.de/DE/Aussenpolitik/ KulturDialog/InterkulturellerDialog/IslamDialog_node.html. 
universellen Menschenrechten und Pluralismus werden dabei häufig als "westlich" und damit "unislamisch" abgelehnt. Ziel des Dialogs ist, die Verständigung zwischen dem "Westen" und der islamischen Welt sowie innergesellschaftlichen Pluralismus und den Abbau antiwestlicher Stereotypen und Feindbilder in islamisch geprägten Gesellschaften zu fördern. “156

In ähnlicher Weise wie bereits im dritten Abschnitt dieses Kapitels anhand der dort analysierten Regierungsdokumente wird wiederum sichtbar, wie stark eine angenommene Grenzlinie zwischen dem »Westen « und der »islamischen Welt« die Wahrnehmungsmuster außenpolitischer Eliten von der Weltordnung nach dem Kalten Krieg prägt. Gleichfalls zeigt sich an diesem Beispiel das Wirken der dichotomischen Logik in der Konstruktion eines »kulturellen Wir«, das nunmehr eben den »Westen« umfasst. Dies wird in einer Beschreibung des eigenen Arbeitsauftrages, die der Beauftragte für den Dialog mit der islamischen Welt im Jahr 2004 verfasste, besonders deutlich:

"Das Ziel unserer Dialogaktivitäten ist es, die Glaubwürdigkeit unserer Wertvorstellungen zu stärken. Es gilt, in der skeptisch eingestellten islamischen Welt den Nachweis zu liefern, dass wir der Debatte, die von islamischen und islamistischen Gruppen geführt werden (sic), nicht ausweichen. Wir sollten uns dafür interessieren, die muslimischen Erfahrungen mit der "Moderne" westlicher Prägung zu hören und uns grundsätzlich bereit zeigen, auch Modifikationen an unseren eigenen Positionen im Sinne eines wirklich globalen Demokratieverständnisses vorzunehmen. «" ${ }^{157}$

Sichtbar wird hier wiederum, wie sich Konzepte von »unseren Wertevorstellungen« oder der »Moderne westlicher Prägung« gerade in der Abgrenzung von dem Gegenkonzept »islamische Welt« hervorrufen lassen.

Nun ist es nicht mein Anliegen, die Existenz von Differenzen etwa zwischen Deutschland und dem Oman in Frage zu stellen. Tatsächlich sind bestimmte politische, gesellschaftliche und ökonomische Prozesse in vielen europäischen Ländern anders verlaufen als in den Ländern Nordafrikas, des Nahen oder Mittleren Ostens - nicht zuletzt da europäische koloniale und imperiale

156 | Ebd. Anhand dieses Zitat wird nicht nur deutlich, wie "Islam" und "Westen" als eindeutig voneinander abgegrenzt konstruiert werden, sondern dass es aus dieser Perspektive die islamische Welt ist, die das "Problem" darstellt. So ist etwa keine Rede von den Kriegen, die westliche Staaten im Mittleren Osten führen oder auch von einer wachsenden Islam-Feindschaft in den europäischen und nordamerikanischen Gesellschaften - Entwicklungen, aus denen sich durchaus ein Diskussions- und Handlungsbedarf ableiten lassen könnte.

157 | Auswärtiges Amt (Hrsg.): Dialogue with the Islamic World / Dialog mit der islamischen Welt. Berlin 2006, S. 49f. 
Projekte dortige Entwicklungen hemmten, ganz unterbrachen oder langwierige Konflikte auslösten. Gleichfalls ist es richtig, dass etwa die Dominanz unterschiedlicher religiöser Systeme zumindest das westliche Europa einerseits und große Teile der »islamischen Welt« andererseits in einen jeweiligen historischen und kulturellen Kontext gerückt haben. Ebenso lässt sich nicht verleugnen, dass Versuche zur Politisierung der Vorstellung eines grundlegenden Gegensatzes zwischen dem »Islam « und dem »Westen « durch unterschiedliche Akteure in den letzten Jahren eine zum Teil nachhaltige Wirkung entfaltet haben. Das Problem in der Konzipierung eines Politikbereichs Dialog mit der islamischen Welt besteht aber darin, dass hier Grenzen und Unterschiede in der Tendenz essentialisiert werden - anders formuliert: der widersprüchliche Verlauf vieler dieser politischen und gesellschaftlichen Prozesse in den einzelnen Ländern, die Debatten und internen Kämpfe, die sich jeweils mit ihnen verknüpften, die inneren Brüche und Ambivalenzen, die mitunter großen Unterschiede im Verlauf solcher Prozesse innerhalb des »Westens« oder des Nahen Ostens, die multiplen Verflechtungen und Austauschbeziehungen, die sowohl Europa als auch islamische Länder nachhaltig geprägt und geformt haben oder auch die vielfältigen Präsenzen von Muslimen in westlichen Gesellschaften werden durch einen solchen Ansatz bereits durch die epistemologische Grundordnung ausgeblendet. Vor diesem Hintergrund stellt sich die Frage, ob durch eine solche praktische Übersetzung der dichotomischen Logik in ein politisches Programm das Problem eines Auseinanderdriftens zweier geopolitischer Räume, das eigentlich entschärft werden soll, nicht eher verfestigt wird: Eben durch die Festschreibung des Bildes von einer grundlegenden Grenze, die beiden Regionen jeweils eine nach »Innen « homogene und nach »Außen« abgrenzbare Form gibt.

\section{Skepsis gegenüber der epistemologischen Ordnung}

Das Vermögen zur Skalierung des »inneren Raums« unterstreicht noch einmal die Wirkmächtigkeit der dichotomischen Logik, die das politische Narrativ strukturiert, Wissensbestände ordnet und Sprechpositionen definiert. Aus diesen Beobachtungen folgt aber nicht, dass Skepsis oder Kritik gegenüber den Effekten dieser Logik ausgeschlossen sind. So äußerte eine Mitarbeiterin des Referats Islamdialog im Auswärtigen Amt mir gegenüber, dass man eigentlich mit den dualen Denkmodellen brechen müsse, um die ursprüngliche Absicht, durch die Stärkung des Dialogs mit der islamischen Welt einen Gegenentwurf zur These vom clash of civilizations zu entwickeln, realisieren zu können. Gerade in der Begründung dieses Politikschwerpunktes falle man aber immer wieder in dichotomische und zugleich wertende Argumentationsfiguren - etwa von der »Modernisierungskrise in der islamischen Welt« oder vom »Westen« 
als der selbstverständlichen universalen Norm für Entwicklung und Demokratie -, die einen »Dialog auf Augenhöhe« schwierig machten. ${ }^{158}$

Eine kritische Haltung gegenüber den Grundfiguren und Denklogiken, die den Ansatz eines Europäisch-Islamischen Kulturdialogs durchziehen, findet sich bis hin zu führenden Mitgliedern der policy community. So formulierte etwa Minister Steinmeier seine Skepsis:

"Ich frage mich im übrigen manchmal, ob wir mit dem fast als Parallelaktion zum ,Krieg gegen den Terrorismus gestarteten Dialog mit dem Islam beziehungsweise mit der islamischen Welt nicht der Stellvertreter- und Avantgardestrategie der islamischen Fundis ein wenig auf den Leim gehen. Wir laufen Gefahr, als Einheit zu behandeln, was der terroristische Islamismus erst zu einer schmieden will."159

Steinmeier regte an, die zu diesem Zeitpunkt etablierte Figur vom Dialog der Kulturen durch das Idealbild einer Kultur des Dialogs als Orientierungsgröße Auswärtiger Kulturpolitik zu ersetzen, um die Vorstellung von sich gegenüberstehenden zivilisatorischen Blöcken abzumildern:

"Wenn ich in Zentralasien mit Regierungsvertretern rede oder in Afghanistan mit einer Lehrerin an einer von der EU finanzierten Mädchenschule, dann führe ich keinen Dialog mit dem Islam, auch wenn meine kulturelle Prägung eine andere sein wird als die der Leute, mit denen ich spreche. Wir haben schon etwas gemeinsam, bevor wir unser Gespräch beginnen, sei es die Regierungsverantwortung in unserem Staat, sei es das Interesse an Bildung und Ausbildung. Verantwortung und Interesse führen uns zusammen und sie bestimmen auch das Thema. Wir wollen auf etwas hinaus, ja im gar nicht so seltenen Fall: sogar gemeinsam etwas erreichen."

Bemerkenswert ist, dass auch Minister Steinmeier - trotz dieser grundlegenden Skepsis - an dem Instrument des Islamdialogs festhielt und somit auch einer dichotomischen Logik, der er kritisch gegenübersteht, ihren Entfaltungsbereich beließ. Als ein Fazit lässt sich formulieren, dass sich gerade aus dem Zusammenwirken von diskursiven und materiellen Ordnungen, von langfristigen politischen Narrativen und dem Selbstverständnis der policy community, von Förderprogrammen und Veranstaltungsformaten eine gewissermaßen systemische Wirkmächtigkeit der etablierten epistemologischen Grundfigur ergibt. Das Bild von einer Grenze zwischen dem inneren und äußeren Raum der Kultur, die es zu bearbeiten und zu überschreiten gilt, besitzt in dem Po-

158 | Feldnotizen vom August 2008.

159 | "Der Dialog der Kulturen: eine Herausforderung für die deutsche Außenpolitik" Rede von Bundesaußenminister Steinmeier auf Einladung der EKD am 21.11.2006; auch das folgende Zitat. 
litikbereich eine weitgehend naturalisierte Plausibilität, der sich Vertreter der policy community gerade in Momenten der öffentlichen Repräsentation und Legitimierung Auswärtiger Kulturpolitik offensichtlich nur schwer entziehen können. Formen der Skalierung des inneren Raums werte ich nicht nur als einen weiteren Ausdruck genau dieser hohen Plausibilität, sondern sie verweisen zugleich auf die Schwierigkeit, innerhalb des offiziellen Narrativs eine Sprechposition zu definieren, die ohne die Vorstellungen von einer »eigenen Kultur« oder von einem »kulturellen Wir« auskommt.

Zugleich deutet sich an, dass skeptische Potenziale gegenüber der strukturierenden Kraft der dichotomischen Logik und epistemologischen Grundfigur gerade in konkreten kulturpolitischen Praxis- und Übersetzungsfeldern entstehen können. Dies zeigt sich an dem letzten Zitat des Außenministers ebenso wie in Äußerungen meiner Gesprächspartnerin aus dem Arbeitsbereich Islamdialog, die ihr Vorgehen als ein »learning by doing « charakterisierte - also als eine mitunter experimentelle Suche nach Wissen, geeigneten Kooperationspartnern, Herangehensweisen oder Projektformaten, die »nicht akademisch sind, sondern Handeln ermöglichen. «160 Der Anspruch und der Auftrag in sehr diversen lokalen Kontexten präsent zu sein, Dialogprozesse mit den unterschiedlichsten Partnern zu initiieren und hierbei jeweils relevante Anschlüsse zu erzielen, scheint eine zumindest partielle Reflexivierung bestimmter Grundfiguren des politischen Narrativs zu ermöglichen. Aus dem Aufeinandertreffen der etablierten epistemologischen Ordnung und den Erfordernissen der praktischen Arbeit können somit Reibungen entstehen, deren Rückwirkungen auf die imaginäre Dimension des Politikbereichs es im Auge zu behalten gilt. In der Konsequenz ist davon auszugehen, dass sich eine deutsche Kulturmittlerin in Ramallah oder Sarajevo in eben diesem Wechselspiel aus routinierter Reproduktion und situativer Flexibilisierung der dichotomischen Logik, aus der fraglosen Repräsentation einer »eigenen Kultur« und der alltäglichen Orientierung an den konkreten Problemstellungen projektbezogener Kooperation bewegt. Reibungen zwischen epistemologischen Ordnungen und anwendungsorientierten Handlungslogiken stellen im folgenden Kapitel eine wichtige Untersuchungsperspektive dar. Bevor ich mich aber dieser Wissensund Übersetzungsarbeit in Ramallah, Tel Aviv und Sarajevo zuwende, möchte ich noch einen kurzen Blick auf die asymmetrischen Relationen zwischen den politischen Zentren und Mittlerorganisationen als einer zentralen Rahmenbedingung für die translokale Mobilisierung politischer Zielsetzungen und somit für kulturpolitische Vermittlung insgesamt werfen.

160 | Feldnotizen vom August 2008. 


\section{Asymmetrien und Handlungsspielräume: RELATIONEN ZWISCHEN POLITISCHEN ZENTREN UND KULTURMITTLERN}

Betrachtet man Auswärtige Kulturpolitik mit Pierre Bourdieu als ein »Feld von aktuellen und potentiellen Kräften«, das durch Kämpfe »um den Erhalt oder die Veränderung der Konfiguration dieser Kräfte « ${ }^{161}$ gebildet wird, so zeigt sich, dass die »Relationen « zwischen den wichtigen Akteuren des Politikbereichs durch eine grundlegende Asymmetrie geprägt sind: Die politischen Zentren in Berlin ${ }^{162}$ verfügen über die elementaren »Trümpfe «, die das »Spiel« - also die Konkurrenzkämpfe um Zugänge zu diesem Feld, um Gelder und Positionen - überhaupt erst ermöglichen.

\section{Trümpfe und Potenziale:}

\section{Strukturelle Grundlagen der Dominanz der politischen Zentren}

Hierzu zählt erstens das Potenzial, den »offiziellen Diskurs « ${ }^{163}$ zu setzen, also Auswärtige Kulturpolitik als einen eigenständigen, sichtbaren und zusammenhängenden Politikbereich zu konstituieren. Hierbei geht es mir weniger um die konkrete Ausgestaltung eines inzwischen vielstimmigen Diskurses oder um die Entwicklung einzelner Argumentationslinien, wie sie etwa im Rahmen der Berliner Konferenzen zutage getreten sind, sondern um das Vermögen zu einem grundlegenden »Erkenntnisakt, dem Anerkennung zu Teil wird « und der hierdurch gewissermaßen eine »objektive« Sicht der Dinge zu formulieren vermag. Die politischen Zentren verfügen über die Macht, Auswärtige Kulturpolitik als einen öffentlich gewünschten und dauerhaften Politikbereich zu konstituieren, die elementaren »Probleme«, die es zu bearbeiten gilt, zu definieren und einen »offiziellen Standpunkt« zu ihrer Legitimierung

161 | Pierre Bourdieu \& Loic Wacquant: Die Ziele der reflexiven Soziologie. ChicagoSeminar, Winter 1987. In: Dies.: Reflexive Anthropologie, S. 95-249; S. 132.

162 | Zu den "politischen Zentren" rechne ich die Instanzen, die an der Bewilligung und Vergabe der Bundesmittel beteiligt sind: in erster Linie das Auswärtige Amt und den Deutschen Bundestag, aber auch die weiteren fünf Ministerien, die (in einem deutlich geringeren) Umfang gleichfalls Gelder der Auswärtigen Kulturpolitik verteilen. Siehe zu einer Auflistung dieser Ministerien und der innen jeweils zur Verfügung stehenden Mittel für den Untersuchungszeitraum: Deutscher Bundestag (Hrsg.): Antwort der Bundesregierung auf die Große Anfrage der Abgeordneten Dr. Uschi Eid et al., S. 24.

163 | Hier und in den nächsten Zitaten beziehe ich mich auf Überlegungen von Pierre Bourdieu zum "offiziellen Diskurs", der hier an Aaron Cicourel anschließt: Pierre Bourdieu: Sozialer Raum und symbolische Macht. In: Ders.: Rede und Antwort. Frankfurt 1992, S. 135-154, S. 150. 
festzuschreiben. Ebenso liegt hier das Vermögen, Auswärtige Kulturpolitik als eine eigenständige politische Konstruktion und Struktur wieder gänzlich aufzulösen.

Ein zweiter wesentlicher Trumpf besteht in der Entscheidungsgewalt über die Vergabe der öffentlichen Gelder, welche die internationale kulturelle Vermittlungsarbeit in dieser institutionalisierten Form überhaupt erst ermöglichen. Im Wechselspiel zwischen Parlament und Auswärtigem Amt wird nicht nur grundsätzlich über die verfügbaren Mittel entschieden, sondern auch über die konkrete Ausstattung einzelner Mittlerorganisationen, die Setzung regionaler oder inhaltlicher Schwerpunkte sowie über zeitlich begrenzte besondere Förderprogramme. Diese budgetären Entscheidungen haben direkte Auswirkungen auf die Arbeit der Mittlerorganisationen und lassen somit die Asymmetrien besonders deutlich zutage treten: Die, über die Jahre hinweg betrachtet, eigentlich eher moderaten Kürzungen zur Zeit der rotgrünen Regierung, ${ }^{164}$ die mit der gesamtpolitischen Zielsetzung der »Haushaltskonsolidierung « begründet wurden, konnten etwa vom Goethe-Institut nicht durch andere Geldquellen ausgeglichen werden und schlugen sich entsprechend in der Schließung einzelner Institute nieder. ${ }^{165}$ Die Aufstockungen der Mittel in der ersten Amtsperiode Frank-Walter Steinmeiers ${ }^{166}$ führten hingegen zu Neugründungen von Goethe-Instituten oder anderen Formen kulturpolitischer

164 | Zwischen 1999 und 2005 sanken die Ausgaben des Bundes für AKBP in einem überschaubaren Umfang von ca. 1,15 Milliarden Euro auf 1,092 Milliarden Euro. Der Anteil des Auswärtigen Amtes sank im gleichen Zeitraum deutlicher von etwa 657 Millionen Euro auf 601 Millionen Euro. Die Budgetentwicklung unterschied sich dabei von Jahr zu Jahr: So sanken die Gesamtausgaben 2000 im Vergleich zum Vorjahr um 3,2\%, um im folgenden Jahr wieder um 2,6\% zu steigen. Siehe: Deutscher Bundestag (Hrsg.): Antwort der Bundesregierung auf die Große Anfrage der Abgeordneten Dr. Uschi Eid et al., S. 24.

165 | Entsprechend der gleichen Antwort der Bundesregierung sanken die Bundesmittel für das Goethe-Institut zwischen 1993 und 1994 deutlich von ca. 169 Millionen Euro auf 158,7 Millionen Euro oder zwischen 2004 und 2005 von ca. 169 Millionen Euro auf 159,5 Millionen Euro. Allerdings gab es in anderen Zeiträumen auch deutliche Zuwächse, etwa zwischen 2000 und 2001 von 150,75 Millionen Euro auf etwa 165 Millionen Euro und dann auch zur Zeit der Großen Koalition (s.u.). Ebd., S. 27.

166 | Zwischen 2005 und 2009 stiegen die Ausgaben des Bundes für AKBP deutlich von 1,092 Milliarden Euro auf 1,436 Milliarden Euro. Die darin enthaltenen Mittel des Auswärtigen Amtes wuchsen im gleichen Zeitraum von 601 Millionen Euro auf 726 Millionen Euro (in diesem Fall ohne Ausgaben des Deutschen Archäologischen Instituts). Angaben entsprechend der Jahresberichte der Bundesregierung zur Auswärtigen Kulturpolitik, online einsehbar unter: http://www.ifa.de/kultur-und-aussenpolitik/basistexte-und-bibliografien/berichte-zur-akbp.html (letzter Zugriff am 10. August 2017). 
Vertretungen in von Seiten der Politik vorgegebenen Weltregionen. So lässt sich zuspitzen, dass es diesen Bereich der internationalen kulturellen Vermittlung in dieser Form ohne öffentliche Gelder schlichtweg nicht gäbe. Die Abhängigkeit der Arbeit der Kulturmittler lässt sich anhand der Zusammensetzung des Budgets des Goethe-Instituts demonstrieren: Im Jahre 2009 betrug der Anteil des Auswärtigen Amtes zur Finanzierung der Auslandsaktivitäten des Instituts knapp $77 \% .^{167}$

Einen dritten Trumpf - das Vermögen, Verfahren oder Techniken zur Ordnung des Wissens zu etablieren - werde ich nach einer Darstellung der Beziehungsstruktur zwischen Auswärtigen Amt und Kulturmittlern untersuchen.

\section{Abhängigkeit und Freiräume: Wechselspiele der Macht zwischen Auswärtigem Amt und Kulturmittlern}

Aus Perspektive des Auswärtigen Amtes stellt sich - trotz solcher Trümpfe gerade aufgrund des globalen und translokalen Charakters des kulturpolitischen Akteurs- und Handlungsfeldes die Frage nach den Möglichkeiten einer Steuerung, Ordnung und Zielausrichtung des Politikbereichs: Über welche Beziehungsmuster, Regierungstechniken und bürokratische Arrangements lassen sich hier etwa ein organisatorischer und inhaltlicher Zusammenhang herstellen oder politische Zielvorstellungen mobilisieren?

Die auffälligste strukturelle Anordnung des Politikbereichs - das enge und kontinuierliche Zusammenspiel zwischen einem staatlichen Ministerium und einer überschaubaren Anzahl formal unabhängiger Mittlerorganisationen lässt sich als eine Reaktion auf dieses »Problem« interpretieren: Sein Kern wird durch dauerhafte institutionelle Relationen gebildet, die ich unter Rückgriff auf Richard Rottenburgs Studie zur Entwicklungszusammenarbeit als »Prinzipal-Agent-Konstellation« bezeichnen möchte. ${ }^{168}$ Es sind zwei Besonder-

167 | Die Zuwendungen des Auswärtigen Amtes an das Goethe-Institut betrugen in diesem Jahr 222,5 Millionen Euro. Hinzu kamen 6,1 Millionen Euro an Zuschüssen anderer Ministerien und öffentlicher Stellen. Den zweitgrößten Anteil am Budget bilden die primär durch Sprachkurse und Prüfungsgebühren erzielten Eigeneinnahmen in einer Höhe von 56,1 Millionen Euro (= 19\% der gesamten Einnahmen). Spenden oder Sponsoring - und somit nicht-öffentliche Gelder - machen mit 4,5 Millionen Euro (=1,6\% der gesamten Einnahmen) einen eher geringen Anteil aus. Die Goethe-Institute in Deutschland arbeiten laut Rechenschaftsbericht kostendeckend und wirtschaften in einem von den Auslandsinstituten getrennten Finanzkreislauf. Siehe hierzu: Goethe-Institut: Rechenschaftsbericht 2009/2010. München 2010, S. 19.

168 | Richard Rottenburg: Weit hergeholte Fakten, S. 38. Rottenburg bezieht sich wiederum auf: Kenneth J. Arrow: The economics of agency. In: John W. Pratt \& Richard J. Zeckhauser (Hrsg.): The structure of business. Boston 1991 (1985), S. 37-51. 
heiten, die sich durch diesen Begriff herausstellen lassen - diese Beziehungen sind zugleich durch eine »klare Hierarchie« und ein »prinzipielles Kontrollproblem« gekennzeichnet: Einerseits befinden sich die Mittlerorganisationen als »Agenten « in einer existenziellen Abhängigkeit von ihrem »Prinzipal« und Auftraggeber, dem Auswärtigen Amt, der - in Abstimmung mit den anderen maßgeblichen Akteuren des politischen Feldes - über die grundlegende Fortführung oder inhaltliche Ausrichtung des Politikbereichs entscheiden und über die Verteilung der finanziellen Mittel bestimmen kann; andererseits verfügen sie über einen »nicht aufholbaren Wissensvorsprung«, was die praktische Um- und Übersetzung Auswärtiger Kulturpolitik in den Zielregionen betrifft. Die Mittlerorganisationen bringen spezifische Erfahrungen, lokale Kontakte und Wissensbestände in die Beziehung ein, die sie für das Auswärtige Amt praktisch unverzichtbar machen. Gleichzeitig ist das Ministerium bereits aus logistischen Gründen in der Beurteilung der Zielerfüllung im hohen Maße auf die Selbstauskünfte ihrer »Agenten« angewiesen. Aus dieser »asymmetrischen Verteilung relevanter Informationen« entsteht für die Mittlerorganisationen ein Potenzial, sich aus der Abhängigkeit von ihrem Prinzipal ein Stück weit zu lösen und ihr Handlungsvermögen somit zu vergrößern - ein Potenzial, das in der Antwort des Leiters eines Goethe-Instituts auf meine Frage nach seinen Handlungsspielräumen deutlich zum Ausdruck gebracht wird:

"Also inhaltlich bin ich ziemlich frei. Doch. Es ist das Schöne an diesem Job, dass man sich der Kontrolle ein wenig entziehen kann. Anders als beispielsweise ein Theaterintendant in Deutschland, der ganz nah an den Kontrollorganen sitzt, müssen sich meine Kontrollorgane ja mehr oder weniger auf das verlassen, was ich innen erzähle und deshalb kann ich inhaltlich wirklich- Eigentlich habe ich eine ganz, ganz große Freiheit. Das hat natürlich auch damit zu tun, wie viel Vertrauen man sich erwirbt. Bei denen, die einen möglicherweise kontrollieren wollen. “169

In diesem Interviewausschnitt klingt das Problem einer asymmetrischen Informationsverteilung in translokal verzweigten Organisationsfeldern an, dem politische Zentren - laut Richard Rottenburg - durch eine doppelte Strategie begegnen können: »Man führt ein angemessen dichtes Kontrollsystem ein und gleichzeitig versucht man, wechselseitiges Vertrauen aufzubauen. «170

Die verlässliche und kontinuierliche Privilegierung der Mittlerorganisationen in der Vergabe der materiellen Ressourcen lässt sich als ein wesentlicher Baustein in diesem Wechselspiel aus Vertrauen und Kontrolle verstehen: Das Goethe-Institut und die weiteren Mittler können - auch trotz jährlicher Budgetschwankungen - im Prinzip auf die substanzielle Förderung ihrer Aktivitäten

169 | Interview vom 10. Dezember 2008.

170 | Richard Rottenburg: Weit hergeholte Fakten, S. 38. 
durch öffentliche Gelder zählen. So dokumentieren die Berichte der Bundesregierung zur Auswärtigen Kulturpolitik, dass Jahr für Jahr über 90\% des Kulturhaushaltes des Auswärtigen Amtes den großen Mittlerorganisationen zugeteilt werden. ${ }^{171}$ Der Großteil der finanziellen Mittel des Auswärtigen Amtes wird also seit Jahren an die gleichen Partner vergeben; im Hinblick auf die Verteilung der Gelder und somit der zentralen Ressource, die kulturelle Vermittlung in ihrer aktuellen Form ermöglicht, stellt sich das Akteursfeld - trotz der Rhetorik der Öffnung, die etwa die Berliner Konferenzen prägte - als eher exklusiv, abgeschlossen und klar umgrenzt dar. Andere Institutionen kultureller oder wissenschaftlicher Produktion werden durch diese Organisationsformen von der Teilnahme am Konkurrenzkampf um den Löwenanteil der finanziellen Mittel weitgehend ausgeschlossen.

Im Rahmen dieser institutionellen Arrangements lässt sich das Wechselspiel zwischen Vertrauen und Kontrolle realisieren: Die restriktive Beschränkung des Zugangs zu dem Großteil der finanziellen Mittel auf eine kleine Anzahl von Partnerorganisationen einerseits sowie der Aufbau langfristiger, exklusiver Beziehungen andererseits bedeuten für die Mittlerorganisationen gleichzeitig eine relative Planungssicherheit, die Eröffnung von inhaltlichen Gestaltungsfreiräumen, die Ausschaltung einer breiteren Konkurrenz und die Reproduktion ihres grundlegenden Abhängigkeitsverhältnisses vom Auswärtigen Amt. Aus Perspektive des politischen Zentrums vereinfacht diese Struktur zweifelsohne die Steuerung des Feldes, da der Kreis der Ansprechpartner überschaubar bleibt, langfristige Verbindlichkeiten entstehen und sich somit zugleich Vertrauen herstellen und auch informelle Kontrollmechanismen etablieren lassen. Dieses Wechselspiel vor dem Hintergrund einer grundlegenden Machtasymmetrie verdeutlichte eine Diplomatin in einer deutschen Auslandsvertretung, mit der ich während meiner Feldforschung ein Interview führen konnte:

171 Der Anteil des Auswärtigen Amtes an den gesamten Bundesmitteln für AKBP betrug im Jahr 2009 58,3\%. Hiervon erhielt das Goethe-Institut 30,2\%; die Institutionen des Auslandsschulwesens 29,2\%; der DAAD 23,5\%; die Alexander von Humboldt-Stiftung 5,4\% und "Sonstiges" 10,6\%. Zur Rubrik "Sonstiges" zählen unter anderem die Zuwendungen an kleinere Mittlerorganisationen - etwa an das Institut für Auslandsbeziehungen - aber auch erforderliche Baumaßnahmen.

Auch die Mittel des Bundesbeauftragten der Bundesregierung für Kultur und Medien (BKM) - der etwa im Jahr 2009 mit 19,9\% der gesamten Mittel über das zweitgrößte Budget im Bereich AKBP verfügte - werden in strukturell ähnlicher Weise an die zentraIen Mittlerorganisationen - bspw. an die Deutsche Welle oder an die Bundeskulturstiftung - verteilt. Siehe hierzu: Bericht der Bundesregierung zur Auswärtigen Kulturpolitik 2009/2010, S. 11. 
"Wir haben schon auch eine gewisse Beobachtungsfunktion. Also wenn jetzt irgendeiner der Mittler völligen Mist bauen würde oder Dinge machen würden, die gegen Grundzüge der deutschen Außenpolitik gehen, dann würden wir da sicher intervenieren, das heißt, wir würden das über das Auswärtige Amt melden. Denn letztlich sind ja alle diese Mittler vom Auswärtigen Amt abhängig, finanziell abhängig: Goethe, DAAD, ZfA usw. Das sind letztlich alles Mittel aus dem Auswärtigen Amt. Aber da besteht hier eigentlich kein Bedarf. “172

Die eingespielten Relationen zwischen den zentralen Akteuren machen direkte Interventionen also nur in Ausnahmefällen notwendig. Als einen weiteren Trumpf kann das Auswärtige Amt in der alltäglichen Steuerung des Politikbereichs auf das Vermögen zurückgreifen, Verfahren zur Ordnung des Wissens $\mathrm{zu}$ setzen und hierdurch zugleich kontinuierliche »Übersetzungsketten « 173 zwischen politischen Zentren und den Einsatzregionen zu etablieren.

\section{Rechen(schafts)zentren: Knotenpunkte zur Ordnung des Wissens in einem translokalen politischen Feld}

Richard Rottenburg hat in seiner Studie gleichfalls den Begriff des Rechen(schafts)zentrums genutzt, um Institutionen $\mathrm{zu}$ beschreiben, in denen Informationen aus den unterschiedlichsten Orten eines weltweit verzweigten Politikbereichs zusammenfließen und in einen neuen Kontext gestellt werden können. Demnach entsteht hier - etwa mittels der Kombination von Projektberichten aus verschiedenen Ländern - ein neues Wissen, durch welches die Beamten oder die politische Leitung eines Ministeriums »Zusammenhänge« erkennen, »auf die man an keinem der getrennten Orte hätte kommen können«. Rechen(schafts)zentren lassen sich somit als »Knoten« in globalen Netzwerken verstehen, die solche Informationen nicht nur passiv ordnen, sondern zugleich über die Macht verfügen, »Verfahrensregeln« zu etablieren, an die sich andere Akteure halten müssen. Gerade in global vernetzten Feldern besteht ihre Funktion in den parallelen Bemühungen, einerseits »die Welt« - etwa durch bürokratische Verfahren - »berechenbar und dadurch auf Distanz kontrollierbar zu machen« sowie andererseits Rechenschaft - beispielsweise über die Kosten und Aktivitäten eines translokalen Politikbereichs - gegenüber der Öffentlichkeit abzulegen. ${ }^{174}$

172 | Interview vom 2.12.2008.

173 | Auch der Begriff der "Übersetzungskette" wird hier im Anschluss an Richard Rottenburg genutzt. Siehe hierzu insbesondere: Richard Rottenburg: Weit hergeholte Fakten, S. 17, S. $112 \mathrm{ff}$. und S. $227 \mathrm{ff}$.

174 | Richard Rottenburg: Weit hergeholte Fakten, S. 121f. Den Begriff Rechen(schafts)zentren entwickelt Rottenburg im Anschluss an Bruno Latour: Science in action. How to follow scientists and engineers through society. Cambridge 1987. 
Die Position des Auswärtigen Amtes innerhalb des Akteursfeldes der Auswärtigen Kulturpolitik lässt sich als ein solches Rechen(schafts)zentrum verstehen: Hier werden öffentliche Zuwendungen verbucht und legitimiert; hier fließt Wissen von den Aktivitäten der Mittlerorganisationen in den unterschiedlichsten Ländern, über die Anzahl der Kursteilnehmer und Bibliotheksentleihungen, über Kooperationen und vergebene Stipendien zusammen; hier können diese Informationen nach den jeweiligen Erfordernissen von politischen Entscheidungsträgerinnen, administrativen Prozessen oder von Belangen der Öffentlichkeitsarbeit - etwa nach Land, Zielsetzung oder Grad der Kostendeckung - zusammengeführt werden. Die Macht des Auswärtigen Amtes als einem Rechen(schafts)zentrum zeigt sich in dem Vermögen, sowohl politische Zielvorgaben als auch bürokratische Regeln und Wissensordnungen festzulegen, denen sich die Mitspieler nicht entziehen können.

In den letzten Jahren forcierte das Auswärtige Amt - neben den beschriebenen inhaltlichen Erweiterungen Auswärtiger Kulturpolitik - gleichfalls Prozesse eines strukturellen Wandels, durch die nicht nur neue Steuerungstechniken und Legitimierungsverfahren, sondern auch verstärkt eine Sprache und Rationalität von Unternehmensberatern Eingang in den Politikbereich gefunden haben. So ist etwa in der Konzeption 2000 die Rede von der Notwendigkeit einer »Verbesserung der Effizienz auf der Basis interner und externer Evaluation « und des »allmählichen Abbaus eigener Strukturen ${ }^{175}$ unter gleichzeitiger Verlagerung auf lokale, regionale und privatwirtschaftliche Trägerstrukturen« sowie von dem »enormen Rationalisierungs-, aber auch Neugestaltungspotential«, das sich aus den »Fortschritten in der Kommunikations- und Medientechnologie « ergebe. Die Forderung nach der »Verringerung der Personal- und Sachkosten« des Goethe-Instituts kann dabei als Auftrag zur Reduzierung regulärer Beschäftigungsverhältnisse gelesen werden. ${ }^{176}$ Begründet wird dieser Reformbedarf dabei mit dem Ziel der »allgemeinen Haushaltskonsolidierung «: »Um die anstehenden neuen Aufgaben trotz knapper Mittel bewältigen zu können, gilt es Ressourcen zu bündeln, die Effizienz zu erhöhen, zuguns-

175 | "Eigene Strukturen" bedeutet in diesem Fall staatliche (oder staatlich finanzierte) Strukturen.

176 | Entsprechend wird der Abbau regulärer Beschäftigungsverhältnisse in den deutschen Goethe-Instituten im Rechenschaftsbericht 2009/2010 als Erfolg verbucht: "Grundsätzlich führt die Region vor dem Hintergrund einer Risikovorsorge eine straffe Personalpolitik. Ziel ist es, dauerhaft die Kosten zu senken, um weiterhin konkurrenzfähig zu bleiben und die Krisenfestigkeit zu stärken. Im Unterrichtsbereich werden im Regelfall ausscheidende feste Lehrer/innen durch Honorarkräfte ersetzt. "(Ebd., S. 13) Es sei nur am Rande festgehalten, dass im Rahmen dieser durch einen grünen und einen sozialdemokratischen Außenminister angestoßenen Reformprozesse die Prekarisierung von Beschäftigungsverhältnissen zum "Erfolg" wird. 
ten prioritärer Bereiche Mittel umzuschichten und verstärkt nichtstaatliche Träger für die Auslandskulturarbeit zu gewinnen. ${ }^{177}$

Für das Goethe-Institut wurden hieraus umfassende Veränderungsprozesse abgeleitet, die eine Flexibilisierung im Umgang mit öffentlichen Geldern ebenso beinhalten sollten wie eine Reduzierung der Aufgaben und Mitarbeiterzahlen in der Münchner Zentrale. Stattdessen wurden die I3 Regionen ${ }^{178}$ des Goethe-Institutes gestärkt, indem man Entscheidungskompetenzen über inhaltliche Schwerpunkte, Programme und die Verteilung der Gelder hierher verlagerte. Hierdurch sollten gleichzeitig bürokratische Prozeduren vereinfacht und eine stärkere Orientierung der Programmarbeit an regionalen Rahmenbedingungen erreicht werden. Eine entscheidende Rolle spielen hierbei neue Formen von Papieren, die zwischen dem Vorstand des Goethe-Instituts und dem Auswärtigen Amt, beziehungsweise zwischen dem Vorstand und den Regionalleitern sowie zwischen den Institutsleitern einer Region für mehrere Jahre ausgehandelt werden. Von besonderer Bedeutung sind hierbei die Zielvereinbarung und die Regionalstrategien. ${ }^{179}$ In der Selbstdarstellung des GoetheInstitutes klingen diese komplexen Verfahren der Umsetzung, Administration und Dokumentation Auswärtiger Kulturpolitik folgendermaßen:

"Auf Basis der mit dem Auswärtigen Amt getroffenen Zielvereinbarung und der daraus abgeleiteten, zwischen Vorstand und Regionalleitungen festgelegten regionalen Vorhaben werden in weiten Teilen Entscheidungen direkt in den einzelnen Instituten bzw. Regionen getroffen. So werden Entscheidungsträger und Multiplikatoren aus den Sektoren Bildung, Kultur und Wissenschaft sowie Nachwuchseliten und an Deutschland interessierte Personen entsprechend den lokalen Bedingungen gezielt erreicht. “ ${ }^{180}$

Dieses Zitat verdeutlicht nicht nur die Funktion des Auswärtigen Amtes als dem relevanten Rechen(schafts)zentrum dieses Politikbereichs, sondern ver-

177 | Alle Zitate: Auswärtiges Amt: Auswärtige Kulturpolitik - Konzeption 2000, S. 3-6. 178 | Diese 13 Regionen sind zum Zeitpunkt meiner Feldforschungen (2008/09): Nordwesteuropa, Deutschland, Südwesteuropa, Mittelosteuropa, Südosteuropa, Osteuropa/ Zentralasien, Südasien, Südostasien/Australien/Neuseeland, Ostasien, Nordafrika/ Nahost, Subsahara/Afrika, Nordamerika und Südamerika. Im Zentrum dieser Regionen steht ein Regionalinstitut, das die Arbeit und die Verteilung der Gelder in der Region koordiniert. Ramallah und Sarajevo sind den Regionen Nordafrika/Nahost bzw. Südosteuropa zugeordnet. Die zuständigen Regionalinstitute befinden sich in Kairo und Athen. 179 | Die im Dezember 2007 vereinbarte Zielvereinbarung zwischen dem Auswärtigen Amt und dem Goethe-Institut e.V. besaß eine Gültigkeit für die Jahre 2008-2010. Die Regionalstrategien der für diese Arbeit relevanten Regionen Nordafrika/Nahost sowie Südosteuropa wurden gleichfalls für diesen Dreijahreszeitraum verabschiedet.

180 | Goethe-Institut: Rechenschaftsbericht 2009/2010, S. 3. 
weist zugleich auf das offizielle Narrativ einer systematischen Übersetzung und Übertragung zentraler politischer Zielsetzungen in die spezifischen lokalen Arbeitskontexte des globalen Netzwerkes.

\section{Übersetzungsketten: Verbindungslinien zwischen politischen Zentren und lokalen Handlungskontexten}

Entlang solcher bürokratischer Verfahren und Vereinbarungen werden zwei gegenläufige Übersetzungsketten sichtbar, durch die Wissensbestände nicht nur translokal wandern können, sondern die zugleich zu dem Idealbild eines kohärenten Politikbereichs beitragen.

Demnach werden die politischen Zielsetzungen der Bundesregierung in Zielvereinbarungen zwischen Auswärtigem Amt und Kulturmittlern übertragen. Hieran anknüpfend formuliert der Vorstand des Goethe-Institutes »strategische Ziele«, die sich auf die gesamte Institution beziehen und sich in den einzelnen Regionalstrategien wiederfinden müssen. Dabei liegt es an dem Leitungspersonal einer jeweiligen Region zu entscheiden, wie die strategischen Ziele auf das Einsatzgebiet bezogen werden: In der Konkretisierung der politischen Zielsetzungen in den Regionalstrategien entsteht also ein Spielraum, der mit regionalen Erfordernissen und Erfahrungen gefüllt werden kann. Auf Basis der strategischen Ziele einigen sich die Institutsleiterinnen auf »Regionalziele«, die wiederum zu den regionalen »Projekten« - also mehrjährigen, übergreifenden Schwerpunktthemen - führen. Die lokalen »Maßnahmen« der einzelnen Goethe-Institute sollen sich aus diesen regionalen Projekten ableiten. Diese Übersetzungskette lässt sich also als ein Prozess der zunehmenden Konkretisierung und Lokalisierung der eher allgemein gehaltenen Zielsetzungen des politischen Zentrums verstehen. In der Idealvorstellung sind somit den Aktivitäten eines Goethe-Instituts als Ergebnis eines mehrstufigen Übersetzungsprozesses sowohl die politischen Zielsetzungen als auch die jeweilige lokale Situation eingeschrieben.

In die andere Richtung verläuft eine Kette von Berichten, die letztlich in der Kulturabteilung des Auswärtigen Amtes mündet: Die einzelnen Goethe-Institute dokumentieren ihre Arbeit - auf Basis der in den Regionalstrategien beschlossenen Projekte und Zielsetzungen - schriftlich und mündlich gegenüber der Regionalleiterin, die wiederum die Aktivitäten der Region gegenüber dem Vorstand des gesamten Goethe-Institutes legitimiert. Der Vorstand erstellt dann auf Basis der Darstellungen aus allen Regionen einen Jahresbericht, dessen Form in der Zielvereinbarung mit dem Auswärtigen Amt festgelegt ist. Dieser Bericht enthält etwa »eine zusammenfassende Bewertung der Zielerreichung im Berichtsjahr« sowie eine »Bewertung über die Erreichung der einzelnen Ziele«, die etwa durch »einen Abgleich der IST-SOLL-Werte für 
jedes Ziel und dessen Indikatoren « zu leisten ist. ${ }^{181}$ Dem Idealbild nach landen also im Auswärtigen Amt zunehmend verdichtete, an den politischen Zielsetzungen ausgerichtete Informationen der einzelnen Mittlerorganisationen, die hier wiederum neu kombiniert und etwa der politischen oder medialen Öffentlichkeit als übergreifende Aussagen zu den kulturpolitischen Aktivitäten Deutschlands in einer Region präsentiert werden können. ${ }^{182}$

Ein leitender Beamter des Auswärtigen Amtes formulierte mir gegenüber, dass diese Übertragungswege und Referenzpapiere von zentraler Bedeutung für die eigene Zielvorstellung einer »strategischen Steuerung « der Mittlerorganisationen sind. Gerade die Zielvereinbarungen bilden demnach ein zentrales Instrument, da in ihnen gleichzeitig Rahmenbedingungen und überprüfbare Zielsetzungen formuliert sowie weitgehende operative Freiräume für die Mittler verabredet werden können. In der Praxis bedeute dies etwa, dass Mitarbeiterinnen der Abteilung Kultur und Kommunikation des Auswärtigen Amtes bei den Planungsgesprächen in den einzelnen Regionen des Goethe-Institutes anwesend seien, im Detail aber nicht eingriffen. Eventuelle Interventionen orientierten sich ausschließlich an den Zielvereinbarungen. ${ }^{183}$

Zugleich wurde in diesem Gespräch deutlich, dass diese Übersetzungsketten den politischen Zentren Bahnen zur Verfügung stellen, über die neue Schlüsselbegriffe, Zielvorstellungen, Sonderprogramme oder Schwerpunktthemen weltweit, durch das gesamte Netzwerk hindurch bis zu den einzelnen Goethe-Instituten oder Repräsentanten anderer Mittlerorganisationen mobi-

181 | Abgedruckt als Zitat aus der Zielvereinbarung 2008-2010 zwischen dem Auswärtigen Amt und dem Goethe-Institut e.V. in: Goethe-Institut: Rechenschaftsbericht 2009/2010, S. 6.

182 | Hierbei gilt es zu beachten, dass es Repräsentationen von Aktivitäten sind, die durch solche Übersetzungsketten wandern und nicht die Aktivitäten selbst: Ähnlich wie die durch Richard Rottenburg untersuchte Entwicklungszusammenarbeit, erweist sich die Auswärtige Kulturpolitik somit als ein Feld, in dem "Repräsentationen aufwendig fabriziert werden “ (Richard Rottenburg: Weit hergeholte Fakten, S. 14.) und translokal wandern. Die Feststellung, dass die komplexen Verfahren zur Dokumentation und Repräsentation Auswärtiger Kulturpolitik maßgeblich durch die Vorgaben des Auswärtigen Amtes strukturiert werden, bedeutet daher nicht zwangsläufig, dass die tatsächlichen Aktivitäten vor Ort in jedem Fall so eng an die politischen Zielsetzungen gekoppelt sind, wie es auf Basis eines Idealbildes erscheinen mag: Ein bürokratisches Verfahren, das innerhalb eines weitverzweigten Netzwerkes Berichte und Dokumentationen einfordert, kann auch Handlungsspielräume für die einzelnen Akteure in ihren lokalen Kontexten eröffnen, sofern sie den geforderten Formen des Berichtsystems Genüge leisten. Siehe hierzu auch Kapitel III.3-5.

183 | Feldnotizen vom 6. August 2008. 
lisiert werden können. ${ }^{184}$ Übersetzungsketten stellen somit zentrale Mechanismen zur Verknüpfung von politischen Diskursen und konkreten Arbeitsfeldern der Kulturmittler dar. In welchem Umfang diese bürokratischen Verfahren und Verbindungslinien die alltägliche Arbeit vor Ort tatsächlich bestimmen, bleibt dabei eine empirische Frage. Denn in diesen lokalen Handlungsfeldern kommen andere Erwartungen, Erfordernisse und Zielsetzungen ins Spiel, die von einem deutschen Ministerium nicht vorgegeben werden können. Die kulturpolitische Vermittlungsarbeit beschränkt sich daher nicht alleine auf eine möglichst stringente Übersetzung der Zielsetzungen deutscher Auswärtiger Kulturpolitik, sondern erfordert auch deren Verknüpfung mit anderen Wissensbeständen, Akteursfeldern und Handlungslogiken. Dennoch bilden die asymmetrischen Beziehungen zwischen politischen Zentren und Mittlerorganisationen zweifelsohne eine relevante Rahmenbedingung für die alltägliche Projektarbeit in den translokalen Praxis- und Übersetzungsfeldern, die sich um Goethe-Institute im Ausland entwickeln. Diese Wissensarbeit deutscher Kulturmittler in Sarajevo und Ramallah steht im Mittelpunkt des folgenden Kapitels.

184 | Mein Gesprächspartner erläuterte mir dies exemplarisch durch den Verweis auf umfangreiche Vereinbarungen, die das Auswärtige Amt mit den unterschiedlichen Kulturmittlern treffen konnte, um die politische Initiative zur Stärkung des Deutschunterrichts in Schulen im Ausland "PASCH in konkrete Aktivitäten und Kooperationen umzusetzen. 



\section{III.Translokale Formationen \\ Wissens- und Übersetzungsarbeit in Ramallah und Sarajevo}

Es bleibt die Frage, welche Relevanz die nunmehr freigelegten imaginären Dimensionen Auswärtiger Kulturpolitik mit ihren Argumentationsfiguren, politischen Rationalitäten und epistemologischen Ordnungen im Arbeitsalltag deutscher Kulturmittler besitzen. Diese Frage nach den Beziehungen zwischen Diskursen und Praxen rückt nun in den Vordergrund der Analyse. Ich verfolge hierzu die diskursive Formation einer Auswärtigen Kulturpolitik als Konfliktprävention, die Schlüsselbegriffe Deutschlandbilder vermitteln und Dialog sowie Angehörige der policy community nach Ramallah, Sarajevo und Tel Aviv. Die kulturpolitischen Handlungsfelder, die durch die Mobilisierung von Akteuren, materiellen Ressourcen und Zielsetzungen einer deutschen Politik an diesen Orten entstehen, bilden hierbei den Untersuchungsrahmen. Neben meinen ethnografischen Beobachtungen in den Goethe-Instituten in Ramallah und Sarajevo stellen Interviews mit Besucherinnen und Projektpartnern dieser Institute sowie mit Kulturmittlern, Diplomatinnen und Vertreterinnen deutscher politischer Stiftungen in Bosnien-Herzegowina, den besetzten Palästinensischen Gebieten und in Israel die empirische Basis für die nun folgende Textteile dar. Zwei analytische Perspektiven strukturieren dieses Kapitel:

Zum einen untersuche ich kulturpolitische Vermittlung als eine Wissensarbeit, die durchgängig Übersetzungen zwischen zwei Polen herzustellen versucht: den politischen Zentren in Deutschland mit ihren Zielvorgaben, Denklogiken und Interessenskonjunkturen einerseits und den lokalen Kontexten mit ihren jeweiligen materiellen Rahmenbedingungen, kulturpolitischen Erfordernissen und hier situierten Diskursen andererseits. Im zweiten Abschnitt dieses Kapitels schlage ich translokale Praxis- und Übersetzungsfelder als ein Analysemodell vor, um Grundlagen und Effekte einer solchen Wissensarbeit gerade auch vergleichend in den Blick nehmen zu können. Auf dieser Basis arbeite ich in den folgenden Abschnitten (III.3-5) vier unterschiedliche Strategien kulturpolitischer Übersetzung heraus, die diese beiden Pole auf eine jeweils andere Weise zueinander in Beziehung setzen und hierbei immer stabile und 
flüchtige Elemente - also etwa dauerhafte Infrastrukturen und temporäre Projekte - zusammenfügen; ich zeichne unterschiedliche Subjektpositionen und asymmetrische Beziehungen nach, die diese Arbeitskontexte prägen; und ich analysiere die konkreten Projektformate, diskursiven Anschlüsse und eventuellen Reibungen, die aus dieser alltäglichen Übersetzungsarbeit hervorgehen.

Zum anderen frage ich nach den Repräsentationen und Positionierungen eines europäischen Nationalstaates im Modus der Kultur, die durch diese translokale Wissensarbeit hervorgerufen und somit auf globalen Bühnen platziert werden. Hierbei geht es im Kern um die Analyse des Narrativs vom Nationalen, das im Zuge der Präsenzen, Alltagspraxen und Interaktionsmodi deutscher Kulturmittler an ihren Einsatzorten entsteht - insbesondere auch, da die Herstellung von Deutschlandbezügen weiterhin ein Schlüsselmotiv zur Begründung der eigenen Arbeit darstellt. Vor diesem Hintergrund lässt sich einerseits aufzeigen, dass ein solches Narrativ auf eine jeweils spezifische Weise aus teils altbekannten, teils flexiblen Bestandteilen zusammengefügt werden muss, um in einem lokalen Kontext auf Interesse zu stoßen und zugleich relevante kulturpolitische Effekte herbeizuführen. Andererseits arbeite ich Reibungen, Ambivalenzen und Bruchlinien im Hinblick auf den Zielhorizont einer Auswärtigen Kulturpolitik als Konfliktprävention heraus, die sich aus der fortwährenden Mobilisierung eines Narrativs vom Nationalen mit den ihm eingeschriebenen Botschaften von Differenz, Grenzen und beschränkten Zuständigkeiten ergeben.

Ich beginne dieses Kapitel aber mit einer kurzen Darstellung meiner ersten Eindrücke bei meiner Ankunft in Ramallah und Sarajevo. Dieser Abschnitt ist als eine Einführung in die beiden lokalen Kontexte angelegt, in denen die in den anschließenden Textteilen diskutierte Wissens- und Übersetzungsarbeit situiert ist.

\section{Lokale Kontexte: Ramallah und Sarajevo aus der Perspektive eines Anreisenden}

Meine Feldforschungen in Ramallah und Sarajevo begannen jeweils mit einer Anreise aus Berlin. Für meinen Aufenthalt in den besetzten Palästinensischen Gebieten flog ich Ende August 2008 nach Tel Aviv und fuhr nach zwei Tagen Zwischenstation in Jerusalem weiter nach Ramallah. Meine Forschung in Bosnien-Herzegowina begann Anfang Mai 2009 mit einem Flug nach Sarajevo. Am Flugplatz nahm ich ein Taxi, das mich zu meinem ersten Quartier am nahe gelegenen westlichen Stadtrand brachte. Beide Male näherte ich mich den Zentren der Städte - und somit auch den lokalen Goethe-Instituten - zunächst mit einem öffentlichen Verkehrsmittel. 


\section{Kontakt- und Sperrzonen: Materielle und kategoriale Trennlinien im urbanen Raum Ramallah/Jerusalem}

Im Falle von Ramallah beginnt die Fahrt auf einem Platz unweit des Damaskus-Tors der Jerusalemer Altstadt. Grün-weiße Kleinbusse bieten hier nicht nur einen einfachen Zugang zu den palästinensischen Gemeinden des Westjordanlandes, sondern gleichfalls zu einer Art Parallelwelt zu der kulturell und politisch dominanten israelisch-jüdischen Stadtgesellschaft. Dies wird mir etwa deutlich, als ich von Ramallah aus eine Tagung in Jerusalem besuche und hier bei einem Abendempfang ins Gespräch komme. Ein langjähriger Bewohner Westjerusalems zeigt sich erstaunt, als ich ihm erzähle, dass arabische Linienbusse Jerusalem und die Städte der Westbank regulär und kontinuierlich miteinander verbinden. Er demonstriert hierdurch nicht nur ein bemerkenswertes Unwissen im Hinblick auf den Stadtraum, den er bewohnt, sondern verdeutlicht zugleich, dass er eine klare Trennung als Normalfall oder als einen anzustrebenden Idealzustand betrachtet - eine Trennung zwischen Räumen und Bevölkerungsgruppen, die auch in den besagten Kleinbussen spürbar wird: Jüdische Israelis gehören niemals zu den Fahrgästen, obwohl etwa die Busse nach Ramallah oder Bethlehem den Großteil ihres Weges innerhalb Jerusalems zurücklegen.

Die Busse nach Ramallah durchqueren Ostjerusalem nach Norden und passieren hierbei ehemalige - inzwischen weitgehend verwaiste - Hauptgeschäftsstraßen des arabischen Jerusalem, bekannte historische Viertel wie die American Colony und alte arabische Nachbarschaften wie etwa Beit Hanina. Die Stadtlandschaft ist zunehmend durchzogen von jüdischen Neubaugebieten, die sich als Ausdruck des Anspruchs des Staates Israel, auch das annektierte Ostjerusalem dauerhaft in die eigene Hauptstadt $\mathrm{zu}$ integrieren, lesen lassen.

Kurz nach Beginn meiner ersten Fahrt wird der Bus von einem temporären Militär- oder Polizeiposten herausgewunken. Die Israelis kontrollieren die Fahrgäste, interessieren sich wenig für die Reisepässe von mir und zwei Italienern und fordern einige der Palästinenser auf, auszusteigen. In Ostjerusalem und in der Westbank - so wird bereits an dieser Szene deutlich - begegnen sich auf engem Raum Menschen, die über unterschiedliche Status verfügen, die im Zuge von staatlichen Handlungen und Politiken kategorisiert und Gruppen zugeordnet werden: ${ }^{1}$ jüdische Israelis, Araber mit israelischer Staatsangehörigkeit, Inhaber einer sogenannten Jerusalem ID (arabische Bewohner von Ost-

1 Ich beschreibe hier Regeln und Statusunterschiede, wie sie sich in der zweiten Jahreshälfte 2008 darstellten und Gültigkeit besaßen und die sich in ihrem konkreten Zuschnitt oder ihrer Ausgestaltung seitdem verändert haben können, ohne das Grundprinzip des Besatzungsregimes zu modifizieren. 
jerusalem), jüdische Siedler, palästinensische Bewohnerinnen der Westbank, Inhaber unterschiedlicher ausländischer Reisepässe - die Papiere entscheiden über Zugänge, Mobilität, Aufenthaltsberechtigungen oder Behandlungsformen durch israelische Sicherheitskräfte. So ist es israelischen Staatsbürgern durch israelische Gesetze in der Regel untersagt, Territorien unter palästinensischer Selbstverwaltung - also etwa Ramallah oder Bethlehem - als Zivilisten zu betreten; arabische Bewohner des Westjordanlandes dürfen wiederum Israel und Ostjerusalem nur mit einer speziellen Berechtigung betreten; die jüdischen Siedler bewegen sich hingegen selbstverständlich durch das Westjordanland und lassen sich in der Regel dabei auch nicht durch Checkpoints aufhalten - häufig auf eigenen Straßen und in eigenen Fahrzeugen; die arabischen Bewohner Ostjerusalems dürfen sich sowohl in Israel als auch im Westjordanland aufhalten; Bürger westlicher Staaten können gleichfalls Trennmauern und Checkpoints in der Regel in beide Richtungen überqueren. Den urbanen Raum zwischen der Jerusalemer Altstadt und Ramallah durchqueren diese unterschiedlichen »Kategorien« vergleichsweise häufig - eine Kontaktund zugleich Sperrzone von Ungleichen. Vier kurze ethnografische Beobachtungen, um die Bedeutung dieser Status für den Alltag und das soziale Leben in dieser Kontakt- und Sperrzone zu verdeutlichen:

- Wenn ein Bus auf seiner Fahrt von Ramallah nach Jerusalem den Checkpoint Qalandia überquert, entscheiden die Dokumente und somit der Status der Fahrgäste darüber, wer im Bus sitzen bleiben darf (Inhaber westlicher Staatsbürgerschaften, Träger einer Jerusalem ID, Fahrgäste unter- oder oberhalb gewisser Altersgrenzen...) und wer aussteigen muss, um den Checkpoint zu Fuß durch einen »Terminal« zu überqueren (erwachsene Bewohner der Westbank...).

- Wenn eine jüdischer Siedlerin und eine palästinensische Bewohnerin im Westjordanland in einen Konflikt geraten, so unterliegen sie unterschiedlichen Rechtssystemen: der Status und die Rechte der Siedlerin richten sich nach dem israelischen Zivilrecht, der Status und die Rechte der Palästinenserin nach israelischem Militärrecht.

- Ein Künstler und Galerist in Ramallah erzählt mir, dass er die Stadt seit neun Jahren nicht verlassen hat. Er stammt aus Gaza und befürchtet, dorthin gegen seinen Willen deportiert zu werden, wenn er an einem israelischen Checkpoint kontrolliert würde.

- Den palästinensischen Mitarbeitern des Goethe-Instituts ist es im Unterschied zu ihren deutschen Kollegen nicht erlaubt, den Flughafen in Tel Aviv zu nutzen, wenn sie etwa arbeitsbedingt nach Deutschland fliegen. Sie sind gezwungen, von Amman in Jordanien abzufliegen. Das gilt auch dann, wenn sie als gebürtige Palästinenser deutsche Staatsbürger geworden sind. 
Den markantesten Einschnitt auf der Fahrt nach Ramallah bildet die israelische Mauer, die hier arabische Nachbarschaften voneinander trennt, ehemalige Durchgangsstraßen blockiert und Ramallah von seinem historischen urbanen Kontext abschließt. Der Bus durchfährt den Checkpoint Qalandia auf der Fahrt nach Ramallah in der Regel unkontrolliert - der üblichste Zugangsweg, der aber zu bestimmten Anlässen - etwa zu hohen jüdischen Feiertagen - von israelischer Seite geschlossen werden kann. Jenseits des Checkpoints: ein Stau von Autos, deren Fahrer auf ein Kommando der israelischen Soldatinnen zur Weiterfahrt nach Jerusalem warten; ein vernachlässigter, zugemüllter und sandiger urbaner Raum; auf der Mauer Farbdrucke mit Silhouetten von palästinensischen Gefangenen oder Mahatma Gandhi, Graffitis mit politischen Botschaften: »CTRL + ALT + DELETE «, »I am not a terrorist «, »Free Palestine« oder »End the Occupation«; das Flüchtlingslager Qalandia, an dem die Straße nach Ramallah vorbeiführt; oberhalb der Straße und des Lagers auf einer Anhebung - in Ruf- und Sichtweite - die jüdischen Siedlungen Kokhav Ya'akov und Psagot aus weißen Einfamilienhäusern mit roten Dächern. Drei, vielleicht vier Kilometer nach dem Checkpoint beendet der Bus seine Fahrt in einem Parkhaus im Stadtzentrum von Ramallah und entlässt seine Passagiere in das immer quirlige, immer volle Straßenleben einer arabischen Kleinstadt, die zum provisorischen Zentrum der palästinensischen Selbstverwaltung geworden ist. Einige Meter von der Endhaltestelle entfernt stößt man auf den Hauptplatz der Stadt - vier Statuen von Löwen in seiner Mitte - und von hier führt eine Straße zu einem hellen, dreistöckigen Steinhaus, das durch eine deutsche, eine französische und eine EU-Fahne sichtbar markiert ist: das Deutsch-Französische-Kulturzentrum, das gemeinsam durch das Goethe-Institut und das Centre Culturel Français gebildet wird.

\section{Historische Schichtungen und europäische Resonanzräume: Urbane Ordnungen in Sarajevo}

In Sarajevo ist es eine Straßenbahn, die ich neun Monate später für meine erste Fahrt aus einer, am westlichen Ende der Stadt, unweit des Flughafens gelegenen dorfähnlichen Siedlung in das Stadtzentrum nutze. In diesem Vorort steht, umgeben von Einfamilienhäusern, ein Seminar- und Tagungshaus, das durch die deutsche Initiative Schüler Helfen Leben errichtet worden ist und in dem ich die ersten Tage meines Aufenthaltes verbringe. Am anderen, dem östlichen Ende von Sarajevo - in einem Talkessel, an drei Seiten von Bergen umgeben - befindet sich die Altstadt, die zugleich das historische Zentrum und den baulichen Abschluss der Stadt darstellt. Dazwischen liegen etwa I2 Kilometer einer in die Länge gestreckten, vielfältig strukturierten und historisch geschichteten Stadtlandschaft, die sich an dem Flüsschen Miljacka entlangzieht und erst im Osten etwas in die Breite geht. Deutlich sichtbar ist dem urbanen 
Raum seine Vergangenheit mit ihren unterschiedlichen politischen Ordnungen und Systemen, mit ihren Brüchen und imperialen Bezügen materiell und symbolisch eingeschrieben. Eine Straßenbahnlinie bildet gemeinsam mit der parallel verlaufenden Hauptverkehrsstraße die wichtigste Lebensader der Stadt und zugleich das Bindeglied zwischen ihren unterschiedlichen Teilen.

Diese Straßenbahn durchquert zunächst sozialistische Urbanisierungszonen, die den größten Teil des Stadtgebietes einnehmen: Plattenbausiedlungen, die sich - mittels rechtwinkliger Querstraßen erreichbar - über mehrere Kilometer hinziehen, durchsetzt von sozialistischen Funktionsbauten, einem Freiluftmarkt, kleinen farbigen Kiosken, einigen Hochhäusern, Sportstätten, vereinzelten Kriegsruinen, kürzlich errichteten Einkaufszentren im Stil des globalen Kapitalismus sowie von einer ebenfalls neuen, durch saudische Geldgeber finanzierten - in Bosnien-Herzegowina architektonisch ungewöhnlichen - Großmoschee.

Auch wenn die Ausgestaltung dieser Stadtteile eindeutig ihre lokalen Besonderheiten besitzt, so erscheint mir auf meiner ersten Fahrt ins Stadtzentrum diese materielle Welt des Postsozialismus aufgrund ihrer Ähnlichkeiten $\mathrm{zu}$ anderen Orten Mittelost- und Südosteuropas vertraut: Die Spuren der sozialistischen Moderne und ihrer Transformation, die Straßenbahn als urbanes Bindeglied, die slawische Sprache, in der sich die Fahrgäste unterhalten und die gleichfalls auf Plakaten und Anzeigentafeln zu finden ist, bilden Bezugspunkte für die spontane Einordnung der neuen Umgebung in einen europäischen Referenzrahmen - nicht aufgrund einer kulturellen Substanz, anhand derer sich »Europa « und »Nicht-Europa« eindeutig voneinander abgrenzen ließen, sondern aufgrund der sichtbaren Präsenz einer Vielzahl von historisch gewachsenen Bezügen, Resonanzräumen und Zeichensystemen, durch die sich ein Ort graduell mit Vorstellungen von »Europäizität« verbinden lässt.

Die Fahrt geht weiter zu älteren Vierteln, die städtebaulich auf die österreichisch-ungarische Präsenz in Bosnien verweisen: die Höhe der Häuser wird einheitlicher; hier finden sich repräsentative Verwaltungsgebäude und eine Hauptpost, eine Oper, mehrere katholische und orthodoxe Kirchen, eine große Synagoge, angelegte Stadtplätze und Parks mit Cafés, Geschäftsstraßen mit flanierenden Passanten sowie die Brücke über die Miljacka, von der aus im Jahr I9I4 der österreichische Thronfolger Franz Ferdinand und seine Frau erschossen wurden und Sarajevo als Folge kurzfristig in den Fokus der Weltpolitik rückte. An dieser Stelle befindet sich nicht nur eine Straßenbahnstation, sondern zugleich eine Gedenktafel und ein städtisches Museum, das an dieses Ereignis erinnert - weitere Verweise auf den mitteleuropäischen Kontext der Stadt.

Im Anschluss durchquert die Tram die ältesten Stadtteile, in denen die osmanische Vergangenheit sichtbar wird: etwa durch einige historische Moscheen, deren Architekten auch in Istanbul oder Damaskus ähnliche Bau- 
werke geschaffen haben, durch die basarartige Struktur einer Fußgängerzone mit Cafés, Restaurants, Kleingewerbe und Souvenirgeschäften, durch den zentralen Platz mit einem Brunnen aus türkischer Zeit und durch ein großes Gebäude, das die österreichisch-ungarischen Autoritäten explizit in einem repräsentativen, »orientalischen Stil« errichten ließen und das zunächst als Stadtverwaltung, später als Nationalbibliothek genutzt wurde - ein von den die Altstadt umgebenden Bergen weithin sichtbares Bauwerk, das von den Belagerern Sarajevos in den I990er Jahren beschossen und massiv beschädigt wurde und dessen Ruine das allmähliche bauliche Ende der Stadt markiert. Weiter nach Osten führt die Straße in die Berge hinaus; die Straßenbahn fährt hier einen Halbkreis, um in Richtung Westen zurückzukehren und den gleichen, historisch geschichteten Stadtraum erneut zu durchfahren; hier geht das Stadtzentrum in die älteren, sich die Hügel hochziehenden Wohngebiete über, die nach und nach den Charakter von Bergdörfern annehmen; und etwa hier befindet sich in einem zweistöckigen, älteren Stadthaus aus weißen Mauern und dunklem Holz auch das Goethe-Institut: »Völlig falsch gelegen«, sagt mir ein bosnischer Mitarbeiter an meinem ersten Arbeitstag, »denn hier ist die Stadt vorbei

\section{Analyserahmen: Translokale Praxis- Und ÜBersetzungsfelder}

\section{Erste Einblicke: Das Eindringen einer deutschen Policy in lokale Handlungskontexte}

Diese Bemerkung zur Lage des Goethe-Institutes fällt zu Beginn meines Forschungsaufenthaltes in Sarajevo, als zwei bosnische Mitarbeiter mir Grundzüge und Rahmenbedingungen ihrer Arbeit erklären. Bereits in diesem ersten Gespräch wird deutlich: Goethe-Institute stellen Kontexte dar, über die eine deutsche Policy mit ihren Akteuren, Zielvorstellungen und Rationalitäten in einen lokalen Raum eindringt und mit den Realitäten vor Ort verwoben wird. Zugleich vermitteln mir meine Gesprächspartner einen ersten Eindruck von den Faktoren und Problemstellungen, die aus ihrer Perspektive diese kulturpolitische Übersetzungsarbeit im institutionellen Alltag prägen - etwa die Frage, ob man sich duzt oder siezt: ${ }^{2}$

Wir duzen uns hier alle untereinander, sagen sie, nur die beiden "Entsandten" würden gesiezt. Diese wiederum würden die "Einheimischen" duzen. Allerdings gäbe es da

2 | Diese und die folgenden Passagen entstammen meinen Feldnotizen vom 11. Mai 2009. 
Unterschiede, da einige "Einheimische" geäußert hätten, dass sie gesiezt werden wollen und das würde dann auch von den "Entsandten" beachtet. Aber es würde dennoch etwas aussagen, kommentiert einer meiner Gesprächspartner und fügt hinzu, er wolle eigentlich nicht gleich am Anfang schon motzen. Im Laufe der Unterhaltung wird klar, dass "duzen " hier "Vorname und Sie " meint. Dennoch bleibt ein Unterschied: Die beiden deutschen Leiterinnen nehmen sich das Recht, die lokalen Mitarbeiter mit Vornamen anzusprechen, erwarten aber selbstverständlich, von diesen "Ortskräften “ ${ }^{3}$ mit Nachnamen adressiert zu werden.

Ein erster Faktor sind interne Hierarchien und Dynamiken innerhalb eines Goethe-Instituts, die sich hier andeuten. Hierzu passt auch die Bemerkung, dass als ein Effekt des politisch formulierten Spargebots - gerade »Ortskräfte« häufig nur »halbe Stellen« hätten, mitunter verschiedene Positionen kombinierten und zum Teil nur befristet angestellt seien. Meine Gesprächspartner erzählen von einer »permanenten Überforderung«, die sich aus dieser Personalsituation ergebe: »Man hechelt von Projekt zu Projekt, ist immer komplett mit dem Laufenden befasst und kommt eigentlich nie dazu, sich etwas fundiert zu erarbeiten und entsprechend zu planen.«

Diese Überforderung verschärfe sich eher noch durch Sondermittel, die den Goethe-Instituten in der Region in den letzten Jahren etwa aus dem Stabilitätspakt für Südosteuropa ${ }^{4}$ zur Verfügung standen: Das Budget für die Programmarbeit habe sich hierdurch temporär substanziell erhöht; ${ }^{5}$ die Zahl der Mitarbei-

3 | "Ortskraft" ist der innerhalb des Goethe-Instituts übliche Begriff, um lokale Mitarbeiter von dem entsandten deutschen Leitungspersonal abzusetzen. Ich werde diese Bezeichnungen und die sich daraus ergebenden internen Hierarchien im 3. Abschnitt dieses Kapitels diskutieren.

4 | "Stabilitätspakt für Südosteuropa» ist die Bezeichnung für eine 1999 getroffene Vereinbarung der internationalen Staatengemeinschaft, um Bemühungen zur Friedenssicherung und Stabilisierung in der Region zu bündeln und zu koordinieren. Über dieses Instrument wurden auch finanzielle Mittel zur Förderung unterschiedlicher Projekte zur Verfügung gestellt. Die Goethe-Institute in der Region konnten Anträge stellen und somit erhebliche Sondermittel für ihre Aktivitäten erhalten. Der "Stabilitätspakt" wurde 2008 durch den "Regionalen Kooperationsrat für Südosteuropa" abgelöst. Siehe für eine Übersicht zu den Zielsetzungen, der Struktur und den Arbeitsweisen des Pakts: Marie-Janine Calic: Der Stabilitätspakt für Südosteuropa. Eine erste Bilanz. In: Aus Politik und Zeitgeschichte B13-14/2001, S. 9-16; einsehbar unter: http://www.bpb.de/apuz/ 26356/der-stabilitaetspakt-fuer-suedosteuropa?p=all. (letzter Zugriff am 6. November 2017).

5 | Nach Auskunft meiner Gesprächspartner lagen die Sondermittel, die das Goethe-Institut in Sarajevo noch im Jahr 2006 aus dem Stabilitätspakt für die eigene Programmarbeit nutzen konnte, bei etwa 100.000 Euro; zum Zeitpunkt meiner Forschung 
ter, die diese Projekte dann stemmen müssen, bleibe aber in der Regel gleich. Dieser Verweis auf den Stabilitätspakt führt auch zu einem zweiten Faktor, der die Arbeit von Kulturmittlern prägt: Veränderungen im Hinblick auf die Aufmerksamkeit, die Südosteuropa aus Deutschland entgegengebracht wird:

Unter Rotgrün habe es ein starkes Interesse an den Entwicklungen in Bosnien gegeben; das sei unter der neuen Regierung deutlich abgeflaut. Entsprechend hätten sie vor einigen Jahren auch mehr in Richtung Konfliktprävention gearbeitet - nicht zuletzt, da es die Stabilitätspaktgelder möglich machten, auch "ohne direkten Deutschlandbezug" zu arbeiten und stärker "Kulturentwicklungsprojekte" zu machen. Jetzt würden sie wieder eher "klassische Goethe-Arbeit« machen - also Projekte mit Deutschlandbezug, zur Vermittlung eines Deutschlandbildes usw.

Von Interesse sind hier nicht nur die Schwankungen der Programmgelder, die mit solchen Konjunkturen der politischen Aufmerksamkeit in Beziehung stehen, sondern auch die Handlungsspielräume, die sich durch Zweckbindungen solcher Mittel eröffnen oder schließen. Die Bearbeitung der Zielvorstellung Konfliktprävention tritt hier eher als eine an Sondermittel gekoppelte Ergänzung des eigentlichen Kernauftrages Herstellung von Deutschlandbezügen hervor. Meine Gesprächspartner erwähnen explizit zwei solcher Stabilitätspaktprojekte, die sie mit meinem Forschungsinteresse in Verbindung bringen: Zum einen das Projekt think $\mathrm{BiH},{ }^{6}$ das darauf abzielte, durch Plakate, Radiospots und andere Elemente einer Werbekampagne die Identifikation mit dem Gesamtstaat Bosnien-Herzegowina zu vergrößern. Denn eine solche Identifizierung gäbe es in dem fragmentierten Land zu wenig, erklären sie mir das Ausgangsproblem und fügen hinzu, das Projekt habe »nicht funktioniert« - auch weil man dieses komplexe Thema letztlich wieder »nur antippen« konnte. Zum anderen berichten sie von einem mehrjährigen Kooperationsprojekt mit der Organisation für Sicherheit und Zusammenarbeit in Europa zur Konzipierung eines überkonfessionellen Faches Kultur der Religionen, das in den Schulen des ganzen Landes unterrichtet werden soll:

Dieses Projekt erweise sich als sehr kompliziert, etwa weil es in Bosnien-Herzegowina insgesamt 13 Bildungsminister gäbe, mit denen die Verhandlungen für die Einführung eines neuen Faches geführt werden müssten. Sie seien auf ziemliche Widerstände

im Jahr 2009 standen demnach noch etwa 25.000 Euro zur Verfügung. Die regulären Gelder für die Programmarbeit betrugen laut Auskunft meiner Gesprächspartner in diesem Jahr etwa 75.000 Euro. Diese Aussagen demonstrieren entsprechend die Dimensionen eines erhöhten Mittelzuflusses aufgrund eines temporär gesteigerten politischen Interesses.

6 | "BiH» ist die gängige Abkürzung für Bosnien-Herzegowina. 
gestoßen, gerade auch da die drei Religionsgemeinschaften Angst hätten, durch ein solches überkonfessionelles Fach ihren Einfluss zu verlieren; auch die Religionslehrer fürchteten, dass sie ihre Arbeit verlören, wenn "Kultur der Religionen“ als verpflichtendes Unterrichtsfach eingeführt würde.

Hier zeigen sich drei weitere Faktoren, welche die kulturpolitische Übersetzungs- und Vermittlungsarbeit formen: Erstens können Projekte eines Goethe-Instituts weitgehende Interventionen in den lokalen Kontext darstellen - hier in Gestalt des Bemühens, ein neues Schulfach erst zu konzipieren und dann in den Schulen zu implementieren; zweitens wird sichtbar, dass solche Interventionen häufig die Mobilisierung politischer Konzepte oder Zukunftsvisionen beinhalten - in den genannten Projekten etwa von einer »kohärenten Staatlichkeit« oder von »Toleranz« und »Versöhnung«, die aus einer Vermittlung von Wissen über religiöse Diversität erwachsen sollen; und drittens deuten sich Komplikationen, Alltagsprobleme oder Reibungen an, die entstehen können, wenn ein solches Projekt auf die konkreten Realitäten, Erwartungen und Eigenlogiken vor Ort trifft.

\section{Temporäre Formationen: Stabile Strukturen und flüchtige Elemente}

Im Zuge meiner Forschung habe ich einen Analyserahmen entwickelt, um ein solches Eindringen einer deutschen Policy in lokale Handlungskontexte ebenso in den Blick zu nehmen wie die Übersetzungsarbeit, die sich an die hiermit verbundenen Mobilisierungen von politischen Zielen, Schlüsselbegriffen und Ressourcen anschließt. Dieser Analyserahmen soll es ermöglichen, den Politikbereich nunmehr aus der Perspektive der translokalen Handlungsfelder, die er weltweit eröffnet, ethnografisch zu erschließen und hierbei insbesondere eines ihrer zentralen Charakteristika zu fokussieren: das Zusammenwirken der relativ stabilen Strukturen Auswärtiger Kulturpolitik mit eher flüchtigen Elementen, das sich sowohl als Funktionsbedingung als auch als Effekt kulturpolitischer Vermittlungsarbeit beobachten lässt. Zu den stabilen Strukturen gehören etwa die kontinuierliche finanzielle Ausstattung mit öffentlichen Geldern, das weltumspannende institutionelle Netz der Mittlerorganisationen oder auch die grundlegenden Prinzipien und diskursiven Figuren zur Legitimierung des Politikbereichs; ${ }^{7} \mathrm{zu}$ den flüchtigen Elementen zähle ich Projekt-

7 | Hierdurch möchte ich nicht sagen, dass die finanziellen Zuwendungen an die Mittlerorganisationen Jahr für Jahr gleich ausfallen oder die Formulierungen von Zielsetzungen identisch bleiben. So wurde im Kapitel II.6 bereits dargestellt, dass es durchaus Schwankungen in der finanziellen Ausstattung des Politikbereichs gibt, die etwa zu Schließungen oder Neueröffnungen von Goethe-Instituten an bestimmten Orten und somit zu Veränderungen in der Ausgestaltung des Netzwerkes führen können. Gleich- 
ideen, temporäre Kooperationspartner, mobile Metaphern und Wissensbestände oder zeitlich begrenzt verfügbare Finanzierungsquellen, die im Zuge der kulturpolitischen Arbeit aktiviert oder einbezogen werden. Von einem herausgehobenen Interesse ist hierbei, wie sich im Rahmen einer Veranstaltung, eines Förderprogramms, einer Kooperation oder eines Netzwerkes stabile Strukturen und flüchtige Elemente auf eine jeweils spezifische Weise zusammenfügen und als temporäre Formationen vielfältige kulturpolitische Effekte hervorrufen. Ich spreche von translokalen Praxis- und Übersetzungsfeldern, um eben diese Prozesse des Zusammenfügens analysieren zu können. »Projekte« bilden hierbei nicht nur den üblichen Rahmen, in dem unterschiedliche lokale oder institutionelle Kontexte in Beziehung gebracht, Bedeutungen ausgehandelt, Ressourcen genutzt und Übersetzungspraxen entwickelt werden; sie bieten zugleich einen geeigneten Einstieg, um translokale Praxis- und Übersetzungsfelder ethnografisch zu untersuchen. Mein Verständnis von diesem Analyserahmen möchte ich nun anhand des Projektes der »deutsch-palästinensischen Seifenoper«, das bereits zu Beginn dieser Studie Erwähnung fand, erläutern. ${ }^{8}$

\section{Das Beispiel Matabb: Die "palästinensische Soap" als ein translokales Praxis- und Übersetzungsfeld}

Diese Serie entstand im Rahmen eines Projektes mit europäischen Fördergeldern und lokalen Verankerungen, mit translokalen Kooperationsbeziehungen und Wissenstransfer, mit internen Hierarchien und einem komplexen »sozialen Leben «. ${ }^{9}$ Zur Realisierung von Matabb bildete sich eine temporäre Formation, in der die Serie ihre narrative Struktur und filmische Gestalt erhielt. Die Charaktere und ihre Geschichten, die Plots und die politischen Haltungen, die Matabb auszeichnen, verweisen auf den organisatorischen Rahmen einer

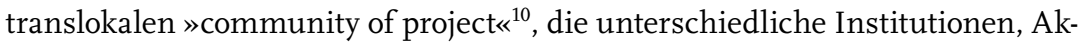

zeitig ließ sich dort aufzeigen, dass es ein grundlegendes und langfristiges politisches Bekenntnis zu den übergreifenden Zielsetzungen und zentralen Akteuren des Politikbereichs gibt, das verlässliche und relativ stabile Strukturen mit sich bringt.

8 Die folgende Beschreibung basiert auf meinen Feldnotizen aus dem September 2008 sowie auf Gesprächen, die ich im Rahmen meiner Feldforschung in Ramallah mit unterschiedlichen Projektbeteiligten führen konnte.

9 | Steven Sampson: The Social Life of Projects: Importing Civil Society to Albania. In: Chris Hann \& Elizabeth Dunn (Hrsg.): Civil Society. Challenging Western Models. London 1996, S. 121-142.

10 | Manfred Faßler: Globalisierung und Geosozialität. In: Herbert Willems (Hrsg.): Lehr(er)buch Soziologie. Für die pädagogischen und soziologischen Studiengänge. Wiesbaden 2008, S. 455-485. 
teure, Wissensbestände und Ressourcen temporär miteinander verband. Im Zentrum dieses sozialen Gefüges stand ein $»$ Kulturformat $\aleph^{11}$ - die Seifenoper -, das offenbar global verstanden wird und Akteure an unterschiedlichen Orten der Welt zu mobilisieren vermag. Für einen begrenzten Zeitraum bildeten das Goethe-Institut in Ramallah als Initiator, das Institute of Modern Media der Al-Quds-Universität als wichtigster lokaler Partner, die Europäische Union und die deutsche Gesellschaft für technische Zusammenarbeit als Geldgeber, Produktionsmitarbeiter und Drehbuchautorinnen der deutschen Fernsehserien Dahoam is Dahoam und Lindenstraße als Mentorinnen und Werkstattleiter sowie palästinensische Schauspieler, Produktionsleiter, Kamera- und Tonleute, ein Regisseur usw. einen verdichteten Kommunikations- und Handlungsraum, der nach Beendigung des Projektes wieder auseinanderlief. Trotz ihrer Flüchtigkeit erwies sich diese translokale Formation als in vielfacher Hinsicht produktiv: Die Charaktere der Serie und ihre Konflikte wurden etwa im Zusammenspiel von deutschen und palästinensischen Akteuren entworfen und konkretisiert; palästinensische Teilnehmer an einem Drehbuchworkshop diskutierten grundlegende gesellschaftliche und politische Problemstellungen und deren mögliche Personifizierung; deutsche Drehbuchautorinnen vermittelten in diesem Rahmen strukturelle und dramaturgische Regeln des Formats »Soap-Opera« und verdichteten später die Probleme und Charaktere zu einem Handlungsstrang mit Episoden; der Leiter des Goethe-Instituts fungierte als Co-Autor des Drehbuchs; der palästinensische Regisseur milderte die Konflikte der Charaktere, um lokale Tabus zu streifen, aber nicht zu brechen und verfasste die Dialoge. Somit bildete sich ein temporäres Praxis- und Übersetzungsfeld, in dem das Format einer gesellschaftskritischen Seifenoper in die politische und soziale Komplexität des palästinensischen Alltags übertragen wurde. Durch das Endprodukt wurden gleichfalls die sozialen und politischen Problemkonstellationen eines lokalen Raums - der besetzten Palästinensischen Gebiete - auf eine spezifische Weise gerahmt und in der Folge über das Internet, durch Vorführungen in unterschiedlichen Goethe-Instituten und auf Festivals international verbreitet.

Diese translokale Formation erweitert sich mit der Fertigstellung von $\mathrm{Ma}$ tabb um mediale Akteure. Nicht nur die Tagesthemen, sondern auch Printmedien berichten von der Lindenstraße in Ramallah. Unter der Überschrift Mutter Beimer trägt jetzt Kopftuch spürt etwa Spiegel Online den Deutschlandbezügen des Projekts nach:

11 | Siehe zum Begriff "Kulturformat»: Wolfgang Kaschuba: Europäisierung als kulturalistisches Projekt? Ethnologische Beobachtungen. In: Hans Joas \& Friedrich Jaeger (Hrsg.): Europa im Spiegel der Kulturwissenschaften. Baden-Baden 2008, S. 204-225. 
"Um Erfahrungen zu sammeln, haben sich der Produzent vom Goethe-Institut und der palästinensische Regisseur George Khleifi von der Al-Quds-Universität vor einem Jahr nach Köln-Bocklemünd aufgemacht, um drei Tage lang die Dreharbeiten zur Lindenstraße، zu beobachten. 'Die Bedingungen sind nicht übertragbarı seufzt Majari [...] 'Die Lindenstraße hat für eine Folge mehr Geld als wir für die ganze Serie`, sagt er; 170.000 Euro spendierten Deutschland und die EU. Kein Wunder, dass auch ein bisschen Deutschland drinsteckt in der Serie. Die NGO-Kulisse sieht aus wie das Goethe-Institut mit ordentlich aufgereihten Aktenordnern und Pinnwänden - was nicht zuletzt daran liegt, dass ein Teil der Ausstattung tatsächlich von dort stammt. " ${ }^{12}$

Die medialen Berichte erzählen somit - teils wohlwollend, teils mit ironischem Unterton - von der Übersetzung des »deutschen Formats« einer gesellschaftskritischen Seifenoper in einen anderen politischen und kulturellen Kontext. Neben einzelnen Plots und Serienfiguren sind es die geringeren finanziellen Möglichkeiten, der Zwang zu künstlerischen und technischen Improvisationen sowie eher stereotypisierende Verweise auf die politischen Rahmenbedingungen über die das Projekt in die deutsche Öffentlichkeit rückübertragen wird:

"Man muss sich an die Ästhetik gewöhnen`, gibt auch Farid Majari zu, Leiter des Goethe-Instituts in Ramallah und neuerdings Serienproduzent. Es ist ja auch nicht einfach, mit einer Handvoll Laienschauspielern, nur einer Kamera und einem Kameramann, der sonst Kriegsreportagen dreht. Ganz abgesehen davon, dass Ramallah eben nicht Köln-Bocklemünd ist, weshalb die Kulissen in einem ehemaligen Fitnessstudio untergebracht und die Möbel aus Pressholz sind. Auch Außendrehs sind heikel, weil palästinensische Jugendliche in Anwesenheit einer Kamera gerne 'Nieder mit Israelı skandieren, es könnte ja CNN sein. "13

In der Süddeutschen Zeitung tritt noch stärker eine kulturalistische Argumentationslinie hinzu, um den Entstehungskontext von Matabb als einen von Deutschland deutlich unterschiedenen zu präsentieren: »Die Version der deutschen >Lindenstraße < birgt einigen Sprengstoff für die palästinensische Gesellschaft, die wie alle arabischen Gesellschaften an Tabus strikt festhält. « ${ }^{14}$

12 | "Palästinensische 'Lindenstraßeı: Mutter Beimer trägt jetzt Kopftuch", erschienen auf Spiegel Online am 27. August 2008. Der Bericht in den Tagesthemen fand bereits in der Einleitung Erwähnung. Auch Zeit Online veröffentlichte am 25. August 2008 einen Artikel unter dem Titel "Lindenstraße in Ramallah".

13 | "Palästinensische 'Lindenstraße: Mutter Beimer trägt jetzt Kopftuch", auf Spiegel Online.

14 | "Bremsschwellen im Alltagsleben. Das Goethe-Institut in Ramallah produziert die erste palästinensische Seifenoper mit dem Titel 'Matabbı", in: Süddeutsche Zeitung vom 26. August 2008. 
Anhand dieser Beobachtungen und medialen Berichte lassen sich einige Dimensionen des translokalen Praxis- und Übersetzungsfeldes, das sich um Matabb entwickelt hat, festhalten: die Relevanz der stabilen Struktur Auswärtiger Kulturpolitik, die zu der lokalen Präsenz des Goethe-Instituts und somit zum Ausgangspunkt des Projektes geführt hat; die temporären Verknüpfungen zwischen ganz spezifischen lokalen und institutionellen Kontexten, unterschiedlichen Akteuren, Wissensbeständen und finanziellen Ressourcen; die Produktivität dieser Konstellation, die sich nicht nur im filmischen Ergebnis zeigt, sondern etwa auch in den Aushandlungsprozessen zu Plot, Problemkonstellationen und Charakteren der Serie; die Reproduktion von kulturalisierenden Wahrnehmungsmustern zu geografischen Räumen, politischen Konstellationen oder »anderen« Lebensweisen und somit die Mobilisierung von symbolischen Bedeutungen; sowie die Reibungen, die aus solchen translokalen Übersetzungen entstehen können. Es handelt sich hierbei um Dimensionen, die in Variationen auch für die Analyse anderer translokaler Praxis- und Übersetzungsfelder von Relevanz sind.

\section{Träger sozialen Wandels und Arenen kultureller Produktion: Projekte als Felder kultur- und sozialanthropologischer Forschung}

Eben diese translokale Formation macht Matabb auch über das Endprodukt hinaus für eine empirische Untersuchung Auswärtiger Kulturpolitik interessant und bietet zugleich einen möglichen Anknüpfungspunkt für eine Analyse der »kulturellen Konsequenzen von Globalisierungsprozessen.« ${ }^{15} \mathrm{Im}$ Zentrum stand hier ein Projekt und somit eine Organisationsform, die - so hat es gerade auch die jüngere sozial- und kulturanthropologische Forschung gezeigt - viele Felder inter-/transnationaler Kooperation und Verflechtung maßgeblich strukturiert. ${ }^{16}$ »Projekte « lassen sich als »temporäre Handlungszusammenhänge von Akteuren, die ein gemeinsames Interesse realisieren und dann

15 | Gisela Welz: "Sighting / Siting globalization. "Gegenstandskonstruktion und Feldbegriff einer ethnographischen Globalisierungsforschung. In: Sonja Windmüller, Beate Binder \& Thomas Hengartner (Hrsg.): Kultur - Forschung: Zum Profil einer volkskundlichen Kulturwissenschaft. Münster u.a. 2009, S. 195-210, S. 195.

16 | Siehe exemplarisch: Kimberley Coles: Democratic Designs. International Intervention and Electoral Practices in Postwar Bosnia-Hercegovina. Ann Arbor 2007; Ruth Mandel: Seeding civil society. In: Chris Hann: Postsocialism. Ideals, ideologies and practices in Eurasia. London, New York 2002, S. 279- 296; Shalini Randeria \& Ciara Grunder: The (Un)Making of Policy in the Shadow of the World Bank: Infrastructure Development, Urban Resettlement and the Cunning State in India. In: Cris Shore, Susan Wright \& Davide Però (Hrsg.): Policy Worlds, S. 187-204; Steven Sampson: Beyond transition: rethinking elite configurations in the Balkans. In: Chris Hann: Postsocialism, S. 297-316; 
wieder auseinandergehen «, verstehen ${ }^{17}$ Sie stellen flexible, nach außen nicht eindeutig abgeschlossene Einheiten dar, in denen sich nicht nur internationaler kultureller Dialog, sondern auch Entwicklungszusammenarbeit, die Förderung von Demokratisierungsprozessen oder wirtschaftspolitische Restrukturierungsprogramme konkret ereignen. Projekte geben somit der Flüchtigkeit translokaler Interaktionen eine vorübergehende Form und bieten daher auch eine Zugriffsmöglichkeit, um globalen Verknüpfungen und Machtfeldern ethnografisch nachzuspüren. Hierbei sind zwei Aspekte von besonderer Relevanz, die sich anhand von Matabb bereits andeuteten:

Einerseits lassen sich Projekte als Träger sozialen Wandels untersuchen. ${ }^{18}$ Internationale Organisationen, nationalstaatliche Regierungen, NGOs oder Stiftungen initiieren ihre transnationalen Projekte zumeist mit der Zielsetzung, Transformationsprozesse anzustoßen oder zu unterstützen. Im Zentrum stehen häufig global mobile Werte oder gegenwärtige Zielhorizonte der »internationalen Gemeinschaft«, die in diesem Rahmen eine Operationalisierung erfahren und in den Zielregionen etwa zu Stabilisierung, guter Regierungsführung, Menschenrechten oder Aussöhnung führen sollen. ${ }^{19}$ Solche Projekte stellen somit immer Interventionen in lokale Kontexte und hier situierte Diskussionen dar, in die sie politische Absichten und Vorstellungen von einer »wünschenswerten Zukunft« transportieren. Insbesondere in »Transformationsländern « oder »Konfliktgebieten« kommt es zu Zusammenballungen solcher Projekte, die weitgehend von einem externen Mittelzufluss abhängig sind. $\mathrm{Zu}$ den sichtbaren Konsequenzen solcher Interventionen gehören das Auftauchen einer mobilen Projektelite, die Etablierung einer besonderen Projektsprache, die etwa zur Verfassung von Anträgen und Berichten genutzt wird, oder auch die Entstehung eines neuen Arbeitsmarktes an den Einsatzorten. Gleichzeitig ergeben sich aus der Mobilisierung politischer Zielhorizonte innerhalb der lokalen Handlungsfelder häufig »Reibungen«, die zu nicht-intendierten Effekten führen können. ${ }^{20}$

Gisela Welz \& Annina Lottermann (Hrsg.): Projekte der Europäisierung. Kulturanthropologische Forschungsperspektiven. Frankfurt 2009.

17 | Gisela Welz \& Annina Lottermann: Projekte der Europäisierung. Einleitung. In: Dies. (Hrsg.): Projekte der Europäisierung, S. 11-16, S. $13 \mathrm{f}$.

18 Siehe hierzu etwa: Sally Falk Moore: Comparisons: Possible and Impossible. In: The Annual Review of Anthropology, 34 (2005), S. 2-11.

19 | David Mosse: Politics and Ethics: Ethnographies of Expert Knowledge and Professional Identities. In: Cris Shore, Susan Wright \& Davide Però (Hrsg.): Policy Worlds, S. 50-67.

20 | "Reibungen" nach der bereits zitierten Studie von Anna Lowenhaupt Tsing: Friction. Auch die Formulierung "arena of cultural production « erfolgt in Anlehnung an Tsing, die sie aber nicht auf "Projekte" bezieht. Ebd., S. 51. 
Andererseits bilden Projekte Arenen kultureller Produktion, wie sich am Beispiel von Matabb gut verdeutlichen lässt. Die Produktivität dieser temporären Formation zeigt sich nicht nur an dem filmischen Ergebnis, sondern insbesondere auch an Bedeutungen, Wertigkeiten und Wahrnehmungsmustern, die in diesem Rahmen ausgehandelt, als »soziale Tatsachen« gesetzt und vermittelt wurden. Dies gilt zunächst für die Herstellung unterschiedlicher Raumbilder und -bezüge: Neben den translokalen Verflechtungen in der Produktionsphase ist es vor allem die Konstruktion des lokalen Raums durch die Inszenierung bestimmter Orte und Geschichten sowie durch die Einbeziehung politischer und sozialer Problemlagen, die in dieser Serie hervorsticht. Gleichzeitig werden durch Matabb gewisse Idealvorstellungen von der individuellen und kollektiven Lebensführung aufgerufen und anhand der Beziehungen und Auseinandersetzungen der Protagonisten vermittelt. Als Leitgedanken durchziehen beispielsweise die Ideale der Gleichberechtigung der Geschlechter oder der friedlichen Konfliktlösung die Narration.

Auf dieser Basis betrachte ich in dieser Studie Projekte als den üblichen Kern der translokalen Praxis- und Übersetzungsfelder, die sich im Zuge der Umsetzung Auswärtiger Kulturpolitik entwickeln. Von einem besonderen Interesse sind hierbei die Reibungen, die aus dem Aufeinandertreffen von mobilen Schlüsselbegriffen, Idealvorstellungen, Personengruppen und lokalen Kontexten entstehen. Solche Reibungen zeigten sich gleich zu Beginn meiner Forschung in Ramallah anhand der Versuche, das filmische Produkt von $\mathrm{Ma}$ tabb in den lokalen Kontext einzuarbeiten.

\section{Reibungen: Wissen und Nicht-Wissen in der kulturpolitischen Vermittlungsarbeit}

Mit Matabb begann meine Forschung in den Palästinensischen Gebieten. Zwar hatte ich nicht an der Planung und Umsetzung des Projektes teilgenommen; von dem Schreibtisch, den ich am 2. September 2008 in der Programmabteilung des Goethe-Instituts bezog, konnte ich aber die Diskussionen der Mitarbeiterinnen zu unerwarteten Entwicklungen beobachten: Das letzte Glied einer Übersetzungskette zur Realisierung der Projektziele von Matabb - die Verankerung der fertiggestellten Serie in den lokalen Kontext - drohte zu scheitern.

Das Kalkül des Goethe-Instituts und seiner Partner hatte darin bestanden, $M a t a b b$ während der ersten zehn Tage des Ramadan über den palästinensischen Fernsehsender PBC auszustrahlen. Der Ramadan ist in Palästina Seifenoper-Zeit: Nach einem langen Tag des Fastens kommen die muslimischen Familien mit Sonnuntergang zu einem üppigen Mahl zusammen und parallel verfolgt man gemeinsam Abend für Abend die Episoden zumeist syrischer, 
ägyptischer oder türkischer Serien. ${ }^{21}$ Matabb - so war es der Wunsch der Macher - sollte in diese jährliche Primetime für Soap Operas platziert werden. An die Stelle der Verwicklungen aus dem kolonialen Damaskus - in einer der populärsten Serien - könnten somit lokale Geschichten treten. Der Anspruch, durch Matabb »Tabus « zu thematisieren und lokale Diskussionen zu beeinflussen, sollte durch eine solche breite Verankerung realisiert werden. Und mehr noch: Die Projektziele beinhalteten die Hoffnung, dass sich über das Satellitenprogramm der $\mathrm{PBC}$ auch das Publikum in den arabischen Nachbarstaaten für die palästinensische Soap begeistern ließe. In dem Antrag an die Europäische Union zur Finanzierung der Serie wurde sogar die Erwartung formuliert, dass hierüber auch kommerzielle Partner zur Fortführung von Matabb gefunden werden könnten.

Am ersten Abend des Ramadan 2008 geschieht nichts dergleichen. Die Serie wird trotz der Vereinbarungen mit einem Programmdirektor des Fernsehsenders, trotz der - aus Perspektive des Goethe-Instituts - rechtzeitigen Übergabe der DVDs an den Sender und trotz öffentlicher Ankündigungen nicht ausgestrahlt. Am nächsten Morgen - meinem ersten Arbeitstag - bestimmt dieses Thema die Unterhaltungen und den Arbeitsrhythmus im Goethe-Institut: Die zuständige Mitarbeiterin telefoniert durchgängig mit Projektpartnern, Geldgebern, Journalisten und dem Institutsdirektor, der zu diesem Zeitpunkt im Urlaub ist. Im Laufe des Tages betreten unterschiedliche Mitarbeiter die Programmabteilung und berichten von Gesprächen mit Freunden oder Partnern des Instituts, die zum vorgesehenen Zeitpunkt PBC einschalteten und über die Nicht-Ausstrahlung erstaunt waren. Auch eine Erklärung oder Information zu dieser Programmänderung hat es offenbar nicht gegeben. Vor meinen Augen entfaltet sich ein Gewebe aus Spekulationen, Vermutungen und Halb-Wissen, um die überraschenden Entwicklungen zu interpretieren. Alle diese Deutungsversuche zeichnen sich durch ihren spekulativen Charakter, ihre eher situative Überzeugungskraft und letztlich fehlende Klarheit über die »wahren Gründe« aus.

Da ich gerade meine ersten Tage im Institut verbringe, ist es wenig verwunderlich, dass mir die Zusammenhänge schwer durchschaubar erscheinen. Aber auch den Mitarbeitern bleiben offensichtlich nicht nur die Gründe für die Nicht-Ausstrahlung unklar, sondern zunächst auch die Frage, wer eigentlich die Absage einer Vereinbarung zwischen einem deutschen Kulturinstitut und einem palästinensischen Fernsehsender veranlassen kann: So hat eine Mit-

21 Siehe hierzu etwa den Artikel "Türkische Fernsehserien. Eine kulturelle Revolution " in der FAZ vom 20. November 2012. Diese Textpassagen geben den Stand der Dinge zurzeit meiner Feldforschung im Jahr 2008 wieder und beziehen eventuelle Veränderungen durch später einsetzende, dramatische politische Entwicklungen in den genannten Ländern nicht ein. 
arbeiterin von einer »Kommission « gehört, welche innerhalb oder im Umfeld des Fernsehsenders die Serie erst noch »überprüfen« müsse. Sofern nichts zu beanstanden sei, könne Matabb dann in einigen Tagen gesendet werden. An anderer Stelle wird auf einen Präsidentenberater verwiesen, der die Ausstrahlung gestoppt habe. Das Wort von der »Zensur« macht nun die Runde durch das Institut. Ein kritischer Artikel, den ein bekannter Journalist angeblich geschrieben habe, wird zwischenzeitlich als eine andere Ursache angeführt. Ebenso vielfältig sind die Gerüchte über die angeblich monierten Stellen der Serie: Ein Mitarbeiter hat gehört, die PLO sei als zu korrupt dargestellt; eine andere Mitarbeiterin liefert den Hinweis, die Israelis und Europäer seien zu positiv, Palästinenser hingegen zu negativ gezeichnet; dem Regisseur der Serie wird zugetragen, eine Szene, in der Palästinenser israelischen Soldaten Blumen reichten, habe die Ausstrahlung verhindert. Die Ratlosigkeit nimmt zu, da es entweder solche Sequenzen gar nicht gibt oder die Kritikpunkte als inhaltlich unbegründet erscheinen: Missverständnisse und Unkenntnis werden neben politischer Einflussnahme und Unprofessionalität des Fernsehsenders als Ursachen für die Nicht-Ausstrahlung diskutiert.

Nach seiner Rückkehr aus dem Urlaub beginnt der Institutsdirektor, aktiver auf die Absage zu reagieren: Kurzfristig wird eine abendliche Ausstrahlung der Serie im Veranstaltungsraum des Deutsch-Französischen Kulturzentrums $^{22}$ angesetzt. Ebenso kurzfristig werden Besucher und Partner des Goethe-Instituts, Angehörige der Kulturszene und der internationalen Community in Ramallah zu diesen Vorführungen eingeladen, die um die zwanzig Personen anziehen. Eine Pressemitteilung wird verfasst, in der die Verantwortung für die Nicht-Ausstrahlung und somit das drohende Scheitern einer breiteren lokalen Verankerung des Projektes alleine dem palästinensischen Sender zugewiesen wird:

"The PBC, however, cancelled 'Matabb , without prior notice replacing it with another local TV serial. As-to-date no official explanation has been provided by the PBC explaining its action. The PBC cancellation came as a complete surprise to the Producers who granted the PBC, upon its request, the right to broadcast the new TV serial before other broadcasters. The PBC had ample time to review the tapes of the serial and its trailer which were delivered in due time.

Yahia Barakat, Director General of Programming at the PBC, who had been the Goethe Institute's liaison at the PBC was abroad on a trip when the show was cancelled. Upon his return, Mr. Barakat informed the Goethe-Institute that some scenes in the serial had raised a red flag with the authorities. He referred to one passage in which the PLO is

22 Das Goethe-Institut und das Centre Culturel Français in Ramallah teilen nicht nur Räumlichkeiten, sondern positionieren sich - zumindest dem Ideal nach - als ein integriertes Kulturzentrum in der Stadt. 
allegedly mentioned as corrupt. In this relatively marginal scene the PLO is investigating fraud during the Lebanese civil war and is therefore not depicted in a negative way. On September 7, Mr. Barakat told the Goethe-Institute's director that a committee will review the series and make a decision on whether to air it at the end of Ramadan. "23

Palästinensische Kooperationspartner oder Besucherinnen des Goethe-Instituts teilen zumeist die Einschätzung des PBC als technisch und organisatorisch unprofessionell oder politisch kontrolliert und entwickeln doch gleichzeitig partiell Verständnis für eine kritische Haltung gegenüber Matabb. Die Serie sei »voller Stereotype«, sagt die Mitarbeiterin einer NGO, »und es sind die europäischen Stereotype über die Region, nicht die arabischen«. Das drohende Scheitern der lokalen Verankerung erscheint in solchen Aussagen nicht als ein bloßes organisatorisches Problem, sondern lässt sich demnach auch über inhaltliche Hintergründe deuten. In eine ähnliche Richtung weist die Verärgerung zweier palästinensischer Journalisten über die erste Szene der Serie: Eine Gruppe von Jugendlichen wirft hier Molotow-Cocktails auf ein gerade entstehendes Theatergebäude in Jenin. Matabb reproduziere hierdurch - so die beiden Journalisten - die negativen Vorurteile von »Gewalt und Terror«, die westliche Akteure gegenüber Palästina hegten.

Auch die lokalen Mitarbeiter des Goethe-Instituts beginnen nun zaghaft Kritik am eigenen Vorgehen zu äußern: Hatte man die DVDs tatsächlich zeitig genug an den Fernsehsender übergeben? Und hatte man hier mit der richtigen Person die Absprachen getroffen? Und war der örtliche Sender PBC überhaupt der passende Partner in Anbetracht der Tatsache, dass das lokale Fernsehpublikum primär Satellitenprogramme aus den arabischen Nachbarstaaten rezipiert? Als eine »Ortskraft« mir gegenüber formuliert, eigentlich hätten die Palästinenser keine Lust, sich auch noch im Ramadan mit ihrer politischen Misere zu befassen, stelle ich mir die Frage, ob sich das Problem der lokalen Verankerung von Matabb nicht zumindest zum Teil als ein internes Übersetzungsproblem zwischen deutschen und palästinensischen Mitarbeitern im Goethe-Institut erklären lässt. Die innere Distanz und kritische Haltung, die lokale Akteure gegenüber den »wohlwollenden Interventionen« deutscher Kulturpolitik einnehmen können, verdeutlicht die gleiche Mitarbeiterin, nachdem sie in der Zeitung einen Artikel über ein deutsch-israelisches militärisches Kooperationsprojekt gelesen hat: »Den Israelis schicken die Deutschen U-Boote, wir bekommen eine Soap.«

Diese Beobachtungen verdeutlichen, wie die Um- und Übersetzungen von Zielen, Plänen und Absprachen in der alltäglichen Arbeit eines Kulturinsti-

23 Goethe-Institut Palestine: Press Release September 8, 2008. Palestinian-German TV Serial "Matabb" (Speed Bump) cancelled by Palestinian Broadcasting Corporation $(\mathrm{PBC})$. 
tuts ins Stocken geraten können. Anna Lowenhaupt Tsing hat vorgeschlagen, solche »Reibungen« als ethnografische Ansatzpunkte zu nutzen, um globale Verknüpfungen sowie die neuen kulturellen und politischen Arrangements, die aus ihnen erwachsen, zu untersuchen - Reibungen, die etwa aus dem Eindringen von mobilen universalen Begriffen und Wertvorstellungen in lokale Räume entstehen; oder Reibungen, die sich im Aufeinandertreffen von Projekten und Machtkonstellationen mit ihren jeweils unterschiedlichen räumlichen Verankerungen zeigen: »Attention to friction opens the possibility of an ethnographic account of global interconnection. Abstract claims about the global can be studied as they operate in the world. We might thus ask about universals not as truths or lies but as sticky engagements. ${ }^{24}$

Reibungen - das zeigt das Beispiel von Matabb - stellen sich in der alltäglichen Arbeit eines Kulturinstituts gerade im Zuge der erforderlichen Kooperationen und Interaktionen her: Die Mitarbeiterinnen sehen sich in der Bearbeitung der Probleme und Verwicklungen um die Seifenoper immer wieder gezwungen, durch partiell unbekanntes Territorium zu navigieren. Deutungen der jüngsten Entwicklungen in einer Kooperation oder Entscheidungen zu den nächsten Organisationsschritten basieren häufig auf unsicheren Vermutungen oder »Nicht-Wissen« - etwa über die Reaktionsweisen von Partnern, über bestimmte politische oder gesellschaftliche Rahmenbedingungen und deren Auswirkung auf den Projektablauf oder auch über die Effekte der eigenen Arbeit.

Diese Konfrontation mit Nicht-Wissen zeigt sich zunächst im institutionellen Alltag: Mitunter mangelt es an sprachlichen Kompetenzen, um sich etwa in dem komplexen Umfeld eines deutsch-französischen Kulturzentrums als Teil einer englischsprachigen internationalen Community in einem arabischsprachigen Kontext unter einer hebräischsprachigen Besatzung eine Situation, einen Zeitungsartikel zu erschließen oder ein Papier termingerecht in der jeweils erforderlichen Sprache zu verfassen. Häufig sind es aber auch ganz alltagspraktische und logistische Fragestellungen, zu denen Mitarbeitern ein Wissensfragment fehlt, um ein Vorgehen fundiert festzulegen: Wer ist die richtige Person, um die Ausstrahlung einer Fernsehproduktion im palästinensischen Fernsehen zu vereinbaren? Wen gilt es zu kontaktieren, wenn eine solche Absprache nicht eingehalten wird? Wird es gelingen, eine Person oder erforderliche Materialien trotz aller - in ihrem aktuellen Ausmaß unvorhersehbaren - Mobilitätsbeschränkungen durch die israelische Besatzung rechtzeitig zu einem Projektort zu bekommen? Und: Ist es angemessen, auf einem Oktoberfest vor dem Institut Bier auszuschenken und Schweinewürstchen zu servieren? Welche Konsequenzen können daraus entstehen? Es sind solche mitunter sehr kleinteiligen Fragen, die in zeitraubenden Diskussionen zwi-

24 | Anna Lowenhaupt Tsing: Friction, S. 6. 
schen entsandten und lokalen Mitarbeiterinnen, die Hierarchieleitern hinauf und hinab und unter Hinzuziehung externer »Experten« diskutiert und entschieden werden.

Die Bedeutung von Nicht-Wissen zeigt sich aber auch in der Umsetzung umfassender politischer Zielvorstellungen oder in der grundsätzlichen Verständigung über die Ergebnisse der eigenen Arbeit: »Wie sich Zivilgesellschaft hier wirklich stärken lässt, weiß doch kein Mensch«, formulierte mir gegenüber etwa ein Kulturmittler in Ramallah. Und die im Abschnitt I.I zitierten Positionen zu Konfliktprävention als einer Zielsetzung Auswärtiger Kulturpolitik weisen in die gleiche Richtung: Sowohl in der Bearbeitung von Alltagsfragen als auch anhand der längerfristigen Zielvorstellungen, sowohl in logistischen Problemstellungen als auch in Einschätzungen zum politischen und kulturellen Kontext wird deutlich, dass es sich bei der praktischen Umsetzung Auswärtiger Kulturpolitik in »Konfliktgebieten« um eine anspruchsvolle Arbeit entlang der Grenze von »Wissen« und »Nicht-Wissen« handelt, in deren Realisierung spekulative Vermutungen oder direkte Unkenntnis durch unterschiedliche Praxisformen in - zumindest situativ gültiges - »Wissen« verwandelt werden.

\section{Verorten, Verankern, Anschlüsse schaffen, Verknüpfen: Strategien kulturpolitischer Wissens- und Übersetzungsarbeit}

Vor diesem Hintergrund bildet die Wissensarbeit von Kulturmittlern, neben Projekten, den zweiten Schwerpunkte, um in den verbleibenden Teilen dieses Buches das Eindringen einer deutschen Policy in unterschiedliche lokale Kontexte ethnografisch nachzuzeichnen. Wissensarbeit betrachte ich dabei als Übersetzungsarbeit, die kulturpolitische Diskurse und Alltagspraxen direkt zueinander in Beziehung bringt und in diesem Rahmen immer auch stabile Strukturen und flüchtige Elemente zu translokalen Formationen zusammenfügt. Mit diesem Fokus schließe ich an Positionen an, die »übersetzen« einerseits als ein zentrales Problem jedweder inter-/transnationaler Kooperation betrachten und andererseits »Übersetzungspraxen« als einen privilegierten empirischen Zugriff zur Erforschung solcher Felder nutzen. ${ }^{25}$ Richard Rottenburg formuliert hierzu grundsätzlich:

„Übersetzen findet statt, wenn: eine Idee oder Sache aus einem in ein anderes Idiom, aus einer in eine andere Kultur über-tragen wird; eine Idee oder Sache durch eine andere er-setzt wird; eine Idee oder Sache mit einer anderen so verbunden wird, dass sie

25 Siehe hierzu etwa: Richard Rottenburg: Weit hergeholte Fakten; Bruno Latour: Reassembling the Social. An Introduction to Actor-Network-Theory. Oxford 2005; Barbara Czarniawska: A City reframed. Managing Warsaw in the 1990s. Amsterdam 2000. 
dadurch weiter reicht oder stärker wird (wie es beispielsweise beim Flaschenzug oder der Fahrradkette der Fall ist); eine Idee sich in eine Praxis oder eine Sache materialisiert und umgekehrt. Alle diese Bedeutungen haben einen gemeinsamen Nenner: Übersetzen bringt Getrenntes zusammen, setzt es dadurch in Relation, vermittelt zwischen zwei Elementen, macht sie kompatibel und komensurabel."²6

»Übersetzen« meint hier einen parallelen Prozess von Mobilisierung und Transformationen, der immer auch kreative Potenziale und produktive Effekte mit sich bringt:

"Damit Ideen (ebenso Modelle und Artefakte) translokal zirkulieren - von einer in die andere soziale Welt, von einem in den anderen Bezugsrahmen -, müssen sie von jemandem aufgegriffen, angeeignet und verändert werden. [...] Bei jedem Übersetzungsakt wird (performativ) unvermeidlich etwas weggelassen und etwas anderes hinzugefügt, sonst würde die Kette der Übersetzungen abreißen. Stärker formuliert: Jeder Übersetzungsakt ist auch ein Schöpfungsakt, der etwas hervorbringt, was es vorher nicht gegeben hat. " ${ }^{27}$

Rottenburg schlägt vor, die translokale Zirkulation von Ideen, Modellen oder Artefakten durch »Übersetzungsketten« im Rahmen von »Aushandlungszonen « ${ }^{28}$ zu untersuchen - also anhand von konkreten Interaktionen, Verhandlungen oder auch bürokratischen Akten, in denen Wissen zugleich mobilisiert und verändert wird. Mein Vorschlag, translokale Praxis- und Übersetzungsfelder als Analyserahmen für die Umsetzung Auswärtiger Kulturpolitik zu nutzen und hierbei gerade auch Wissensarbeit zu fokussieren, ist deutlich von diesen Überlegungen inspiriert. Allerdings betone ich insbesondere das letzte Glied der mehrteiligen Übersetzungsketten zwischen den »Rechen(schafts)zentren « und lokalen Handlungsfeldern, die Rottenburg in den Blick nimmt, und frage zugleich nach den vielfältigen translokalen Verwebungen und Formationen, die hierbei im Zusammenspiel unterschiedlicher Akteure, Wissensbestände, institutioneller Kontexte oder Ressourcen entstehen und daher nicht alleine auf die Zentren deutscher Auswärtiger Kulturpolitik verweisen.

Im Rahmen meiner ethnografischen Arbeit ließ sich die vielfältige translokale Wissensarbeit zu vier Strategien kulturpolitischer Übersetzung verdichten. »Strategien« verstehe ich dabei nicht als von isolierten Individuen oder Kleingruppen intentional erdachte, zu Papier gebrachte Überlegungen zu einer systematischen und passgenauen Umsetzung zuvor klar formulierter politischer

26 | Richard Rottenburg: Weit hergeholte Fakten, S. 15.

27 | Ebd., S. 16.

28 | So der Begriff, den Richard Rottenburg nutzt: Ebd., S. $159 \mathrm{ff}$. 
Zielvorstellungen, sondern »praxeologisch « und »prozessual ${ }^{29}$ Es geht mir um die relationalen Fähigkeiten, die sich im Zuge von alltäglichen Handlungen und Interaktionen in den translokalen Praxis- und Übersetzungsfeldern herausbilden, verdichten und Kulturmittlern - trotz ihres partiellen Nicht-Wissens - Orientierung und Positionierung an ihren Einsatzorten ermöglichen. Hierbei betrachte ich die alltägliche Auseinandersetzung mit den Reibungen und Problemen, die sich aus der Einarbeitung von Elementen der deutschen Auswärtigen Kulturpolitik in lokale Handlungskontexte ergeben, als besonders produktiv. Entsprechend gehe ich davon aus, dass sich diese relationalen Fähigkeiten etwa im routinierten oder reflexiven Umgang mit politisch formulierten Zielvorstellungen, in der praktischen Aneignung oder Veränderung zentraler diskursiver Figuren oder in der kleinteiligen Verkopplung solcher kulturpolitischer Diskurse mit Erwartungen und Begebenheiten vor Ort sukzessive entwickeln. Die konkrete Ausgestaltung solcher Strategien kann dabei lokal von sehr unterschiedlichen Rahmenbedingungen abhängen - einige dieser Faktoren sind zu Beginn des Abschnittes oder anhand der Darstellungen zu Matabb bereits angeklungen. Entscheidend ist, dass ich die translokalen Arbeitskontexte mit ihren Akteuren, Infrastrukturen, Ressourcen, Narrationen, Wissensbeständen, Konflikten, Hierarchien und Aufmerksamkeitskonjunkturen als koproduzierend im Hinblick auf die Herausbildung dieser relationalen Fähigkeiten betrachte.

Auf dieser Basis möchte ich nun vier Strategien kulturpolitischer Übersetzung voneinander differenzieren, welche die beiden zentralen Pole im Arbeitsalltag der Kulturmittler - die politischen Zentren einerseits, die lokalen Handlungskontexte andererseits - auf eine jeweils spezifische Weise zueinander in Beziehung setzen. Die folgende Auflistung ist dabei nicht als eine erschöpfende Typologie voneinander eindeutig abgrenzbarer Herangehensweisen gedacht, sondern als Ausdruck einer analytischen Perspektive, die nach kontinuierlichen Bezugspunkten und habitualisierten Vorgehensweisen im alltäglichen Fluss der Programmarbeit fragt. Entsprechend werde ich aufzeigen, dass diese Strategien innerhalb der translokalen Praxis- und Übersetzungsfelder nicht exklusiv, sondern parallel, kombiniert und überlappend zur Anwendung kommen:

29 | Inspiriert durch Pierre Bourdieu, der etwa das "Prinzip der Strategien " bezeichnet als den "mpraktischen Sinn، oder, wenn man will, das, was man auch sspielerisches Gespür nennen könnte: der gekonnte praktische Umgang mit der immanenten Logik eines Spiels, die praktische Beherrschung der inm innewohnenden Notwendigkeit - und dieser 'Sinn, wird durch Spielerfahrung erworben und funktioniert jenseits des Bewusstseins und des diskursiven Denkens. "Siehe: Pierre Bourdieu: Von der Regel zu den Strategien. In: Ders.: Rede und Antwort, S. 79-98, S. 81. 
i. Mit Verorten benenne ich Vorgehensweisen, die im Kern darauf abzielen, im Rahmen einer Policy neue Präsenzen zu schaffen. Hierzu zählen etwa die Planungs-, Diskussions- und Realisierungsprozesse, die zur Neugründung eines Goethe-Instituts oder einer anderen kulturpolitischen Vertretung an einem konkreten Ort führen. Der Begriff umfasst aber auch die übliche Praxis der »Entsendung« von Deutschen an solche Standorte und die sich daran anschließende Wissensarbeit, mit denen sich diese Kulturmittler in ihrem neuen Arbeitskontext zu etablieren versuchen. Solche Strategien des Verortens werde ich insbesondere im folgenden Abschnitt (III. 3) illustrieren, indem ich Praxen, Kontexte und Vorgehensweisen von Leitern eines Goethe-Instituts, sich nach einer Entsendung ihr neues Feld zu erschließen, vorstelle.

ii. Strategien des Verankerns bauen auf solchen Verortungen auf, gehen aber einen Schritt weiter. Ich verstehe hierunter den letzten Bestandteil einer Übersetzungskette, dem innerhalb der Auswärtigen Kulturpolitik eine besondere Bedeutung zukommt. Denn unabhängig davon, ob eine politisch oder institutionell formulierte Zielsetzung lautet, ein zeitgenössisches Deutschlandbild zu vermitteln, die deutsche Sprache zu fördern, zur Konfliktprävention beizutragen oder Zivilgesellschaft zu stärken - in all diesen Fällen besteht die Intention darin, an einem jeweiligen Einsatzort nachhaltige soziale, kulturelle oder politische Effekte zu erzielen. Strategien des Verankerns zeigen sich somit etwa in den praktischen Bemühungen, Kooperationen aufzubauen, Kultur- oder Bildungseinrichtungen zu unterstützen, Deutschunterricht an Schulen zu etablieren, Bildungseliten für Deutschland zu gewinnen oder eine bestimmte diskursive Position in den lokalen Kontext einzubringen - kurz: ein ethnografischer Fokus auf Verankerungspraxen führt direkt zu den Effekten und möglichen Reibungen, die sich aus dem Einarbeiten von politischen Zielsetzungen in lokale Handlungskontexte ergeben. Strategien des Verankerns werde ich primär im vierten Abschnitt dieses Kapitels diskutieren.

iii. Strategien zur Schaffung von Anschlüssen zielen hingegen darauf ab, durch die Mobilisierung wirkmächtiger Metaphern oder Narrative neue Beziehungen und Handlungsfelder zu eröffnen. Solche Strategien zeigen sich etwa in den Bemühungen, Bestände des Narrativs vom Nationalen translokal anschlussfähig zu machen, indem man sie in innovative Programmformate, relevante Themenfelder oder breitere Netzwerke einbringt. Sie finden somit in allen Projekten Anwendung, in denen die Herstellung von Deutschlandbezügen auf neue Kooperationsformen oder inhaltliche Verbindungen angelegt ist. Im vierten Abschnitt dieses Kapitels werde ich darüber hinaus eine weitere Ausformung dieser Strategie herausarbeiten: Hier zeichne ich nach, wie sich durch die Mobilisierung von »Europa« als einer 
kulturpolitischen Metapher neuartige Referenzrahmen und Handlungsoptionen für die Arbeit eines deutschen Kulturinstituts hervorrufen lassen.

iv. Strategien des Verknüpfens entstehen aus der Notwendigkeit, zwischen den mitunter weit auseinanderstrebenden Erfordernissen, Rationalitäten und Diskursen in den unterschiedlichen lokalen und institutionellen Kontexten, die in den kulturpolitischen Praxis- und Übersetzungsfeldern in Kontakt geraten, Verbindungen herzustellen. So müssen Kulturmittler in ihrer Programmarbeit nicht nur die Intentionen ihrer politischen Auftraggeber und ihres Arbeitgebers einbeziehen, sondern gleichfalls mit den Erwartungen ihrer lokalen Kooperationspartner oder den dominanten Positionen innerhalb der medialen Öffentlichkeiten - sowohl in Deutschland als auch an den Einsatzorten - jonglieren. Als Folge der Mobilisierung der neuen Policy lässt sich eine weiter gesteigerte Komplexität dieser kulturpolitischen Übersetzungsarbeit ausmachen: Kulturmittler wenden sich verstärkt den lokalen Präsenzen und Bruchlinien der »Konflikte« zu, suchen nach passenden Programmformaten und werden zugleich häufiger mit den asymmetrischen Rahmenbedingungen und Reibungspotenzialen ihrer Arbeit konfrontiert. Die Verknüpfungsarbeit, die sich aus dieser Gemengelage entwickelt, steht im Zentrum des fünften Abschnitts.

In der Gesamtschau werde ich aufzeigen, wie diese Strategien kulturpolitischer Übersetzung Diskurse und Alltagspraxen auf flexible Weise miteinander verbinden, stabile Strukturen und eher flüchtige Elemente zusammenfügen und zugleich Navigationen durch partiell unbekanntes Gelände ermöglichen. Die verbleibenden Textteile sind der Darstellung dieser anspruchsvollen Wissensarbeit, die in den translokalen Praxis- und Übersetzungsfeldern einer Auswärtigen Kulturpolitik als Konfliktprävention vonstattengeht, gewidmet.

\section{SUbJEKTPOSITIONEN UND WISSENSHIERARCHIEN: Entsendungen als Strategien des Verortens}

Die Schaffung von Präsenzen bildet zumeist Ausgangspunkt und Grundlage für die kulturpolitische Praxis. Entsprechend beginne ich meine Analyse der translokalen Wissens- und Übersetzungsarbeit mit Strategien des Verortens. Die folgende Darstellung eröffnet zugleich einen Blick in die alltäglichen Arbeitskontexte von Goethe-Instituten im Ausland und somit in die Innenräume translokaler Praxis- und Übersetzungsfelder. Hierzu einige allgemeine Bemerkungen vorweg: Organisatorisch unterteilt sich ein solches Institut in der Regel in die Bereiche Programmabteilung, Spracharbeit, Bibliothek und Verwaltung, der neben der Verwaltungsleitung auch Rezeptionisten, Hausmeister, Fahrer und IT zugeordnet sind. In diesem Arbeitsfeld begegnen sich Personen mit 
sehr unterschiedlichen Status, Aufstiegschancen und Verweildauern: lokale Mitarbeiter, die mit längerfristigen oder projektbezogenen Verträgen, die Programmarbeit maßgeblich umsetzen; das aus Deutschland entsandte Leitungspersonal, das nach vier, fünf Jahren an den nächsten Einsatzort weiterzieht; Deutschlehrerinnen, die in der Regel als Honorarkräfte nach Bedarf eingesetzt und entlohnt werden; unbezahlte Praktikanten, die im Rahmen ihrer mehrmonatigen Aufenthalte wichtige Aufgaben übernehmen und den operativen Betrieb substanziell unterstützen. Die politische Forderung nach einer Reduktion der Personalkosten schlägt sich in den Auslandsinstituten auch in einem Abbau von dauerhaften Beschäftigungsverhältnissen (etwa unter den Sprachlehrern) und in einer zunehmenden Übergabe von Abteilungsleiterfunktionen an die deutlich geringer entlohnten $»$ Ortskräfte $\ll^{30}$ nieder.

Gerade unter den Rahmenbedingungen einer Konfliktkonstellation stellt sich die Frage nach dem Umgang mit den Grenzen zwischen »Wissen« und »Nicht-Wissen«, die sich im Arbeitsalltag auftun. Denn die Kooperation von Mitarbeitern mit distinkten Status und lokalen Bezugssystemen führt auch zu einem Aufeinandertreffen hierarchisierter Wissensbestände in einem mitunter schwer zu durchschauenden lokalen Kontext.

\section{Improvisation als kulturpolitischer Arbeitsmodus}

$\mathrm{Zu}$ Beginn meiner Feldforschung in Ramallah ist eine Mitarbeiterin der Programmabteilung - in diesem Fall eine deutsche Doktorandin, die diese Funktion temporär und parallel zu ihrer Forschung in Palästina übernommen hat - mit der Organisation der Lesereise eines deutschen Islamwissenschaftlers beschäftigt. ${ }^{31}$ Die besondere Herausforderung besteht darin, dass der Autor nicht nur in Ramallah, sondern auch in Ostjerusalem und im abgeriegelten Gazastreifen auftreten soll. In einem Pausengespräch kommentiert eine andere Mitarbeiterin, die kürzlich in Gaza zu tun hatte: Ganz sicher sei sie nicht, aber soweit sie wüsste, seien die Checkpoints zwischen Israel und dem Gazastreifen freitags und samstags komplett geschlossen. Die Planerin zeigt sich verzweifelt, da dies bedeuten würde, dass der Autor und die ihn begleitenden Kulturmittler drei Nächte in Gaza schlafen müssten - oder man wirft die ganze Planung wieder um. Die Mitarbeiterinnen diskutieren, wie man an verlässliche Informationen über die mögliche Schließung kommen könnte. In der Programmabteilung finden sich zwei Telefonnummern von israelischen

30| Aus Gründen der Lesbarkeit verzichte ich in den folgenden Textteilen zumeist darauf, den institutsintern gängigen Begriff "Ortskraft" in Anführungszeichen zu setzen, auch wenn ich inn - primär aufgrund seiner in diesem Abschnitt herausgearbeiteten hierarchisierenden Effekte - weiterhin kritisch betrachte.

31 | Für die gesamte folgende Sequenz: Feldnotizen vom 4. September 2008. 
Soldaten, die an Checkpoints ihren Dienst tun und sich zu einer informellen Informationsweitergabe bereit erklärt hatten. Auf einen Anruf reagieren sie nicht. Schließlich fragt man im deutschen Vertretungsbüro in Ramallah nach. Die zuständige Person dort gibt nach Rücksprache »unter Vorbehalt« die Auskunft, dass der Checkpoint zumindest am Freitagmorgen offen sein müsste. Später kommt die Mitarbeiterin auf diese Sequenz zu sprechen und verbindet sie mit dem anstehenden Wechsel in der Institutsleitung:

MA: "Der neue Institutsleiter wird es sehr schwer haben."

JA: "Warum?"

MA: "Weil man hier nichts planen kann. Und weil er nicht weiß, wie die Dinge hier laufen und wen man fragen kann. Selbst die Leute, die man dann fragt, wissen es nicht. Hier kann man einfach nichts planen. (Pause) Ich würde den Job nicht machen wollen."

JA: "Warum nicht?"

MA: "Weil ich immer nicht weiß, was ich machen soll."

JA: "Aber du machst doch die ganze Zeit."

MA: "Ja, aber ich weiß immer nicht, wie ich mit solchen Situationen umgehen soll."

Diese Szene verdeutlicht zunächst die Verunsicherung, die eine Konfrontation mit dem eigenen Nicht-Wissen in der alläglichen Organisationsarbeit in politisch komplexen Einsatzgebieten hervorrufen kann. Sie illustriert zweitens einen Modus der Improvisation, den ich gerade in Ramallah häufig beobachten konnte: Das Ziel, ein definiertes Kulturformat - also etwa eine Lesung - in einen lokalen Kontext zu übertragen, führt zu Reibungen, die eine kleinteilige Vorgehensweise, durch die das notwendige Wissen zum Teil erst im Tun erworben wird, erforderlich machen. »Improvisation« - so hat es Ignacio Farías in einem anderen Zusammenhang formuliert - »sollte nicht als bloßes planloses oder unkompetentes Agieren verstanden werden, sondern als die einzige mögliche Handlungsform in ungewissen Situationen.« Dieser Arbeitsmodus baue durchaus auf Kompetenzen und etablierten Modellen auf, führe aber in deren Übertragung in ungewissen Situationen zu neuen Praxen, »Strategien, Produkten und Formationen «. ${ }^{32}$ Und drittens verweist diese Sequenz auf die Rolle des Institutsleiters als einer zentralen Subjektposition, die durch die Auswärtige Kulturpolitik hervorgerufen wird. Denn ein spezifisches Arrangement des Politikbereichs besteht in dem System der Rotation, durch welches die fest angestellten, deutschen Mitarbeiter in Leitungsfunktionen auf die weltweiten Standorte des Goethe-Instituts verteilt werden. Die Aufenthaltsdauer einer Institutsleiterin ist somit auf einige Jahre begrenzt - anders formuliert: das

32 Ignacio Farías: Die Improvisation einer Politik. Katastrophenbewältigung, neoliberale Experimente und die Grenzen ökonomischen Wissens. In: Jens Adam \& Asta Vonderau (Hrsg.): Formationen des Politischen, S. 153-181, S. $154 \mathrm{f}$. 
Goethe-Institut versetzt sein Leitungspersonal im Hinblick auf die lokalen Gegebenheiten, Diskussionen, Sprachen und Kulturszenen an den jeweils neuen Einsatzorten systematisch und regelmäßig in einen Zustand weitgehender Inkompetenz. Zwar finden sich im Kreise der rotierenden Mitarbeiter natürlich die unterschiedlichsten, fundierten und sehr spezifischen sprachlichen und regionalen Kenntnisse. Die Versetzungspraxis bringt es aber mit sich, dass auch eine Person, die im Rahmen ihres Studiums etwa intensiv Serbokroatisch oder Hebräisch gelernt hat, sich nach vier oder fünf Jahren der Berufstätigkeit in einem anschlussfähigen Umfeld in einem sprachlich unvertrauten Kontext wiederfinden wird. In der Konsequenz bedeutete dies, dass keiner der Institutsleiter, denen ich im Rahmen meiner Forschung an den Standorten Ramallah, Sarajevo, Jerusalem und Tel Aviv begegnet bin, über hinreichende Kenntnisse der jeweiligen lokalen Sprache verfügte, um ein inhaltliches Gespräch auf Bosnisch, Arabisch oder Hebräisch führen zu können. Auch Begrüßungen und Einführungen zu Veranstaltungen wurden in der Regel auf Deutsch oder Englisch gehalten. Gespräche mit Projektpartnern, an denen ich in Ramallah oder Sarajevo teilnehmen konnte, fanden entweder in diesen Sprachen statt oder mussten durch lokale Mitarbeiter gedolmetscht werden.

Die Arbeit von entsandten Institutsleitern an ihren Einsatzorten bewegt sich entsprechend in einem elementaren Spannungsverhältnis: Einerseits fehlt ihnen - gerade zu Beginn ihres Einsatzes - ein grundlegendes lokales Orientierungswissen, so dass sie immer wieder zu tastenden, improvisierenden Vorgehensweisen gezwungen sind; andererseits nehmen sie in ihrem Arbeitsfeld von Beginn an eine herausgehobene und leitende Position ein. In der Konsequenz müssen sie Entscheidungen zu aktuellen Sachverhalten, Kooperationen oder Programmschwerpunkten fällen, ohne deren Rahmenbedingungen und Folgewirkungen auf Basis eigener lokaler Erfahrungen und Kompetenzen wirklich einschätzen zu können. Einige der Organisationweisen und internen Arrangements des Goethe-Instituts lassen sich als Versuch verstehen, Stabilität zu schaffen und somit die Notwendigkeit zur Improvisation zu kaschieren oder abzumildern. Hierzu zählt etwa, den Entsandten Ortskräfte zur Seite zu stellen, die über spezifische Kenntnisse zu den lokalen Gegebenheiten, Akteursfeldern und Kulturszenen verfügen, zugleich aber durch ihren nachgeordneten Status und ihren Arbeitsauftrag auf eben diesen engen geografischen Rahmen festgelegt sind. Aber auch die Entsandten selbst entwickeln Strategien, um mit ihrem anfänglichen Nicht-Wissen umzugehen und sich sukzessive in einem neuen Kontext zu verorten. Kulturpolitische Übersetzungsarbeit stellt sich als ein Wechselspiel aus Improvisation und Routine dar. 


\section{Der Reiz des Entdeckens: Kosmopolitische Erzählungen und ethnografische Praxen}

Wenn ich die Leiter von Goethe-Instituten danach frage, wie sie sich nach einer Rotation ihr neues Arbeitsumfeld erschließen, so erzählen sie mir Geschichten einer praxisnahen Erarbeitung, individuellen Aneignung und allmählichen Durchdringung eines Raums, den sie zunächst als »fremd « erlebten. Die Institutsleiterin aus Sarajevo entwickelt im Rahmen unseres Interviews hierzu das Bild einer Art persönlicher Transformation, um ihren Bericht von der allmählichen Verortung in dem »fremden Feld « zu illustrieren:

"Ich komme hierher wie so ein Baby. Also ich bin hier kulturell einfach neu geboren. Ich habe keine Ahnung und man muss sozusagen ein neues Team bilden und auch den Mitarbeitern vor Ort sagen: Ich bin auf Ihre Unterstützung und auf Ihren Blick in das Land angewiesen. Zuhören! Also erst mal im Institut und dann aus dem Institut heraus; und dann zu sagen: Welche Partner sind bisher wichtig gewesen? ${ }^{33}$

Das Bild von der kulturellen Neugeburt verweist auf die eigene Wahrnehmung einer anfänglichen Unselbständigkeit im Umgang mit der neuen lokalen Umgebung ebenso wie auf einen Prozess des Erwachsenwerdens, der in der Einschätzung meiner Gesprächspartnerin nach etwa einem Jahr zu einer Art Statuspassage führt:

IL: "Da gibt es ganz unterschiedliche Schritte. So bestimmte Dinge passieren in den ersten Tagen und ersten Wochen. Also auch, dass man sich in der Stadt orientieren kann, dass man ein Bild davon hat, wie Sachen zueinander liegen, dass man das Gefühl hat, die wichtigsten Partner kenne ich jetzt. Aber so ein Kulturleben hat einen Rhythmus und das ist ein Jahresrhythmus und morgen ist mein erstes Jahr zu Ende und ich merke, dass ich jetzt sozusagen erwachsen geworden bin. Das geht ja nicht von einem Tag auf den anderen. Natürlich entwickle ich mich auch noch weiter. [...] Hier gibt es für mich noch sehr, sehr viel zu entdecken, aber ich stehe jetzt auf sicherem Grund. So würde ich es vielleicht beschreiben."

JA: "Was gibt Ihnen das Gefühl, auf sicherem Grund zu stehen?"

IL: "Dass ich das Gefühl habe, dass ich mich jetzt auskenne, dass ich wesentliche Dinge in meine Überlegungen einbeziehen kann, dass ich Leute habe, die ich befragen kann, die ich um Rat fragen kann, dass ich einschätzen kann, wenn Fragen aus dem Land nach Zusammenarbeit an mich herangetragen werden, ob das für mich relevant ist oder nicht. Am Anfang kann ich das gar nicht einschätzen: Wer ist das? Ist das ein wichtiges Thema? Jetzt habe ich da schon- Ich habe Urteilsvermögen entwickelt."

33 | Interview vom 23. Juli 2009; auch die folgenden drei Sequenzen. 
Im Kern berichtet die Institutsleiterin hier von der Entwicklung ihrer Fähigkeiten zur Orientierung in Raum und Zeit des lokalen Feldes, die maßgeblich durch praktische Erfahrungen erfolgt. Hierbei geht es grundlegend um ein Vertrautwerden mit der materiellen Beschaffenheit des neuen urbanen Kontextes, zugleich aber um ein Verständnis für seine sozialen und kulturellen Dimensionen: Denn das Gefühl »jetzt auf sicherem Grund « zu stehen, beruht gerade auch auf tragfähigen Kontakten, die sie im Laufe des Jahres knüpfen konnte sowie auf der gewachsenen Sicherheit, die »Bedeutung « bestimmter Themen und Fragestellungen vor dem Hintergrund lokaler kultureller Debatten und Erfordernisse einschätzen zu können.

Die Entwicklung eines eigenen »Urteilsvermögens« verläuft dabei stark über konkrete Begegnungen, die sich zu einem Prozess der allmählichen Reifung zusammensetzen. Erste Besuche bei Kulturschaffenden oder Repräsentanten von Kultureinrichtungen dienen somit nicht nur dem persönlichen Kennenlernen, sondern zugleich dem Vertrautwerden mit den lokalen Arbeitskontexten und institutionellen Ordnungen:

"Ja, also das ist ganz klar: Wenn ich das erste Mal da bin, dann gehe ich zu denjenigen. ${ }^{34}$ Also erst mal ist es eine Referenz, [...] ein Stück Respekt und dass ich auch komme und erst mal den Ort, wo diese Institution, die Person lebt, arbeitet, wirkt, kennenlerne, weil ich dann auch eine bessere Einschätzung davon habe, wo ein Anknüpfungspunkt ist, wie die Situation ist."

Die Bedeutung solcher Besuche liegt also in praktischen Lernschritten, die auf Beobachtungen und Interaktionen beruhen und letztlich das Vermögen vergrößern, sich eigenständig und somit »erwachsen« in den Logiken und Diskursen des lokalen Feldes zu bewegen:

"Ich lerne dadurch sehr viel und das ist wichtig, dass ich sehe: Wie ist ein Theater untergebracht? Wie ist die Institution untergebracht? Wo in der Stadt sind sie? Wo sind die anderen Orte? Einfach nur, weil die Adressen sind schon interessant, und in was für Gebäuden sind die untergebracht? Haben die ein großes oder kleines Büro? Wie viele Mitarbeiter haben die? Und dann auch von sich erzählen- [...] Das gibt ja ganz, ganz viele Informationen. Mein Institut, das kenne ich. Also das ist meine eigene Neugier. Das ist Teil des Eingewöhnens und es ist auch ein Signal, dass ich erst mal zuhören möchte, um etwas von der Seite zu erfahren."

An solchen Erzählungen fiel mir die Kombination von Beobachtung, Erfahrung und Gespräch auf. Bemerkenswert erschien mir hieran insbesondere eine gewisse Ähnlichkeit zu den ethnografischen Praxen, mit denen sich eine

34 | "Denjenigen« meint hier die etablierten lokalen Partner des Goethe-Instituts. 
Anthropologin ein neues Forschungsfeld nach und nach erschließt. Diesen Zusammenhang möchte ich anhand einer längeren Passage aus dem Interview, das ich mit dem Leiter des Goethe-Instituts in Tel Aviv führen konnte, genauer herausarbeiten und anschließend interpretieren:

JA: "Aber stochert man zu Beginn nicht sehr in einem unbekannten Feld?"

IL: "Aber doch völlig. Aber doch völlig."

JA: "Und?"

IL: "Man kommt irgendwohin. Man liest vorher ganz, ganz viele Bücher und man hat natürlich sowieso ein Vorurteil, ja, weil man weiß ja alles aus der Presse und hat ein Bild von dem Land. Und dann liest man noch mehr und dann wird das Bild schärfer. Und dann kommt man irgendwohin und dann ist alles natürlich ganz anders. Weil die Wirklichkeit selten mit dem, was jemand anders darüber berichtet hat, übereinstimmt." [...]

JA: "Sie haben gesagt, es sieht dann alles ganz anders aus, wenn man dann hier ankommt. Wie haben Sie sich das Feld erschlossen? Also, wie erschließt sich Innen das Feld, wenn Sie als Institutsleiter dann hier neu ankommen und feststellen, es sieht anders aus-"

$[\ldots]$

IL: "Machen wir es ganz praktisch [...] Ich komme hierhin. Ich kenne keinen Menschen. Und habe aber langjährige Mitarbeiter hier und mache zunächst einmal- lerne zunächst einmal die großen Partner, die Traditionspartner kennen, mit denen wir schon seit 20 Jahren zusammenarbeiten."

JA: "Begleitet von den Mitarbeitern, oder?"

IL: "Begleitet oder auch unbegleitet, also, das kommt ganz drauf an. Und dann fängt man- man hat ja Programmvorgaben, die der Vorgänger schon angeschoben hat. Das heißt, das erste halbe Jahr stößt man sowieso nichts an, weil das Programm geplant ist. Und in diesem halben Jahr hat man Zeit genug, Leute kennenzulernen, Gedanken, erste Gedanken zu entwickeln. Was ich dann halt mit großer Leidenschaft tue. Ich bezeichne mich immer gerne als 'cultural streetworker. Ich repräsentiere nicht, sondern ich gehe wirklich durch die Stadt. Ich bin unausgesetzt auf der Suche nach Orten, die noch keiner gefunden hat. Und das macht die Arbeit dann spannend. Dann komme ich in Bereiche rein, die auch hier nicht so wirklich vertraut sind, ja, weil das ist- also gerade Israel ist eine sehr dynamische Gesellschaft. Die verändert sich ununterbrochen. Und Sie müssen dauernd unterwegs sein, sonst kriegen Sie es nicht mit. Sonst kriegen Sie es nicht mit. Es kommt ja erschwerend hinzu, dass ich selbst nach drei Jahren diese Sprache nicht spreche und von lesen-«

JA: "Gar nicht, oder?"

IL: "Also ja, ich kann mich auf dem Markt durchschlagen und komme im Alltag durch. Aber, ich kann ja nichts lesen, also respektive, ich muss mich konzentrieren, wenn ich ein Wort entziffern will. Das heißt, Sie kriegen überhaupt keine Information en passant mit. Das ist, also das ist nur in arabischen Ländern ähnlich. Oder in Ländern, die wirklich ganz andere Schriftsysteme haben. Und ist für mich auch eine neue Erfahrung, dass ich 
beim Flanieren nichts en passant wahrnehmen kann, ja. Das heißt, ich muss mir jede Information gezielt holen."

JA: "Das heißt, Sie müssen andere Zeichen lesen als Schriftzeichen."

IL: "Ja. In der Tat und ich muss einfach viel reden. Ich muss ein gutes Netzwerk haben von Leuten, die mir Informationen zutragen. Weil ich kriege es einfach nicht mit. Bis ich einen Wochenendveranstaltungskalender gelesen habe, ist das Wochenende vorbei. Das heißt, ich muss einfach wissen, was vorgeht. Und das ist nur über so ein Informationsnetzwerk möglich. Das baut man sich dann langsam auf und dann sucht man sich Vertrauensleute, also Menschen, mit denen man gut auskommt, wo man weiß- [...] wo man merkt, man hat gleiche Ansätze, also man hat Ansätze, über die man reden kann. ${ }^{35}$

Die Ähnlichkeit zu ethnografischen Arbeitsweisen lässt sich anhand dieser Erzählung gut verdeutlichen: Mein Gesprächspartner beginnt bereits aus der Ferne, sich dem zukünftigen Feld literarisch und über Medienberichte zu nähern; er verweist auf Praxen des Beobachtens und des aktiven Sehens, gerade zu Beginn der Entsendung; er erzählt von Wahrnehmungsspaziergängen zur Erkundung des Stadtraums; er betont die Bedeutung von lokalen Experten, die ihn - ähnlich wie die Schlüsselinformanten einer Anthropologin - in die örtlichen Diskurse und Szenen einführen; er deutet Strategien an, durch die sich kulturelle Zeichen und Bedeutungen auch ohne Sprachkenntnisse entschlüsseln und interpretieren lassen; und er berichtet von Praxen der (semi-)aktiven und zugleich beobachtenden Teilnahme an der Realisierung von Programmen des Vorgängers, die ihm eine sukzessive Sozialisation in den neuen lokalen Kontext und seinen kulturellen Referenzrahmen ermöglichen. Die prägnante - und individuell gewählte - Selbstbezeichnung als »cultural streetworker« unterstreicht hier noch einmal die Bedeutung, die mein Gesprächspartner selbst der kontinuierlichen sinnlichen Wahrnehmung und Erfahrung des urbanen Raums als Basis zur allmählichen Erschließung seines neuen Arbeitsumfeldes beimisst. Weitere Analogien bestehen in der längerfristigen körperlichen Präsenz, in der Betonung von Interaktion und Gesprächen mit lokalen Akteuren sowie in einem routinierten Umgang mit Nicht-Wissen, um sich einen lokalen Kontext sukzessive zu erschließen. ${ }^{36}$

35 | Interview vom 10. Dezember 2008.

36 | An die Anschlussfähigkeit dieser Beobachtungen an die Diskussionen zu "paraethnografischen Praxen " sei an dieser Stelle kurz erinnert, siehe: Douglas R. Holmes \& George E. Marcus: Cultures of Expertise and the Management of Globalization: Toward the Re-functioning of Ethnography. In: Aihwa Ong \& Stephen J. Collier (Hrsg.): Global Assemblages, S. 235-252. 
Aus diesen Sequenzen möchte ich zwei weiterführende Schlussfolgerungen zur Subjektposition eines entsandten Institutsleiters sowie zu den Rahmenbedingungen und Bedeutungen von Strategien des Verortens ableiten:

Zunächst lassen sich - im Anschluss an Ulf Hannerz - gerade solche Ähnlichkeiten zwischen den Arbeitspraxen von Anthropologinnen und anderen Akteursgruppen als mögliche Zugänge für eine ethnografische Forschung in translokalen Feldern festhalten. In seiner Studie zu Auslandskorrespondenten entwickelt Hannerz die methodische Vorgehensweise des »studying sideways«, um ein Tätigkeitsfeld zu untersuchen, das gewisse Parallelitäten zu seinem eigenen aufweist:

"Like anthropologists, news media foreign correspondents report from one part of the world to another. We share the condition of being in a transnational contact zone, engaged there in reporting, representing, interpreting - generally managing meaning across distances, although (in part, at least) with different interests, under different constraints. ${ }^{37}$

Die Interviewausschnitte zeigen, dass sich auch in den Arbeitsformen, Herangehensweisen und Handlungsräumen von Kulturmittlern und Anthropologen solche Berührungspunkte finden lassen. Letztlich sind die Arbeitsfelder aller drei Akteursgruppen wesentlich von translokalen Wissenspraxen geprägt - anders formuliert: Auslandskorrespondentinnen, entsandte Kulturmittlerinnen und Anthropologinnen sind demnach in einer vergleichbaren Form - wenn auch mit jeweils spezifischen Ausprägungen und anderen Zielsetzungen mit dem Erwerb, der Verarbeitung und der Mobilisierung von ortsbezogenen Wissensbeständen beschäftigt. Das ethnografische Feld ist in solchen Fällen entsprechend nicht »fremd«, sondern durch eine Vielzahl von Analogien, Bezugspunkten und »Spiegelungen« geprägt. Der Fokus auf solche Berührungspunkte und Ähnlichkeiten - im Sinne eines »studying sideways « - schärft den Blick für unterschiedliche »transnationale Kontaktzonen«, in denen Akteursgruppen parallel oder im Austausch agieren, Wissensbestände und Ortsbezüge zusammenfließen und an denen sich somit Aushandlungen und Verflechtungen als Effekte von Globalisierungsprozessen beobachten lassen. Die Kontexte und Handlungsfelder, in denen sich eine Institutsleiterin zu verorten versucht, können entsprechend auch als solche Kontaktzonen verstanden und $\mathrm{zu}$ ähnlich gelagerten Feldern analytisch in Beziehung gesetzt werden.

37 | Ulf Hannerz: Foreign News. Exploring the World of Foreign Correspondents. Chicago 2004, S. 3. Hannerz spricht hier auch von einer "Spiegelung" der Anthropologie in den translokalen Arbeits- und Wissenspaxen von Auslandskorrespondenten: "anthropology looks into its own mirror", ebd., S. 4. 
Die zweite Schlussfolgerung knüpft an diese Beobachtungen an und zielt auf die Logiken und Hierarchien, die solche transnationalen Kontaktzonen durchziehen. Die Erzählungen meiner Gesprächspartner zu ihren praktischen Vorgehensweisen und Verortungen in einem neuen Arbeitskontext machen eine Subjektposition sichtbar, die spürbar an narrative Traditionen eines westlichen Kosmopolitismus anschließt: Die Betonung der eigenen Neugier, Lernfähigkeit und Offenheit gegenüber »kultureller Differenz«, die Verweise auf das Interesse an der praktischen Begegnung und der produktiven Auseinandersetzung mit den »Anderen«, der Glaube an die Möglichkeit der Verständigung sowie die Muße, einem solchen Prozess Zeit und Raum zu geben, sind solche Motive einer »kosmopolitischen Erzählung«, die klassischerweise in den Berichten von - in der Regel westlichen - »Weltreisenden «, etwa »in Gestalt des Künstlers, des Wissenschaftlers und insbesondere des Ethnologen « ${ }^{38}$ anzutreffen waren. Regina Römhild und Michael Westrich haben kürzlich daran erinnert, dass wiederum Ulf Hannerz diesen, im Kontext von Kolonialismus und europäischer Dominanz entstandenen Kosmopolitismus als ein »imperiales Modell« beschrieben hat, »das denjenigen offensteht, die in der privilegierten Position sind, die Welt zu erkunden, zu verstehen und sich wissend anzueignen «. ${ }^{39}$ Der westliche Kosmopolit befindet sich demnach in einer exklusiven epistemologischen Position, von der aus sich das in unterschiedlichen lokalen Kontexten gesammelte Wissen wahrnehmen, kategorisieren und in eine »universale« Perspektive einordnen lässt. Diese Ausformung des Kosmopolitismus setzt demnach zwei, in einem hierarchischen Verhältnis stehende Rollen voraus: die »Anderen«, die »Fremdes an anderen Orten lokal repräsentieren«, sowie »mobile Reisende, die ihr Wissen über Andere in kulturelles Kapital [...] transferieren können «, das sich in anderen Kontexten zum Einsatz bringen lässt.

In den Erzählungen der Institutsleiter zu ihren Verortungen in neuen lokalen Kontexten sind Motive und Selbstverständnis dieser zweiten Rolle deutlich angeklungen. Die hierarchische Konstellation, die diese Subjektposition erst ermöglicht, werde ich in den folgenden Textteilen näher untersuchen. Denn das translokal mobile Subjekt, das sich Wissen aus einer privilegierten Position

38 | Regina Römhild \& Michael Westrich: Kosmopolitismus an der Grenze. Der Mittelmeerraum als Laboratorium für transversalen Gemeinsinn. In: Kathrin Audehm \& Iris Clemens (Hrsg.): GemeinSinn (= Zeitschrift für Kulturwissenschaften 2/2013), S. 8598, S. 92. Siehe auch: Ulf Hannerz: Cosmopolitans and Locals in World Culture. In: Mike Featherstone (Hrsg.): Global Culture. Nationalism, Globalization and Modernity. London 1990, S. 237-251.

39 | Regina Römhild \& Michael Westrich: Kosmopolitismus an der Grenze, S. 92; auch das folgende Zitat. 
aneignet, erfordert auch hier »Andere «, die in ihrem Aktionsradius weitgehend auf den lokalen Raum beschränkt bleiben.

\section{Asymmetrische Kooperationen: Begründungsfiguren für institutionelle Hierarchien}

Die »Anderen « sind im internen Arbeitskontext eines Goethe-Institutes zunächst die »Ortskräfte « - also die lokalen Mitarbeiter, die im Vergleich zu dem rotierenden Leitungspersonal im Hinblick auf Status, Bezahlung, Entscheidungsbefugnisse und Aufstiegschancen substanziell schlechter gestellt sind. Die Institutsleiter verwiesen in meinen Interviews durchgängig auf die Unverzichtbarkeit dieser Ortskräfte, um die eigenen Defizite an ortsspezifischen Kenntnissen und Kontakten auszugleichen - anders formuliert: die translokale Mobilität der deutschen Kulturmittler baut auf asymmetrischen Kooperationen mit institutionell verorteten »lokalen Anderen« auf. Gerade aufgrund der Prominenz der Metapher vom Dialog auf Augenhöhe in den offiziellen Legitimierungen Auswärtiger Kulturpolitik erstaunte mich, dass diese grundlegenden internen Hierarchien von meinen Gesprächspartnern kaum problematisiert wurden. Das mobile, örtlich flexible deutsche Leitungspersonal einerseits und die ihnen nachgeordneten Ortskräfte stellen zwei weitgehend naturalisierte Subjektpositionen dar, die nicht nur in den Aussagen der Institutsleiter, sondern gerade auch im Arbeitsalltag immer wieder aufs Neue Bestätigung erfahren.

Während meiner Feldforschung interessierte mich, wie die Institutsleiter ihre herausgehobene Position und die damit verbundenen internen Asymmetrien - gerade vor dem Hintergrund ihres Nicht-Wissens zu Beginn eines Einsatzes - wahrnehmen und erklären. Aus meinen Interviews lassen sich zwei zentrale Argumentationslinien herausarbeiten, welche eine Notwendigkeit der Rotation betonen und in der Konsequenz die damit verbundene hierarchische Konstellation normalisieren: Zum einen zeigt sich eine Tendenz zur Verfremdung der Einsatzorte und zur Betonung von »Distanz« oder »Differenz« als unverzichtbarem Ausgangspunkt für die eigene Arbeit. Zum anderen verweisen meine Gesprächspartner auf spezifische Kenntnisse, Erfahrungen, Ortsbezüge und Perspektiven, die sich gerade aus ihrer translokalen Mobilität ergeben - kurz: Wissensbestände und Erfahrungshorizonte, die sie deutlich von ihren lokalen Mitarbeitern unterscheiden. Beide Argumentationslinien führen zu einer - in der Konsequenz kaum überwindbaren - hierarchischen Abgrenzung zwischen den Subjektpositionen »entsandtes Leitungspersonal « und »Ortskraft«. Diese Zusammenhänge möchte ich nun anhand von drei Interviewsequenzen erläutern. 


\section{(i) "Immer ein Dialogpartner bleiben"}

Der Sinn der Rotationen ergibt sich aus Perspektive meiner Gesprächspartner primär aus den inhaltlichen Impulsen, die von einem solchen Wechsel ausgehen. Die Ankunft einer neuen Direktorin ist im Verständnis der Institutsleiterin in Sarajevo ein Moment, »an dem die Sachen, die bisher gelaufen sind, auf den Prüfstand kommen«. In der Programmarbeit kämen »neue Saiten zum Klingen «; eine »eigene Handschrift« müsse erkennbar werden, um das aufwendige Verfahren der Rotation legitimieren zu können: »Das kostet extrem viel Geld, das muss also in irgendeiner Form neue Impulse geben.«

An solchen Erklärungen zeigt sich zugleich die Routiniertheit, mit der das entsandte Leitungspersonal eine herausgehobene und bestimmende Position in dem neuen Arbeitskontext beansprucht und einnimmt. So formuliert der Institutsleiter aus Tel Aviv:

"Es tut der Arbeit auch gut, wenn es regelmäßige Paradigmenwechsel an den Instituten gibt. Denn die Richtung des Instituts ist deutlich geprägt durch den Institutsleiter bei uns. Wir haben alle unsere eigenen Vorstellungen von der Welt. Wir haben den Rahmen unserer allgemeinen Strategie, unserer Grundsatzpapiere, aber den füllen wir ganz selbständig aus." ${ }^{40}$

Die Unterstützung durch die nachgeordneten Ortskräfte und somit asymmetrische Kooperationen bilden hierbei eine fraglose Voraussetzung, um diese bestimmende Position einnehmen zu können. Aus der Perspektive meines Gesprächspartners in Tel Aviv führt ein längerer Aufenthalt an einem Einsatzort zwar zu einer durchaus positiven wachsenden Vertrautheit mit dem lokalen Kontext, bringt aber zugleich ein grundlegendes Problem mit sich:

"Das ist hier etwas, vor dem wir uns hüten müssen: Zu viel Empathie - also Sympathie schon - aber zu viel Empathie mit dem Gastland zu entwickeln. Das ist ja auch der Grund, weshalb wir durch die Welt reisen; dass wir uns nicht zu stark akkulturieren, sondern immer ein Dialogpartner bleiben. Also unsere Position nicht der des Gastlandes angleichen. Sie merken das sehr deutlich, wenn Sie ein paar Jahre irgendwo sind, wie stark Sie akkulturieren. Und wie Sie - vielleicht Ihre eigene Position nicht vergessen aber doch viel mehr Verständnis haben und die Diskurse nicht mehr so spannend und spitz und scharf werden, wie sie mal am Anfang gewesen sind."

In dieser Deutung stehen also eine zu starke »Empathie« oder Identifikation mit dem Einsatzort der Anforderung, als entsandter Institutsleiter »Dialogpartner zu bleiben«, entgegen. Distanz erscheint hier als eine Voraussetzung, um überhaupt in Dialog treten zu können: »Die wachsende Vertrautheit darf

40 | Interview vom 10. Dezember 2008; auch die folgenden beiden Zitate. 
nicht zur Aufgabe seiner eigenen Position führen. Denn wir sind in einer Dialogfunktion hier und ein Dialog funktioniert nur, wenn es klar definierte Positionen gibt, ja. Und nicht, wenn einer immer abnickt.«

Hier ist zunächst die spezifische Bezugnahme auf Dialog und somit auf einen kulturpolitischen Schlüsselbegriff bemerkenswert. Denn die Subjektposition eines entsandten Institutsleiters wird in diesen Stellungnahmen mit einem Verständnis von Dialog verbunden, das eine gewisse kulturelle oder auch soziale »Fremdheit« als notwendigen Ausgangspunkt betrachtet, um in einen produktiven Austausch treten zu können. Die Vertrautheit mit einem Ort oder spezifische ortsbezogene Kompetenzen werden hier weniger als eine unverzichtbare Ressource für eine inhaltlich fundierte Kooperation betrachtet, sondern eher als ein Hindernis für einen »Dialog«, der »klar definierte Positionen« braucht. Von Relevanz sind dabei die Effekte einer solchen Argumentationslinie: Einerseits wird in solchen Aussagen das klassische Bild von abgrenzbaren kulturellen Einheiten, die es in einen Austausch zu bringen gilt, aufgerufen und - etwa statt der Vorstellung von historisch gewachsenen Verflechtungen und alltäglichen Verknüpfungen zwischen verschiedenen Orten und Kulturschaffenden als einem Regelfall zeitgenössischer kultureller Produktion - als Ausgangspunkt der kulturpolitischen Vermittlungsarbeit normalisiert. Andererseits lässt sich durch genau dieses Verständnis von Dialog begründen, warum die Position einer Institutsleiterin durch eine aus Deutschland entsandte Person besetzt werden muss - anders formuliert: die translokale Rotation und die Entsendungen von Deutschen sind in diesem Verständnis also Voraussetzungen, um eine grundlegende Zielsetzung Auswärtiger Kulturpolitik umsetzen zu können. In diese Richtung argumentiert auch meine Gesprächspartnerin in Sarajevo: Lokalen Mitarbeitern fehle die notwendige Distanz und Unabhängigkeit, um eine »objektive Programmarbeit« zu realisieren. Zusammengefasst zeigt sich, dass eine spezifische Übersetzung der Metapher vom Dialog nicht nur einen Beitrag zur Konstituierung der Subjektposition eines Institutsleiters und somit zur Verortung der Entsandten in ihren neuen lokalen Kontexten darstellt, sondern zugleich in der Arbeitspraxis zur Legitimierung von internen Asymmetrien zwischen translokal mobilen Deutschen und den »lokalen Anderen« herangezogen werden kann.

\section{(ii) "Ich-Gesellschaft" versus "Wir-Gesellschaft"}

Die zweite Sequenz verdeutlicht die privilegierte Position der Entsandten, translokal gesammeltes Wissen aus einer distanzierten Perspektive hierarchisierend zu ordnen und lokale Arbeitskontexte hierdurch zugleich zu »verfremden«. Auf die Frage, ob nach einer Rotation die Vertrautheit mit dem bekannten institutionellen Kontext oder die Wahrnehmung der neuen, unbekannten Rahmenbedingungen überwiegt, erklärt mir die Institutsleiterin in Sarajevo: 
IL: "Das ist mein viertes Auslandsinstitut - und das war Syrien, Ghana, Pakistan und jetzt Bosnien-Herzegowina - und ich kann immer noch sagen, dass die Unterschiede von jedem einzelnen Land zu Deutschland größer waren als die Unterschiede untereinander. Das hört sich komisch an. Ich möchte das vielleicht an drei Beispielen klar machen: In Deutschland gibt es einen grundsätzlich anderen Familienbegriff als in all diesen vier Ländern. Also Deutschland würde ich- man sagt immer, es ist eine ,Ich-Gesellschaft und die anderen Gesellschaften sind 'Wir-Gesellschaften`. Da tritt das Individuum meistens in einen familiären Zusammenhang. Der kann größer oder kleiner sein, und es ist auch kulturell anders definiert, aber das Individuum tritt in einem 'Wir, was meistens einen verwandtschaftlichen Rahmen hat, zurück und richtet danach auch sein Agieren aus. Und in Deutschland würde das Ilch meistens Vorrang haben. Dann der Umgang mit Zeit ist auch etwas, worin sich Deutschland gravierend von allen Ländern unterscheidet."

JA: "In welchem Sinne?"

IL: "In Deutschland hat eine Stunde 60 Minuten und in all den Ländern, wo ich bisher war, kann das eben weniger und mehr sein. Je nachdem wie es gerade passt. Das kann vielleicht in Ghana einen größeren Rahmen haben, als es in Bosnien-Herzegowina ist, aber das insgesamt Zeit nicht so nach dem Takt geht, das trifft auch auf Bosnien-Herzegowina zu. Also wann fängt man mit einer Veranstaltung an: Es ist schon europäischer als die anderen drei Länder. Und das andere ist die Rolle, die Religion spielt. Das ist das dritte Feld. In allen Ländern, wo ich bisher war, ist Religion im Leben und Alltag prägend und etwas, was öffentlich gemacht wird. In Deutschland würde ich nie jemanden fragen: 'Welcher Religion gehören Sie an? Hier ist man- das ist ein Land, wo das noch zuletzt ein Argument war, ob ich meinen Nachbarn bekriege oder nicht. Oft wird das auch instrumentalisiert, aber es spielt eine Rolle. Dieses Land ist religiös sehr gespalten, aber auch in den anderen Ländern ist Religion etwas, was den Tag bestimmt: Wann man betet, mit wem man wo betet, Geburt, Hochzeit, Sterben sind öffentliche Veranstaltungen und in allen Gesellschaften kann man sich kaum vorstellen, dass man nicht glaubt. Ob das jetzt christlicher Glaube ist oder muslimischer Glaube ist dann schon wieder weniger wichtig. " ${ }^{41}$

In dieser Sequenz erläutert meine Gesprächspartnerin also auf Basis ihrer langjährigen Berufserfahrung ihren Eindruck einer grundlegenden kulturellen oder zivilisatorischen Differenz zwischen Deutschland einerseits und allen ihren bisherigen Einsatzorten andererseits. Die Einsatzorte werden als substanziell »anders« dargestellt und hierdurch trennscharf von »Deutschland « und dem dort verorteten »kulturellen Wir« abgegrenzt. Verflechtungen zwischen den unterschiedlichen geografischen Räumen geraten in dieser Argumentationslogik ebenso in den Hintergrund wie sozialer Wandel, politische Transformationen oder ökonomische Entwicklungen, die auch Grundbegriffe

41 | Interview vom 23. Juli 2009. 
wie »Familie«, »Zeit« oder »Religion« in einem jeweiligen staatlichen und gesellschaftlichen Kontext erfassen und somit - ob nun in Deutschland, Ghana, Syrien oder Bosnien-Herzegowina - zu dynamischen und umstrittenen Kategorien machen. Anfangszeiten einer Veranstaltung oder Bezugnahmen auf »Religion « werden hier weniger aus konkreten Kontexten und Problemlagen heraus gedeutet, sondern als Ausdruck von grundlegenden Unterschieden zwischen kulturellen oder gesellschaftlichen Einheiten wahrgenommen. In solchen Aussagen zeigt sich entsprechend das Wirken einer dichotomischen Logik im Umgang mit »Kultur«, die im Rahmen dieser Arbeit bereits als ein Grundprinzip des offiziellen Narrativs Auswärtiger Kulturpolitik herausgearbeitet worden ist. Sichtbar wird zugleich die Nähe dieses Wahrnehmungs- und Ordnungsmusters zu Blickregimen, die im Kontext des europäischen Kolonialismus entstanden sind, eine zivilisatorische Teilung in den "Westen « und den »Rest « vornahmen ${ }^{42}$ und als Konsequenz in (post-)kolonialen Gesellschaften unterschiedliche Formen der Rückständigkeit, der substanziellen kulturellen Differenz sowie der geringeren Autonomie und Entwicklungsfähigkeit von Individuen zu erkennen meinten. Der Verweis auf eine engere Einbindung des Einzelnen in Familienverbände und ein - in dieser Sequenz - eher unaufgeklärt wirkendes Verhältnis zu Religion sind dabei ebenso typische und etablierte Motive wie die Vorstellung einer anderen Zeitlichkeit in den Ländern des globalen Südens. Eine asymmetrische Weltordnung - so mein Argument - hat also nicht nur Eingang in den klassischen westlichen Kosmopolitismus gefunden, sondern lässt sich gleichfalls in der Subjektposition von deutschen entsandten Kulturmittlern nachzeichnen.

Nun geht es mir nicht darum, solche Aussagen einfach als falsch, überholt oder unreflektiert darzustellen. Auch ist es nicht meine Intention, die Wahrnehmungen einer spezifischen Person zu kritisieren, nicht zuletzt da mir ähnliche Aussagen im Rahmen meiner Feldforschungen in Goethe-Instituten mehrfach begegnet und fraglos auch in anderen deutschen Institutionen anzutreffen sind. Die Möglichkeiten, eine solche Position der unbeteiligten Kritik zu beziehen, erscheinen mir auch deshalb begrenzt, da der Blick in transnationale Kontaktzonen hier wiederum Analogien zwischen kulturpolitischen und anthropologischen Wissenspraxen zutage treten lässt. Denn schließlich werden in dieser Sequenz Argumentationsfiguren sichtbar, die zu den traditionelle Wissensbeständen der Ethnologie gehören. Auch wenn Anthropologinnen schon seit einiger Zeit solche Vorstellungen - etwa von der engen Bindung zwischen Kultur, Territorium und Gruppe oder von einem dichotomischen Unterschied zwischen Tradition und Moderne - vielstimmig reflektieren und sich um die Erarbeitung neuer konzeptioneller Zugänge bemühen, so gilt

42 Stuart Hall: Der Westen und der Rest. Diskurs und Macht. In: Ders.: Rassismus und kulturelle Identität, S. 137-179. 
es dennoch anzuerkennen, dass meine Gesprächspartnerin hier Wahrnehmungsmuster anlegt, an deren Etablierung die anthropologischen Disziplinen als Co-Produzenten kolonialer Epistemologien maßgeblich beteiligt waren. ${ }^{43}$ Entsprechend interessiert mich an dieser Stelle vielmehr die Frage, welche Rückschlüsse sich aus solchen Sprechweisen auf die kulturellen Logiken und Hierarchien, die translokale Praxis- und Übersetzungsfelder Auswärtiger Kulturpolitik prägen, ziehen lassen.

Hierzu ist zunächst die Reflexion der Interviewsituation von Relevanz: Schließlich gaben meine Fragen den Anlass dazu, dass die Institutsleiterin die Vielfalt ihrer Beobachtungen, Erfahrungen und Gespräche an unterschiedlichen Orten in die Form einer Antwort brachte und hierdurch kategorisierte. Eine solche Aufforderung zur Ordnung des translokal erworbenen Wissens und somit zur Erklärung des eigenen Tuns gegenüber Besuchern von außen erscheint mir als nicht untypisch für den Arbeitsalltag von entsandten Kulturmittlern. Denn sie operieren an Schnittstellen unterschiedlicher Handlungs- und Diskursfelder und sehen sich mit den interessierten Fragen von Mitarbeiterinnen des Auswärtigen Amtes, Bundestagsabgeordneten, Journalisten, eingeladenen Künstlerinnen oder eben Anthropologen konfrontiert. An diesen Schnittstellen entstehen Strategien des Verknüpfens, die ich im Abschnitt III.5 genauer untersuchen werde. Hier möchte ich festhalten, dass solche Verweise auf Differenz, das Denken in einer dichotomischen Logik, die zwischen einem »kulturellen Wir« und den »Anderen« unterscheidet, offenbar plausible Argumentationsfiguren darstellen, um Wissensbestände zwischen den unterschiedlichen Kontexten, die in einer transnationalen Kontaktzone zusammenkommen, zu vermitteln. Hier wird einerseits nochmals die privilegierte Subjektposition eines entsandten Kulturmittlers sichtbar, die eine solche Ordnung, Hierarchisierung und Bewertung von lokalen Erfahrungen und Wissensbeständen aus einer vergleichenden Perspektive ermöglicht. Andererseits zeigt sich, dass die Strategien des Verortens der mobilen Institutsleiter nicht nur auf improvisierenden Vorgehensweisen einer schrittweisen Erarbeitung eines neuen lokalen Kontextes beruhen, sondern zugleich solche Formen der Distanzierung von einem aktuellen Einsatzort beinhalten.

\section{(iii) "In einem anderen Orbit"}

Die internen Asymmetrien zwischen Entsandten und Ortskräften lassen sich nicht nur durch die Betonung von »kultureller Differenz« oder der Notwendigkeit von »Distanz« als Voraussetzung für Dialog begründen. Regelmäßig verweisen meine Gesprächspartnerinnen auf die spezifischen Erfahrungen, Kompetenzen und Perspektiven, die sie im Rahmen ihrer professionellen Mo-

43 | Johannes Fabian: Time and the Other: How Anthropology Makes Its Object. New York 1983. 
bilität entwickeln. Translokales Wissen und translokale Bezugssysteme - beides im Rahmen der Rotation erworben - zeigen sich hier als ein kulturelles Kapital, über welches die Entsandten im Unterschied zu den Ortskräften verfügen. Die Institutsleiterin in Sarajevo entwirft wiederum ein einprägsames Bild, um diese Differenz zu illustrieren:

"Also Entsandter oder nicht Entsandter ist keine Frage des Passes, sondern ist eine Frage, in was für einem Orbit ich mich bewege und eine ,Ortskraft - das sagt das Wort schon - ist sozusagen an dem Ort verortet und ist unendlich wichtig, weil, wenn ich ankomme, ich auf diese Kenntnis angewiesen bin. Auf der anderen Seite ist es aber auch sehr wichtig, dass man über diesen Tellerrand hinwegschauen kann und die Arbeit, die wir tun in einem regionalen und in einem globalen Zusammenhang sehen kann und auch verbunden mit dem Auftraggeber, der auch unser Zuwendungsgeber ist. Und aus diesem Spannungsfeld erwächst Produktivität. ${ }^{44}$

»Entsandte« und »Ortskräfte« beschreiben also trotz eines gemeinsamen Arbeitsalltags gewissermaßen distinkte Flugbahnen. Sie agieren einerseits in unterschiedlichen räumlichen Bezugssystemen und nehmen hier andererseits Positionen ein, von denen aus sich deutlich andere Sichtfelder öffnen. Das rotierende Leitungspersonal verfügt im Hinblick auf ihren jeweiligen Einsatzort - so lässt sich das Bild vom »Orbit« interpretieren - zugleich über einen »Blick von oben«, durch den das konkrete Tun im lokalen Feld in einen breiteren geografischen und kulturpolitischen Kontext gerückt werden kann, über einen »Blick von außen«, der Routinen vor Ort in Frage stellt und neue Ideen einbringt sowie über einen »Blick aus der Bewegung «, der neue translokale Verknüpfungen ermöglicht und hierdurch das räumliche Bezugssystem eines Goethe-Instituts dynamisiert. Einen »Blick von innen«, der für die kulturpolitische Übersetzungsarbeit gleichfalls unverzichtbar ist, steuern demnach die Ortskräfte bei. Die internen Asymmetrien erfahren auch durch dieses Bild eine Normalisierung: Die andere Verortung in den Praxis- und Übersetzungsfeldern Auswärtiger Kulturpolitik, die anderen Perspektiven, Netzwerke und Aktionsradien plausibilisieren hier die Grenzziehung und Statusunterschiede zwischen beiden Berufsgruppen.

Der »andere Orbit« steht aber nicht nur für die potenziell globale Mobilität, sondern verweist zugleich auf das professionelle Netzwerk der Entsandten und somit auf den kontinuierlichen Rahmen ihrer Arbeit. Die wiederum translokale - und zugleich in Deutschland zentrierte - Struktur des Goethe-Instituts bietet der Institutsleiterin Hilfsmittel, die sie zur Annäherung an ein neues lokales Handlungsfeld nutzt: Zunächst die durch das entsandte Lei-

44 | Interview vom 23. Juli 2009. 
tungspersonal in einer Region kollegial erarbeitete $»$ Regionalstrategie ${ }^{45}$, die meiner Gesprächspartnerin die Identifizierung bestimmter »Aktionsfelder« und »Themen« in Sarajevo erleichterte. Sie betont aber auch die regelmäßigen, formalisierten Treffen und informellen Begegnungen mit den anderen Institutsleitern, durch die sie - gerade zu Beginn einer Entsendung - direkt und im persönlichen Austausch mit den institutionellen Netzwerken, Diskursen, Arbeitsschwerpunkten und Problemfeldern in der Region Südosteuropa vertraut gemacht wurde. Diese Einbindung der Entsandten in einen distinkten sozialen Rahmen hat für die Verortung in einem neuen lokalen Arbeitskontext zumindest drei Konsequenzen:

Zunächst bedeutet dies, dass die entsandten Kulturmittler mit einem Status ausgestattet werden, der nicht primär vor Ort verdient werden muss, sondern sich gewissermaßen aus der institutionellen Bindung in den lokalen Arbeitskontext übertragen lässt. Zweitens ist davon auszugehen, dass die Zielsetzungen und institutionellen Interessen des Goethe-Instituts - und somit im Prinzip auch die Rationalitäten Auswärtiger Kulturpolitik - immer schon als eine relevante Orientierungsgröße in den Praktiken, Herangehensweisen und Präsenzen des deutschen Leitungspersonals inkorporiert sind. Und drittens kommt dieses in einen direkten Kontakt zu einem generalisierbaren Wissen, das sich von den »nur« lokalen Perspektiven der Ortskräfte abgrenzen lässt. Dies zeigt sich in der Aussage einer weiteren Entsandten in Sarajevo, die ich frage, ob sie in ihrer institutionellen Ausbildung mit spezifischen Kompetenzen zur Arbeit in (Post-)Konfliktkonstellationen ausgestattet worden sei - eine Frage, die sie mit dem Hinweis auf ihr Selbstverständnis als »Generalistin« verneint:

"Denn das Goethe-Institut ist ja [...] selbstverständlich kein Akteur, der nur dort agiert wo es Konfliktsituationen gibt oder der in Postkonfliktländern eingesetzt wird, um auch durch Kulturarbeit Konflikte zu moderieren oder einen Demokratisierungsprozess zu konsolidieren, sondern das Goethe-Institut arbeitet weltweit und wir werden für eine Arbeit am Goethe-Institut weltweit ausgebildet und wir werden ausgebildet dazu, die Ziele des Goethe-Instituts umzusetzen in jedem Land und in jedem Arbeitszusammenhang des Goethe-Institutes und ich denke, dass nur durch eine solche Professionalisierung und auch Generalisierung in der Ausbildung die hohe Qualität der Arbeit an den Goethe-Instituten vor Ort gewährleistet werden kann. « ${ }^{46}$

Im Rahmen meiner Forschung konnte ich mehrfach beobachten, wie Entsandte ihren lokalen Mitarbeitern einen entsprechenden Weitblick und die Fähigkeit, ihr alltägliches Tun in die breitere Programmatik des Goethe-Instituts

45 | Siehe zur Funktion der "Regionalstrategien « des Goethe-Instituts Kapitel II.6.

46 | Interview vom 25. Juli 2009. 
oder der deutschen Auswärtigen Kulturpolitik insgesamt einzuordnen, absprachen. Die Frage, »in was für einem Orbit ich mich bewege«, führt somit auch zu einer institutionalisierten Hierarchisierung von Wissensbeständen, die eine der Grundlagen für die internen Asymmetrien darstellt. Wenn es um die Umsetzung der Ziele des Goethe-Institutes und der übergeordneten Intentionen Auswärtiger Kulturpolitik geht, werden ortsspezifische Kompetenzen und lokales Wissen im Vergleich zu dem generalisierbaren, institutionell vermittelten und translokal gesammelten Wissen der Entsandten offensichtlich als deutlich nachgeordnet eingeschätzt. Auf Basis dieser Beobachtungen drängt sich die Frage auf, ob eine kulturpolitische Praxis, die tatsächlich darauf ausgerichtet wäre, die Zielvorstellung Konfliktprävention an den Einsatzorten ins Zentrum zu rücken, nicht zu einer grundlegenden Infragestellung genau dieser Hierarchisierungen und einer substanziellen Aufwertung lokaler Wissensbestände führen müsste - anders formuliert: liegen die Grenzen einer Auswärtigen Kulturpolitik als Konfliktprävention nicht vielleicht gerade in diesen Wissenshierarchien, in denen sich die Rangordnungen zwischen Entsandten und Ortskräften widerspiegeln, begründet? Und lassen sich Konfliktkonstellationen, die gerade auch durch die Asymmetrien einer (post-)kolonialen Weltordnung hervorgerufen werden, glaubwürdig mittels institutionalisierter Hierarchien bearbeiten, in denen sich offensichtlich genau diese Ungleichheiten niedergeschlagen haben?

\section{Kontrapunkte: Rotationen und ihre Effekte aus Perspektive eines lokalen Mitarbeiters}

Keine Hierarchie lässt sich als ein eindimensionales System von Dominanz und Unterordnung verstehen. Auch über Jahrzehnte gewachsene und fest etablierte asymmetrische Ordnungen sind relationale Gefüge, die Spielräume für Aushandlungen, Kritik und Veränderungen eröffnen. Dieser Aspekt klingt etwa an, wenn die Institutsleiterin in Sarajevo von einem »Tauschhandel« zwischen deutschem Leitungspersonal und lokalen Mitarbeitern spricht, der durch die Entsendungen hervorgerufen wird: »Also, ich bekomme im Prinzip eine Einführung in die Landeskunde und Kultur des jeweiligen Landes und bringe aber genau dieses Wissen mit, womit ich dann, sagen wir mal, das Institut an bestimmte Entwicklungen anschließe, dem Institut neue Impulse gebe oder interne Veränderungen anstoßen kann. « ${ }^{47}$

Später bringt sie die Machtpotenziale, die sich aus dem relationalen Charakter eines solchen »Tauschhandels« für die lokalen Mitarbeiter ergeben, explizit zum Ausdruck: »Aber wenn ich mit meinen Mitarbeitern nicht pfleglich umgehe- die können mich hier brutal an die Wand fahren lassen und sagen:

47 | Interview vom 23. Juli 2009; auch das folgende Zitat. 
>Lass die mal wurschteln.< Also indem sie etwa keine Informationen weitergeben [...] Also es ist sehr, sehr wichtig, dass die Mitarbeiter bereit sind, einen an die Hand zu nehmen.«

Vor diesem Hintergrund stellt sich die Frage, wie lokale Mitarbeiterinnen diese institutionalisierten Relationen sowie die damit verbundenen Hierarchisierungen von Personen und ihren Wissensbeständen erleben. Welche Erfahrungen machen sie mit den Rotationen des Leitungspersonals und den damit verbundenen Praxen des Verortens? Und welche Handlungsspielräume ergeben sich für sie trotz dieser asymmetrischen Ordnungen?

Zum Abschluss möchte ich solche Fragestellungen anhand der Positionen eines lokalen Mitarbeiters in der Programmabteilung in Sarajevo diskutieren. Mein Gesprächspartner stand zum Zeitpunkt des Interviews im Begriff, das Goethe-Institut zu verlassen, da es ihm als Ortskraft keine befriedigende Entwicklungs- und Aufstiegsperspektive anbot. Seine Kritik an institutionellen Arbeitsformen sowie die Betonung von Problemfeldern in den internen Kooperationen fielen vor diesem Hintergrund sicherlich etwas dezidierter aus. Daher ist mir die Vorbemerkung wichtig, dass es mir hier nicht darum geht, die Zusammenarbeit zwischen den Mitarbeitern in einem spezifischen Institut als besonders schwierig darzustellen. Gleichfalls ist es nicht meine Intention, den Eindruck zu vermitteln, die Relationen zwischen Entsandten und Ortskräften seien primär problemdurchzogen oder immer ähnlich gelagert. Während meiner Feldforschungen konnte ich ganz im Gegenteil von Seiten der lokalen Mitarbeiter die unterschiedlichsten Umgangsformen mit internen Hierarchien beobachten: offener Widerspruch oder stille Verschleppung als Reaktionen auf Arbeitsanweisungen der Institutsleiter; Infragestellungen der Kompetenzen der Entsandten im Kreise der lokalen Mitarbeiterinnen; interne Aushandlungen, etwa um Arbeitsabläufe kooperativ abzustimmen oder Machtkonstellationen ein Stück weit zu verschieben; den Ausdruck einer offensichtlichen Zufriedenheit mit der eigenen Position, die trotz aller Asymmetrien dennoch als interessant und in Anbetracht des lokalen Arbeitsmarktes als vergleichsweise gesichert erlebt wurde; oder die Einnahme einer pragmatischen Haltung, aus der die Arbeit im Goethe-Institut einfach als ein Job - nicht mehr und nicht weniger - erschien. Ich privilegiere in den folgenden Passagen also die kritischen Positionen eines Mitarbeiters, die ich aber ebenso wie die Haltungen der Institutsleiter als sozial situiert betrachte - anders ausgedrückt: keine der Aussagen ist a priori mit einer größeren Legitimität oder einem höheren Wahrheitsgehalt ausgestattet als die anderen; jede dieser Stellungnahmen reflektiert die Position einer Sprecherin innerhalb des Arbeitskontextes sowie die Relationen, die sich zwischen den institutionell erschaffenen Subjektpositionen entfalten. Gerade durch die Kombination unterschiedlicher Perspektiven sollten sich die Reibungsflächen verdeutlichen lassen, die durch die Entsendungen und das Aufeinandertreffen verschiedener Personengruppen und Wissensbestän- 
de entstehen. Diese Reibungen ermöglichen wiederum eine vertiefende analytische Perspektive auf die kulturellen Logiken und Machtrelationen, welche Goethe-Institute - und somit die Innenräume translokaler Praxis- und Übersetzungsfelder Auswärtiger Kulturpolitik - auch über eine jeweilige Situation hinaus prägen.

Klingen die Berichte der Institutsleiter zumeist wie kosmopolitische Narrative von der schrittweisen Entdeckung und produktiven Erarbeitung neuer kultureller Kontexte, so werden in den Aussagen des lokalen Mitarbeiters aus Sarajevo eher die problematischen Effekte dieser Entsendepraxis für ein Institut, die Ortskräfte und die lokalen Kooperationspartner greifbar. Der institutionell in Kauf genommene Mangel an lokalem Wissen und Bezügen des Leitungspersonals stellt hierbei einen zentralen Ansatzpunkt zur Kritik dar:

"Ein Problem ist die Frage, wie das Goethe-Institut strukturiert ist. Also dass es nicht unbedingt das richtige Personal - meiner Ansicht nach - für solche Stellen hat; dass dieses Rotationssystem auch viele negative Sachen mit sich bringt und keine wirklichen Vorbereitungen bei den Mitarbeitern stattfinden, wie etwa bei den politischen Stiftungen teilweise. [...] Also die Leute werden einfach wie Diplomaten durch die Welt geschickt und das setzt einfach Grenzen für die ersten zwei Jahre der Arbeit, meiner Ansicht nach. Dann kann man zwei Jahre lang inhaltlich vielleicht arbeiten, wenn das Interesse sehr groß ist, und nach zwei Jahren ist ja dann auch schon wieder Schluss. Also dann kommt der Nächste, der auch wieder - bis er auf dem Niveau ist - bestimmt eineinhalb Jahre hier arbeiten muss. " 48

Die relative Unkenntnis eines Entsandten von den lokalen Gegebenheiten wird hier also als ein relevantes Hemmnis dargestellt, das gerade ein ernsthaftes inhaltliches Arbeiten erschwert. Die Ankunft eines neuen Leiters liest sich hier weniger als ein Moment neuer Perspektiven und produktiver Impulse, sondern als eine relevante Begrenzung der Potenziale einer Programmabteilung eine Begrenzung, die erst nach anderthalb oder zwei Jahren der Einarbeitung ausgeglichen werden kann. Bemerkenswert ist, dass mein Gesprächspartner von konkreten Personen abstrahiert und stattdessen institutionelle Faktoren anführt: einerseits die mangelnde Eignung des entsandten Leitungspersonals für einen jeweiligen Einsatzort; andererseits die - im Vergleich zu anderen Organisationen - schlechte inhaltliche Vorbereitung der Entsandten.

Die gewissermaßen systemisch hervorgerufene Kombination von hierarchischer Überordnung und Defiziten an lokalem Wissen produziert aus der Perspektive meines Gesprächspartners eine Reihe von Reibungsflächen. Im Hinblick auf die alltägliche Zusammenarbeit zwischen Entsandten und Orts-

48 | Interview vom 27. Juli 2009; auch alle folgenden Zitate in diesem Abschnitt. 
kräften betont er etwa die brüchige Autorität einer Führungsperson, der es an ortsbezogenen Wissensbeständen, Erfahrungen und Kontakten fehlt:

"Also, es ist auch problematisch, mit einem Leiter zu arbeiten, der weniger Kenntnisse hat als man selbst - also auf jeden Fall eine Zeit lang. Es ist dann auch schwer, gegenüber solchen Personen Autorität zu akzeptieren, wenn deren Einschätzung - hängt vom Leiter ab - teilweise falsch ist, einfach. Also wenn seine Einschätzungen nicht dem entsprechen, wie die Realität ist."

Diese Erfahrungs- und Wissensdefizite eines neuen Leiters können gleichfalls dazu führen, dass sich ein lokaler Mitarbeiter dazu gezwungen sieht, seine institutionell vorgesehene - und honorierte - Position zu überschreiten: »Es ist so ein Verantwortungszwang. Du musst Verantwortung übernehmen, obwohl du das vielleicht gar nicht - von deiner Position her, wie sie rein rechtlich definiert ist - machen solltest.«

Vor diesem Hintergrund mag es für einen lokalen Mitarbeiter besonders problematisch erscheinen, wenn sich eine neue Institutsleitung nicht in existente Arbeitsabläufe oder Programmentscheidungen einfügt, sondern ihr Potenzial zur Dominanz realisiert - etwa wenn alltägliche Arbeitsformen nicht im Team vereinbart, sondern autoritär vorgegeben werden. So berichtet mein Gesprächspartner von einer Erfahrung in der Büroorganisation:

"Wir hatten ein, meiner Ansicht nach, ein gutes Ablagesystem bei uns im Schränkchen mit Ordnern. [...] Obwohl das auch schon letztes Jahr, bevor sie kam, für 2009 vorbereitet war mit neuen Ordnern, [...] wollte die neue Institutsleitung nicht mit diesem System arbeiten und hat gemeint: 'Nee, wir arbeiten jetzt mit meinem System, mit meinen Ablagen. [...] Das fand ich schon problematisch einfach, dass es wirklich in die Details reingeht. Also dass einfach ganze Arbeitsweisen total geändert werden, also nicht irgendwie adaptiert werden, sondern geändert werden."

Gleichfalls kann die Intention der Institutsleiter, an einem neuen Einsatzort in der Programmarbeit einen »Paradigmenwechsel« herbeizuführen, bisherige Kooperationen »auf den Prüfstand zu stellen« oder »neue Saiten zum Klingen zu bringen« aus der Perspektive eines lokalen Mitarbeiters als Geringschätzung der eigenen Erfahrungsbestände erlebt werden. So berichtet mein Gesprächspartner von dem Wunsch der neuen Institutsleitung, mit einem großen Kulturfestival zusammenzuarbeiten, obwohl sich die Programmabteilung zuvor - inhaltlich, strategisch und organisatorisch begründet - eindeutig dagegen entschieden hatte:

"Der Außenblick ist: Wir wollen mit einem großen Festival arbeiten. Ich komme jetzt neu in ein Land, ich bin jetzt die neue Institutsleitung- natürlich soll man auch mit den 
wichtigen Kulturinstituten arbeiten, präsent sein, sichtbar sein. Aber auf der anderen Seite fehlen halt diese ganzen inhaltlichen Kenntnisse und wenn man sich die nicht im Detail holt, sondern einfach sich ein bisschen anhört und dann gleich losarbeitet, dann kommt es zu vielen Problemen."

Das Aufeinandertreffen von mobilen Entsandten und Ortskräften, das die Institutsleiter häufig als produktiv beschreiben, stellt sich für diesen lokalen Mitarbeiter - zumindest in seiner gegenwärtig praktizierten Form - also eher als eine Quelle von Reibungen und Problemen dar. Die institutionalisierten Asymmetrien, die in solchen Aussagen greifbar werden, bilden entsprechend einen wesentlichen Grund, dass mein Gesprächspartner sich zunehmend von der ihm zugewiesenen Subjektposition einer Ortskraft distanziert:

"Es ist auch ein Druck, dass man sich immer wieder adaptieren muss, weil ein neuer Leiter unglaublich das Institut verändert. Also ich hab das nur einmal jetzt gemacht und das ist mir jetzt eigentlich genug. Ich möchte das nicht noch ein zweites Mal machen. Das ist schon eine zu große Veränderung einfach, wenn man mich fragt, weil es geht wirklich in Banalitäten rein."

Aber auch im Hinblick auf die Potenziale von Kooperationen oder die Möglichkeiten eines Dialogs entwickelt mein Gesprächspartner eine kritische Perspektive: Demnach würden lokale Kooperationspartner spürbar unterscheiden zwischen solchen Entsandten, die »Insider-Kenntnisse« besäßen und die lokale Sprache beherrschten sowie anderen, die über solche Kompetenzen nicht verfügten:

"Und man merkt halt den Unterschied [...] wie die Personen auch akzeptiert werden von Seiten der Partner. Also da ist, von Seiten der Lokalen her, eine sehr starke Grenzeinstellung: ja nicht zu weit gehen, weil die Person versteht uns auch nicht und viel Skepsis von Seiten der lokalen Leute, was den Entsandten die Arbeit noch schwieriger macht, also diese Ausgangsbasis sehr, sehr stark erschwert, meiner Ansicht nach."

Die bereits diskutierte Vorstellung von Institutsleitern, Dialog ließe sich nur unter den Bedingungen von »Distanz« und eines »Außenblicks« realisieren, wird in dieser Position also gewissermaßen konterkariert: »Lokale Leute«, die gerade in einem Interventionsgebiet wie Bosnien-Herzegowina kontinuierlich mit einer Vielzahl von internationalen Mitarbeitern der unterschiedlichsten Organisationen konfrontiert sind, reagieren demnach auf die häufig fehlenden lokalen Wissensbestände und sprachlichen Kompetenzen ihrer Gesprächspartner eher mit Rückzug. Aus dieser Perspektive rufen die institutionell hergestellte Distanz der Entsandten und die Betonung ihrer kulturellen Differenz also eher Abgrenzung und Zurückhaltung hervor, anstatt zu dem produkti- 
ven Austausch zu führen, den sich die Institutsleiter erhoffen. Gerade auch vor dem Hintergrund meiner ethnografischen Beobachtungen ließe sich die hier angeführte »Skepsis der lokalen Leute« als Ausdruck einer Ernüchterung interpretieren: Auch die Kooperationspartner entwickeln Routinen im Umgang mit den immer wieder neuen Entsandten, die - trotz ihrer begrenzten Wissens- und Erfahrungsbestände zu den lokalen Gegebenheiten - zumeist über einen hohen sozialen und ökonomischen Status verfügen und etwa über die Fortsetzung oder die Konditionen einer Zusammenarbeit entscheiden können. Die Begegnungen in translokalen Praxis- und Übersetzungsfeldern haben demnach also nicht zwangsläufig Produktivität oder Verständigung zur Folge, sondern gerade deren erkennbar asymmetrischer Charakter kann Distanzierungen hervorrufen und Grenzen reproduzieren. Die notwendigen Ausgangslagen oder Rahmenbedingungen für einen gelingenden Dialog - so lässt sich festhalten - können offensichtlich sehr unterschiedlich eingeschätzt werden.

Um zum Abschluss noch einmal auf die internen Kooperationen zwischen Entsandten und Ortskräften sowie auf die Perspektive eines lokalen Mitarbeiters auf die konkreten Ausformungen der Entsendepraxis zurückzukommen: Im Rahmen unseres Gespräches wird deutlich, dass mein Interviewpartner das System der Rotationen nicht per se ablehnt. So betont er, dass »man vielleicht auch einen verfälschten Blick (hat), wenn man als Ortskraft zu lange da ist. Also so ein Außenblick ist ja auch gut.« Die kritische Haltung zu den institutionellen Ordnungen und Verfahren, die ich in diesem Abschnitt dargestellt habe, erklärt sich eher aus der spezifischen Kombination der beschriebenen Hierarchisierungen von Personen und Wissensbeständen mit den ausgrenzenden Logiken der Rekrutierung des rotierenden Leitungspersonal. Denn der Aufstieg eines »nicht-deutschen « lokalen Mitarbeiters in den »anderen Orbit« der »Entsandten« ist, zumindest zum Zeitpunkt unseres Interviews, - gänzlich unabhängig von dem individuellen Leistungsvermögen, den Kompetenzen, Bildungsbiografien oder Ambitionen - aus formellen Gründen ausgeschlossen. Es ist diese institutionelle Praxis, die mein Gesprächspartner in Frage stellt:

"Ich habe gegen das Rotationssystem als System nichts. Ich finde das auch gut, eigentlich. Es müsste einen Austausch geben, aber die Frage ist einfach: Wie will man es schaffen, dass die Leute - schon bevor sie kommen - zum jeweiligen Land passen? Muss es denn wirklich fest angestellte Goethe-Mitarbeiter geben, die da durch die Welt wie Diplomaten rotieren? Kann es nicht einfach einen Institutsleiter geben, der aus Bosnien stammt und der dann halt vier Jahre in Sarajevo oder danach vier Jahre in Belgrad ist und danach vier Jahre in Zagreb ist, der die Region kennt?" 
Die internen Reibungen, die anhand der Aussagen meines Gesprächspartners greifbar werden, lenken somit den Blick auf eine relevante Rahmenbedingung des Innenraums der translokalen Praxis- und Übersetzungsfelder, die im Umfeld eines Goethe-Instituts im Ausland entstehen: Der Zugang zu der privilegierten Gruppe des rotierenden Leitungspersonals bleibt »Deutschen« vorbehalten. Auch wenn die Institutsleiterin in Sarajevo in einem bereits zitierten Interviewausschnitt formuliert, »Entsandter oder nicht Entsandter ist keine Frage des Passes«, so gilt es festzuhalten, dass die internen Hierarchien eines Goethe-Instituts im Ausland eindeutig eine ethnische Komponente besitzen: Zwar ist es richtig, dass ortsansässige Deutsche die Position einer Ortskraft einnehmen können; die »lokalen Anderen« besitzen aber keine Zugangsmöglichkeiten zu der Position eines entsandten Institutsleiters und werden somit institutionell nicht nur dauerhaft in einem jeweiligen lokalen Kontext, sondern zugleich in einer nachgeordneten Rolle verortet.

Die Problematik dieser Praxis wird umso sichtbarer, wenn man die Biografien dieser »Ortskräfte« genauer betrachtet: In Sarajevo und Ramallah setzte sich diese Gruppe - zumindest der mit inhaltlichen Aufgaben betrauten Mitarbeiter - zumeist aus Personen zusammen, die als Kriegsflüchtlinge viele Jahre in Deutschland verbracht hatten oder als Nachfahren von Migranten primär dort aufgewachsen waren. Die »Rückkehr« in die Länder, in denen sie dem Goethe-Institut als Ortskräfte zur Verfügung stehen, verlief gerade bei der ersten Gruppe in der Regel zumeist nicht freiwillig. In der Konsequenz greifen deutsche Kulturmittler also auf eine Gruppe zurück, die häufig ihre Schulbildung und/oder ihr Studium in Deutschland absolviert hat und routiniert zwischen den Sprachen und kulturellen Kontexten zu wechseln und zu übersetzen vermag - anders formuliert: »Ortskraft« erweist sich als ein Mythos, als eine institutionalisierte Subjektposition, die »lokale Andere« nicht nur fraglos produziert, sondern ihnen ebenso fraglos eine nachgeordnete Rolle mit einer vergleichsweise geringen Bezahlung und schlechten Aufstiegschancen zuweist. Diese transnationalen Biografien mit ihren spezifischen Erfahrungen und Verwebungen mit Deutschland - so lässt sich zugespitzt festhalten - sind aus Perspektive des Goethe-Instituts offenbar nicht geeignet, das »kulturelle Wir« auf den Bühnen Auswärtiger Kulturpolitik in herausgehobener Position zu repräsentieren. Hierzu braucht es weiterhin die inkorporierte Ethnizität von »Deutschen«, die sich durch die Entsendung des Leitungspersonals in den Zielregionen verorten lässt.

\section{Das Nationale als hierarchisierende Kategorie}

Es geht mir an dieser Stelle nicht darum, Hierarchien innerhalb des Goethe-Instituts als erklärungsbedürftigen Sonderfall darzustellen. Ganz im Gegenteil lässt sich im Anschluss an Pierre Bourdieu formulieren, dass es in einer hie- 
rarchisierten Welt keine Institutionen gibt, die nicht hierarchisiert wären und nicht Hierarchien und soziale Abstände zum Ausdruck brächten. ${ }^{49}$ Allerdings ist es von Interesse, auf Basis welcher Rationalitäten, Mechanismen oder Prinzipien eine jeweilige institutionelle Hierarchie funktioniert und welche Effekte hieraus entstehen. Ein Befund erscheint besonders relevant: Das Nationale kann sich gerade in der Realisierung und Begründung von institutionellen Verortungspraxen als eine distanzierende und hierarchisierende Kategorie reproduzieren.

Dies zeigt sich deutlich anhand der institutionell erschaffenen Subjektposition von translokal mobilen, deutschen Entsandten, die sich ohne die enge Kooperation und kontinuierliche Interaktion mit nachgeordneten, im lokalen Raum verankerten Ortskräften nicht realisieren ließ. Die Asymmetrien zwischen diesen beiden Personengruppen manifestieren sich nicht nur in den substanziellen Unterschieden in der Entlohnung, im Status und in den Aufstiegschancen, sondern insbesondere auch in der Hierarchisierung von Wissensbeständen, die an das »imperiale Modell« eines westlichen Kosmopolitismus erinnert: Auf der einen Seite steht das aus dem Zentrum entsandte, mit generalisierbarem Wissen ausgestattete Leitungspersonal, das sich den unterschiedlichen Einsatzorten zumeist mit einer relativen Unkenntnis, aber mit Interesse und Offenheit nähert und die im Rahmen einer Berufsbiografie an unterschiedlichen Orten gesammelten Erfahrungen aus einer privilegierten Position wahrnehmen, einordnen und kategorisieren kann. Auf der anderen Seite finden sich die lokalen Mitarbeiter, die das ebenso unverzichtbare wie in den gegenwärtigen institutionellen Hierarchien - nachrangige ortsbezogene Wissen beisteuern, die in ihrem Alltag in der Regel auf eben diesen lokalen Arbeitskontext festgelegt bleiben und denen somit sowohl eine vergleichbare kosmopolitische Erfahrung als auch der Zugang zu den Positionen des rotierenden Führungspersonals systemisch verwehrt wird - kurz: eine Personengruppe, die institutionell zu »lokalen Anderen« gemacht wird. Auch wenn sich die Machtrelationen zwischen beiden Gruppen nicht eindimensional darstellen und auch wenn unterschiedliche Individuen diese Subjektpositionen in der Praxis sicherlich verschieden ausgestalten können, so gilt es festzuhalten, dass sich die Interaktionen im Innenraum eines kulturpolitischen Praxis- und Übersetzungsfeldes nicht als ein Dialog auf Augenhöhe, sondern als eine Begegnung von Ungleichen realisieren.

In den Begründungen dieser Entsendepraxis und der damit verbundenen internen Hierarchien durch die Institutsleiter zeigt sich das Wirken der dichotomischen Logik, die bereits als ein strukturierendes Muster der Diskurse im

49 | Bei Bourdieu heißt es: "In einer hierarchisierten Gesellschaft gibt es keinen Raum, der nicht hierarchisiert wäre und nicht Hierarchien und soziale Abstände zum Ausdruck brächte."In: Pierre Bourdieu: Ortseffekte, S. 160. 
politischen Zentrum herausgearbeitet worden war. Sowohl in der Betonung von »Distanz« und der Notwendigkeit der Bewahrung einer »eigenen Position« als Voraussetzung für fruchtbare Dialoge als auch in den Erklärungen zu den grundlegenden Differenzen zwischen Deutschland und verschiedenen lokalen Kontexten klingen etablierte Argumentationsfiguren an: Die Vorstellung von abgrenzbaren kulturellen Einheiten, die es in einen Austausch zu bringen gilt und das Bild von einem »kulturellen Wir«, das sich von den »Anderen« unterscheidet, werden hier als weitgehend normalisierte Ausgangspunkte Auswärtiger Kulturpolitik nochmals sichtbar. Die Praxis der Entsendungen lässt sich vor diesem Hintergrund nicht nur als eine Form der Verortung eines besonders qualifizierten Führungspersonals an den Einsatzorten verstehen, sondern zugleich als Strategien zur Schaffung von körperlichen Repräsentationen des Nationalen - denn in der Subjektposition der Entsandten ist »Deutschsein « inkorporiert. Vor diesem Hintergrund mag es zunächst paradox erscheinen, dass sich diese Subjektposition zugleich translokal informiert darstellt: »Deutschsein« betritt die Bühnen Auswärtiger Kulturpolitik in Sarajevo, Ramallah oder Tel Aviv in einem kosmopolitischen Gewand, das zwar fraglos Offenheit und Weltläufigkeit repräsentiert, zugleich aber die Asymmetrien zu den unverzichtbaren Ortskräften nochmals untermauert. Das Nationale wird hier also auch in seiner translokalen Flexibilisierung zu einer Kategorie, die Differenz naturalisiert, Zwischenpositionen marginalisiert und Hierarchien begründet.

Gerade im Hinblick auf die Zielvorstellung einer Auswärtigen Kulturpolitik als Konfliktprävention stellt sich die Frage, ob sich hier Herangehensweisen andeuten, die auch in der Programmarbeit eher Distanz zu den Einsatzgebieten aufbauen und sich durch den Rückgriff auf Bestände des Nationalen aus den komplexen Gefügen eines lokal greifbaren Konfliktes herauszuziehen versuchen. In den folgenden Abschnitten werde ich die Effekte solcher Mobilisierungen des Nationalen sowie der Asymmetrien, die Praxis- und Übersetzungsfelder Auswärtiger Kulturpolitik in Konfliktgebieten durchziehen, anhand von Beispielen aus der Projektarbeit in Ramallah und Sarajevo vertiefend untersuchen.

\section{DeutschlandBezüge UNd Ihre Wirkungsketten: Verankern und ANSChLÜSSE Schaffen als Strategien KULTURPOLITISCHER ÜBERSETZUNG}

Sind kulturpolitische Präsenzen erst einmal geschaffen, so rückt die Frage nach den Arbeitsweisen, Formaten und Kontaktzonen, über die sich Zielvorstellungen, Metaphern und Programme der deutschen Auswärtigen Kulturpolitik an den Einsatzorten verankern lassen, in den Vordergrund. Ich werde in den folgenden Textpassagen herausarbeiten, dass es den Kulturmittlern hier- 
bei explizit nicht um ein bloßes Abwerfen vorgefertigter Pakete geht, sondern um die Initiierung von Wirkungsketten: Ihre Arbeit zielt darauf ab, Begegnungsräume zu öffnen, Diskurse zu verändern, kulturelle Praktiken hervorzurufen oder auch Infrastrukturen zu stärken - anders formuliert: sie wollen Anschlüsse schaffen, deren Effekte über einen jeweiligen Projektrahmen hinausreichen. Entscheidend ist hierbei, dass Bezugnahmen auf die Bestände des symbolischen Raums des Nationalen ihre Formate auch weiterhin maßgeblich ausstatten und zugleich Ausgangspunkte für die intendierten Wirkungsketten darstellen. Allerdings verlaufen diese Referenzen immer als Übersetzungen. Das Nationale wird gewissermaßen passend gemacht, indem man es reflexiv auf lokale Interessen, Bedürfnisse und Erwartungen bezieht. In den translokalen Praxis- und Übersetzungsfeldern Auswärtiger Kulturpolitik konfiguriert sich das Nationale somit gerade auch durch parallele Konstruktionen des Lokalen auf eine jeweils spezifische Weise.

\section{"Was brauchen wir hier?": Deutschlandbezüge translokal übersetzen}

So erklärt mir der Institutsleiter in Ramallah, »dass reine deutsche Kulturprogramme hier nicht laufen«. Es gebe dafür ein kleines Publikum, aber zu wenige interessierte Personen, um eine solche Programmausrichtung zu begründen. Man müsse daher »Bezüge schaffen« - auf den Punkt gebracht: »Deutsche Kulturschaffende kommen hierher und erarbeiten gemeinsam mit palästinensischen Kulturschaffenden etwas Neues.«Dies sei »eine relativ neue Erkenntnis«, die jetzt seine Arbeit bestimme..$^{50}$ In unserem Interview erläutert er mir diese zunehmende Orientierung der Programmarbeit an lokalen Erfordernissen und Erwartungen:

"Und wir haben dann einfach gesagt, wir machen nicht mehr die tollen Projekte- unsere Zentrale hat ja bis vor kurzem noch - macht das immer noch, aber weniger - auch selber produziert [...] sehr schöne, interessante Ausstellungen oder Musik oder Kunstprojekte, die aber hier nicht greifen, die hier kein Publikum finden. Wir haben diese Ausstellungen dann teilweise hierher bestellt, für viel Geld, haben die ins Haus gehängt. [...] Dann kamen auch fünf Leute zu einem Empfang, oder zehn, aber es war nicht wirklich erfolgreich. Und dann haben wir halt überlegt: Was brauchen wir hier?„ 51

Kulturformate, die zentral in Deutschland geplant und erstellt werden, um dann global durch die Goethe-Institute zu wandern, stoßen in Ramallah demnach auf ein zu geringes Interesse. Die Frage »Was brauchen wir hier? ver-

$50 \mid$ Feldnotizen vom 12. November 2008.

51 | Interview vom 4. Dezember 2008; auch das folgende Zitat. 
weist auf die Konsequenzen, die aus dieser Beobachtung gezogen wurden: eine zunehmende Befragung des lokalen Kontextes und eine aktive Einbeziehung lokaler Kulturschaffender als Grundelemente der Programmarbeit:

"Also haben wir gesagt, wir machen lieber Sachen, bei denen wir was zu bieten haben, wir etwas hierher bringen können: Wir machen Workshops, etwa mit Filmemachern, aus denen etwas Gemeinsames, Neues entsteht. Das erreicht dann am Ende ein größeres Publikum [...] wir haben dann ein Projekt am Ende, in dem Palästinenser die Hauptschaffenden sind und die ziehen allemal ein Publikum an. Also einfach auch im eigenen Interesse, dass wir Projekte machen, die wahrgenommen werden, mussten wir Projekte machen, die sehr weitgehend von Palästinensern gemacht wurden."

Die wachsende Bedeutung dialogischer oder kooperierender Arbeitsweisen erklärt mein Gesprächspartner hier also gerade auch aus einem Eigeninteresse. Die Wirkungen sind größer und die Positionierung des Instituts im lokalen Kontext verläuft erfolgreicher, wenn Palästinenser stärker als Akteure direkt in die Programmarbeit involviert werden.

Ähnlich argumentiert die Institutsleiterin in Sarajevo. Ihre Konzentration auf zwei der übergreifenden Themenfelder, welche die Regionalstrategie des Goethe-Institutes anbietet - Erinnern und Vergessen und Südosteuropa im Spannungsfeld zwischen Nationalismen und der Europäischen Integration -, begründet sie gleichfalls aus ihren Wahrnehmungen von Diskussionen und Notwendigkeiten vor Ort:

"Andere Felder spielen natürlich auch eine Rolle, aber das ist dann schon eher in einem globalen Sinn, also ob ich jetzt nach 'Menschenrechten ' gucke oder ,Genderfragen ‘ behandle. Das ist für mich jetzt nicht so spezifisch für hier. Da würde ich mich auch an anderen Orten wiederfinden. Aber das sind eigentlich die beiden Felder, von denen ich sagen kann, das ist relevant für hier, das ist spezifisch für hier. « ${ }^{52}$

Gleichzeitig betont sie, dass es in diesen Wahrnehmungen durchaus Differenzen zu den Einschätzungen ihrer bosnischen Kooperationspartner geben kann:

"Für mich ist Bosnien-Herzegowina noch ein zutiefst traumatisiertes Land, das mit den Auswirkungen des Krieges noch lange nicht fertig ist. Signifikant ist, dass jeder einem sagt: 'Ach, das haben wir doch alles schon aufgearbeitet. Wir müssen jetzt nicht noch die 50. Ausstellung machen oder das 50. Projekt zu diesem Themar - dass man das eher von sich wegschiebt, ist ja ein bekanntes Muster, das wir aus unserer eigenen Geschichte kennen."

52 | Interview vom 23. Juli 2009; auch die folgenden beiden Sequenzen. 
Offensichtlich sind die Wahrnehmungen von den lokalen Notwendigkeiten, auf die es durch Kulturformate zu reagieren gilt, nicht von vorneherein gegeben, sondern sie bilden sich im Zuge der Auseinandersetzungen mit dem Einsatzort sukzessive heraus. Das »Lokale« als eine zentrale Orientierungsgröße für die Gestaltung der Programmarbeit zeigt sich hier als eine partiell flexible, durchaus auch umstrittene Kategorie. In der gleichen Sequenz benennt meine Gesprächspartnerin auch den zweiten, von Standort zu Standort konstanten Orientierungspunkt: projektbezogene Bezugnahmen auf »Deutschland« und hier situierte Erfahrungen oder kulturelle Entwicklungen. So führt sie als weiteren Grund für die Privilegierung der beiden genannten Themenkomplexe an:

"[...] da haben wir auch als deutsches Kulturinstitut etwas dazu zu sagen, etwas dazu zu bringen. Und zwar in beiden Feldern. Einmal, dass man sagt: Wie geht Deutschland mit solchen Fragen um? Also, dass man quasi das als Matrix anbietet. [...] Es gibt sicher auch andere virulente Themen, wo ich sagen kann, da können wir jetzt relativ wenig dazu beitragen, den Schuh zieh ich mir jetzt nicht an, weil ich eigentlich nicht weiß, warum Deutschland daran anknüpfen soll.“

Die Suche nach Deutschlandbezügen zeigt sich hier als ein plausibles Selektionskriterium für die Programmgestaltung eines deutschen Kulturinstituts, durch das sich auch Stringenz und Ordnung in die prinzipiell unendliche Vielfalt möglicher kultureller Aktivitäten bringen lässt. Bei solchen Referenzen geht es - zumindest dem eigenen Idealbild nach - allerdings weniger um eine einseitige Repräsentation statischer nationalkultureller Bestände, sondern vielmehr um eine translokale Verbindung von Diskursen und ästhetischen Positionen oder auch um eine dialogische Spiegelung von historischen Erfahrungen in Bosnien-Herzegowina und Deutschland. Inwiefern dies in der Praxis gelingt, werde ich in den folgenden Passagen dieser Studie untersuchen. Bereits jetzt lässt sich festhalten, dass eine weitgehend naturalisierte Orientierung an dem Zielhorizont Herstellung von Deutschlandbezügen die Formate und Strategien deutscher Kulturmittler maßgeblich präkonfiguriert; gleichzeitig werden die damit verbundenen Bezugnahmen auf Bestände des Nationalen in der Regel auf Basis von parallel verlaufenden Konstruktionen des Lokalen perspektiviert. Im Arbeitsalltag spiegelt sich dieser Zusammenhang in einem reflexiven Changieren der Kulturmittler zwischen den beiden illustrativen Fragen »Was brauchen wir hier? « und »Wie geht Deutschland mit solchen Fragen um?« wider, die an einem jeweiligen Standort oder im Rahmen eines Projektes auf unterschiedliche Weisen aufeinander bezogen werden können - auch diesen Aspekt werde ich nun anhand des empirischen Materials verdeutlichen. 


\section{"Deutsche Impulse setzen": Repräsentationen des Nationalen (trans-)lokal verankern}

Die bleibende Bedeutung der Orientierungsgröße Deutschlandbezüge erläutert mir der Institutsleiter in Ramallah in Abgrenzung zu seinen französischen Partnern, mit denen er innerhalb des Deutsch-Französischen Kulturzentrums im Arbeitsalltag eng verbunden ist: Die französischen Kulturmittler würden direkt palästinensische Künstler fördern, etwa durch die Finanzierung von Materialien oder indem sie deren Werke in den unterschiedlichen französischen Kulturinstituten innerhalb des fragmentierten Territoriums ausstellten und hierdurch zu einem innerpalästinensischen Austausch beitrügen. Auch wenn sich diese Herangehensweise als erfolgreich erweise - da sich ein ausländisches Kulturzentrum hierdurch als zugänglicher und kooperativer Akteur in der lokalen Kulturszene verankern könne -, käme sie für ihn nicht in Frage, da eben die unverzichtbare Herstellung eines - wie auch immer gearteten - Bezuges nach Deutschland in einer solchen Aktivität fehle. ${ }^{53}$

Dieses Leitmotiv findet sich - aufgrund seiner Zentralität im Selbstverständnis der Kulturmittler von ihrem Arbeitsauftrag, in der sich die Zentralität dieses Zielhorizontes im politischen Narrativ widerspiegelt - auch in den anderen Strategien kulturpolitischer Übersetzung als Referenzpunkt. In einigen Fällen führt es aber auch weiterhin direkt zu Formaten zur Repräsentation des Nationalen und somit zu einem eher klassischen Verständnis von den adäquaten Verankerungen eines nationalen Kulturinstitutes im Ausland. So spricht die Leiterin in Sarajevo etwa regelmäßig von der Zielvorstellung, »einen deutschen Impuls« im bosnischen Kulturleben zu setzen: etwa indem man »deutsch« zu dem zentralen Selektionskriterium für Filmproduktionen macht, die man zu einer Deutschen Filmwoche zusammenstellt und durch mehrere, gerade auch kleinere Städte touren lässt. Die zuständige Mitarbeiterin bezeichnet dieses eher einfache Projekt als ihren aktuellen »Favoriten, weil es einfach so gut ankommt, durch die Bevölkerung sehr dankbar aufgenommen wird «, ${ }^{54}$ also eine große Breitenwirkung erzielt und zugleich auf den Mangel an kulturellen Angeboten in der bosnischen Provinz reagiert. Die Intention, einen »deutschen Impuls zu setzen«, zeigt sich auch in einer anderen Vorgehensweise: Das Goethe-Institut bietet den etablierten Kulturfestivals in Sarajevo, dem Kinder-

53 | Feldnotizen vom 29. September 2008. Palästinensische Kulturschaffende bestätigten mir gegenüber die Einschätzung des deutschen Institutsleiters, dass gerade diese direkte und handfeste Förderung lokaler Künstler vor Ort sehr geschätzt wird - insbesondere auch, da die französischen Kulturzentren in unterschiedlichen Städten des historischen Palästinas dazu beitrügen, die durch Besatzung und Konflikt fragmentierten palästinensischen Kulturszenen in Austausch zu bringen.

54 | Interview vom 18. Juli 2009. 
theaterfestival in Banja Luka oder auch den Organisatoren einer Europäischen Filmwoche Unterstützung durch die Finanzierung eines »deutschen Beitrags« an. Im Selbstverständnis der Kulturmittler besteht ihre Zuständigkeit in einer solchen Zusammenarbeit also darin, den Auftritt deutscher Musikerinnen, die Lesungen deutscher Schriftsteller, die Präsentation von deutschen Filmen, Theaterproduktionen oder von Werken deutscher Künstlerinnen zu ermöglichen und somit einen wahrnehmbaren Bezug nach Deutschland im lokalen Kulturleben zu verankern. Die Frage, was es braucht, damit dieser unverzichtbare Deutschlandbezug gegeben ist, lässt sich dabei flexibel beantworten: So schlägt die Leiterin des Tanzfestivals in Sarajevo im Jahr 2009 etwa einen französischen Tänzer und Choreographen, der seit Jahren primär in Deutschland lebt und arbeitet, als möglichen »deutschen Beitrag« vor. Als Reaktion erklärt mir die Institutsleiterin, dass

die Staatsbürgerschaft eines Künstlers nebensächlich sei. Sie interessiere, wo das künstlerische Schaffen seine Basis habe. Gerade im Bereich Tanz verfüge Deutschland über eine sehr lebendige Szene, aber ein Großteil der Tänzer käme aus dem Ausland. Es sei die Aufgabe des Goethe-Instituts, die künstlerischen Entwicklungen, die in Deutschland passieren und von dort ihren Ausgang nehmen, zu präsentieren und nicht eine an Staatsangehörigkeit gebundene Kultur. Man würde die ganze Dynamik in diesem Bereich nicht einfangen, wenn man nur "Deutsche" einlüde. ${ }^{55}$

Ein französischer Tänzer kann also durchaus geeignet sein, einen »deutschen Impuls« in Bosnien zu setzen. ${ }^{56}$ In einem anderen Zusammenhang argumentiert die gleiche Leiterin gegen den Vorschlag eines lokalen Mitarbeiters, den Film eines polnischen Regisseurs als »deutschen Beitrag « zu der Europäischen Filmwoche in Sarajevo einzuladen. Dieser Regisseur habe zwar in Deutschland studiert, die Bindung seines künstlerischen Schaffens an Deutschland erscheine ihr aber zu schwach; entsprechend bittet sie um die Suche nach einer Alternative. Deutschlandbezug stellt sich hier also nicht als eine eindeutige Größe dar, sondern zumindest partiell als eine situative und relationale Kategorie, die in einem jeweiligen Projektzusammenhang unterschiedlich begründet werden kann. Repräsentationen des Nationalen im Modus der Kultur werden somit immer in den situierten Wissenspraxen eines Goethe-Institutes formiert diesen Prozess der Zusammenfügung des Nationalen möchte ich nun an einem weiteren Beispiel ausführlicher verdeutlichen.

55 | Feldnotizen vom 26. Mai 2009.

56 | Der Auftritt dieses Künstlers scheiterte dann an dem Experten-Votum der zuständigen Fachabteilung des Goethe-Instituts in München, die sein Schaffen in den letzten Jahren als "wenig prägend" einschätzte. 


\section{Das Nationale zusammenfügen: Ein Oktoberfest in Ramallah}

Im Oktober 2008 organisiert das Goethe-Institut in Ramallah einen Deutschen Tag, der in erster Linie der »Werbung für die deutsche Sprache « dienen soll. ${ }^{57}$ Der Institutsleiter formuliert die Intention, durch die Zusammenstellung unterschiedlicher Aktivitäten, Angebote und kultureller Darbietungen die Arbeit des Instituts und insbesondere die Möglichkeiten des deutschen Sprachunterrichts in der Stadt greifbar zu machen. Die Vorgabe lautet, einen Tag zu gestalten, der »als deutsche Veranstaltung erkennbar ist « und hierzu auch die Straße vor dem Deutsch-Französischen Kulturzentrum als urbane Bühne einbezieht. Das Team sieht sich vor die Aufgabe gestellt, einen temporären Erlebnis- und Bildraum zu erschaffen, der »deutsch « repräsentiert und - etwa im Hinblick auf zukünftige Anmeldungen zu Sprachkursen - möglichst erfolgreich im lokalen Kontext verankert.

In der Vorbereitung entfaltet sich innerhalb des Instituts ein informeller Diskussionsprozess, welche kulturellen Bestände, Referenzpunkte, alltäglichen Dinge oder Aktivitäten zu einer solchen Repräsentation des Nationalen geeignet sind: Unhinterfragt ist hierbei die »deutsche Sprache«, die etwa durch »Schnuppersprachkurse« in das Programm integriert und somit als Kernbestand des Angebotes des Goethe-Institutes in den Vordergrund gerückt wird. Der Name Oktoberfest, der im Laufe der Vorbereitungen an die Stelle von Deutscher Tag tritt, lässt sich als pragmatischer Versuch einer Rahmung der Veranstaltung durch die assoziative Bezugnahme auf ein etabliertes, auch in Palästina bekanntes, »deutsches Kulturformat« verstehen. Debatten entwickeln sich etwa um die Zusammenstellung des Programms, des Speiseangebots oder um die Ausgestaltung des Veranstaltungsortes. Schnell steht fest, dass zu Beginn ein Kammerkonzert klassischer (europäischer) Musik im Eingangsbereich des Kulturzentrums stattfinden soll - aber ist es sinnvoll, diese Darbietung »westlicher Musik« mit einem Konzert traditioneller arabischer Musik zu kombinieren? Oder wird hierdurch der zu repräsentierende kulturelle Raum zu sehr überschritten? Der relationale und situative Charakter des Zusammenfügens nationaler Repräsentationen zeigt sich darin, dass der Institutsleiter an einem Tag für und an einem anderen Tag gegen eine solche Einbeziehung arabischer Musik plädiert. Ähnliche Diskussionen entfalten sich um das Speiseangebot: Welches Essen lässt sich als »deutsch« codieren und ist gleichzeitig für ein mehrheitlich muslimisches Publikum attraktiv? »Gehören Schweinewürstchen zwingend dazu«, wie ein deutscher Koch vor Ort betont? Ist es notwendig und angemessen, Bier öffentlich auszuschenken, um auch in Palästina dem Ruf eines Oktoberfestes gerecht zu werden? Ein Restaurantbesitzer, der

57 | Feldnotizen vom 16. September 2008. 
zwischenzeitlich als Caterer vorgesehen war, gibt dem Organisationsteam die Aufforderung »keep it German « mit auf den Weg, erbittet entsprechende Rezepte und bringt hierdurch eine Erwartung zum Ausdruck: »Deutsch« sollte an diesem Tag distinkt erlebbar sein, gerade auch, um sich von anderen Veranstaltungen in der Stadt zu unterscheiden. Das Speise- und Getränkeangebot - Hühnchen, Lammwürstchen, Kartoffel- und Krautsalat, Kaffee und Kuchen, Bier im Hinterhof - kristallisiert sich im Rahmen solcher Debatten heraus und sollte sich während der Veranstaltung als lokal anschlussfähig erweisen. Auch im Hinblick auf die Schmückung des Veranstaltungsortes kommt es zu Diskussionen: So formuliert eine Vertreterin des deutschen Auslandsschulwesens vor Ort den Vorschlag, während des Festes schwarze, rote und goldene Luftballons steigen zu lassen. Der Institutsleiter lehnt diese Idee als »zu staatstragend « $\mathrm{ab}$ und setzt seine Vorstellungen einer ästhetischen Sprache, die eher auf kulturelle Bestände, Assoziationen, spielerische Angebote und direkte Begegnungen statt auf staatliche Symbole zurückgreift, durch.

In der Zusammenstellung des Programms und somit auch von Repräsentationen des Nationalen erweist sich die Suche nach pragmatischen Lösungen unter den spezifischen lokalen Rahmenbedingungen als eine prägende Herangehensweise: Schüler und Lehrerinnen von Schulen, an denen Deutsch unterrichtet wird, werden eingeladen, auf der Bühne ein Lied oder einen Sketch auf Deutsch vorzuführen; die Leiterin eines Erzählworkshops im Goethe-Institut wird gebeten, mit ihren Schülerinnen deutsche Märchen einzuüben und auf dem Fest (auf Arabisch) darzubieten; die europäischen und amerikanischen Lehrer einer lokalen Musikschule werden angefragt, als Ensemble klassische europäische Musik zur Aufführung zu bringen; Projekte des Goethe-Institutes oder anderer deutscher Bildungsakteure sollen dem Publikum vorgestellt werden; das Bier wird nicht aus Deutschland importiert, sondern bei einer lokalen Brauerei bestellt - kurz: der erlebbare Raum kultureller Repräsentationen wird hier in einem großen Umfang durch Rückgriffe auf bestehende Partnerschaften und Bezugnahmen auf Angebote vor Ort zusammengesetzt. Das Bemühen um alltagspraktische Übersetzungen kultureller Repräsentationen des Nationalen in einen lokalen Referenzrahmen stellt sich als zentrale Strategie des Verankerns dar. Diese Vorgehensweise möchte ich nun anhand von drei Beispielen weiter illustrieren: der Produktion von Werbematerialien, der Entwicklung von Argumentationsfiguren zur Begründung der Attraktivität der deutschen Sprache sowie anhand der Auswahl einer Sängerin als Hauptprogrammpunkt.

Das Projektteam erstellt zur Bewerbung des Oktoberfestes einen Radiospot, der eine Sequenz eines bekannten ägyptischen Films nutzt. Hierin wird dem Hauptcharakter vor seiner geplanten Hinrichtung ein letzter Wunsch gewährt. Seine Antwort »Deutsch lernen« erklärt sich durch die in Ländern des Nahen Ostens offenbar verbreitete Ansicht, dass das Erlernen der deutschen 
Sprache besonders kompliziert und langwierig sei; der Moment der Tötung würde entsprechend relevant verzögert, wie mir eine lokale Mitarbeiterin erläutert. ${ }^{58}$ Das Goethe-Institut versucht hier also, seinen zentralen Arbeitsauftrag - Verbreitung der deutschen Sprache - mittels medial produzierter Bilder in lokale Vorstellungswelten einzuarbeiten. Ein ähnliches Bemühen zeigt sich in den Diskussionen zur Erstellung einer Broschüre, die für das Sprachkursprogramm des Instituts werben und während des Oktoberfestes verbreitet werden sollte. Hierzu konsultiert das Team zunächst durch große Goethe-Institute in Nordamerika produzierte Beispiele, deren - in der Einschätzung der Kulturmittler gelungene - urbane und multiethnische Ikonografie aber als unpassend für den lokalen Kontext eingeschätzt wird: »Hier gibt es einfach nicht solche Gesichter«, kommentiert die zuständige Mitarbeiterin und formuliert die Sorge, dass man das palästinensische Publikum durch eine solche importierte Bildsprache eher irritieren könne. ${ }^{59}$ Sie entwickelt die Idee, die von Israel errichtete Mauer als Motiv aufzugreifen und auf dem Titelbild ihre Überwindung durch Goethe und Marianne als Repräsentanten für Deutschland und Frankreich darzustellen - eine Vorstellung, die sich, nicht zuletzt aufgrund eines zunehmenden Zeitdruckes, im weiteren Planungsprozess nicht umsetzen lässt. Auch im Hinblick auf die Begründung der Attraktivität der deutschen Sprache wird versucht, Argumentationsfiguren zu entwickeln, die an lokale Bedeutungssysteme anknüpfen. Hier setzt sich zunehmend die Betonung von »Karriere, Industrie, Jobs und Opportunities «, ${ }^{60}$ die sich durch Deutsch eröffnen könnten, gegenüber der zunächst geplanten Verweise auf Goethe, Kant und Einstein als prominente deutschsprachige Individuen durch. »Deutsch« wird hier weniger als symbolischer Gewinn, sondern primär als eine verwertbare Option auf beruflichen Erfolg und Mobilität in den ökonomisch und politisch instabilen lokalen Kontext Palästina übersetzt. Das Projektteam knüpft somit an die Erfahrung an, dass die Sprachkurse in Ramallah primär von jungen Männern besucht werden, die ein Studium in Deutschland anstreben. Entscheidend erscheint mir hierbei, dass solche Übersetzungen weder willkürlich noch unausweichlich sind: In Abhängigkeit von dem Format, auf das man hinarbeitet, den beteiligten Personen und deren Expertisen, den finanziellen Ressourcen und dem zeitlichen Rahmen könnten auch andere Assoziationsketten oder Ikonografien entwickelt werden, die jedoch an bekannte Bilder oder plausible Argumentationsfiguren anknüpfen müssen. Sichtbar wird hier das kleinteilige Zusammenfügen kultureller Repräsentationen, das sich im Zuge von relationalen, situativen, pragmatischen, mitunter auch zufälligen Übersetzungen in lokale Referenzrahmen realisiert - Übersetzungen, die dennoch

58 | Feldnotizen vom 16. September 2008.

59 | Feldnotizen vom 22. September 2008.

60 | So der Institutsleiter, Feldnotizen vom 22. September 2008. 
zugleich durch Bezugnahmen auf etablierte Bestände des historisch gewachsenen symbolischen Raums des Nationalen unterlegt sind.

Diesen Zusammenhang möchte ich nun anhand der internen Diskussionen, welche der Einladung einer Sängerin als Hauptprogrammpunkt des Oktoberfestes vorausgingen, vertiefen. Während einer Besprechung formuliert der Institutsleiter, dass es eigentlich egal sei, wen man aus Deutschland hole; die Leute hier kämen nicht. Einen Unterschied mache es nur, wenn man Künstler mit Beziehung zur Region oder Musik mit »orientalischem Einschlag« präsentiere. ${ }^{61}$ Diese ernüchternde, aus der Erfahrung gewonnene Einschätzung zu der begrenzten Anschlussfähigkeit von »deutscher Musik« bildet den Ausgangspunkt für die Suche nach einem dennoch geeigneten Beitrag. Zunächst verhandelt das Team mit einer Sängerin aus Berlin, die mit palästinensischen Eltern und einem partiell arabisch gesungenen Programm zwei Aspekte mitbringt, die sie auch in der Einschätzung der lokalen Programmitarbeiterin für ein palästinensisches Publikum interessant macht. Dieser Versuch, eine lokal kompatible Ausformung des Nationalen in Ramallah zu verankern, gerät ins Stocken, als die Sängerin mitteilen lässt, dass sie als »gläubige Muslima« nicht an einem Ort auftreten wolle, an dem Bier ausgeschenkt wird - eine Begründung, die selbst die muslimischen Mitarbeiter des Instituts überrascht. ${ }^{62}$

Nach dieser Absage veranlasst der Institutsleiter eine Anfrage an die Fachabteilung Musik der Zentrale des Goethe-Instituts in München nach einem deutschen Beitrag mit »etwas simpel ausgedrückt: orientalischem Hintergrund, da einfach deutsche Musik in Ramallah nicht zieht«. Der Ansprechpartner in München rät wiederum zu einer Berliner Sängerin, die inzwischen in Istanbul lebt - eine Empfehlung, die auf Zustimmung stößt: Dem Institutsleiter erscheint die »selbstbewusste Weiblichkeit« dieser Künstlerin als angemessene Repräsentationsform in der »nicht so konservativen Stadt « Ramallah; auch die lokale Mitarbeiterin hält diese Wahl für anschlussfähig, wenn auch aus anderen Gründen: »Türkisches « sei gerade in Mode, auch wenn man während der osmanischen Zeit schlechte Erfahrungen gemacht habe. Gerade eine türkische Soap sei aktuell sehr populär.

Entsprechend wird die Sängerin Aziza A nach Ramallah eingeladen und tritt mit einem deutsch-türkischen Programm am Abend des Oktoberfestes auf einer kleinen Bühne vor dem Goethe-Institut auf. Am Tag zuvor wird ein Workshop improvisiert, in dem Aziza mit zwei palästinensischen Hip Hop-Sängern ein Lied erarbeitet, das die drei am Ende des Konzertes gemeinsam vorführen. Das Publikum reagiert freundlich, wenn auch ohne Euphorie.

61 | Feldnotizen vom 16. September 2008.

62 | Feldnotizen vom 18. September 2008; auch die restlichen Verweise und Zitate in diesem Textteil. 
Von Interesse sind dieser Auftritt und seine Vorbereitungen, da hier Orientalisierung und Dialogisierung als zwei mögliche Modi zur Verankerung von kulturellen Repräsentationen des Nationalen im lokalen Kontext Ramallah greifbar werden: Deutsch-türkische Hybridität, biografische Bezüge der Künstlerinnen in die - hier eher flexibel verstandene - Region und »orientalische Klänge« werden zu Bestandteilen, um »deutsche Musik« anschlussfähig zu machen; durch die gemeinsame Arbeit und Performance mit zwei palästinensischen Musikern bleibt es nicht bei einer vorgefertigten Darbietung, sondern Begegnung und Kooperation werden zu Teilaspekten der hier zusammengefügten Repräsentation des Nationalen. Orientalisierung und Dialogisierung verweisen zugleich auf Vorgehensweisen, die zwar auf Verankerungen ausgerichtet sind, aber durch ihr Bemühen, translokale Anschlüsse zu schaffen, einen Schritt weiter gehen. Diese dritte Strategie kulturpolitischer Übersetzung möchte ich nun diskutieren.

\section{Anschlussfähige Formate: Gemeinsame Lernprozesse, produktive Netzwerke, nachhaltige Wirkungen}

Selbst wenn das Zusammentreffen von Aziza A mit palästinensischen Musikern sehr kurzfristig angesetzt wurde und in einem improvisierten Rahmen stattfand, so verweist dieses Beispiel dennoch auf die Bedeutung, die solchen dialogischen Formaten für die lokale Verankerung der Programme eines Goethe-Instituts zukommt: Der gemeinsame Auftritt der drei Sänger wurde von vielen Besuchern als der Höhepunkt des gesamten Oktoberfestes betrachtet. Gleichfalls sprechen die deutschen Kulturmittler häufig von Kooperation, Begegnung oder Wechselseitigkeit, um mir die Spezifik ihrer Arbeit zu erklären. So erläutert mir der Institutsleiter in Ramallah seine Herangehensweisen nochmals in Abgrenzung zu seinen französischen Partnern. Diese würden zumeist eher einfache, relativ kostengünstige, aber durchaus sichtbare Veranstaltungen mit lokalen Kulturschaffenden organisieren: etwa ein Konzert mit Musiklehrern, die bereits in Bildungseinrichtungen vor Ort arbeiten. Dieser Ansatz habe durchaus seine Berechtigung, da zu diesen Anlässen wichtige Personen - also »Multiplikatoren und Meinungsbildner in Palästina« - zusammenkämen: »Das kann man jetzt gar nicht sagen, dass das falsch ist. Wir haben uns nur anders entschieden. Wir haben gesagt, wir machen weniger von diesen kleineren Veranstaltungen. $\ll^{63}$ Stattdessen organisiere man »weitergehende « Formate, im eigenen Verständnis anspruchsvollere Koproduktionen, die »irgendwohin führen müssen « - also nachhaltige Wirkungen hervorrufen, die nicht auf das jeweilige Projekt beschränkt bleiben: »Bei dem französischen Ansatz sieht man sehr viel, bei uns macht man was gemeinsam. Man muss

63 | Interview vom 4. Dezember 2008; auch die folgenden beiden Sequenzen. 
sich mit anstrengen, muss selber was machen und das dürfte zumindest inhaltlich nachhaltiger wirken. [...] Also wir geben den Leuten die Chance, mit uns gemeinsam etwas zu machen.«

Im Rahmen solcher Kooperationen sollen nicht nur gemeinsam erarbeitete Produkte entstehen, sondern vor allem wechselseitige Lernprozesse Raum finden:

"Was die Franzosen auch wieder machen, ist: Sie geben Leuten Geld, um alleine etwas zu machen - etwa um Bilder zu malen, ein Stipendium, die Rahmen und die Farben. Auch das wollen wir nicht. Wir wollen, dass sie mit uns gemeinsam etwas machen, dass sie uns kennenlernen, mehr über uns lernen, aber eben in gemeinsamen Prozessen. Aber auch so, dass wir über sie lernen."

Kooperative Herangehensweisen werden hier nicht nur als alltagstaugliche Strategien zur lokalen Verankerung kulturpolitischer Programme betrachtet, sondern dienen zugleich als Distinktionsmittel: Mein Gesprächspartner verweist auf Idealvorstellungen von den eigenen Vorgehensweisen, um die Spezifik der deutschen Kulturarbeit - in Abgrenzung zu den französischen Kollegen - herauszustellen. Sequenzen aus meinem Interview mit dem Institutsleiter in Tel Aviv vermitteln einen Eindruck davon, wie sich solche Idealbilder in konkrete Arbeitspraxis und Projektformate übersetzen lassen: »Ich habe einen Künstler jetzt vor zwei Monaten da gehabt. Daraus ist innerhalb einer Woche ein so produktives Netzwerk entstanden, die Ideen austauschen, die Sachen miteinander entwickeln- das sind auch keine unnahbaren Künstler. Das sind also eben die, die wirklich noch arbeiten. ${ }^{64}$

Die Einladung eines deutschen Künstlers erfolgt hier also explizit nicht zur Präsentation bereits existenter Werke, sondern mit der Intention, künstlerische Praxis in translokalen Kontexten neu zu verflechten: $\gg$ Da brauche ich keinen [Name eines berühmten Künstlers] hierher zu bringen, der sowieso mit niemand zusammenarbeitet. Da bringe ich einen jungen Künstler und schon passiert etwas. Da muss ich keine große Kunst machen, sondern ich muss diese Netzwerke schaffen.«

In der folgenden Sequenz wird noch deutlicher, wie eine solche Initiierung von produktiven Netzwerken im Rahmen eines Projektes verlaufen kann:

"Ich muss die Netzwerke miteinander verbinden. Was ich jetzt mache- ein sehr aufwendiges Projekt, auch finanziell sehr aufwendig, im Vorlauf wahnsinnig aufwendig: Neue Musik! Neue Musik kann man über ein Konzert verbreiten. Es passiert aber nichts. Da ist ein Konzert, eine kleine Gruppe, die dahin geht, die sich das anhören und das mit nach Hause nehmen oder auch nicht. Ich habe aber folgendes gemacht: Ich habe ein

64 | Interview vom 10. Dezember 2008; auch die folgenden drei Sequenzen. 
junges israelisches, Tel Aviver Ensemble mit einem jungen, engagierten Berliner Ensemble zusammengebracht. Die haben sich ein Jahr lang ausgetauscht, dann haben sie sich getroffen. Sie haben ein gemeinsames Programm entwickelt, also, indem sie ihre Repertoires austauschen, ihre Erfahrungen mit Musik austauschen. Und daraus sind Auftragskompositionen entstanden, und es gibt einen Wechsel im Repertoire. Die Israelis spielen deutsche Komponisten, die Deutschen spielen israelische Komponisten, auf die sie sonst nicht gekommen wären. Da verzahnt sich dann Kultur. Und da wird es auch produktiv, denn die nehmen das ja auch mit auf ihre nächste Tour. Wenn die dann nach Amerika gehen, spielt das deutsche Ensemble auf einmal einen israelischen Komponisten und umgekehrt. Und dann habe ich eine Wirkung. Nicht nur, dass neue Werke entstanden sind - auf die die Welt sicher auch verzichten kann - aber ich weiß, die arbeiten weiter miteinander. Die werden sich in anderen Ensembles wiederfinden. Die empfehlen einander hier und da und dort. Und so entstehen Netzwerke, die einfach auch ästhetisch fruchtbar werden."

Mein Gesprächspartner verweist hier besonders prägnant auf die Initiierung translokaler Wirkungsketten als einer zentralen Zielvorstellung für seine Arbeit. Die Verankerung eines Goethe-Instituts in seinem lokalen Kontext betrachtet auch er hierzu als eine Voraussetzung:

"Nie etwas zwingen. [...] etwas zwingen, heißt: Ich lasse jetzt hier ein UF0 landen, ich hole einfach eine deutsche Combo hierhin und lass die spielen. Und eigentlich interessiert sich keiner dafür, weil es der Bedürfnislage nicht entspricht. Ich kann noch so guten Willens sein. Wenn ich meine Wünsche nicht mit den lokalen Bedürfnissen verquicke, erreiche ich niemanden; erreiche ich niemanden außer denen, die es sowieso schon wussten. Das heißt, also jedes Projekt, jedes Programm ist das Ergebnis oder eine Zwischenstation eines offenen Prozesses. Das kann von allen möglichen Seiten ausgehen."

Anhand solcher Zitate wird ein Spannungsverhältnis sichtbar, das die Übersetzungsarbeit deutscher Kulturmittler grundlegend durchzieht: Einerseits zeigt sich auch hier die bleibende Wirkmächtigkeit der dichotomischen Logik, welche die imaginären Dimensionen Auswärtiger Kulturpolitik elementar strukturiert (Kapitel II.5). Denn die Künstlerinnen und Szenen, die es zu vernetzen gilt, werden immer auch durch nationale Kategorien verortet, ausgewählt und differenziert. Andererseits tritt zutage, welche Relevanz die Kulturmittler der Entwicklung unterschiedlicher Formate, die sich an den Idealvorstellungen von Dialog, Wechselseitigkeit oder Koproduktion orientieren, in der Arbeitspraxis beimessen - und zwar sowohl um sich an einem Einsatzort erfolgreich positionieren als auch um sich in die offenen Prozesse, in denen Kultur sich verzahnt, einschalten zu können. Von einem weiterführenden Interesse ist hierbei, inwiefern eine nationalstaatlich initiierte Kulturpolitik gerade durch dieses 
Wechselspiel in ihren translokalen Kontaktzonen und Übersetzungsfeldern an Anschlussfähigkeit gewinnen kann.

\section{Reskalierungen des Nationalen: Deutschlandbezüge erweitern, verflechten, inkorporieren}

Diese Frage möchte ich nun anhand von drei Projekten aus Bosnien-Herzegowina diskutieren, indem ich aufzeige, wie das Leitmotiv Deutschlandbezüge herstellen eben durch diese Suche nach dialogischen Formaten und die beabsichtigte Initiierung von translokalen Wirkungsketten eine Flexibilisierung und in gewisser Hinsicht auch eine Reskalierung erfahren kann. Gleichzeitig wird sichtbar, wie sich diese zentrale Orientierungsgröße durch eine Inkorporierung von Rationalitäten, Zielsetzungen und Leitvorstellungen des Politikbereichs in die Projektarbeit trotz solcher Öffnungen auch weiterhin reproduziert.

Im ersten Beispiel wird das Grundmodell einer bilateralen Begegnung um eine französische Komponente ergänzt. Für den Herbst 2009 entwickelt das Goethe-Institut gemeinsam mit der Kulturabteilung der französischen Botschaft ein vierwöchiges Artists-in-Residence-Programm, zu dem zwei deutsche und zwei französische Künstler nach Banja Luka eingeladen werden. Unter dem Titel Art and its context(s) sollen hierbei neue Werke entstehen, die zum Projektabschluss im lokalen Museum für zeitgenössische Kunst ausgestellt werden. Der Projekttitel transportiert dabei die Erwartung, dass sich die Künstlerinnen in ihren Arbeiten nicht nur aufeinander beziehen, sondern zugleich mit den Realitäten vor Ort - etwa der prekären finanziellen Situation des Museums und des Kunstbetriebs insgesamt - auseinandersetzen. Gleichfalls wird durch ein gemeinsames Budget, über dessen Aufteilung sich die Künstler selbst einigen müssen, ein praktischer Impuls zur Kooperation gesetzt. ${ }^{65}$

Zur lokalen Verankerung des Projektes wird eine temporäre Formation ins Leben gerufen, die neben dem Goethe-Institut, der französischen Botschaft, den Künstlern und dem Museum in Banja Luka auch einen Straßburger Kunstverein und das Zentrum für zeitgenössische Kunst in Sarajevo, deren Leiterin die Ausstellung kuratiert, umfasst. Ein Kalkül der Initiatorinnen besteht hierbei darin, Akteurinnen aus den Hauptstädten der beiden zunehmend voneinander abgegrenzten - Landesteile Bosnien-Herzegowinas in einen direkten Arbeitskontakt zu bringen. Ermöglicht wird diese Kooperation durch Sondermittel, welche die Regierungen Deutschlands und Frankreichs für die Durchführung von gemeinsamen Vorhaben ihrer Kulturmittler in Drittstaaten zur Verfügung stellen. Es ist aber nicht alleine der pragmatische Rückgriff auf eine zusätzliche Geldquelle, der das Zustandekommen dieses Projektformats erklärt; die Institutsleiterin verweist mir gegenüber auf

65 | Feldnotizen vom 11. Juni 2009. 
die imaginären Dimensionen - Verständigung, Versöhnung, Dialog -, die sich durch eine solche Kooperation zweier ehemals verfeindeter Nachbarstaaten in der Postkonfliktkonstellation Bosnien-Herzegowina aktivieren ließen - anders formuliert: ein deutsch-französisches Projekt ermöglicht in diesem lokalen Kontext Anschlüsse, die sich durch Deutschlandbezüge alleine nicht herstellen ließen. In dem Projektdesign zeigen sich somit nicht nur die Erweiterung dieser Orientierungsgröße auf eine trilaterale Konstellation, sondern zugleich eine direkte Inkorporation von wirkmächtigen diskursiven Figuren - Verständigung durch Dialog - und etablierten Erzählsträngen - hier die beispielhafte Erfolgsgeschichte der deutsch-französischen Versöhnung - des offiziellen Narrativs Auswärtiger Kulturpolitik.

Im zweiten Beispiel manifestiert sich eine andere Form der Reskalierung: Unter dem Titel Zukunftswerkstatt werden junge Wissenschaftler aus den Ländern des ehemaligen Jugoslawiens, aus Österreich, Deutschland und der Schweiz, die - etwa als Historikerinnen oder Kulturwissenschaftler - zu Südosteuropa forschen, zusammengebracht. Im Zentrum stehen hierbei vier mehrtägige inhaltliche Treffen, die nacheinander in Zagreb (2008), Belgrad (2009), Sarajevo (20I0) und Regensburg (20II) stattfinden. Die Goethe-Institute an diesen Standorten arbeiten hierzu mit einer Reihe von Professoren und wissenschaftlichen Instituten in den beteiligten Ländern zusammen. Durch dieses Format sollen erstens junge Wissenschaftler zu einer »neuen Generation von Südosteuropa-Forschern« nachhaltig vernetzt werden; zweitens geht es den Initiatoren um eine Verflechtung und Internationalisierung der Wissenschaftslandschaften in den Ländern des ehemaligen Jugoslawiens; und drittens wird eine Öffnung der - als Folge von Krieg und Konflikten - zunehmend auf Abgrenzung und Exklusivität angelegten nationalen Identitäts- und Geschichtsnarrative in der Region angestrebt. ${ }^{66}$ Realisieren lässt sich dieses Projekt durch Sondermittel aus dem Stabilitätspakt für Südosteuropa, die gerade auch für die Förderung des Austauschs in der Region zur Verfügung gestellt worden waren. Die Institutsleiterin in Sarajevo erklärt mir in diesem Zusammenhang, dass sie die Unterstützung von Begegnungen und Kooperationen von Personen aus unterschiedlichen Ländern Südosteuropas als eine zentrale Aufgabe des Goethe-Instituts betrachte, da die Kriege hier viele vormals etablierte Kontakte zerstört hätten. ${ }^{67}$ Zwar distanziert sich meine Gesprächspartnerin von dem ihr »zu hochtrabend« klingenden Zielhorizont Konflikt-

66 | Hier im Rückgriff auf eine Projektseite des Goethe-Instituts, die im Sommer 2014 noch online einsehbar war, auf die digitale Anthologie Re/Visions, die aus den Zukunftswerkstätten entstanden ist: http://www.kakanien-revisited.at/beitr/re_visions/ SBernasconi1.pdf sowie auf meine Feldnotizen aus dem Frühling/Sommer 2009, die Vorgespräche zu der dritten Werkstatt dokumentieren.

67 | Feldnotizen vom 22. Juni 2009. 
prävention; dennoch zeigt sich hier zumindest der Versuch, im Rahmen der Programmarbeit pragmatische und zukunftsorientierte Umgangsformen mit den Effekten eines Konflikts zu entwickeln - eine Intention, die nicht auf die Zukunftswerkstatt beschränkt bleibt, sondern sich in unterschiedliche Projekte zur Förderung regionaler Vernetzungen übersetzt.

Die intendierten Verflechtungen und Anschlüsse führen im dritten Beispiel noch über diese regionale Dimension hinaus. Im Zentrum steht hierbei mit der Sevdahlinka »eine alte Liedform, die aus der Begegnung zwischen osmanischer Musik und sephardischen Romanzas entstanden ist und sich mit einheimischen Instrumenten durch fünf Jahrhunderte im Balkan gehalten hat ${ }^{68}{ }^{6}$ Das Goethe-Institut und ein deutsch-türkischer Produzent laden Musiker aus elf Ländern dazu ein, »klassische Kompositionen neu einzuspielen«. Als Ergebnis entsteht eine CD, welche die Sevdahlinka als bosnischen Beitrag zum Genre der Weltmusik neu zu positionieren versucht. Das gleichfalls aus Mitteln des Stabilitätspaktes für Südosteuropa finanzierte Projekt zielt somit nicht nur auf internationalen Austausch und ein gemeinsam erarbeitetes Produkt, sondern auf die Wiederentdeckung, globale Aneignung und dialogische Transformation einer lokal situierten musikalischen Praxis:

"Auf diese Weise befreien wir die Sevdahlinka aus den Fesseln einer allein auf Bosnien lokalisierbaren und konservativen Liedform. Wir lassen sie für sich selber sprechen: als eine wunderbar zeitlose Musik, die herausragende junge Interpreten unter Serben, Bosniaken, Makedoniern, Kroaten und anderen Völkern des Balkans hat und Musiker rund um den Globus zu emotional tiefen musikalischen Dialogen inspirieren kann."

In dem gleichen Einleitungstext zu dieser $\mathrm{CD}$ bezeichnet der damalige Institutsleiter das Projekt als einen Beitrag zur »zivilgesellschaftlichen Vertrauensbildung durch die Einbindung der Künste«, die eine wichtige Zielsetzung der Arbeit des Goethe-Instituts in Bosnien darstelle. Die Sevdahlinka könne eine »kulturelle Klammer zwischen den Bevölkerungsgruppen der erst zehn Jahre alten Nachkriegsgesellschaft« darstellen. Auch an diesem Beispiel wird somit sichtbar, wie etablierte politische Rationalitäten oder mobile Metaphern im Rahmen eines Projektes aufgegriffen und in lokale Handlungskontexte eingearbeitet werden.

Auf dieser Basis lassen sich drei Aspekte zu einem Zwischenfazit zusammenführen: Zunächst gilt es festzuhalten, wie stark die Schlüsselbegriffe,

68 So zu lesen in dem einführenden Text des damaligen Institutsleiters in dem Begleitheft der CD, die im Rahmen dieses Projektes produziert worden ist: Goethe-Institut Bosnien und Herzegowina \& Yaman d.o.o.: Bentbasa. Sevdalinka - Bosanska ljubavna pjesma / Bosnische Liebeslieder / Bosnian Love Songs. Sarajevo 2006. Auch die folgenden Zitate entstammen diesem Text. 
Grundprinzipien und Leitmotive des politischen Narrativs die Projekt- und Übersetzungsarbeit der Kulturmittler präkonfigurieren. Sichtbar wird ein Arbeitsmodus, der zentrale Bestandteile der imaginären Dimension Auswärtiger Kulturpolitik in konkrete Formate inkorporiert und hierdurch zugleich zur Kohärenz des translokalen Politikbereiches beiträgt. Zweitens sind die genauen Ausformungen solcher Bezugnahmen in der Regel aber nicht unausweichlich oder gar zentral vorgegeben. Innerhalb des etablierten Rahmennarrativs sind flexible Übersetzungen möglich. Diese Vielfalt ergibt sich sicherlich aus den Interessen und Arbeitsschwerpunkten des Leitungspersonals oder aus zeitlich begrenzten Themenkonjunkturen. Sie ergibt sich aber vor allem aus der Intention, dialogische Formate zu entwickeln, die mit lokalen Notwendigkeiten, Realitäten und Diskussionen korrespondieren sowie zugleich möglichst nachhaltige Wirkungsketten hervorrufen sollen. Aus dieser Arbeitspraxis gehen temporäre Formationen hervor, die Fragestellungen, Wissensbestände, Kooperationspartner, institutionelle oder lokale Kontexte, Ressourcen und Künstlerinnen auf eine jeweilige Weise verbinden. Zielsetzungen der deutschen Auswärtigen Kulturpolitik werden in diesem Rahmen weniger linear umgesetzt, sondern durch habitualisierte Bezugnahmen zirkulär mobilisiert. Drittens zeigt sich das Vermögen, Deutschlandbezüge herstellen als zentrale Orientierungsgröße im politischen Narrativ und im Selbstverständnis der policy community in der Projektarbeit flexibel aufzugreifen und ihre Anschlussfähigkeit durch Verflechtungen kultureller oder wissenschaftlicher Praxis oder Reskalierungen der Bezugsgrößen zu erweitern. Gleichzeitig stellen sich Deutschlandbezüge durch die beschriebene Inkorporierung von politischen Zielvorstellungen fraglos auch dann her, wenn sie in der inhaltlichen oder personellen Zusammenstellung eines Projektes nicht zentral erscheinen. Den Aspekt der neuartigen Anschlüsse, die sich für eine nationalstaatlich initiierte Kulturpolitik durch die Reskalierung ihrer Bezugsgrößen schaffen lassen, werde ich in der letzten Passage dieses Abschnitts anhand eines weiteren Beispiels aus Sarajevo vertiefen.

\section{Geteilte Geschichten: Europa als kulturpolitische Metapher}

»Wenn ich über >Europa< komme, komme ich nicht von außen, sondern bin schon dabei ${ }^{69}$ erläutert mir die Institutsleiterin die Potenziale einer spezifischen Form der Positionierung als deutsche Kulturmittlerin in Sarajevo, die an ihrem vorherigen Einsatzort in Pakistan nicht gegeben waren. Denn hier begegnet sie »der europäischen Kultur in ihrer besonderen, bosnischen Ausprägung«. Aus dieser Beobachtung zieht meine Gesprächspartnerin zumindest zwei Schlussfolgerungen: Zum einen gestattet diese Konstellation Formen des

69 | Interview vom 23. Juli 2009; auch die folgenden Zitate in diesem Textteil. 
Dialogs, in denen es - anders als etwa in arabischen oder afrikanischen Ländern - nicht zuerst um die Herstellung von Gleichwertigkeit gehen müsse: »In Europa hat man schon eher das Gefühl, man verhandelt auf Augenhöhe. Man nimmt das einfach an. An anderen Orten muss man das erst mal klarstellen.« Zum anderen »stellt sich die deutsche Kulturarbeit hier nicht als nationale Kulturarbeit dar, sondern als Teil eines facettenreichen Europas«. Das Bild eines Dialogs auf Augenhöhe geht also mit der Tendenz einher, Repräsentationen des Nationalen zu europäisieren - etwa indem etablierte Vorstellungen von einem »inneren« und einem »äußeren Raum der Kultur« sukzessive zu einem gemeinsamen Kontext verbunden werden:

"Vieles was man als deutsch ansehen würde, ist erst mal europäisch. Man teilt ja eine große gemeinsame Geschichte. Also, als ich in mein erstes Institut im Ausland, nach Syrien gegangen bin und mir dort in der Bibliothek anschaue, was für klassische Musik-CDs wir da haben, da hatte ich das Gefühl, da fehlt unheimlich viel von meinem Erbe. Ich wollte schon wild protestieren, was das für eine einseitige Auswahl ist oder warum da so viele Sachen fehlen bis mir klar geworden ist: Ich trage, was klassische Musik angeht, ein europäisches Erbe in mir und da werden jetzt, weil wir ein deutsches Kulturinstitut sind, Dinge getrennt, die aber einfach epochenmäßig zusammengehören. Ich würde nie sagen, wenn ich an klassische Musik denke: 'Was ist mein deutsches Erbe? Das ist nun vielleicht extrem ein europäisches Erbe, aber das würde ich bei ganz vielen Sachen sagen: Philosophie oder wenn ich an die Aufklärung denke. Das ist europäisch. Wenn ich das dann zur Diskussion stelle, dann komme ich vielleicht eher auf einen deutschen Denker der Aufklärung als auf einen französischen, aber der ist nur zu denken in dem europäischen Kontext."

Diese Entwicklung, einstmals national codierte Bestände stärker als Bestandteile einer »gemeinsamen Geschichte « und eines »geteilten europäischen Erbes« $\mathrm{zu}$ verstehen, verdeutlicht meine Gesprächspartnerin anhand eines weiteren Erlebnisses am Goethe-Institut in Syrien: Ein Vertreter der österreichischen Botschaft sei bei ihnen vorstellig geworden, da sie im Rahmen einer Veranstaltung Musik von Mozart aufführten. Dies wurde damals offenbar als übergriffig erlebt, sei aber heute in dieser Form kaum noch denkbar. ${ }^{70}$ In der Konsequenz formuliert die Institutsleiterin ein zeitgenössisches Idealbild von ihrer Arbeit als deutscher Kulturmittlerin in Bosnien, das deutlich an das von der Europäischen Union etablierte Leitmotiv von der Einheit in Vielfalt ${ }^{71}$ erinnert: »Ich mache das deutsche Fenster auf. Aber es ist natürlich sehr schön, wenn ich das im europäischen Chor mache und die anderen Fenster sind auch auf.«

70| Feldnotizen vom 6. April 2012.

71 Siehe hierzu: Cris Shore: Building Europe. The Cultural Politics of European Integration. London, New York 2000. 
Von Interesse ist, ob und auf welche Weise sich solche Positionen auch in der Entwicklung von Projektformaten, Organisations- und Arbeitsweisen niederschlagen - anders formuliert: welche neuen Anschlüsse lassen sich durch die Mobilisierung von Europa als einer kulturpolitischen Metapher schaffen? Vor diesem Hintergrund sticht zunächst die Betonung des »EUNIC-Clusters« in Sarajevo als »ein ganz wichtiges Instrument« hervor - also der lokalen Variante des Organisationsrahmens, den sich die offiziell beauftragten Kulturinstitute von EU-Mitgliedstaaten gegeben haben, um auf europäischer Ebene präsent zu sein und sich zugleich an jeweiligen Standorten auszutauschen. ${ }^{72}$ In Sarajevo nehmen auch die Kulturabteilungen der Botschaften - inklusive der Schweiz an den Treffen und Aktivitäten dieses Clusters teil; lokale Partner sind von dieser Organisationsform hingegen ausgeschlossen. Europa konstituiert sich hier also durchaus vielstimmig, wenngleich ohne Vertreterinnen Bosnien-Herzegowinas sowie im Regelfall ohne die kulturpolitischen Repräsentanten von Nicht-Mitgliedstaaten der EU. Aber auch innerhalb dieser präkonfigurierten Diversität zeigt sich Europa als ein asymmetrisches Gebilde: etwa aufgrund der Tatsache, dass Deutschland, Frankreich und Großbritannien durch die Kulturabteilungen der Botschaften und die Kulturinstitute doppelt vertreten sind; oder aufgrund der substanziellen Unterschiede zwischen den Mitteln, die den Botschaften von Griechenland, Portugal oder Ungarn einerseits und dem staatlich beauftragten deutschen Kulturinstitut andererseits für die Realisierung von Programmen zur Verfügung stehen. Sichtbar werden solche Asymmetrien zugleich in den Potenzialen, eigene Zielsetzungen oder Aktivitäten in einen europäischen Rahmen zu stellen: In der Arbeitsgruppe »Sprache«, die sich zu einer koordinierten Umsetzung der europäischen Anforderungen an den Fremdsprachenunterricht und der Vorbereitung von lokalen Aktivitäten zum Europäischen Tag der Sprachen gebildet hat, sind nur Deutsche, Franzosen und Briten vertreten. Als die deutsche Institutsleiterin die Vertreterin der griechischen Botschaft zu einer Mitarbeit mit dem Hinweis einlädt, es ließen sich sicher Interessenten an einem griechischen Sprachunterricht in Sarajevo finden, antwortet diese: »I doubt it. $\ll^{73}$

Die Relevanz dieser europäischen Organisationsform für die kulturpolitische Praxis möchte ich am Beispiel eines Projektes erläutern: Während meiner Feldforschung in Sarajevo nutzt die Institutsleiterin dieses Cluster, um eines ihrer Herzensthemen in Bosnien-Herzegowina zu verankern. Den Ausgangspunkt bildet das Netzwerk INPUT, das sich im Jahr I977 als ein globaler Zusammenschluss von Regisseuren, Produzentinnen und Redakteuren öffentlicher Fernsehprogramme gebildet hat. Dieses Netzwerk organisiert jährlich

72 | "EUNIC" steht für "European Union National Institutes for Culture «, siehe hierzu: https://www.eunicglobal.eu (letzter Zugriff am 4. November 2017).

73 | Feldnotizen vom 26. Mai 2009. 
Konferenzen, zu denen - laut Selbstbeschreibung - über Ioo० »television professionals« aus über 50 Ländern an wechselnden Orten zusammenkommen, um eine Woche lang die besten und interessantesten Fernsehproduktionen zu sehen, sowie um neue Trends, Probleme, Themen, Methoden und Techniken in diesem Feld zu diskutieren. ${ }^{74}$ Meine Gesprächspartnerin hatte dazu beigetragen, Ghana und Pakistan - zwei ihrer vorherigen Standorte - in dieses Netzwerk zu integrieren, indem sie etwa die Reise von Fernsehmachern zu den Konferenzen finanzierte oder Produktionen aus diesen Ländern in das Programm einzubringen versuchte. Ein gängiges Format zu einer solchen Verknüpfung stellt die Organisation einer Mini-INPUT dar. Dabei handelt es sich um eine mehrtägige Veranstaltung, auf der ein lokales Publikum eine kleine Auswahl der Beiträge schaut und diskutiert, die zuvor auf der globalen Konferenz gezeigt worden waren. Auf Basis ihrer bisherigen Erfahrung kam die Institutsleiterin bereits mit der Absicht nach Sarajevo, nach Möglichkeit auch Bosnien-Herzegowina durch die Organisation solcher Mini-INPUTs sowie durch die Entsendung von bosnischen Vertretern und Beiträgen an dieses globale Netzwerk anzudocken. Um diese Idee zu realisieren, gründet sie innerhalb des lokalen EUNIC-Clusters eine Arbeitsgruppe, die über mehrere Jahre hinweg Mini-INPUT-Konferenzen in Sarajevo organisieren wird. Projekt und Themenfeld erhalten einen europäischen Rahmen, der sich zunächst in der Organisationsform zeigt. Fünf oder sechs Partner treten gemeinsam als Projektteam auf, finanzieren die Anreise von Gästen aus ihren jeweiligen Ländern und vergrößern hierdurch nicht nur das Projektbudget:

"Das stärkt die Glaubwürdigkeit. Also wenn ich die Mini-INPUT nur als deutsches Kulturinstitut machen würde, dann wäre das eine schwächere Performance als wenn man das in einem europäischen Zusammenhang macht. Ganz abgesehen davon, dass man als Organisationsteam auch potenter ist, wenn es zum Beispiel darum geht, Leute einzuladen. Aber von der politischen Glaubwürdigkeit her ist mir das fast noch wichtiger." " ${ }^{75}$

$\mathrm{Zu}$ dieser größeren Glaubwürdigkeit trägt auch eine Europäisierung des Gegenstandes bei: Denn im Zuge des Projektes wird öffentlich-rechtliches Fernsehen als ein »europäisches Kulturgut« definiert, das - ob als Anspruch oder Wirklichkeit - in einem europäischen Land existieren sollte und zugleich in gewisser Hinsicht dessen Europäizität demonstriert. Dieses gemeinsame Kulturgut kann aber - und das ist zentral - sehr unterschiedlich verstanden und realisiert werden. Nach Auffassung der Institutsleiterin vergrößert sich die Glaubwürdigkeit dieses Formats für ein lokales Publikum gerade dadurch,

74 | So in etwa die Selbstbeschreibung auf der Homepage: http://input-tv.org/about (letzter Zugriff am 4. November 2017).

75 | Interview vom 23. Juli 2009. 
dass auf den Mini-INPUTs Tendenzen einer politischen Einflussnahme in Italien oder anderen EU-Staaten kritisch diskutiert werden - anders ausgedrückt: die Probleme des öffentlichen Fernsehens in Bosnien-Herzegowina zwischen chronischer Mittelknappheit, politischem Klientelismus und ethnonationaler Zersplitterung lassen sich in einen europäischen Rahmen heben und bearbeiten. Sie erscheinen vor diesem Hintergrund weniger als »defizitärer Sonderfall«, als wenn man die schwierigen lokalen Realitäten alleine als ein deutsches Kulturzentrum aufgreifen würde. Der Bezug auf Europa lässt mehr Variationen, Differenzen, weniger hierarchisierte Dialoge zu und ermöglicht zugleich die bereits zitierte Positionierung, trotz einer Entsendung aus Deutschland nach Bosnien »nicht von außen zu kommen«.

Anhand dieses Projektes wird somit deutlich, wie sich ein deutsches Kulturinstitut neue inhaltliche und organisatorische Anschlussmöglichkeiten schafft, indem es Europa als eine kulturpolitische Metapher mobilisiert und hierdurch unterschiedliche Raumbezüge miteinander verbindet: die lokale Situation des öffentlich-rechtlichen Fernsehens als Ausgangsproblem; die translokalen Erfahrungen der Institutsleiterin mit einem globalen Netzwerk; die Entwicklung eines europäischen Organisationsformats, um dieses Themenfeld lokal zu verankern; das Bemühen des Projektteams, zu den Mini-Inputs nicht nur Vertreterinnen unterschiedlicher Sender im fragmentierten Bosnien-Herzegowina, sondern auch aus anderen Nachfolgestaaten Jugoslawiens zusammenzubringen und der Veranstaltung somit eine regionale Dimension zu geben; das Vermögen eines deutschen Kulturinstituts, sich als Veranstaltungsort eines solchen europäischen Projektes mit globalen Verbindungen in der Stadt Sarajevo zu positionieren - kurz: durch Bezugnahmen auf Europa lässt sich der Referenzrahmen für die Repräsentations- und Übersetzungsarbeit deutscher Kulturmittler projektbezogen reskalieren. Gleichzeitig erweist sich »Europäisierung « hier als ein Prozess, der die situative Verbindung sehr unterschiedlicher Raumbezüge ermöglicht.

Diese bemerkenswerte Anschlussfähigkeit erklärt sich auch daraus, dass Sarajevo als ein Kreuzungspunkt unterschiedlicher europäischer Narrative und zum Teil widersprüchlicher »Projekte der Europäisierung « ${ }^{76}$ hierfür einen ausgeprägten Resonanzraum bietet - ein Tatbestand, der auch in den Praxisund Übersetzungsfeldern, die das Goethe-Institut umgeben, greifbar wird. Auffällig ist, dass die Stadt, ihre historische Entwicklung und kulturelle Spezifik in den Kontaktzonen, in denen sich lokale und externe Wahrnehmungsmuster begegnen, immer wieder und explizit eng mit Europa verknüpft wird. Ich möchte dies durch vier kurze Verweise andeuten:

76 | So der Titel des bereits zitierten, durch Gisela Welz \& Annina Lottermann herausgegebenen Sammelbandes. 
i. So beschreibt der Schriftsteller Dževad Karahasan in seinem Buch über die Belagerung, das »Hotel Europa« als »das technische und semantische Zentrum der Stadt Sarajevo « - also als den Ort, an dem sich »der türkische und der österreichisch-ungarische Teil der Stadt« begegnen, »Mitteleuropa« und »Orient « einander durchdringen und somit die besondere »Identität Sarajevos « ästhetisch erfahrbar wird..$^{77}$ Die Zerstörung des Hotels durch die Belagerer erscheint hier als Sinnbild für einen Angriff auf die spezifische Europäizität der Stadt und ein Verständnis von Europa, das auf Begegnung, Verflechtung und Zusammenleben in Diversität aufbaut.

ii. Im Kontext von Veranstaltungen, die das Goethe-Institut zum 20. Jahrestag des Falls der Berliner Mauer in Sarajevo organisiert, wird mehrfach die Formulierung genutzt, »die Trümmer der Berliner Mauer seien den Bosniern auf die Köpfe gefallen ${ }^{78}$ - ein Sprachbild, das auf innereuropäische Verflechtungen ebenso verweist wie auf die asymmetrischen Effekte, die aus der geopolitischen Transformation des Kontinents nach Ende des Sozialismus für unterschiedliche Regionen folgten.

iii. Im Rahmen einer öffentlichen Projektpräsentation des geplanten Museums der Belagerung im Jahr 2012 erklärt einer der Ideengeber, die Bewohner von Sarajevo hätten durch ihr Bleiben während des Krieges die Multikulturalität der Stadt und somit explizit »europäische Werte« verteidigt. Daher erwarte er, dass sich Europa heute solidarisch mit der Stadt zeige, auch wenn es während der Belagerung genau diese Solidarität verweigert habe. ${ }^{79}$

iv. In einem Interview erklärt mir ein Theaterregisseur, Sarajevo sei eine der wenigen »emblematischen Städte des 20. Jahrhunderts, insbesondere in Bezug auf Europa ${ }^{80}$ In den folgenden Gesprächspassagen verweist er auf unterschiedliche Momente der Stadtgeschichte, an denen die Entwicklungen und Brüche der jüngeren europäischen Geschichte in verdichteter Form greifbar werden: das Attentat auf das österreichische Thronfolgerpaar I9I4 mit all seinen Konsequenzen; die weitgehende Ermordung der jüdischen Bevölkerung im Zuge der Besatzung im Zweiten Weltkrieg und die hieraus folgende Zerstörung des Charakters der Stadt als eines der großen jüdischen Zentren; die ausgreifende Erweiterung Sarajevos im Kontext der sozialistischen Moderne und deren Urbanisierungspolitiken; die kosmopolitische Atmosphäre während der Olympischen Spiele r984; und die Normalität eines Lebens in Diversität, das vor den I99oer Jahren niemand »Multikulturalität« genannt habe, da es ein im Alltag »integrated organic cultural concept« gewesen sei. Auch in diesem Gespräch zeigt sich der letz-

77 | Dževad Karahasan: Tagebuch der Aussiedlung. Klagenfurt 1993, S. $91 \mathrm{ff}$.

78 | Feldnotizen vom November 2009.

79 | Feldnotizen vom 5. April 2012.

80 | Interview vom 16. November 2009; auch die folgende Sequenz. 
te Krieg - hier repräsentiert durch die Zerstörung der bosnischen Nationalbibliothek - als der virulenteste Punkt der metaphorischen Verknüpfung der Stadt mit »Europa«: »And that's the major blow - on the cultural level, not only to the city, but also to Europe. Because the bombing of the library, which wasn't a military target, was specifically aimed to destroy the memory of the multicultural way in this city, in this country, and therefore in Europe. So there was a twofold genocide in this country: Genocide against the Bosnian Muslims, but also the genocide against the multicultural idea of living together. Despite that, during the war, a handful of the artists kept this multicultural idea alive. And to this day, we have a cultural pluralism in this city.«

Sichtbar wird an diesen Beispielen, dass Europa für bosnische Kulturschaffende keine Leerformel und auch keinen alltagsfernen Gegenstand politischer Reden darstellt, sondern eine historisch geschichtete, umstrittene und durchaus wertvolle Kategorie, über deren Mobilisierung sich in transnationalen Kontaktzonen ambivalente Effekte erzielen lassen. In Sarajevo steht Europa gewissermaßen existenziell zur Debatte: Es zeigte sich bedroht durch die ethnonationalistischen Angriffe der Belagerer auf die »organische«, alläglich gelebte Diversität, aber auch durch die Zögerlichkeit der europäischen Staaten, die umschlossene Stadt zu unterstützen - eine Erfahrung, die bosnische Kulturschaffende in Gesprächen mit Westeuropäern häufig situativ eine Position moralischer Überlegenheit als »Verteidiger europäischer Werte« beanspruchen lässt, auch um von den Entsandten internationaler Organisationen ein größeres finanzielles Engagement oder geringere Eingriffe in die inhaltliche Arbeit einzufordern. Es ist naheliegend, solche Positionierungen nicht alleine als eine Reaktion auf die lokale Erfahrung von Krieg und Belagerung zu erklären, sondern auch als Ausdruck der historischen Marginalisierungen des Landes und seines strukturellen Ausschlusses aus dem gegenwärtigen politischen Projekt der Europäischen Integration zu begreifen. Entsprechend hat Kimberley Coles herausgearbeitet, dass aktuelle Formen der Exklusion zwar fraglos in etablierten Beschreibungstraditionen des »Balkans« als »strukturell gehasstes Alter Ego Europas « ${ }^{81}$ gründen, insbesondere aber auch in dem alltäglichen Zusammentreffen von »internationals« und »locals« im Zuge der unterschiedlichen Stabilisierungsmissionen der »internationalen Gemeinschaft« reproduziert werden:

81 So eine Formulierung von Maria Todorova, auf deren Studie sich Kimberley Coles beruft: Maria Todorova: Die Erfindung des Balkans. Europas bequemes Vorurteil. Darmstadt 1999, S. 37. 
"However, in conducting research on democratization among internationals in Bosnia-Herzegovina, I found that they were highly ambivalent about the Europe enlargement project they worked towards: they were simultaneously drawn to and repelled by the process and idea. They viewed Bosnia as geographically in . Europe and explicitly worked towards the goal of political, social and economic inclusion into a common, unified, post-Cold War Europe, yet constructed boundaries that maintained difference. Boundary shifts kept the Bosnian categorically separate, as non-European (or not yet fully European) and as lacking, in some cases inherently, certain requisite features of a cosmopolitan pan-European mentality." ${ }^{82}$

Wahrnehmungen von Bosnien als einem ambivalenten Zwischenraum werden demnach genau in den Arbeitsfeldern reaktiviert, die eigentlich auf alltägliche Kooperation ausgerichtet sind und sich an der Zielvorstellung einer Heranführung des Landes an die EU orientieren. Dies erklärt sich zum einen aus dem unterschiedlichen Status der lokalen und internationalen Mitarbeiter, die hier Tag für Tag zusammenkommen; zum anderen zeigt sich in solchen Kontexten die Dominanz eines Verständnisses von Europa, das maßgeblich an westlichen Erfahrungen entwickelt worden ist und bestimmte politische Realitäten, Einstellungen oder Verhaltensweisen in Bosnien als »rückständig«, als »not yet fully European« erscheinen lässt.

Ähnlich wie die Forschungspartner von Kimberley Coles erklärt mir die Leiterin des Goethe-Instituts in Sarajevo, dass sie ihre Präsenz und Aktivitäten vor Ort in den größeren Kontext des internationalen Projektes der Europäisierung des Landes stellen möchte: Auf einer Fortbildung zur Öffentlichkeitsarbeit sei sie aufgefordert worden, einen eingängigen Leitsatz zur Beschreibung ihrer Tätigkeit zu entwickeln. Dort habe sie formuliert, dass die gesamte Arbeit des Goethe-Instituts im Land darauf ausgerichtet sein sollte, »Bosnien-Herzegowina auf dem Weg in die EU zu begleiten«. Aus diesem Grund sei es für sie von so zentraler Bedeutung, europäische Perspektiven und Rahmen für ihre Programme zu entwickeln. Die Mini-Inputs, die Zukunftswerkstatt oder das deutsch-französische Artists-in-Residence-Projekt in Banja Luka sind nur drei von vielen Beispielen, in denen Europa als eine verbindende Metapher, ein Organisationsrahmen oder ein Zielhorizont aktiviert wird. Vor dem Hintergrund von Coles' Beobachtungen mag es kaum überraschen, dass solche Bezüge auch hier ambivalente Effekte hervorrufen können: So nutzt meine Gesprächspartnerin Verweise auf die europäische Öffnung, die Deutschland in den letzten Jahrzehnten durchlaufen habe, um Differenz zu den kleinteiligen

82 | Kimberley Coles: Ambivalent Builders: Europeanization, the Production of Difference and Internationals in Bosnia-Herzegovina. In: Xavier Bougarel, Elissa Helms \& Ger Duijzings (Hrsg.): The New Bosnian Mosaic. Identities, Memories and Moral Claims in a Post-War Society. Aldershot 2007, S. 255-272.S. 257. 
ethnonationalen Identitätspolitiken in Bosnien zu markieren und somit die aktuelle »Europafähigkeit« des Landes in Frage zu stellen:

"Also wenn ich vorhin gesagt habe, dass es wichtig ist, dass ich hier Kulturpolitik nicht im deutschnationalen Sinne mache, sondern dass ich's gut finde, dass man das in einem europäischen Kontext macht, so ist das ein Stück Wertedialog. Dass ich sage, ihr verrennt euch da in nationale Abgrenzungen, wenn ihr wirklich Teil von Europa werden wollt, dann muss euch klar werden, dass eine nationale Einkastelung da kontraproduktiv ist. Und dass kann man aber nicht sagen, indem ich jetzt den Zeigefinger erhebe, sondern indem ich das einfach vorlebe und sage, das Deutsche geht nicht verloren, wenn ich es in einen europäischen Kontext setze, sondern es gewinnt eigentlich dadurch. " 83

Von Interesse sind solche Äußerungen zunächst, da meine Gesprächspartnerin die Repräsentation eines »europäischen Deutschlands« und die Unterstützung der Integration Bosnien-Herzegowinas in die Europäische Union als sinnvolle Übersetzungen der kulturpolitischen Zielvorstellung Konfliktprävention durch Wertedialog in den lokalen Handlungskontext benennt. Die diskursive Formation, die den Ausgangspunkt dieser Studie bildet, wird hier also durch die Mobilisierung einer europäisierten Version der etablierten Orientierungsgröße Deutschlandbezug sowie durch ein Andocken an einen großflächigen politischen Prozess aufgegriffen. Zugleich werden an diesem Zitat einige der Ambivalenzen der Projekte der Europäisierung, die deutsche Kulturmittler in Sarajevo aktivieren, sichtbar: Denn es enthält - trotz der geäußerten Zurückhaltung gegenüber Bevormundungen - die Perspektive, dass eine bestimmte, (west-)europäische Entwicklung den Orientierungspunkt und Normalfall darstellt. Hierbei gerät in den Hintergrund, dass sich Europa eben auch in den bosnischen Erfahrungen mit postsozialistischer Transformation, Krieg und Fragmentierung konstituiert, dass die Instabilitäten in diesem Teil des Kontinentes und die Europäische Integration in anderen Teilen eng miteinander verwoben sind, dass ethnonationale Projekte auch in Westeuropa zunehmend Präsenz und Raum gewinnen oder das Europäisierung nicht zwingend Einschluss und Öffnung bedeutet, sondern immer auch Potenziale der Exklusion und Marginalisierung mit sich bringt.

Somit bleibt festzuhalten, dass die Mobilisierung von Europa als einer kulturpolitischen Metapher die Anschlussmöglichkeiten einer nationalstaatlich initiierten Kulturpolitik maßgeblich erweitert und auch den intendierten Wirkungsketten eine neue Richtung zu geben vermag. Die zentrale Orientierungsgröße Deutschlandbezüge herstellen verschwindet hierdurch nicht, erscheint aber durch situative Reskalierungen des Referenzrahmens deutlich flexibler und stärker mit anderen Raumprojekten verwoben. Die Trennungen der wei-

83 | Interview vom 23. Juli 2009. 
terhin wirkmächtigen dichotomischen Logik des politischen Narrativs lassen sich somit zumindest im Hinblick auf Europa durch Bilder von einer »geteilten Geschichte« und einem »gemeinsamen Erbe« relativieren. Im Rückgriff auf Shalini Randerias - bereits in der Einleitung diskutierten - Bemerkungen zu der doppelten Bedeutung des deutschen Wortes »geteilt« - »shared « und »divided« in der englischen Übersetzung - lässt sich die Frage formulieren, ob und in welchem Umfang, die Brüche, Asymmetrien und Spaltungen, die Europa bis in die Gegenwart teilen, in diesen Projektformaten Berücksichtigung finden. Hier ergibt sich ein ambivalentes, wenngleich produktives Bild: Die privilegierte Position einer deutschen Kulturmittlerin spiegelt sich in dem Vermögen wider, eine bestimmte Perspektive auf die Grundlagen und den Verlauf europäischer Integration als Norm zu setzen; gleichzeitig eröffnen gerade Bezugnahmen auf Europa für bosnische Kooperationspartner einen Artikulationsraum, um alternative Erfahrungen und Gegenpositionen einzubringen. Die Asymmetrien innerhalb der kulturpolitischen Praxis- und Übersetzungsfelder werden hierdurch nicht aus den Angeln gehoben, aber zumindest kritisch perspektiviert.

\section{KonfliktPrävention Und IhRE ReibUngsflächen: VERKNÜPFEN ALS NAVIGIERENDE WISSENSARBEIT}

Die vielfältigen Herausforderungen einer kulturpolitischen Übersetzungsarbeit verdichten sich durch das Auftauchen der Begründungsfigur Auswärtige Kulturpolitik als Konfliktprävention. Auch wenn die etablierte Orientierungsgröße Deutschlandbezüge herstellen fraglos bereits auf die Suche nach lokal funktionierenden Anschlüssen ausgerichtet ist, so verbleibt die Vermittlungspraxis hier doch weitgehend in einem gesicherten Rahmen: Ein solcher programmatischer Fokus eines deutschen Kulturinstituts besitzt für Mitarbeiterinnen, Projektpartner und Besucherinnen eine direkte Plausibilität. Die Zielvorstellung Konfliktprävention mobilisiert hingegen - bei aller Vagheit - den Auftrag, sich explizit den Bruchlinien, Problemlagen und Defiziten an den Einsatzorten zuzuwenden und Möglichkeiten kulturpolitischer Interventionen in umstrittene Felder auszuloten.

Kulturmittler reagieren auf diese Aufforderung einerseits mit der Entwicklung neuer Zugänge und Formate, andererseits aber auch mit Skepsis: Verfügen wir tatsächlich über die Kompetenzen, finanziellen Mittel und das Standing, um in Konfliktkonstellationen nachhaltige Effekte zu erzielen? Besitzen unsere Leitbegriffe an den Einsatzorten die gleichen, positiven Konnotationen? Wie sollen wir mit fehlenden staatlichen Strukturen, materiellen Ressourcen und unzureichenden Planungssicherheiten umgehen? Und anhand welcher Kriterien lässt sich feststellen, ob unsere Maßnahmen erfolgreich sind? Sol- 
che Fragen verweisen auf Problematisierungen, die in der Arbeitspraxis an den Reibungsflächen unterschiedlicher Diskurse, Erwartungen, Denklogiken und Materialitäten entstehen. Verknüpfen betrachte ich als einen vierten Übersetzungsmodus, der trotz solcher Reibungen translokale Verbindungen herzustellen versucht und zugleich Navigationen durch asymmetrische Felder und ihre diskursiven Brechungen ermöglicht.

Die Untersuchung dieser Verknüpfungsarbeit steht nun im Zentrum. Hierzu wende ich mich zunächst den Präsenzen der »Konflikte« im Arbeitsumfeld der Goethe-Institute zu, um ein klareres Bild von den Problemlagen, mit denen eine Auswärtige Kulturpolitik als Konfliktprävention umgehen müsste, zu zeichnen. Hierauf aufbauend diskutiere ich einige der Ausgangspunkte, Vorgehensweisen und Formate, die Kulturmittler entwickeln, um solche Präsenzen aufzugreifen und mit den Zielvorstellungen dieser Policy zu verknüpfen.

\section{Komplexe Gefüge: Konflikte als kulturpolitische Arbeitskontexte}

Anthropologinnen haben an medialen Berichten und politischen Debatten zu den gewaltdurchzogenen Auseinandersetzungen, welche die Welt seit Ende des Kalten Krieges prägen, häufig eine Tendenz zur Vergegenständlichung moniert. Demnach dominieren Vorstellungen von »Konflikten« als zeitlich, geografisch und kausal eingrenzbaren Phänomenen mit isolierbaren »Problemen« solche Diskussionen. Zugleich würden zumeist interessengeleitete, politisch gesetzte »offizielle Wahrheiten « - etwa von den Ursachen, Konfliktlinien, Anfangs- und Endpunkten, von Schuld und Verantwortung für eine Auseinandersetzung - unkritisch reproduziert; hegemoniale Narrative erschienen als unhinterfragbare und gültige Tatsachenbeschreibungen. Solche Repräsentationen trügen ebenfalls dazu bei, Konflikte in bestimmten Regionen zu verorten und transportierten somit die Botschaft, dass zentrale Probleme der gegenwärtigen Welt(un)ordnung nicht »bei uns« - in den westlichen Zentren - liegen, sondern eben in Palästina, Israel, Bosnien-Herzegowina oder andernorts. $^{84}$

84 Zum Einstieg in Fragestellungen und Vorgehensweisen einer Anthropologie politischer Gewalt siehe etwa: Antonius C. G. M. Robben \& Carolyn Nordstrom: Introduction: The Anthropology and Ethnography of Violence and Sociopolitical Conflict. In: Carolyn Nordstrom \& Antonius C. G. M. Robben (Hrsg.): Fieldwork under Fire. Contempory Studies of Violence and Survival. Berkeley, Los Angeles, London 1995, S. 1-23 sowie: Nancy Scheper-Hughes \& Philippe Bourgois: Introduction: Making Sense of Violence. In: Dies. (Hrsg.): Violence in War and Peace. An Anthology. Malden, Oxford, Carlton 2004, S. 1-31. Beide Autorenteams formulieren kritische Positionen zu einer konzeptionellen "Vergegenständlichung" von "Gewalt". 
In meiner Suche nach Spuren und Bedeutungsebenen der Konfliktkonstellationen im Arbeitsalltag der beiden Goethe-Institute habe ich mich von solchen Kritiken inspirieren lassen und - als eine Art Gegenentwurf - ein Konzept von »Konflikten« als vielfältig geschichteten, translokal verwobenen Gefügen ${ }^{85}$ entwickelt: Gefüge aus unterschiedlichen Akteuren, Interessen, politischen und ökonomischen Rationalitäten, physischen Akten, translokalen Relationen, diskursiven Deutungen und Verarbeitungen sowie aus vielfältigen materiellen, politischen und kulturellen Effekten; Gefüge, die zumeist tief in den Verflechtungen, Asymmetrien und Austauschbeziehungen einer (post-)kolonialen Welt verankert sind und alleine deshalb immer auch globale Phänomene darstellen; Gefüge, deren Ausmaß, deren Verknüpfungen, deren jeweilige Vehemenz und zu einem bestimmten Zeitpunkt dominante Ausdrucksform Wandlungen unterliegen; Gefüge, in denen die sichtbare physische Gewalt immer nur die

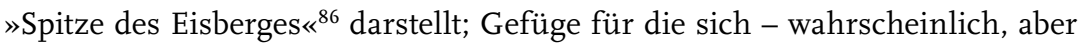
auch das wäre zu diskutieren - ein jeweiliger makropolitischer Kern, ein makropolitisches Problem identifizieren lässt, dessen Auswirkungen und Effekte aber räumlich und zeitlich weiter reichen und Eigenlogiken produzieren, die sicherlich nicht durch einen Fokus auf ein solches Zentrum alleine verstanden werden können; ${ }^{87}$ Gefüge, die sich also nicht räumlich eindeutig begrenzen lassen, aber dennoch bestimmte lokale Kontexte durchziehen und sich hier durch ihre Verwebungen mit situierten Wahrnehmungsmustern, gesellschaftlichen Debatten und Ritualisierungen des Alltags zu einer grundlegenden

85 | In der Konzipierung von Konflikten als "komplexe Gefüge" greife ich sowohl auf Positionen einer Anthropologie politischer Gewalt als auch auf Diskussionen zu "(globalen) Assemblagen « zurück. Siehe zum ersten Punkt neben den genannten Texten von Robben/Nordstrom und Scheper-Hughes/Bourgois auch: Carolyn Nordstrom: A Different Kind of War Story. Philadelphia 1997, insbesondere ihre Bemerkungen zu "warscapes", S. 37f.; zur "ethnography of a warzone ", S. 78ff. und zur Reproduktion der Gewalt auf den unterschiedlichen Ebenen des gesellschaftlichen Lebens, S. 214. Siehe zu (globalen) Assemblagen exemplarisch: Stephen J. Collier \& Aihwa Ong: Global Assemblages, Anthropological Problems. In: Aihwa Ong \& Stephen J. Collier (Hrsg.): Global Assemblages. Technology, Politics, and Ethics as Anthropological Problems. Malden, Oxford, Carlton 2005, S. 3-21 und: Saskia Sassen: Das Paradox des Nationalen. Territorium, Autorität und Rechte im globalen Zeitalter. Frankfurt 2008.

86 | Philippe Bourgois: Recognizing Invisible Violence: A Thirty-Year Ethnographic Retrospective. In: Barbara Rylko-Bauer, Linda Whiteford \& Paul Farmer (Hrsg.): Global Health in Times of Violence. Santa Fe 2009, S. 17.

87 | Siehe zum Verhältnis zwischen makropolitischem Zentrum und mikropolitischen Konstellationen, Kämpfen und Effekten zur Erklärung von Konfliktverläufen exemplarisch: Stephen C. Lubkemann: Culture in Chaos. An Anthropology of the Social Condition in War. Chicago, London 2008. 
Rahmenbedingung menschlicher Existenz verdichten können. ${ }^{88}$ Anhand von drei Dimensionen möchte ich nun die vielfältigen und dynamischen Präsenzen, die solche komplexen Gefüge in den translokalen Praxis- und Übersetzungsfeldern der Goethe-Institute in Ramallah und Sarajevo besitzen können, diskutieren.

\section{(i) Materielle Spuren und Alltagsgespräche}

So schlagen sie sich zunächst in materiellen Spuren im Raum nieder. In Ramallah sind es Grenzen, Mauern, Schranken und Zugangsbeschränkungen, die räumliche Ordnungen schaffen und Menschen mit unterschiedlichen Status ausstatten. Keine Besucherin des Goethe-Instituts, die den Weg zwischen Jerusalem und Ramallah überwindet, kann diese materiellen Effekte der Besatzung übersehen. Der israelische Architekt Eyal Weizman bezeichnet diese variablen Materialitäten und kontinuierlichen Verschiebungen von Grenzen als »elastische Geografien« der Okkupation, in denen

"triviale Elemente der Städteplanung und Architektur zu taktischen Instrumenten und zu Mitteln der Enteignung geworden (sind). Unter dem israelischen Regime einer unberechenbaren Besatzung werden das Leben der Palästinenser, ihr Besitz und ihre politischen Rechte regelmäßig verletzt, und das nicht nur durch die häufigen Übergriffe der israelischen Armee, sondern auch durch einen Prozess, der ihre Umgebung in unvorhersehbarer Weise laufend umgestaltet und sie dabei immer enger einschnürt. “ ${ }^{89}$

Die räumlichen Ordnungen aus Mauern, Zäunen, Checkpoints, Armee-Stützpunkten, jüdischen Siedlungen, unterbrochenen Zugangswegen oder exklusiven Siedlerstraßen sind dabei nicht nur der sichtbare Ausdruck eines asymmetrischen Konfliktverlaufs und der politischen Rationalitäten der Besatzung, sondern stellen zugleich die zentralen Rahmenbedingungen des Alltags sowie des gesellschaftlichen und kulturellen Lebens - und somit auch der Arbeit eines Goethe-Instituts - im Westjordanland dar.

In Sarajevo stechen zunächst verbliebene Ruinen und die Vielzahl von Einschusslöchern in Häusern als materielle Spuren des Konfliktes ins Auge. Daneben sind es hier aber vor allem arrangierte materielle Erinnerungsorte, die im urbanen Raum auf den Krieg verweisen: etwa ein als nationale Gedenkstätte angelegter Friedhof für die gefallenen Verteidiger der belagerten Stadt; mit

88 | Siehe zu den unterschiedlichen Dimensionen oder Schichten von Gewalt, sowie der Konzipierung von "Gewalt" als einem fließenden, gesellschaftliche Kontexte und Räume durchdringenden Phänomen: Antonius C. G. M. Robben \& Carolyn Nordstrom: Introduction, S. 5.

89 | Eyal Weizman: Sperrzonen. Israels Architektur der Besatzung. Hamburg 2008, S. $10 f$. 
roter Farbe gefüllte, künstlerisch transformierte Einschusslöcher in Straßen; oder ein Denkmal für die 64 Opfer eines Granateneinschlags in der zentralen Markthalle, der die NATO im Februar 1994 schließlich doch zu dem aktiven Beschuss der Belagerer bewegt haben soll. Die materiellen Spuren im Stadtbild verweisen hier also auf die Vergangenheit und zugleich auf lokale Gedächtnispolitiken: verbliebene Schäden einerseits; materialisierte Formen der Erinnerung, denen eine in der Stadt etablierte, offizielle Deutung der Geschichte eingeschrieben ist, andererseits. Auf dieser Basis lässt sich in Sarajevo keine mit Ramallah vergleichbare kontinuierliche und gegenwärtige Präsenz eines gewaltdurchzogenen Konflikts ausmachen. Ein Besucherin erhält hier als ersten Eindruck: Die Schlachten sind gekämpft, die Toten begraben, die Schuldigen benannt.

Die komplexen Gefüge werden gleichfalls in Alltagsgesprächen greifbar. In Ramallah antwortet eine Rezeptionistin auf meine Frage nach der Auslastung ihres Hotels: Es laufe ganz gut, »am wichtigsten ist, dass >sie< den Iran nicht angreifen«. Sie verweist hierdurch auf die permanente Präsenz des Konfliktes in Form einer kontinuierlichen Unsicherheit sowie der Befürchtung, dass sich Gewalt im Prinzip jederzeit realisieren und den Alltag, die individuellen und ökonomischen Lebensprojekte beschädigen oder zerstören kann - auch dann, wenn man nicht selbst zu ihrem Ziel wird: Falls Israel den Iran angreifen sollte - so die Befürchtung meiner Gesprächspartnerin - würden ausländische Besucher das Westjordanland zunehmend meiden und sich die Lebensumstände aufgrund eines noch strengeren Besatzungsregimes weiter erschweren. Die strukturellen Dimensionen dieser Konstellation ruft eine Mitarbeiterin des Goethe-Instituts auf: »Die Lage in den Palästinensischen Gebieten wird immer schlimmer - trotz oder wegen der jahrelangen Verhandlungen«, und hebt dabei den zunehmenden Wassermangel in vielen palästinensischen Gemeinden hervor - ein direkter Effekt der Besatzung, da das Wasser aus dem Westjordanland bevorzugt von den jüdischen Siedlungen genutzt oder nach Israel geleitet werde, um von dort wieder teurer an palästinensische Haushalte verkauft zu werden - sofern etwas übrig geblieben sei. Ein Kollege spricht von den möglichen direkten Auswirkungen des Konflikts auf die Projektarbeit des Goethe-Instituts und bringt hierdurch zugleich die kontinuierliche Präsenz der Besatzung im alltäglichen Denken und Sprechen zum Ausdruck: »Ein israelischer Jeep auf Al-Manara und unsere ganze schöne Planung ist nichts mehr wert. $\ll^{90}$

In Sarajevo erzählen Gesprächspartner häufig bereits in ersten Begegnungen von ihrem Aufenthalt als Kriegsflüchtlinge in Deutschland, von erzwungenen Umsiedlungen innerhalb Bosniens oder auch von den Hindernissen,

90 | Feldnotizen vom 4. Oktober 2008; bei Al-Manara handelt es sich um den Hauptplatz von Ramallah. 
nach Ende der Kampfhandlungen an ihre ursprünglichen Wohnorte zurückzukehren. Sie benennen hierdurch einen nachhaltigen Effekt der Gewalt: Das Land ist heute - demografisch, symbolisch, politisch - sehr viel deutlicher entlang von ethnoreligiösen Prinzipien geteilt als vor dem Krieg. In eine ähnliche Richtung weisen die häufigen Klagen über die gegenwärtigen Blockaden politischer Entscheidungen und Entwicklungen durch die Machtspiele der ethnonationalen Eliten - eine Konstellation, die in der Regel mit den »eingefrorenen Fronten « des Krieges, aber auch mit den politischen Ambivalenzen des international vermittelten Friedensschlusses von Dayton - die territoriale Einheit des Landes zu bewahren, aber die ethnonationale Segregation zum zentralen politischen Ordnungsprinzip zu erheben - in Zusammenhang gebracht wird. Auch wenn die dysfunktionale, stagnierende politische Gegenwart in Bosnien-Herzegowina zumeist als eine Konsequenz des Krieges dargestellt wird, so verweisen Gesprächspartner hier praktisch nie auf Alltagsstrategien, die im Umgang mit akuten, potenziell gewalttätigen Ausformungen des Konflikts entwickelt werden müssten.

Bereits erste Alltagsgespräche fördern somit in Ramallah und Sarajevo andere temporale Strukturen, also eine jeweils andere Verwebung der Vorstellungen von Vergangenheit, Gegenwart und Zukunft mit der lokal situierten Konfliktkonstellation zutage: Die Erfahrung von Gewalt bildet in Sarajevo das prägende Moment für die Wahrnehmung, Deutung und Narrativierung der Vergangenheit und wird vereinzelt für eine imaginierte Zukunft nicht ausgeschlossen. ${ }^{91}$ Für die praktische Gestaltung der Gegenwart spielt sie hingegen nicht die gleiche Rolle wie in Ramallah, wo sie - durch die Möglichkeit nächtlicher incursions, der Demütigung an Checkpoints, immer neue Mobilitätsbeschränkungen, außerordentliche »Strafaktionen« des israelischen Militärs in den besetzten Gebieten, durch die Auswirkungen innerpalästinensischer oder internationaler Konflikte - den Alltag grundlegend strukturiert.

\section{(ii) Umstrittene Territorien und symbolische Raumordnungen}

Anhand solcher materieller Spuren und Alltagsgespräche lässt sich verdeutlichen: Die Kategorie »Raum« ist im Westjordanland und in Bosnien-Herzegowina auf eine grundlegende und zutiefst alltagsrelevante Weise umstritten.

91 | Ein lokaler Mitarbeiter des Goethe-Institutes äußerte mir gegenüber: "Wir bereiten uns auf einen neuen Krieg vor. Es ist unmöglich, dass die Serben damit davon kommen." Und: "Dayton hat den Konflikt nicht beendet, sondern nur gestoppt. Es ist wie beim Fußball: Jetzt steht es 1:0, aber jedes Spiel hat zwei Halbzeiten." (Feldnotizen vom 10. Juni 2009) Es handelt sich bei dieser Aussage um den einzigen mir gegenüber geäußerten direkten Verweis auf die Möglichkeit eines Gewaltausbruchs, der darüber hinaus auch nicht als Determinante des Alltags angeführt wird, sondern als zukünftige Revanche imaginiert wird. 
Beide Territorien sind durch Gewaltgeschichten codiert und von kaum miteinander zu vereinbarenden politischen Ansprüchen durchzogen; sie werden durch bewegliche Grenzen neu strukturiert und einzelne Orte von ihren historisch gewachsenen Bezugspunkten abgetrennt. Bewohnerinnen und Bewohner solcher Gebiete kommen nicht darum herum, in ihrer Alltagsgestaltung Lebensräume mit wechselnden Bedeutungen zu verknüpfen und hierdurch mentale Karten zu entwickeln, mit denen sie durch ihre Umgebung hindurch navigieren. Symbolische Raumordnungen verstehe ich vor diesem Hintergrund als kollektiv erschaffene, dynamische Positionierungs- und Orientierungsfelder. Auch wenn sich politische Projekte, Konfliktverläufe oder historisch gewachsene Raumnarrative hierbei als besonders wirkmächtig erweisen, so verfügen Individuen und Gruppen dennoch über Handlungspotenziale, sich - etwa durch alltägliche, künstlerische oder politische Praxen - unterschiedlich zu ihnen in Beziehung zu setzen, sie kritisch zu kommentieren oder situativ zu unterlaufen. Solche Auseinandersetzungen um die Relationen zwischen Räumen, Menschen und Bedeutungen durchziehen auch die Arbeitskontexte der beiden Goethe-Institute.

In Ramallah gibt es keine symbolische Ordnung des Territoriums, keine Positionierung oder mentale Orientierung im Raum jenseits der Besatzung. In Alltagsgesprächen mit Mitarbeiterinnen oder Partnern des Goethe-Instituts zeigen sich in diesem Zusammenhang zwei Pole: eine routiniert erscheinende Orientierung an den räumlichen Vorgaben der Besatzungsmacht einerseits und die explizite Darstellung der jetzigen territorialen Ordnung als einen illegitimen, anormalen und zugleich temporären Zustand andererseits. So nimmt etwa eine Schauspielerin aus Ramallah, die an einem Projekt des Goethe-Instituts in Bethlehem teilnehmen möchte, den langen Umweg einer östlichen Umfahrung Jerusalems in Kauf, da die israelischen Autoritäten ihr den direkten Weg verweigern. Eine Mobilität jenseits der Grenzen von Ramallah führt also zwangsläufig zu einer praktischen Orientierung an den politisch produzierten Raumordnungen. Ein anderer Projektteilnehmer nutzt später die Gelegenheit, um nicht einfach nur die Illegitimität der Besatzung des Westjordanlandes, des Gazastreifens und Ostjerusalems zu betonen, sondern mich zugleich auf die »eigentlichen«, arabischen Namen einzelner israelischer Städte hinzuweisen und sie somit als Teile eines imaginären, gewissermaßen zeitlosen Palästinas zu markieren. Zwischen diesen beiden Polen lassen sich die unterschiedlichsten Formen der Anpassung und Resistenz in Alltagsgesprächen und -situationen beobachten:

- die üblichste Form der Distanzierung besteht darin, Israel nicht beim Namen zu nennen und stattdessen routiniert von " 48 Palestine« zu sprechen, um die Gebiete des »historischen Palästinas« zu bezeichnen, die 1948 Teil des Staates Israel wurden - eine Sprechweise, die auch 
viele entsandte Mitarbeiter internationaler Organisationen in Besprechungen mit Palästinensern übernehmen, selbst wenn sie im Gespräch untereinander ebenso selbstverständlich von »Israel« reden;

- auf einer Fahrt von Ramallah nach Bethlehem fordert mich eine Projektpartnerin während der Kontrolle an einem Checkpoint auf, unfreundlich zu den Soldaten zu sein, um ihnen klar zu machen, dass »wir ihre Besatzung nicht akzeptieren«;

- viel Aufmerksamkeit erhalten im Umfeld des Goethe-Instituts ritualisierte Formen des Protests einzelner palästinensischer Dörfer gegen den weiteren Bau der Mauer;

- im Zuge meiner häufigen Überquerungen von Checkpoints überrascht mich aber eher die Ruhe, mit der die Palästinenser die permanenten Beschränkungen und Maßregelungen zumeist über sich ergehen lassen - ein Eindruck, der durch eine lokale Mitarbeiterin des Goethe-Instituts gespiegelt wird: »Ihr Europäer würdet eine solche Behandlung noch viel weniger ertragen als wir.«

Symbolische Raumordnungen werden insbesondere auch in Diskussionen zu möglichen zukünftigen Entwicklungsszenarien für das Territorium Israel/Palästina aufgerufen. Während meiner Feldforschung bildet hierbei die - auch international gesetzte - »Zweitstaatenlösung « den häufigsten Referenzpunkt im Arbeitsumfeld des Goethe-Instituts: also die Vorstellung der Möglichkeit einer klaren Trennung der beiden Kollektive »Palästinenser « und »(jüdische) Israelis« entlang »eindeutiger« räumlicher Ordnungsprinzipien. Gelegentlich klingt die Zukunftsvision eines gemeinsamen politischen Kontextes und somit einer Ersetzung des Prinzips der nationalen Selbstbestimmung durch die Forderung nach gleichen Bürgerrechten für alle an. Ermutigt durch solche Diskussionen schlage ich einem Gesprächspartner, der mich nach meinem Eindruck von der »Lage« fragt, die »Einstaatenlösung « vor. »Nein, das würde niemals funktionieren«, antwortet er mir etwas erschrocken, »die Zweistaatenlösung ist die einzige Möglichkeit«. Ich verstand seine Skepsis nach Jahrzehnten des Konflikts, der einseitigen Dominanz und wechselseitigen physischen Gewalt; gleichzeitig erscheint die Annahme immer unwahrscheinlicher, dass stattdessen eine Zweistaatenlösung funktionieren würde - zu asymmetrisch die Machtverhältnisse zwischen den beiden projizierten Einheiten, zu eng der Raum, zu verflochten die Siedlungsmuster, zu unvereinbar die politisch-geografischen Konzepte von »Israel « und »Palästina «. ${ }^{92}$ Festzuhalten bleibt, dass die unbeantwortete Frage einer zukünftigen Ordnung des Raums und des Zusammenlebens im Territorium zwischen Mittelmeer und Jordan für die Be-

92 | Eyal Weizman: Sperrzonen, S. 23. 
sucherinnen, Partner und Mitarbeiterinnen des Goethe-Instituts eine zentrale Rahmenbedingung jedweder kulturpolitischer Projektarbeit darstellt.

Auch in Sarajevo scheint in vielen Gesprächen die Frage nach den Beziehungen zwischen - entlang ethnischer und religiöser Kategorien konstruierten - Kollektiven und Territorien hindurch: Der Zusammenbruch der gewohnten staatlichen Ordnung, massive Formen politischer Gewalt, ein Friedensschluss, der die Fronten und Logiken des Krieges nicht wirklich aufgelöst hat, sowie die gegenwärtigen Effekte ethnonationaler Politiken bilden wiederkehrende Elemente in den Positionierungen meiner Gesprächspartner zu den symbolischen Ordnungen eines vielfältig umstrittenen Raums. Häufig wird auch hier eine grundlegende Spannung sichtbar: Auf der einen Seite verweisen sie regelmäßig auf die Anomalie der aktuellen politischen und geografischen Ordnung; auf der anderen Seite zeigt sich die faktische Anerkennung der Wirkmächtigkeit ethnonationaler Prinzipien. So erklärt mir ein Projektpartner des Goethe-Instituts die politische Situation folgendermaßen: »Wahlen in Bosnien bedeuten: >Bitte entscheiden Sie sich zwischen dieser Serviette<. Das mag paradox klingen, aber so ist es: >Entscheiden Sie sich zwischen dieser Serviette <aber es gibt nur eine Serviette! «93

Das ethnonationale Prinzip wird hier zugleich als absurd und wirkmächtig herausgestellt. Wahlen bedeuten demnach nicht die Entscheidung zwischen unterschiedlichen politischen Konzepten und Entwicklungsmodellen für das staatliche Territorium, sondern das zwangsläufige Votum für eine Partei, welche die »eigene« ethnische Gruppe vertritt - oder anders formuliert: die politische Nachkriegsordnung verlangt von den Bürgerinnen und Bürgern, sich als ethnisierte Personen in einem von Trennlinien durchzogenen öffentlichen Raum zu positionieren. Als Gegenpol zu dieser als anormal oder dysfunktional wahrgenommenen Gegenwart treten in solchen Gesprächen primär zwei Referenzpunkte für »Normalität « hervor: einerseits die Erinnerung an die territoriale Ordnung des sozialistischen Jugoslawiens als einem zwar verschwundenen, rückblickend aber vernünftig erscheinenden räumlichen Bezugssystems; andererseits die - inzwischen in eine imaginäre Zukunft gerückte - Vorstellung eines demokratischen, territorial und politisch integrierten Bosnien-Herzegowina in einem offenen europäischen Kontext, wie es durch die Unabhängigkeitserklärung in den frühen I99oer Jahren projiziert worden war. ${ }^{94}$

Die Wirkmächtigkeit ethnonationaler Politiken für alltägliche und symbolische Raumordnungen lässt sich anhand der Äußerungen eines bosnischen

93 | Feldnotizen vom 19. Juni 2009.

94 | Zu einem ähnlichen Befund kommt: Stef Jansen: Yearnings in the Meantime. 'Normal Livesı and the State in a Sarajevo Apartment Complex. London, New York 2015. Siehe zur Frage nach "Normalität" in der belagerten Stadt: Ivana Maček: Sarajevo under Siege. Anthropology in Wartime. Philadelphia 2009. 
Mitarbeiters des Goethe-Instituts weiter verdeutlichen. Demnach prägen die zentralen Teilungsprinzipien des Konflikts bis heute »jeden Aspekt des Alltags«. So finde man in den Geschäften von Sarajevo kein Mineralwasser aus dem serbischen Landesteil, da man keine Waren aus der anderen »Entität « ${ }^{95}$ verkaufe. Ebenso käme es praktisch nicht vor, dass ein Einwohner Sarajevos das günstigste Mobilfunknetz Bosniens wähle, da es aus der Republika Srpska kommt: »Die Leute sind so stark von dem >Problem< besessen, dass sie nicht mal so was machen«, fasst mein Gesprächspartner die Effekte des Konfliktes auf Konsumentscheidungen und mentale Karten zusammen.

Die Frage nach den alltäglichen Navigationen durch codierte Räume möchte ich am Beispiel der Stadt Sarajevo selbst vertiefen. Anhand einer längeren ethnografischen Sequenz lässt sich aufzeigen, wie hier ein historisch etabliertes urbanes Narrativ Reflexionen und Positionierungen zu einem umstrittenen Territorium präkonfiguriert: Am Abend des 20. Jahrestages des Beginns der Belagerung von Sarajevo im April 20I2 treffe ich mich mit drei Journalisten in einer Altstadtbar. ${ }^{96}$ Unser Gespräch kreist um die zentrale Gedenkveranstaltung, die tagsüber das Leben in der Innenstadt bestimmt hat. Auf der gesperrten Hauptstraße wurden II.54I rote Plastikstühle aufgestellt; jeder Stuhl stand für einen Toten der Belagerung. Die Stühle waren zu einer Bühne hin ausgerichtet, auf der - so das Konzept der Veranstaltung - ein Konzert für die Toten gegeben wurde. Entsprechend blieben die Stühle leer für die, die fehlten. Die Bewohner Sarajevos erschienen zahlreich, standen und liefen auf den Bürgersteigen und Plätzen an den Rändern des Arrangements und wurden somit zum Teil der Gedenkveranstaltung. Aus der Vogelperspektive setzten sich die Stühle zu dem Bild einer roten Linie, die sich mehrere Kilometer durch die Stadt zog, zusammen - ein Bild, das über Sarajevo hinaus Wirkung entfaltete und etwa von ausländischen Zeitungen und Fernsehsendern genutzt wurde, um an den Jahrestag zu erinnern. ${ }^{97}$ Als eine besondere Anekdote berichten die Journalisten, dass die Stühle in Serbien gekauft worden seien, da hier - nach Auskunft der Veranstalter - eine Firma das günstigste Angebot erstellt hatte. Auf den Stühlen sei »Made in Serbia « zu lesen gewesen und nun werde in der Stadt diskutiert, ob dies nicht einen Affront für die Hinterbliebenen darstelle. Unser abendliches Gespräch steht unter dem Eindruck dieses Ereignisses als einer der Journalisten nach den Beziehungen zwischen Gewalt und der Stadt Sarajevo fragt und hierdurch Motive zur symbolischen Ordnung des urbanen Territoriums aufruft. Er erzählt, dass er zur Vorbereitung auf den Jahrestag Ivo

95 | "Entität" bezeichnet die beiden durch den Friedensschluss von Dayton geschaffenen Landesteile - "Föderation Bosnien und Herzegowina" und "Republika Srpska" -, deren Grenze in etwa dem Frontverlauf gegen Ende des Krieges entspricht.

96 | Für die gesamte folgende Sequenz: Feldnotizen vom 6. April 2012.

97 | Siehe hierzu das Coverbild dieses Buches. 
Andrić' Roman Das Fräulein wieder gelesen habe. Ihn interessierten hierbei vor allem die Thematisierungen eines besonderen Hasses, den der Autor mit Sarajevo in Verbindung bringt - so etwa in den Beschreibungen des Ausbruchs von Unruhen nach der Ermordung des österreichischen Thronfolgerpaars im Jahr I9I4:

"Die Angehörigen der drei Hauptkonfessionen hassen einander von der Geburt bis zum Tode, vernunftlos und abgrundtief, und sie übertragen diesen Haß auch aufs Jenseits, das sie als ihren Ruhm und Sieg und als Niederlage und Schande des andersgläubigen Nachbarn auffassen. Sie werden geboren, wachsen und sterben in diesem Haß, in diesem wirklich physischen Abscheu vor dem Andersgläubigen; oft vergeht ihr ganzes Leben, ohne daß sich innen Gelegenheit bietet, diesen Haß in seiner ganzen Stärke und Schrecklichkeit zu beweisen, wenn aber anläßlich eines bedeutenden Ereignisses die feste Ordnung der Dinge ins Schwanken gerät und Verstand und Gesetz für einige Stunden oder Tage außer Kraft gesetzt sind, dann ergießt sich diese Horde, beziehungsweise ein Teil von ihr, nachdem sie endlich einen tauglichen Grund gefunden hat, über diese Stadt, die sonst wegen ihrer feinen Liebenswürdigkeit im gesellschaftlichen Leben und ihrer schmeichelhaften Redeweisen bekannt ist. " ${ }^{98}$

Der Journalist lenkt unsere Aufmerksamkeit auf drei Aspekte, die mir für die Frage nach mentalen Karten und symbolischen Raumordnungen wichtig erscheinen: Zunächst stehe der Hass bei Andrić immer im Zusammenhang mit der Vielfalt, welche die Stadt charakterisiere - also die Vielfalt der historischen Spuren und imperialen Bezüge, vor allem aber die Vielfalt der religiösen Gruppen, die den städtischen Raum und Alltag miteinander teilen. Zweitens beschreibe Andrić eine Art Latenz des Hasses - der Hass sei also untergründig auch dann vorhanden, wenn das allägliche Zusammenleben von Freundlichkeit und Respekt geprägt sei und breche aus, sobald sich ein Anlass finde. Und drittens komme dieser Hass in den Texten von Andrić häufig »die Berge hinab«. Diese letzte Beobachtung eröffnet die Möglichkeit, die Frage nach der Beziehung zwischen »Vielfalt«, »Hass« und »Stadt« breiter zu diskutieren, da die Feindseligkeit in diesem Bild nicht primär innerhalb des städtischen Territoriums, sondern außerhalb oder an seinen Rändern angesiedelt wird. Und tatsächlich kreist das Gespräch eben um diese Beziehung: Soll man davon ausgehen, dass aus dem alltäglichen Zusammenleben unterschiedlicher Gruppen Hass entsteht? Oder ist es nicht eher so, dass die Stadt aufgrund der Normalität einer gelebten Diversität den Hass von außen auf sich zieht? Liegt das Problem vielleicht bei einzelnen Personen, die »aus den Bergen « nach Sarajevo gezogen sind, hier lange lebten, mit der ungewohnten Vielfalt nicht zurechtkamen und

98 | Ivo Andrić: Das Fräulein. Frankfurt 2003, S. 108. 
daher schließlich zurück in die Berge gingen, um die Stadt von außen anzugreifen?

Wir kommen in unserem Gespräch nicht zu einer einheitlichen Position. Wie so häufig, wenn ich in Sarajevo nach Gründen für den Krieg frage, kombinieren meine Gesprächspartner Bezugnahmen auf das offizielle Narrativ von dem Angriff von außen mit einer gewissen Ratlosigkeit, wie sich die Vehemenz der Gewalt nach Jahrzehnten des Zusammenlebens denn nun wirklich erklären lässt. Symbolische Ordnungen des urbanen Territoriums zeigen sich in dieser Sequenz als Knotenpunkte eines dynamischen Prozesses, in dem die Verknüpfung einer bestimmten Motivik mit der Stadt Sarajevo aufgerufen und fortentwickelt wird - eine Motivik, die in Variationen sowie mit unterschiedlichen Gewichtungen und Schlussfolgerungen in literarischen Texten ebenso zu finden ist wie in Erinnerungspolitiken, Gedenkveranstaltungen, touristischen Materialien und Alltagsdiskussionen. Im Zentrum steht das Bild von der alltäglichen Vielfalt als einem lokalen Spezifikum, das häufig positiv konnotiert wird, in anderen Situationen aber als »Problem« erscheinen kann. So berichten mir etwa einerseits Angehörige der kulturellen Elite ${ }^{99}$ mehrfach von der eigenen Sozialisation in einem Stadtraum, der durch das enge Nebeneinander von katholischer und orthodoxer Kirche, von Moschee und Synagoge geprägt war und zu einer Art inkorporierter Selbstverständlichkeit im Umgang mit Vielfalt als verbindender Erfahrung der Bewohner Sarajevos führte. Ein Festivaldirektor erzählt darüber hinaus, dass in den ersten Monaten der Belagerung die Zahl der Ehen zwischen Angehörigen unterschiedlicher Religionen deutlich angestiegen sei und sah hierin Akte eines zivilen Protests gegen die gewalttätige Infragestellung der Diversität. Andererseits finden sich Problematisierungen dieser Vielfalt nicht nur durch das Bild von der Aggression von außen, in literarischen Texten oder in den wirkmächtigen Deutungen des Konflikts als einen Ausbruch »uralten Hasses« durch internationale Beobachter, sondern gleichfalls im urbanen Alltag: So betonen einige Gesprächspartnerinnen, dass - im Zuge der grundlegenden Veränderung der Bevölkerungszusammensetzung als Folge des Krieges - eine zunehmende Dominanz der muslimischen Gruppe und die Marginalisierung der Angehörigen anderer Konfessionen als Probleme des städtischen Zusammenlebens hervortreten.

Ivana Maček hat in ihrem Buch Sarajevo under Siege ethnografisch dicht einen symbolischen und politischen Machtkampf analysiert, der die Stadt in der Gegenwart prägt und in dessen Zentrum eben die Frage nach dem Umgang mit Diversität steht - einen Machtkampf, den Maček in Alltagssituationen ebenso nachzeichnet wie in Geschichtspolitiken und Formen der Erin-

99 | Hierunter verstehe ich Personen, die im kulturellen Leben Sarajevos eine gestaltende Rolle spielen, also etwa die Leiter wichtiger Festivals, Theater- oder Kunstvereine. 
nerung, in städtischen oder staatlichen Verwaltungsakten. ${ }^{100}$ Maček zeigt, wie sich die Bewohner in diesem Spannungsfeld positionieren müssen und genau hier liegt der Anknüpfungspunkt für mein Interesse an den Dynamiken sүmbolischer Raumordnungen und ihrer Relevanz für kulturpolitische Übersetzungsarbeit: Etablierte Raumnarrative, Deutungen des »Konflikts«, politische Ordnungsvorstellungen und Alltagserfahrungen verbinden sich zu einem Positionierungs- und Orientierungsfeld, in dem Ambivalenzen - etwa die Frage nach der Beziehung zwischen Vielfalt, Stadt und Gewalt - bestehen bleiben und situativ verschieden interpretiert und mobilisiert werden können.

\section{(iii) Fragmentierte Souveränitäten und die Problematisierung von Staatlichkeit}

Eine weitere Präsenzform der komplexen Gefüge besteht in den multiplen Machteffekten, die Individuen, Gruppen und Arbeitskontexte an Orten wie Ramallah oder Sarajevo berühren. Gerade nach dem Ende militärischer Auseinandersetzungen sehen sich die Bewohner hier mit dem Wirken eines Geflechts aus internationalen Missionen, humanitären Einsätzen, Nichtregierungsorganisationen, staatlichen und kommunalen Administrationen konfrontiert, die wesentliche Beiträge zum Wiederaufbau oder zur Stabilisierung von Alltagen leisten, aber in ihren jeweiligen Arbeitsschwerpunkten und Zuständigkeiten für die Menschen, die von ihnen adressiert werden, häufig undurchschaubar bleiben. ${ }^{101}$ Exemplarisch sei an dieser Stelle darauf verwiesen, dass in den Palästinensischen Gebieten etwa seit Jahrzehnten weite Teile der Gesundheitsversorgung und des Bildungswesens durch unterschiedliche Agenturen der UNO oder das Internationale Komitee vom Roten Kreuz getragen und organisiert werden. In Bosnien-Herzegowina waren militärische Missionen - zuerst unter Leitung der NATO, später der EU - sowie die Errichtung einer internationalen Polizeitruppe zentral für die Durchsetzung des Friedensabkommens von Dayton. Darüber hinaus überwacht das Büro des Hohen Repräsentanten für Bosnien-Herzegowina ${ }^{102}$ seit 1995 auf Basis einer Resolution des UN-Sicherheitsrates die Durchsetzung der zivilen Aspekte des Friedensvertrages und kann hierzu direkt in politische Entscheidungen intervenieren, die aus seiner Perspektive der friedlichen Entwicklung des Landes schaden. Eine umfangreiche Mission der Organisation für Sicherheit und Zusammenarbeit in Europa ${ }^{103}$ übernahm umfassende Zuständigkeiten für die Durchführung der

100 | Ivana Maček: Sarajevo under Siege, S. 190.

101 | Siehe hierzu Elizabeth Dunns Konzept der "Adhocracy«: Elizabeth Cullen Dunn: The Chaos of Humanitarian Aid. Adhocracy in the Republic of Georgia. In: Humanity, 3,1 (Spring 2012), S. 1-23.

102 | Siehe hierzu: http://www.ohr.int (letzter Zugriff 11. August 2017).

103 | Siehe hierzu: http://www.osce.org/bih (letzter Zugriff 11. August 2017). 
ersten Wahlen nach dem Krieg, den Wiederaufbau einer Strafgerichtsbarkeit, die Entwicklung des Bildungssystems, die Durchsetzung von Regelungen des Minderheitenschutzes und vieles mehr. Solche internationalen Missionen sind auf vielfältige Weise mit lokalen politischen Prozessen - aber auch etwa mit zivilgesellschaftlichen Initiativen - verwoben und tragen zur Herausbildung fragmentierter Souveränitäten bei, die sich in den kulturpolitischen Arbeitskontexten eines Goethe-Institutes direkt in kontinuierlichen Problematisierungen von Staatlichkeit niederschlagen.

In Ramallah erscheint die Lage aufgrund der jahrzehntelangen israelischen Besetzung auf den ersten Blick deutlich prekärer: Hier üben die Besatzungsmacht, unterschiedliche internationale Organisationen und die palästinensische Selbstverwaltung eine Vielzahl von »staatlichen Effekten «104 aus, ohne dass ein Staat als ein kontinuierliches, Rechenschaft schuldiges Gegenüber für die Menschen greifbar wäre. In der Konsequenz leben Palästinenser in einem Zustand potenzieller Rechtlosigkeit und weitgehender Unsicherheit - ihr Status, ihr Besitz, ihre Anwesenheit, Mobilität und körperliche Integrität sind durch die Möglichkeit von Eingriffen militärischer oder politischer Macht durchgängig bedroht. Die Zielvorstellungen der internationalen Gemeinschaft, durch ihre Interventionen zum Aufbau einer funktionierenden Staatlichkeit beizutragen, erweisen sich aus dieser Perspektive weitgehend als Fiktion und Beschwörungsformel. ${ }^{105}$

An zwei Beispielen lässt sich aufzeigen, wie diese Konstellation im Arbeitsumfeld des Goethe-Instituts als »Problem« aufgerufen wird: Für eine Veranstaltung im Freien hat sich das Institut Plastiktische und -stühle ausgeliehen. Noch am gleichen Abend bittet der Vermieter um seine Bezahlung. Auf den Vorschlag der deutschen Mitarbeiter, die Angelegenheit am nächsten Tag zu regeln, reagiert er unzufrieden. Der Fahrer des Goethe-Instituts, der die Unterhaltung übersetzt, erklärt: »Ihr dürft nicht vergessen, dass wir hier keinen Staat haben « - und keine verlässlichen Mechanismen im Konfliktfall zu seinem Recht zu kommen, ließe sich hinzufügen. Auch der Leiter des Goethe-Instituts kommt direkt auf das Fehlen staatlicher Strukturen zu sprechen, als ich ihn nach den Besonderheiten der Arbeit vor Ort frage: Palästina habe keine Grenzen; Einreise und Aufenthaltsstatus von Mitarbeiterinnen, Praktikanten oder Gästen liefen immer über Israel, das entsprechende Genehmigungen verweigern könne.

104 Michel-Rolph Trouillot: The Anthropology of the State in the Age of Globalization. Close Encounters of the Deceptive Kind. In: Current Anthropology, 43, 1 (February 2001), S. 125-138.

105 | Lori Allen: The Rise and Fall of Human Rights. Cynicism and Politics in Occupied Palestine. Stanford 2013. 
In Sarajevo beziehen sich Problematisierungen von Staatlichkeit zumeist auf die sichtbare »Auffächerung staatlicher Souveränität «, ${ }^{106}$ auf die damit verbundene Dysfunktionalität staatlicher Prozesse sowie auf die Sorge um die dauerhafte Integrität der unterschiedlichen Landesteile. Der Friedensvertrag von Dayton hat ein kompliziertes politisches Gebilde geschaffen, welches das Land nicht nur in zwei »Entitäten« mit weitgehenden Entscheidungsbefugnissen strukturiert, sondern die eine Entität - die Föderation Bosnien und Herzegowi$n a-$ nochmals in ro Kantone mit jeweiligen Regierungen unterteilt. Hieraus ergibt sich eine Schwäche gesamtstaatlicher Strukturen sowie die Möglichkeit ihrer Blockade durch die Repräsentanten der einzelnen Gebietskörperschaften und der unterschiedlichen ethnonationalen Gruppen. Hinzu kommt die Ausübung von Souveränitätsrechten durch internationale Akteure, die sich an der Rhetorik und Zielvorstellung einer gesamtstaatlichen Integrität orientieren. So wurden etwa staatliche Symbole, eine gemeinsame Währung, landesübergreifende Autonummernschilder und eine gemeinsame Armee durch Interventionen der internationalen Gemeinschaft eingeführt. Die Brüchigkeit dieser staatlichen Konstellation zeigt sich im Alltag etwa in der zweiten, serbischen Entität: die Symbole des Gesamtstaates werden hier praktisch nicht gezeigt, stattdessen trifft man hier auf eine eigene Fahne sowie auf Schilder mit Willkommensgrüßen in der Republika Srpska, sobald man die Entitätsgrenze überquert hat. Auch in kroatisch dominierten Gebieten der Föderation wird die Fahne des Nachbarstaates Kroatien vielfach im öffentlichen Raum genutzt.

Die häufigen Klagen über die Dysfunktionalität und Brüchigkeit der bosnischen Staatlichkeit lassen sich anhand einer bereits erwähnten ethnografischen Sequenz illustrieren: Die Bezugnahmen auf das Buch Das Fräulein in der Altstadtbar hatte mit einer anderen Stelle ihren Anfang genommen. Während unseres Gespräches wirft der Journalist ein, Ivo Andrić formuliere in dem Buch, dass sich in Sarajevo eine »türkische Scheu vor der Arbeit, ein slawischer Hang zum Extremen und ein falsches Verständnis von österreichischen staatlichen Organisationsformen« auf fatale Weise kreuzten. »Genau das ist bis heute der Fall« - kommentiert der Journalist sarkastisch überspitzt - »die Leute wollen nicht arbeiten, der Hang zum Extremen zeigte sich im Krieg und wir haben keinen Staat! «107

106 | Siehe zu dem Begriff "Auffächerung staatlicher Souveränität«: Aihwa Ong: Flexible Staatsbürgerschaften. Die kulturelle Logik von Transnationalität. Frankfurt 2005, S. 296.

107 | Wie ich später feststellte, findet sich bei Andrić selbst - zumindest in der deutschen Übersetzung - kein so expliziter Verweis auf ein "falsches Verständnis von Staatlichkeit" - ein bemerkenswerter Tatbestand, der aber den ethnografischen Befund der Problematisierung der bosnischen Staatlichkeit im Jahre 2012 nicht in Frage stellt. An der Stelle, die der Journalist vermutlich im Sinn hatte, heißt es: "Unsere bürgerliche 
Diese Auffächerung staatlicher Souveränität wird auch innerhalb des Goethe-Instituts in der Gestaltung des Raums oder von Arbeitspraxen sichtbar: Im Büro der Leiterin der Sprachabteilung hängen etwa die Fotos der dreizehn Bildungsminister des Landes. ${ }^{108}$ Sie erzählt mir, dass sie mit all diesen Ministerien in Kontakt treten muss, wenn sie landesweit einen neuen Impuls im Deutschunterricht setzen oder ein Fortbildungsangebot anbieten will. Gleichfalls nennt die Leiterin des Instituts die Schwäche des Gesamtstaates als ein zentrales Problem ihrer Arbeit: Projekte würden nicht aufgegriffen und liefen ins Leere; niemand fühle sich für das »gemeinsame Kulturgut« verantwortlich; gesamtstaatliche Kultureinrichtungen - etwa die Nationalgalerie oder das Filmarchiv - bekämen kein oder kaum Budget und seien daher nicht arbeitsfähig: »Das Ich ist hier nicht definiert«, kommentiert sie - es ist also unklar, wer Entscheidungen trifft, wer Leitungspositionen besetzt, wer Verantwortung auf gesamtstaatlicher Ebene übernimmt und wer damit als kulturpolitischer Repräsentant Bosnien-Herzegowinas eine Sprecherposition übernehmen kann.

Auf Basis solcher Beobachtungen lässt sich festhalten: In den kulturpolitischen Arbeitsfeldern, die ein Goethe-Institut in Ramallah oder Sarajevo eröffnet, begegnen sich auch sehr unterschiedliche Formationen von Staatlichkeit. Die finanzielle, politische und infrastrukturelle Potenz Deutschlands wird in solchen Kontexten - ob intendiert oder nicht - besonders deutlich und konfiguriert eine grundlegend asymmetrische Ausgangslage kulturpolitischer Begegnung - ein Aspekt, auf den ich sogleich zurückkommen werde.

\section{Zwischenfazit: Kulturpolitische Verknüpfungsarbeit in Zeiten des "Not-War-Not-Peace"}

Die amerikanische Kulturanthropologin Carolyn Nordstrom spricht in ihrem Buch Shadows of War von Zuständen oder Zeiten eines »Not-War-Not-Peace«, um regionale und politische Kontexte zu beschreiben, in denen wirkmächtige offizielle Diskurse nach Phasen massiver Gewalt einen Friedens- oder Versöhnungsprozess verorten, ethnografische Beobachtungen und informelle Gespräche aber die vielfältige Präsenz der unterschiedlichen Dimensionen

Welt, die ohnehin mit den türkischen Gewohnheiten der Faulheit und dem slawischen Bedürfnis nach Ausschweifungen erblich belastet ist, hat dazu noch die österreichischen formalen Begriffe von der Gesellschaft und den gesellschaftlichen Verpflichtungen übernommen, nach denen das persönliche Ansehen und die Klassenwürde des Menschen auf einer bestimmten Zahl unnützer, sinnloser Ausgaben, oft auf einem leeren, lächerlichen, geist- und geschmacklosen Luxus beruhen."In: Ivo Andrić: Das Fräulein, S. $64 \mathrm{f}$.

108 Es handelte sich hierbei um die Bildungsminister der beiden Entitäten, der zehn Kantone der Föderation Bosnien und Herzegowina sowie des Sonderverwaltungsgebietes "Distrikt Brčko". 
und Effekte von Konflikten und Gewalt aufzeigen. ${ }^{109}$ Im Anschluss an Michael Taussig thematisiert sie die Etablierung »öffentlicher Geheimnisse «110 in politischen oder medialen Repräsentationen solcher Kontexte: Man kenne die Realität, spreche über sie aber in der Regel nicht öffentlich. Nordstrom betont insbesondere die Rolle der internationalen Gemeinschaft, die Missionen entsendet, Hilfsmaschinerien in Gang bringt, transnationale Bürokratien etabliert, Wiederaufbau- oder Staatswerdungsprozesse finanziert und ein eventuelles Scheitern solcher Bemühungen so lange wie möglich auszublenden versucht - auch um Einflusssphären, die eigene Reputation oder persönliche Karrieren nicht $\mathrm{zu}$ gefährden: »With this vast peace-brokering network in place, how do you admit it isn't working? «111

Bosnien-Herzegowina und Israel/Palästina unterscheiden sich in vieler Hinsicht voneinander - etwa im Hinblick auf die machtpolitischen Konstellationen und die geopolitischen Positionen, auf die Temporalität des Konflikts, auf den Charakter und die Historizität der Auseinandersetzung oder auch im Hinblick auf die Alltagsrelevanz und die Aktualität von physischer und militärischer Gewalt - und beide Kontexte unterscheiden sich wiederum sehr deutlich von den Kriegszonen des südlichen Afrikas oder Sri Lankas, die Carolyn Nordstrom erforscht. Dennoch erscheint mir die Vorstellung eines Zustands des »Not-War-Not-Peace « geeignet, um bei aller Unterschiedlichkeit zwei anschlussfähige Aspekte herauszustellen: erstens eine bleibende politische Brüchigkeit und Infragestellung von Staatlichkeit als Konsequenz gewalttätiger Auseinandersetzungen, in deren Regulierungsversuche internationale Akteure vielfältig und massiv eingebunden sind; und zweitens eine gewisse Diskrepanz zwischen den offiziellen Diskursen von Friedens- oder Stabilisierungsprozessen einerseits und den lokalen Alltagswelten andererseits, die mit der jeweiligen Konfliktkonstellation und ihren Effekten komplex verwoben sind: Die Schatten der Konflikte verdunkeln weiterhin und nachhaltig die Leben der Menschen in Ramallah und Sarajevo. ${ }^{112}$

Für eine Analyse Auswärtiger Kulturpolitik als Konfliktprävention stellt sich die Frage, ob und in welcher Weise deutsche Kulturmittler Zugänge zu solchen lokalen Präsenzen komplexer Gefüge suchen und mit den Rationalitäten, diskursiven Figuren und Ressourcen dieser Policy zu verknüpfen vermögen. In

109 | Carolyn Nordstrom: Shadows of War, Violence, Power, and International Profiteering in the twenty-first Century. Berkeley, Los Angeles, London 2004, S. $165 \mathrm{ff}$.

110 | Ebd., S. 168, bezugnehmend auf: Michael Taussig: Colonialism, Shamanism, and the Wild Man. Chicago 1987.

111 | Carolyn Nordstrom: Shadows of War, S. 170.

112 Nordstrom spricht zur Beschreibung der Alltagsrealitäten in Angola von dem "shadow of war": "People watched the shadow of war move across the horizon to cloud their lives, their hands empty of the tools they needed to stop it." In: Ebd., S. 171. 
den verbleibenden Textteilen dieses Abschnitts werde ich exemplarisch einige der Positionen, Umgangsweisen und Projektformate, die innerhalb der Goethe-Institute in Sarajevo und Ramallah hierzu entwickelt werden, aber auch einige der Reibungen, Asymmetrien und blinden Flecken, die im Zuge dieser Arbeit hervortreten, diskutieren.

\section{Unbeantwortete Fragen: "Wie wären Gesellschaften, wenn es uns nicht gäbe?"}

Kurz vor seiner Versetzung nach Beirut schreibt der Institutsleiter in Ramallah in einem Abschiedseditorial für die Programmzeitschrift des Deutsch-Französischen Kulturzentrums:

"Eine Herausforderung stellen nicht nur die durch die Besatzung erschwerten Arbeitsbedingungen dar, sondern die für mich unbeantwortete Frage, ob unsere Kulturarbeit langfristig helfen kann, ein freies, demokratisches Palästina ins Leben zu rufen oder ob sie - im Gegenteil - hilft, den Anschein der Normalität in einer jedem Völkerrecht spottenden Situation aufrechtzuerhalten. Ihr bewundernswertes Interesse an Kunst auch in Zeiten der Okkupation hat unser gesamtes Team jedoch darin bestärkt, weiterzumachen. Eines Tages wird Palästina frei sein, und es macht einen großen Unterschied, ob seine Jugend nur mit der alltäglichen Gewalt oder auch mit Filmen, Theater und Literatur aufgewachsen ist. ${ }^{113}$

Im weiteren Verlauf des Textes finden zwei aktuelle Projekte als Beispiele für die Bearbeitung der hier aufgerufenen Problemstellung Erwähnung: die Renovierung und Inbetriebnahme eines Kinos in der Stadt Jenin im nördlichen Westjordanland sowie die Eröffnung einer Bibliothek »im von der Außenwelt völlig abgeschlossenen Gaza«. Bemerkenswert ist diese Passage, da hier zumindest vier zentrale Elemente der Übersetzung einer Auswärtigen Kulturpolitik als Konfliktprävention in Arbeitspraxis sichtbar werden: Zunächst zeigen sich Bezugnahmen auf offiziell gemachte Zielhorizonte der internationalen Gemeinschaft - hier die »Staatswerdung Palästinas«, in wie weite Ferne sie auch gerückt sein mag - als erhoffter Beitrag zur »Konfliktlösung«; zweitens ruft der Institutsleiter mit dem Verweis auf die »jedem Völkerrecht spottende Situation « lokal situierte Wahrnehmungs- und Sprechformen zur Beschreibung des politischen Kontextes auf, ohne dabei die Grenzen des Sagbaren innerhalb deutscher Diskussionsfelder zu deutlich zu überschreiten; drittens werden konkrete Projekte als mögliche Interventionen in diese konfliktdurchzogenen Arbeitsfelder angeführt; und viertens scheint die Unsicherheit eines

113 | Fareed Majari: Editorial. In: Manara, Monatszeitung des Deutsch-Französischen Kulturzentrums, 2008-09, 12-1, S. 3. 
Kulturmittlers hindurch, ob die kulturpolitischen Präsenzen und Maßnahmen tatsächlich dazu beitragen, erwünschte Veränderungen voranzutreiben oder nicht eher gegenteilige Effekte hervorrufen. Als ich den Institutsleiter explizit nach der Bedeutung der Zielvorstellung Konfliktprävention für seine Arbeit frage, kommt er insbesondere auf diesen letzten Aspekt zu sprechen:

IL: "Also das ist wieder der Punkt. Wir können sagen, dass die Konfliktprävention durch die Politik oft nicht funktioniert und dass man dann die Kultur befragt, ob sie das machen kann, das macht aus meiner Sicht Sinn."

JA: "Macht Sinn, oder nicht?"

IL: "Macht Sinn, dass man sagt, wenn wir in der Politik scheitern und das direkte Gespräch nirgendwohin führt, versuchen wir es mit weichen Kanälen und das ist die Kultur. Das ist sicher überlegenswert. Also da kann man nichts gegen sagen. Ob das funktioniert- das Problem ist, wir haben keine Messinstrumente. Es gibt eigentlich auch keine Parameter, die da auf Erfolg oder Misserfolg hinweisen. Wie kann man nachweisen, ob hier in der Region das Konfliktpotenzial deutlich weniger geworden ist, weil wir Kulturarbeit machen? Wir wissen nicht, wie es wäre, wenn es uns nicht gäbe." ${ }^{114}$

Hier zeigt sich zunächst, dass der Institutsleiter zentrale Denkfiguren, die im Zuge des Auftauchens einer Auswärtigen Kulturpolitik als Konfliktprävention mobilisiert worden sind, als Bezugspunkte akzeptiert. Seine Formulierungen erinnern an Reden des Außenministers oder auch an die Aussagen meiner Gesprächspartner in Berlin: »Kultur macht Dinge möglich, die politisch nicht laufen«, erklärte mir etwa der Leiter der Abteilung für Kultur und Kommunikation des Auswärtigen Amtes zu Beginn meiner Forschung. Gleichzeitig benennt der Institutsleiter ein zentrales Übersetzungsproblem dieser Policy: Wie lassen sich schlüssige Beziehungen zwischen Konfliktverläufen und kulturpolitischen Aktivitäten herstellen? Durch einen Verweis auf standardisierte Verfahren der UNO, aktuelle Entwicklungen möglichst realitätsnah zu dokumentieren, führt er diese Schwierigkeit weiter aus:

"Hier gibt es natürlich ein hoch und runter von Konfliktparametern. Da gibt es von der hiesigen UN-Vertretung diese Sicherheitslisten: wie viele Steinwürfe, wie viele Molotowcocktails, wie viele Kassam-Raketen pro Tag? Das ist genau in Excel-Sheets aufgelistet und da kann man sagen, das geht hoch oder runter, aber das hat nun wirklich nichts mit uns zu tun. Da spielen ganz andere Faktoren eine Rolle und mit Sicherheit nicht die Kultur. Also von daher würde ich sagen, wir können diese Frage, schlicht und einfach nicht beantworten."

114 | Interview vom 4. Dezember 2008; auch die folgenden beiden Zitate. 
Mein Gesprächspartner verbindet hier also eine grundsätzliche Bejahung einer »Befragung der Kultur« in politisch umstrittenen Feldern mit Zweifeln im Hinblick auf zu direkte Kausalitätserwartungen, die in den Formulierungen einer Auswärtigen Kulturpolitik als Konfliktprävention mitschwingen können. In diese Skepsis fließen sicherlich Erfahrungen mit der Vielschichtigkeit und den unvorhersehbaren Verläufen eines Konfliktes ebenso ein wie das Wissen von der Begrenztheit der Mittel und den Interventionsmöglichkeiten eines deutschen Kulturinstituts. Zugleich treten hier aber auch unterschiedliche Vorstellungen von der Steuerbarkeit und Zielgerichtetheit einer Kulturpolitik hervor, die im Zuge ihres Wanderns durch translokale Übersetzungsketten aufeinandertreffen. Unabhängig von der neuen Figur Konfliktprävention sehen sich die Institutsleiter zum Zeitpunkt meiner Feldforschungen mit den wachsenden Erwartungen der politischen Auftraggeber und Ministerialbürokratien konfrontiert, die Schwerpunkte der Goethe-Institute innerhalb einer Region stärker zu erklären und an übergreifenden Zielvereinbarungen auszurichten. Auch wenn mein Gesprächspartner entsprechende Fragen nach messbaren Wirkungen, auflistbaren Ergebnissen oder produktiven Kooperationen zwischen einzelnen Instituten im Prinzip als legitim betrachtet, so führen sie in der Praxis dennoch zu Reibungen und Leerstellen:

"Das ist ja auch eine richtige Überlegung von ihrer Position aus. Das sollte sein, wie beim Airbus, das heißt: Man macht etwas gemeinsam. Also beim Airbus werden Bauteile aus Toulouse mit einem Riesen-Airbus nach Hamburg geflogen und da an die Tragflächen geschraubt. [...] Und ein Airbus, der muss halt fliegen, während hier jedes Institut seine Sache machen kann und wenn es gute Presse kriegt, dann reicht das auch. Der Airbus, der fliegt nicht, wenn inm die Flügel nicht angeschraubt werden. Das merkst du dann schon. Während hier merkst du es nicht, also hier kannst du trotzdem starten. Das ist der Unterschied in der Kulturarbeit überhaupt, dass gut und schlecht nicht so voneinander zu unterscheiden sind, dass es keine wirklichen Qualitätsmerkmale gibt, dass auch der Wirkungsgrad nicht messbar ist, weil sich die Frage immer nur umgekehrt stellt: Wie wären Gesellschaften, wenn es uns nicht gäbe?»

Festzuhalten bleibt, dass im Zuge der translokalen Wissens- und Übersetzungsarbeit einer Auswärtigen Kulturpolitik als Konfliktprävention unterschiedliche Rationalitätsformen in Kontakt geraten. Das Bild vom Airbus steht hier für die Bestrebungen politischer Entscheidungsträger und Ministerialbürokratien, eine größere Planbarkeit, Lesbarkeit und Zielgerichtetheit kulturpolitischer Arbeitsprozesse innerhalb des globalen Netzwerkes herzustellen. Aus der Perspektive meines Gesprächspartners können sich Diskrepanzen ergeben, wenn solche Bestrebungen auf die Eigenwirklichkeit jedweder lokal situierter Kulturarbeit, die weitgehende Unvorhersehbarkeit kultureller Prozesse oder das Problem der Messbarkeit der Effekte kulturpolitischer Interventionen 
treffen. Im Laufe dieses Abschnitts wird deutlich werden, dass Kulturmittler diese Kontingenz ihrer Projektarbeit weniger als Störungen einer stringenten Policy betrachten, sondern gerade in ihr zentrale Potenziale kulturpolitischer Präsenzen in Konfliktgebieten verorten. Zunächst möchte ich aber Goethe-Institute als Kontexte untersuchen, in denen solche mitunter widerstrebende Rationalitätsformen in einen direkten Kontakt geraten und zugleich verknüpft werden müssen, um eine translokale Kulturarbeit überhaupt realisieren zu können.

\section{Reibungsflächen und Anknüpfungspunkte: Was bedeutet Dialog in einem besetzten Territorium?}

In Ramallah sind Kulturmittler häufiger mit ausformulierten Erwartungen politischer oder medialer Akteure aus Deutschland konfrontiert als in Sarajevo. Gleichzeitig sind hier die Präsenzen und Brechungen der Konfliktkonstellation gegenwärtig deutlich akuter. Entsprechend größer sind die Potenziale zur Kollision unterschiedlicher Rationalitätsformen im Arbeitsalltag eines deutschen Kulturinstituts. Verknüpfungspraxen zielen hier darauf ab, zwischen unterschiedlichen Zielvorstellungen, Diskursen und den »facts on the ground « auszutarieren - etwa wenn im Zuge einer Nahost-Reise von Bundestagsabgeordneten konkrete Vorstellungen und Bewertungen an die Kulturmittler herangetragen werden:

IL: "Ich weiß nicht, ob du dabei warst, als dieser Unterausschuss für Kultur hier war. Da kam dann wirklich sehr massiv der Einwand: Was Sie hier machen, ist nicht gut. Kultur muss Brücken schlagen.ı Und da sagen wir: , Nein, das funktioniert aus unserer Erfahrung so nicht. Es wird abgelehnt. Was aber funktionieren könnte: Wir fördern solche Kräfte innerhalb dieser Gesellschaft, die bereit sind, Brücken zu schlagen. Wir können keine Brücken zwischen Palästinensern und Israelis schlagen, schon gar nicht als Deutsche. Also das ist so durchsichtig, dass es der letzte Idiot kapiert, warum wir das machen: dass das uns selber gut tut, aber nicht denen. Das ist wirklich eine Form, sich besser zu fühlen."

JA: „Inwiefern?"

IL: "Na klar, weil wir eine Geschichte haben und wir wollen hier was gut machen und deshalb diesen Konflikt, bei dem wir schon das Gefühl haben, dass wir etwas beigetragen haben historisch - und das ist ja nun auch wirklich so - da wollen wir was gut machen und dafür sagen wir, was die Palästinenser zu tun haben und die Israelis auch. Das ist durchsichtig, das will keiner. ${ }^{115}$

115 | Interview vom 4. Dezember 2008; auch die folgenden drei Zitate. 
Zwei Rahmenbedingungen einer kulturpolitischen Verknüpfungsarbeit treten hier hervor: Einerseits zeigen sich historische und politische Präkonfigurationen translokaler Praxis- und Übersetzungsfelder. Diese sind vielleicht nirgendwo so stark, wie in dem Verhältnis zu Israel, das hier die Erwartungen der Besucher an eine Kulturarbeit in Palästina überlagert. Deren Wert bemisst sich demnach primär an ihrem Beitrag zur israelisch-palästinensischen Verständigung. Aus Perspektive meines Gesprächspartners liegt die Problematik hierbei in der ausgeprägten Mobilisierung einer deutschen Befindlichkeit, die sich letztlich als kontraproduktiv herausstellt: Sie wird von den lokalen Partnern als naive und paternalistische Selbstbespiegelung durchschaut. Andererseits verweist er auf seine erfahrungsbasierten Vorstellungen von funktionierenden Herangehensweisen, die sich gerade in Abgrenzung zu dieser Position herauskristallisieren. Dieser zweite Punkt wird anhand seiner Einschätzungen zu dem Schlüsselbegriff der neuen Policy noch deutlicher:

"Also was nicht funktioniert ist Konfliktprävention, indem man mit Israelis und Palästinensern arbeitet. Das kann man vergessen. Das wird von beiden Seiten als aufoktroyiert, paternalistisch, herablassend empfunden und ich meine zu Recht. Das funktioniert nicht, das wird abgewiesen und unterminiert sogar unseren Status hier."

Eine Übersetzung von Konfliktprävention in »israelisch-palästinensische Dialogprojekte« - gemäß den Erwartungen deutscher politischer Entscheidungsträger - stößt also vor Ort auf Ablehnung und schadet sogar der eigenen Reputation im direkten Arbeitsumfeld. Entsprechend formuliert der Institutsleiter einen Gegenentwurf, der Rationalitätsformen und Schlüsselbegriffe des politischen Narrativs in Deutschland durchaus aufgreift, aber stärker an lokal situierten Haltungen und Diskursen ausrichtet:

"Anders könnte es funktionieren, nämlich, dass man sagt, wir fördern hier - nicht in Israel, da gibt es das andere Goethe-Institut - wir fördern hier Kräfte, die für eine Zivilgesellschaft, für eine demokratische Gesellschaft sind. Das sind nun auch Kräfte, die eher an einem Dialog interessiert sind, als die Kräfte, die Befreiungskampf als Djihad sehen. Das kann natürlich sein, dass wir sagen, wir fördern Kräfte, mit denen es Israel leichter hat, einen guten Gesprächspartner zu finden. Wenn Israel das möchte.“

Dialog, oder die Förderung von Demokratie und Zivilgesellschaft bilden auch hier Orientierungsgrößen, werden aber zurückhaltender ausformuliert: Im Mittelpunkt steht demnach die Entwicklung von Potenzialen zum Dialog innerhalb der palästinensischen Gesellschaft - ob diese Potenziale in einem folgenden Schritt zur Bearbeitung der Konfliktkonstellation aufgegriffen werden, liegt nicht in der eigenen Hand. 
Solche Unterschiede in den Auffassungen zu dem Auftrag und dem Vermögen eines deutschen Kulturinstitutes oder zu den Übersetzungsmöglichkeiten kulturpolitischer Schlüsselbegriffe lassen sich sehr deutlich mit divergierenden Einschätzungen zu der Gestalt des »Konfliktes« in Zusammenhang bringen:

"Das ist ja auch dieser Quatsch mit dem ,Brückending؛: , Wir wissen zu wenig übereinander, wir reden zu wenig miteinander. Nein, das ist es nicht. Die Israelis wissen genug über die Palästinenser, die Palästinenser genug über die Israelis. Und wenn sie es nicht wissen, dann wollen sie es nicht wissen, dann nützen wir ihnen auch nichts mehr. Weil die Leute sind beratungsresistent. Die erreichen wir mit unseren Kulturaktionen auch nicht mehr."

Nach Auffassung des Institutsleiters geht es an seinem Einsatzort also nicht um die Frage der Bearbeitung eines »Kommunikationsproblems«, sondern um einen »politischen Konflikt, in dem ein Land ein anderes Land besetzt hält« - eine Einschätzung, die von der Vertreterin des DAAD in Ostjerusalem, geteilt wird:

"Also, ich denke, wir haben hier einen Konflikt, der nicht Resultat des mangelnden menschlichen Verständnisses ist, sondern hier geht es um einen massiven, konkreten Konflikt um Land, um politische Herrschaft. Und diesen Konflikt kann ich nicht lösen, indem ich Leute zusammenbringe. [...] Wir können noch so viele Freundschaften haben, wenn ich den Konflikt nicht löse - also sprich, die Besatzung nicht beende - dann komme ich nie weiter. ${ }^{116}$

Beide Kulturmittler erklären in diesen Sequenzen den israelisch-palästinensischen Konflikt primär als eine politische Konfiguration; beide grenzen sich hierdurch zugleich von den häufig medial oder politisch mobilisierten Erwartungen an konfliktpräventive Effekte von Dialog- oder Begegnungsprojekten $\mathrm{ab}$ und geben den Kernauftrag zur Konfliktlösung somit gewissermaßen von der Kultur an die Politik zurück. Auf dieser Basis lassen sich zugleich Rückschlüsse auf Verknüpfen als einen Modus kulturpolitischer Übersetzung ziehen. Denn aus solchen Sequenzen sprechen die ausgeprägten Verwebungen von Kulturmittlern mit unterschiedlichen Präsenzen der Konfliktkonstellation in ihren Arbeitskontexten - Verwebungen, die zu Perspektiven und Erfahrungen führen, die deutsche Parlamentarier kaum machen, und die es einzubeziehen gilt, wenn man seinen Status und seine Handlungsfähigkeit in den Kulturszenen vor Ort nicht gefährden möchte.

Die Herausforderungen einer kulturpolitischen Verknüpfungsarbeit, die sich aus dem Aufeinandertreffen divergierender Positionen und Rationalitä-

116 | Interview vom 5. Dezember 2008. 
ten ergeben, lassen sich gut anhand lokaler Diskussionen um den Begriff der »Normalisierung« als einem Gegenpol zu der Zielvorstellung »Brücken bauen « herausstellen. Vielfach berichten mir deutsche Entsandte, dass die große Mehrheit palästinensischer Kulturschaffender oder zivilgesellschaftlicher Akteure einer Teilnahme an öffentlich sichtbaren Dialogprojekten ablehnend gegenübersteht, solange der Staat Israel seine Besatzungs- und Siedlungspolitik nicht nachhaltig verändert und hierdurch überhaupt die Voraussetzung für eine gleichberechtigte Begegnung schafft. Die politisch mobilisierte Idealvorstellung eines »israelisch-palästinensischen Dialogs« bleibt aus dieser Perspektive - etwa aufgrund der fortwährenden Asymmetrien in der Verfügung über politische und militärische Gewalt sowie in der Beschränkung von Zugängen und Mobilität - eine weitgehend irreale Fiktion. Ein Stiftungsmitarbeiter bezeichnet die Sorge palästinensischer Künstlerinnen, durch die Beteiligung an Austauschprojekten mit israelischen Organisationen zu einer »Normalisierung « des permanenten Ausnahmezustandes der Besatzung beizutragen, als »Geschäftsgrundlage« unter seinen palästinensischen Partnern, die seine eigene Arbeit entsprechend bestimmt. Diese Position lässt sich gut anhand der Äußerungen einer Schauspielerin erläutern, die ich nach ihrer Haltung zu eventuellen Dialogprojekten des Goethe-Instituts frage:

"It would collect only a minority of the minority of the minority of Palestinians. [...] If you ask how many artists in the country or writers are interested in doing something together with Israel: You'll find only one person, maybe half a person. [...] I mean the ınormalization`, you know: We decide to go now and make a project, to make music. Music is free, so let's do music, Palestinians and Israelis. We go to Berlin and make a performance. And we meet and we stay in this nice fancy hotel and we eat together and we might fall in love as if nothing is happening in Gaza. This is what I mean by normalization. There is a great range of Palestinians against this idea: let's act under the title of art that everything is $\mathrm{OK}$, because art is liberal and free. $1 \%$, maybe, out of all the Palestinian artists are accepting the ,normalization`, but the rest are not. “ ${ }^{117}$

Dialogprojekte erscheinen aus dieser Perspektive also als mögliche Beiträge zur Normalisierung eines politisch geschaffenen Ausnahmezustandes, in dem »es keine Normalität gibt« - wie es der Institutsleiter einmal formuliert hat. Auch wenn sich zweifelsohne Beispiele finden lassen, in denen einzelne palästinensische Künstlerinnen oder Intellektuelle Argumentationslinien entwickeln, um diese dominante Position zu umgehen, so laufen sie hierbei immer Gefahr, sich in ihrem eigenen Arbeitskontext zu marginalisieren. Gleiches gilt für deutsche Entsandte, die zu offensiv für Dialogformate werben. Dennoch können Kulturmittler, die im Rahmen einer staatlichen Policy operieren, die

117 | Interview vom 29. November 2008. 
Erwartungen und Rationalitäten deutscher Parlamentarier nicht einfach ignorieren. Entlang der Mobilisierung eines kulturpolitischen Schlüsselbegriffs ergeben sich an unterschiedlichen Passagepunkten Kollisionsmöglichkeiten, die es durch translokale Verknüpfungspraxen zu vermeiden gilt.

\section{Navigieren lernen: Reflexive Wissensarbeit und die Entstehung eines translokalen Gespürs}

Kulturmittler müssen in ihrem Arbeitsalltag vielfältige diskursive Klippen umschiffen. Eine Verknüpfungsarbeit wird erforderlich, da diese Klippen in sehr unterschiedlichen lokalen und institutionellen Kontexten situiert sein können. Gerade an Einsatzorten in Konfliktgebieten ähnelt die Projektarbeit mitunter einem Navigieren durch unruhige, partiell unbekannte Gewässer. Eine jeweilige Bewegung kann sich ebenso als fatal erweisen wie neue Freiräume eröffnen. Im Herbst 2008 führt etwa die Einladung eines skandalumwobenen Berliner »Gewaltrappers« zu einer European-Palestinian Hip Hop Concert Tour durch das Westjordanland zu einem Eklat: Ein Bundestagsabgeordneter veröffentlicht eine rügende Stellungnahme, Medien berichten kritisch und der Präsident des Goethe-Instituts reagiert mit einer ungewöhnlich starken Distanzierung von seinen Mitarbeitern in Ramallah. Einige Jahre zuvor wurde ein früherer Institutsleiter - bereits nach seiner Rotation nach Damaskus - durch die damalige Präsidentin getadelt und nach Indien strafversetzt: Er hatte auf einer Berliner Diskussionsveranstaltung Verständnis für die Ehrung palästinensischer Selbstmordattentäter als »Freiheitskämpfer« innerhalb ihrer lokalen Gemeinschaften geäußert. ${ }^{118}$ Für beide Fälle lässt sich formulieren, dass die Kulturmittler lokal situierte Bedürfnislagen oder Diskurse gegenüber den Rationalitäten und Erwartungen, die deutsche politische und mediale Öffentlichkeiten dominieren, privilegiert haben - eine Form der Verknüpfung, die offensichtlich Reibungen innerhalb der translokalen Praxis- und Übersetzungsfelder auslösen kann. Auch wenn die Arbeit der Kulturmittler zumeist darauf ausgerichtet ist, es nicht zu solchen Eklats kommen zu lassen, so riskieren sie mitunter doch Reibungen oder rufen sie sogar gezielt hervor. Dabei entwickeln sie im Zuge ihres Aufenthaltes an einem Einsatzort sukzessive Expertisen, diskursive Klippen zu erahnen und Kollisionen zu vermeiden, aber auch eine größere Souveränität, mit diesen Gefahrenpotenzialen umzugehen und sogar für die eigene Arbeit produktiv zu machen - das Spektrum ihrer Verknüpfungspraxen erweitert sich. In der folgenden Sequenz berichtet mir die Repräsentantin des DAAD in Ostjerusalem von ihrer demonstrativen Distanzierung von dem Auftreten eines deutschen Gastes in den Palästinensischen Gebieten:

118 | www.tagesspiegel.de/kultur/jenseits-der-frommen-wuensche/373590.html (letzter Zugriff am 30. Juli 2017). 
"Es gibt so richtige Karikaturen: Wir hatten mal einen Vertreter einer politischer Partei ich will jetzt nicht nennen von welcher -, der zu einem Empfang - es war sogar ein Dinner - mit geladenen Gästen, alles Palästinenser, mit dem deutschen Vertreter in den Palästinensischen Gebieten eingeladen war. Und diese Person hat es dann tatsächlich fertig gebracht zu sagen: IIch bin hier, um den Holocaust aufzuarbeiten. Und dann sagt natürlich jeder Palästinenser: I'm sorry, bist du hier nicht am falschen Platz?، Gegen dieses Karikaturbild ist es natürlich für ungeheuer viele Leute hier wichtig, wenn sie jemanden treffen, bei dem sie merken, der oder die kennt den Konflikt und der oder die bezieht dann auch mal Position und bezieht dann auch zum Beispiel mal eine Position, die vielleicht auch dann in Deutschland selber nicht so ohne Weiteres akzeptiert wird. " ${ }^{119}$

Auch eine solche kritische Haltung gegenüber dem Auftreten eines hochrangigen deutschen Gastes stellt eine Verknüpfungspraxis dar: Vielleicht riskiert meine Gesprächspartnerin durch ihre explizite Solidarisierung mit lokal situierten Wahrnehmungen Verbindungen zu deutschen Entscheidungsträgern zu verschließen; gleichzeitig eröffnen sich aber neue Vernetzungspotenziale, die andere, besatzungskritische Positionen aus Deutschland, Palästina und eventuell auch Israel in einen Resonanzraum bringen können.

Im Laufe meiner Forschung konnte ich in vielen Momenten beobachten, wie Kulturmittler divergierende Erwartungen und Rationalitäten reflexiv aufeinander zu beziehen versuchen und hierbei zwischen den Modi des Verknüpfens changieren. Eine Variante besteht in der dezenten Provokation: Der deutsche Kulturstaatsminister kommt im Rahmen einer mehrtägigen Reise durch Israel zu einer kurzen Stippvisite nach Ramallah. ${ }^{120}$ In einem Gespräch mit palästinensischen Kulturschaffenden stellt der Institutsleiter eine Teilnehmerin aus Jerusalem als »eine Vertreterin aus der Hauptstadt Palästinas« vor und konfrontiert den Besucher hierdurch mit einer Gegenposition zu der »offiziell gemachten«, israelischen Sichtweise von Jerusalem als »ewiger und unteilbarer Hauptstadt eines jüdischen Staates «. ${ }^{121}$ Eine andere Variante verläuft als umfassende Unterrichtung: Während des Besuches einer Bundestagsabgeordneten in Ramallah nimmt sich der Institutsleiter viel Zeit, um im direkten Gespräch auf Basis seiner Erfahrungen und Alltagsbeobachtungen die lokalen Realitäten kleinteilig und nüchtern zu erläutern. Er nutzt hierdurch die Gelegenheit, seine Sicht auf die Gründe und Verantwortlichkeiten für das Stocken des »Friedensprozesses« im deutschen politischen Feld zu

119 | Interview vom 5. Dezember 2008.

120 | Feldnotizen vom 22. Oktober 2008.

121 | So ein Beschluss der israelischen Knesset aus dem Jahr 1980, der seither vielfach durch israelische Regierungen und internationale Besucher - trotz anderslautender Rechtslage - wiederholt wurde. 
platzieren. ${ }^{122}$ Eine dritte Variante zeigt sich in dem Bemühen, Journalistinnen oder Workshopleiterinnen direkt mit den Alltagsrealitäten der Besatzung zu konfrontieren - etwa indem man sie während ihres Aufenthaltes nicht immer mit dem gekennzeichneten Institutsbus über einen Diplomatenübergang zwischen Ramallah und Jerusalem transportiert, sondern zumindest einmal mit einem öffentlichen Verkehrsmittel über normale Checkpoints fahren lässt. In all diesen Modi geht es darum, in Deutschland situierte Positionen mit lokalen Wahrnehmungen oder Alltagsfakten zu verknüpfen und hierdurch eine neue Perspektive auf den israelisch-palästinensischen Konflikt zu eröffnen.

Aber auch in der Zusammenarbeit mit lokalen Partnern und Öffentlichkeiten lassen sich solche reflexiven Wissenspraxen beobachten. Die Kulturmittler kennen und achten die Dominanz einer ablehnenden Haltung gegenüber Kooperationen mit Israel, bemühen sich in konkreten Projektkonstellationen aber dennoch immer wieder um deren Flexibilisierung und diskursive Verschiebung. Sie greifen hierbei die Zielvorstellung einer Auswärtigen Kulturpolitik als Konfliktprävention auf, suchen aber nach Anknüpfungspunkten, die im lokalen Kontext funktionieren könnten. Kulturpolitische Übersetzungen verlaufen hier als ein pragmatisches, translokal informiertes und inkrementelles Navigieren durch die Rationalitäten und Erfordernisse unterschiedlicher Diskussions- und Handlungsfelder. Die folgende Interviewsequenz mit einer Bibliothekarin des Goethe-Instituts in Ramallah vermittelt einen Eindruck von dieser navigierenden Verknüpfungsarbeit. Im Mittelpunkt steht Felix, ein kleiner Hase und Held eines deutschen Kinderbuches, das ins Arabische übersetzt worden ist - nur leider durch einen in Israel angesiedelten Verlag:

MA: "Jetzt legt man mir ein tolles Kinderbuch auf den Tisch: 'Felix', mit Briefen drinnen und so, ins Arabische übersetzt. Aber das Buch hat ein Verlag in Israel produziert. Und das ist schon ein Problem. Also ich, wenn ich hierherkomme, stelle ich mir natürlich vor, das ist ja eigentlich ideal: Mit dem Buch arbeitest du schon einmal hier mit einer Kindergruppe und das haben wir auch gemacht. Die Kinder haben dann Briefe- Also das ist ein Hase, der geht auf dem Flughafen verloren und aus Versehen endet er im Flugzeug und seine Besitzerin, die kleine Sophie, steht da und er ist weg. Und dann schreibt er ihr immer Briefe aus Tokio und aus Hongkong und so. Und die Briefe stecken da im Buch drinnen. Und dann haben wir mit den Kindern gearbeitet und haben gesagt: 'Also inr könnt auch Briefe jetzt an den Felix schreiben، und dann schreiben die an Felix: ,Halt doch auch mal, komm doch bitte, halt doch auch mal hier, komm doch mal hierher, du brauchst keine Angst haben. Du wirst viele Leute mit Gewehren sehen. Hab keine Angst, wir hätten so gerne, dass du kommst und wir erzählen dir dann, wie das hier alles so ist und so. Gut, das war eigentlich ein guter Anfang. Ich hatte mir vorgestellt, ich könnte-

122 | Feldnotizen vom 19. November 2008. 
Also in Jerusalem, fanden sie das halt auch so toll im Institut, ${ }^{123}$ ob wir es nicht hinkriegen, dass eine Gruppe von Kindern auf der israelischen Seite sich mit einer Gruppe von Kindern von hier schreibt."

JA: "Hebräischsprachige Kinder, oder-?"

MA: "Ja, ja. Aber es ist gaaanz schwierig, Jens. Du, ich habe auch eben bei der Aktion versucht, ein palästinensisches Bildungsinstitut miteinzubeziehen und die haben gesagt: 'Also wir schicken euch unsere Kinder und ihr könnt natürlich damit arbeiten und alles. Aber bitte schreibt nicht, dass wir da als Partner mit drinnen stecken. Oder du musst halt Lehrer oder Eltern finden, die das mitvollziehen, was du dann da als Strategie hast. Oder jetzt habe ich das Buch auf der Buchmesse hier ausgelegt. Es gibt Leute, die zeigen dir: 'Aber das ist doch ein israelischer Verlag." "

JA: "Wie weit seid ihr damit gekommen mit dieser Idee, dass sich israelische und arabische Kinder Briefe schreiben?"

MA: "Ich bin noch nicht viel weiter- Ich habe es noch nicht ganz aufgegeben. Aber, weißt du, das ist dann halt auch sehr aufwendig. Da brauchst du dann wahnsinnig viel Zeit dafür, um das durchzuziehen."

JA: "Was ist das Argument, warum wollen die damit nicht in Beziehung gesetzt werden, dieses Bildungsinstitut?"

MA: "Du weißt, das Grundproblem hier ist, dass dir die Palästinenser sagen: ,Wir haben 40 Jahre lang versucht - also mit Unterbrechungen - mit unseren Nachbarn zu reden und zu irgendwelchen Vereinbarungen zu kommen. Es gibt $x$ Vereinbarungen, die auf dem Tisch liegen schriftlich und immer sind sie geplatzt und so, oder: ,Und wir wollen nicht mehr.ı [...]

JA: "Und kannst du das nachvollziehen?"

MA: "Ja, ich kann es schon nachvollziehen. Ob ich es richtig finde, ist eine andere Frage." JA: "Findest du es richtig, oder-?"

MA: "Ich finde es nicht ganz richtig. Und ich meine, es gibt ja weiterhin Initiativen auf beiden Seiten und auch gemeinsame Initiativen. Aber generell ist das die Situation. “ ${ }^{124}$

Der Arbeitsalltag einer Kulturmittlerin zeigt sich hier als ein projektbezogenes Zusammenfügen von kulturpolitischen Zielvorstellungen, diskursiven Positionen, Materialien, lokalen Organisationen und Akteursgruppen - ein Zusammenfügen, das gleichzeitig in Abhängigkeit von ganz handfesten Faktoren wie Zeit, Geld oder einem Mitarbeiterschlüssel geschieht. Sichtbar wird hierbei die vorsichtige Suche nach Möglichkeiten des Verknüpfens, die ich als »translokales Gespür« bezeichne - eine erfahrungsbasierte Fertigkeit, die sich in dieser Dichte erst im Laufe eines längeren Einsatzes an den Schnittstellen unterschiedlicher lokaler und institutioneller Felder herausbilden kann. Auf

123 | Also im Goethe-Institut in Westjerusalem, das ein jüdisch-israelisches Publikum adressiert.

124 | Interview vom 22. November 2008. 
Basis dieses Gespürs vermag meine Gesprächspartnerin mögliche Reibungen ihrer Programmvorschläge vorauszuahnen und kann dennoch zu der Entscheidung kommen, sie situativ zu riskieren - oder auch nicht. Lokal dominante Positionen werden nachvollzogen, aber nicht zwingend übernommen; sie können aus einer Distanz betrachtet oder auch als Produkt der Dynamiken innerhalb einer Partnerorganisation erkannt werden. Begriffe und diskursive Begrenzungen, die im ersten Kontakt unumstößlich erschienen, bilden weiterhin die Arbeitsgrundlage, lassen sich im Laufe einer zunehmenden Vertrautheit mit den lokalen Gegebenheiten und einer wachsenden Verflechtung mit den Präsenzen des Konflikts aber vorsichtig austarieren.

\section{Problematisieren als Wissensmodus: "Was kann man mit den Mitteln der Kultur hier erreichen?"}

Solche Navigationen erfordern eine kontinuierliche Auseinandersetzung mit den politischen, diskursiven oder infrastrukturellen Gegebenheiten an den Einsatzorten: Ein neuer Wissens- und Arbeitsmodus taucht auf, wenn Kulturmittler Rationalitäten und Schlüsselbegriffe einer Auswärtigen Kulturpolitik als Konfliktprävention aufgreifen. Lokal wahrnehmbare Konfliktlinien, Defizite oder Reibungsflächen erscheinen nicht einfach als schwierige Rahmenbedingungen, sondern rücken explizit ins Zentrum der Programmarbeit. Die Kulturmittler entwickeln Herangehensweisen, die ihre Einsatzorte nach den multiplen Präsenzen und Effekten der »Konflikte« befragen und auf dieser Basis Möglichkeiten temporärer Interventionen ausloten. Den Ausgangspunkt bildet hierbei zumeist die Identifizierung einer Irritation oder Stagnation, einer defizitären Infrastruktur oder bedrohlichen Entwicklung, die im Rahmen von Diskussionen, Kooperationen und einem jeweiligen Projektformat als lokal situierte Probleme moduliert werden. Ich bezeichne eine solche Arbeitspraxis als »problematisieren « und untersuche sie nun als einen Übersetzungsmodus der neuen Policy.

»Was kann man mit den Mitteln der Kultur hier erreichen?« formulierte der Leiter der Abteilung für Kultur und Kommunikation des Auswärtigen Amtes in einem bereits zitierten Gespräch eine zentrale Fragestellung für eine Kulturpolitik, die sich Konfliktprävention auf die Fahnen geschrieben hat. Während meiner Feldforschung habe ich Gelegenheit, einen Besuch dieses hohen Beamten in Ramallah zu beobachten. Ein Treffen mit lokalen Kulturschaffenden diskutiere ich in dem folgenden Abschnitt als eine exemplarische Konstellation, in der dieser neue Wissens- und Arbeitsmodus wirksam wird und zugleich die Basis bildet, um in einen dichten Austausch zu treten. Vor diesem Hintergrund erscheinen drei Fragestellungen besonders relevant: Welche thematischen Zuschnitte kristallisieren sich im Zuge solcher Problematisierungen als mögliche Anknüpfungspunkte für kulturpolitische Interventionen 
heraus? Welche Rolle spielen hierbei die Asymmetrien, die translokale Praxisund Übersetzungsfelder durchziehen? Und welche Effekte sollen durch solche Interventionen im Modus des Problematisierens hervorgerufen werden?

\section{Ungleiche Partnerschaften: "Ihr schuldet uns etwas, denn wir haben nichts!"}

In diesen Praxis- und Übersetzungsfeldern wirken Asymmetrien, die sich insbesondere aus dem Zusammentreffen unterschiedlicher Konfigurationen von Staatlichkeit ergeben. Im Rahmen dieser Untersuchung wurde bereits herausgearbeitet, dass sich die Präsenz deutscher Kulturmittler an Orten wie Ramallah oder Sarajevo als Ausdruck der politischen und ökonomischen Potenziale eines europäischen Nationalstaates verstehen lässt, der für sich in Anspruch nimmt, jenseits seiner Grenzen kulturpolitische Effekte zu erzielen. Gerade aus dieser Perspektive erscheinen die lokalen Ausformungen von Staatlichkeit häufig als fragmentiert oder defizitär und zugleich als Hindernis für die Orientierung an den Schlüsselmetaphern des Politikbereichs, die auf Partnerschaft und einen Dialog auf Augenhöhe abzielen. Eine solche Problematisierung von Staatlichkeit wird etwa anhand der Antwort des Institutsleiters in Ramallah greifbar, als ich ihn nach den zentralen kulturpolitischen Rahmenbedingungen an seinem Einsatzort frage: »Palästina ist ein Entwicklungsland «, das »nur in einem sehr beschränkten Maße über einen eigenen Haushalt verfügt [...] keinen eigenen Staat, keine Außengrenzen hat und damit nur sehr beschränkt überhaupt Partner sein kann. ${ }^{125}$

Eine solche Ausgangslage bringt für deutsche Kulturmittler zumindest zwei Schwierigkeiten mit sich. Zum einen ist es kaum möglich, substanzielle »Partnerleistungen« einzufordern, die im Selbstverständnis des Goethe-Instituts und auch seiner politischen Kontrollinstanzen eigentlich Voraussetzungen für eine Zusammenarbeit darstellen: »Nein, man kann einem nackten Mann nicht in die Tasche greifen. Das ist klar. Du kannst keine Partnerleistungen fordern, wo kein Partnergeld da ist. Wenn es Geld gibt, ja. Aber Palästina hat nun mal einen kaputten Haushalt.«

Zum anderen lässt sich schwerlich die ebenfalls projizierte »Nachhaltigkeit« für die, zumeist als »Impulse « angelegten, eigenen Programme erreichen. Intendierte Wirkungsketten werden aufgrund fehlender staatlicher und lokaler Strukturen sowie finanzieller Kapazitäten unterbrochen oder kommen überhaupt gar nicht erst in Gang:

"Sobald man Mittel wieder abzieht, sich anderen Themenstellungen zuwendet oder Personal reduziert, bricht alles, was man geschaffen hat, in sich zusammen. [...] Wir

125 | Interview vom 4. Dezember 2008; auch die folgenden drei Zitate. 
haben nicht die Chance, irgendwann mal davon auszugehen, dass die Palästinenser Projekte, die wir angeregt haben, Knowhow, das wir geschaffen haben, auf eigene Faust weiterführen."

Lokale Partnerorganisationen des Goethe-Institutes sind vor diesem Hintergrund auf die finanziellen Mittel eines wechselhaften, translokalen Projektemarktes angewiesen, um ihre Vorhaben umsetzen zu können:

"Wir haben immer die Situation, dass unsere Partner dann den nächsten Sponsoren angehen und von einer Stiftung an die nächste weitergereicht werden. Keine Stiftung ist bereit, etwa ein Bildungsprogramm mehr als ein Jahr oder, wenn es hoch kommt, für drei Jahre zu fördern. Danach sagen die: 'So, jetzt müsst ihr auf eigenen Füßen stehen`, mit einigem Recht auch. Das tun sie aber nicht und dann muss der palästinensische Partner zum nächsten EU-Land oder zu den Amerikanern gehen oder das ganze Projekt unter einem anderen Namen noch einmal neu verkaufen."

Deutsche Kulturmittler haben hingegen einen kontinuierlichen Zugriff auf staatlicherseits bereitgestellte Ressourcen, infrastrukturelle Arrangements und Netzwerke, die sie im Verhältnis zu den meisten ihrer Projektpartner mit einem gesicherten Status, einem langfristigen Planungsvermögen und größeren Handlungsoptionen ausstatten - ob intendiert oder nicht, in ihrer Arbeitspraxis produzieren sie Machteffekte, die von lokalen Akteuren durchaus erkannt werden: So finden sich in meinen Interviews mit Kulturschaffenden in Ramallah und Sarajevo immer wieder Klagen über die unerwartete, einseitige Beendigung einer langjährigen Zusammenarbeit durch die Goethe-Institute, über die Fixierung deutscher Kulturmittler auf die Realisierung ihrer eigenen Programme, die nicht immer als wirklich dringlich anerkannt werden, oder auch über deren Erwartung, dass lokale Akteure unbezahlt zu solchen Projekten beitragen. Gleichzeitig lässt sich beobachten, wie sich gerade entlang der Intention, sich lokal situierten Konfliktlinien zuzuwenden, temporäre Übersetzungsräume öffnen, in denen Positionen zu den Problemstellungen oder Bedürfnissen vor Ort, zu Interventionsmöglichkeiten oder den Modi der Zusammenarbeit in Bewegung geraten können. Diese Dynamik möchte ich nun anhand einer Begegnung erläutern. ${ }^{126}$

Im Rahmen einer Reise durch Israel und die besetzten Gebiete besucht der Leiter der Abteilung für Kultur und Kommunikation des Auswärtigen Amtes das Goethe-Institut in Ramallah. Am nächsten Tag wird er nach Jenin weiterreisen, um dort die Möglichkeiten zur Unterstützung eines anspruchsvollen infrastrukturellen Projektes zu prüfen. Eine deutsch-palästinensische Initiative plant hier die Renovierung und Eröffnung eines ehemaligen Kinos und hat

126 | Für die gesamte folgende Sequenz: Feldtagebuch vom 21. November 2008. 
das Amt um eine substanzielle Förderung gebeten. Eine translokale community of project aus Kulturschaffenden, zivilgesellschaftlichen Aktivistinnen, Unternehmen und Politikern hat sich zur Realisierung des Cinema Jenin gebildet. ${ }^{127}$ Auch das lokale Goethe-Institut hat sich an diese Initiative angedockt, nicht zuletzt da in diesem Rahmen ein Standort für die eigenen Sprachkurse und Kulturprogramme im nördlichen Westjordanland entstehen würde. Vor diesem Hintergrund hat der Institutsleiter einige seiner Partner aus dem Bereich Film zu einem Gespräch gebeten. Es bildet sich ein temporärer Übersetzungsraum, in dem lokal situierte Probleme konfiguriert werden, deren Zuschnitt und Verknüpfungspotenziale sich entlang von vier Positionen und einem Eklat illustrieren lassen:

$\mathrm{Zu}$ Beginn rahmt der Abteilungsleiter das Treffen mit Bezugnahmen auf bekannte Argumentationsfiguren einer konfliktpräventiven Kulturpolitik. Er spricht von der »Kultur, die auch unter schwierigen politischen Bedingungen ihren Wert hat« und verweist auf die ursprünglich unwahrscheinliche Erfolgsgeschichte der deutsch-französischen Versöhnung als beispielhaft für andere, gegenwärtig unlösbar erscheinende Konfliktkonstellationen. Anschließend positioniert er sich zum einen als Vertreter einer finanzstarken Institution, indem er aktuell wachsende Budgets hervorhebt, die auch kulturpolitischen Aktivitäten in den Palästinensischen Gebieten zugutekommen sollen; zum anderen betont er, dass er sich im Hinblick auf eine mögliche Förderung des Cinema Jenin einen Rat erhoffe und versetzt somit seine palästinensischen Gesprächspartner in die Rolle von lokalen Experten.

In einer ersten Reaktion bringt der Direktor des zu diesem Zeitpunkt einzigen Kinos und Theaters im Westjordanland mit permanenter Spielstätte zunächst seine Freude über das geplante Projekt zum Ausdruck, um im Anschluss primär über die Defizite im lokalen Kulturleben, über unbeantwortete Bedürfnisse und das unzureichende Engagement internationaler Akteure in den Palästinensischen Gebieten zu klagen. Verweise auf fehlende Infrastrukturen und den Verlust kultureller Kompetenzen stehen dabei im Zentrum: Alle Kinos des Westjordanlandes seien während der ersten Intifada geschlossen worden. Hierdurch seien nicht nur Kinos als Institutionen verschwunden, sondern auch die einfache Gewohnheit ins Kino zu gehen oder grundlegendes Wissen über diese Kunstform und kulturelle Praxis. Als Konsequenz würde sich die palästinensische Gesellschaft gegenwärtig kulturell zurückentwickeln, ein Teil radikalisiere sich. Für die Entfaltung »eines gesünderen kulturellen Lebens« bräuchte es nachhaltige Investitionen in kulturelle Infrastrukturen und in den Aufbau einer umfassenden kulturellen Bildung in den Schulen. Aus dem palästinensischen Haushalt ließen sich diese Bedarfe nicht decken und auch die Arbeit der eher kleinen ausländischen Kulturinstitute

127 | Siehe hierzu: www.cinemajenin.org (letzter Zugriff am 4. November 2017). 
reiche nicht aus. Entsprechend fordert er ein deutlich substanzielleres Engagement europäischer Regierungen in den besetzten Gebieten: »Wir haben nicht, was wir haben sollten«, fasst er zusammen.

Die zweite palästinensische Gesprächspartnerin stellt sich als Leiterin des einzigen Frauenfilmfestivals in der arabischen Welt vor und knüpft zumindest in der Beurteilung grundlegender kultureller Dynamiken an die zuvor geäußerten Einschätzungen an: Sie spricht von einer »kulturellen Regression « in den Palästinensischen Gebieten und beklagt den zunehmenden Rückzug der Menschen in immer kleinteiligere Räume und Gemeinschaften. Ihre Projekte reagierten auf diese Entwicklung, indem sie gerade auch in den abgelegenen Städten des besetzten Territoriums auf die Öffnung von Diskussionsräumen ausgerichtet seien, die den Aufbau einer »Zivilgesellschaft, die Differenz akzeptiert«, unterstützten. Im Hinblick auf die infrastrukturellen Defizite relativiert sie das Bild hingegen etwas: Die zwölf lokalen Universitäten, an denen das Festivalprogramm gezeigt würde, verfügten alle über voll ausgestattete Auditorien. Sie lenkt den Blick vielmehr auf fehlende Verbindungen zwischen unterschiedlichen lokalen Organisationen. Es gebe eine ganze Reihe von Medienabteilungen mit zeitgemäßer technischer Ausrüstung; jeder dieser Einrichtungen fehle aber zumindest ein relevanter Baustein. Aus ihrer Perspektive müsse es eher darum gehen, verschiedene Akteure durch »strategische Interventionen « zusammenzubringen, als ganz neue Institutionen und Infrastrukturen aufzubauen. Einen Grund zur Klage sieht sie in den immer nur projektbezogenen, in ihren Augen strategielosen Förderpolitiken ausländischer Geldgeber, die ihr nicht nur viel Zeit raubten, sondern eine langfristige Planung unmöglich machten.

Der Institutsleiter nutzt die Gelegenheit, um die Zielvorstellung »Nachhaltigkeit« als zentrales Problem seiner Arbeit zu markieren. Am Beispiel der Soap-Opera Matabb erläutert er, dass es keine lokalen Strukturen gäbe, die einen solchen Impuls aufgreifen und fortführen könnten. Seine Kooperationspartner rieben sich im »tagtäglichen Kleinklein« auf. Der Theaterdirektor flöge etwa persönlich nach Kairo, um dort einen Film für sein Kino abzuholen, da es für die besetzten Gebiete kein Verleihsystem gäbe. Vor diesem Hintergrund hätten sie als Goethe-Institut vor Ort aufgehört, »hier eine Lesung und dort ein Konzert zu organisieren«, sondern stattdessen strategisch entschieden, sich ganz auf »Kino und Medienentwicklung zu konzentrieren «. Sie brächten etwa Ausrüstung ins Land, organisierten dann aber auch Ton- oder Kameraworkshops, um zu unterrichten, wie man diese Ausrüstung nutzt. Die Musikszene könne sich nun berechtigterweise beschweren, dass sie vernachlässigt werde, aber bei limitierten Ressourcen sei es unerlässlich, solche Schwerpunkte zu definieren.

Gegen Ende des Gespräches kommt es zu einem Eklat, als der Gast aus Berlin erwähnt, dass man in internationalen Gremien häufig höre, Palästina 
sei »overfunded«. Es gebe zwei Regionen über die das so gesagt werde: Afghanistan und Palästina. Der Theaterdirektor reagiert verärgert und mobilisiert Bilder von einer historischen Schuld. Europa unterstütze Israel seit Jahrzehnten, etwa mit der Zahlung von einigen hundert Millionen Euro. Israel sei nun stabil und würde ihnen alles wegnehmen, sogar das Wasser. Vor diesem Hintergrund trage Europa dafür Verantwortung, hier Stabilität zu schaffen: »Ihr habt uns zerstört, ihr schuldet uns etwas, denn wir haben nichts«, erklärt er und schließt: »No, we are not overfunded.« Der Abteilungsleiter versucht die Wogen zu glätten: Es sei nicht seine Haltung, dass Palästina »overfunded « sei, sondern eine international weitverbreitete Position. In Anbetracht der vielen lokal tätigen NGOs käme man allerdings nicht umhin, den deutschen Steuerzahlern zu erklären, warum man sich noch für ein weiteres Projekt in den Palästinensischen Gebieten engagiere. Er äußert Verständnis für die Reaktion und versucht, einen situativen Konsens herzustellen. Man bestätigt sich wechselseitig, dass die Förderung einzelner Initiativen nicht ausreiche, um den massiven Bedarf an Infrastrukturen und kultureller Bildung zu sättigen. Man trennt sich freundlich, aber letztlich unverbindlich.

Anhand dieser Sequenz lässt sich verdeutlichen: Im Zuge der Mobilisierung von Zielvorstellungen einer Auswärtigen Kulturpolitik als Konfliktprävention bilden sich neuartige Übersetzungsräume heraus, in denen sich Perspektiven auf Einsatzorte und kulturpolitische Aufgaben partiell verlagern und neu verweben. Zwar führte auch das langfristig etablierte Prinzip der kulturellen Selbstdarstellung bereits $\mathrm{zu}$ einer Suche nach lokal funktionierenden Anschlüssen, die aber zumeist in dem angestammten konzeptionellen Rahmen einer nationalstaatlichen Kulturpolitik verblieb: Die - wenn auch in Form und Inhalt zunehmend flexible - Vermittlung von Beständen des Narrativs vom Nationalen stand dabei immer im Zentrum. Eine neue Dynamik der zunehmenden inhaltlichen Öffnung und Pluralisierung kulturpolitischer Praxis- und Übersetzungsräume lässt sich aus der letzten Sequenz zumindest anhand von drei Aspekten herauslesen: Erstens zeigt sich die Erörterung lokal situierter Probleme als eine treibende Kraft der Zusammenkunft eines translokalen Akteursfeldes, das - je nach konkretem Setting - deutsche Kulturmittler, Diplomaten, Policymaker, lokale Partner, »Ortskräfte«, externe Experten und andere Personen umfassen kann. Gemeinsam bilden sie einen temporären Praxis- und Übersetzungsraum, in dem Positionen aus unterschiedlichen lokalen und institutionellen Kontexten vorgebracht, aufgegriffen, weiterentwickelt oder fallen gelassen werden können. »Probleme« werden in diesem Rahmen in einem jeweils spezifischen Ausschnitt konfiguriert und gerade auch im Hinblick auf mögliche kulturpolitische Maßnahmen diskutiert. Die praxisorientierte Suche nach geeigneten Projekt- und Kooperationsformaten, durch die sich kulturpolitische Zielvorstellungen und Ressourcen mit lokal situierten Diskursen, Erwartungen und Defiziten verknüpfen lassen, gehört entsprechend zu solchen 
Erörterungen dazu. Zweitens treten Potenziale zur Verschiebung von Zuständigkeiten hervor: Infrastrukturelle Mängel, fehlende finanzielle Ressourcen, unzureichende staatliche Strukturen, der Verlust kultureller Kompetenzen oder besorgniserregende gesellschaftspolitische Entwicklungen geraten in den Fokus einer nationalstaatlichen Kulturpolitik, die zunehmend Interventionsmöglichkeiten in Konfliktkonstellationen prüft. Hierdurch ist nicht gesagt, dass deutsche Kulturmittler gewillt oder in der Lage wären, Verantwortung für die Bearbeitung all dieser Problemlagen zu übernehmen - aber die thematischen Schwerpunkte und Grenzen möglicher Handlungsfelder konfigurieren sich doch deutlich anders als im Rahmen einer Kulturpolitik, die im Kern auf Selbstdarstellung ausgerichtet blieb. Und drittens eröffnen sich in diesen verlagerten Übersetzungsfeldern Potenziale, Asymmetrien innerhalb von kulturpolitischen Kooperationen als »Problem« zu markieren. So wehrt sich der Theaterdirektor hier nicht nur gegen das hierarchisierende Bild von einem übermäßigen und einseitigen Kapitalfluss in die besetzten Palästinensischen Gebiete, sondern unterstreicht, wie stark europäische Akteure in die Entstehung, den historischen Verlauf und die gegenwärtige Realität eines Konfliktes verwoben sind, der gerade durch externe Beobachter zumeist als lokal verortet und somit geografisch eingrenzbar betrachtet wird. Verweise auf globale Verflechtungen und Machtfelder perspektivieren das »Problem« hier auf eine andere Weise und verlagern zugleich Fragen nach Verantwortlichkeiten und einem angemessenen Engagement westlicher Staaten. Welche Konsequenzen aus einer solchen situativen Konfrontation entstehen, bliebe zu prüfen: Sicherlich zeigt sich die anhaltende Wirkmächtigkeit der Asymmetrien, die ein solches Aufeinandertreffen durchziehen, in dem Vermögen des Gastes aus Berlin, die Begegnung mit unverbindlichen Äußerungen $\mathrm{zu}$ beenden oder in der bleibenden Dominanz eines politischen Narrativs, dass die Bereitstellung von Geldern auch weiterhin weniger als ein Recht der Bewohnerinnen und Bewohner geopolitischer Konfliktkonstellationen, sondern primär als Ausdruck deutscher Wohltätigkeit darstellt.

\section{Asymmetrische Problematisierungen: "Das ist etwas anderes, das sollten wir hier draußen lassen."}

Im Laufe meiner Feldforschung bin ich eher vereinzelt solchen direkten Konfrontationen begegnet, die auch die breiteren Rahmenbedingungen kulturpolitischer Kooperationen als »Probleme« markieren. Sehr viel häufiger schälen sich Herangehensweisen heraus, die primär auf lokale Ausschnitte abzielen und in denen sich die Machtgefüge etablierter institutioneller Ordnungen und Muster der Ressourcenverteilung widerspiegeln. Es zeigt sich eine Tendenz zur asymmetrischen Problematisierung, die ich nun an einem Beispiel aus Sarajevo erläutern möchte. 
Hier nehme ich an den Vorbereitungen zu einer Tagung mit dem Arbeitstitel Sprach(en)politik teil, die mit der Beobachtung einer Dynamik des sprachlichen Auseinanderdriftens ihren Ausgang nimmt. Denn im Zuge des Zerfalls Jugoslawiens wurde auch die zuvor offiziell unumstrittene Idee einer gemeinsamen Sprache, welche die Menschen in Bosnien-Herzegowina, Kroatien, Montenegro und Serbien unabhängig von ihren ethnonationalen Zugehörigkeiten miteinander teilen, hinterfragt und bekämpft. In den nunmehr unabhängigen Staaten gewannen Positionen an Bedeutung, die sprachliche Eigenständigkeiten behaupteten und die Betonung oder Schaffung von Differenzen förderten. Diese Dynamiken zeigen sich etwa anhand von Sanktionierungen zuvor selbstverständlicher Variationen des Serbokroatischen durch nationalstaatliche Sprachpolitiken und erweisen sich insbesondere für Bosnien-Herzegowina als problematisch, da hier Bosnisch, Kroatisch und Serbisch offiziell als Staatssprachen definiert worden sind. In der Konsequenz bewegen sich viele Bürgerinnen und staatliche Institutionen in einem Spannungsfeld zwischen dem Festhalten an der Vorstellung grenz- und gruppenübergreifender sprachlicher Zusammengehörigkeit und der Orientierung an neuen lingualen Standards und Differenzierungen, die primär durch die Nachbarländer Serbien und Kroatien gesetzt werden. Auch im Arbeitsalltag des Goethe-Instituts entfalten solche Dynamiken ihre Wirksamkeit: Eine bosnische Mitarbeiterin berichtet mir irritiert, dass eine Gemeindeverwaltung aus dem kroatisch dominierten Landesteil ein in der »lokalen Sprache« verfasstes Schreiben unbearbeitet zurückgesandt und mit der Aufforderung versehen habe, es noch einmal »auf Kroatisch« zu schicken. Verwundert reagiert man auch auf die Reaktion der Kolleginnen am Goethe-Institut in Zagreb, denen man vorgeschlagen hatte, im Rahmen eines Kooperationsprojektes nur eine Übersetzung aus dem Deutschen anzufertigen: »Wenn man die Texte in Kroatien als Provokation verstehen solle, dann könne man die Texte aus Bosnien nehmen.«128 Die Mitarbeiter des Goethe-Institutes in Sarajevo werden in solchen Situationen damit konfrontiert, dass ihr pragmatisches Festhalten an der Idee einer gemeinsamen und geteilten Sprache im postjugoslawischen Raum andernorts auf Ablehnung stoßen kann - ein Pragmatismus, der allerdings von vielen ihrer lokalen Projektpartner geteilt wird: »Rein linguistisch betrachtet, handelt es sich um eine Sprache«, erklärt mir ein Professor für Philosophie, der regelmäßig deutsche Texte übersetzt. Je nach Standort des Verlages, der seine Übersetzungen veröffentlicht, nenne er die Zielsprache »Kroatisch, Serbisch oder Bosnisch - einen substanziellen Unterschied gebe es aus seiner Perspektive

128 | Feldnotizen vom 23. Juni 2009. Viele Personen im Umfeld des Goethe-Instituts sprechen häufig von der "lokalen Sprache“, um den Zwang einer ethnonationalen Kategorisierung zu umgehen. 
dabei nicht. ${ }^{129}$ Ähnlich positioniert sich ein Mitarbeiter des bosnischen Fernsehens, der das lokale Publikum und internationale Kollegen auf der bereits diskutierten Mini-Input Konferenz mit den Worten willkommen heißt: »Ich begrüße sie gerade in vier Sprachen gleichzeitig. $\ll^{130}$

So lassen sich die Hintergründe des Projektes Sprach(en)politik kurz andeuten. Durch diese Tagung greifen die Kulturmittler also einen langfristigen Effekt des gewaltvollen Auseinanderbrechens Jugoslawiens auf und stellen ihn ins Zentrum eines Programmformats: »Sprache« ist sowohl auf einer grundlegenden als auch auf einer ganz alläglichen Ebene zu einem Problem geworden; die Nutzung bestimmter Worte oder die schlichte Frage nach der Benennung der lokalen Sprache können sich als ein Politikum erweisen. Die Institutsleiterin erklärt mir die Bedeutung des Projektes gerade aus den weitreichenden Bezügen des Themas:

"[...] weil um diese Sprachenfrage kreist so viel und die ist ja auch nur ein Symbol für diese- Eigentlich die Grundfrage an diesem Institut, in diesem Land ist die Identität und es gibt keine identitätsverbindende Klammer, die die Menschen in diesem Land in einer ihrer verschiedenen Rollen sagen lässt: 'Wir gehören einer gemeinsamen Identität an.` Und was mich sehr umtreibt ist, dass hier, was Identitäten angeht, einem Reinheitsgedanken gehuldigt wird, den ich schlichtweg beklemmend finde." ${ }^{131}$

Das sprachliche Auseinanderdriften wird hier also als Ausdruck einer tieferen, letztlich identitären Dynamik verstanden: der Omnipräsenz und Wirkmächtigkeit eines ethnonationalen Teilungsprinzips, das auch nach Ende des Krieges politische Öffentlichkeiten und Alltagskonstellationen zu strukturieren vermag. Vor diesem Hintergrund interessiert mich die Frage, welcher Ausschnitt und welche Herangehensweisen entwickelt werden, um dieses Thema kulturpolitisch bearbeitbar zu machen. Konfiguriert sich Sprach(en)politik eher als ein lokal situiertes und somit eingrenzbares Problem oder entwickelt sich über diese Themensetzung ein Raum, in dem großflächigere Dynamiken, translokale Verflechtungen oder Parallelentwicklungen zur Sprache kommen können? Zur Diskussion dieser Frage lohnt der Blick auf das Veranstaltungsformat, das im Zuge der Vorbereitungen entwickelt wird und in vier Konferenzblöcken die »Kontrastierung von Perspektiven aus den »deutschsprachigen Ländern und Bosnien-Herzegowina« vorsieht: Zunächst sollen in zwei sprachwissenschaftlichen Panels zu den »Fakten« im deutschen Sprachraum und vor Ort »Grundlagen« erarbeitet werden, um auf dieser Basis in zwei wei-

129 | Feldnotizen vom 16. November 2009.

130 | Feldnotizen vom 22. November 2009. Er meint hiermit: Bosnisch, Kroatisch, Serbisch und Montenegrinisch.

131 | Interview vom 23. Juli 2009. 
teren Blöcken den jeweiligen »sprachpolitischen Umgang « mit dieser Lage zu diskutieren. Der Teilnehmerkreis soll sich aus Wissenschaftlern eben aus den deutschsprachigen Ländern und aus der Region zusammensetzen. Zwei Beobachtungen sind hierbei von Interesse: Zum einen zeigt sich das etablierte Prinzip der - in diesem Falle sprachräumlich erweiterten - Deutschlandbezüge auch hier als normalisierter Modus, um lokale Konstellationen weiträumiger zu verknüpfen. Zum anderen stechen gerade in dieser Kombination Asymmetrien hervor, die sich anhand meiner Feldnotizen zu einem Planungstreffen verdeutlichen lassen:

Während des Gesprächs sagt die Institutsleiterin Sprachpolitik sei in Deutschland, Österreich und der Schweiz unstrittig. Es gebe hier keine Kontroverse; aufgrund der "Fakten" habe man sich zu einem pragmatischen Umgang mit "Sprache" entschieden. Hier sei es im Prinzip so gelaufen, dass man sich gemeinsam auf eine Standardsprache geeinigt habe und den "Rest" als akzeptierte Varianzen betrachte. Der lokale Mitarbeiter lässt einfließen, dass es im jugoslawischen Raum 150 Jahre lang eigentlich genauso gewesen sei - bis zum Krieg eben. ${ }^{132}$

Bemerkenswert erscheint hier zunächst die Charakterisierung des deutschsprachigen Raums als pragmatisch, faktenorientiert und konsensorientiert, die bestimmte Entwicklungen in Bosnien-Herzegowina besonders deutlich als erklärungsbedürftig hervorhebt - anders formuliert: dem Projekt wird eine eindimensionale, asymmetrische Blickrichtung von einem vernunftgeprägten Ruhepol auf den problematischen Sonderfall eingeschrieben. Durch eine solche Perspektivierung gerät aus dem Blick, dass auch die gegenwärtig »unstrittig « erscheinenden kulturellen Konstellationen in Mitteleuropa erst im Zuge von machtvollen, häufig gewaltdurchzogenen Homogenisierungspolitiken erschaffen worden sind. Auch der Hinweis des lokalen Mitarbeiters, dass in beiden Regionen in der Entwicklung sprachlicher Standards durchaus ähnliche Prinzipien wirksam waren, findet in der Projektplanung keine weitere Beachtung. Die Perspektive einer asymmetrischen Problematisierung und damit verbundene Ausblendungen zeigen sich auch an einem zweiten Aspekt:

Die Institutsleiterin bemerkt, dass es in Deutschland eigentlich keine Sprachpolitik gäbe. An dieser Stelle merke ich an, dass man von "Migranten " kontinuierlich verlange, dass sie Deutsch lernen; ihre häufige Mehrsprachigkeit wird eher als Problem markiert. Und wenn sie die deutsche Staatsbürgerschaft annehmen wollen, so müssen sie als Bedingung entsprechende Sprachkenntnisse nachweisen. Handelt es sich hierbei nicht um eine Sprachpolitik, frage ich. Es wird deutlich, dass der Institutsleiterin meine An-

132 | Feldnotizen vom 8. Juni 2009; auch die folgende Sequenz. 
merkung missfällt. Sie sagt, da gehe es um "Migranten ", das sei etwas anderes und das sollten wir hier draußen lassen.

Auch wenn man anerkennt, dass es zu den kontinuierlichen Herausforderungen einer Kulturmittlerin gehört, alleine schon aus Praktikabilitätsgründen einen jeweiligen Projektzuschnitt zu begrenzen und in diesem Rahmen mögliche Erweiterungen auszuschließen, erscheint diese Position fragwürdig. Denn gerade am Beispiel des Umgangs mit Migration lässt sich aufzeigen, wie vehement auch in den deutschsprachigen Ländern Politiken initiiert werden, um das historisch etablierte Selbstbild von der »gemeinsamen und geteilten Sprache« in der Gegenwart intakt zu halten. Gleichfalls würde das Bild von einem besonders pragmatischen Umgang mit Sprache auf einer deutschen Ausländerbehörde schnell Schaden nehmen: Trotz der alltäglichen Multilingualität dominieren hier die Vorstellungen einer sprachlichen Norm und Homogenität die Begegnungen und bürokratischen Akte. Gerade in Anbetracht der Dauerhaftigkeit von Migrationsbewegungen wirkt die Formulierung, wir sollten Migranten hier draußen lassen, somit nicht nur merkwürdig antiquiert, sondern sie bedeutet zugleich, eine zentrale Dynamik, die auch in Deutschland gegenwärtig kontinuierlich sprach- und bildungspolitische Interventionen hervorruft, auszublenden. Asymmetrien zeigen sich hierbei in dem Vermögen, einen Blickwinkel auszuwählen, der in der Bearbeitung eines Themenfeldes den lokalen Kontext einseitig problematisiert, anstatt Verflechtungen und Parallelentwicklungen herauszuarbeiten; sie zeigen sich aber auch in den Infrastrukturen und Ressourcen, die einer deutschen Kulturmittlerin zur Verfügung stehen, um eine jeweilige Problematisierung zum Ausgangspunkt eines Programmformats zu machen - eine Rahmenbedingung, auf die palästinensische oder bosnische Kulturschaffende nicht in gleicher Weise zugreifen können. Solche asymmetrischen Problematisierungen - so lässt sich auf Basis meiner Feldforschungen formulieren - prägen bisher viele der Projekte, die sich explizit den Konfliktkonstellationen zuwenden. Vor diesem Hintergrund stellt sich die Frage, ob nicht die gezielte Erarbeitung von symmetrischen Herangehensweisen und thematischen Zuschnitten eine explizite Aufmerksamkeit erfahren müsste, um die Intentionen einer Auswärtigen Kulturpolitik als Konfliktprävention glaubhaft in lokale Handlungskontexte zu übersetzen.

\section{Evozierte Effekte: Infrastrukturen entwickeln, Menschen mobilisieren, Diskurse öffnen}

Trotz solcher Kritikpunkte gilt es festzuhalten, dass die Bezugnahmen auf Rationalitäten und Zielvorstellungen der neuen Policy fraglos zu einer tieferen und kontinuierlichen Auseinandersetzung mit den fragilen politischen Realitäten an den Einsatzorten, zu einer Verbreiterung des Themenspektrums in 
der Programmarbeit sowie zu einer Pluralisierung von Projekt- und Kooperationsformaten geführt haben. Gleichzeitig generieren die hieraus hervorgehenden Praxen des Verknüpfens vielfältige neue Wissensbestände - etwa zu der Komplexität eines Konfliktes, zu der Brüchigkeit und lokalen Situiertheit von scheinbar universellen Begriffen wie Dialog oder Verständigung, zu den vielfältigen Klippen, die es selbst bei der translokalen Arbeit mit einem Kinderbuch zu umschiffen gilt, zu den Erwartungen lokaler Projektpartner an kulturpolitische Kooperationen oder auch zu den fehlenden Kriterien, um den »Erfolg « einer jeweiligen Maßnahme zur Konfliktbearbeitung messen zu können. Gerade diese erfahrungsbasierten Zweifel an eindeutigen Kausalbeziehungen zwischen Projekten und Konfliktverläufen lenken die Aufmerksamkeit auf die Frage, welche Vorstellungen von wünschenswerten Effekten stattdessen hervorgerufen werden: Welche Wirkungen erhoffen sich die Kulturmittler von ihrer Verknüpfungsarbeit und inwiefern korrespondieren ihre Intentionen mit den Erwartungen ihrer Kooperationspartner? Im Rahmen meiner Feldforschung konnte ich vielfach ein Wechselspiel zwischen effektvollen Interventionen und demonstrativen Distanzierungen beobachten, das ich nun in seinen Ambivalenzen diskutieren möchte.

Als ich die Institutsleiterin in Sarajevo nach ihren Zielvorstellungen für das Projekt Sprach(en)politik frage, erwähnt sie ein Treffen mit Vertreterinnen der schweizerischen und österreichischen Botschaften, die man in diesem Fall als Kooperationspartner gewinnen konnte. Hierbei habe sich gezeigt, dass die Botschaftsmitarbeiterinnen einen »politischen Zugang « zu dem Thema wählten: Klare Zielsetzungen und definierte Resultate stünden für sie im Zentrum. Sie selbst suche hingegen einen »kulturellen Zugang«, der ein »offenes Forum « ins Leben rufen solle und auf die Initiierung »ergebnisoffener Erkenntnisgewinnungsprozesse« ausgerichtet sei:

"Also ich gehe nicht hin - und das ist ein Unterschied zu den beteiligten Botschaften - , dass diese Sprachenpolitikkonferenz ein Ergebnis haben muss, das ich jetzt schon im Kopf habe. Ich glaube, das wäre ein völliges Missverständnis. Aber dass man überhaupt dieses Forum bietet und sich überlegt, wen lädt man denn jetzt an einen Tisch, die sonst nicht miteinander sprechen oder man lädt Leute ein, die sonst nicht zusammenkommen und das ist wie eine Versuchsanordnung. In der Hoffnung, dass man damit etwas positiv verändert in jedem Einzelnen und da neue Horizonte eröffnet.. ${ }^{133}$

Diese Passage erscheint mir signifikant, da sie in zwei Positionen mit vielen Äußerungen, die ich während meiner Feldforschungen sammeln konnte, korrespondiert: zum einen in der Orientierung an der Idealvorstellung einer »Kultur, die nicht der Politik unterworfen ist«, wie es der Institutsleiter in Ramallah

133 | Interview vom 23. Juli 2009. 
in einem unserer Gespräche formuliert hat:, ${ }^{134}$ zum anderen in der Selbstbeschränkung auf die Rolle einer »Impulsgeberin «, die durchaus Wirkungsketten hervorzurufen versucht, ohne aber deren Verläufe vorherzubestimmen.

Aus der ersten Position spricht die Erwartung, dass gerade die Suche nach einem »neutralen Standpunkt«, der Unabhängigkeit gegenüber allen politischen Akteuren bewahrt, neue Perspektiven, Gesprächskonstellationen, diskursive oder zivilgesellschaftliche Anschlüsse möglich macht - eine Erwartung, die auch der Abteilungsleiter aus dem Auswärtigen Amt in seinen mehrfach zitierten Formulierungen zu den besonderen Potenzialen von »Kultur« mobilisiert. Genau diese Äußerungen eines hochrangingen Politikgestalters fördern dabei zugleich die Ambivalenzen eines »kulturellen Zugangs « zutage. Kulturmittler, die sich in ihrer Arbeit verstärkt den politischen Brechungen und Präsenzen gewaltdurchzogener Konflikte zuwenden, sind auf zumindest zweierlei Weise in politische Prozesse verstrickt: einerseits durch ihre Entsendung und Ausstattung im Rahmen einer nationalstaatlichen Kulturpolitik; andererseits durch die Intention, im Rahmen ihrer Programmarbeit an den Einsatzorten explizit auch politisch umkämpfte Felder zu betreten. So nachvollziehbar der Anspruch auf »Politikferne« für die Gestaltung der Programmarbeit auch ist, er bringt das Risiko mit sich, die politischen Verflechtungen und Wirkungen sowohl der eigenen Praxis als auch der gewählten Themenfelder auszublenden.

Die zweite Position steht mit dieser Intention in engem Zusammenhang: Sie beruht auf der sicherlich realistischen Einschätzung meiner Gesprächspartnerin, dass sie als Kulturmittlerin, »anders als etwa die OSZE, über kein politisches Mandat verfügt«, das es ihr erlaube, ein Projekt »politisch zu flankieren $\ll^{135}$ - etwa indem man grundlegende Veränderungen in der institutionellen Verfasstheit Bosnien-Herzegowinas, die Durchführung einer Schulreform oder die dauerhafte Finanzierung einer lokalen Kultureinrichtung aus dem Staatshaushalt einfordere: »Wenn sich die politischen Köpfe treffen, dann haben wir keine Stimme«, fügt sie hinzu. Dennoch können solche Selbstbeschränkungen auf die Rolle einer »neutralen Mediatorin « mitunter als Versuche erscheinen, sich aus den möglichen Effekten der eigenen Interventionen wieder herauszuziehen und somit auch einer längerfristigen Verantwortung für die erschaffenen Konstellationen aus dem Weg zu gehen. Denn gerade Formate, die Präsenzen der Konflikte aufgreifen, zielen häufig auf die Initiierung von Wirkungsketten ab, die deutlich über einen Projektzeitraum hinausgehen. So formulieren die Kulturmittler in Sarajevo etwa die weitreichende Hoffnung, durch ihre Konferenz die Entwicklung einer neuen »Standardsprache« zu unterstützen - »einer Standardsprache, die von allen „Volksgruppen<

134 | Feldnotizen vom 29. September 2008.

135 | Feldnotizen vom 9. Juni 2009; auch das folgende Zitat. 
akzeptiert wird und in der Folge auch als Basis für eine Verständigung der verschiedenen Nachfolgestaaten Jugoslawiens zur Verfügung stehen könnte ${ }^{136}$ Das Team ist sich über die »politische Sprengkraft« des Themas im Klaren und nimmt sie in diesem Fall als notwendig oder sogar produktiv in Kauf. In Ramallah erläutert mir der Institutsleiter, dass er selbst bei der Organisation eines einzelnen Workshops zur Vermittlung eines sehr spezifischen Wissens großflächigere Entwicklungen im Blick hat:

"Kulturarbeit braucht auch technisches Wissen. [...] In der Filmproduktion muss man wissen, wie man Ton macht, wie man Licht macht. Das bringen wir hierher. Aber uns interessiert natürlich das Produkt, das am Ende hergestellt wird. Wir würden es nicht dabei belassen, jetzt nur Tontechnik zu üben. Sondern wir sind daran interessiert, dass daraus ein spannendes, kreatives palästinensisches Kino entsteht, das Filme macht, die diskutiert werden und Diskussionsprozesse, Diskurse in Gang setzt, die sonst nicht in Gang gesetzt würden. «137

Auswärtige Kulturpolitik übersetzt sich in solchen Sequenzen gerade auch in Bilder von effektvollen Interventionen und wünschenswerten Wirkungsketten. Die Hoffnung zu längerfristigen Veränderungen, wachsenden Expertisen oder der Entstehung konkreter materieller Produkte beizutragen, findet sich - trotz der häufig parallelen Betonung der »Ergebnisoffenheit« - immer wieder in den Erklärungen zu einem Projekten. Von Relevanz erscheint mir an dieser Stelle, dass die lokalen Kooperationspartner genau solche spürbaren Effekte einfordern - anders formuliert: im Zuge der neuen Policy erweist sich das Evozieren potenzieller Wirkungsketten als ein wesentlicher Modus translokaler Verknüpfungsarbeit, in dem auch widerstrebende Diskurse, Positionen und Erwartungen situativ zusammenfinden können. Die so mobilisierten Vorstellungen von wünschenswerten Effekten kulturpolitischer Kooperationen lassen sich drei Aspirationen zuordnen, die in einem jeweiligen Praxis- und Übersetzungsfeld unterschiedlich gewichtet und kombiniert werden können.

Eine erste Aspiration dreht sich um die Frage nach möglichen Beiträgen zur Entwicklung kultureller Infrastrukturen in den konfliktdurchzogenen Einsatzgebieten. Diese zeigt sich etwa anhand der Diskussionen um das Cinema Jenin, dessen Förderanfrage den zentralen Anlass für die Reise des Abteilungsleiters gebildet hat. Der Theaterdirektor bringt bei diesem Treffen aber auch das Projekt zur Gründung einer Schauspielschule in Ramallah zur Sprache, das er zu diesem Zeitpunkt gemeinsam mit deutschen Partnern voranzubringen versucht und als besonders dringlich erachtet. Seine Forderung nach einer massiven Unterstützung kultureller Infrastrukturen erklärt er mir auch

136 | Feldnotizen vom 8. Juni 2009.

137 | Interview vom 4. Dezember 2008. 
in einem späteren Interview mit deren grundlegenden Bedeutung für gesellschaftliche Entwicklungen:

"We have to rebuild the Palestinian culture through students and through infrastructures, through building cultural infrastructures [...] which means cinemas, theatres, schools. We have to go to the schools and to teach - teaching is very important and not to leave the schools in the hands of the radicals, because the radicals will bring the children up to be radicals one day and we don't like that, we have to oppose this and we don't have the tools to oppose this, because nobody is standing with us. "138

Kulturelle Infrastrukturen stellen aus dieser Perspektive also keinen Selbstzweck und auch kein von den Realitäten abgehobenes Statussymbol dar, sondern sie bilden die unverzichtbare Basis für gehaltvolle Lernprozesse und die Stärkung von Potenzialen zum Widerstand gegen lokal wirksame Radikalisierungstendenzen. Deutsche Kulturmittler erkennen zumeist das Problem der fehlenden infrastrukturellen Grundlagen für symmetrische Kooperationsprojekte und somit im Prinzip auch den Bedarf einer substanziellen Förderung durch ausländische Geldgeber an. In Sarajevo bemüht sich das Goethe-Institut etwa um die Unterstützung des nationalen Filmarchivs Kinoteka, dessen Finanzierung durch die eigentlich zuständigen, aber besonders schwachen gesamtstaatlichen Strukturen zumeist ausbleibt. Es fehlt an technischem Gerät, um die archivierten Filme vor dem Verfall zu bewahren und selbst an Mitteln, um den Alltagsbetrieb aufrechtzuerhalten. Ein Förderantrag des Goethe-Instituts an das Auswärtige Amt zielt darauf ab, das Filmarchiv in einen arbeitsfähigen Zustand zu versetzen und die bedrohten Bestände zu retten. Er wird bezeichnenderweise mit dem Argument begründet, dass Bosnien-Herzegowina solche Institutionen brauche, um zur »Teilnahme an einem Dialog auf Augenhöhe überhaupt in der Lage zu sein «. ${ }^{139}$ Denn diese kulturpolitische Idealvorstellung erweist sich im Projektalltag als unerreichbare Illusion, wenn die Mitarbeiterinnen einer potenziellen Partnerinstitution nicht einmal ihre Löhne erhalten. Gleichzeitig zögern die Kulturmittler, ihre Programmgelder durch langfristige Kooperationen mit einer bestimmten Institution zu binden. Die Institutsleiterin in Sarajevo erklärt mir diese Haltung über den Wunsch, möglichst breite und kontinuierlich innovative Programme zu entwickeln, der sich nur durch zeitlich begrenzte, immer wieder neue Partnerschaften realisieren lasse. Stärker als ihr Kollege in Ramallah fordert sie außerdem, dass lokale Gemeinschaften und staatliche Stellen Verantwortung für den Aufbau und dauerhaften Unterhalt kultureller Institutionen übernehmen müssten

138 | Interview vom 2. Dezember 2008.

139 | Feldnotizen vom 10. Juli 2009. 
und positioniert sich hierdurch als eine »externe Akteurin« mit begrenzten Zuständigkeiten. ${ }^{140}$

Im Hinblick auf die Unterstützung kultureller Infrastrukturen tut sich also ein Spannung auf: Ihr Fehlen oder ihr ungesicherter Status behindern nicht nur die Entwicklung lokaler kultureller Szenen, sondern auch die Möglichkeit gleichberechtigter Kooperationsprojekte; langfristige institutionelle Förderungen würden mit den naturalisierten Vorstellungen von den Aufgaben einer deutschen Auswärtigen Kulturpolitik brechen und letztlich andere Muster der Ressourcenverteilung erfordern. Mögliche Umgangsweisen mit diesem Zielkonflikt bestehen in der Etablierung von - in der Regel zeitlich begrenzten Sonderförderprogrammen und der Gewährung einer »Anschubfinanzierung« für bauliche Maßnahmen oder grundlegende technische Ausstattungen, die eine Institution in einen arbeitsfähigen Zustand versetzen soll. Die Hoffnung, dass lokale Instanzen im Anschluss den Unterhalt übernehmen, bricht sich häufig an den Realitäten fragmentierter Staatlichkeit. So ließ sich etwa das Cinema Jenin trotz einer mehrjährigen substanziellen Sonderförderung durch das Auswärtige Amt und trotz des stetigen Engagements eines internationalen zivilgesellschaftlichen Unterstützerkreises nicht als zweites permanentes Kino im Westjordanland etablieren. In einem Interneteintrag Cinema Jenin is closing its doors forever berichtet die Initiative vom harschen Ende ihrer jahrelangen Bemühungen: Ein Investor habe das Gebäude gekauft und werde dort nach dem Abriss der Spielstätte ein Einkaufszentrum errichten; Gespräche mit der palästinensischen Autonomiebehörde und dem Auswärtigen Amt zu einem möglichen Erwerb des Kinos durch lokale Instanzen seien ins Leere gelaufen. ${ }^{141}$ Und auch im Hinblick auf die Kinoteka in Sarajevo fallen die Ergebnisse nach einer mehrjährigen Förderung ernüchternd aus. Die zentralen Projektziele - die Arbeitsfähigkeit der Institution herzustellen und die archivischen Bestände zu sichern - seien nicht erreicht worden, erklärt mir die Institutsleiterin als ich sie drei Jahre nach meiner Feldforschung noch einmal zu diesem Engagement befrage. Das teure technische Gerät zur Rettung der Filme habe sich aufgrund der räumlichen Bedingungen als nicht einsetzbar erwiesen; an der fehlenden finanziellen Ausstattung durch staatliche Instanzen habe sich nichts geändert. Meine Gesprächspartnerin zieht dennoch keine negative Bilanz: »Was wir tun konnten, haben wir getan. Wir haben Grundlagen geschaffen, auf denen nun weitergearbeitet werden kann.«Als ausländisches Kulturinstitut könne man nur »Angebote machen « und müsse akzeptieren, »dass das Land nicht weiter daran arbeiten will «. ${ }^{142}$ Diese demonstrative Distanzierung von einem eigenen Projektes verweist aus meiner Perspektive auf die Rahmenbedingungen

140 | Feldnotizen vom 10. April 2012.

141 | www.cinemajenin.org (letzter Zugriff am 4. November 2017).

142 | Feldnotizen vom 6. April 2012; auch die folgende Gesprächsnotiz. 
solcher Kooperationen: Die deutsche Auswärtige Kulturpolitik ist mit ihren gegenwärtig etablierten institutionellen Ordnungen, Förderlogiken und Vorstellungen von begrenzten Zuständigkeiten nur sehr bedingt in der Lage, kulturelle Infrastrukturen in Zonen fragmentierter Staatlichkeit nachhaltig zu unterstützen.

Im weiteren Gesprächsverlauf lenkt die Institutsleiterin die Aufmerksamkeit von den Problemen der Entwicklung von Infrastrukturen auf die zweite kulturpolitische Aspiration, die auf mobilisierende Effekte abzielt:

Sie erzählt von einem jungen Mitarbeiter der Kinoteka, dem sie ein Praktikum im Bundesarchiv in Berlin ermöglicht haben - man wisse nicht, was er in der Zukunft daraus mache. In der Bewertung eines solchen Projektes müsse man "von den Menschen ausgehen" und "die Bewertungen anders setzen“. Die Zahl der gereinigten Filme in der Kinoteka habe sich durch die Kooperation nicht verändert; Kulturarbeit lasse sich aber nicht in Zahlen darstellen. Sie wolle nicht primär die institutionellen Folgen fokussieren, sondern die individuellen Entwicklungsschritte eines Mitarbeiters und die möglichen langfristigen, aktuell nicht kontrollierbaren Effekte: „Wenn man bei Kulturarbeit keine Zeit hat, dann soll man es besser gleich lassen."

Mögliche, bisher nicht vollzogene Entwicklungsschritte eines Individuums werden hier vorgebracht, um einen Projektverlauf trotz aller Abweichungen und Enttäuschungen dennoch als lohnenswert und ertragreich darzustellen. Meine Gesprächspartnerin entwickelt hierzu Vorstellungen von aktuell weder plan- noch vorhersehbaren Wirkungen, die sich aus den neuen Erfahrungen, Expertisen und Begegnungen des Archivmitarbeiters ergeben können. Produktive Effekte werden hier also aus einer parallelen Mobilisierung von Menschen und Wissensbeständen erwartet. Die bereits zitierten Hoffnungen der Institutsleiterin, im Rahmen der Tagung zur Sprach(en)politik ein »Forum« anzubieten, in dem sich »in jedem Einzelnen etwas positiv verändert« zeugen ebenso von dieser Aspiration. In die gleiche Richtung weist auch der Wunsch ihres Kollegen in Ramallah, durch die Organisation von Ton- oder Kameraworkshops zur Entwicklung eines spannenden palästinensischen Kinos beizutragen und letztlich neue Diskussionsprozesse in Gang zu setzen. An diesen Beispielen zeigt sich zugleich eine enge Beziehung zwischen der zweiten und dritten kulturpolitischen Aspiration: Die Hoffnung auf weitergehende diskursive und zivilgesellschaftliche Effekte, die sich an einem letzten Projektbeispiel verdeutlichen lässt. ${ }^{143}$

Die Festivaldirektorin aus Ramallah, die bereits im Gespräch mit dem Berliner Gast die Ausrichtung ihrer Arbeit auf die Entwicklung einer diversen

143 | Die gesamte folgende Passage auf Basis unseres Interviews vom 3. Dezember 2008. 
Zivilgesellschaft herausgestellt hat, berichtet mir von ihrer aktuellen Kooperation mit dem Goethe-Institut. Gemeinsam hat man fünf junge Palästinenserinnen und einen jungen Palästinenser durch den Prozess der Realisierung und öffentlichen Präsentation ihrer ersten Kurzfilme begleitet. In diesem Rahmen ging es einerseits um Maßnahmen zur Befähigung dieser konkreten Personen: Technisches Gerät wurde zur Verfügung gestellt, wesentliche Expertisen durch eine ganze Reihe von Workshops und individuelles Coaching vermittelt. Andererseits zielte das Vorhaben auf zivilgesellschaftliche Interventionen, die bereits mit der Themensetzung ihren Anfang nahmen: Die Prozesse, in denen Mädchen innerhalb einer patriarchalen Gesellschaft zu Mädchen und allmählich zu Erwachsenen werden sowie der Blick auf das andere Geschlecht sollten aus Sicht der Initiatoren im Zentrum der filmischen Arbeiten stehen. Das Projekt mündete in sieben Kurzfilmen, die von den alltäglichen Zwängen und Konflikten junger Palästinenserinnen erzählen, gleichzeitig aber von den vorsichtigen Versuchen der Protagonistinnen, enge gesellschaftliche Vorgaben und Rollenerwartungen vorsichtig zu verschieben. ${ }^{144}$ Die Filme wurden im Herbst 2008 unter dem Titel Confession als Eröffnungsprogramm des vierten Women's Film Festival in Palestine an zehn Universitäten in den gesamten besetzten Gebieten gezeigt. Meine Gesprächspartnerin berichtet von den »ekstatischen Reaktionen « des studentischen Publikums, die sie sich gerade mit den diskursiven Potenzialen der Filme erklärt: »Apparently they generated discussions about issues that are hidden in Palestinian society. Issues that youth are encountering, that we as adults don't want to talk about.« ${ }^{145}$

Diese Potenziale ergeben sich aus ihrer Perspektive aus einer dosierten Kritik an kulturellen oder gesellschaftlichen Rahmenbedingungen, die durch die Filme transportiert wird: »The response is beyond the imagination, because the films are daring but they are within the cultural parameters. So they have not overstepped a redline, whereby they could be dismissed.«

Eine solche Ablehnung ließ sich auch deshalb vermeiden, weil die Geschichten hier von den eigenen Schwestern, Töchtern oder Cousinen erzählt wurden, die man darüber hinaus bewusst aus Gemeinden in der Peripherie des Westjordanlandes rekrutiert habe. Zwei Aspekte erscheinen mir an diesem Beispiel von Relevanz: Zum einen zeigt sich besonders deutlich die Erwartung, dass die intensive Arbeit mit einer überschaubaren Gruppe angehender Filmemacherinnen zu mehr führen soll als zu individuellen Kompetenzfortschritten; zum anderen werden Bemühungen um eine lokale Situierung diskursiver Interventionen sichtbar, die auf kontinuierlichen Beobachtungen und Analysen gesellschaftlicher Entwicklungen in den besetzten Gebieten beru-

144 | Siehe hierzu die DVD "Confession «; herausgegeben durch: Goethe-Institut Ramallah \& Shashat im Jahr 2008; Produzenten: Fareed Majari und Alia Arasoughly.

145 | Interview vom 3. Dezember 2008; auch die folgenden Zitate. 
hen. Beides fließt zusammen in der grundlegenden Programmatik des Festivals und seiner Trägerorganisation, die mir die Leiterin als »promoting social change through culture « mit dem besonderen Fokus auf »social change in terms of perceptions of women and women's lives « beschreibt. Den Gegenpol ihrer Arbeit bildet demnach die zunehmende Ausbreitung einer »siege mentality«, die zu einem Rückzug in immer kleinteiligere Gemeinschaften, einem dramatischen Zerfall des sozialen Zusammenhalts und der Ausbreitung traditionaler Geschlechterrollen führe - mit weitreichenden Folgen, auch über innerpalästinensische Dynamiken hinaus: »[...] because what's happening with the checkpoints and the wall is that Palestinian communities are becoming more and more segregated and if one village cannot communicate with the next village: how can we expect it to be a part of a global dialogue?»

In solchen Betonungen von Dialog, der Wertschätzung von grenzüberschreitenden Verbindungen und in der Hoffnung auf weitergehende zivilgesellschaftliche Effekte eines kleinteiligen Projekts treten Verknüpfungspotenziale mit einer Auswärtigen Kulturpolitik als Konfliktprävention deutlich zutage. Ihre Wortwahl und Vorstellungen von möglichen Wirkungsketten, die sich durch Confession hervorrufen lassen, korrespondieren spürbar mit Erklärungen deutscher Kulturmittler in Ramallah, Sarajevo und Tel Aviv, die im Laufe dieser Studie diskutiert worden sind:

"And I think that when you begin opening up spaces for difference internally - that is not threatening and that is not dangerous and that leads to open discussion of difference and disagreement that you can disagree verbally and nothing will happen; you don't have to go kill the other person because you disagree, ideologically or any way - then I think it promotes an atmosphere of culture interaction, which is really the final safety place for all of us, who are quite different than each other: 'This is, difference, so what? But I think it's very important to begin at that grassroots level that to be different is OK. To discuss ideas that you don't agree with, but that really challenge you is also OK. And I think this is what is happening with the screenings of 'Confession.."

Die Konstellationen einer Verknüpfungsarbeit, in denen Kulturmittler und ihre Projektpartner lokal greifbare Präsenzen der Konflikte und mobile Metaphern aufeinander beziehen, neue Themenfelder und Projektformate entwickeln sowie Vorstellungen von wünschenswerten Effekten evozieren, erweisen sich vor diesem Hintergrund als besonders produktive Übersetzungsfelder der neuen Policy. Entsprechend zieht meine Gesprächspartnerin trotz ihrer Klagen über die fehlendende Langfristigkeit und strategische Herangehensweise in der Arbeit des Goethe-Instituts ein verhalten positives Fazit aus ihrer Kooperation: 
"And I think that this kind of approach of Goethe in terms of building infrastructures here and promoting an intercultural Palestinian dialogue is very, very important, because it's the only way to open up to wider issues, for example that if you cannot discuss something that is happening in Palestine that challenges you a little bit, how can you talk about global intercultural dialogue or intercultural dialogue between Europe and us - so to speak - or between fundamentalist ideas and European ideas." 



\section{Schluss}

Die diskursive Formation Auswärtige Kulturpolitik als Konfliktprävention hat auf vielfältige Weise in die Arbeitspraxis von Goethe-Instituten Eingang gefunden - so lautet ein grundlegendes Fazit dieser Studie: Die Kulturmittler setzen sich kontinuierlich mit den lokalen Präsenzen und Auswirkungen der Konfliktkonstellationen auseinander; sie wenden sich infrastrukturellen Defiziten und Hindernissen für die Entfaltung lokaler kultureller Szenen zu; sie entwickeln neue Formate und Herangehensweisen, um auch kontroverse Themen in ihrer Programmarbeit aufzugreifen (Kapitel III.5). Gleichzeitig bleibt eine Reserviertheit gegenüber der neuen Zielsetzung. Diese zeigt sich etwa in zurückhaltenden Äußerungen zu dem eigenen Vermögen, Konfliktverläufe spürbar zu beeinflussen oder einen messbaren Beitrag zu einer - wie auch immer gearteten - Konfliktprävention zu leisten. So beantwortet die Institutsleiterin in Sarajevo meine Frage nach ihren Möglichkeiten, in einen Konflikt hineinzuwirken, folgendermaßen:

"Ich habe ein Problem mit dem Wort 'wirken. Also, ich mache das mal für Pakistan: In Pakistan war es ganz wichtig, dass wir überhaupt da waren, gerade als die Zeiten extrem schwer waren: 'Es ist wichtig, dass ihr uns nicht im Stich lasst. ( Das war die Botschaft, gerade von den Intellektuellen dort: 'Nicht, ihr habt uns nicht aufgegeben. Ihr erkennt noch, dass es im Land nicht nur solche Leute gibt, von denen man in den Nachrichten hört, sondern es gibt hier Anknüpfungspunkter und das ist extrem wichtig, auch für das Selbstverständnis des Landes. Ob das dann schon eine Wirkung ist, weiß ich nicht, aber das war eine ganz elementare Sache. ${ }^{1}$

Hier zeigt sich ein Ideal- und Selbstbild, an dem sich deutsche Kulturmittler etwa zehn Jahre nach dem Auftauchen der neuen diskursiven Formation orientieren: langfristig präsent sein - auch wenn es schwierig wird -, Interesse am Austausch zeigen, kreative Prozesse und Begegnungen ermöglichen, Kooperationen von Künstlerinnen und Verflechtungen zwischen kulturellen Sze-

1 | Interview vom 23. Juli 2009. 
nen anbahnen, die Entwicklung von Infrastrukturen nach Möglichkeit unterstützen, Diskussionen und unterschiedlichen Perspektiven Raum geben, um »etwas gemeinsam zu machen « und »voneinander zu lernen $\ll^{2}$ - nicht mehr und nicht weniger.

Die Zurückhaltung gegenüber einer noch direkteren Indienstnahme für politisch formulierte Zielsetzungen, die in solchen Positionierungen zumeist enthalten ist, wirkt aus zumindest zwei Gründen schlüssig: Zum einen spricht hieraus eine realitätsnahe Einschätzung der eigenen Kompetenzen und eingespielten Arbeitsweisen, der Begrenzungen der finanziellen Mittel und personellen Ressourcen eines Goethe-Instituts sowie des Status und des Kernauftrages von Kulturmittlern. Zum anderen zeigen sich hier Bestände eines erfahrungsbasierten Wissens von den komplexen, historisch geschichteten und translokal verwobenen Gefügen, in denen sich Dialog, Verständigung oder Stabilisierung nicht einfach als Fließbandprodukte planen, transferieren oder umsetzen lassen. Bereits der Versuch, eine überschaubare Institution wie die Kinoteka in Sarajevo in einen arbeitsfähigen Zustand zu versetzen, führt zu Aufgabenstellungen, Problemen und Verwicklungen, die ein ausländisches Kulturinstitut an den Rand seiner Leistungsfähigkeit und der etablierten Vorstellungen von seinen Zuständigkeiten bringen können (Kapitel III.5).

Zugleich habe ich im Rahmen dieser Studie die möglichen Ausblendungen einer solchen Positionierung aufgezeigt: Denn schließlich operieren Kulturmittler immer im Rahmen einer staatlich finanzierten Policy und tragen fraglos zur Mobilisierung ihrer Schlüsselbegriffe, epistemologischen Ordnungen und Rationalitäten bei. Ebenso rufen sie unterschiedliche Machteffekte an ihren Einsatzorten hervor - etwa wenn sie einzelne Themen privilegieren, bestimmte Vorstellungen zum Geschlechterverhältnis oder zu dem Zusammenleben nach einem Krieg einbringen, über die Dauer von Kooperationen entscheiden oder Asymmetrien im Verhältnis zu ihren Mitarbeitern und Partnern reproduzieren. Eine Positionierung als »neutraler Akteur« oder die Selbstbeschränkung auf »kulturelle Zugänge « zu umstrittenen Themen erscheint vor diesem Hintergrund fragwürdig.

Ich möchte nun abschließend solche Positionierungen und mit ihnen verbundene Dynamiken der Ausblendung vor dem Hintergrund der zentralen Spannung diskutieren, die Auswärtige Kulturpolitik als Konfliktprävention aus meiner Perspektive durchzieht und die bereits Eingang in den Titel dieses Buches gefunden hat. Denn aus ihnen spricht die bleibende Verwobenheit des Politikbereiches in den Ordnungen des Nationalen als einem weiterhin zentralen Teilungsprinzip der Welt ebenso wie die wachsende Bereitschaft, sich explizit lokal wirksamen Bruchlinien oder Konfliktkonstellationen zuzuwenden und

2| So zwei der bereits zitierten praxisnahen Zielvorstellungen des Institutsleiters in Ramallah. 
in deren Bearbeitung nicht zuletzt Erfahrungen und Perspektiven miteinander $z u$ teilen. Als Ergebnis meiner Untersuchung werde ich nun vier zentrale Effekte dieser Spannung in den translokalen Praxis- und Übersetzungsfeldern Auswärtiger Kulturpolitik herausarbeiten.

\section{(i) Die Pluralisierung der Wissens- und Arbeitsmodi}

Eine erste Konsequenz liegt in der spürbaren Verbreiterung der Herangehensweisen oder Modi, über die kulturpolitische Vermittlungspraxis erfolgt. Zwar wurde sichtbar, dass inzwischen auch die Mobilisierung der etablierten Zielsetzung Herstellung von Deutschlandbezügen an den einzelnen Standorten zu einer anspruchsvollen Suche nach jeweils funktionierenden Anschlüssen und Formen der Verankerung führt (Kapitel III.4). Die Relevanz von »Deutschland « und seiner kulturellen Angebote erklärt sich gewissermaßen nicht von selbst, sondern muss gemäß historisch präkonfigurierter Referenzräume und aktueller Bedürfnislagen immer wieder neu ausgehandelt werden. Dennoch hat sich die Tendenz, sich den lokalen Realitäten gerade auch in ihrer Brüchigkeit und Komplexität zuzuwenden, durch die neue Zielvorstellung verstärkt. Die Pluralisierung der Arbeitsweisen, Wissensmodi und Projektformate, die sich im Kapitel III.5 herauskristallisierte, deute ich als Konsequenz der politisch geforderten Auseinandersetzung mit den Bruchlinien und Konfliktkonstellationen einer geteilten Welt - nicht zuletzt da sich für die Kulturmittler aus diesem Perspektivwandel eine kontinuierliche Konfrontation mit dem eigenen Nicht-Wissen ergibt. Das Ausmaß dieses Nicht-Wissens und seine Bedeutung für den Arbeitsalltag ist dabei von Standort zu Standort verschieden. Beides hängt von der lokalen Gestalt und den Verläufen eines Konflikts ebenso ab wie von den internen Kompetenzverteilungen und dem Innovationsgrad der Projektformate, die eine jeweilige Programmabteilung entwickelt. Trotz dieser Unterschiede gilt es einen übergreifenden Effekt der diskursiven Formation Auswärtige Kulturpolitik als Konfliktprävention festzuhalten: Die Auseinandersetzung mit strittigen Themen, lokal situierten Bedürfnissen und Diskussionen sowie mit Fragen nach Wirkungen und den Bedingungen von Wechselseitigkeit sind stärker in den Vordergrund gerückt - eine Entwicklung, die für Kulturmittler die Inkorporation vielfältiger Wissensformen notwendig macht. Ich betrachte diese alltägliche Arbeit an den Grenzlinien von Wissen und Nicht-Wissen als eine wesentliche Antriebskraft für die Entwicklung neuer Arbeitsmodi in der translokalen Übersetzungspraxis. Entsprechend habe ich im gesamten Kapitel III nachgezeichnet, wie etablierte Herangehensweisen eines deutschen Kulturinstituts im Ausland - repräsentieren, vermitteln oder kooperieren - durch stärker suchende Wissens- und Arbeitsmodi ergänzt worden sind: Am Beispiel der Diskussionen zu den Schließzeiten eines israelischen Checkpoints habe ich improvisieren als einen Arbeitsmodus diskutiert, in dem kleinteiliges, dennoch unverzichtbares Wissen erst im Tun erworben und mit den 
Routinen vertrauter Projektformate, institutioneller Hierarchien oder Abläufe verbunden wird (Kapitel III.3); navigieren erwies sich als eine tastende und erfahrungsbasierte Vorgehensweise, um die diskursiven und mikropolitischen Klippen in der Verknüpfung sehr unterschiedlicher lokaler und diskursiver Felder zu umschiffen; und problematisieren zeigte sich als ein Arbeitsmodus, der lokal situierte Präsenzen und Effekte der »Konflikte « im Hinblick auf mögliche kulturpolitische Interventionen befragt (Kapitel III.5). In all diesen Herangehensweisen spiegeln sich die politisch formulierten Aspirationen, sich den Teilungen der gegenwärtigen Welt zuzuwenden; gleichzeitig eröffnen sie Potenziale, Wissen von den Lebens- und Arbeitsbedingungen entlang einiger der globalen Bruchlinien bis in die Zentren des Politikbereichs zu tragen.

\section{(ii) Die Flüchtigkeit kulturpolitischer Interventionen}

Im Rahmen dieses Buches wurde ein Moment der Flüchtigkeit in den translokalen Praxis- und Übersetzungsfeldern sichtbar, das sich gleichfalls mit der zentralen Spannung Auswärtiger Kulturpolitik als Konfliktprävention in Verbindung bringen lässt. Diese Flüchtigkeit zeigt sich zunächst an der Vielzahl von Formaten, die darauf abzielen, in komplexen Debatten oder zu vielschichtigen Problemfeldern - etwa dem sprachlichen Auseinanderdriften in den Nachfolgestaaten des ehemaligen Jugoslawien - einen »Impuls« zu setzen. Insbesondere in Sarajevo stellte sich die Programmarbeit als eine Aneinanderreihung inhaltlich sehr unterschiedlich ausgerichteter Aktivitäten dar. Ein lokaler Mitarbeiter bedauerte diesen Tatbestand als ein permanentes »Antippen«, die Institutsleiterin verwies hingegen auf eine strategische Entscheidung: Gerade das »Zusammenspiel der Themen« in Kombination mit der dauerhaften Präsenz vor Ort sei der wesentliche Beitrag des Goethe-Instituts zur Entwicklung des kulturellen Lebens in Bosnien-Herzegowina. Selbst wenn man diese Einschätzung teilt, stellt sich die Frage, ob sich im Rahmen von kurzfristigen Beschäftigungen eine inhaltliche Dichte erreichen lässt, welche die Diskussionen zu komplexen Themen tatsächlich nachhaltig vorantreibt. Eine Tendenz zur Flüchtigkeit zeigt sich selbst in Projekten, die sich aus Perspektive der Kulturmittler bereits als außerordentlich aufwendig und langfristig darstellen: Die Seifenoper Matabb konnte nach zehn Episoden nicht fortgesetzt, eine professionelle Vermarktung oder Verstetigung des Projektes nicht geleistet werden; der transnationale Unterstützerkreis für das Cinema Jenin stand trotz einer außerordentlichen Förderung durch das Auswärtige Amt nach einigen Jahren vor dem Aus; und in Sarajevo wurde die Zusammenarbeit mit der Kinoteka nach drei Jahren beendet, auch wenn sich die ursprünglichen Projektziele offensichtlich nicht realisiert hatten. Die Begründungen meiner Gesprächspartner habe ich im Rahmen dieser Studie vorgestellt: die fragmentierte Staatlichkeit am Einsatzort; die fehlenden finanziellen Kapazitäten der lokalen Autoritäten oder ihre mangelnde Bereitschaft, Impulse aufzugreifen; die Er- 
schöpfung zivilgesellschaftlicher Kapazitäten als Folge langandauernder, auch eingefrorener Konflikte; die prekären Arbeitsbedingungen lokaler Partner; die Begrenztheit der finanziellen Möglichkeiten eines Goethe-Instituts. Jeder dieser Gründe ist zweifelsohne plausibel und dennoch erscheint es mir zentral, sie auch als Hinweise zu deuten, dass bisher nicht hinreichend Infrastrukturen, Ressourcen und Kooperationsmodelle zur Verfügung stehen, um den Aspirationen einer Auswärtigen Kulturpolitik als Konfliktprävention gerecht zu werden. Der Politikbereich bleibt deutlich in den Ordnungen des Nationalen mit ihren Vorstellungen von begrenzten Zuständigkeiten und Verantwortungen verhaftet.

\section{(iii) Asymmetrien in den translokalen Praxis und Übersetzungsfeldern}

Diese Verhaftung unterstützt gleichfalls die Reproduktion von Asymmetrien im Zuge der kulturpolitischen Vermittlungs- und Projektarbeit. So ließ sich herausarbeiten, dass die krassen Hierarchien zwischen »Entsandten« und »Ortskräften«, die ein Goethe-Institut intern strukturieren, gerade auch durch unterschiedliche Bewertungen ihres jeweiligen Wissens und ihrer Beziehungen $\mathrm{zu}$ »Deutschland « legitimiert werden (Kapitel III.3). Die Orts- und Sprachkenntnisse der lokalen Mitarbeiter gelten zwar als unverzichtbar für die Planung und Realisierung der Programmarbeit, werden aber dennoch den global einsetzbaren, generalisierten Expertisen der Entsandten nachgeordnet - ein Befund, der im Hinblick auf eine nachhaltige Operationalisierung Auswärtiger Kulturpolitik als Konfliktprävention bemerkenswert erscheint: Denn hier müsste es aus meiner Perspektive darum gehen, gerade lokal situiertes Wissen zu den Präsenzen und Effekten der komplexen Gefüge, von den teilweise unsichtbaren Bruchlinien und globalen Verflechtungen, von den häufig ambivalenten Potenzialen zu einer »Verständigung « sowie von den elementaren Bedürfnissen örtlicher Kulturszenen zum eigentlichen Ausgangspunkt der Arbeitspraxis zu machen. Dies bedeutet nicht, dass die translokalen Raumbezüge und Erfahrungen der Entsandten, ihr »Blick von außen« auf lokale Problemlagen nicht gleichfalls wertvoll sind. Aber eine symmetrischere Verzahnung dieser beiden Wissensformen und ihrer Träger erschiene als angemessen.

Kritik ruft gleichfalls hervor, dass diese Hierarchien auch auf eng verstandenen, normalisierten Repräsentationslogiken des Nationalen aufbauen. Denn zumindest an den Standorten in Ramallah und Sarajevo verfügen die »Ortskräfte« aufgrund von Migrations-, Flucht- oder Bildungsbiografien über verdichtete - wenn auch brüchigere - Bezüge nach Deutschland und hiermit verbunden über ein routiniertes Vermögen, zwischen unterschiedlichen sprachlichen und kulturellen Kontexten zu übersetzen. Sie beziehen somit intermediäre Positionen in den Ordnungen des Nationalen, aufgrund derer sie aus Perspektive der institutionellen und politischen Entscheidungsträger offenbar 
nicht als geeignet gelten, »Deutschland « auf den Bühnen kultureller Selbstdarstellung in einer herausgehobenen Position zu repräsentieren. Hierzu ist auch weiterhin die inkorporierte Ethnizität des entsandten deutschen Leitungspersonals erforderlich - ein Deutschsein, das allerdings durch die translokale Mobilität ein kosmopolitisches Gewand erhält und sich genau hierdurch nochmals von der Subjektposition einer »Ortskraft« absetzen lässt.

In den translokalen Praxis- und Übersetzungsfeldern werden gleichfalls Asymmetrien reproduziert, die noch stärker auf eine ungleiche Weltordnung verweisen. So verdeutlichen die Aussagen meiner Gesprächspartner vielfach die Relevanz der strukturell ungleichen Ausgangsbedingungen in Kooperationsprojekten zwischen deutschen Kulturmittlern und lokalen Kulturschaffenden in Konfliktregionen. Hier traten insbesondere die Effekte verschiedener Formen von Staatlichkeit und ihrer jeweiligen ökonomischen und politischen Potenziale hervor, die sich in den unterschiedlichen Status und Handlungsspielräumen der beteiligten Personen niederschlagen. Die Schwierigkeiten, eine - im institutionellen Selbstverständnis der Kulturmittler eigentlich erforderliche - Partnerleistung « einzufordern, in Kontexten fragmentierter Staatlichkeit »Nachhaltigkeit« für die eigenen Aktivitäten zu erreichen, oder überhaupt in offiziellen Strukturen ein entscheidungsmächtiges »Gegenüber« zu finden, wurden mehrfach herausgestellt (Kapitel III.5). Es handelt sich hierbei um Rahmenbedingungen, die auch die alltägliche Zusammenarbeit durchziehen, wie ich an einer letzten Sequenz aus Sarajevo illustrieren möchte: Hier nehme ich an Planungsgesprächen zu einer Ausstellung teil, die als Kooperation zwischen dem Goethe-Institut, einer deutschen Galerie, einem Museum in Sarajevo und einer lokalen Kuratorin realisiert werden soll. Mehrfach fragt die Institutsleiterin die Kuratorin nach ihrer »Partnerleistung « und ruft hierdurch einen Moment seltener Vehemenz hervor: Ihre Gesprächspartnerin erwidert, sie habe seit Februar keinen Lohn mehr erhalten. Entsprechend sei sie als Direktorin ihrer Organisation eigentlich eine Freiwillige. Alles was sie beitragen könne, seien ihre Kompetenzen und ihre professionelle Arbeit. ${ }^{3}$

Im Unterschied zu dieser Kuratorin verfügt das entsandte Leitungspersonal eines Goethe-Instituts über Festanstellungen mit besonderen Auslandszuschlägen, die in Arbeitskontexten wie Sarajevo oder Ramallah nicht nur ein komfortables, sondern ein durchaus repräsentatives Leben ermöglichen; die Kontinuität und Verlässlichkeit der Förderung des Goethe-Instituts durch öffentliche Gelder wurden im Kapitel II.6 herausgearbeitet. Auch wenn man diese Rahmenbedingungen sicherlich positiv bewerten kann, da sie eine deutsche Arbeitnehmerin in ihrer globalen Mobilität absichern und der Institution eine langfristige Planung ermöglichen, so führen sie in der Zusammenarbeit mit Partnern in instabileren, ökonomisch schwächeren Regionen gewissermaßen

3 | Feldnotizen vom 17. Juli 2009. 
systemisch zu Reibungen: Denn in solchen Kooperationen begegnen sich Organisation und Personen, deren finanzieller Rückhalt, Absicherung und Planungszeiträume sich grundsätzlich voneinander unterscheiden. Gerade wenn man davon ausgeht, dass die ungleiche Verteilung von Wohlstand, Ressourcen, Gewalt und Sicherheit maßgeblich zur Konfliktträchtigkeit der zeitgenössischen Welt beiträgt, stellt sich die Frage, ob sich die neuen kulturpolitischen Zielvorstellungen in der Arbeitspraxis glaubhaft aufgreifen lassen, wenn man solche strukturellen Asymmetrien auch institutionell reproduziert und weitgehend unthematisiert lässt. Aus meiner Perspektive müsste es im Rahmen einer Auswärtigen Kulturpolitik als Konfliktprävention ganz im Gegenteil explizit um die Entwicklung von Verfahren und Herangehensweisen gehen, die eben diese Hierarchien, ihre Effekte und Möglichkeiten ihrer Bearbeitung in den Mittelpunkt stellen.

\section{(iv) Die Naturalisierung kulturpolitischer Grundfiguren}

Im Laufe dieses Buches wurde deutlich, wie stark Ordnung des Nationalen auch weiterhin die zentralen epistemologischen Grundfiguren und politischen Rationalitäten prägen, die dem Politikbereich langfristig eingelagert sind. So ließ sich durch eine Analyse der imaginären Dimension Auswärtiger Kulturpolitik die Wirkmächtigkeit einer dichotomischen Logik herausarbeiten, die einerseits immer wieder das Bild von einem umgrenzten, distinkten kulturellen Raum als Ausgangspunkt der Vermittlungsarbeit hervorruft und andererseits durchgängig zu Dialog, Begegnung oder Austausch und somit zu einer routinierten Überschreitung dieser Grenze auffordert (Kapitel II.5). Ich habe aufgezeigt, wie etablierte Grundfiguren des Nationalen - etwa die Vorstellungen von »kultureller Differenz«, »nationaler Spezifik« oder der Existenz eines »kulturellen Wir«, das sich den »Anderen« mitteilen möchte - als Folge dieser Logik eine Rezentrierung erfahren und dennoch gleichzeitig flexibilisiert werden können. Denn es geht im Selbstverständnis der policy community ja nicht um die Darstellung von statischen nationalkulturellen Inhalten oder um die Inszenierung von unüberbrückbaren Grenzen, sondern ganz im Gegenteil um translokale Zusammenarbeit, um die Verflechtung kultureller Szenen und um die Dialogisierung von Perspektiven auf zeitgenössische kulturelle Entwicklungen. Eine zentrale Beobachtung bestand darin, dass die genannten Grundfiguren des Nationalen dennoch - wenn auch in sich verändernden inhaltlichen Ausformungen - auch innerhalb der translokalen Praxis- und Übersetzungsfelder als kaum hinterfragte Ausgangspunkte erhalten bleiben. So ermöglicht es diese dichotomische Logik den deutschen Kulturmittlern, sich in ihrem Arbeitsalltag situativ auf das »Eigene « zurückzuziehen oder auf die Grenzen der eigenen Zuständigkeit und Verantwortung für lokale Konstellationen zu verweisen. Solche Tendenzen zeigten sich im Rahmen meiner Untersuchung etwa in Aussagen meiner Gesprächspartner, in denen sie 
»Differenz«, »Fremdheit« oder einen »Blick von außen« als notwendige Ausgangsbedingung für produktive Dialoge benannten oder ihre Einsatzorte über (post-)koloniale Blickregime exotisierten und somit als grundlegend »anders« darstellten (Kapitel III.3).

Auch wenn man anerkennt, dass deutsche Kulturmittler solche Positionen im Zuge einer langjährigen beruflichen Erfahrung entwickelt haben, lassen sie sich dennoch einer kritischen Befragung unterziehen. Einen ersten Anlass bietet die weitgehend naturalisierte Ausgangsvermutung von Differenz als einer erforderlichen Grundkonstellation für Dialog. In Abgrenzung hierzu möchte ich argumentieren, dass eine kulturpolitische Praxis, die unter den Rahmenbedingungen eines Konfliktes tatsächlich auf eine Kooperation auf Augenhöhe ausgerichtet wäre, sich explizit am Idealbild einer symmetrischen Bearbeitung zuvor gemeinsam identifizierter »Probleme«, Fragestellungen oder Themenschwerpunkte orientieren müsste. Dies würde in der Konsequenz bedeuten, explizit Projektformate, Akteurskonstellationen und Sprechweisen zu erarbeiten, welche »Distanz« sukzessive reduzieren und somit auch Vorstellungen von »Differenz« relativieren. Auf dieser Basis würde ich dafür plädieren, Verflechtung an die Stelle von Dialog als Leitmotiv für die kulturpolitische Praxis zu setzen, um dem Tatbestand Rechnung zu tragen, dass sich in den translokalen Praxis- und Übersetzungsfeldern nicht getrennte, unabhängige Einheiten im Gespräch begegnen, sondern Zeitgenossen gemeinsam die kulturellen Bedingungen einer im doppelten Sinne geteilten Welt auszuloten versuchen.

Eine zweite kritische Perspektive zielt auf noch weitgehendere globale Effekte eines solchen Festhaltens an den etablierten Grundfiguren der Differenz, Grenze und Spezifik. Im Anschluss an Ulrich Beck formuliere ich, dass gerade die Zentrierung von Beständen des Nationalen in den kulturpolitischen Diskurs- und Praxisfeldern dazu beiträgt, die angeführten Asymmetrien einer ungleichen Weltordnung als gegeben zu naturalisieren. In einem bereits zitierten Essay fragt Beck, was eigentlich die »globale Ungleichheitsordnung legitim und stabil (macht)«, um das »Nationalstaatsprinzip« in politischer Herrschaft und Soziologie als den zentralen Faktor zu identifizieren:

"Nationale Grenzen trennen scharf zwischen politisch relevanter und irrelevanter Ungleichheit. Ungleichheiten innerhalb nationaler Gesellschaften werden in der Wahrnehmung enorm vergrößert; gleichzeitig werden Ungleichheiten zwischen nationalen Gesellschaften ausgeblendet. Die ,Legitimation، globaler Ungleichheiten beruht also auf institutionalisiertem Wegsehen. Der nationale Blick 'befreit، vom Blick auf das Elend der Welt. Er operiert mittels einer doppelten Exklusion: Er exkludiert die Exkludierten. “ ${ }^{4}$

4 | Ulrich Beck: Die Neuvermessung der Ungleichheit unter den Menschen, S. 13. Siehe hierzu auch die Überlegungen im Kapitel I.3 dieses Buches. 
Der Vorwurf eines »institutionalisierten Wegsehens« trifft auf die Akteursund Diskussionsfelder Auswärtiger Kulturpolitik sicherlich weniger zu als auf das Zusammenwirken von staatlicher Herrschaft mit dem »methodologischen Nationalismus « ${ }^{5}$ der etablierten Sozialwissenschaften, das Ulrich Beck hier kritisiert. Denn schließlich schafft dieser Politikbereich weltweit Präsenzen, Arbeitskontexte und Interaktionsräume - wenn auch nicht in den globalen Elendsregionen, so doch zumindest entlang von einigen Bruchlinien der gegenwärtigen Weltordnung. Dennoch zeigten sich während meiner Forschung vielfach Momente einer institutionalisierten Distanzierung oder Formen eines routinierten Sich-Herausziehens, die aus meiner Perspektive gerade auf Basis der naturalisierten Grundfiguren des Nationalen möglich sind. Denn die Vorstellungen von einer umgrenzbaren »nationalen Spezifik« oder die Subjektposition eines »Entsandten«, der am Einsatzort ein anderes »kulturelles Kollektiv« vertritt, legen es nahe, den Standpunkt eines »externen Partners und Wohltäters « mit eingeschränkten Zuständigkeiten für lokal sichtbare Konflikte, Mängel oder Exklusionen zu beziehen. Auch wenn eine Institutsleiterin die systemischen Ungleichheiten als zentrales Hindernis für eine nachhaltige Arbeit »auf Augenhöhe« anerkennt und verstärkt nach Möglichkeiten für eine Unterstützung kultureller Infrastrukturen sucht (Kapitel III.5), so bleibt es zumeist bei Formen der Zusammenarbeit, aus denen sich die deutschen Kulturmittler jederzeit einseitig zurückziehen können, um »neue Impulse« zu setzen, andere Themen zu bearbeiten und neue Partnerschaften einzugehen. Die asymmetrischen Arbeitsbedingungen werden entsprechend eher als lokale Problemlagen oder Defizite betrachtet und weniger als Ausdruck von transnationalen Geweben, in denen man auch als deutscher Akteur immer schon verfangen ist.

Aus meiner Perspektive trägt die Wirkmächtigkeit der weitgehend naturalisierten Grundfiguren des Nationalen wesentlich dazu bei, solche situativen Distanzierungen von den lokalen Arbeitskontexten plausibel zu machen. Das Nationale bildet hier einen zugleich konzeptionellen wie praktischen Rückzugsraum, durch den sich der Druck zu einer radikaleren Bearbeitung von Asymmetrien und zu einem expliziten Sich-Einlassen auf die konfliktdurchzogenen lokalen Konstellationen reduziert. Eine alternative Positionierung deutscher Kulturmittler als dauerhaft involvierte Teilhaber einer community of transformation, die an der Kreation (trans)lokaler Dynamiken beteiligt sind und - gemeinsam mit anderen Akteuren - für deren Entwicklungsrichtung, Erfolge und Misserfolge auch Verantwortung tragen, verliert vor diesem Hintergrund an Dringlichkeit. Auf dieser Basis möchte ich argumentieren, dass eine kulturpolitische Praxis, die sich tatsächlich zentral an dem Leitmotiv Konfliktprävention orientierte, einen deutlicheren Bruch mit dem Nationalen

5 | Siehe hierzu etwa: Ebd., S. $16 \mathrm{ff}$. 
als elementarem Bezugssystem und Schlüsselbestand des offiziellen Narrativs Auswärtiger Kulturpolitik erforderlich machte. Ein solcher Bruch würde es aus meiner Perspektive mit sich bringen, kulturpolitische Vermittlungsarbeit nicht länger als Instrumente der Selbstdarstellung und auch nicht als Wohltätigkeit eines europäischen Nationalstaates zu verstehen, sondern als einen Beitrag, um Menschen in Konfliktregionen die Wahrnehmung ihrer bedrohten Rechte auf Gestaltung lokal situierter kultureller Prozesse und auf Teilhabe an globalen kulturellen Dynamiken zu ermöglichen.

\section{Das Zusammenfügen einer Policy: Formierungsprozesse und Wissensarbeit}

Der konzeptionell-methodische Vorschlag dieser Studie lautete, die Formierungsprozesse einer Policy entlang des Auftauchens und der Mobilisierung einer neuen diskursiven Figur zu untersuchen. ${ }^{6}$ Hierdurch rückten Prozesse und Praxen des Zusammenfügens von politischen Zielvorstellungen, Wissensformen, materiellen Ressourcen, infrastrukturellen Arrangements, lokalen und institutionellen Kontexten in den Mittelpunkt der Analyse. Vor diesem Hintergrund war von besonderem Interesse, ob und in welcher Weise sich die politischen Narrative im Arbeitsalltag der Kulturmittler niederschlagen. Aufgrund ihrer Bedeutung für die Verbindung von Diskursen und Praxis wurde Wissens- und Übersetzungsarbeit zur zentralen analytischen Zielgröße dieser Untersuchung.

Im Rahmen meiner Forschung bin ich zwei zentralen Metaphern des offiziellen Narrativs - Deutschlandbilder vermitteln und Dialog - sowie der neuen diskursiven Formation Auswärtige Kulturpolitik als Konfliktprävention an die beiden Standorte Ramallah und Sarajevo gefolgt. Meine Beobachtungen in den translokalen Praxis- und Übersetzungsfeldern, die sich hier um die Goethe-Institute bilden, förderten vielfach zutage, wie Argumentationsfiguren und Schlüsselbegriffe aus den politischen Zentren durch Wissenspraxen aufgegriffen und mit lokalen Erfordernissen, Erwartungen und Logiken verknüpft werden (Kapitel III) - anders formuliert: diskursive und nicht-diskursive Elemente werden erst im Rahmen einer translokalen Projektarbeit zu einer Policy zusammengefügt. Der Umgang mit solchen Kernbeständen des politischen Narrativs stellt sich dabei nicht als eine lineare Umsetzung dar. Zwar rahmen die etablierten Ausgangspunkte und Denklogiken seiner beiden langfristig etablierten Leitmotive - also des Prinzips der kulturellen Selbstdarstellung und des Prinzips der Wechselseitigkeit - (Kapitel II.3) deutlich das Selbstverständnis der Kulturmittler und ihre Praxis vor Ort. Auch neue politisch gesetzte Begrifflich-

6 | Siehe hierzu auch den bereits in der Einleitung erwähnten, durch Jens Adam \& Asta Vonderau herausgegebenen Sammelband "Formationen des Politischen". 
keiten bilden Referenzpunkte für die alltägliche Projektarbeit, insbesondere wenn sie durch Sonderförderprogramme begleitet werden. Schlüsselbegriffe, Zielhorizonte und politische Rationalitäten sind somit spürbar in die Formate der Vermittlung inkorporiert. Auf dieser Basis ließen sich vier Strategien kulturpolitischer Übersetzung herausarbeiten, in denen sich die kontinuierlichen Verbindungsleistungen zwischen politischen Zentren und kulturpolitischen Settings an den Einsatzorten auf eine jeweils spezifische Weise verdichten (Kapitel III.2-5). So wurde sichtbar, dass die Bezugnahmen auf das politische Narrativ nicht zu vorhersehbaren oder gleichbleibenden Ergebnissen führen, sondern ganz im Gegenteil zu einer Vielzahl von Praxen, Projektarten und Kooperationsmodellen - um es noch einmal im Anschluss an Richard Rottenburgs Beobachtungen zur Entwicklungszusammenarbeit auszudrücken: Diskurs und Praxis erweisen sich als »lose gekoppelt«? Hierfür lassen sich drei Gründe ausmachen:

Zunächst zeigte sich, dass übergreifende Zielsetzungen auch innerhalb der Auswärtigen Kulturpolitik Inkonsistenzen und Widersprüchlichkeiten produzieren. So waren meine Gesprächspartner häufig verunsichert, wie die neue Figur Konfliktprävention zu verstehen ist und in welchem Verhältnis sie zu etablierten Zielvorstellungen steht; sie fragten sich, über welche Formate oder Herangehensweisen sie sich in ihre Projektarbeit integrieren lässt; sie monierten fehlende Kriterien oder Messinstrumente für »Erfolg « und verwiesen auf die Begrenzungen der Mittel und Einflussmöglichkeiten eines Goethe-Instituts; sie verdeutlichten Kollisionen zwischen dieser Zielvorstellung und den »realen« politischen Verhandlungsprozessen, Entscheidungen sowie anderen Grundsätzen; sie verteidigten eine Kontingenz und eventuelle Ziellosigkeit kultureller Arbeit als wünschenswert. Die inkohärenten, manchmal verwirrenden oder nicht-intendierten Effekte, die im Zuge der Formierung einer neuen Policy hervorgerufen werden, traten vielfach zutage.

Zweitens wurde deutlich, dass auch in der kulturpolitischen Vermittlungsarbeit »Momente der unausweichlichen Unbestimmtheit« entstehen, wenn Zielvorstellungen des offiziellen Narrativs durch Übersetzungsketten wandern und hierbei in den unterschiedlichen lokalen oder institutionellen Kontexten aufgegriffen und übertragen werden. So habe ich nachgezeichnet, durch welch unterschiedliche Inhalte, Verbindungen, Herangehensweisen oder Formate sich das Leitmotiv Herstellung von Deutschlandbezügen in Sarajevo und Ramallah verankern lässt (Kapitel III.4). Ein ähnlicher Befund ergab sich aus der Dar-

7 | Richard Rottenburg: Weit hergeholte Fakten, S. 97ff. An dieser Stelle spricht Rottenburg auch von der "Inkonsistenz" politischer Narrative, bzw. von den "Widersprüchlichkeiten", die sie produzieren. Die folgenden Zitate "Momente der unausweichlichen Unbestimmtheit" und "andere Optionen..." gleichfalls im Anschluss an Richard Rottenburg, siehe hierzu: Ebd., S. 231. 
stellung der vielfältigen Aushandlungen und Navigationen, die Kulturmittler in den besetzten Palästinensischen Gebieten realisieren, um die Schlüsselmetapher Dialog translokal zu verknüpfen (Kapitel III.5). Auch wenn die Auseinandersetzung mit den politisch gesetzten Zielsetzungen unausweichlich ist, so hätte es dennoch in jedem dieser Fälle auch »andere Optionen gegeben, als die, die man tatsächlich gewählt hat«.

Und drittens ist der kreative Umgang deutscher Kulturmittler mit offiziellen Begrifflichkeiten auch in den politischen Entscheidungszentren durchaus erwünscht - zumindest so lange sie sich innerhalb der gesetzten Rahmenbedingungen bewegen. Dies zeigte sich etwa anhand der Erklärungen des Abteilungsleiters im Auswärtigen Amt zur Idealvorstellung einer »strategischen Steuerung «: die Ministerialbeamten greifen in die Details der Programmgestaltung nicht ein; eventuelle Interventionen werden an den Zielvereinbarungen ausgerichtet (Kapitel II.6). Gleichfalls wurden während meiner Forschung vielfach Wertschätzungen der Freiräume, Kontakte und Perspektiven, die sich aus einem »kulturellen Zugang« zu kontroversen Themen ergeben können, formuliert. Die Überzeugung von den besonderen Potenzialen der Kultur, in umstrittenen Feldern Wirkung zu erzielen, lässt sich zu den naturalisierten Wissensbeständen des Politikbereichs zählen.

Die Beziehungen zwischen dem politischen Narrativ und der Wissensarbeit zeigen sich in den translokalen Praxis- und Übersetzungsfeldern somit zwar »lose gekoppelt«, sind aber dennoch präkonfiguriert: Die herausgearbeiteten Prinzipien und politischen Rationalitäten zur Legitimierung Auswärtiger Kulturpolitik (Kapitel II.2-3) stellen Leitplanken dar, die selten überschritten werden, zugleich aber in dem so gesetzten Rahmen Freiräume für Experimente, lokal anschlussfähige Sprech- oder Arbeitsweisen und somit für eine bemerkenswerte Diversifizierung kulturpolitischer Übersetzungspraxen eröffnen. Diese Beobachtungen spiegeln sich in den Formierungsprinzipien der policy community wider: Ein zentrales Element stellt ein Habitus kultureller Repräsentation dar (Kapitel II.4 und III.3), dem die Denklogiken und Grundfiguren des politischen Narrativs inkorporiert sind, der aber zugleich flexible Verknüpfungen mit translokalen Formationen ermöglicht - ein Habitus, den man als Kulturmittlerin erlernen muss, um sich nicht zu verhalten, »wie ein Hund in einem Kegelspiel, einem Spiel, dessen implizite Regeln er nicht kennt «. ${ }^{8}$ Diese Aufgabe wird dadurch einfacher, dass es sich - aller Rhetorik zur Öffnung zum Trotz - bei den offiziell beauftragten kulturpolitischen Repräsentanten um ein übersichtliches und exklusives Akteursfeld handelt: Die Position einer Institutsleiterin bleibt bisher nicht nur »Ortskräften« verschlossen, sondern wird in der Regel aus dem Kreis der dauerhaften Stammmitarbeiter besetzt - ein Organisationsprinzip, das die »strategische Steuerung« und die Herstel-

8 | Pierre Bourdieu: Das politische Feld, S. 47. 
lung von Kohärenz in einem weltweit vernetzten Politikbereich sicherlich erleichtert, aber zugleich eine deutlichere Infragestellung und Transformation der etablierten diskursiven und infrastrukturellen Grundfiguren des Politikbereichs unwahrscheinlich macht.

\section{Potenziale der Kosmopolitisierung}

Im Rahmen dieser Untersuchung ließ sich das Wirken einer Formierungsmacht herausarbeiten, die diskursive Figuren, politische »Probleme«, Ressourcen, Orte und Akteure zu einer Policy zusammenzufügen vermag (Kapitel II.4). Hierbei erwiesen sich zwei Effekte als besonders relevant: Zum einen trat das Potenzial zutage, Grundbegriffe und Denkmuster, über die kulturelle Vermittlung und Selbstdarstellung konzipiert und praktiziert werden können, zu etablieren, zu verändern oder zu bestätigen; zum anderen ließ sich die Formierung eines weltweit verflochtenen Netzwerkes aus kulturpolitischen Präsenzen nachzeichnen, das wirkmächtige Bahnen zur Mobilisierung von Schlüsselbegriffen, politischen Zielvorstellungen und Rationalitäten anbietet. Die Policy eröffnet somit präkonfigurierte Wege, über die sich Kulturaustausch realisieren kann. Die Formierungsmacht legt epistemologische und infrastrukturelle Ordnungen übereinander und trägt hierdurch dazu bei, Alternativen auszuschließen: etwa andere Grundbegriffe, Problemdefinitionen oder Kooperationsmodelle, die sich stärker an einem postnationalen Verständnis von Kultur, an nomadischen kulturellen Praxen oder an einer postkolonialen Kritik orientieren würden (Kapitel II.4-6).

Zugleich wurde sichtbar, dass diese relativ stabilen Elemente im Rahmen einer alltäglichen Organisations- und Wissensarbeit an den unterschiedlichen Einsatzorten in temporäre Arrangements eingebracht werden. Eben das Zusammenwirken von längerfristigen Infrastrukturen und diskursiven Formationen mit solchen flüchtigeren Komponenten eines »Projektes« - also jeweiligen Kooperationspartnern, Fragestellungen, Veranstaltungsorten, Formaten oder Finanzierungsquellen - ließ sich durch das Analysemodell translokale Praxis- und Übersetzungsfelder in den Blick nehmen (Kapitel III.2). Hier bilden sich kulturpolitische Vermittlungsräume, in denen sich auch eine Dynamik zwischen Authentisierung und translokaler Flexibilisierung von Beständen des Nationalen beobachten lässt: Einerseits erfahren Vorstellungen von einer »nationalen Spezifik«, die Sprechposition eines »kulturellen Wir« oder die Annahme von »Grenzen« der eigenen Zuständigkeit immer wieder aufs Neue eine Bestätigung als plausible Ausgangspunkte der Vermittlungsarbeit; andererseits gilt es, an einem jeweiligen Einsatzort unterschiedliche Erfahrungen, Repräsentationsformen, Bilder und Erwartungen aufzugreifen, um ein Narrativ vom Nationalen jenseits seines eigentlichen Territoriums anschlussfähig zu machen. In dieser Dynamik werden Transformationen des Nationalen sichtbar, die ich nun 
abschließend unter dem Begriff Potenziale der Kosmopolitisierung diskutieren möchte. Ich knüpfe hierzu an sozialwissenschaftliche und kulturanthropologische Positionen an, die »Kosmopolitisierung « als einen durch die Macht der Verhältnisse erzwungenen Prozess und somit nicht als Ausdruck einer normativen Haltung einzelner Personen verstehen: ${ }^{9}$ Reale Entgrenzungen, Mobilitäten und globale Risiken führen demnach in lokalen Räumen oder politischen Öffentlichkeiten zu einer »unmittelbaren, unabweisbaren Konfrontation« mit Problemstellungen, Akteuren oder Konflikten, die sich kaum noch innerhalb von etablierten Begrifflichkeiten und Ordnungsmustern - etwa »Nation«, »Identität«, »Zugehörigkeit« oder »Kultur« - verstehen und bearbeiten lassen. Langfristig gesetzte Kategorien der Selbstwahrnehmung und politischen Auseinandersetzung geraten unter Druck, der zu ihrer - zunächst unintendierten - sukzessiven Öffnung oder Flexibilisierung führen kann. Kosmopolitisierung erscheint aus dieser Perspektive nicht als »eine reflexive Eigenleistung der daran beteiligten Akteure«, sondern als »eine ungewollte Nebenfolge« von Handlungen, politischen Projekten oder Migrationsbewegungen, die an einem Ort oder in einer politischen Arena eine eigentlich unbeabsichtigte Wirksamkeit entfalten. Als maßgebliche Effekte können hieraus Potenziale zur »Erweiterung politischer Diskurse und Handlungsräume« entstehen.

Durch meine gesamte Untersuchung hindurch wurde deutlich, wie Repräsentationen des Nationalen im Zuge der Diskussions- und Praxisfelder Auswärtiger Kulturpolitik mit unterschiedlichen Raumbezügen, politischen Projekten, kulturellen Referenzrahmen und lokal situierten Wahrnehmungsmustern in Austausch geraten. Gegenwärtige Transformationen des Nationalen lassen sich gerade auf solche Konfrontationen zurückführen. Hier entstehen Potenziale der Kosmopolitisierung, die ich entlang von vier Mikro-Dynamiken diskutieren möchte: Zunächst sind es die neuen, universalen Zielhorizonte Konfliktprävention oder Förderung der Menschenrechte, die etablierte Ordnungen des Nationalen herausfordern und den Politikbereich zumindest potenziell auf eine Bearbeitung von Problemlagen einer geteilten Welt hin ausrichten; zwei-

9 | Siehe etwa: Ulrich Beck: Der kosmopolitische Blick oder Krieg ist Frieden. Frankfurt 2004; Ulrich Beck \& Natan Sznaider: Unpacking Cosmopolitanism for the Social Sciences: A Research Agenda. In: British Journal of Sociology 57.1 (2006), S. 1-23; Steven Vertovec \& Robin Cohen (Hrsg.): Conceiving Cosmopolitanism. Theory, Context, and Practice. Oxford 2002; Regina Römhild: Aus der Perspektive der Migration. Die Kosmopolitisierung Europas. In: Sabine Hess, Jana Binder \& Johannes Moser (Hrsg.): No integration?! Kulturwissenschaftliche Beiträge zur Integrationsdebatte in Europa. Bielefeld 2009, S. 225-238. Die Zitate in diesem Absatz entstammen diesem letzten Beitrag von Regina Römhild und finden sich auf S. 231; mit der Ausnahme von "eine ungewollte Nebenfolge" - ein Begriff, der von Ulrich Beck geprägt wurde und sich etwa in "Der kosmopolitische Blick" auf Seite 30 findet. 
tens zeigten sich gerade in Sarajevo Tendenzen zur Europäisierung von Projektformaten und Sprechpositionen, die hergebrachte Vorstellungen von Grenzen und Differenz zwischen nationalkulturellen Räumen flexibilisieren und stattdessen deren Verflechtungen stärker in den Vordergrund rücken; drittens wurde anhand von kritischen Bemerkungen lokaler Mitarbeiter oder Projektpartnerinnen deutlich, wie die kontinuierliche Auseinandersetzung mit den allagspraktischen Problemen, narrativen Brechungen, politischen Dissonanzen und geopolitischen Grenzlinien in einem »Konfliktgebiet« die Legitimität einer Kulturpolitik, der es im Kern um eine Herstellung von Deutschlandbezügen geht, in Frage stellt; und viertens zeigte sich anhand einer Vielzahl von Beispielen der Druck, Deutschlandbezüge - und somit die mobilisierten Bestände des Nationalen - immer wieder neu zu konfigurieren, um in einem jeweiligen lokalen Kontext überhaupt relevante Anschlüsse hervorrufen zu können. Potenziale der Kosmopolitisierung ergeben sich aus den ganz praktischen Problemstellungen, Übersetzungen, Reibungen und Effekten, die im Zuge solcher Konfrontationen hervorgerufen werden.

In den Diskussionen, vor allem aber in den Praxis- und Übersetzungsfeldern des Politikbereichs zeigen sich also vielfältige Bewegungen der Flexibilisierung, die aber bisher nicht zu einer nachhaltigen Infragestellung seiner grundlegenden epistemologischen Ordnungen und institutionellen Arrangements geführt haben - eine Beobachtung, die zu einer abschließenden kritischen Bemerkung Anlass bietet: Der kamerunische Historiker und Philosoph Achille Mbembe hat kürzlich darauf hingewiesen, »dass dem europäischen Denken in seiner gesamten Geschichte die Tendenz innewohnt, Identität nicht im Sinne gemeinsamer Zugehörigkeit zu ein und derselben Welt zu verstehen, sondern im Sinne eines selbstbezüglichen Verhältnisses [...]«, in dem eine »Logik der Autofiktion, der Selbstbetrachtung, also der Abschließung« wirksam wird. ${ }^{10}$ Dieser postkoloniale Blick auf Grundfiguren der europäischen Moderne eröffnet die Möglichkeit, die elementaren Ordnungen der Auswärtigen Kulturpolitik Deutschlands nach der Jahrtausendwende nochmals kritisch zu beleuchten: Denn durch meine Studie wurde sichtbar, dass die dichotomische Logik, die ihr offizielles Narrativ strukturiert, - trotz ihrer deutlichen Ausrichtung auf Dialog - kontinuierlich die Vorstellung einer Grenze zwischen einem inneren und einem äußeren Raum der Kultur reproduziert: Die Intention, der Welt etwas von »unserer Kultur«, »unseren Werten« oder »unserer Art, zu sein« mitzuteilen, ist als Dreh- und Angelpunkt des Politikbereichs erhalten geblieben. Das Auftauchen von Konfliktprävention in kulturpolitischen Foren hat somit zwar fraglos zu einer Vielzahl von diskursiven Öffnungen, von neuen Formaten und Wissenspraxen geführt; der grundlegende Paradigmenwechsel, der sich I999 kurzzeitig anzudeuten schien, ist aber ausgeblieben. Eine

10 | Achille Mbembe: Kritik der schwarzen Vernunft. Frankfurt 2014, S. $11 f$. 
kulturpolitische Arbeit, welche die Konfliktträchtigkeit der gegenwärtigen Formen des globalen Zusammenlebens tatsächlich in ihr Zentrum stellte, müsste aus meiner Perspektive bedeuten, eben die Vorstellung einer gemeinsamen Zugehörigkeit zu ein und derselben Welt zugleich zu ihrem Ausgangspunkt und ihrem Zielhorizont zu nehmen. Die Potenziale der Kosmopolitisierung, die sich bisher in den translokalen Praxis- und Übersetzungsfeldern Auswärtiger Kulturpolitik eher ereignen, als dass sie wirklich intendiert wären, könnten hierzu einen zentralen Beitrag liefern: Es gälte sie reflexiv voranzutreiben und für die Neuausrichtung des Narrativs, der Infrastrukturen und der Organisationsformate des Politikbereichs heranzuziehen - immer in dem Bewusstsein, dass wir diese Welt auch deshalb teilen, weil wir die Nebenfolgen der Policies, die wir mobilisieren, nicht kennen. Europäische Staaten haben zumindest über zwei Jahrhunderte eine Vielzahl von Bürokratien, Museen, Bildungseinrichtungen und Kulturinstitutionen aufgebaut, um Ordnungen des Nationalen als Refugien von Differenz auszugestalten. Es ist an der Zeit, Institutionen und Infrastrukturen zu entwickeln, in denen sich kosmopolitische Wissens- und Handlungsräume erarbeiten lassen, welche die Konflikte, Brüche und Ungleichheiten einer geteilten Welt als ihren integrativen Bestandteil und nicht als äußerlich wahrnehmen. 


\section{Anhang}

\section{Feldtagebücher Und Interviews}

\section{Feldtagebücher}

Feldtagebuch Berlin, März 2007 - Oktober 2010, 369 Seiten.

Feldtagebuch Ramallah, August - Dezember 2008, 562 Seiten.

Feldtagebuch Sarajevo, Mai - Juli 2009, November 2009 \& April 20I2, 669 Seiten.

\section{Interviewpartner/innen in Ramallah, Sarajevo, Jerusalem und Tel Aviv}

Für diese Studie führte ich in den Jahren 2008/o9 insgesamt 45 semi-strukturierte Interviews mit Mitarbeiterinnen und Mitarbeitern der Goethe-Institute in Ramallah, Sarajevo, Jerusalem und Tel Aviv, mit Entsandten anderer deutscher Mittlerorganisationen und politischer Stiftungen in Bosnien-Herzegowina und in den besetzten Palästinensischen Gebieten, mit Diplomatinnen an den dortigen deutschen Auslandsvertretungen, mit Projektpartnerinnen und Projektpartnern der Goethe-Institute und anderen Kulturschaffenden in Ramallah und Sarajevo. Weiterhin flossen Hintergrundgespräche mit fünf Mitarbeiterinnen und Mitarbeitern des Auswärtigen Amtes in Berlin sowie mit sechs Mitarbeiterinnen und Mitarbeitern von Mittlerorganisationen in Stuttgart und Berlin in diese Untersuchung mit ein. 


\section{Materialien}

\section{Zeitungsartikel und andere Medienberichte}

»Ach, Albanien! >Perspektive Europa<: ein literarisch-politisches Dichtertreffen in der Akademie der Künste Berlin.« In: Der Tagesspiegel vom 4. Juni 2007.

»Bremsschwellen im Alltagsleben. Das Goethe-Institut in Ramallah produziert die erste palästinensische Seifenoper mit dem Titel >Matabb«. In: Süddeutsche Zeitung vom 26. August 2008.

»Ein Kino für den Frieden. Neue Hoffnung für Nahost.« Bericht in der Fernsehsendung »Aspekte« (ZDF) vom I3. August 2010.

»Europas bedrückende Erbschaft. Das Jahrhundert von Auschwitz ist noch nicht zu Ende: Rede zum Auftakt des Berliner Kongresses >Perspektive Europa« von Imre Kertész. In: Süddeutsche Zeitung vom 2./3. Juni 2007. »Lindenstraße in Palästina: Matabb: Die Palästinensersoap.« Bericht in den Tagesthemen (ARD) vom 3I. August 2008.

»Lindenstraße in Ramallah«. In: ZEIT ONLINE vom 25. August 2008, siehe: http://www.zeit.de/online/2008/35/nahost-lindenstrasse-in-ramallah (Zugriff: 24.II.20I7).

»Machen wir kein Drama draus. Oder doch? Wie in der Berliner Akademie der Künste Imre Kertész und Frank-Walter Steinmeier nach europäischen Perspektiven suchten.«In: Die Welt vom 4. Juni 2007.

»Nennen wir uns einfach Herakles. Die Akademie der Künste fragte recht allgemein nach der >Perspektive Europa «« In: Berliner Zeitung vom 4. Juni 2007.

»Olé kommt von Allah. Ohne Fraktionszwang: Eine Berliner Europa-Konferenz.« In: Frankfurter Allgemeine Zeitung vom 4. Juni 2007.

»Palästinensische >Lindenstraße $<$ Mutter Beimer trägt jetzt Kopftuch.« In: SPIEGEL ONLINE vom 27. August 2008, siehe: http://www.spiegel.de/ kultur/gesellschaft/palaestinensische-lindenstrasse-mutter-beimertraegt-jetzt-kopftuch-a-574772.html (Zugriff: 24.II.20I7).

»Perspektive Europa<. Klaus Staeck vor sseiner Akademie der Künste in Berlin.« In: Portal der DEUTSCHEN WELLE www.dw-world.de vom 4. Juni 2007.

»Renovated Cinema to bring new life to Jenin.« In: Homepage von BBC News am 28. Januar 20I0, siehe:

http://news.bbc.co.uk/2/hi/middle_east/8480852.stm (Zugriff: 24.II.20I7).

»Türkische Fernsehserien. Eine kulturelle Revolution.«In: Frankfurter Allgemeine Zeitung vom 20. November 20I2, siehe:

http://www.faz.net/aktuell/feuilleton/medien/tuerkische-fernsehserien -eine-kulturelle-revolution-II963627.html (Zugriff: 24-II.20I7). 


\section{Publikationen und Arbeitsmaterialien des Goethe-Instituts und anderer Kulturinstitutionen}

Akademie der Künste: Perspektive Europa. I. - 2. Juni 2007. Eine Veranstaltung der Akademie der Künste in Zusammenarbeit mit dem Auswärtigen Amt. Programmheft. Berlin 2007.

Bartmann, Christoph, Carola Dürr \& Hans-Georg Knopp (Hrsg.): Wiedervorlage: Nationalkultur. Variationen über ein neuralgisches Thema. Dokumentation der Tagung. Göttingen 20Iо; mit den zitierten Beiträgen von: Christoph Bartmann \& Hans-Georg Knopp, S. 7-ı; Carola Dürr, S. II-I6; Klaus-Dieter Lehmann, S. 27-33; und Frank-Walter Steinmeier, S. I7-25.

Bernasconi, Sara \& Elena Messner: Re/Visions. Digitale Anthologie zu dem Projekt »Zukunftswerkstatt« des Goethe-Instituts; siehe: http://www.kakanien-revisited.at/beitr/re_visions/SBernasconiı.pdf (Zugriff: 24.II.20I7).

Deutsch-Französisches Kulturzentrum in Ramallah: Manara. Monatszeitung. Ausgaben vom August 2008 - Januar 2009.

Goethe-Institut: Rechenschaftsbericht 2009/20Iо. München 2010.

Goethe-Institut \& Staatliche Museen zu Berlin: Wiedervorlage: Nationalkultur. 24. - 26. April 2008 auf der Museumsinsel Berlin. Programmablauf. Berlin 2008.

Goethe-Institut Bosnien und Herzegowina \& Yaman d.o.o.: Bentbasa. Sevdalinka - Bosanska ljubavna pjesma / Bosnische Liebeslieder / Bosnian Love Songs. CD. Sarajevo 2006.

Goethe-Institut Bosnien und Herzegowina: Mini-INPUT Bosnia and Herzegovina. February 20-22, 2009 at the Goethe-Institute in Sarajevo. Best of INPUT 2008, Joburg, Programmheft. Sarajevo 2009.

Goethe-Institut Bosnien und Herzegowina: Mini-INPUT Bosnia and Herzegovina. November I9-2I, 2009 at the Goethe-Institute in Sarajevo. Best of INPUT 2009, Warsaw, Programmheft. Sarajevo 2009.

Goethe-Institut Bosnien und Herzegowina: Mini-INPUT Bosnia and Herzegovina. November $18-20,2010$ at the Goethe-Institute in Sarajevo. Best of INPUT 20I0, Budapest, Programmheft. Sarajevo 2010.

Goethe-Institut Bosnien und Herzegowina: Mini-INPUT Bosnia and Herzegovina. November I7-I9, 20II, Art House Cinema Kriterion Sarajevo. Best of INPUT 2oII, Seoul, Programmheft. Sarajevo 2011.

Goethe-Institut Bosnien und Herzegowina, Österreichische Botschaft \& Schweizer Botschaft: Sprach(en)politik in Bosnien und Herzegowina und im deutschsprachigen Raum. Sammelband zur gleichnamigen Konferenz vom 22. März 20II in Sarajevo. Sarajevo 20II.

Goethe-Institut Palestine: Press Release September 8, 2008. Palestinian-German TV Serial »Matabb«« (Speed Bump) cancelled by Palestinian Broadcasting Corporation (PBC). 
Goethe-Institut Ramallah: »Matabb«, zehnteilige Fernsehserie. Produzent: Fareed Majari; Regisseur: George Khleifi. Io DVDs. Ramallah 2008. Die Episoden sind online verfügbar unter: http://www.goethe.de/ins/ps/ram/ prj/mat/enindex.htm (Zugriff: 24.II.20I7).

Goethe-Institut Ramallah \& Shashat: Confession. Seven short videos. Produzenten: Fareed Majari \& Alia Arasoughly. DVD. Ramallah 2008.

Institut für Auslandsbeziehungen: Zeitschrift für Kulturaustausch; Schwerpunktthema: »Mit Kultur gegen Krisen. Kulturdialog als Mittel der Konfliktprävention«, 5I, 2 (200I).

\section{Regierungsdokumente, politische Papiere und offizielle Berichte}

Annan, Kofi: Facing the humanitarian challenge: towards a culture of prevention. New York I999.

Annan, Kofi: Prevention of armed conflict. United Nations. New York 200I (= S/200I/574), siehe: http://www.securitycouncilreport.org/atf/cf/\%7B65BFCF9B-6D27-4E9C-8CD3-CF6E4FF96FF9\%7D/CPR\%20S\%202001\%20 574.pdf (Zugriff: 24.II.20I7).

Auswärtiges Amt: Leitsätze für die auswärtige Kulturpolitik. Bonn I970; siehe: http://www.ifa.de/fileadmin/pdf/aa/akbp_leitsaetzer974.pdf (Zugriff: 24.II.20I7).

Auswärtiges Amt: Wo die Musik spielt. Kultur und Kommunikation in der deutschen Außenpolitik. Berlin (ohne Jahr).

Auswärtiges Amt - Kulturabteilung: Auswärtige Kulturpolitik - Konzeption 2000. Berlin 2000, siehe: http://www.ifa.de/fileadmin/pdf/aa/akbp_konzeption2000.pdf (Zugriff: 24.II. 20I7).

Auswärtiges Amt (Hrsg.): 5. Bericht der Bundesregierung zur Auswärtigen Kulturpolitik 2000. Berlin 200I, siehe: http://www.ifa.de/fileadmin/pdf/ aa/akbp_bericht2000.pdf (Zugriff: 24.II.20I7).

Auswärtiges Amt (Hrsg.): 6. Bericht der Bundesregierung zur Auswärtigen Kulturpolitik 200I. Berlin 2002, siehe: http://www.ifa.de/fileadmin/pdf/ aa/akbp_bericht200I.pdf (Zugriff: 24.II.20I7).

Auswärtiges Amt (Hrsg.): Bericht der Bundesregierung zur Auswärtigen Kulturpolitik 2002. Berlin 2003, siehe: http://www.ifa.de/fileadmin/pdf/aa/ akbp_bericht2002.pdf (Zugriff: 24.II.20I7).

Auswärtiges Amt (Hrsg.): Bericht der Bundesregierung zur Auswärtigen Kulturpolitik 2003. Berlin 2004, siehe: http:/www.ifa.de/fileadmin/pdf/aa/ akbp_bericht2003.pdf (Zugriff: 24.II.20I7).

Auswärtiges Amt (Hrsg.): Bericht der Bundesregierung zur Auswärtigen Kulturpolitik 2004. Berlin 2005, siehe: http://www.ifa.de/fileadmin/pdf/aa/ akbp_bericht2004.pdf (Zugriff: 24.II.20I7). 
Auswärtiges Amt (Hrsg.): Bericht der Bundesregierung zur Auswärtigen Kulturpolitik 2005/2006. Berlin 2006, siehe: http://www.ifa.de/fileadmin/ pdf/aa/akbp_bericht2005-06.pdf (Zugriff: 24.II.20I7).

Auswärtiges Amt (Hrsg.): Dialogue with the Islamic World / Dialog mit der islamischen Welt. Berlin 2006; siehe: http:/www.ifa.de/fileadmin/pdf/aa/ islamdialog2005.pdf (Zugriff: 24.II.20I7).

Auswärtiges Amt (Hrsg.): Menschen bewegen - Kultur und Bildung in der deutschen Außenpolitik, Berlin 25. - 26. Oktober 2006, Konferenzdokumentation. Berlin 2006.

Auwärtiges Amt: »Der Dialog der Kulturen: eine Herausforderung für die deutsche Außenpolitik« - Rede von Bundesaußenminister Steinmeier auf Einladung der EKD am 2I.II.2006. Berlin 2006; siehe: https://www.auswaertiges-amt.de/de/Newsroom/o6II2 I-bischhofsdinner/2I9502 (Zugriff: 24.II.2OI7).

Auswärtiges Amt (Hrsg.): Grußwort von Bundesaußenminister Steinmeier anlässlich der Veranstaltung »Perspektive Europa« in der Akademie der Künste zu Berlin. Berlin I. Juni 2007.

Auswärtiges Amt (Hrsg.): Bericht der Bundesregierung zur Auswärtigen Kulturpolitik 2006/2007. Berlin 2007, siehe: http://www.ifa.de/fileadmin/ pdf/aa/akbp_bericht2006-07.pdf (Zugriff: 24.II.20I7).

Auswärtiges Amt (Hrsg.): Menschen bewegen - Kulturpolitik im Zeitalter der Globalisierung, Berlin, I8.-26. April 2009. Dokumentation der Konferenz, des Kulturabends und der Tage der Auswärtigen Kulturpolitik. Berlin 2009.

Auswärtiges Amt (Hrsg.): Bericht der Bundesregierung zur Auswärtigen Kulturpolitik 2008/2009. Berlin 20I0, siehe: http://www.ifa.de/fileadmin/ pdf/aa/akbp_bericht2008-09.pdf (Zugriff: 24.II.20I7).

Auswärtiges Amt (Hrsg.): Bericht der Bundesregierung zur Auswärtigen Kultur- und Bildungspolitik 2009/20Io. Berlin 20II, siehe: http://www.ifa.de/ fileadmin/pdf/aa/akbp_bericht2009-Io.pdf (Zugriff: 24.II.20I7).

Boutros-Ghali, Boutros: An Agenda for Peace. Preventive diplomacy, peacemaking and peace-keeping. United Nations, New York I992 (= S/24III), siehe: http://www.securitycouncilreport.org/atf/cf/\%7B65BFCF9B-6D27-4E9C8CD3-CF6E4FF96FF9\%7D/Disarm\%20S24III.pdf Zugriff: 24.II.2OI7).

Boutros-Ghali, Boutros: Supplement to an Agenda for Peace. United Nations, New York I995 (= Si995/I).

Deutscher Bundestag (Hrsg.): Bericht der Enquete-Kommission Auswärtige Kulturpolitik gemäß Beschluß des Deutschen Bundestages vom 23. Februar I973, Drucksache 7/4I2I. Bonn 1975; siehe: http://dip2r.bundestag.de/ dip2I/btd/07/04I/0704I2I.pdf (Zugriff: 24.II.20I7).

Deutscher Bundestag (Hrsg.): Stellungnahme der Bundesregierung zu dem Bericht der Enquete-Kommission »Auswärtige Kulturpolitik« des Deut- 
schen Bundestages. Drucksache 8/927. Bonn 1977; siehe: http://dip2r.bundestag.de/dip2I/btd/08/009/0800927.pdf (Zugriff: 24.II.20I7).

Deutscher Bundestag (Hrsg.): Antwort der Bundesregierung auf die Große Anfrage der Abgeordneten Dr. Uschi Eid, Marieluise Beck (Bremen), Birgitt Bender, weiterer Abgeordneter und der Fraktion Bündnis 90/Die Grünen, Drucksache I6/4024. Berlin 2007; online einsehbar unter: http://dip2I. bundestag.de/dip2I/btd/I6/040/1604024.pdf (Zugriff: 24.II.20I7).

\section{LiteratuR}

Adam, Jens: »Kaum noch normale Berliner«. Stadtethnologische Erkundungen in einem »sozialen Problemquartier«. Münster 2005.

Adam, Jens \& Asta Vonderau (Hrsg.): Formationen des Politischen. Anthropologie politischer Felder. Bielefeld 20I4.

Adam, Jens \& Asta Vonderau: Formationen des Politischen. Überlegungen zu einer Anthropologie politischer Felder. In: Dies. (Hrsg.): Formationen des Politischen. Anthropologie politischer Felder. Bielefeld 20I4, S. 7-32.

Allen, Lori: The Rise and Fall of Human Rights. Cynicism and Politics in Occupied Palestine. Stanford 20I3.

Anderson, Benedict: Die Erfindung der Nation. Zur Karriere eines folgengreichen Konzepts. Berlin I988.

Andrić, Ivo: Buffet Titanic. Erzählungen. München 2000.

Andrić, Ivo: Das Fräulein. Frankfurt 2003.

Appadurai, Arjun (Hrsg.): The Social Life of Things: Commodities in Cultural Perspective. Cambridge, New York 1986.

Appadurai, Arjun: Modernity at Large. Cultural Dimensions of Globalization. Minneapolis 1996.

Appadurai, Arjun: Die Geographie des Zorns. Frankfurt 2009.

Arrow, Kenneth J.: The economics of agency. In: John W. Pratt \& Richard J. Zeckhauser (Hrsg.): The structure of business. Boston I99I (I985), S. 37-5I. Barsamian, David \& Edward W. Said: Culture and Resistance. Conversations with Edward W. Said. Cambridge 2003.

Barth, Fredrik: Introduction. In: Ders. (Hrsg.): Ethnic Groups and Boundaries. The Social Organization of Culture Difference. Boston, Oslo et al. I969, S. 9-38.

Bauman, Zygmunt: After the Nation State - What? In: Ders.: Globalization.

The Human Consequences. Cambridge 1998, S. 55-76.

Beck, Ulrich: Was ist Globalisierung? Frankfurt 1998.

Beck, Ulrich: Der kosmopolitische Blick oder Krieg ist Frieden. Frankfurt 2004. 
Beck, Ulrich \& Natan Sznaider: Unpacking Cosmopolitanism for the Social Sciences: A Research Agenda. In: British Journal of Sociology 57,I (2006), S. I-23.

Beck, Ulrich: Die Neuvermessung der Ungleichheit unter den Menschen: Soziologische Aufklärung im 2I. Jahrhundert. Frankfurt 2008.

Binder, Beate: 50. Wiederkehr des Kriegsendes: Zur Konstruktion der Gedächtnislandschaft Berlin. In: Beate Binder, Wolfgang Kaschuba \& Peter Niedermüller (Hrsg.): Inszenierungen des Nationalen. Geschichte, Kultur und die Politik der Identitäten am Ende des 20. Jahrhunderts. Köln, Weimar, Wien 200I, S. 285-308.

Binder, Beate: Streitfall Stadtmitte. Der Berliner Schlossplatz. Köln, Weimar, Wien 2009.

Binder, Beate: Troubling policies. Gender und queertheoretische Interventionen in die Anthropology of Policy. In: Jens Adam \& Asta Vonderau (Hrsg.): Formationen des Politischen. Anthropologie politischer Felder. Bielefeld 2014 , S. 363-386.

Binder, Beate, Wolfgang Kaschuba \& Peter Niedermüller (Hrsg.): Inszenierungen des Nationalen. Geschichte, Kultur und die Politik der Identitäten am Ende des 20. Jahrhunderts. Köln, Weimar, Wien 200 .

Binder, Beate, Peter Niedermüller \& Wolfgang Kaschuba: Inszenierungen des Nationalen - einige einleitende Bemerkungen. In: Beate Binder, Wolfgang Kaschuba \& Peter Niedermüller (Hrsg.): Inszenierungen des Nationalen. Geschichte, Kultur und die Politik der Identitäten am Ende des 20. Jahrhunderts. Köln, Weimar, Wien 200I, S. 7-I5.

Borneman, John \& Nick Fowler: Europeanization. In: Annual Review of Anthropology 26, (I997), S. 487-5I4.

Bougarel, Xavier, Elissa Helms \& Ger Duijzings: Introduction. In: Dies. (Hrsg.): The New Bosnian Mosaic. Identities, Memories and Moral Claims in a PostWar Society. Aldershot 2007, S. I-35.

Bourdieu, Pierre: Von der Regel zu den Strategien. In: Ders.: Rede und Antwort. Frankfurt i992, S. 79-98.

Bourdieu, Pierre: Sozialer Raum und symbolische Macht. In: Ders.: Rede und Antwort. Frankfurt I992, S. I35-I54.

Bourdieu, Pierre: Ortseffekte. In: Pierre Bourdieu et al.: Das Elend der Welt. Zeugnisse und Diagnosen alltäglichen Leidens an der Gesellschaft. Konstanz 1997, S. 159-167.

Bourdieu, Pierre: Das politische Feld. Zur Kritik der politischen Vernunft. Konstanz 200I.

Bourdieu, Pierre: Was heißt sprechen? Zur Ökonomie des sprachlichen Tausches. Wien 2005 (I. Auflage r990).

Bourdieu, Pierre: Die Praxis der reflexiven Anthropologie. Einleitung zum Seminar an der École des hautes études en sciences sociales, Paris, Oktober 
I987. In: Pierre Bourdieu \& Loïc J. D. Wacquant: Reflexive Anthropologie. Frankfurt 2006, S. 25I-294.

Bourdieu, Pierre: Das Haus oder die verkehrte Welt. In: Ders.: Entwurf einer Theorie der Praxis auf der ethnologischen Grundlage der kabylischen Gesellschaft. Frankfurt 2009 (I. Auflage I979), S. 48-65.

Bourdieu, Pierre \& Loic Wacquant: Die Ziele der reflexiven Soziologie. Chicago-Seminar, Winter 1987. In: Dies.: Reflexive Anthropologie. Frankfurt 2006, S. 95-249.

Bourgois, Philippe: Recognizing Invisible Violence: A Thirty-Year Ethnographic Retrospective. In: Barbara Rylko-Bauer, Linda Whiteford \& Paul Farmer (Hrsg.): Global Health in Times of Violence. Santa Fe 2009, S. I8-40.

Brubaker, Rogers, Margit Feischmidt, Jon Fox \& Liana Grancea: Nationalist Politics and Everyday Ethnicity in a Transylvanian Town. Princeton, Oxford 2006.

Calic, Marie-Janine: Der Stabilitätspakt für Südosteuropa. Eine erste Bilanz. In: Aus Politik und Zeitgeschichte Bi3-I4 (200I), S. 9-16; siehe: http://www. bpb.de/apuz/26356/der-stabilitaetspakt-fuer-suedosteuropa?p=all.(Zugriff: 23.II.20I7).

Chakrabarty, Dipesh: Europa provinzialisieren. Postkolonialität und die Kritik der Geschichte. In: Sebastian Conrad \& Shalini Randeria (Hrsg.): Jenseits des Eurozentrismus. Postkoloniale Perspektiven in den Geschichts- und Kulturwissenschaften. Frankfurt, New York 2002, S. 283-312.

Coles, Kimberley: Democratic Designs. International Intervention and Electoral Practices in Postwar Bosnia-Herzegovina. Ann Arbor 2007.

Coles, Kimberley: Ambivalent Builders: Europeanization, the Production of Difference and Internationals in Bosnia-Herzegovina, in: Xavier Bougarel, Elissa Helms \& Ger Duijzings (Hrsg.): The New Bosnian Mosaic. Identities, Memories and Moral Claims in a Post-War Society. Aldershot 2007, S. 255-272.

Collier, Stephen J. \& Aihwa Ong: Global Assemblages, Anthropological Problems. In: Aihwa Ong \& Stephen J. Collier (Hrsg.): Global Assemblages. Technology, Politics, and Ethics as Anthropological Problems. Malden, Oxford, Carlton 2005, S. 3-2I.

Conrad, Sebastian \& Shalini Randeria: Einleitung. Geteilte Geschichten - Europa in einer postkolonialen Welt. In: Dies. (Hrsg.): Jenseits des Eurozentrismus. Postkoloniale Perspektiven in den Geschichts- und Kulturwissenschaften. Frankfurt, New York 2002, S. 9-49.

Czarniawska, Barbara: A City reframed. Managing Warsaw in the I99os. Amsterdam 2000.

Delpla, Isabelle: In the Midst of Injustice: The ICTY from the Perspective of some Victim Associations. In: Xavier Bougarel, Elissa Helms \& Ger Duijzings (Hrsg.): The New Bosnian Mosaic. Identities, Memories and Moral Claims in a Post-War Society. Aldershot 2007, S. 2II-234. 
Dreyfus, Hubert \& Paul Rabinow: Michel Foucault. Jenseits von Strukturalismus und Hermeneutik. Weinheim i994.

Düwell, Kurt: Zwischen Propaganda und Friedensarbeit - Ioo Jahre Geschichte der deutschen Auswärtigen Kulturpolitik. In: Kurt-Jürgen Maaß (Hrsg.): Kultur und Außenpolitik. Handbuch für Studium und Praxis. Baden-Baden 2009 (2. vollständig überarbeitete und erweiterte Auflage), S. 6I-III.

Dunn, Elizabeth Cullen: The Chaos of Humanitarian Aid. Adhocracy in the Republic of Georgia. In: Humanity, 3,I (Spring 20I2), S. I-23.

Ernst, Marcel: Der deutsche »Dialog mit der islamischen Welt«. Diskurse deutscher Auswärtiger Kultur- und Bildungspolitik im Maghreb. Bielefeld 20I4.

Fabian, Johannes: Time and the Other: How Anthropology Makes Its Object. New York i983.

Farías, Ignacio: Die Improvisation einer Politik. Katastrophenbewältigung, neoliberale Experimente und die Grenzen ökonomischen Wissens. In: Jens Adam \& Asta Vonderau (Hrsg.): Formationen des Politischen. Anthropologie politischer Felder. Bielefeld 20I4, S. I53-I8I.

Farmer, Paul: An Anthropology of Structural Violence. In: Current Anthropology, 45, 3 (June 2004), S. 305-325.

Fassin, Didier \& Mariella Pandolfi (Hrsg.): Contemporary States of Emergency. The Politics of Military and Humanitarian Interventions. New York 2013.

Faßler, Manfred: Globalisierung und Geosozialität. In: Herbert Willems (Hrsg.): Lehr(er)buch Soziologie. Für die pädagogischen und soziologischen Studiengänge. Wiesbaden 2008, S. 455-485.

Feischmidt, Margit: Symbolische Kämpfe der Nationalisierung. Die Auseinandersetzung um ein Nationaldenkmal im multiethnischen Ort Cluj. In: Beate Binder, Wolfgang Kaschuba \& Peter Niedermüller (Hrsg.): Inszenierungen des Nationalen. Geschichte, Kultur und die Politik der Identitäten am Ende des 20. Jahrhunderts. Köln, Weimar, Wien 200I, S. 263-284.

Feldman, Gregory: Illuminating the Apparatus: Steps toward a Nonlocal Ethnography of Global Governance. In: Cris Shore, Susan Wright \& Davide Però (Hrsg.): Policy Worlds. Anthropology and the Analysis of Contemporary Power. Oxford 20II, S. 32-49.

Feldman, Gregory: The Migration Apparatus. Security, Labor, and Policymaking in the European Union. Stanford 2012.

Foucault, Michel: Archäologie des Wissens. Frankfurt I98I.

Foucault, Michel: Für eine Kritik der politischen Vernunft. In: Lettre International, I (I988), S. 58-66.

Foucault, Michel: »Le jeu de Michel Foucault« In: Ders.: Dits et écrits II, Paris I994, S. 298-329.

Gellner, Ernest: Nationalismus und Moderne. Berlin I99I.

Girard, Monique \& David Stark: Heterarchies of Value: Distributing Intelligence and Organizing Diversity in a New Media Startup. In: Aihwa Ong \& Stephen 
J. Collier (Hrsg.): Global Assemblages: Technology, Politics, and Ethics as Anthropological Problems. Malden, Oxford, Carlton 2005, S. 293-319.

Götz, Irene: Deutsche Identitäten. Die Wiederentdeckung des Nationalen nach I989. Köln, Weimar, Wien 20II.

Habermas, Jürgen: Jenseits des Nationalstaates? Bemerkungen zu Folgeproblemen der wirtschaftlichen Globalisierung. In: Ulrich Beck (Hrsg.): Politik der Globalisierung. Frankfurt 1998, S. 67-84.

Hall, Stuart: Der Westen und der Rest. Diskurs und Macht. In: Ders.: Rassismus und kulturelle Identität (= Ausgewählte Schriften 2). Hamburg I994, S. 137-I79.

Hall, Stuart: Die Frage der kulturellen Identität. In: Ders.: Rassismus und kulturelle Identität (= Ausgewählte Schriften 2). Hamburg 1994, S. I80-222.

Hannerz, Ulf: Cosmopolitans and Locals in World Culture. In: Mike Featherstone (Hrsg.): Global Culture. Nationalism, Globalization and Modernity. London I990, S. 237-25I.

Hannerz, Ulf: Transnational Connections. Culture, people, places. London, New York 1996.

Hannerz, Ulf: Foreign News. Exploring the World of Foreign Correspondents. Chicago 2004.

Harvey, David: The Condition of Post-Modernity. Oxford I989.

Harvey, Penelope: Hybrids of Modernity. Anthropology, the nation state and the universal exhibition. London, New York 1996.

Hobsbawm, Eric: Nationen und Nationalismus. Mythos und Realität seit I780. München 1996.

Holmes, Douglas R. \& George E. Marcus: Cultures of Expertise and the Management of Globalization: Toward the Re-Functioning of Ethnography. In: Aihwa Ong \& Stephen J. Collier (Hrsg.): Global Assemblages: Technology, Politics, and Ethics as Anthropological Problems. Malden, Oxford, Carlton 2005 , S. 235-252.

Hyatt, Susan Brin: Poverty in a >post-welfare< landscape: Tenant management policies, self-governance and the democratization of knowledge in Great Britain. In: Cris Shore \& Susan Wright (Hrsg.): Anthropology of Policy. Critical perspectives on governance and power. London, New York I997, S. $217-238$.

Jansen, Stef: Remembering with a Difference: Clashing Memories of Bosnian Conflict in Everyday Life. In: Xavier Bougarel, Elissa Helms \& Ger Duijzings (Hrsg.): The New Bosnian Mosaic. Identities, Memories and Moral Claims in a Post-War Society. Aldershot 2007, S. 193-208.

Jansen, Stef: Yearnings in the Meantime. >Normal Lives< and the State in a Sarajevo Apartment Complex. London, New York 20I5.

Jordan, Glen \& Chris Weedon: Cultural Politics: Class, Gender, Race and the Postmodern World. Oxford 1995. 
Kaldor, Mary: Neue und alte Kriege: Organisierte Gewalt im Zeitalter der Globalisierung. Frankfurt 2000.

Karahasan, Dževad: Tagebuch der Aussiedlung. Klagenfurt I993.

Kaschuba, Wolfgang: Geschichtspolitik und Identitätspolitik. Nationale und ethnische Diskurse im Kulturvergleich. In: Beate Binder, Wolfgang Kaschuba \& Peter Niedermüller (Hrsg.): Inszenierungen des Nationalen. Geschichte, Kultur und die Politik der Identitäten am Ende des 20. Jahrhunderts. Köln, Weimar, Wien 2001, S. 19-42.

Kaschuba, Wolfgang: Europäisierung als kulturalistisches Projekt? Ethnologische Beobachtungen. In: Hans Joas \& Friedrich Jaeger (Hrsg.): Europa im Spiegel der Kulturwissenschaften. Baden-Baden 2008, S. 204-225.

Kolind, Torsten: In Search of >Decent People<: Resistance to the Ethnicization of Everyday Life among the Muslims of Stolac. In: Xavier Bougarel, Elissa Helms \& Ger Duijzings (Hrsg.): The New Bosnian Mosaic. Identities, Memories and Moral Claims in a Post-War Society. Aldershot 2007, S. I23-I38.

Latour, Bruno: Science in action. How to follow scientists and engineers through society. Cambridge i987.

Latour, Bruno: Aramis or the Love of Technology. Cambridge, MA I996.

Latour, Bruno: Reassembling the Social. An Introduction to Actor-Network-Theory. Oxford 2005 .

Lemke, Thomas, Susanne Krasmann \& Ulrich Bröckling: Gouvernementalität, Neoliberalismus und Selbsttechnologien. Eine Einleitung. In: Ulrich Bröckling, Susanne Krasmann \& Thomas Lemke (Hrsg.): Gouvernementalität der Gegenwart. Studien zur Ökonomisierung des Sozialen. Frankfurt 2000, S. 7-40.

Löfgren, Orvar: Moving Metaphors. In: Per Olof Berg, Anders Linde-Laursen \& Orvar Löfgren (Hrsg.): Invoking a Transnational Metropolis: The Making of the Oresund Region. Lund 2000, S. 27-53.

Lowenhaupt Tsing, Anna: Friction. An Ethnography of Global Connection. Princeton, Oxford 2005.

Lubkemann, Stephen C.: Culture in Chaos. An Anthropology of the Social Condition in War. Chicago, London 2008.

Maaß, Kurt-Jürgen: Überblick: Ziele und Instrumente der Auswärtigen Kulturpolitik. In: Ders. (Hrsg.): Kultur und Außenpolitik. Handbuch für Studium und Praxis. Baden-Baden 2009 (2. vollständig überarbeitete und erweiterte Auflage), S. 25-32.

Maček, Ivana: >Imitation of Life $<$ Negotiating Normality in Sarajevo under Siege. In: Xavier Bougarel, Elissa Helms \& Ger Duijzings (Hrsg.): The New Bosnian Mosaic. Identities, Memories and Moral Claims in a Post-War Society. Aldershot 2007, S. 39-57.

Maček, Ivana: Sarajevo under Siege. Anthropology in Wartime. Philadelphia 2009. 
Macey, Eva: The cultural politics of populism: Celebrating Canadian national identity. In: Cris Shore \& Susan Wright (Hrsg.): Anthropology of Policy. Critical perspectives on governance and power. London, New York I997, S. I36-I64.

Malcom, Noel: Bosnia. A Short History. London, Oxford 2002.

Mandel, Ruth: Seeding civil society. In: Chris Hann (Hrsg.): Postsocialism. Ideals, ideologies and practices in Eurasia. London, New York 2002, S. 279-296.

Marcus, George: Ethnography in/of the World System. The Emergence of multi-sited Ethnography. Zuerst erschienen in: Annual Review of Anthropology 24 (I995).

Mbembe, Achille: Necropolitics. In: Public Culture, I5, I (2003), S. II-40.

Mbembe, Achille: Kritik der schwarzen Vernunft. Frankfurt 2014.

Moore, Sally Falk: Comparisons: Possible and Impossible. In: The Annual Review of Anthropology, 34 (2005), S. 2-II.

Mosse, David: Politics and Ethics: Ethnographies of Expert Knowledge and Professional Identities. In: Cris Shore, Susan Wright \& Davide Però (Hrsg.): Policy Worlds. Anthropology and the Analysis of Contemporary Power. Oxford 20II, S. 50-67.

Müller, Birgit: The Elephant in the Room: Multistakeholder Dialogue on Agricultural Biotechnology in the Food and Agriculture Organization. In: Cris Shore, Susan Wright \& Davide Però (Hrsg.): Policy Worlds. Anthropology and the Analysis of Contemporary Power. Oxford 20II, S. 282-299.

Münkler, Herfried: Die neuen Kriege. Reinbek bei Hamburg 2002.

Nassehi, Armin: Das stahlharte Gehäuse der Zugehörigkeit. Unschärfen im Diskurs um die »multikulturelle Gesellschaft«. In: Ders. (Hrsg.): Nation, Ethnie, Minderheit. Beiträge zur Aktualität ethnischer Konflikte. Köln, Weimar, Wien I997, S. I77-208.

Niedermüller, Peter: Diskurs, Kultur, Politik. Zur Herausbildung der Nationalkultur in Ungarn. In: Beate Binder, Wolfgang Kaschuba \& Peter Niedermüller (Hrsg.): Inszenierungen des Nationalen. Geschichte, Kultur und die Politik der Identitäten am Ende des 20. Jahrhunderts. Köln, Weimar, Wien 2001, S. I67-I85.

Nielsen, Gritt B.: Peopling Policy: On Conflicting Subjectivities of Fee-Paying Students. In: Cris Shore, Susan Wright \& Davide Però (Hrsg.): Policy Worlds. Anthropology and the Analysis of Contemporary Power. Oxford 20II, S. 68-85.

Nordstrom, Carolyn: A Different Kind of War Story. Philadelphia I997.

Nordstrom, Carolyn: Shadows of War. Violence, Power, and International Profiteering in the twenty-first Century. Berkeley, Los Angeles, London 2004.

Nordstrom, Carolyn: Global Outlaws. Crime, Money, and Power in the Contemporary World. Berkeley, Los Angeles, London 2007.

Ong, Aihwa: Flexible Staatsbürgerschaften. Die kulturelle Logik von Transnationalität. Frankfurt 2005 . 
Ong, Aihwa \& Stephen J. Collier (Hrsg.): Global Assemblages. Technology, Politics, and Ethics as Anthropological Problems. Malden, Oxford, Carlton 2005. Osterhammel, Jürgen: Die Verwandlung der Welt. Eine Geschichte des I9. Jahrhunderts. München 20II (Sonderausgabe, Erstausgabe 2009).

Pandolfi, Mariella: From Paradox to Paradigm. The Permanent State of Emergency in the Balkans. In: Didier Fassin \& Mariella Pandolfi (Hrsg.): Contemporary States of Emergency. The Politics of Military and Humanitarian Interventions. New York 2013, S. I53-I72.

Però, Davide: Migrants' Practices of Citizenship and Policy Change. In: Cris Shore, Susan Wright \& Davide Però (Hrsg.): Policy Worlds. Anthropology and the Analysis of Contemporary Power. Oxford 20II, S. 244-263.

Rabinow, Paul: Anthropologie der Vernunft. Studien zu Wissenschaft und Lebensführung. Frankfurt I994.

Randeria, Shalini: Geteilte Geschichten und verwobene Modernen. In: Jörn Rüsen, Hanna Leitgeb \& Norbert Jegelka (Hrsg.): Zukunftsentwürfe: Ideen für eine Kultur der Veränderung. Frankfurt, New York I999, S. 87-96.

Randeria, Shalini \& Ciara Grunder: The (Un)Making of Policy in the Shadow of the World Bank: Infrastructure Development, Urban Resettlement and the Cunning State in India. In: Cris Shore, Susan Wright \& Davide Però (Hrsg.): Policy Worlds. Anthropology and the Analysis of Contemporary Power. Oxford 20II, S. I87-204.

Robben, Antonius C. G. M. \& Carolyn Nordstrom: Introduction: The Anthropology and Ethnography of Violence and Sociopolitical Conflict. In: Carolyn Nordstrom \& Antonius C. G. M. Robben (Hrsg.): Fieldwork under Fire. Contempory Studies of Violence and Survival. Berkeley, Los Angeles, London I995, S. I-23.

Römhild, Regina: Alte Träume, neue Praktiken: Migration und Kosmopolitismus an den Grenzen Europas. In: Transit Migration Forschungsgruppe (Hrsg.): Turbulente Ränder. Neue Perspektiven auf Migration an den Grenzen Europas. Bielefeld 2007, S. 2II-222.

Römhild, Regina: Aus der Perspektive der Migration. Die Kosmopolitisierung Europas. In: Sabine Hess, Jana Binder \& Johannes Moser (Hrsg.): No integration?! Kulturwissenschaftliche Beiträge zur Integrationsdebatte in Europa. Bielefeld 2009, S. 225-238.

Römhild, Regina: Transnationale Migration und soziokulturelle Transformation: Die Kosmopolitisierung der Gesellschaft; online veröffentlicht im Jahr 20II unter: https://heimatkunde.boell.de/20II/05/I8/transnationale-migration-und-soziokulturelle-transformation-die-kosmopolitisierung-der (Zugriff: 24.II.2OI7).

Römhild, Regina \& Michael Westrich: Kosmopolitismus an der Grenze. Der Mittelmeerraum als Laboratorium für transversalen Gemeinsinn. In: Ka- 
thrin Audehm \& Iris Clemens (Hrsg.): GemeinSinn (= Zeitschrift für Kulturwissenschaften 2/2013), S. 85-98.

Rottenburg, Richard: Weit hergeholte Fakten. Eine Parabel der Entwicklungshilfe. Stuttgart 2002.

Said, Edward W.: Orientalism. New York 1978.

Said, Edward W.: Culture and Imperialism. New York 1994.

Said, Edward W.: From Oslo to Iraq and the Roadmap. London 2004.

Sampson, Steven: The Social Life of Projects: Importing Civil Society to Albania. In: Chris Hann \& Elizabeth Dunn (Hrsg.): Civil Society. Challenging Western Models. London I996, S. I2I-I42.

Sampson, Steven: Beyond transition: rethinking elite configurations in the Balkans. In: Chris Hann (Hrsg): Postsocialism. Ideals, ideologies and practices in Eurasia. London, New York 2002, S. 297-316.

Sampson, Steven: Weak States, Uncivil Societies and Thousands of NGOs Western Democracy Export as Benevolent Colonialism in the Balkans; veröffentlicht im Jahr 2002 auf: http://www.anthrobase.com/Txt/S/Sampson_S_oI. htm (Zugriff Juni 20I5).

Sassen, Saskia: Das Paradox des Nationalen. Territorium, Autorität und Rechte im globalen Zeitalter. Frankfurt 2008.

Sassen, Saskia: The global inside the national. A research agenda for sociology. In: Sociopedia.isa 20IO,I, S. 3-Io.

Scheper-Hughes, Nancy \& Philippe Bourgois: Introduction: Making Sense of Violence. In: Dies. (Hrsg.): Violence in War and Peace. An Anthology. Malden, Oxford, Carlton 2004, S. I-3I.

Schneider, Wolfgang: Vom Export zum Netzwerk, vom Event zur Intervention. Zum Wandel Auswärtiger Kulturpolitik. In: Ders. (Hrsg.): Auswärtige Kulturpolitik. Dialog als Auftrag - Partnerschaft als Prinzip. Bonn 2008, S. I3-3I.

Schwenzer, Victoria: Bei Bohne. Einblicke in ein dörfliches Industriearbeitermilieu. In: Institut für Europäische Ethnologie der Humboldt Universität zu Berlin \& Sorbisches Institut Bautzen (Hrsg.): Skizzen aus der Lausitz. Region und Lebenswelt im Umbruch. Köln, Weimar, Wien I997, S. 269-292.

Segal, Daniel A. \& Richard Handler: How European is nationalism? In: Social Analysis, 32 (Dezember 1992), S. I-I5.

Shore, Cris: Building Europe. The Cultural Politics of European Integration. London, New York 2000.

Shore, Cris \& Susan Wright (Hrsg.): Anthropology of Policy. Critical perspectives on governance and power. London, New York 1997.

Shore, Cris \& Susan Wright: Policy: A new field of anthropology. In: Dies. (Hrsg.): Anthropology of Policy. Critical perspectives on governance and power. London, New York I997, S. 3-40.

Shore, Cris, Susan Wright \& Davide Però (Hrsg.): Policy Worlds. Anthropology and the Analysis of Contemporary Power. New York, Oxford 20II. 
Shore, Cris \& Susan Wright: Introduction. Conceptualising Policy: Technologies of Governance and the Politics of Visibility. In: Cris Shore, Susan Wright \& Davide Però (Hrsg.): Policy Worlds. Anthropology and the Analysis of Contemporary Power. New York, Oxford 20II, S. I-25.

Taussig, Michael: Colonialism, Shamanism, and the Wild Man. Chicago I987. Todorova, Maria: Die Erfindung des Balkans. Europas bequemes Vorurteil. Darmstadt I999.

Trouillot, Michel-Rolph: The Anthropology of the State in the Age of Globalization. Close Encounters of the Deceptive Kind. In: Current Anthropology, 43, I (February 200I), S. I25-I38.

Vertovec, Steven \& Robin Cohen (Hrsg.): Conceiving Cosmopolitanism. Theory, Context, and Practice. Oxford 2002.

Vonderau, Asta: Leben im »neuen Europa«. Konsum, Lebensstile und Körpertechniken im Postsozialismus. Bielefeld 2 oro.

Weber, Eugen: Peasants into Frenchmen. The Modernization of Rural France, I870-I9I4. London I977.

Wedel, Janine: Collision and Collusion. The Strange Case of Western Aid to Eastern Europe. New York 200I.

Weizman, Eyal: Sperrzonen. Israels Architektur der Besatzung. Hamburg 2008.

Wellgraf, Stefan: Das Ende der Hauptschule in Berlin. Zur ideologischen Dimension von Bildungsmythen. In: Jens Adam \& Asta Vonderau (Hrsg.): Formationen des Politischen. Anthropologie politischer Felder. Bielefeld 2014, S. 35-65.

Welz, Gisela: »Sighting / Siting globalization.« Gegenstandskonstruktion und Feldbegriff einer ethnographischen Globalisierungsforschung. In: Sonja Windmüller, Beate Binder \& Thomas Hengartner (Hrsg.): Kultur - Forschung: Zum Profil einer volkskundlichen Kulturwissenschaft. Münster u.a. 2009, S. I95-210.

Welz, Gisela \& Annina Lottermann (Hrsg.): Projekte der Europäisierung. Kulturanthropologische Forschungsperspektiven. Frankfurt 2009.

Welz, Gisela \& Annina Lottermann: Einleitung. In: Dies. (Hrsg.): Projekte der Europäisierung. Kulturanthropologische Forschungsperspektiven. Frankfurt 2009, S. II-I6.

Wright, Susan \& Sue Reinhold: >Studying throughく: A Strategy for Studying Political Transformation. Or Sex, Lies and British Politics. In: Cris Shore, Susan Wright \& Davide Però (Hrsg.): Policy Worlds. Anthropology and the Analysis of Contemporary Power. Oxford 20II, S. 86-IO4.

Zinn, Dorothy Louise: The Case of Scanzano: Raison d'État and the Reasons for a Rebellion. In: Cris Shore, Susan Wright \& Davide Però (Hrsg.): Policy Worlds. Anthropology and the Analysis of Contemporary Power. Oxford 2OII, S. 227-243. 



\section{Danksagung}

Diese Studie entstand als Dissertation am InStitut FÜr EuropäISChe EthnoLOGIE an der Philosophischen Fakultät der Hum Boldt-Universität ZU BerLin. Sie wurde dort durch Prof. Dr. Wolfgang Kaschuba und Prof. Dr. Beate Binder betreut. Mein wissenschaftliches Interesse an der Auswärtigen Kulturpolitik hat sich in meiner Zeit als Stipendiat der Robert Bosch Stiftung im Rahmen des Programms Robert Bosch Kulturmanager in Mittel- und Osteuropa herausgebildet. Die wichtigste empirische Grundlage dieser Untersuchung stellten zwei ethnografische Feldforschungen an den Goethe-Instituten in Ramallah und Sarajevo dar. Die Förderung durch das Studienwerk der HeinRICH-BöLL-STIFTUNG bot den unverzichtbaren finanziellen Rahmen für ihre Realisierung. Ein weiterer Feldaufenthalt in Sarajevo wurde durch ein Stipendium des Schroubek Fonds Östliches Europa ermöglicht. Große Teile des Textes konnte ich als Mitarbeiter am Leibniz-Zentrum Moderner Orient verfassen. Die fertiggestellte Dissertation wurde im Jahr 2016 mit dem IFA-FoRSCHUngSPReis Auswärtige Kulturpolitik ausgezeichnet.

All diesen Institutionen und den dort tätigen Personen danke ich herzlich für die Ermöglichung und Unterstützung dieser Arbeit, den kontinuierlichen Austausch sowie die geduldige Begleitung. 


\section{Politikwissenschaft}

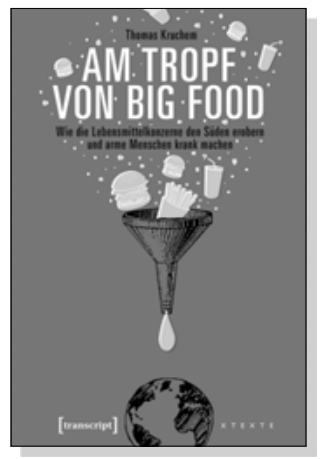

Thomas Kruchem

Am Tropf von Big Food

Wie die Lebensmittelkonzerne den Süden erobern und arme Menschen krank machen

Juni 2017, 214 S., kart., zahlr. Abb.

$19,99 €(D E), 978-3-8376-3965-0$

E-Book

PDF: $16,99 €(D E)$, ISBN 978-3-8394-3965-4

EPUB: $16,99 €(D E)$, ISBN 978-3-7328-3965-0

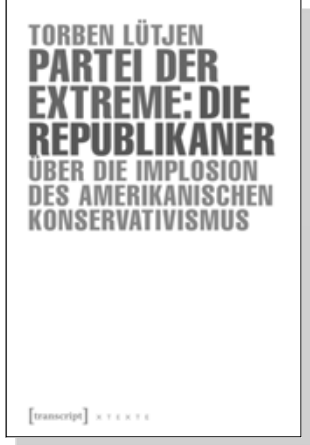

Torben Lütjen

Partei der Extreme: Die Republikaner

Über die Implosion des amerikanischen Konservativismus

2016, 148 S., kart.

$14,99 €(D E), 978-3-8376-3609-3$

E-Book

PDF: $12,99 €$ (DE), ISBN 978-3-8394-3609-7

EPUB: $12,99 €(D E)$, ISBN 978-3-7328-3609-3

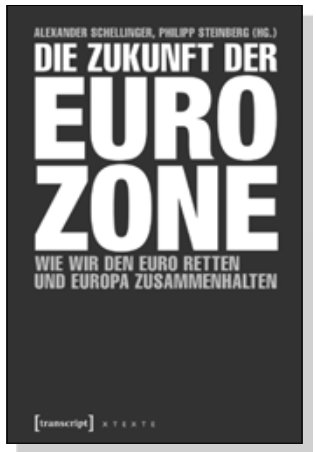

Alexander Schellinger, Philipp Steinberg (Hg.)

\section{Die Zukunft der Eurozone}

Wie wir den Euro retten und Europa zusammenhalten

2016, 222 S., kart.

$19,99 €(D E), 978-3-8376-3636-9$

E-Book

PDF: $17,99 €(D E)$, ISBN 978-3-8394-3636-3

EPUB: $17,99 €(D E)$, ISBN 978-3-7328-3636-9 


\section{Politikwissenschaft}

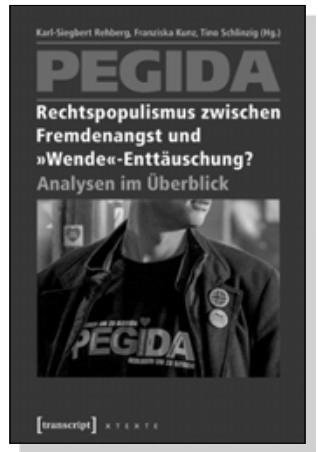

Karl-Siegbert Rehberg, Franziska Kunz, Tino Schlinzig (Hg.)

PEGIDA - Rechtspopulismus zwischen

Fremdenangst und "Wende"-Enttäuschung?

Analysen im Überblick

2016, 384 S., kart.

$29,99 €(D E), 978-3-8376-3658-1$

E-Book

PDF: $26,99 €$ (DE), ISBN 978-3-8394-3658-5

EPUB: $26,99 €(D E)$, ISBN 978-3-7328-3658-1

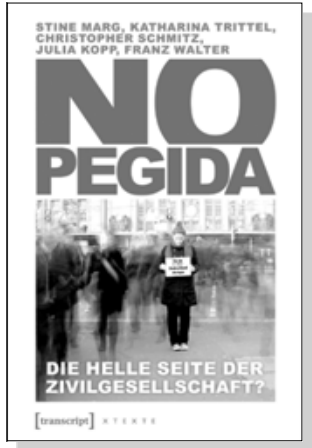

Stine Marg, Katharina Trittel,

Christopher Schmitz, Julia Kopp, Franz Walter

\section{NoPegida}

Die helle Seite der Zivilgesellschaft?

2016, 168 S., kart.

$19,99 €(D E), 978-3-8376-3506-5$

E-Book

PDF: $17,99 €$ (DE), ISBN 978-3-8394-3506-9

EPUB: 17,99€ (DE), ISBN 978-3-7328-3506-5

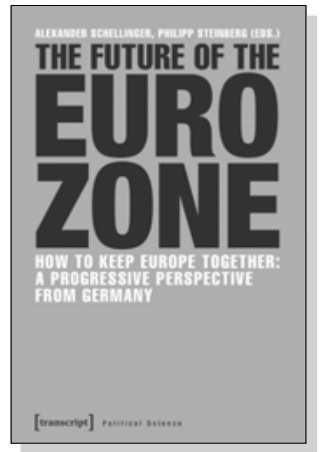

Alexander Schellinger, Philipp Steinberg (eds.)

The Future of the Eurozone

How to Keep Europe Together:

A Progressive Perspective from Germany

October 2017, 202 p., pb.

$29,99 €(D E), 978-3-8376-4081-6$

E-Book

PDF: $26,99 €$ (DE), ISBN 978-3-8394-4081-0

EPUB: $26,99 €(D E)$, ISBN 978-3-7328-4081-6 
